\title{
Systematic analysis of heterochromatin modification readout
}

\author{
Dissertation \\ to acquire the doctoral degree in mathematics and natural science \\ 'Doctor rerum naturalium' \\ at the Georg-August-Universität Göttingen \\ in the doctoral degree programme Biomolecules: Structure - Function - Dynamics \\ at the Georg-August University \\ School of Science (GAUSS) \\ Submitted by \\ Nadin Zimmermann \\ born in \\ Berlin, Germany
}

Göttingen, 2016 


\section{Thesis Committee}

Dr. Wolfgang Fischle, Chromatin Biochemistry, Max Planck Institute for Biophysical Chemistry

Prof. Dr. Holger Stark, Dep. of Structural Dynamics, Max Planck Institute for Biophysical Chemistry

Prof. Dr. André Fischer, Dep. for Psychiatry and Psychotherapy, University Medical Center, German Center for Neurodegenerative Diseases

Members of the examination board:

Referee: Dr. Wolfgang Fischle, Chromatin Biochemistry, Max Planck Institute for Biophysical Chemistry

Co-referee: Prof. Dr. Holger Stark, Dep. of Structural Dynamics, Max Planck Institute for Biophysical Chemistry

Other members of the Examination Board:

Prof. Dr. André Fischer, Dep. for Psychiatry and Psychotherapy, University Medical Center, German Center for Neurodegenerative Diseases

Prof. Dr. Henning Urlaub, Research Group Mass Spectrometry, Max Planck Institute for Biophysical Chemistry

Prof. Dr. Detlef Doenecke, Dept. of Molecular Biology, University Medical Center Göttingen

Prof. Dr. Steven Johnsen, Clinic for General, Visceral and Pediatric Surgery, University Medical Center Göttingen

Date of the oral examination: $15^{\text {th }}$ June 2016 


\section{Affidavit}

I hereby declare that the presented thesis "Systematic analysis of heterochromatin modification readout" has been written independently and with no other sources and aids than quoted.

Göttingen, $10^{\text {th }}$ May 2016

Nadin Zimmermann 



\section{Acknowledgements}

First and foremost, I would like to express my gratitude to my mentors, Dr. Wolfgang Fischle and Prof. Dr. Henning Urlaub. Thank you for entrusting me this project, for your continuous support, guidance and your enthusiasm.

I am grateful to the members of my thesis committee - Prof. Dr. Holger Stark and Prof. Dr. André Fischer for their interest in the project, the helpful discussions and suggestions. I also thank Prof. Dr. Detlef Doenecke and Prof. Dr. Steven Johnsen for their time as part of my thesis examination committee.

I want to thank the GGNB for their generous financial support, informative lectures, highly supportive method courses as well as inspiring retreats.

My sincere thanks to all the present and past members of the Chromatin Biochemistry group for providing a great working atmosphere. I particularly thank my supervisor Miro sharing all his knowledge about the project, for all the difficult discussions, his support, his patience, and his trust. I am grateful to Alexandra for sharing her knowledge of protein biochemistry and for never running out of answers to all my questions. I thank Kyoko for all the fruitful discussions. I am especially grateful to Alexandra, Henriette und Maria for their friendship and the valuable hours spend outside of the lab.

I am very grateful to all the members of the Mass Spectrometry group for their support during the last four years. I want to thank Henning and Christof, for their support, the many fruitful cigarettediscussions, for Esprit, barbeques and many more. I also thank Uwe, Moni and Annika for their help and the introduction to the mass spectrometers.

I am thankful to Thomas Conrad for providing and culturing HeLa cells for me.

I thank the people from the climbing gym for giving me good reasons to leave the lab and the opportunity to clear my head. I also want to thank all my friends supporting me from far away over all the years.

I am very thankful to Cyrille for his support, the helpful discussions, his patience and all the chocolate he provided the last months. 
I owe my gratitude to my family. In particular I want to thank my parents and my sister for constantly supporting and encouraging me to go all the way and for cheering me up during difficult times. 


\section{Table of contents}

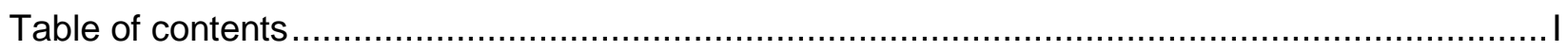

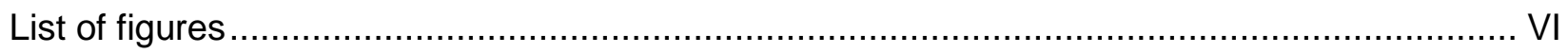

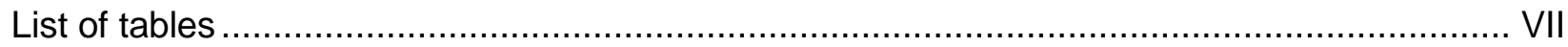

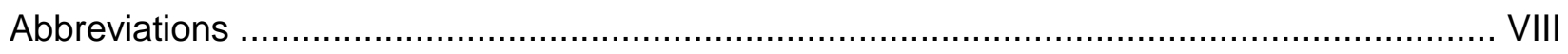

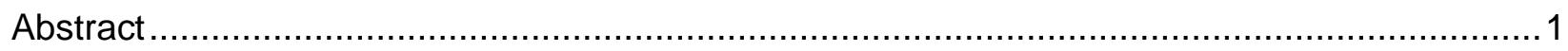

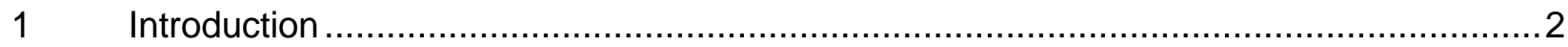

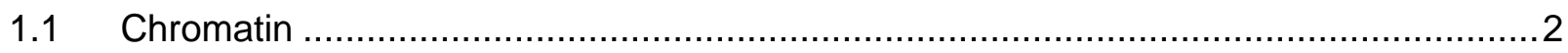

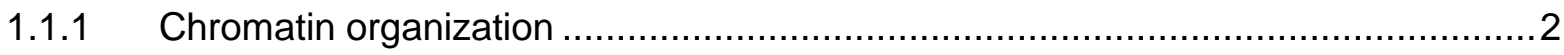

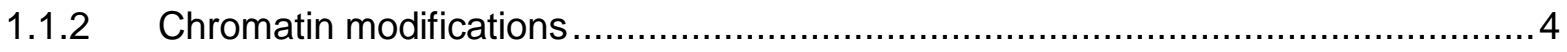

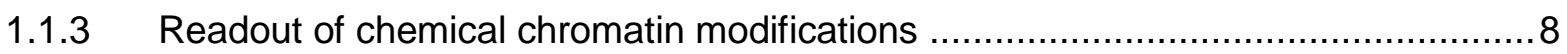

1.1.4 Multivalent readout and crosstalk of chemical chromatin modifications ...............11

1.1.5 Chromatin arrays for systematic analysis of chemical modification readout..........13

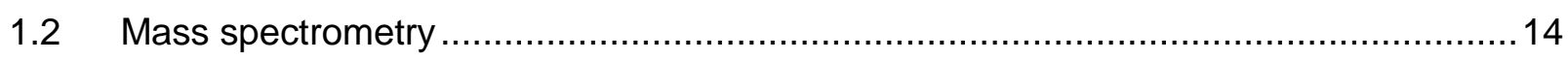

1.2.1 Mass spectrometry based protein identification of complex samples ....................14

1.2.2 Relative quantification by Stable Isotope Labeling by Amino acids in Cell culture

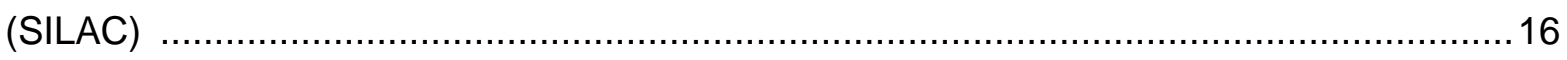

1.2.3 Protein-protein cross-linking mass spectrometry ……....................................17

$1.3 \quad H y p o t h e s i s$ and objectives of the presented thesis ..................................................19

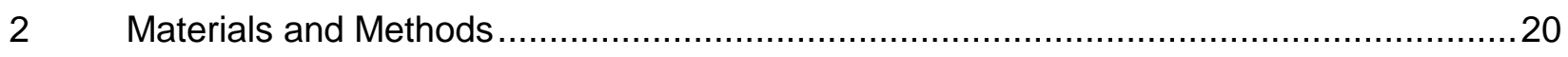

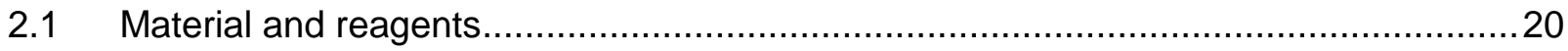

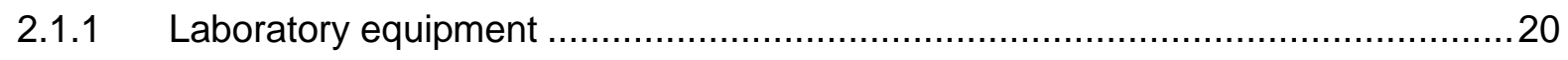

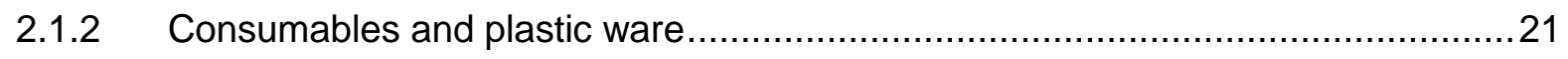

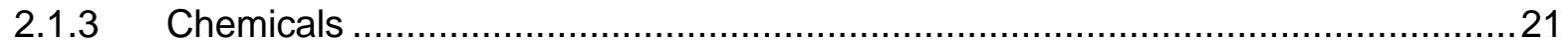

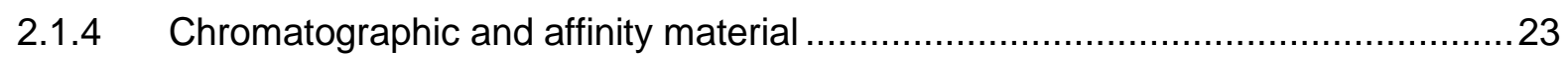

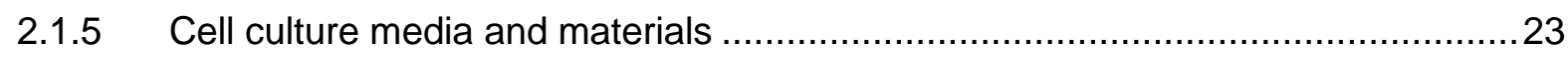

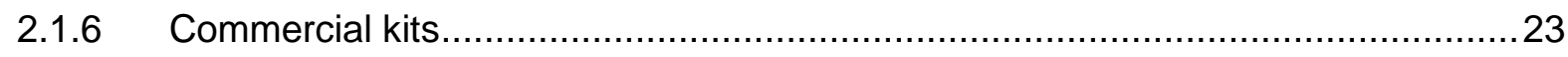

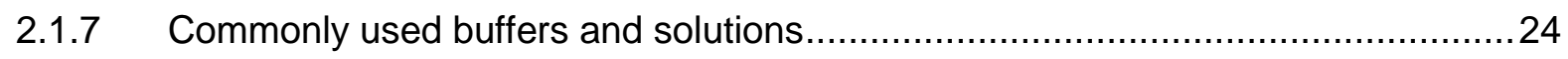




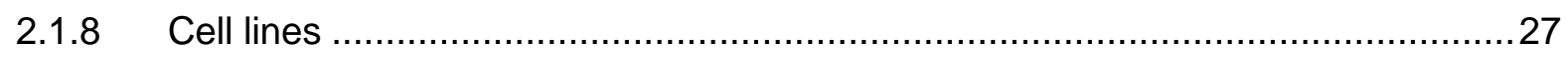

2.1.9 Chemically competent Escherichia coli strains ...........................................28

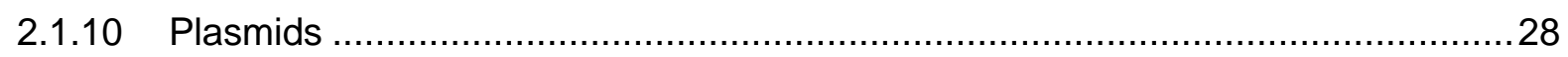

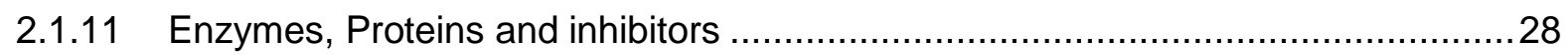

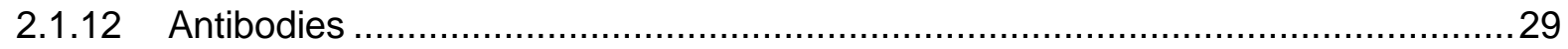

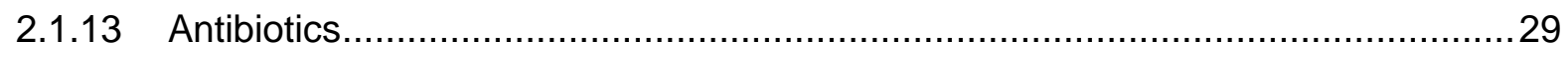

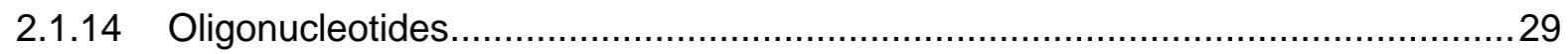

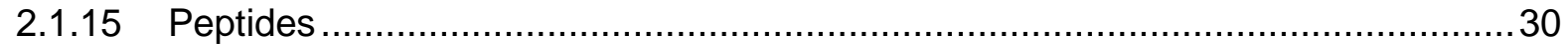

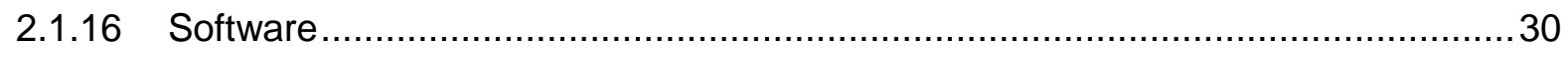

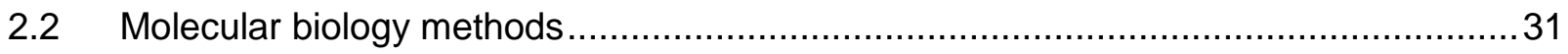

2.2.1 Transformation of chemically competent bacteria.............................................

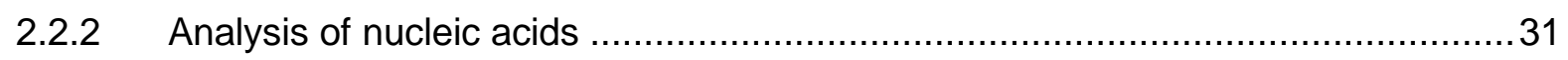

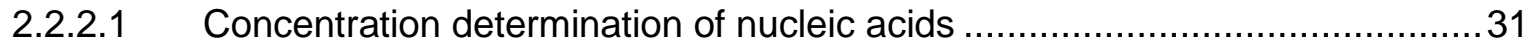

2.2.2.2 Nucleic acid gel electrophoresis ...............................................................

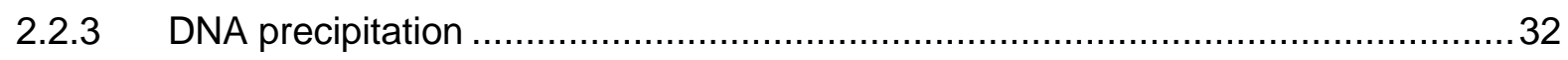

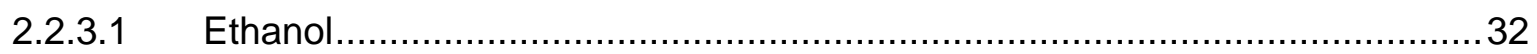

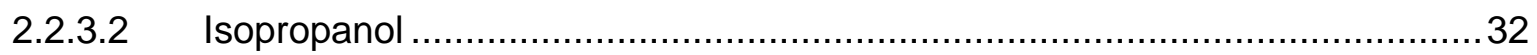

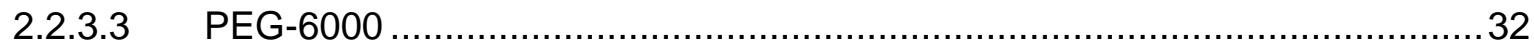

2.2.4 Preparation of DNA templates for chromatin array assembly .............................32

2.2.4.1 Preparation of biotinylated 12×200-601 DNA template ................................32

2.2.4.2 Methylation of biotinylated 12x200-601DNA template .................................33

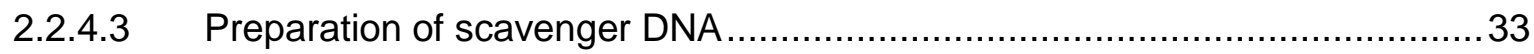

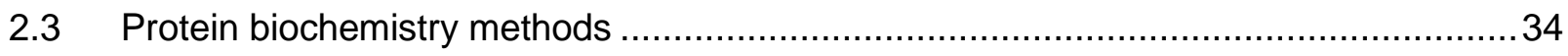

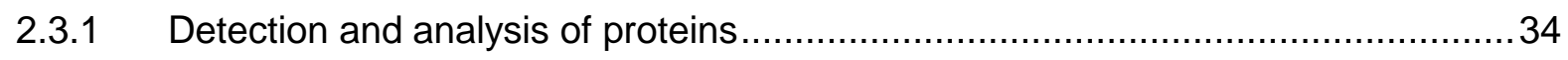

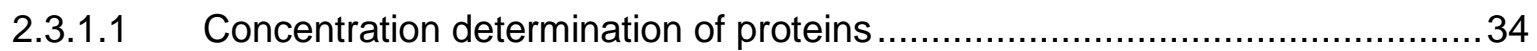

2.3.1.2 Sodium dodecyl sulfate polyacrylamide gel electrophoresis (SDS-PAGE).....35

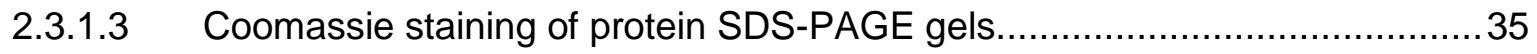

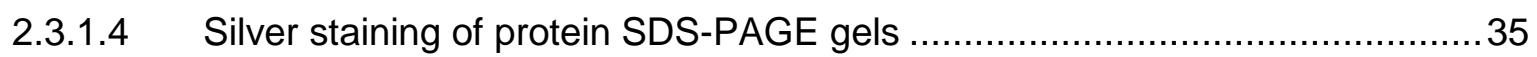

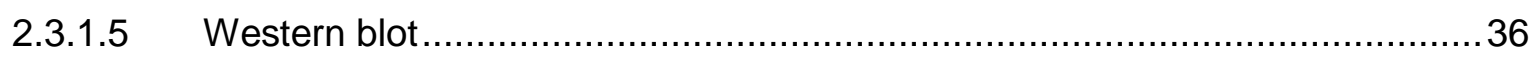

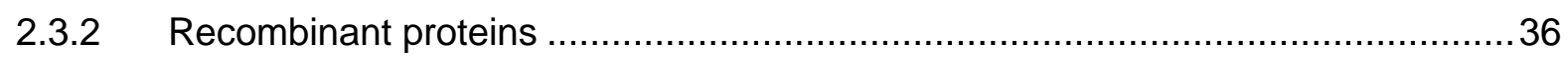

2.3.2.1 Expression and purification of recombinant histone proteins .........................36

2.3.2.2 Introduction of posttranslational modifications by native chemical ligation .....37 
2.3.2.3 Introduction of methyl lysine analogs 38

2.3.3 Preparation of recombinant chromatin. 38

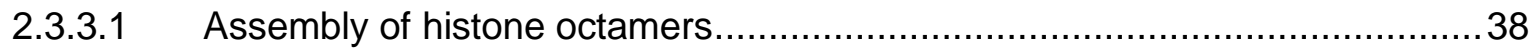

2.3.3.2 Chromatin reconstitution by salt dialysis ............................................... 39

2.3.4 Molecular characterization of recombinant chromatin ..................................... 39

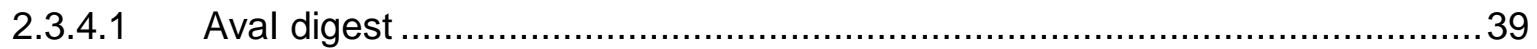

2.3.4.2 Analytical ultracentrifugation....................................................... 40

$2.4 \quad$ Cell culture and metabolic labeling ............................................................ 40

2.4.1 Stable isotope labeling by amino acids in cell culture (SILAC) of HeLa S3 cells ... 40

2.4.2 Preparation of nuclear extracts ............................................................. 40

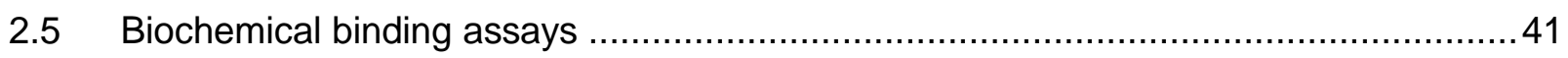

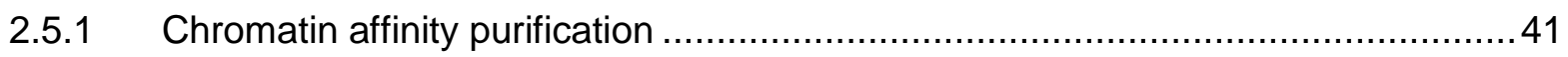

2.5.2 Protein-protein cross-linking on beads ........................................................ 42

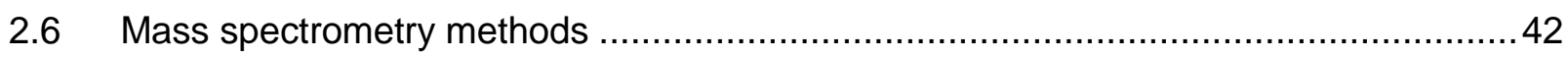

2.6.1 In-gel proteolysis of proteins and peptide extraction ...................................42

2.6.2 In-solution proteolysis of proteins …........................................................ 43

2.6.3 LC-MS/MS analysis of peptides on LTQ-Orbitrap .....................................43

2.6.4 LC-MS/MS analysis of peptides on QExactive.......................................... 44

2.6.5 Molecular weight determination of histone proteins .................................... 45

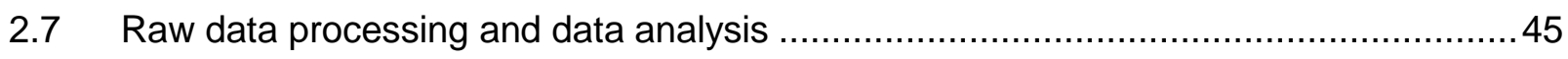

2.7.1 Mass spectrometric raw data processing with MaxQuant ............................... 45

2.7.2 Mass spectrometric raw data processing with pLink .....................................45

2.7.3 Data filtering and visualization of quantified MS data................................... 46

2.7.4 Protein-protein interaction analysis ................................................... 47

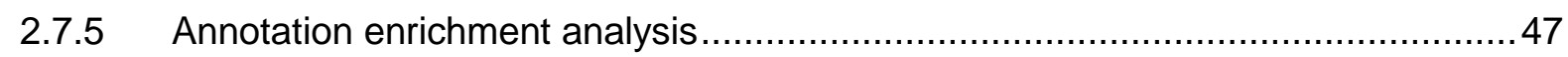

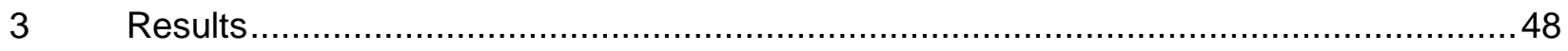

3.1 12mer nucleosomal arrays are of reproducible quality independent of incorporated modifications.

3.1.1 Native chemical ligation and alkylation of cysteine residues successfully introduce PTMs to recombinant Xenopus laevis histones .48 
3.1.2 Octamer-DNA molar ratios from 1.1 to 1.3 assemble fully saturated, reproducible nucleosomal arrays .50

3.2 The statistical tool "significance A" determines significant protein fold enrichment cutoffs .50

3.3 ChAP coupled to quantitative MS enables the investigation of chromatin-protein interactomics and provides insights into chromatin modification crosstalk. .55

3.3.1 Individual chromatin modification states recruit specific chromatin-binding interactomes... 56

3.3.2 The methylation degrees of lysine residues affect protein binding to different extends . .72

3.3.3 Two chromatin modifications in trans do not only constitute the sum of their single counterparts but demonstrate an independent impact on chromatin-binding interactomes. 74

3.3.3.1 Chromatin arrays carrying a combination of H3K9me3 and H4K2Ome3 result

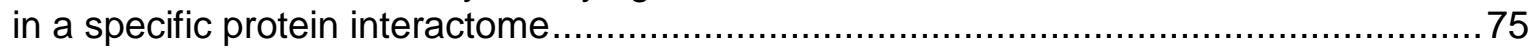

3.3.3.2 The double modification $\mathrm{H} 3 \mathrm{~K} 9 \mathrm{me} 3 \mid \mathrm{H} 4 \mathrm{~K} 20 \mathrm{me} 3$ indicates positive and negative crosstalk 76

3.3.3.3 Communication of histone and DNA modifications is indicated by positive and negative crosstalk 79

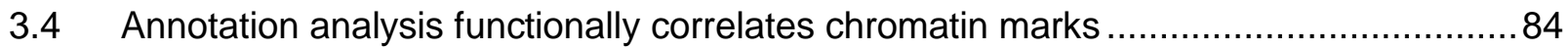

3.5 Protein-protein cross-linking of the chromatin-binding interactome ............................. 88

3.5.1 Protein-protein cross-linking on assembled chromatin arrays .............................. 88

3.5.2 Protein-protein cross-links of complex protein samples are specifically detected by the search algorithm pLink in combination with a reference database ..............................93

3.6 ChAP-MS coupled with $\mathrm{XL}$ provides information of the binding hierarchy beyond primary binding proteins recruited to chromatin. .95

4 Discussion 99

4.1 Individual chromatin-binding interactomes are comparable based on significant enrichment cutoffs 99

4.2 Interactomics of chromatin-binding proteins provide new insights into the functional impact of individual chromatin modifications 100

4.3 Protein-binding interactomes of modified chromatin arrays display only moderate overlap compared to those revealed with different modified templates 105

4.4 Individual heterochromatic marks display positive as well as negative functional correlation. 106 
4.5 Combinatorial readout in trans provides new insights into crosstalk between chemical

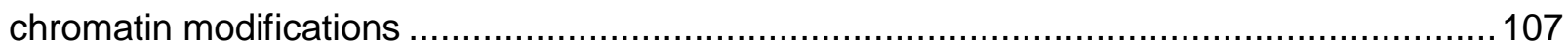

4.6 Protein-protein cross-linking coupled to MS maps the binding hierarchy of chromatin-

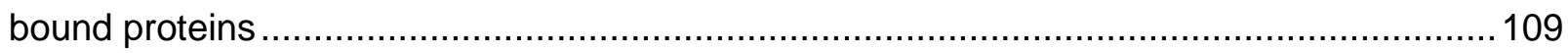

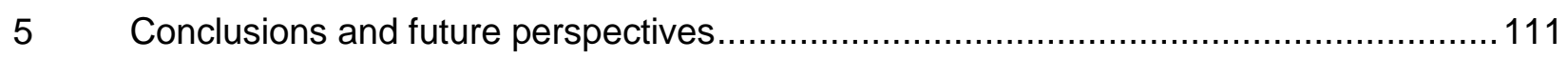

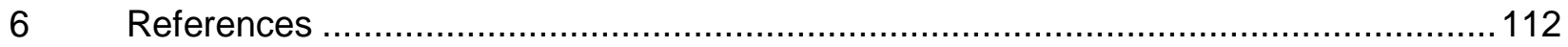

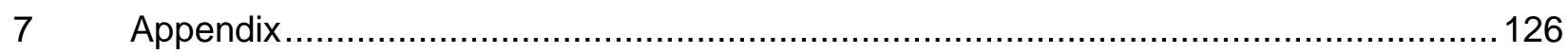

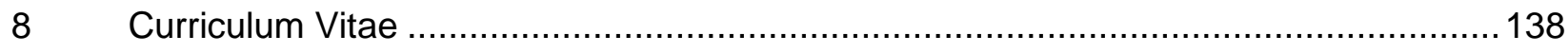




\section{List of figures}

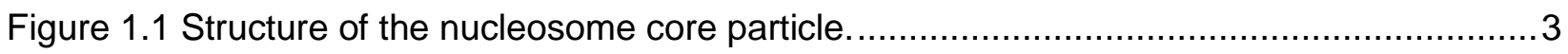

Figure 1.2 Combinatorial readout of posttranslational histone modifications............................12

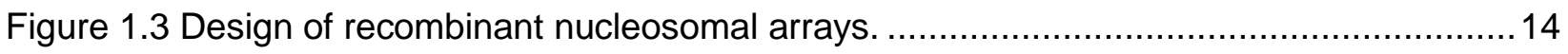

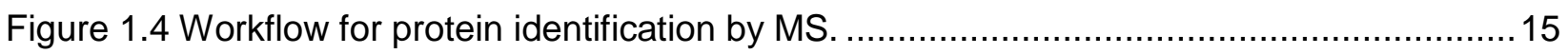

Figure 3.1 Introduction of posttranslational histone modifications. ........................................49

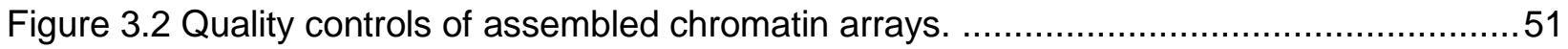

Figure 3.3 Workflow of SILAC-based chromatin affinity purification coupled with mass

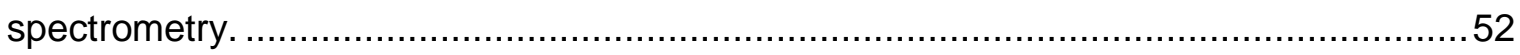

Figure 3.4 Determination and visualization of significantly regulated proteins using the example

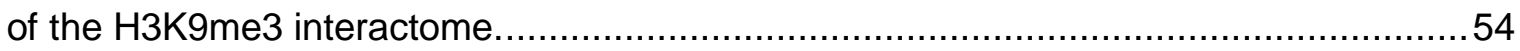

Figure 3.5 Protein-binding interactomes of H3K9me1 and H3K9me2 chromatin.......................57

Figure 3.6 Protein-binding interactome of H3K9me3-modified chromatin. ..............................62

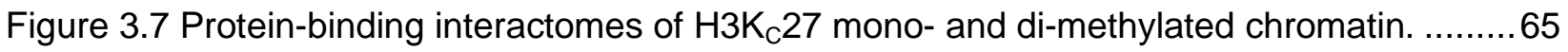

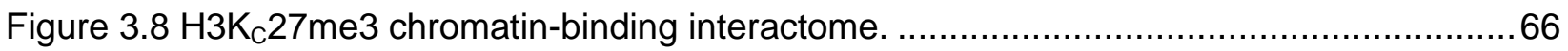

Figure 3.9 Chromatin-associated protein interactomes of histone H4K20 mono- and tri-

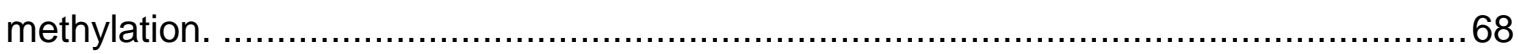

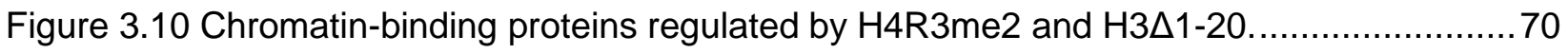

Figure 3.11 Protein-binding interactome of chromatin containing methylated DNA...................71

Figure 3.12 Overlap of proteins regulated by the methylation degrees of H3K9 ......................72

Figure 3.13 Overlap of significant proteins regulated by different methylation states. ................74

Figure 3.14 Protein-binding interactome of H3K9me3|H4K20me3-modified chromatin...............76

Figure 3.15 Comparison of significant protein binding to chromatin affected by $\mathrm{H} 3 \mathrm{~K} 9 \mathrm{me}$,

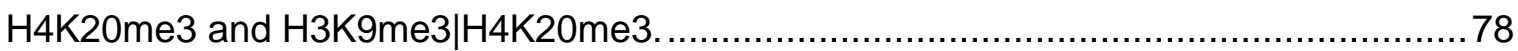

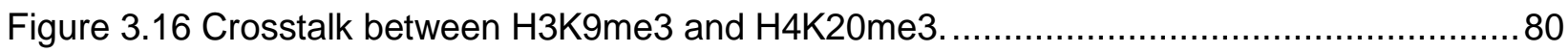

Figure 3.17 Chromatin-binding interactome of $\mathrm{H} 3 \mathrm{~K} 9 \mathrm{me} / \mathrm{meCpG}$ in comparison to the individual

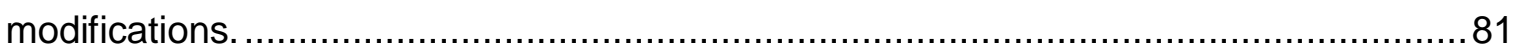

Figure 3.18 Crosstalk of H3K9me3 and CpG-methylated DNA ….................................... 83

Figure 3.19 Annotation enrichment analyses of biological pathways and GO terms of molecular

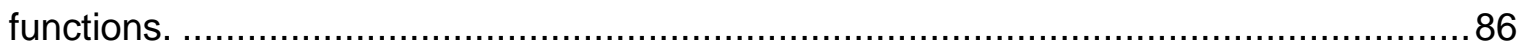

Figure 3.20 General workflow of protein-protein cross-link coupled with ChAP-MS. .................89

Figure 3.21 Quality control of reconstituted nucleosomal arrays.............................................90

Figure 3.22 Enrichment for protein-protein cross-links by size exclusion chromatography (SEC).

Figure 3.23 Chemical cross-links of peptides using the cross-linker BS3 ............................92

Figure 3.24 Protein-protein cross-links in the context of three-dimensional protein structures. ..94 
Figure 3.25 Protein-protein cross-link analysis of the core nucleosome................................96

Figure 3.26 Interaction network of cross-linked factors associated with chromatin. .98

\section{List of tables}

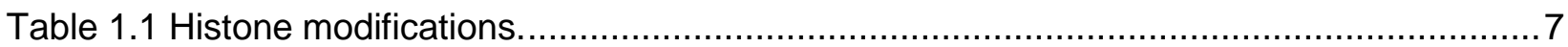

Table 2.1 Plasmids used for chromatin reconstitution and protein expression. .......................28

Table 2.2 Antibodies and antisera used for western blot analysis. ......................................29

Table 2.3 Peptides used for native chemical ligation. ......................................................... 30

Table 2.4 Values used for the determination of histone concentrations. ................................34

Table 3.1 Proteins recruited and excluded from binding to chemically modified chromatin. .......59 


\section{Abbreviations}

\begin{tabular}{|c|c|c|c|}
\hline aa & amino acid(s) & $\min$ & minute(s) \\
\hline $\mathrm{ACN}$ & acetonitrile & MLA & methyl lysine analog \\
\hline APS & ammonium peroxodisulfate & MPAA & 4-Mercaptophenylacetic acid \\
\hline ATP & adenosine triphosphate & MS & mass spectrometry \\
\hline bp & base pairs & MS/MS & tandem mass spectrometry \\
\hline BS3 & bis(sulfosuccinimidyl) suberate & Mw & molecular weight \\
\hline BSA & bovine serum albumin & MWC & molecular weight cut-off \\
\hline C & Celsius & NCL & native chemical ligation \\
\hline ChAP & chromatin affinity purification & NE & nuclear extract \\
\hline ChIP & chromatin immunoprecipitation & OD & optical density \\
\hline $\mathrm{Da}$ & Dalton $(\mathrm{g} / \mathrm{mol})$ & PAGE & polyacrylamide gel electrophoresis \\
\hline DDA & data-dependent acquisition & PBS & phosphate buffered saline \\
\hline $\mathrm{ddH} 2 \mathrm{O}$ & double distilled water (sterilized) & $\mathrm{PCl}$ & phenol:chloroform:isoamyl alcohol \\
\hline DMEM & Dulbecco's Modified Eagle's medium & PEG & polyethylene glycol \\
\hline DNA & deoxyribonucleic acid & PMSF & phenylmethylsulfonyl fluoride \\
\hline ds & double-stranded & ppm & parts per million \\
\hline DTT & dithiothreitol & PTM & posttranslational modification \\
\hline E.coli & Escherichia coli & RNA & ribonucleic acid \\
\hline e.g. & for example, exempli gratia & $\mathrm{RP}$ & reversed phase \\
\hline EDTA & ethylenediaminetetraacetic acid & rpm & revolutions per minute \\
\hline ESI & electrospray ionization & Rt & room temperature \\
\hline et al. & and others, et alii & s & second(s) \\
\hline FA & formic acid & SDS & sodium dodecyl sulfate \\
\hline FBS & fetal bovine serum & SEC & size exclusion chromatography \\
\hline FW HM & full width at half maximum & SILAC & $\begin{array}{l}\text { stable isotope labeling by amino acids } \\
\text { in cell culture }\end{array}$ \\
\hline g & gram or gravity force & TBE & tris/borate/EDTA \\
\hline $\mathrm{h}$ & hour & TEMED & $\mathrm{N}, \mathrm{N}, \mathrm{N}^{\prime}, \mathrm{N}^{\prime}$-tetramethylethylenediamine \\
\hline HEPES & $\begin{array}{l}\text { 4-(2-Hydroxyethyl)-1- } \\
\text { piperazineethanesulfonic acid }\end{array}$ & Tris & tris-(hydroxymethyl) aminomethane \\
\hline HPLC & high pressure liquid chromatography & TSS & transcription start site \\
\hline IAA & iodacetamide & UV & ultraviolet \\
\hline k & kilo & $\mathrm{V}$ & volt \\
\hline KDM & lysine demethylase & $\mathrm{v} / \mathrm{v}$ & volume per volume \\
\hline KMT & lysine methyltransferase & $w / v$ & weight per volume \\
\hline । & liter & WT & wild type \\
\hline LC & liquid chromatography & $X$. laevis & Xenopus laevis \\
\hline LTQ & linear trap quadropole & $\mathrm{XL}$ & cross-link \\
\hline $\mathrm{m}$ & milli or meter & $\alpha$ & anti-/antibody \\
\hline M & molar & $\Delta$ & delta (deletion) \\
\hline $\mathrm{m} / \mathrm{z}$ & mass-to-charge ratio & $\mu$ & micro \\
\hline
\end{tabular}





\section{Abstract}

Modifications of the building units of chromatin - the histone proteins and DNA - direct the functional readout of the genome and are part of the so called "epigenome" (Fischle et al., 2003b; Kouzarides, 2007). Several studies have shown that specialized proteins recognize these chemical modifications and thereby define the functional state of chromatin (Bartke et al., 2010; Nikolov et al., 2011; Vermeulen et al., 2010). Several individual chromatin-binding factors have been analyzed in detail. Also, proteomics approaches have taken inventory of factors interacting with single chromatin modifications. However, the full set of factors specific to particular modified chromatin domains, such as for example eu-and heterochromatin, is not known yet.

In this thesis, I report my findings from a systematic in vitro chromatin affinity purification approach in combination with quantitative mass spectrometry (Nikolov et al., 2011) that significantly expands the list of proteins regulated by heterochromatic modifications, both alone and in combination. The results provide a comprehensive catalogue of proteins specifically regulated in their chromatin binding by specific modification patterns. Moreover, the datasets highlight novel biological functions associated with individual modifications and reveal unpredicted functional relationships of the investigated chromatin modifications.

The analysis of the interactomes of combinatorially modified chromatin identifies for the first time proteins whose binding properties to chromatin depend on the cooperative action of two modifications. The results provide novel insights into the communication between two chromatin modifications, namely positive and negative crosstalk.

Furthermore, I introduce a new experimental workflow combining chromatin affinity purification and cross-linking mass spectrometry. This workflow maps physical protein-protein interactions sites of chromatin-bound proteins and provides first insights into the hierarchy of protein recruitment to chromatin.

Overall, my findings demonstrate that by using a template that mimics the native form of chromatin, which is not accessible by the widely used reductionist approaches, chromatin modifications can be analyzed in multiple dimensions. In combination with the new methods established here, my work will help to improve our understanding of epigenetic regulation of chromatin-associated processes. 


\section{Introduction}

Epigenetic is the scientific field, which investigates heritable changes in gene expression that do not result from changes of the underlying DNA sequence, in other words an alteration of the phenotype by retaining the genotype. The term was first introduced by $\mathrm{C}$. Waddington in the 1950s (Waddington, 1953) and referred in general to molecular processes affecting gene activity resulting in a particular phenotype. Over time, the definition of epigenetics narrowed and was defined as "An epigenetic trait is a stably heritable phenotype resulting from changes in a chromosome without alterations in the DNA sequence." in 2009 (Berger et al., 2009). Those changes of chromatin can occur at several levels. Currently considered in literature are DNA methylation, covalent posttranslational histone modifications, histone variants and non-coding RNA associated silencing. All these processes are thought to be involved in the establishment and maintenance of certain chromatin states that alter chromatin structure and consequently gene regulatory processes.

\subsection{Chromatin}

\subsubsection{Chromatin organization}

The eukaryotic genome is located in the cell nucleus in form of a highly compacted structure called chromatin, made of DNA and its associated molecules including proteins and RNA. In the cell, chromatin exists at several levels of compaction, each of them regulating the accessibility of the DNA template to the transcription machinery and gene expression factors.

The first level of chromatin compaction consists in the linear arrangement of repeating uniform units of DNA and histone octamers. Histone octamers consist of two copies of each of the four core histone proteins $\mathrm{H} 2 \mathrm{~A}, \mathrm{H} 2 \mathrm{~B}, \mathrm{H} 3$ and $\mathrm{H} 4$, around which 147 base pairs (bp) of a left-handed DNA superhelix are wrapped in a 1.65 turn (Luger et al., 1997). These uniform units represent the basic packaging unit of chromatin and are referred to as nucleosome core particles (NCPs) (Kornberg, 1974; Olins and Olins, 1974). Histones are 11-15 kDa in size and very basic proteins. They belong to the most conserved proteins among eukaryotes. All core histones exhibit a common structural feature called the histone fold motif comprising three $\alpha$-helices that are connected by two loops. These motifs mediate the protein-protein interactions between the histone pairs $\mathrm{H} 2 \mathrm{~A}-\mathrm{H} 2 \mathrm{~B}$ and $\mathrm{H} 3-\mathrm{H} 4$ as well as the interaction between each histone pair and the DNA wrapped around the nucleosome. In presence of DNA or high salt concentration a tetramer of two copies of $\mathrm{H} 3$ and $\mathrm{H} 4$ and two $\mathrm{H} 2 \mathrm{~A}-\mathrm{H} 2 \mathrm{~B}$ dimers self-assemble to a stable histone octamer (Luger et al., 1997). In contrast to the histone fold motif, the $\mathrm{N}$ - and C-terminal tails of histones 
are far less structured. They form flexible and accessible domains protruding out of the nucleosome core particle and mediate multiple interactions with the neighboring nucleosomes as well as with non-histone proteins.

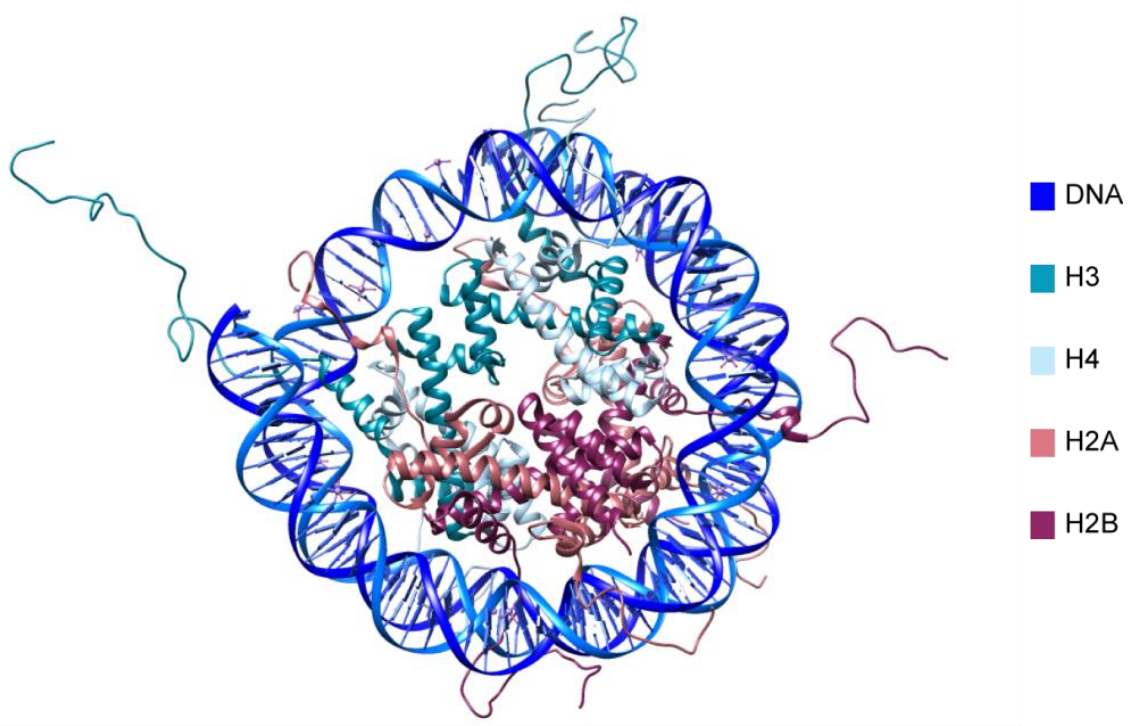

Figure 1.1 Structure of the nucleosome core particle.

Crystal structure at 1.9- $\AA$ (PDB_1KX5 (Davey et al., 2002)) visualized using Xlink Analyzer and Chimera (Kosinski et al., 2015; Pettersen et al., 2004). 147 bp of DNA (blue) is surrounding the histone octamer, which consists of two copies of each of the histones H2A (salmon), H2B (pink), H3 (turquois) and H4 (light blue).

The next level of chromatin organization is stabilized by nucleosome to nucleosome arrangements. Nucleosomes are formed by both the nucleosome core particles and a linker DNA varying in length from 10 to $70 \mathrm{bp}$. Under physiological salt conditions and the presence of divalent cations an array of nucleosomes folds into a helical fiber of approximately $30 \mathrm{~nm}$ diameter. However, the detailed structure and the in vivo relevance of the $30 \mathrm{~nm}$ fiber is controversial and still under discussion (Kruithof et al., 2009; Schalch et al., 2005; Tremethick, 2007). Chromatin organization on levels beyond the $30 \mathrm{~nm}$ fiber is even less understood. Higher order compaction forming tertiary chromatin structures presumably involves inter-array interactions e.g. looping of chromatin fibers.

A fifth histone type, histone $\mathrm{H} 1$, contributes to stabilize the $30 \mathrm{~nm}$ fiber and higher order structures of chromatin by interacting with both, the linker DNA and the nucleosome core particles in a 1:1 ratio (NCP:H1). Nevertheless, it was shown that nucleosomal arrays fold into compact states even in absence of H1 (McBryant and Hansen, 2012; Woodcock et al., 2006).

Chromatin structure is not only influenced by the presence of $\mathrm{H} 1$. Variance in length and sequence of nucleosomal repeats, the presence of different histone variants and the pattern of posttranslational modifications (PTMs) of histones and DNA influence the structural formation of chromatin. 
The structure of chromosomes can be generally distinguished in eu- and heterochromatin. The differentiation is ascribed to the heterogeneous staining intensities of chromosome areas observed with DNA staining dyes (Heitz, 1928). Heterochromatin represents chromosome regions formed by highly condensed chromatin and characterized by an intense DNA staining. These heterochromatic regions are thought to be distinctive for silenced gene loci (Dillon and Festenstein, 2002; Elgin and Grewal, 2003). Heterochromatin can be further distinguished into facultative and constitutive heterochromatin. Facultative heterochromatin mainly includes gene loci that are silenced in a time and tissue specific manner whereas constitutive heterochromatin is constituted by gene poor regions like repetitive satellite sequences, telomeres and centromeres. In contrast, euchromatin has a more relaxed structure. In consequence, staining by DNA specific dyes is less intense than the one observed for heterochromatin. The vast majority of transcriptionally active genes is present in euchromatin whose loose structure is thought to facilitate its accessibility for transcriptional factors. This explains the observations that constitutive heterochromatin remains condensed during the complete cell cycle while euchromatin undergoes decondensation during interphase.

The structural organization of chromatin is much more complex than the historical staining initially suggested and exists in many structural subtypes, which appeared to play key roles in the regulation of essential genomic functions like transcription, replication or DNA repair.

\subsubsection{Chromatin modifications}

PTMs of histones are one of the key mechanisms regulating chromatin structure and function. PTMs that occur on histones are biochemically diverse and include for instant serine/threonine/tyrosine phosphorylation, lysine acetylation, lysine and arginine methylation, lysine ubiquitination, lysine sumoylation, ADP-ribosylation, arginine citrullination and proline isomerization (Rothbart and Strahl, 2014). More than 150 different histone modification sites have been identified so far. The vast majority of modification sites are found within the $\mathrm{N}$ terminal tails of histones although the number and relevance of posttranslational modifications at the histone globular domains are of increasing interest (Lawrence et al., 2016). Not only the different positions of histone modifications compose the degree of diversity, but also the level of the modification state. Methylation can occur in three degrees for lysine (mono-, di- and tri-) and arginine (mono-, symmetric and asymmetric di-) residues.

A model of how histone PTMs function in regulating chromatin structure was postulated with the histone code (Jenuwein and Allis, 2001; Strahl and Allis, 2000). This concept is based on antagonistic acting enzymes such as kinases/phosphatases, histone acetyltransferases (HATs)/histone deacetylases (HDACs) and lysine methyltransferases (KMTs)/lysine 
demethylases (KDMs) that establish specific histone modification patterns, which are recognized and interpreted by non-histone proteins called histone modification readers.

The large variety of histone modifications provides a high potential for the regulation of chromatin organization and function. Histone modifications have been linked to cellular processes involved in transcriptional regulation, cell cycle process, DNA replication, DNA repair, splicing, epigenetic silencing and others (Izzo and Schneider, 2010; Kouzarides, 2007). Chromatin organization can be directly modulated by histone modifications that affect the interactions between nucleosomes. For instance, the incorporation of histone H4 lysine 16 acetylation (H4K16ac) was shown to reduce chromatin compaction and increase transcription (Akhtar and Becker, 2000; Shogren-Knaak et al., 2006) whereas H4K20 di- and tri-methylation have been shown to enhance chromatin condensation in vitro (Lu et al., 2008). In addition to these direct effects, histone modifications can act indirectly on chromatin organization by promoting or preventing the recruitment of chromatin binding proteins. Basically all DNA processes can be influenced by the regulation of chromatin access of chromatin modifying protein complexes or single factors, transcription factors and effector proteins that activate downstream signaling (Margueron et al., 2005; Torres and Fujimori, 2015; Wysocka et al., 2006).

\section{Genomic distribution of histone modifications}

The local enrichment of certain histone modification patterns is known to correlate with structural regions along the genome. Some histone modifications are highly enriched in heterochromatin and depleted from euchromatin while others are specific for euchromatin. Moreover, histone modification patterns correlate also with functional genomic elements along these chromatin subtypes. This observation allows using them as markers to differentiate certain genomic regions.

For example, in euchromatin histone marks like tri-methylation of histone $\mathrm{H} 3$ at lysine 4 (H3K4me3) and H3K27 acetylation are highly enriched at transcriptional start sites (TSS) of active genes (Kimura, 2013) while H3K36me3 and H3K4me1 are distributed within the gene body (Kimura, 2013). In contrast, heterochromatic inactive gene loci are mainly characterized by high levels of H3K9me3 and H3K27me3 as well as the presence of H3K9me2 and H3K27me2 (Kimura, 2013; Wang et al., 2009). Thus, it appears that inactive genes are depleted from active histone marks such as H3K4 methylation while in turn active gene loci appear to be mainly free of histone modifications specific for silenced genes. Nevertheless, those general rules can deviate depending on cell type and/or developmental stage. For example, in undifferentiated stem cells, bivalent chromatin domains were found to be simultaneously marked by both 
H3K4me3 and H3K27me3, characteristic histone marks for eu- and heterochromatin, respectively (Bernstein et al., 2006; Vastenhouw and Schier, 2012). Those domains with a dual chromatin identity might have a potential function in the fine regulation of gene expression during embryonic development by the capacity of switching between a H3K4me3 transcriptionally active state and a H3K27me3 silenced gene state.

The development of techniques such as ChIP-on-chip and ChIP-Seq have considerably contributed to deepen our understanding of the global chromatin landscape by providing genome wide pictures of the histone mark distribution (Barski et al., 2007; Mikkelsen et al., 2007; Wang et al., 2008).

It was observed that histone lysine acetylation is generally found at transcribed regions of active genes, which links this modification to active transcription and gene expression in a more general way (Cui and Shi, 2016; Ucar et al., 2011; Wang et al., 2008). A set of distinct histone modifications is also generally linked to heterochromatin. H3K9me3, H4K20me3 and H3K27me3 have been characterized as predominant constitutive heterochromatin marks (Bannister and Kouzarides, 2011; Mikkelsen et al., 2007). In agreement with previous reports, which associated H3K9me3 and H4K20me3 with silencing of centromeres, transposons and tandem repeats, genome wide mapping revealed a strong enrichment of these marks at telomeric, satellite and long terminal repeats (Mikkelsen et al., 2007). The group of identified repressive marks included next to the tri-methylation sites of H3K9, H4K2O and H3K27 H3K27me2 and H3K9me2 while most other modifications correlated with transcriptional activation (Barski et al., 2007; Cui and Shi, 2016; Wang et al., 2008). Within heterochromatin not only constitutive regions are marked by histone modifications. H3K27me3 is the hallmark of facultative heterochromatin that includes regulatory chromatin elements like boundary elements and insulators (Bannister and Kouzarides, 2011; Van Bortle et al., 2012) but has also been detected at inactive enhancer elements. Surprising and in strong contrast to their di- and tri-methylation counterparts, the mono-methylated states of H3K9, H3K27 and H4K20 were found to be located mainly at active promoter sites and within the gene body, suggesting an association with transcriptional activity. Nevertheless, all three mono-methylation states have been already associated in the context of transcriptional repression. H3K27me1 was even shown to be significantly present in heterochromatin (Jacob et al., 2010).

Another histone modification assumed to be associated with heterochromatin is the symmetrical di-methylation of arginine 3 of histone H4 (H4R3me2). Genome wide analysis showed no prediction for either, active or silenced promoters (Barski et al., 2007). However, several studies associated H4R3me2 with transcriptional repression (Cui and Shi, 2016; Hou et al., 2008; Litt et al., 2009; Xu et al., 2010; Zhao et al., 2009). 
Table 1.1 Histone modifications.

The table represents selected $\mathrm{N}$-terminal histone modifications and their associated functions. The information of the table is based on (Izzo and Schneider, 2010; Lawrence et al., 2016).

\begin{tabular}{|c|c|c|}
\hline histone & modification & function \\
\hline $\mathrm{H} 2 \mathrm{~A}$ & $\begin{array}{l}\text { H2AK4/5ac } \\
\text { H2AK7ac } \\
\text { H2AS1P } \\
\text { H2AK119P } \\
\text { H2AK119uq }\end{array}$ & $\begin{array}{l}\text { transcriptional activation } \\
\text { transcriptional activation } \\
\text { mitosis; chromatin assembly } \\
\text { spermatogenesis } \\
\text { transcriptional repression }\end{array}$ \\
\hline $\mathrm{H} 2 \mathrm{~B}$ & $\begin{array}{c}\text { H2BK5ac } \\
\text { H2BK11/12ac } \\
\text { H2BK15/16ac } \\
\text { H2BS14P } \\
\text { H2BK120uq } \\
\text { H2BK123uq }\end{array}$ & $\begin{array}{l}\text { transcriptional activation } \\
\text { transcriptional activation } \\
\text { transcriptional activation } \\
\text { apoptosis } \\
\text { spermatogenesis/meiosis } \\
\text { transcriptional activation }\end{array}$ \\
\hline $\mathrm{H} 3$ & $\begin{array}{c}\text { H3K4me2 } \\
\text { H3K4me3 } \\
\text { H3K9me1 } \\
\text { H3K9me2 } \\
\text { H3K9me3 } \\
\text { H3K27me1 } \\
\text { H3K27me2 } \\
\text { H3K27me3 } \\
\text { H3K36me3 } \\
\text { H3R17me } \\
\text { H3K4ac } \\
\text { H3K9ac } \\
\text { H3K14ac } \\
\text { H3K23ac } \\
\text { H3K27ac } \\
\text { H3S10P } \\
\text { H3t11/S28P }\end{array}$ & $\begin{array}{l}\text { permissive euchromatin } \\
\text { transcriptional elongation; active euchromatin } \\
\text { transcriptional activation/repression } \\
\text { transcriptional repression } \\
\text { transcriptional repression; imprinting; DNA methylation } \\
\text { transcriptional activation/repression } \\
\text { transcriptional activation/repression } \\
\text { transcriptional silencing; X-inactivation; bivalent genes/gene poising } \\
\text { transcriptional elongation } \\
\text { transcriptional activation } \\
\text { transcriptional activation } \\
\text { histone deposition; transcriptional activation } \\
\text { transcriptional activation; DNA repair } \\
\text { transcriptional activation; DNA repair } \\
\text { transcriptional activation } \\
\text { mitosis; meiosis; transcriptional activation } \\
\text { mitosis }\end{array}$ \\
\hline $\mathrm{H} 4$ & $\begin{array}{l}\text { H4K20me1 } \\
\text { H4K20me3 } \\
\text { H4R3me } \\
\text { H4R3me2s } \\
\text { H4K5ac } \\
\text { H4K8ac } \\
\text { H4K12ac } \\
\text { H4K16ac }\end{array}$ & $\begin{array}{l}\text { transcriptional activation/silencing } \\
\text { heterochromatin } \\
\text { transcriptional activation } \\
\text { transcriptional silencing } \\
\text { histone deposition; transcriptional activation; DNA repair } \\
\text { transcriptional activation; DNA repair; transcriptional elongation } \\
\text { histone deposition; telomeric silencing; transcriptional activation; DNA repair } \\
\text { transcriptional activation; DNA repair }\end{array}$ \\
\hline
\end{tabular}




\section{DNA methylation}

In addition to posttranslational histone modifications chromatin can also be chemically modified on the DNA. In eukaryotes, DNA is predominantly methylated at carbon- 5 of cytosine $(5 \mathrm{mC})$ in the context of $\mathrm{CpG}$ dinucleotides. $\mathrm{CpG}$ dinucleotides are found throughout the genome but are mainly located within $\mathrm{CpG}$ islands, which are characterized as short $\mathrm{CpG}$ rich regions that are often associated with TSSs of promotors, gene bodies and intergenic regions (Jones, 2012). $\mathrm{CpG}$ islands are usually unmethylated and associated with the euchromatic histone modification H3K4me3 (Guenther et al., 2007; Illingworth and Bird, 2009; Mikkelsen et al., 2007). However, most of the genome is CpG-deficient and essentially methylated.

In case DNA methylation of $\mathrm{CpG}$ islands occurs it is generally associated with transcriptional repression and connected with a functional role in imprinting, X-chromosome inactivation and transposon repression (Breiling and Lyko, 2015). Recent studies, however, suggest that DNA methylation can also be associated with promotion of transcription, indicating a far more complex functional diversity (Hu et al., 2013; Jin et al., 2012; Wu et al., 2010).

The establishment of DNA methylation is performed by three DNA methyltransferases comprising DNMT1, which has a strong preference for hemimethylated DNA while the two others, DNMT3A and DNMT3B, do not show such a preference and are therefore known as the de novo methyltransferases (Goll and Bestor, 2005). DNMT3A and DNMT3B establish DNA methylation in early development and together with DNMT1 participate in maintaining DNA methylation (Li et al., 2015; Liao et al., 2015).

\subsubsection{Readout of chemical chromatin modifications}

The dynamic control of chemical modifications of DNA and histones include the action of antagonistic working enzymes referred to as "writers" and "erasers". Those enzymes are targeted to chromatin by "reader" domains that recognize specifically modified (or unmodified) amino acid residues and DNA. "Reader" domains can be found within the protein structure of "writers" and "erasers" or as part of an associated factor.

The most abundant binding domains involved in histone modification readout include bromodomains, Bromo-adjacent homology (BAH) domains, plant homeodomain (PHD) fingers, WD40 repeat domains, the "royal family" modules and 14-3-3 domains.

Bromodomains are acetyl-binding domains that specifically recognize $\varepsilon$-N-acetylated lysine residues (Owen et al., 2000). They are present in a variety of chromatin-associated factors, including HAT and HMT enzymes, ATP-dependent helicases and also transcription factors (Ferri 
et al., 2016). Bromodomains often occur in tandem with other domains within the same protein. They are most frequently found associated together with PHD fingers, but also with PWWP, SET and BAH domains (Sanchez et al., 2014).

$\mathrm{BAH}$ domains have been found to act as protein-protein interaction modules, histone lysine methylation recognition and nucleosome binding modules. There are present in many proteins associated with DNA and histone modifications, such as DNMT1, MTA1 and the origin recognition complex 1 (Orc1) (Yang and $\mathrm{Xu}, 2013$ ). For instance, the $\mathrm{BAH}$ domain was shown to be important in mediating the preferential binding of OCR1 to H4K20me2 (Kuo et al., 2012). $\mathrm{BAH}$ domains are essentially found in factors connected to processes involved in DNA methylation, replication and transcriptional regulation. Therefore it is also not surprising to find them often together with reader modules known to bind histones and DNA, such as Bromo, SANT and PHD domains.

PHD domains are structurally conserved modules that act as readers of methylated lysine on histone tails as well as non-histone proteins (Musselman and Kutateladze, 2011). In rare cases, PHD domains have been shown to interact with methylated arginine and acetylated lysine. For instance, the PHD finger of RAG2 preferentially interact with symmetrically methylated H3R2 whereas the tandem PHD finger of DPF3b was shown to bind to H3K14ac (Ramon-Maiques et al., 2007; Zeng et al., 2010). As most of the other reader modules, PHD fingers are flanked by additional reader domains, which appear to be important in combinatorial readout of histone modifications.

The "royal family" modules are a group of structurally related protein motives, including the Tudor, PWWP, chromatin-binding (Chromo) and malignant brain tumor (MBT) domains that recognize lysine methylation by aromatic cage pockets (Maurer-Stroh et al., 2003). Besides individual exceptions of the PHD and the WD40 domain, Tudor domains are the only arginine binding motives known so far (Gayatri and Bedford, 2014). Some of the proteins harboring a Tudor domain have been shown to interact with specific histone methylation sites as well. While the Tudor domains of PHF1 and PHF19 interact specifically with H3K36me3, the Tudor domains of JMJD2A and UHRF1 can target several modification states, alone or in combination with a second binding motive, respectively (Lu and Wang, 2013).

PWWP domains contain a highly conserved Pro-Trp-Trp-Pro motif and were initially identified as non-specific DNA-binding domains. However, recently the PWWP domain of Brf1 has been demonstrated to specifically associate with H3K36me3 (Vezzoli et al., 2010).

Chromodomains are very well characterized as they were discovered among the first histone binding folds. Although chromodomains are structurally similar, they can be divided into different 
subclasses comprising HP1/CBX chromodomains, chromobarrel, chromo shadow and chromoATPase/helicase-DNA-binding (CHD) domains (Yap and Zhou, 2010). The proteins heterochromatin protein 1 (HP1) and Polycomb were the first factors characterized as specifically recognizing tri-methylated lysine 9 of $\mathrm{H} 3$ and tri-methylated lysine 27 of $\mathrm{H} 3$ via their chromodomain (Patel, 2016; Yap and Zhou, 2010). CBX chromodomains show a similar preference for those two modification marks although they have been additionally functionally associated with H3K27me2. Interestingly, the chromo shadow domains do not interact with methylated histones but were shown to act as protein-protein interaction modules (Brasher et al., 2000; Cowieson et al., 2000) while the MSL3 chromobarrel domain was shown to bind to monomethylation of H4K20 only in the presence of DNA (Kim et al., 2010).

Proteins harboring MBT repeats are functionally involved in processes like mitosis, tumor suppression and preferentially bind to mono- and di-methylated lysines of histones (Bonasio et al., 2010). The MBT modules as well as the other members of the "royal family" can occur and act as tandem reader modules (Patel, 2016).

WDR 40 motifs are another family of modules acting in form of tandem repeats, which bind to methylated as well as unmethylated histones. One of the best studied representatives is the protein WDR5 (Patel, 2016; Yap and Zhou, 2010).

Histone phosphorylation mainly occurs at serine residues that can be recognized by 14-3-3 domains as well as the tandem breast cancer susceptibility (BRCT) domain. For instance, the BRCT domain of the protein Breast cancer associated 1 (BRCA1) has been shown to be important for its function in DNA repair (Glover, 2006; Yap and Zhou, 2010).

$5 \mathrm{mC}$ methylated DNA is also recognized by certain protein-binding motifs. So far, three different binding motifs were identified - the meCpG-binding domain (MBD), SET and RING-associated (SRA) domains and Kaiso and Zpf57 zinc finger proteins (Patel, 2016). Kaiso can recognize symmetrically modified $5 \mathrm{mC}$ in the context of tandem $\mathrm{CpG}$ dinucleotides via a zinc finger motif (Prokhortchouk et al., 2001). In mammals, SRA domains are restricted to the UHRF1 protein family comprising UHRF1 and UHRF2 and have been demonstrated to bind hemimethylated 5mCpG/CpG sites (Bronner et al., 2007; Unoki et al., 2004). MBD domains were shown to recognize symmetrically methylated CpG dinucleotides (Nan et al., 1993; Ohki et al., 2001). Independent of the DNA methyl binding domain, 5mC-binding proteins have been shown to recruit proteins associated with transcriptional repression (Patel, 2016). 


\subsubsection{Multivalent readout and crosstalk of chemical chromatin modifications}

The diversity of recognition of chemical chromatin modifications is already enormous when only considering the range of binding folds identified and their specificity, which is supported by their arrangement within a polypeptide and the flanking amino acid sequences of the PTMs to be recognized. The diversity strongly increases considering additional processes of multivalent readout. The discovery of the linkage of multiple binding domains, either within a single protein or multi protein complexes, strongly supports the assumption of complex readout. In particular, the readout of a certain modification can be influenced by adjacent PTMs offering countless PTM combinations functioning in a synergistically as well as antagonistically manner.

Several mechanisms of combinatorial readout of chromatin modifications are conceivable. The combinatorial readout can either take place on the same histone tail or on different histone tails, referred as in cis and in trans, respectively. Readout in trans happens either on a particular histone or at the nucleosomal level. The latter can be further distinguished between intra- and inter-nucleosomal combinatorial readout. Additionally, multivalence can also occur concomitant DNA and histone recognition. Binding modules involved in combinatorial readout can be coupled by protein-protein interactions in multiple protein complexes or directly within a single protein. Possibilities of protein-binding modes reflecting the introduced mechanisms are illustrated in figure 1.2.

To date, the combinatorial readout in cis and in trans by paired protein-binding modules within one protein could be demonstrated for several proteins. For instant, combinatorial readout in cis via a PHD-Bromo cassette has been shown for TRIM24 and TRIM33, which bind unmodified H3 in parallel with $\mathrm{H} 3 \mathrm{~K} 23 \mathrm{ac}$ and $\mathrm{H} 3 \mathrm{~K} 9 \mathrm{me} 3$ concomitantly with $\mathrm{H} 3 \mathrm{~K} 18 \mathrm{ac}$, respectively (Tsai et al., 2010; Xi et al., 2011). The tandem Tudor-PHD finger cassette of UHRF1 has also been shown to bind the $\mathrm{N}$-terminus of $\mathrm{H} 3$ in cis (Arita et al., 2012). Combinatorial binding in trans is far less studied. One of the best studied examples is the intra-nucleosomal binding of the PHD-Bromo cassette of BPTF that associates with H3K4me3 and H4K16ac (Ruthenburg et al., 2011).

Besides paired protein-binding modules within one protein, crosstalk can be addressed on the level of indirect protein recruitment by protein-protein interactions. Chromatin modifying protein complexes can contain multiple "readers" providing a wide range of binding motifs in combination with "writers" and "erasers". The local accumulation of such complexes provides the advantage of a higher degree of specificity and the ability to establish a complex series of modification patterns. The HBO1 complex for instance contains several PHD finger modules that act cooperatively to regulate its acetyltransferase activity (Musselman et al., 2012; Torres and Fujimori, 2015). In a similar fashion, WD40 repeats, PHD and Tudor domains of the PRC2 
complex regulate its methyltransferase activity. Different posttranslational histone modifications have been shown to specifically recruit those protein complexes and additionally provided first insights into the multivalent impact of DNA and histone modifications (Bartke et al., 2010; Bluhm et al., 2016; Engelen et al., 2015; Kunowska et al., 2015; Nikolov et al., 2011; Vermeulen et al., 2010).

A)

$$
\text { readout of multiple PTMs in cis }
$$

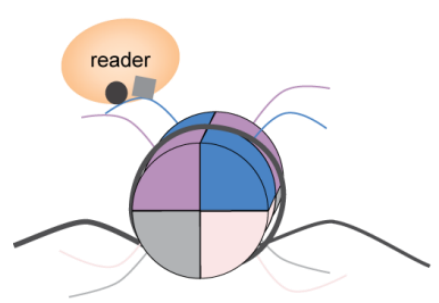

C)
B) combinatorial readout in cis

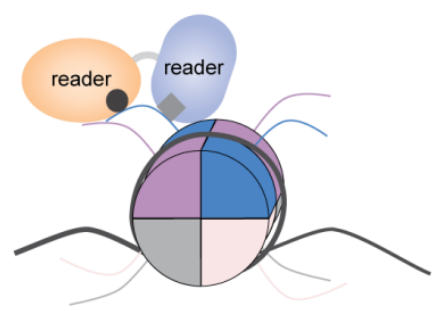
combinatorial readout in trans

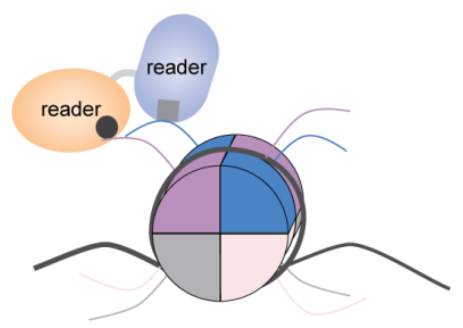

intra-nucleosomal

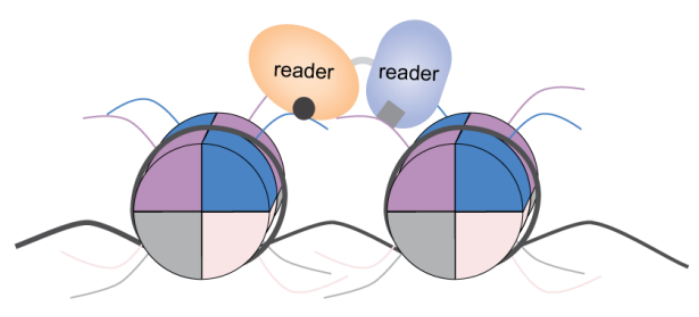

inter-nucleosomal

D) combinatorial readout in trans by a multi protein complex

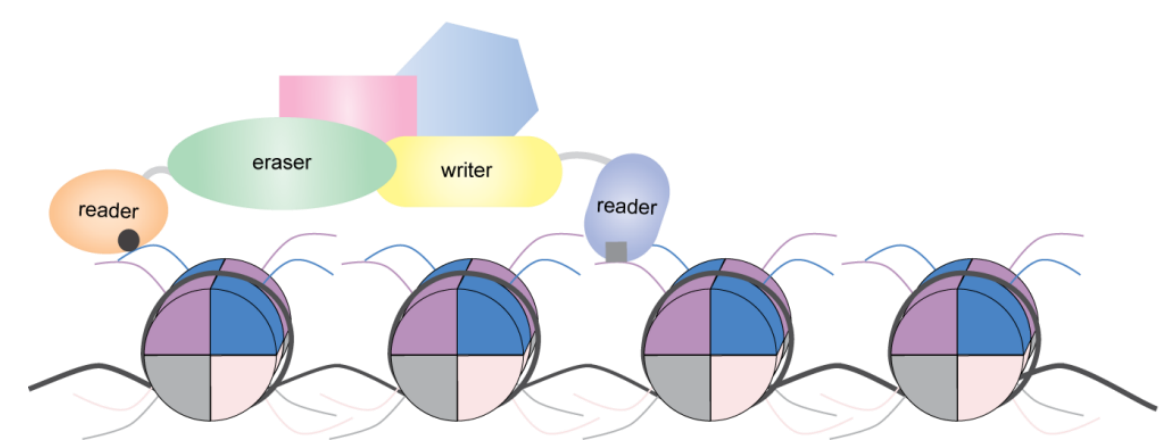

Figure 1.2 Combinatorial readout of posttranslational histone modifications.

A) Readout of two histone modifications on the same histone tail by a single protein. B) Combinatorial readout in cis. Histone PTMs on the same histone tail are recognized by two different proteins. C) Combinatorial readout in trans. The readout of two PTMs at different histone tails can take place either within one nucleosome (intra-nucleosomal) as shown in the left panel or on different nucleosomes (inter- 
nucleosomal) as represented in the right panel. D) Combinatorial readout in trans facilitated by a multi protein complex. The figure is adapted from (Musselman et al., 2012).

Nevertheless, recent knowledge of PTM recognition mechanisms is mainly based on specific binding of single proteins to distinct histone modifications or combinations thereof. The extent of the influence various modification patterns have on protein binding to chromatin and the functional consequences thereof are still not fully understood. Toward understanding the epigenetic language of histone and DNA modifications, the assignment of functional consequences and therefore the identification of the complement of proteins regulated by individual chromatin modifications and combinations is of immense importance.

\subsubsection{Chromatin arrays for systematic analysis of chemical modification readout}

The question of how distinct histone modifications influence the protein recruitment to chromatin and more globally the chromatin interactome consists in the field of epigenetics since many years. To approach this question several strategies have been developed over years. Modified histone $\mathrm{N}$-terminal peptides are one of the major tools used in approaches ranging from array- to affinity-based protein interaction studies (Kim et al., 2006; Wysocka, 2006). In general, affinity purifications strategies enable an unbiased, modification-dependent identification of chromatinassociated factors and macromolecular protein complexes. The experimental design is based on the incubation of unmodified or modified $\mathrm{N}$-terminal histone peptides with nuclear extracts followed by the identification of the bound proteins by mass spectrometric analysis (Wysocka, 2006). Recently, this approach has seen important modification by coupling it with quantitative mass spectrometry (Oda et al., 2010; Vermeulen et al., 2010). However, the characterization of histone PTMs binding proteins has, so far, essentially relied on the use of in vitro synthesized modified histone $\mathrm{N}$-terminal peptides as bait in affinity purification that may not reflect faithfully the diversity and complexity of the PTM-regulated histone interactome. One strategy to overcome this problem has consisted in using mononucleosomes or even oligonucleosomal arrays (Bartke et al., 2010; Nikolov et al., 2011). Nucleosomal arrays are of particular interest as they offer a far more physiologically relevant model compared to the peptide-based strategy. For example, chromatin arrays allow to (i) control histone PTM and underlying DNA sequences, (ii) to investigate the binding of proteins within the nucleosomal context including the identification of proteins binding only in presence of more than one nucleosome, (iii) characterize the crosstalk between histone modifications by inserting two or more PTMs on one single chromatin array, (iv) investigate the crosstalk between PTMs of histones and DNA.

The assembly of homogenous recombinant nucleosomal arrays has been well described in the literature (Dyer et al., 2004; Huynh et al., 2005; Luger et al., 1999). A general overview is shown 
in figure 1.3. The system is based on histone octamers that are uniformly position on a defined DNA template (Huynh et al., 2005).
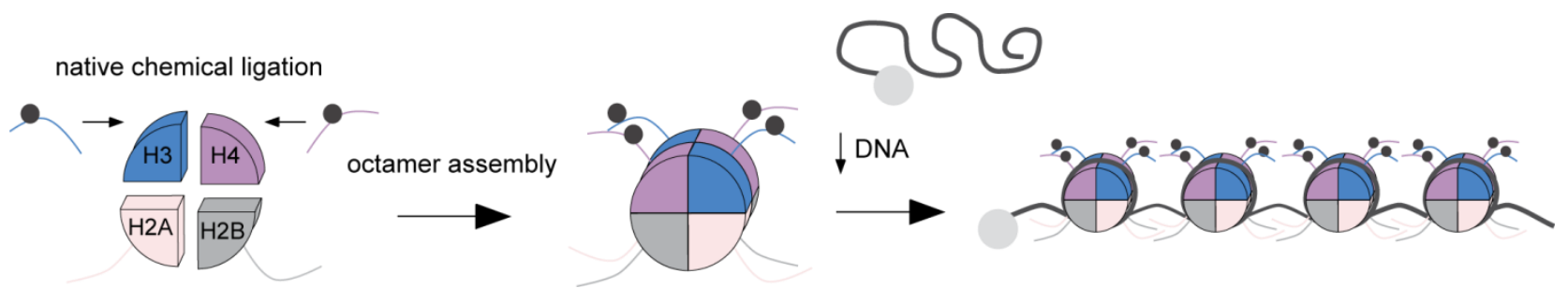

Figure 1.3 Design of recombinant nucleosomal arrays.

Recombinant full length histones, either unmodified or modified by native chemical ligation, were assembled to histone octamers via dialysis against high salt. Biotinylated 12x200x601 DNA templates and histone octamers containing modified histones of choice were mixed and reconstituted to $12 \mathrm{mer}$ chromatin arrays by dialysis over a salt gradient.

The application of chromatin based affinity purification of an unmodified and a chemically modified chromatin array in direct comparison demonstrated that nucleosomal arrays are well suited for the identification of specific protein-binding interactomes of chromatin arrays in the context of defined chemical modifications, when coupled with relative quantification by mass spectrometry (Nikolov et al., 2011). Thus, this method has the potential to elucidate the complex protein interactomes of diverse chromatin modification patterns and can provide insights into epigenetic regulated biological pathways and histone modification crosstalk.

\subsection{Mass spectrometry}

\subsubsection{Mass spectrometry based protein identification of complex samples}

Mass spectrometry is a powerful method especially in terms of global protein identification, which has allowed the characterization of organelle proteomes, signaling pathways and complete protein complexes. Although mass spectrometry has a long history in science, it is only during the last 20 years that it has been developed to the standard method for protein identification and quantification of complex samples in proteomics.

The principle of identification and quantification of molecules by mass spectrometry is based on the mass-to-charge ratio of the molecules. The molecule of interest is transferred into the gas phase and ionized. These ions can be separated according to their mass-to-charge ratio $(\mathrm{m} / \mathrm{z})$ by ion acceleration in an electric or magnetic field. Ion acceleration is later converted in ion current and gives the signal to be detected. 
A standard proteomics workflow for MS based protein identification is carried out by tandem mass spectrometry comprising four main successive steps - (i) protein proteolysis, (ii) $\mathrm{m} / \mathrm{z}$ detection of intact ions, (iii) precursor ion selection and fragmentation, (iv) peptide identification followed by protein identification using protein sequence databases (figure 1.4).

The proteomic workflow starts with the proteolysis of the proteins of interest. For the reason that peptides possess solubility in a wider range of solvents and their masses can be determined with high accuracy, the complex protein sample is proteolytic digested into peptides prior mass spectrometric analysis. Consequently, the degree of complexity increases even more. To ensure a comprehensive, unambiguous identification, including the detection of low-abundance species, a sufficient separation is required. While SDS-PAGE separation can be a first step to reduce the degree of complexity at the protein level, the application of liquid chromatography (LC) is successfully in use to reduce the complexity at the peptide level. The separation is achieved by nanoliter flow rate reversed-phase C18 chromatography coupled to direct elution into an electrospray ionization (ESI) mass spectrometer. Additional LC systems are commonly in use as well as separation based on capillary electrophoresis and the application of multidimensional separation, successfully increasing the number of identified peptides (Di Palma et al., 2012; Heemskerk et al., 2016; Yates, 2004).

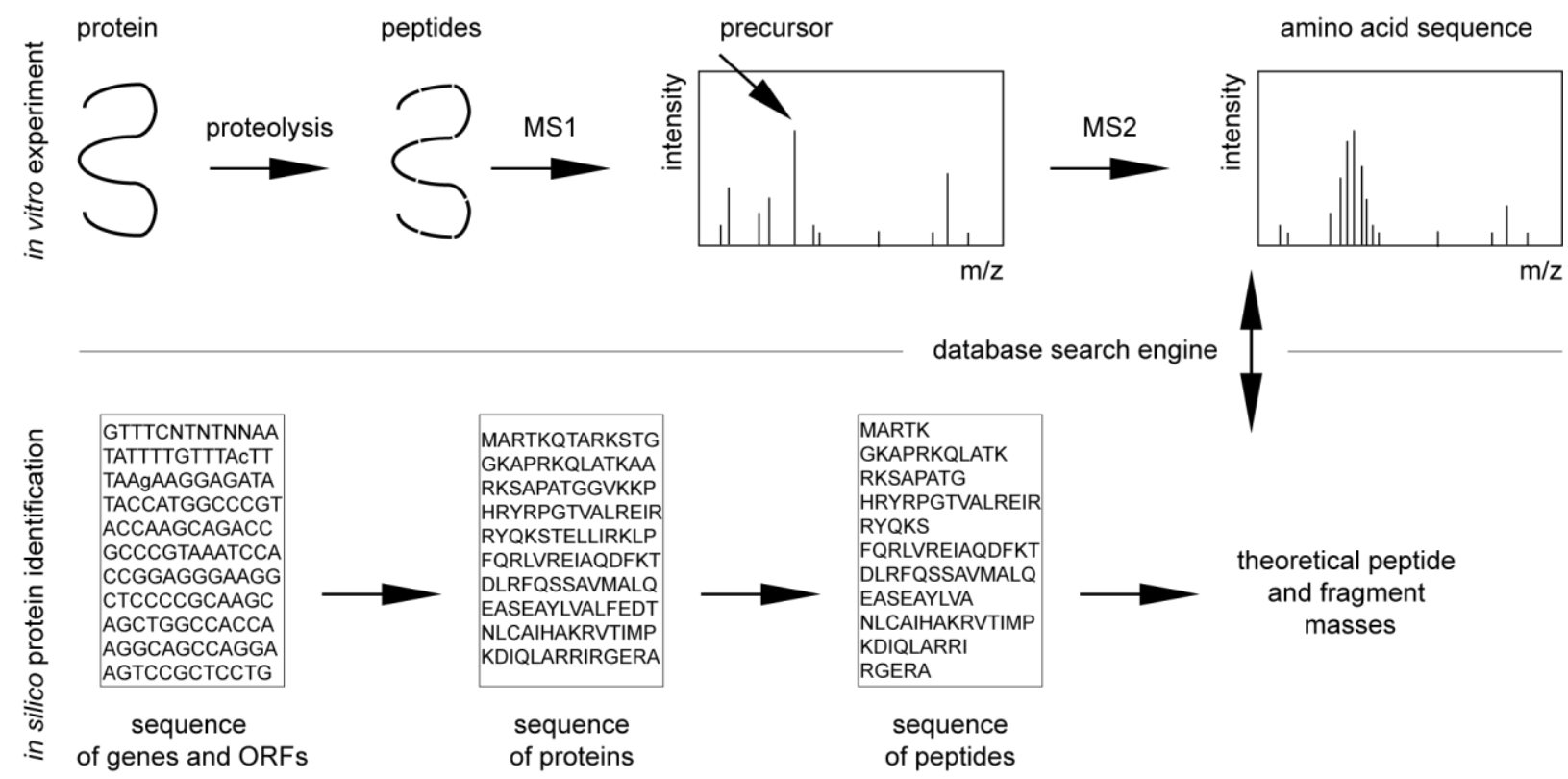

Figure 1.4 Workflow for protein identification by MS.

Proteins are hydrolyzed to peptides using a specific protease. The mass-to-charge ratios of the peptides are determined by mass spectrometry (MS1). Based on the first measurement precursor ions are selected and fragmented (MS2). For protein identification the information obtained from the MS1 and MS2 spectra are compared to the theoretical masses of a database by a search engine. The figure is adapted from (Schmidt, 2010). 
The majority of tandem mass spectrometry experiments in proteomics use data-dependentacquisition (DDA) for detection of $\mathrm{m} / \mathrm{z}$ ratios. In a first step, the MS1 spectrum is generated by a complete scan over a distinct mass range that records $\mathrm{m} / \mathrm{z}$ ratios of all ions eluting from the $\mathrm{LC}$ at a certain time point. The most abundant precursor ions are selected for fragmentation, commonly done by low-energy collision induced dissociation (CID). The scans of the fragmented ions provide the MS2 (or MS/MS) spectra. The DDA cycle acquires data over the length of the LC elution gradient.

The peptide identification is performed in silico by database search engines. A protein sequence database provides the theoretically mass of proteolytically digested peptides. The mass information resulting from the matched MS1 and MS2 spectra is used to search for peptide candidates in the database with corresponding theoretically masses. A certain mass tolerance setting according to the mass accuracy of the mass spectrometer is used and the results are reported according to statistical ranking.

\subsubsection{Relative quantification by Stable Isotope Labeling by Amino acids in Cell culture (SILAC)}

Quantitative mass spectrometry is of major importance in the field of MS based proteomics. Two general approaches have been developed over the last years - stable isotope-based and labelfree methods. Label-free methods are based on comparison of identical peptides and proteins between multiple measurements and rely on either spectrum count or MS1 intensity based approaches (Bantscheff et al., 2012). With vast technical progress, label-free approaches have become more popular nowadays but still struggle with certain drawbacks. They require several replicates and rely on high reproducibility in terms of sample preparation and separation steps prior to mass spectrometric measurements. Stable isotope-based mass spectrometry relies on the nearly identical physicochemical properties of heavy stable isotopes and their corresponding light isotopes. Relative quantification is achieved by comparing the MS intensities of unlabeled peptides to their corresponding heavier isotopic labeled peptides. Consequently, the method allows the measurement of different labeled peptides (and proteins) within the same sample, thus reducing errors from sample preparation and ion suppression by mass spectrometers in different MS runs.

Isotope labels can be incorporated chemically, enzymatically or metabolically (Ong and Mann, 2005). One of the most popular metabolic labeling approaches is Stable Isotope Labeling by Amino acids in cell culture (SILAC) (Ong et al., 2002). The incorporation of heavy isotopes takes place during cellular or organismal growth with the consequence of a certain limitation in the range of applicable samples. This drawback was reduced with the recent development of super SILAC and spike-in SILAC allowing the application of SILAC to tissues and body fluids (Geiger 
et al., 2010; Geiger et al., 2011). Another disadvantage is the number of samples that can be compared using SILAC. While a few years ago the complexity of analysis caused restrictions the actual limitations are reasoned in the limited number of parallel labels for heavy labeled amino acids. Widely in application are the stable isotope labeled amino acids lysine and arginine, containing $\mathrm{C} 13$ and N15. Using labeled lysines and arginines up to 5-plex SILAC experiments can be performed (Ong et al., 2002). Nevertheless, SILAC has proven to be a method of choice as it has been proven to be highly accurate and more robust compared to other stable isotopebased methods and therefore suitable for affinity purification strategies (Chen et al., 2015).

Quantitative MS-based proteomics as large scale MS-based approaches in general require specific algorithms for peptide/protein identification and quantification. MaxQuant is a quantitative software packages that enables protein identification and quantification in the context of SILAC based approaches but also supports quantification of additional label-based and label-free approaches (Cox and Mann, 2008; Cox et al., 2011). The software incorporates all steps of data analysis, starting from processing MS spectra to final statistical evaluation. The analysis pipeline starts with high intensity-weighted estimation of masses for peptide peaks (assignment of 3D peaks based on intensity, $\mathrm{m} / \mathrm{z}$ and elution time), the determination of reliably isotope patterns and the assignment of SILAC peptide pairs. After improvement of mass accuracy, peptide and protein identification is done using an integrated database search engine. The last steps are directed at protein quantification based on the enrichment ratios of individual peptide pairs. The software package Perseus enables statistical evaluation and visualization.

\subsubsection{Protein-protein cross-linking mass spectrometry}

Cross-linking mass spectrometry (XL-MS) is a powerful approach, which allows investigating structural as well as physical interaction sites of proteins. A bifunctional cross-linker is used to covalently link proteins in physical close proximity engaged in non-covalent interactions. Commonly, cross-linkers used in MS possess two cross-linking reactive groups that are connected by a spacer but can have properties like variable spacer length, cleavability, composition, solubility, isotope labeling and photo reactivity (Paramelle et al., 2013). Another very important property is the specificity of a cross-linker that ranges from low to high specificity and can be modulated by altering the cross-linking conditions such as $\mathrm{pH}$ and the protein-crosslinker ratio. One of the most popular cross-linking reagents in use is bis(sulfosuccinimidyl) suberate (BS3). BS3 is a chemical cross-linker with a spacer arm length of $11.4 \AA$ and belongs to the highly reactive $\mathrm{N}$-hydroxysuccinimide (NHS) ester that target nucleophiles. It is a homobifunctional cross-linker that can react with primary amines in the side chains of lysine (K) 
residues and the $\mathrm{N}$-terminus of polypeptides resulting in stable amide bonds (Sinz, 2006; Tran et al., 2016).

After cross-linking, non-cross-linked peptides are still the overrepresented peptide species in a given sample (Sinz, 2006). Thus, compared to linear peptides, cross-linked peptide are less abundant and therefore less likely to be identified by mass spectrometric measurements. To overcome these issues, different enrichment strategies for cross-linking containing species can be applied on the protein as well as on the peptide level. Based on the property of a higher molecular weight of cross-linked species separation according to size can successfully enrich for both, cross-linked proteins and cross-linked peptide pairs. Depending on experimental design and sample preparation SDS-PAGE and size exclusion chromatography are applicable methods on the protein level. Size exclusion chromatography also has been shown to be applicable on the peptide level (Leitner et al., 2012). Alternatively, separation of cross-linked peptides was successfully achieved applying strong cation exchange chromatography as they exhibit more charges compared to linear peptides (Fritzsche et al., 2012).

Still, one of the major challenges in XL-MS is the identification of cross-linked peptides and proteins. Database search engines used for identification of proteins based on linear peptides cannot be applied for the analysis of cross-link spectra. The main reason is due to the fact that both, MS1 and MS2 spectra do not correspond to one peptide but to all possible combinations of cross-linked peptide pairs. The consequence is a dramatic increase of the database used for the search associated with increased risk of random assignments and higher false discovery rates. To date, two data processing tools, xQuest/xProphet and pLink, have been developed that search for cross-links against large databases exceeding a number of 30 to 40 proteins, implementing a false discovery rate control (Purcell et al., 2007; Rinner et al., 2008; Walzthoeni et al., 2012). Both have been proven to be suitable tools, sensitive enough to study proteinstructure and protein-protein-interactions. The disadvantage of $x Q u e s t / x P r o p h e t$ is the necessity of labeled cross-linker, thus pLink was the software used for my studies. 


\subsection{Hypothesis and objectives of the presented thesis}

Chromatin structure adopts two main configurations: a relaxed state containing most of the expressed genes and referred to as euchromatin and a condensed state associated with transcriptional silencing called heterochromatin. Both chromatin states exhibit histone posttranslational modifications found to be specifically associated with either eu- or heterochromatin. These chromatin modifications form a complex language whose understanding has been subject of constant efforts over the past decade. To date, the vast number of histone modification sites that have been identified, their distribution across the genome as well as their specific binders have provided considerable information on how chromatin functions can be regulated. However, a comprehensive overview of the proteomes associated with defined chromatin modifications and their functional outcomes were not achieved so far.

The hypothesis that has set the basis of my thesis work was that the biochemical status of chromatin domains is brought about by single chromatin modifications and combinations thereof that regulate the recruitment and the exclusion of specific networks of chromatin-associated proteins. One important question was whether a set of factors, common to all modifications associated with a given chromatin stage, would be responsible of the eu- or heterochromatin identities.

To address these questions, my primary objective was to characterize the proteomes associated with the majority of the known heterochromatic chromatin modifications and combinations thereof, by using a strategy based on chromatin affinity purification coupled to quantitative mass spectrometry. One of the first aims was to implement a statistical function that defines a fold enrichment cutoff meeting the requirements for the comparison of modification-specific regulated factors identified by individual performed experiments. Proteins regulated by specific modifications provided the basis for the identification of the biological functions of each of the ten heterochromatic modifications and the information regarding their functional relationships. The scope of my work has been broadening by introducing the question whether combinations of two chromatin modifications have an impact on the biochemical status of chromatin domains different from the individual modifications. To investigate this point, the combinations of $\mathrm{H} 3 \mathrm{~K} 9 \mathrm{me} 3$ and H4K20me3 as well as H3K9me3 and $\mathrm{CpG}$ methylated DNA were investigated. Lastly, a new workflow coupling ChAP with cross-linking mass spectrometry was establish in order to map the physical interactions among chromatin-associated proteins with the aim to determine the hierarchy of protein recruitment to chromatin. 


\section{Materials and Methods}

\subsection{Material and reagents}

\subsubsection{Laboratory equipment}

$\begin{array}{ll}\text { ÄKTA Explorer/Purifier/micro } & \text { GE Healthcare, Buckinghamshire (UK) } \\ \text { Balances } & \text { Metler-Toledo, Gießen (DE) } \\ \text { BBD 6220 CO } \text { incubator }_{\text {Beraeus, Hanau (DE) }} \\ \text { Bioreactor 5L with ez-Control } & \text { Applikon, Schiedam (NL) } \\ \text { Centrifuge Cryofuge 6000i } & \text { Heraeus, Hanau (DE) } \\ \text { Centrifuge Sorvall Evolution RC } & \text { Thermo Scientific, Braunschweig (DE) } \\ \text { Centrifuges tabletop 5415R/5810R } & \text { Eppendorf, Hamburg (DE) } \\ \text { ChemiDoc MP System } & \text { Bio-Rad, München (DE) } \\ \text { Electrophoresis power supplies } & \text { Bio-Rad, München (DE) } \\ \text { HP1100 and HP1200 LC systems } & \text { Agilent, Santa Clara (USA) } \\ \text { Laminar flow clean bench } & \text { Heraeus, Nahau (DE) } \\ \text { LTQ-Orbitrap Velos } & \text { Thermo Fischer Scientific, Bremen (DE) } \\ \text { LTQ-XL } & \text { Thermo Fischer Scientific, Bremen (DE) } \\ \text { Mini Trans-Blot system } & \text { Bio-Rad, München (DE) } \\ \text { Mini-PROTEAN Tetra PAGE cell } & \text { Bio-Rad, München (DE) } \\ \text { NanoDrop ND-1000 } & \text { Peqlab, Erlangen (DE) } \\ \text { Peristaltic pump } & \text { Ismatec, Glattburgg (CH) } \\ \text { pH meter } & \text { Metler-Toledo, Gießen (DE) } \\ \text { Q-Exactive } & \text { Thermo Fischer Scientific, Bremen (DE) } \\ \text { Sonication bath SONOREX Super } & \text { BANDELIN Electronic, Berlin (DE) } \\ \text { Sorval SA600 rotor } & \text { Thermo Scientific, Braunschweig (DE) } \\ \text { Sorval SS34 rotor } & \text { Thermo Scientific, Braunschweig (DE) } \\ \text { SpeedVac Savant SPD121P } & \text { Thermo Scientific, Braunschweig (DE) } \\ \text { Sub-Cell-GT agarose gel electrophoresis } & \text { Bio-Rad, München (DE) } \\ \text { Thermocycler epgradientS } & \text { Eppendorf, Hamburg (DE) } \\ \text { Thermomixer Comfort } & \text { Eppendorf, Hamburg (DE) } \\ \text { Water bath TW12 } & \text { Julabo, Selbach (DE) } \\ \text { XCell Sure Lock Mini NuPAGE cell } & \text { Invitrogen, Karlsruhe (DE) } \\ & \end{array}$




\subsubsection{Consumables and plastic ware}

Amicon Ultra centrifugal filter devices

(MWCO 3 and $10 \mathrm{kDa}$ )

Phase Lock Heavy Tubes (2-, 15-, 50 ml)

Slide-A-Lyzer dialysis units and cassettes

(MWCO 3500, 7000, 10000)

Spectra/Por dialysis membrane

(MWCO 3500 and 10000)

\subsubsection{Chemicals}

(2-bromoethyl)-tri-methylammonium bromide

(2-chloroethyl)-dimethylammonium chloride

(2-chloroethyl)-methylammonium chloride

2-mercaptoethanol

4-(2-Hydroxyethyl)-1-piperazineethanesulfonic acid (HEPES)

4-Mercaptophenylacetic acid (MPAA)

Acetic acid

Acetonitrile, LiChrosolv

Acrylamide/Bisacrylamide (37.5:1)

Agarose

Ammonium hydrogen carbonate

Ammonium peroxodisulfate

Bis(sulfosuccinimidyl) suberate (BS3)

Boric acid

Bovine serum albumin (BSA)

Bromophenol blue

Cleland's reagent (DTT, for MS analysis)

Coomassie Briliant Blue G-250

D/L-Methionine

Deoxynucleotide-5'-phosphate (dATP, dCTP, dGTP, dTTP)

Dipotassium hydrogen phosphate $\left(\mathrm{K}_{2} \mathrm{HPO}_{4}\right)$

Dithioerythrol (DTE)

Dithiothreitol (DTT)
Millipore, Billerica (USA)

5PRIME, Hamburg (DE)

Pierce/Thermo Scientific, Rockford (USA)

Spectrum Laboratories, Rancho Domingues (USA)

Sigma-Aldrich, Steinheim (DE)

Merck, Darmstadt (DE)

Karl Industries, Aurora (USA)

Sigma-Aldrich, Steinheim (DE)

VWR, Poole (DE)

Sigma-Aldrich, Steinheim (DE)

Merck, Darmstadt (DE)

Merck, Darmstadt (DE)

Merck, Darmstadt (DE)

Serva, Heidelberg, (DE)

Fluka, Buchs $(\mathrm{CH})$

AppliChem, Darmstadt (DE)

Thermo Scientific, Schwerte (DE)

Merck, Darmstadt (DE)

Sigma-Aldrich, Steinheim (DE)

Serva, Heidelberg (DE)

Calbiochem, Darmstadt (DE)

Fluka, Buchs $(\mathrm{CH})$

Sigma-Aldrich (DE)

Roth, Karlsruhe (DE)

Roth, Karlsruhe (DE)

Roth, Karlsruhe (DE)

Alexis Biochemicals, Farmingdale (USA) 
Ethanol

Ethidium bromide

Ethylendiamine tetraacetate (EDTA)

Formic acid (FA)

Glycerol

Guanidine hydrochloride

Guanidine hydrochloride ( $\geq 99.5 \%$ )

Hydrochloric acid $(37 \% \mathrm{HCl})$

lodacetamide (IAA)

LB Broth

Magnesium chloride $\left(\mathrm{MgCl}_{2}\right)$

Methanol, LiChrosolv

$\mathrm{N}, \mathrm{N}, \mathrm{N}$ ',N'-Tetramethylethylendiamid (TEMED)

Non-fat dry milk powder

Ortho-Phosphoric acid

Phenol:Chlorophorm:Isoamil alcohol (PCl)

[25:24:1]

Phenyl-methylsulfonyl fluoride (PMSF)

Polyethylene glycol 6000 (PEG-6000)

Ponceau S

Potassium chloride $(\mathrm{KCl})$

Potassium dihydrogen phosphate $\left(\mathrm{KH}_{2} \mathrm{PO}_{4}\right)$

RapiGest

S-(5'-adenosyl)-L-methionine (SAM)

Sodium acetate

Sodium azide $\left(\mathrm{NaN}_{3}\right)$

Sodium chloride $(\mathrm{NaCl})$

Sodium dodecyl sulfate (SDS)

Sodium hydrogen phosphate $\left(\mathrm{Na}_{2} \mathrm{HPO}_{4}\right)$

Sodium hydroxide $(\mathrm{NaOH})$

Tris(hydroxymethyl)amino ethane (Tris base)

Triton X-100

Tween-20

Urea

Water, LiChrosolv
Merck, Darmstadt (DE)

Roth, Karlsruhe (DE)

Roth, Karlsruhe (DE)

Fluka, Buchs $(\mathrm{CH})$

Merck, Darmstadt (DE)

Sigma-Aldrich, Steinheim (DE)

Roth, Karlsruhe (DE)

Merck, Darmstadt (DE)

Sigma-Aldrich, Steinheim (DE)

MOBIO, Hamburg (DE)

Merck, Darmstadt (DE)

Merck, Darmstadt (DE)

Sigma-Aldrich, Steinheim $(\mathrm{CH})$

Regilait, Saint-Martin-Belle-Roche (FR)

Merck, Darmstadt (DE)

Roth, Karlsruhe (DE)

Roche, Mannheim (DE)

Merck, Darmstadt (DE)

Sigma-Aldrich, Steinheim (DE)

Merck, Darmstadt (DE)

Roth, Karlsruhe (DE)

Waters (USA)

New England Biolabs, Ipswitch (USA)

Roth, Karlsruhe (DE)

Alfa Aesar, Massachusetts (USA)

Merck, Darmstadt (DE)

VWR, Poole (DE)

Merck, Darmstadt (DE)

Merck, Darmstadt (DE)

Roth, Karlsruhe (DE)

Merck, Darmstadt (DE)

Sigma-Aldrich, Steinheim (DE)

Merck, Darmstadt (DE)

Merck, Darmstadt (DE) 


\subsubsection{Chromatographic and affinity material}

C18 reversed phase precolumn $(0.15 \mathrm{~mm}$ ID $\mathrm{x}$ 20 mm, Reprosil-Pur120 C18-AQ 5 Hm)

HiLoad Superdex 200 10/300

HiLoad Superdex 200 16/60

Superdex 200 Increase 3.2/300

Superdex Peptide 3.2/300

HiTrap SP HP $1 \mathrm{ml}$

PD-10 columns

Q-Sepharose XK26/20

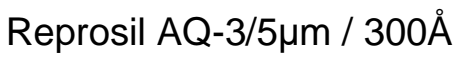

SilicaTip emmiters

SP-Sepharose XK26/20

Streptavidin MagneSphere particles
Dr. Maisch, Ammerbuch-Entringen (DE)

GE Healthcare, Buckinghamshire (UK) GE Healthcare, Buckinghamshire (UK) GE Healthcare, Buckinghamshire (UK) GE Healthcare, Buckinghamshire (UK) GE Healthcare, Buckinghamshire (UK) GE Healthcare, Buckinghamshire (UK) GE Healthcare, Buckinghamshire (UK) Dr.Maisch, Ammerbuch (DE) New Objective, Woburn (USA) GE Healthcare, Buckinghamshire (UK) Promega, Mannheim (DE)

\subsubsection{Cell culture media and materials}

DMEM High Glucose ([w/o] Lys, [w/o] Arg)

Fetal bovine serum, dialyzed

L-Arginine (Arg0)

L-Arginine, ${ }^{13} \mathrm{C}_{6}$ (Arg6)

L-Lysine (Lys0)

L-Lysine, ${ }^{2} \mathrm{D}_{4}$ (Lys4)

Penicillin/Streptomycin $100 \mathrm{x}$
PAA Laboratories, Colbe (DE)

PAA Laboratories, Colbe (DE)

Sigma-Aldrich, Steinheim (DE)

Euriso-top, Saarbrücken (DE)

Sigma-Aldrich, Steinheim (DE)

Euriso-top, Saarbrücken (DE)

PAA Laboratories, Colbe (DE)

\subsubsection{Commercial kits}

$1 \mathrm{~Kb}$ Plus DNA Ladder

ECL Pus Western Blotting Detection System ECL Western Blotting Detection System

Hybond ECL nitrocellulose membrane Hybond $\mathrm{N}+$ membrane Imperial Protein Stain Mini-PROTEAN 4-12\% TGX gels NucleoBond PC 10000
Invitrogen, Karlsruhe (DE)

GE Healthcare, Buckinghamshire (UK) GE Healthcare, Buckinghamshire (UK) GE Healthcare, Buckinghamshire (UK) GE Healthcare, Buckinghamshire (UK) Pierce/Thermo Scientific, Rockford (USA) Bio-Rad, München (DE) Machery\&Nagel, Düren (DE) 
NucleoBond Xtra Midi Plus

NucleoSpin Plasmid

NucleoSpin Extract II

NuPAGE Antioxidant

NuPAGE LDS Sample Buffer (4x)

NuPAGE MOPS SDS Running Buffer (20x)

NuPAGE Novex 4-12\% Bis-Tris gels, $1 \mathrm{~mm}$

NuPAGE Sample Reducing Agent (10x)

SeeBlue Plus2 Protein Standard
Machery\&Nagel, Düren (DE)

Machery\&Nagel, Düren (DE)

Machery\&Nagel, Düren (DE)

Invitrogen, Karlsruhe (DE)

Invitrogen, Karlsruhe (DE)

Invitrogen, Karlsruhe (DE)

Invitrogen, Karlsruhe (DE)

Invitrogen, Karlsruhe (DE)

Invitrogen, Karlsruhe (DE)

\subsubsection{Commonly used buffers and solutions}

10x DNA loading dye

10x PBS

1x PBS-T

10x SDS running buffer

$1 \times$ SDS sample buffer
$30 \%[v / v]$ Glycerol

10 mM EDTA

0.25\% [w/v] Bromophenol blue

$1.37 \mathrm{M} \mathrm{NaCl}$

$27 \mathrm{mM} \mathrm{KCl}$

$100 \mathrm{mM} \mathrm{Na}_{2} \mathrm{HPO}_{4}$

$20 \mathrm{mM} \mathrm{KH}_{2} \mathrm{PO}_{4}$

1x PBS

$0.1 \%[\mathrm{v} / \mathrm{v}]$ Tween-20

$250 \mathrm{mM}$ Tris base

1.92 M Glycine

$1 \%[w / v]$ SDS

$60 \mathrm{mM}$ Tris-HCl (pH 6.8)

$10 \%$ [v/v] Glycerol

$2 \%[w / v]$ SDS

$0.1 \%$ [w/v] Bromphenol blue

150 mM 2-Mercaptoethanol

$890 \mathrm{mM}$ Tris base

890 mM Boric acid 
10x TBE

$1 \times$ Transfer buffer

Chromatin loading buffer

Colloidal Coomassie stain

Injection buffer

MC buffer

PD150 (pull-down wash buffer)
$890 \mathrm{mM}$ Tris base

$890 \mathrm{mM}$ Boric acid

20 mM EDTA NaOH (pH 8.0)

$25 \mathrm{mM}$ Tris base

$0.192 \mathrm{M}$ Glycine

$0.1 \%[\mathrm{w} / \mathrm{v}] \mathrm{SDS}$

$20 \%[\mathrm{v} / \mathrm{v}]$ Methanol

10\% [v/v] Glycerol

1x RB low

$0.08 \%[\mathrm{w} / \mathrm{v}]$ Coomassie Brilliant Blue G 250

$20 \%[\mathrm{v} / \mathrm{v}]$ Methanol

$1.6 \%[\mathrm{v} / \mathrm{v}]$ Ortho-Phosphoric acid

$8 \%[\mathrm{w} / \mathrm{v}]$ Ammonium sulfate

$2 \%[\mathrm{w} / \mathrm{v}]$ SDS

$100 \mathrm{mM} \mathrm{NaCl}$

$10 \mathrm{mM}$ HEPES (pH 7.9)

$50 \mathrm{mM}$ Tris- $\mathrm{HCl}(\mathrm{pH} 7.5)$

$2 \mathrm{mM} \mathrm{MgCl}_{2}$

$10 \mathrm{mM}$ HEPES (pH 7.6)

$10 \mathrm{mM} \mathrm{KOAc}$

$0.5 \mathrm{mM} \mathrm{Mg}(\mathrm{OAc})_{2}$

$5 \mathrm{mM}$ DTT

$20 \mathrm{mM}$ HEPES $\mathrm{NaOH}(\mathrm{pH} 7.9)$

$10 \%[\mathrm{v} / \mathrm{v}]$ Glycerol

$150 \mathrm{mM} \mathrm{NaCl}$

$0.1 \%[\mathrm{v} / \mathrm{v}]$ Triton X-100 
RB High

RB Low

Röder C

Röder D

SAU200

SAU600
$10 \mathrm{mM}$ Tris- $\mathrm{HCl}(\mathrm{pH} 7.5)$

1 mM EDTA NaOH (pH 8.0)

$2 \mathrm{M} \mathrm{NaCl}$

$1 \mathrm{mM}$ DTT

$10 \mathrm{mM}$ Tris- $\mathrm{HCl}(\mathrm{pH} 7.5)$

1 mM EDTA NaOH (pH 8.0)

$12.5 \mathrm{mM} \mathrm{NaCl}$

$1 \mathrm{mM}$ DTT

25\% [v/v] Glycerol

20 mM HEPES (pH 7.9)

$420 \mathrm{mM} \mathrm{NaCl}$

$1.5 \mathrm{mM} \mathrm{MgCl}_{2}$

0.2 mM EDTA NaOH (pH 8.0)

10\% [v/v] Glycerol

20 mM HEPES ( $\mathrm{pH} 7.9$ )

$100 \mathrm{mM} \mathrm{KCl}$

$1.5 \mathrm{mM} \mathrm{MgCl}_{2}$

$0.2 \mathrm{mM}$ EDTA NaOH (pH 8.0)

$0.5 \mathrm{mM}$ DTT

$0.5 \mathrm{mM}$ PMSF

7 M Urea,

$20 \mathrm{mM}$ Sodium acetate ( $\mathrm{pH}$ 5.2)

1 mM EDTA

$200 \mathrm{mM} \mathrm{NaCl}$

2 mM DTT

$7 \mathrm{M}$ Urea

$20 \mathrm{mM}$ Sodium acetate (pH 5.2)

1 mM EDTA

$600 \mathrm{mM} \mathrm{NaCl}$

$2 \mathrm{mM}$ DTT 
TW buffer

Unfolding buffer

Wash buffer

XL-SEC buffer

$1 \mathrm{M}$ Tris- $\mathrm{HCl}$ buffer

1 M HEPES buffer
$50 \mathrm{mM}$ Tris- $\mathrm{HCl}(\mathrm{pH} 7.5)$

$100 \mathrm{mM} \mathrm{NaCl}$

1 mM EDTA

$1 \%[\mathrm{v} / \mathrm{v}]$ Triton X-100

2 mM DTT

$7 \mathrm{M}$ Guanidinium- $\mathrm{HCl}$

$20 \mathrm{mM}$ Tris- $\mathrm{HCl}(\mathrm{pH} 7.5)$

$10 \mathrm{mM}$ DTT

$50 \mathrm{mM}$ Tris- $\mathrm{HCl}(\mathrm{pH} 7.5)$

$100 \mathrm{mM} \mathrm{NaCl}$

1 mM EDTA

1 mM PMSF

$1 \mathrm{mM}$ Benzamidine

2 mM DTT

$0.2 \%[\mathrm{w} / \mathrm{v}] \mathrm{SDS}$

$100 \mathrm{mM} \mathrm{NaCl}$

10 mM HEPES (pH 7.9)

$1 \mathrm{M}$ Tris base, desired $\mathrm{pH}$ adjusted with $37 \%[\mathrm{w} / \mathrm{w}] \mathrm{HCl}$

$1 \mathrm{M}$ HEPES, desired $\mathrm{pH}$ adjusted with $5 \mathrm{M} \mathrm{NaOH}$ or $5 \mathrm{M} \mathrm{KOH}$

\subsubsection{Cell lines}

HeLa S3 (human cervical cancer, Computer cell culture centre, BE) were provided by the facility for Cell Production, Max Planck Institute for Biophysical Chemistry, Göttingen, DE. 


\subsubsection{Chemically competent Escherichia coli strains}

$\begin{array}{ll}\mathrm{DH} 5 \mathrm{\alpha}^{\mathrm{TM}} & \text { Invitrogen, Karlsruhe (DE) } \\ \text { dam-/dcm- } & \text { New England Biolabs, Ipswitch (USA) } \\ \text { BL21-CodonPlus (DE3)-RIL } & \text { Stratagene, La Jolla (USA) }\end{array}$

\subsubsection{Plasmids}

Table 2.1 Plasmids used for chromatin reconstitution and protein expression.

\begin{tabular}{|c|c|c|c|}
\hline Name & Promotor & Resistance & Supplier \\
\hline pET3a_H2A/H2B/H3/H4 & $\mathrm{T} 7$ & Ampicillin & $\begin{array}{l}\text { Karolin Luger, Colorado State Univ., Fort } \\
\text { Collins (USA) (Dyer et al., 2004) }\end{array}$ \\
\hline pET3a_H3A21C $\Delta 1-20$ & T7 & Ampicillin & $\begin{array}{l}\text { Wolfgang Fischle, MPI for Biophysical } \\
\text { Chemistry Göttingen (DE) }\end{array}$ \\
\hline pET3a_H4R23C $\Delta 1-22$ & $\mathrm{~T} 7$ & Ampicillin & $\begin{array}{l}\text { Wolfgang Fischle, MPI for Biophysical } \\
\text { Chemistry Göttingen (DE) }\end{array}$ \\
\hline pET3d_H3K27C C110A & T7 & Ampicillin & $\begin{array}{l}\text { Wolfgang Fischle, MPI for Biophysical } \\
\text { Chemistry Göttingen (DE) }\end{array}$ \\
\hline pUC18_12x200-601 & $\mathrm{T} 7$ & Ampicillin & $\begin{array}{l}\text { Daniela Rhodes, MRC Cambridge (UK) } \\
\text { (Huynh et al., 2005) }\end{array}$ \\
\hline
\end{tabular}

\subsubsection{Enzymes, Proteins and inhibitors}

Antarctic phosphatase

Benzonase

M.SssI CpG methyltransferase

Proteinase Inhibitor Cocktail Complete,

EDTA free

Restriction endonuclease enzymes

T4 DNA ligase

Taq polymerase

Trypsin

Trypsin
New England Biolabs, Ipswitch (USA)

Calbiochem, Darmstadt (DE)

New England Biolabs, Ipswitch (USA)

Roche, Mannheim (DE)

New England Biolabs, Ipswitch (USA)

New England Biolabs, Ipswitch (USA)

Chromatin Biochemistry (MPlbpc) (DE)

Roche, Mannheim (DE)

Promega, Mannheim (DE) 


\subsubsection{Antibodies}

Table 2.2 Antibodies and antisera used for western blot analysis.

\begin{tabular}{|c|c|c|c|}
\hline Name & Host & Supplier & Dilution \\
\hline$\alpha-\mathrm{H}_{3}$ & rabbit, polyclonal & Abcam, ab1791 & $1: 10000$ \\
\hline a-Sept9 & rabbit, polyclonal & $\begin{array}{l}\text { William S. Trimble, SickKids Toronto (CA) } \\
\text { (Surka et al., 2002) }\end{array}$ & $1: 250$ \\
\hline a-Sept2 & rabbit, polyclonal & $\begin{array}{l}\text { William S. Trimble, SickKids Toronto (CA) (Xie } \\
\text { et al., 1999) }\end{array}$ & $1: 250$ \\
\hline a-SF3A3 & rabbit, polyclonal & $\begin{array}{l}\text { Reinhard Lührmann, MPI for Biophysical } \\
\text { Chemistry Göttingen (DE) (Sharma et al., } \\
\text { 2014) }\end{array}$ & $1: 2000$ \\
\hline$\alpha-B d p 1$ & rabbit, polyclonal & $\begin{array}{l}\text { Robert White, University of York (UK) (Fairley } \\
\text { et al., 2003) }\end{array}$ & $1: 500$ \\
\hline a-rabbit HRP & swine, polyclonal & Dako, P0399 & $1: 5000$ \\
\hline
\end{tabular}

\subsubsection{Antibiotics}

Antibiotics:

Ampicillin

Kanamycin

Chloramphenicol

Tetracycline
Final concentrations used:

$100 \mu \mathrm{g} / \mathrm{ml}$

$50 \mu \mathrm{g} / \mathrm{ml}$

$34 \mu \mathrm{g} / \mathrm{ml}$

$12.5 \mu \mathrm{g} / \mathrm{ml}$

\subsubsection{Oligonucleotides}

Oligonucleotides were purchased from SIGMA (Steinheim, DE) and or were kindly provided by Alexandra Stützer (Chromatin Biochemistry Group, Max Planck Institute for Biophysical Chemistry, Göttingen, DE).

- Primers for PCR amplification of scavenger DNA for chromatin array reconstitution: Forward 5' GTTATCCGCTCACAATTCCACACAACATAC 3' Reverse 5' TAATGCAGCTGGCACGACAGGTTTC 3' 
- Oligonucleotides for biotin labeling of 12x200-601 template for chromatin array reconstitution:

EcoRI_3'P 5'-GGGGGGGGATCCGGGGGGGp-3'

EcoRI_5'P_3'bio 5'-pAATTCCCCCCCGGATCCCCCCCC-biotin-3'

\subsubsection{Peptides}

Table 2.3 Peptides used for native chemical ligation.

\begin{tabular}{|c|c|c|c|}
\hline Protein & Sequence & C-terminal & Supplier \\
\hline H3K9me3 & ARTKQTARK $_{\text {me3 }}$ STGGKAPRKQL & $\alpha$-thioester & $\begin{array}{l}\text { Petra Henklein, Charite } \\
\text { Berlin (DE) }\end{array}$ \\
\hline H3K9me2 & ARTKQTARK $_{\text {me2 }}$ STGGKAPRKQL & $\alpha$-thioester & $\begin{array}{l}\text { Petra Henklein, Charite } \\
\text { Berlin (DE) }\end{array}$ \\
\hline H3K9me1 & ARTKQTARK $_{m e 1} \mathrm{STGGKAPRKQL}$ & a-thioester & $\begin{array}{l}\text { Petra Henklein, Charite } \\
\text { Berlin (DE) }\end{array}$ \\
\hline H4K20me3 & SGRGKGGKGLGKGGAKRHRK ${ }_{\text {me3 }} V L$ & $\alpha$-thioester & $\begin{array}{l}\text { Petra Henklein, Charite } \\
\text { Berlin (DE) }\end{array}$ \\
\hline H4K20me1 & SGRGKGGKGLGKGGAKRHRK $\mathrm{me}_{\mathrm{1}} \mathrm{VL}$ & a-thioester & $\begin{array}{l}\text { Petra Henklein, Charite } \\
\text { Berlin (DE) }\end{array}$ \\
\hline H4R3me2 & $\mathrm{SGR}_{\text {me2sym }} \mathrm{GKGGKGLGKGGAKRHRKVL}$ & $\alpha$-thioester & $\begin{array}{l}\text { Petra Henklein, Charite } \\
\text { Berlin (DE) }\end{array}$ \\
\hline
\end{tabular}

\subsubsection{Software}

Adobe Creative Suite 4

Cytoscape 3.3.0

DAVID Bioinformatics Resources 6.6

MaxQuant and Andromeda

Microsoft Office

pLink

ProMass Deconvolution
Adobe Systems, San Hose (USA)

Cytoscape Consortium (Shannon et al., 2003)

National Institute of Allergy and Infectious Diseases (Huang da et al., 2009a, b)

Max Planck Institute for Biochemistry (Cox and Mann, 2008; Cox et al., 2011)

Microsoft Corporation, Redmont (USA)

Broad Institute of Harvard and Massachusetts Institute of Technology, Cambridge (USA) (Purcell et al., 2007)

Thermo Scientific (USA) 
Proteome Discover

$\mathrm{R}$ language for statistical computing

Xlink Analyzer
Thermo Scientific (USA)

R Foundation for Statistical Computing ( $R, 2011)$

(Kosinski et al., 2015; Pettersen et al., 2004)

\subsection{Molecular biology methods}

\subsubsection{Transformation of chemically competent bacteria}

A minimum of $10 \mathrm{ng}$ of the respective plasmids were added to chemical competent cells and incubated for $30 \mathrm{~min}$ on ice. The transformation took place by a heat shock that was introduced by incubating the cell/plasmid mixture for $30 \mathrm{~s}$ at $42{ }^{\circ} \mathrm{C}$. Following, the sample was incubated for $2 \mathrm{~min}$ on ice and $500 \mu \mathrm{l} \mathrm{LB}$-medium were added. The cell suspension was shaken at $37^{\circ} \mathrm{C}$ for 60 min before $100 \mu$ l were plated on antibiotic (2.1.13) containing LB agar plates. The agar plates were incubated overnight at $37^{\circ} \mathrm{C}$.

\subsubsection{Analysis of nucleic acids}

\subsubsection{Concentration determination of nucleic acids}

The concentration of DNA was determined by measuring the absorbance against a reference at $260 \mathrm{~nm}$ using the nanodrop spectrophotometer NanoDrop ND-1000. The DNA concentrations were calculated with the following equation: $1 \mathrm{OD}_{260 \mathrm{~nm}}=50 \mu \mathrm{g} / \mathrm{ml}$ double-stranded DNA (Sambrook, 2001).

\subsubsection{Nucleic acid gel electrophoresis}

DNA fragments were separated according to their size on $0.5 \%$ to $2 \%[\mathrm{w} / \mathrm{v}]$ agarose gels in a TBE (or TB) buffer system (Sambrook, 2001). In general samples containing $300 \mathrm{ng}$ of DNA and $1 \mathrm{x}$ loading dye were loaded onto an agarose gel. Using a horizontal DNA electrophoresis cell (Sub-Cell-GT) $90 \mathrm{~V}$ to $120 \mathrm{~V}$ were applied for approximately $60 \mathrm{~min}$. As a size reference $5 \mu \mathrm{l}$ of a $1 \mathrm{~kb}$ Plus DNA Ladder run with all samples in parallel. Subsequently, the DNA was stained with $0.5 \mu \mathrm{g} / \mathrm{ml}$ ethidium bromide for $20 \mathrm{~min}$ and visualized on the ChemiDoc MP imaging system. 


\subsubsection{DNA precipitation}

\subsubsection{Ethanol}

DNA was incubated with $0.3 \mathrm{M}$ sodium acetate $(\mathrm{pH} 5.2)$ and 3 volumes ice cold ethanol (99\% $[\mathrm{v} / \mathrm{v}])$ overnight at $-20^{\circ} \mathrm{C}$ (Sambrook, 2001). Afterwards, the mixture was centrifuged at $16000 \mathrm{x}$ $\mathrm{g}$ for $30 \mathrm{~min}$ at $4{ }^{\circ} \mathrm{C}$. The pellet was washed with $70 \%[\mathrm{v} / \mathrm{v}]$ ethanol, air dried and dissolved in double-distilled water $\left(\mathrm{ddH}_{2} \mathrm{O}\right)$.

\subsubsection{Isopropanol}

DNA was mixed with $0.3 \mathrm{M}$ sodium acetate $(\mathrm{pH} 5.2)$ and 0.7 volumes isopropanol and centrifuged immediately at $16000 \times \mathrm{g}$ for $30 \mathrm{~min}$ at $4{ }^{\circ} \mathrm{C}$. The pellet was washed with $70 \%[\mathrm{v} / \mathrm{v}$ ] ethanol, air dried and dissolved in $\mathrm{ddH}_{2} \mathrm{O}$ (Sambrook, 2001).

\subsubsection{PEG-6000}

In order to separate large DNA fragments from smaller ones, DNA was sequentially precipitated using PEG (Lis and Schleif, 1975). For this, DNA was incubated with $0.5 \mathrm{M} \mathrm{NaCl}$ and $4 \%$ [v/v] PEG-6000 for $10 \mathrm{~min}$ at RT. The mixture was centrifuged at $16000 \times \mathrm{g}$ for $15 \mathrm{~min}$ at $4{ }^{\circ} \mathrm{C}$. The supernatant was collected and the PEG-6000 concentration was increased in 1\% steps up to $9 \%$ $[\mathrm{v} / \mathrm{v}]$ by repeating the centrifugation step of the resulting supernatants. The pellets resulting from each fraction were washed with ice cold $70 \%[\mathrm{v} / \mathrm{v}]$ ethanol, air dried at RT and dissolved in $\mathrm{ddH}_{2} \mathrm{O}$.

\subsubsection{Preparation of DNA templates for chromatin array assembly}

\subsubsection{Preparation of biotinylated 12x200-601 DNA template}

For amplification of pUC18_12x200-601 (2.1.10) the plasmid was transformed into chemically competent dam-/dcm- E. coli cells (2.1.9). $200 \mathrm{ml}$ ampicillin containing LB medium were inoculated with a single bacteria colony at $37^{\circ} \mathrm{C}$ and $120 \mathrm{rpm}$ overnight. For large scale preparation, 2.5 I of LB medium supplemented with ampicillin were inoculated with $5 \mathrm{ml}$ of the overnight culture. This culture was grown at $37^{\circ} \mathrm{C}$ overnight, shaking at $120 \mathrm{rpm}$. Cells were 
harvested by centrifugation for $15 \mathrm{~min}$ at $6000 \mathrm{xg}$ at $4{ }^{\circ} \mathrm{C}$. Cell lysis and DNA purification were done using the NucleoBond PC 10000 kit according to the manufacturer's instructions.

The 12x200-601 sequence was obtained by digestion of purified plasmid DNA using the restriction endonuclease enzymes Bsrl, EcoRl, Haell, and Ddel. For the digest, $300 \mathrm{U}$ of each enzyme per mg of DNA were used. The reaction took place in 1 x NEBuffer 2.1 (New England Biolabs) at $37^{\circ} \mathrm{C}$ overnight. After inactivation of the restriction endonuclease enzymes (at $80^{\circ} \mathrm{C}$ for $20 \mathrm{~min}$ ) the $2.4 \mathrm{~kb} 12 \times 200-601$ DNA template was separated by PEG precipitation (2.2.3.3).

The 12x200-601 DNA was biotinylated by ligation of a biotinylated ds oligonucleotide to the 5' EcoRI overhang of the DNA. An equimolar mix of the "EcoRI_3'P" and "EcoRI_5'P_3'bio" oligonucleotides were annealed by heating them to $95^{\circ} \mathrm{C}$ and allowing them to cool down to RT. Prior ligation, the 12x200-601 DNA was dephosphorylated using 1 U Antarctic phosphatase per $2 \mu \mathrm{g}$ DNA according to the manufacturer's instructions. The ligation was performed using a tenfold molar excess of the (ds) oligonucleotide. The reaction was carried out with $2.5 \mathrm{U}$ T4 DNA ligase per $1 \mu \mathrm{g}$ of DNA for at least three hours at RT. The biotinylated DNA was extracted by addition of an equal volume of phenol:chlorophorm:isoamyl alcohol [25:24:1] in phase lock heavy tubes followed by isopropanol precipitation (2.2.3.2) of the aqueous phase.

\subsubsection{Methylation of biotinylated 12x200-601DNA template}

CpG sites of the 12x200-601 DNA template were methylated using the M.Sssl methyltransferase. $500 \mu \mathrm{g}$ of DNA were incubated with $240 \mathrm{U}$ of $\mathrm{M}$.Sssl in $50 \mathrm{mM} \mathrm{NaCl}, 10 \mathrm{mM}$ EDTA, $10 \mathrm{mM}$ Tris- $\mathrm{HCl}, \mathrm{pH} 7.9$ and $160 \mu \mathrm{M}$ SAM at $37^{\circ} \mathrm{C}$. After three hours, $80 \mu \mathrm{M}$ SAM and $100 \mathrm{U}$ of M.Sssl were added, the reaction was left to proceed overnight at $37^{\circ} \mathrm{C}$. To ensure complete methylation another $130 \mu \mathrm{M}$ of SAM and $80 \mathrm{U}$ of M.SssI were added and the reaction was incubated for four more hours. The DNA was extracted with PCI [25:24:1] and precipitated using ethanol (2.2.3.1).

The efficiency of methylation was tested using restriction endonucleases unable to cleave at methylated CpG sites, like BsaAl, according to the manufacturer's instructions.

\subsubsection{Preparation of scavenger DNA}

The scavenger DNA is a 147 bp sequence of the backbone of the pUC18_12x200-601 plasmid (2.1.10) that does not contain the 200-601 nucleosome positioning sequence. The DNA was amplified by polymerase chain reaction (PCR) according to the following PCR mix and PCR cycle program: 
PCR mix $(100 \mu \mathrm{l}):$

$100 \mathrm{mM}$

$500 \mathrm{mM}$

$0.8 \%[\mathrm{v} / \mathrm{v}]$

$0.2 \mathrm{mM}$ (each)

$0.2 \mu \mathrm{m}$ (each)

$1.5 \mathrm{mM}$

$50 \mathrm{ng}$

$20 \mathrm{U} / \mathrm{ml}$
Tris- $\mathrm{HCl} \mathrm{pH} 8.8$

$\mathrm{KCl}$

NP-40

dNTP mix

primers

$\mathrm{MgCl}_{2}$

DNA template

Taq polymerase
PCR cycle program:

$\begin{array}{ll}\text { Temperature } & \text { Time } \\ 95^{\circ} \mathrm{C} & 2 \mathrm{~min} \\ 95^{\circ} \mathrm{C} & 30 \mathrm{~s} \\ 68^{\circ} \mathrm{C} & 30 \mathrm{~s} \\ 72{ }^{\circ} \mathrm{C} & 30 \mathrm{~s} \\ 72^{\circ} \mathrm{C} & 1 \mathrm{~min} \\ 10^{\circ} \mathrm{C} & \infty\end{array} 40 \mathrm{x}$

The PCR product was extracted with PCI [25:24:1] and subsequently precipitated with isopropanol (2.2.3.2).

\subsection{Protein biochemistry methods}

\subsubsection{Detection and analysis of proteins}

\subsubsection{Concentration determination of proteins}

The concentration of histone proteins was determined by UV adsorption at $276 \mathrm{~nm}$ on a NanoDrop ND-1000 using published molar extinction coefficients listed in table 2.4 (Luger et al., 1999). The protein concentration of nuclear extracts was measured by UV adsorption at $280 \mathrm{~nm}$ assuming $1 \mathrm{OD}_{280}=1 \mathrm{mg} / \mathrm{ml}$.

Table 2.4 Values used for the determination of histone concentrations.

\begin{tabular}{lll}
\hline Protein & Molecular weight (Da) & Molar extinction coefficient $\left(\mathbf{M}^{-1} \mathbf{c m}^{-1}\right)$ \\
\hline H2A & 13960 & 4050 \\
H2B & 13774 & 6070 \\
H3 & 15273 & 4040 \\
H4 & 11236 & 5400 \\
\hline
\end{tabular}




\subsubsection{Sodium dodecyl sulfate polyacrylamide gel electrophoresis (SDS-PAGE)}

Proteins were separated and visualized by discontinuous polyacrylamide gel electrophoresis in presence of SDS based on the method of Laemmli (Laemmli, 1970) according to standard protocols (Gallagher, 2006). 15\% or $18 \%$ Tris-glycine resolving gels in combination with a $4 \%$ stacking gel were used for the analysis of histone proteins. The gels were cast and run using the Mini-PROTEAN electrophoresis system according to instructions provided by the manufacturer. Before loading the protein samples onto the gel, they were boiled for $5 \mathrm{~min}$ at $95^{\circ} \mathrm{C}$ in SDS sample buffer. As a reference $5 \mu \mathrm{l}$ of SeeBlue Plus2 pre-stained protein standard was used. Protein samples were run in $1 \times$ SDS running buffer at constant current at $15 \mathrm{~mA}(250 \mathrm{~V}$ maximum) per gel until the bromophenol blue front reached the bottom of the gel.

The separation of complex protein mixtures, including samples for mass spectrometry analysis, was performed using NuPAGE Novex 4-12\% Bis-Tris gradient gels in combination with the NuPAGE Pre-cast system according to the manufacturer instructions. For all runs $1 \times$ MOPS buffer, antioxidant, LDS sample buffer and reducing agent were used.

\subsubsection{Coomassie staining of protein SDS-PAGE gels}

Proteins separated on SDS-PAGE gels were either stained overnight with Colloidal Coomassie stain solution or for 10 to 20 min with Imperial Protein Stain. Gels were destained with $\mathrm{dd}_{2} \mathrm{O}$ for at least four hours.

\subsubsection{Silver staining of protein SDS-PAGE gels}

Silver staining was performed according to the protocol of Blum at al. (Blum H., 1987). For silver staining of proteins separated on SDS-PAGE gels all incubation steps took place at RT on a shaker. Gels were incubated in fixative solution $(50 \% \mathrm{MetOH}, 12 \% \mathrm{HOAc})$ overnight. Afterwards, they were incubated twice in $50 \% \mathrm{EtOH}$ followed by an incubation in $30 \% \mathrm{EtOH}$ for 20 min each. Gels were sensitized in $0.8 \mathrm{mM}$ sodium thiosulfate for exactly $60 \mathrm{~s}$. Sodium thiosulfate solution was removed by three wash steps with $\mathrm{ddH}_{2} \mathrm{O}, 20 \mathrm{sec}$ each time. Subsequently, the gels were incubated in silver nitrate solution ( $2 \mathrm{~g} / \mathrm{l}$ silver nitrate, $0.026 \%$ formaldehyde) for $20 \mathrm{~min}$. Before development the gels were washed three times with $\mathrm{dd}_{2} \mathrm{O}$. The water was replaced by developer solution (60 $\mathrm{g} / \mathrm{l} \mathrm{Na} \mathrm{NO}_{3}, 0.0185 \%$ formaldehyde, $16 \mu \mathrm{M}$ sodium thiosulfate) until protein bands on the gel turned brown. The process was terminated by addition of fixative solution. 


\subsubsection{Western blot}

Immunoblotting of protein samples separated by SDS-PAGE was done using the MiniTrans Blot system. Proteins were transferred onto nitrocellulose membranes. For this, the membrane was directly laid onto the SDS-PAGE gel and both were sandwiched between three layers of Whatman paper equilibrated in $1 \times$ transfer buffer. Proteins were blotted onto the membrane in 1 $\mathrm{x}$ transfer buffer for one hour at $100 \mathrm{~V}$ at $4{ }^{\circ} \mathrm{C}$. The membrane was rinsed with $1 \mathrm{x}$ PBS-T and subsequently blocked with $5 \%$ milk for $30 \mathrm{~min}$ at RT. Afterwards, the membrane was incubated with a primary antibody overnight at $4{ }^{\circ} \mathrm{C}$. All antibodies were diluted in $5 \%$ milk according to table 2.2. In order to remove non-bound antibodies, the membrane was washed three times with $1 \mathrm{x}$ PBS-T for 10 minutes each. The membrane was then incubated with a secondary antibody at RT for at least two hours, followed by three wash steps as described above. The detection was done using an enhanced chemiluminescence system (ECL) according to the manufacturer's instructions. The imaging was done using the ChemiDoc MP System.

\subsubsection{Recombinant proteins}

\subsubsection{Expression and purification of recombinant histone proteins}

Purification of Xenopus leavis histones was performed as described before (Luger et al., 1999) with certain alterations.

The respective plasmids (2.1.10) were transformed into BL21-CodonPlus (DE3)-RIL E. coli cells. $200 \mathrm{ml}$ of ampicillin containing 2xYT medium were inoculated with a single bacteria colony overnight at $37^{\circ} \mathrm{C}$ and shaking at $120 \mathrm{rpm}$. The overnight culture was used to inoculate $2 \mathrm{xYT}$ medium (1:1000) that was incubated at $37{ }^{\circ} \mathrm{C}$ and shaking at $120 \mathrm{rpm}$. When the culture reached an $\mathrm{OD}_{600}=0.5$ expression was induced with $0.2 \mathrm{mM}$ IPTG and the culture was incubated for four more hour. Afterwards, cells were harvested by centrifugation at $6000 \times \mathrm{g}$ for $15 \min$ at $4^{\circ} \mathrm{C}$.

In order to purify the histone proteins inclusion bodies were isolated. All steps were performed at $4{ }^{\circ} \mathrm{C}$. The cell pellet resulting from 2 I bacteria culture was resuspended in $100 \mathrm{ml}$ Wash buffer and cells were lysed using a Canadian Press. After 4 cycles the suspension was centrifuged for $20 \mathrm{~min}$ at $23000 \mathrm{x}$. The pellet was resuspended in $100 \mathrm{ml} \mathrm{TW}$ buffer and centrifuged for $20 \mathrm{~min}$ at $23000 \times \mathrm{g}$. The wash step was repeated twice. In order to remove Triton X-100 the inclusion bodies were additionally washed two times with Wash buffer.

To extract histone proteins from inclusion bodies the pellet was soaked with $1 \mathrm{ml}$ DSMO, minced and incubated at RT for $30 \mathrm{~min}$. Next, $30 \mathrm{ml}$ of Unfolding buffer were added. The suspension 
was stirred for $1 \mathrm{~h}$ at RT and then centrifuged at $23000 \times \mathrm{g}$ for $20 \mathrm{~min}$. The resulting pellet was re-extracted by adding another $10 \mathrm{ml}$ of Unfolding buffer for $30 \mathrm{~min}$. After another centrifugation step both supernatants were pooled and dialyzed three times against 2 I of SAU200.

For purification of the histone proteins a Q-Sepharose XK26/20 and a SP-Sepharose XK26/20 column were connected in series and equilibrated with SAU200 before the extracted proteins were loaded onto the column combination. After the input has passed the Q-Sepharose column was disconnected and washing was continued only with the SP-Sepharose column. Histone proteins were eluted applying a linear gradient from $0 \%$ to $100 \%$ of SAU600 over one column volume. Histone containing elution fractions were pooled, dialyzed three times against $2 \mathrm{mM}$ DTT, lyophilized and stored at $-80^{\circ} \mathrm{C}$.

For H4R23C $\Delta 1-22$ additional alterations to the protocol were implemented. The expression of the protein was not induced before the bacteria culture reached an $\mathrm{OD}_{600}=1$ and induction took place for one hour. Protein extraction from inclusion bodies was extended to two hours. H4R23C $\Delta 1-22$ proteins were eluted from the SP column applying a step gradient that was first set to $20 \%$ of SAU600, until the UV absorption at $276 \mathrm{~nm}$ reached again the value that was detected when the column was equilibrated, followed by a gradient from $20 \%$ to $100 \%$ of SAU600 over one column volume. The elution of proteins was visible by two peaks monitored at $276 \mathrm{~nm}$. Elution fractions of the first peak were kept whereas the elution fractions of the second peak were pooled, diluted 1:3 with SAU0 (the same as SAU200 but without $\mathrm{NaCl}$ ) and reloaded onto the newly equilibrated SP column in a second run. H4R23C $\triangle 1-22$ was again eluted with $20 \%$ SAU600. Histone containing elution fractions of the first and the second run were pooled, dialyzed three times against $2 \mathrm{mM}$ DTT, lyophilized and stored at $-80^{\circ} \mathrm{C}$.

\subsubsection{Introduction of posttranslational modifications by native chemical ligation}

The introduction of methylation sites at the $\mathrm{N}$-termini of histone $\mathrm{H} 3$ and histone $\mathrm{H} 4$ was achieved by native chemical ligation as described before (Shogren-Knaak and Peterson, 2004) with a few alterations. Synthetic $\mathrm{N}$-terminal histone polypeptides containing modified amino acid residues were ligated to the truncated histones H3A21C $\Delta 1-20$ or H4R23C $\Delta 1-22$, respectively. The reaction took place using a molar histone:peptide ratio of $1: 5$ with a final peptide concentration of $2 \mathrm{mM}$.

Briefly, the respective amount of histone proteins were dissolved in ligation buffer (6 M guanidinium- $\mathrm{HCl}, 100 \mathrm{mM} \mathrm{KP}$, pH 7.9, $20 \mathrm{mM}$ TECEP, $50 \mathrm{mM}$ MPAA) and added to the lyophilized peptides. The reaction mixture was incubated at RT for at least $40 \mathrm{~h}$ and finally dialyzed three times against SAU200. The reaction mixture was loaded onto a HiTrap SP HP $1 \mathrm{ml}$ column equilibrated with SAU200. The histone proteins were eluted by applying a linear 
gradient from $0 \%$ to $100 \%$ of SAU600 over 20 column volumes. Fractions containing full length histone proteins were pooled, dialyzed three times against $2 \mathrm{mM} \mathrm{DTT}$, lyophilized and stored at - $80{ }^{\circ} \mathrm{C}$. The progress of the reaction was monitored by SDS-PAGE and confirmed by electrospray mass spectrometry.

\subsubsection{Introduction of methyl lysine analogs}

The introduction of methyl lysine analogs (MLA) was achieved by performing an alkylation reaction as described before (Simon, 2010). All methylation states of H3K27 were produced using the histone mutant H3K27C, C110A that was dissolved in Alkylation buffer (4 M guanidine hydrochloride, $1 \mathrm{M}$ HEPES $\mathrm{pH}$ 7.8, $10 \mathrm{mM}$ DL-methionine). All alkylation reactions were performed with $10 \mathrm{mg}$ of H3K27C, C110A in a final volume of $1 \mathrm{ml}$.

The introduction of the tri-methyl lysine analog was performed at $50{ }^{\circ} \mathrm{C}$ in presence of $400 \mathrm{mM}$ (2-bromoethyl)-tri-methylammonium bromide and $20 \mathrm{mM}$ DTT in the dark with occasional flicking. After $5 \mathrm{~h}$ the reaction was quenched with $50 \mu \mathrm{l} 2$-mercaptoethanol for $30 \mathrm{~min}$ at RT. The MLA histones were purified using a PD-10 column according to the manufacturer's instructions using $2 \mathrm{mM}$ 2-mercaptoethanol for equilibration and elution.

The introduction of the di-methyl lysine analog was performed at RT in the presence of $50 \mathrm{mM}$ (2-chloroethyl)-di-methylammonium chloride and $20 \mathrm{mM}$ DTT in the dark with occasional flicking. After $2.5 \mathrm{~h}$ additional $50 \mathrm{mM}$ of (2-chloroethyl)-di-methylammonium chloride were added to the reaction mixture. After a total of $4.5 \mathrm{~h}$ the reaction was quenched with $50 \mu \mathrm{l} 2$-mercaptoethanol for 30 min at RT. The MLA histones were purified using a PD-10 column as described for the trimethyl lysine analog.

The introduction of the mono-methyl lysine analog was performed at RT in the presence of 300 $\mathrm{mM}$ (2-chloroethyl)-methylammonium chloride and $20 \mathrm{mM}$ DTT in the dark with occasional flicking. After $5 \mathrm{~h}$ the reaction was quenched with $50 \mu \mathrm{l} 2$-mercaptoethanol for $30 \mathrm{~min}$ at RT. The MLA histones were purified as described for the tri-methyl lysine analog.

\subsubsection{Preparation of recombinant chromatin}

\subsubsection{Assembly of histone octamers}

The assembly of octamers composed of the histone proteins $\mathrm{H} 2 \mathrm{~A}, \mathrm{H} 2 \mathrm{~B}, \mathrm{H} 3$ and $\mathrm{H} 4$ were performed according to a generalized protocol (Luger et al., 1999). Each of the lyophilized histone proteins were completely dissolved in Unfolding buffer to a concentration of $2 \mathrm{mg} / \mathrm{ml}$. All four histone variants were mixed in equimolar ratios and adjusted to a final concentration of 1 
$\mathrm{mg} / \mathrm{ml}$. The protein mixture was dialyzed three times against 2 I RB high at $4{ }^{\circ} \mathrm{C}$. Afterwards, the assembled octamers were concentrated to a final volume of $50 \mu \mathrm{l}$ using Amicon Ultra centrifugal filter devices and loaded onto a HiLoad 16/60 Superdex 200 prep grade gel filtration column installed on an ÄKTA FPLC system. The octamer-containing fractions were pooled and $50 \%$ $[\mathrm{v} / \mathrm{v}]$ glycerol was added for long term storage at $-20^{\circ} \mathrm{C}$.

\subsubsection{Chromatin reconstitution by salt dialysis}

The reconstitution of oligonucleosomal chromatin arrays composed of 12 nucleosomes was performed according to a previously established protocol by dialysis from high salt to low salt (Luger et al., 1999). The concentration of octamers in RB high buffer was determined by UV spectroscopy $\left(0.45 \mathrm{OD}_{276} \mathrm{~nm}=1 \mathrm{mg} / \mathrm{ml}\right.$ octamer $\left.=92.2 \mu \mathrm{M}\right)$. The DNA template described at 2.2.4.1 and histone octamers (2.3.3.1) were mixed in a molar ratio of 1.1(-1.3):1 (octamer:DNA) in RB high buffer. Additionally, an equimolar amount of scavenger DNA (2.2.4.3) was added to bind excess octamer. The salt gradient from $2 \mathrm{M} \mathrm{NaCl}$ to $12.5 \mathrm{mM} \mathrm{NaCl}$ was achieved by continuously replacing $\mathrm{RB}$ high with $\mathrm{RB}$ low over $30 \mathrm{~h}$ using a peristaltic pump.

The reconstitution was analyzed by agarose gel electrophoresis on a $0.5 \%[\mathrm{w} / \mathrm{v}] \mathrm{gel}$ in $0.2 \times \mathrm{TB}$ buffer at $4{ }^{\circ} \mathrm{C}$. Furthermore, the saturation level of reconstituted chromatin arrays was analyzed by restriction digest using the Aval restriction enzyme and analytical ultracentrifugation (2.3.4.2).

\subsubsection{Molecular characterization of recombinant chromatin}

\subsubsection{Aval digest}

The saturation level of reconstituted chromatin arrays was analyzed by a digest with the restriction enzyme Aval, which cuts within the 200-601 nucleosome positioning sequence of the chromatin DNA template (2.2.4.1) only when not occupied by histone octamers.

$1 \mu \mathrm{g}$ of chromatin arrays (2.3.3.2) and $0.5 \mu \mathrm{g}$ of the DNA template (2.2.4.1) were incubated with $20 \mathrm{U}$ of Aval in a total volume of $30 \mu \mathrm{l}$ for $3 \mathrm{~h}$ at $37^{\circ} \mathrm{C}$ according to the manufacturer instructions. The digested samples were analyzed by agarose gel electrophoresis on a $2 \%$ [w/v] gel. 


\subsubsection{Analytical ultracentrifugation}

For the analysis of oligonucleosomes by the sedimentation velocity method using a Beckmann $\mathrm{XL}$-A centrifuge, $400 \mu \mathrm{l}$ containing $0.5 \mathrm{OD}_{260}$ oligonucleosomes were prepared in RB low buffer. The buffer sector of the cells was filled with $400 \mu \mathrm{l}$ RB low buffer, while the sample sector was filled with $390 \mu \mathrm{l}$ of the chromatin containing solution. A speed of $15.000 \mathrm{rpm}$ was used for sedimenting the oligonucleosomes within a series of 25-35 scans. Data analysis was performed using the software UltraScan II (Demeler). In general, after assignment of meniscus, cell bottom and plateau for each of the analyzed cells, spikes and time-invariant noise as well as unsatisfactory scans (where a sigmoidal sedimentation curve cannot be assigned) were manually removed. Initial sedimentation distribution was calculated for the viscosity of RB low and the sample frictional coefficient. The initial result was used to perform van Holde-Weischet boundary analysis on each of the sedimentation scans. The resulting boundary analysis was used for plotting the distribution of corrected sedimentation coefficients.

\subsection{Cell culture and metabolic labeling}

\subsubsection{Stable isotope labeling by amino acids in cell culture (SILAC) of HeLa S3 cells}

HeLa S3 cells were cultivated as described before (Nikolov et al., 2011). The cells were grown in custom High Glucose Dulbecco's Modified Eagle's Medium (DMEM) lacking arginine and lysine. The medium was supplemented with either light arginine (Arg0) and lysine (Lys0) or heavy amino acids (Arg6 and Lys4) (2.1.5) to a final concentration equivalent to $50 \mathrm{mg} / \mathrm{ml}$ light amino acids. In addition, $1 / 10$ volume of dialyzed fetal bovine serum and $1 \times$ Penicillin/Streptomycin were added to the medium.

The culture was started by bringing $10^{8}$ cells into a spinner flask containing $100 \mathrm{ml}$ medium. Cells were grown at $37{ }^{\circ} \mathrm{C}, 5 \% \mathrm{CO}_{2}$ and $95 \%$ relative humidity. Over at least six passages the culture was expanded to $1.5 \mathrm{I}$ and finally transferred to a $5 \mathrm{I}$ bioreactor where cells were grown under standard conditions $\left(2 \times 10^{6} \mathrm{cells} / \mathrm{ml}\right.$, continuously addition of synthetic air and dissolved oxygen level kept at $\mathrm{pO}_{2}=20$ using a feedback monitoring system).

\subsubsection{Preparation of nuclear extracts}

The preparation of nuclear extracts was done as described before (Dignam et al., 1983). Generally, $10^{10}$ SILAC labeled HeLa S3 cells were harvested by centrifugation for $5 \mathrm{~min}$ at 2000 rpm. The pellet was washed twice with 1 x PBS and resuspended in 1.25 volumes MC buffer 
supplemented with $1 / 500$ volumes $0.25 \mathrm{M}$ DTE and EDTA-free protease inhibitor cocktail according to the manufacturer's instructions. The suspension was incubated on ice for 5 min and homogenized using a $50 \mathrm{ml}$ Dounce homogenizer, performing 18 strokes. Nuclei were pelleted by centrifugation at $18000 \times \mathrm{g}$ for $5 \mathrm{~min}$ at $4{ }^{\circ} \mathrm{C}$. The pellet was resuspended in 1.3 volumes Röder C buffer supplemented with $1 / 500$ volumes 0.25 M DTE and 1/200 volumes $0.1 \mathrm{M}$ PMSF. Nuclei were disrupted using a Dounce homogenizer, performing 20 strokes. The suspension was stirred for $40 \mathrm{~min}$ at $4{ }^{\circ} \mathrm{C}$ and finally centrifuged at $30000 \times \mathrm{g}$ for $30 \mathrm{~min}$ at $4{ }^{\circ} \mathrm{C}$. The supernatant was frozen in liquid nitrogen and stored at $-80^{\circ} \mathrm{C}$.

Three nuclear extracts were prepared from individual cell cultures for each labeling, heavy and light labeled. The three extracts were pooled according to their labeling and dialyzed three times against 2 I of Röder D buffer and centrifuged at $9000 \times \mathrm{g}$ for 2 min at $4{ }^{\circ} \mathrm{C}$. The supernatant was aliquoted, frozen in liquid nitrogen and stored at $-80^{\circ} \mathrm{C}$.

\subsection{Biochemical binding assays}

\subsubsection{Chromatin affinity purification}

Chromatin affinity purification using differentially SILAC-labeled HeLa S3 nuclear extracts was essentially done as described before (Nikolov et al., 2011). All steps were performed at $4{ }^{\circ} \mathrm{C} .40$ $\mu \mathrm{g}$ of chromatin arrays of two different modification states were separately immobilized on $160 \mu \mathrm{l}$ paramagnetic streptavidin coated beads in PD150 buffer supplemented with $1 \mathrm{mg} / \mathrm{ml}$ BSA overnight. Chromatin-bound beads were washed three times with $800 \mu$ I PD150 wash buffer. In parallel, $1 \mathrm{ml}$ of heavy and light labeled nuclear extract was incubated with $40 \mu \mathrm{l}$ paramagnetic streptavidin coated beads for $1 \mathrm{~h}$ to remove unspecific binding proteins. $4 \mathrm{mg}$ of nuclear extract were separated from beads and incubated with chromatin-bound beads. After four hours of incubation, beads were washed three times with PD150 wash buffer and proteins were eluted with 1 x NuPAGE LDS Sample buffer supplemented with $2 \mathrm{mM} \mathrm{MgCl}_{2}$ and $10 \mathrm{mM}$ Tris-HCL pH 7.9. Eluates from pull-downs done with heavy and light nuclear extracts were mixed in a 1:1 ratio. To ensure complete release of all chromatin-bound proteins and to improve the following separation step, the mixed sample was digested with $1250 \mathrm{U}$ benzonase for $45 \mathrm{~min}$ at $37^{\circ} \mathrm{C}$. After addition of $1 \times$ NuPAGE reducing agent the samples were heated at $70{ }^{\circ} \mathrm{C}$ for $10 \mathrm{~min}$ before loading onto a $4 \%$ - 12\% SDS-PAGE gel. After separation, the gel was stained with Coomassie overnight. 


\subsubsection{Protein-protein cross-linking on beads}

$150 \mu \mathrm{g}$ of chromatin arrays (2.3.3.2) were immobilized on $600 \mu \mathrm{l}$ paramagnetic streptavidin coated beads in PD150 buffer overnight. Chromatin-bound beads were washed 3 times with 3 $\mathrm{ml}$ PD150 wash buffer and incubated with $2 \mathrm{ml}$ pre-cleared nuclear extract $(2.5 .1)$ at $4{ }^{\circ} \mathrm{C}$. After $3 \mathrm{~h} 20 \mu \mathrm{M}$ BS3 was added to the sample, which was incubated for $4 \mathrm{~h}$ in total. Chromatin-bound beads were washed once with $3 \mathrm{ml}$ PD150 wash buffer to remove non-specifically bound proteins. To chemically cross-link chromatin-bound proteins beads were incubated twice in $2 \mathrm{ml}$ PD150 wash buffer supplemented with $200 \mu \mathrm{M}$ BS3 for 10 min at RT. Cross-linked proteins and chromatin were eluted from the beads by adding $55 \mu$ Injection buffer and $1250 \mathrm{U}$ benzonase followed by incubation for $45 \mathrm{~min}$ at $37^{\circ} \mathrm{C}$. Afterwards the sample was heated to $70{ }^{\circ} \mathrm{C}$, mixed several times using a vortex mixer, and finally separated from magnetic beads using a magnetic rack.

$50 \mu \mathrm{l}$ of the sample were injected onto a Superdex 200 column equilibrated with XL-SEC buffer. For separation, a flow rate of $55 \mu \mathrm{l} / \mathrm{min}$ was applied and $50 \mu \mathrm{l}$ fractions were collected. Elution fractions were pooled according to the peaks of the elution profile monitored at $215 \mathrm{~nm}$ and analyzed by silver staining of SDS-PAGE gels and western blot analysis.

Proteins of the pooled fractions containing cross-linked material were precipitated using acetone at $-20^{\circ} \mathrm{C}$ overnight. Pellets were resuspended in 1\% RapiGest before tryptic digestion (2.6.2). Subsequently, acetonitrile was added to the digested samples to a final proportion of $10 \%$ [v/v]. Following, the sample was injected onto a Superdex peptide column that was equilibrated with $30 \%$ acetonitrile and $0.1 \%$ formic acid. For separation, a flow rate of $55 \mu \mathrm{l} / \mathrm{min}$ was applied and $50 \mu \mathrm{l}$ fractions were collected. Elution fractions were pooled according to the peaks of the elution profile monitored at $215 \mathrm{~nm}$ and subjected to mass spectrometric analysis (2.6.4).

\subsection{Mass spectrometry methods}

\subsubsection{In-gel proteolysis of proteins and peptide extraction}

In-gel digest of proteins and the following extraction of peptides were essentially done as described before (Shevchenko et al., 2006; Shevchenko et al., 1996). All steps of the in-gel digest were performed at $25^{\circ} \mathrm{C}$ under shaking at $1050 \mathrm{rpm}$. Each SDS-PAGE gel lane was sliced into either 11 or 22 equidistant gel pieces regardless of staining. In order to reduce proteins embedded in gel, slices were dehydrated with acetonitrile and rehydrated with $100 \mathrm{mM}$ dithiothreitol (DTT) in $50 \mathrm{mM}$ ammonium hydrogen carbonate $\left(\mathrm{NH}_{4} \mathrm{HCO}_{3}\right) \mathrm{pH} 8.0$ and incubated at $56{ }^{\circ} \mathrm{C}$ for $50 \mathrm{~min}$. After dehydrating the gel slices in acetonitrile, reduced cysteine residues 
were alkylated in $60 \mathrm{mM}$ iodoacetamide, $50 \mathrm{mM} \mathrm{NH}_{4} \mathrm{HCO}_{3} \mathrm{pH} 8.0$ for 20 min at $26{ }^{\circ} \mathrm{C}$ in the dark. Gel pieces were washed afterwards with $50 \mathrm{mM} \mathrm{NH}_{4} \mathrm{HCO}_{3} \mathrm{pH} 8.0$, which was replaced by acetonitrile to completely dehydrate them. Finally, gel pieces were rehydrated by incubation in $20 \mu \mathrm{l}$ digestion buffer (12.5 $\mathrm{ng} / \mu \mathrm{l}$ trypsin (Roche), $41.7 \mathrm{mM} \mathrm{NH}_{4} \mathrm{HCO}_{3} \mathrm{pH} 8.0,4.2 \mathrm{mM} \mathrm{CaCl}$ ) for $40 \mathrm{~min}$ on ice. $60 \mu \mathrm{l}$ digestion buffer without trypsin were added to the gel pieces and incubated at $37^{\circ} \mathrm{C}$ overnight.

For the extraction of peptides from gel pieces incubation steps were performed at $37^{\circ} \mathrm{C}$, shaking

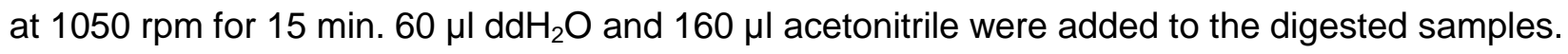
Once gel pieces were shrank, the supernatants were collected and $100 \mu \mathrm{l} \%$ formic acid were added to the dehydrated gel pieces. Subsequently, gel pieces were dehydrated again by adding acetonitrile and the supernatants were collected and pooled with those from the first extraction step. Supernatants were evaporated to dryness in a centrifugal concentrator. Dried peptides were kept at $-20{ }^{\circ} \mathrm{C}$ or were immediately dissolved in loading buffer (5\% [v/v] acetonitrile, $1 \%$ [v/v] formic acid) and analyzed by LC-MS/MS (2.6.3).

\subsubsection{In-solution proteolysis of proteins}

For the in-solution digest of proteins all reagents used were dissolved in $25 \mathrm{mM}$ ammonium bicarbonate $\mathrm{pH}$ 8.5. Proteins precipitated with acetone were dried in a centrifugal concentrator. The pellet was dissolved in $5 \mu \mathrm{l}$ \% RapiGest (sodium 3-[(2-methyl-2-undecyl-1,3-dioxolan-4yl)methoxy]-1-propanesulfonate) (Yu et al., 2003). In order to reduce the proteins $5 \mu \mathrm{l} 50 \mathrm{mM}$ DTT were added and the sample was incubated for $1 \mathrm{~h}$ at $37^{\circ} \mathrm{C}$. Subsequently, $5 \mu \mathrm{l} 100 \mathrm{mM}$ iodoacetamide were added and the sample was incubated for $1 \mathrm{~h}$ at $37^{\circ} \mathrm{C}$ in the dark. The concentration of RapiGest was decreased to $0.1 \%$ by adding $35 \mu \mathrm{l}$ trypsin (Promega) solution corresponding to an enzyme:protein ratio of approximately 1:20 enzyme-to-substrate ratio (w:w). The digest took place at $37^{\circ} \mathrm{C}$ overnight. To decompose the RapiGest the sample was supplemented with $5 \mu \mathrm{l} 40 \%$ TFA and incubated at $37^{\circ} \mathrm{C}$ for two more hours. Finally, the sample was centrifuged at $16000 \times \mathrm{g}$ for $30 \mathrm{~min}$ and the supernatant was transferred to a new tube. Tryptic peptides were dried in a centrifugal concentrator, dissolved in loading buffer (5\% [v/v] acetonitrile, $1 \%[\mathrm{v} / \mathrm{v}]$ formic acid) immediately afterwards and analyzed by LC-MS/MS (2.6.4).

\subsubsection{LC-MS/MS analysis of peptides on LTQ-Orbitrap}

Tryptic peptides from in-gel digestion (2.6.1) were analyzed by nanoflow chromatography coupled to hybrid ion trap-orbitrap mass spectrometry using a vented column setup (Licklider et al., 2002). Dried peptides were dissolved in $20 \mu$ l loading buffer (5\% [v/v] acetonitrile, 1\% [v/v] 
formic acid) and $20 \%$ of the sample was injected and enriched on a custom-packed reversedphase $\mathrm{C} 18$ precolumn, followed by separation on a custom-packed reversed-phase $\mathrm{C} 18$ column (0.075 mm ID x 150 mm, Reprosil-Pur 120 C18-AQ, $3 \mu \mathrm{m})$ using a 90 min linear gradient of 5$35 \%[\mathrm{v} / \mathrm{v}]$ acetonitrile, $0.1 \%[\mathrm{v} / \mathrm{v}]$ formic acid at $300 \mathrm{nl} / \mathrm{min}$. The eluent was analyzed in positive ion mode on a LTQ-Orbitrap Velos hybrid quadrupole/orbitrap mass spectrometer equipped with a Flexlon nanoSpray source with a stainless steel emitter and operated under Excalibur software 2.1.0.1140 to perform data-dependent acquisition. Each experimental cycle consisted of one full MS scan across the $350-1600 \mathrm{~m} / \mathrm{z}$ range that was acquired at a resolution setting of 30,000 FWHM, an automatic gain control target of $10 e^{6}$ and lock mass correction for $\mathrm{m} / \mathrm{z} 441.120025$ from polysiloxane. Up to the 15 most abundant peptide precursors of charge states 2 to 4 above an intensity threshold of $2 \times 10 \mathrm{e}^{3}$ were selected at an isolation width of $2.0 \mathrm{Th}$, fragmented by collision-induced dissociation (CID) using helium as collision gas at a normalized collision energy setting of $37.5 \%$ and an activation time of $10 \mathrm{~ms}$. The product ion spectra were recorded using an AGC target of $2 \times 10 \mathrm{e}^{5}$ and a maximum fill time of $100 \mathrm{~ms}$. Precursor $\mathrm{m} / \mathrm{z}$ values selected for MS/MS were excluded for $30 \mathrm{~s}$ after one occurrence. Three technical replicates were acquired per sample.

In-gel digestions resulted in eleven samples per chromatin affinity purification experiment were measured on a LTQ-Orbitrap Velos using a 90 min total gradient, in-gel digestions resulted in 22 samples were analyzed on a LTQ-Orbitrap XL mass spectrometer using a 50 min gradient.

\subsubsection{LC-MS/MS analysis of peptides on QExactive}

Tryptic in-solution digested peptides (2.6.2) were analyzed by nanoflow chromatography coupled to a hybrid quadrupole-orbitrap mass spectrometer using a vented column setup (Licklider et al., 2002). Samples were injected and enriched on a custom-packed reversed phase-C18 precolumn, followed by separation on a custom-packed reversed-phase $\mathrm{C} 18$ column $(0.075 \mathrm{~mm}$ ID x $150 \mathrm{~mm}$, Reprosil-Pur $120 \mathrm{C18-AQ,} 3 \mu \mathrm{m})$ using a $70 \mathrm{~min}$ total gradient of 5-40\% [v/v] acetonitrile, $0.1 \%[\mathrm{v} / \mathrm{v}]$ formic acid at $300 \mathrm{nl} / \mathrm{min}$. The eluent was analyzed in positive ion mode on a QExactive hybrid quadrupole-orbitrap mass spectrometer equipped with a Flexlon nanoSpray source with a stainless steel emitter and operated under Excalibur software 2.2.0.48 to perform data-dependent acquisition. Each experimental cycle consisted of one full MS scan across the 350-1600 m/z range that was acquired at a resolution setting of 70,000 FWHM, an automatic gain control target of $10 e^{6}$ and lock mass correction for $\mathrm{m} / \mathrm{z} 441.120025$ from polysiloxane. Up to the 15 most abundant peptide precursors of charge states 3 to 7 above an intensity threshold of $2 \times 10 \mathrm{e}^{4}$ were selected at an isolation width of $2.0 \mathrm{Th}$, fragmented by higher energy collisional dissociation (HCD) using nitrogen as collision gas at a normalized collision energy setting of $25 \%$, and their product ion spectra recorded using an AGC target of $2 \times 10 e^{5}$ 
and a maximum fill time of $60 \mathrm{~ms}$. Precursor $\mathrm{m} / \mathrm{z}$ values selected for MS/MS were excluded for $30 \mathrm{~s}$ after one occurrence.

\subsubsection{Molecular weight determination of histone proteins}

Histone proteins were dissolved in $20 \%$ [v/v] acetonitrile and $0.1 \%[\mathrm{v} / \mathrm{v}]$ formic acid and injected to a LTQ XL Linear Ion Trap mass spectrometer. The proteins were analyzed in a scan mode ranging from $500-2000 \mathrm{~m} / \mathrm{z}$. The spectra were extracted and deconvoluted using the Small Protein mode of the ProMass Deconvolution software.

\subsection{Raw data processing and data analysis}

\subsubsection{Mass spectrometric raw data processing with MaxQuant}

Protein identification and quantification of raw data files derived from mass spectrometric measurements $(2.6 .3,2.6 .4)$ were performed using the MaxQuant software, version 1.5.2.8 (Cox and Mann, 2008; Cox et al., 2011). Enzyme specificity was set to trypsin/P at a maximum of two missed cleavages, methionine oxidation was set as variable and cysteine carbamidomethylation was set as fixed modification. All other parameters were set according to the default settings with the following exceptions. For experiments done with SILAC-labeled nuclear extracts ${ }^{13} \mathrm{C}_{6}{ }^{-}$ arginine and ${ }^{13} \mathrm{C}_{4}$-lysine were set as fixed modifications. Additionally, the minimum length of peptides was decreased to 6 and the maximal number of modification per peptide to 4 .

Proteins were identified using the Andromeda algorithm searching against the UniProtKB SwissProt Human protein sequence database 2014.01 (134921 protein entries) supplemented with the MaxQuant common contaminants database (248 entries).

\subsubsection{Mass spectrometric raw data processing with pLink}

In order to identify cross-linked peptides, raw files obtained from mass spectrometric measurements (2.6.4) were transformed into mgf files using Proteome Discover and analyzed by the pLink software version 1.07 (Purcell et al., 2007). Raw files of one experiment were analyzed in parallel and results were summarized in one output table. The ini file used for the data search was configured in the following way. Carbamidomethyl was set as a fixed modification, oxidation, pyro-glutamic acid and $\mathrm{N}$-terminal acetylation were set as variable modifications, the search was done in mode 1 , noninterexport was set as true and the fdr was set to 0.01 . All other settings 
were defined according to the experimental design and/or correspond to the default setting of the example ini file downloaded with the software. Raw files used for the pLink search were also analyzed by MaxQuant (2.7.1). Identified proteins summarized in the proteinGroups.txt of MaxQuant were used as the reverence database for the pLink search. The reverence database included only identified proteins with more than 2 "razor and unique peptides". Additionally, contaminants and reverse hits were excluded. The amino acid sequences of identified histone proteins were exchanged by those from Xenopus laevis (2.1.10). The most abundant proteins of the samples were determined by dividing the number of identified "unique and razor peptides" by the molecular weight of the corresponding proteins. The most abundant proteins were arranged according to the quotient starting with the highest value.

In total, three pLink searches using different databases were performed for each experiment. The first database included the top 100 of the most abundant proteins, the second database was composed of the top 150 most abundant proteins and the third database included all identified proteins of each experiment, respectively. For spectra that were annotated in more than one of the pLink searches the spectrum with the highest score was chosen for further analysis. Those spectra that were annotated in more than one pLink search displaying different outcomes were excluded from further analysis.

Visualization of identified protein-protein cross-links was done using xiNET (Combe et al., 2015). Visualization and analysis of cross-linking data in the context of their three-dimensional protein structures were done using the Xlink Analyzer software (Kosinski et al., 2015; Pettersen et al., 2004).

\subsubsection{Data filtering and visualization of quantified MS data}

The MaxQuant output table proteinGroups.txt was imported to the statistical software package PERSEUS (Cox and Mann, 2008), version 1.5.0.26. Normalized heavy-to-light ratios (H/L) and intensities were extracted and log2-transformed. Only proteins with a ratio count of at least 3 in forward and reverse experiments for a given modification state were considered for quantification. After removal of reverse and contaminant entries, the statistical significance of individual quantification results was assessed using Perseus' "significance A" algorithm using a two-sided False Discovery Rate estimation to a $p$ value of 0.1 and application of a BenjaminiHochberg correction (Cox and Mann, 2008). The processed data were visualized using the open-source statistical software package $R$ (version 3.2.3). For data visualization only, missing values in one of the experiments were imputed to zero. 


\subsubsection{Protein-protein interaction analysis}

Protein-protein interaction analyses were done using the STRING database (Jensen et al., 2009). Leading UniProt IDs reported in the proteinGroups.txt of MaxQuant (2.7.1) were used to identify known and predicted functional and structural interactions (default settings) for the design of protein-protein association networks.

\subsubsection{Annotation enrichment analysis}

Functional annotation enrichment analyses were done using the functional annotation chart of the online software package DAVID (Huang da et al., 2009a, b). Results were obtained by using default settings of the statistical parameters with the exception of the EASE score (enrichment) cutoff, which was set to 0.05. All genome genes of Homo sapiens were used as the background database. The background database was provided by DAVID (Huang da et al., 2009a, b). Terms were annotated to significant enriched and significant depleted proteins of a certain chromatin modification state, respectively. Only enriched annotated terms were included in further analyses. The processed data were visualized using the open-source statistical software package $\mathrm{R}$ (version 3.2.3). 


\section{Results}

Nucleosomal arrays have been successfully used to characterize the diversity and complexity of PTM-regulated protein interactomes associating with chromatin (Nikolov et al., 2011). Nevertheless, comprehensive information regarding the impacted of heterochromatic chromatin modifications on proteins binding specifically to chromatin is still lacking. The current information content rarely allows drawing conclusions about the extent of functional outcomes associated with certain histone modification patterns.

In this study, chromatin affinity purification (ChAP) coupled to quantitative mass spectrometry was optimized predominately in terms of statistical evaluation, to investigate the interactomes brought about by ten different chromatin modifications. Furthermore, the method was extended to not only identify the interactomes in the context of singly-modified chromatin arrays but also the combination of two posttranslational histone modifications. Thus, the extended workflow enabled the investigation of the impact of histone PTMs on protein binding to chromatin and histone modification crosstalk on a global level. Additionally, a workflow combining ChAP, chemical cross-linking and mass spectrometry is introduced. The new combination of experimental steps enabled the identification of site-specific protein-protein interactions. Moreover the assignment of a network on the basis of these physical protein interactions provided information about the hierarchy of protein binding to chromatin.

\subsection{2mer nucleosomal arrays are of reproducible quality independent of incorporated modifications}

In order to expand the extent of modification-specific protein-binding interactomes investigated so far, homogenous, recombinant nucleosomal 12mer arrays carrying different chemical modifications, alone and in combination, were generated. These chromatin arrays were used as templates for affinity purification studies investigating protein-chromatin interactomics in the context of single modification and combinations thereof.

\subsubsection{Native chemical ligation and alkylation of cysteine residues successfully introduce PTMs to recombinant Xenopus laevis histones}

Two different approaches were used to introduce site-specific posttranslational modifications to recombinant Xenopus laevis histones. Modifications within the first 20 amino acids of the $\mathrm{N}$ terminus of histone $\mathrm{H} 3$ and the first 22 amino acids of the $\mathrm{N}$-terminus of histone $\mathrm{H} 4$ were introduced by native chemical ligation (NCL) (Shogren-Knaak and Peterson, 2004). The histone 
methylation sites H3K9me1/-me2/-me3, H4K20me1/-me3 and H4R3me2 were successfully created by NCL. The products of the reactions were visualized by SDS-PAGE and mass spectrometry. This is exemplarily shown for the NCL of H4K20me3 in figure 3.1 A and B.

As a second approach to introduce PTMs, methyl lysine analogs (MLAs) were used. Here, lysine 27 of histone $\mathrm{H} 3$ was modified to $\mathrm{H}_{3} \mathrm{~K}_{\mathrm{c}} 27 \mathrm{me} 1 /-\mathrm{me} 2 /$ and $-\mathrm{me} 3$. The method is based on the alkylation of an artificially introduced cysteine residue. The cysteine undergoes alkylation with an electrophilic ethylamine resulting in aminoethylcysteine, which is an analog of lysine (Simon et al., 2007). The completeness of the MLA reaction was verified by mass spectrometry using ESIMS, exemplarily shown for $\mathrm{H}_{3} \mathrm{~K}_{\mathrm{c}} 27 \mathrm{me} 3$ in figure $3.1 \mathrm{C}$.

A)

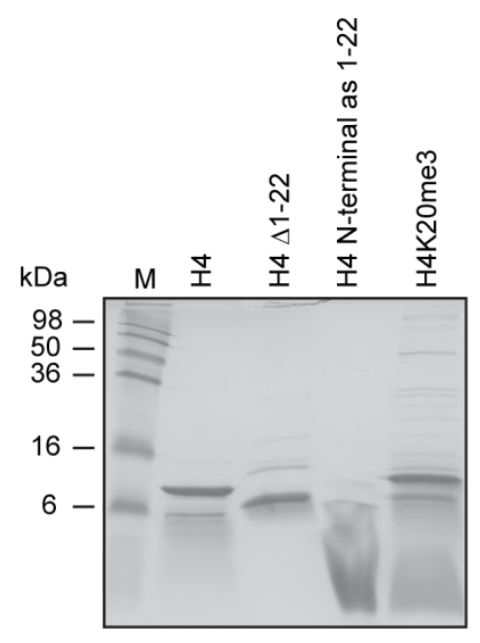

C)

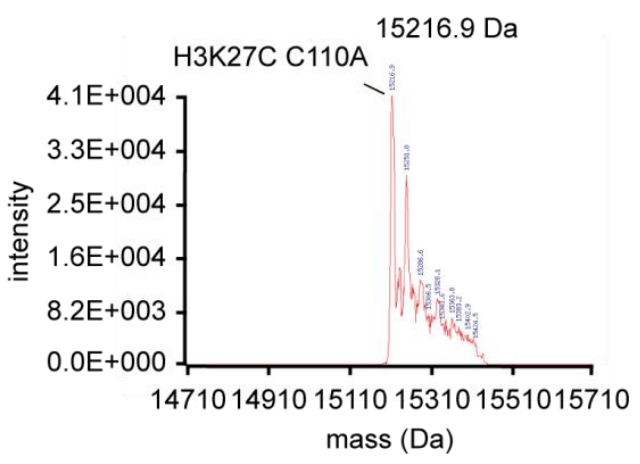

B)
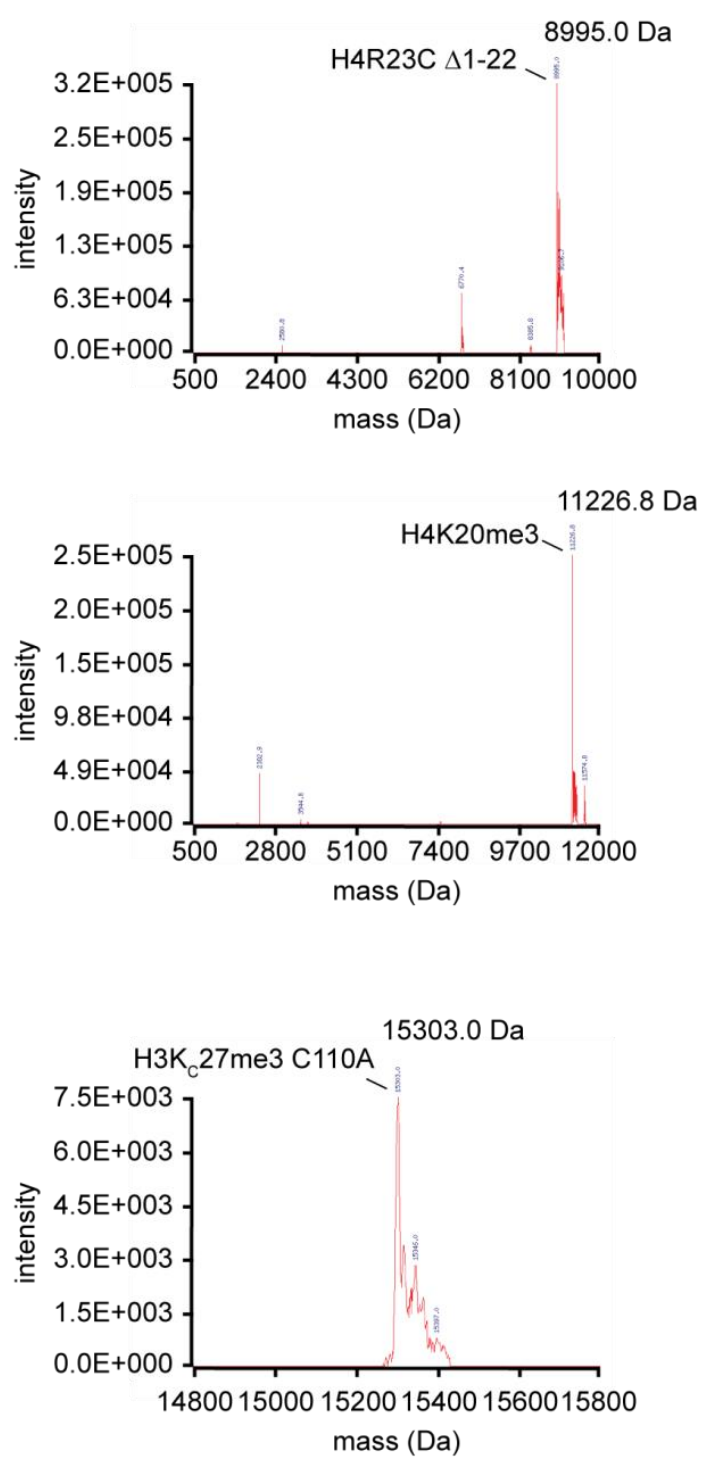

Figure 3.1 Introduction of posttranslational histone modifications.

A) SDS-PAGE analysis of the recombinant histone H4, H4R23C $\Delta 1-22$, the thioester peptide composed of the $\mathrm{N}$-terminal amino acids $1-22$ of $\mathrm{H} 4$ and the ligated product H4K20me3. B) ESI-LTQ mass spectrometric analysis of H4R23C $\Delta 1-22$ (upper part of the panel) and the ligation product H4K20me3 
(lower part of the panel). C) ESI-LTQ mass spectrometric analysis of the non-alkylated H3K27C C110A (left part of the panel) and $\mathrm{H}_{3} \mathrm{~K}_{\mathrm{C}} 27 \mathrm{me} 3$ (right part of the panel).

\subsubsection{Octamer-DNA molar ratios from 1.1 to 1.3 assemble fully saturated, reproducible nucleosomal arrays}

The assembly of nucleosomal arrays is based on histone octamers that uniformly position on a DNA template. The optimal octamer-DNA molar ratio was determined for each chromatin species separately. An octamer-DNA molar ratio from 1.1 up to 1.3 was found to be sufficient to obtain a saturation of more than $90 \%$ of the DNA template with histone octamers. The success of the reconstitution was visualized by native agarose gel electrophoresis (Figure $3.2 \mathrm{~A}$ ). The exact rate of saturation of the nucleosomal arrays was determined by a restriction enzyme digest using Aval (Figure $3.2 \mathrm{~B}$ ). Aval cuts the DNA template of chromatin arrays within the 601 sequence only once per 200 bp repeat. Depending on octamer occupancy Aval digestion results in mononucleosomes and free DNA.

We additionally tested the reproducibility of the chromatin reconstitution method using analytical ultracentrifugation. The distribution of sedimentation coefficients (S) of nucleosomal arrays, either fully or subsaturated (saturation level of $75 \%$ ) were determined by sedimentation velocity and van Holde-Weischet analysis. As shown in figure $3.2 \mathrm{C}$, different fully saturated chromatin arrays displayed a similar sedimentation distribution, mainly in the range between 40 and $50 \mathrm{~S}$. The similar sedimentation coefficient distribution of both replicates demonstrated that the reconstitution method generates highly reproducible chromatin arrays. In comparison, the subsaturated chromatin species showed a distribution of sedimentation coefficients in a lower range, between 30 and $40 \mathrm{~S}$, indicating lower compaction of the chromatin species. The results verified that the saturation level of oligonucleosomal arrays can be reproducibly adjusted over the octamer-DNA molar ratio, generating arrays of comparable quality.

\subsection{The statistical tool "significance A" determines significant protein fold enrichment cutoffs}

To investigate the extent of changes in protein composition and binding to chromatin, affected by distinct chromatin modification patterns, chromatin-based affinity purification in combination with relative quantification by mass spectrometry was established in our lab (Figure 3.3) (Nikolov et al., 2011).

ChAP coupled to quantitative MS is based on the relative quantification of proteins binding to unmodified and modified chromatin arrays. This is possible by using SILAC-labeled HeLa nuclear extracts. For each modification state two biological replicates were performed in parallel 
as a label swap experiment. In the "forward" experiment unmodified chromatin arrays were incubated with light nuclear extract and modified chromatin arrays with heavy isotope-labeled nuclear extract. For the "reverse" experiment modified and unmodified chromatin arrays were incubated with light and heavy nuclear extracts, respectively, thus obtaining inverse enrichment ratios.

A)

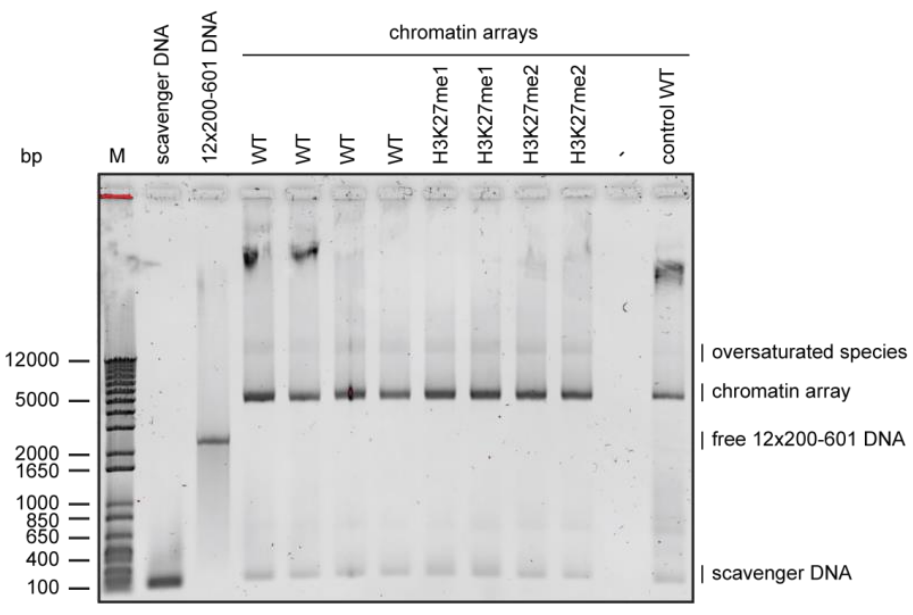

B)

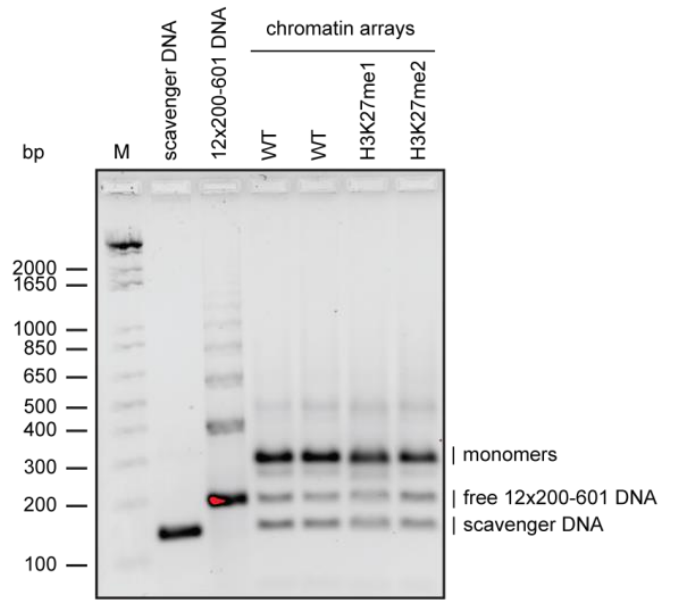

C)

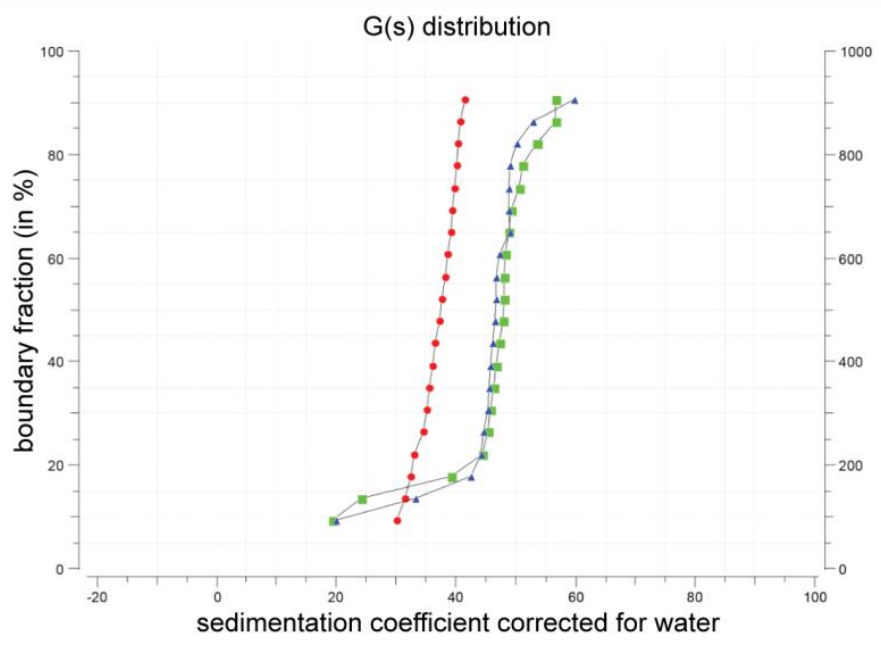

chromatin array $75 \%$ saturated chromatin array fully saturated chromatin array fully saturated

Figure 3.2 Quality controls of assembled chromatin arrays.

A) Native agarose gel electrophoresis of reconstituted chromatin arrays and free DNA. The samples were separated by gel electrophoresis on a $0.5 \%$ agarose gel using $0.2 x$ TB buffer. The gel was stained with ethidium bromide. M defines the DNA size marker. B) Aval digestion of reconstituted chromatin arrays and free DNA. Digested samples were separated by agarose gel electrophoresis on a $2 \%$ agarose gel using TBE buffer. The gel was stained with ethidium bromide. C) Analytical ultracentrifugation of fully saturated and $75 \%$ saturated chromatin arrays. The distribution of sedimentation coefficients was obtained using sedimentation velocity and van Holde-Weischet analysis (analyzed by Dr. Ron Finn, Max Planck Institute for Biophysical Chemistry, Göttingen, Germany). 
The method enables the identification of all proteins binding to chromatin and that are specifically regulated by the underlying chromatin modification. Throughout this study, I will refer to this pool of proteins as the "chromatin-binding interactome". By investigating different modification patterns several interactomes were generated by performing experiments with individual chromatin modifications. Hereby, a statistical evaluation strategy ensuring a valid interpretation of the results not only for individual experiments but also for comparison of different interactomes is of immense importance.

In previous studies, a fixed fold enrichment cutoff was defined and applied to all individual experiments, not meeting the requirements of a significant determination of a fold enrichment cutoff for each individual experiment. To verify significant protein enrichment, I optimized the strategy of statistical evaluation to faithfully investigate and interpret the interactomes brought about by twelve different chromatin modification patterns.

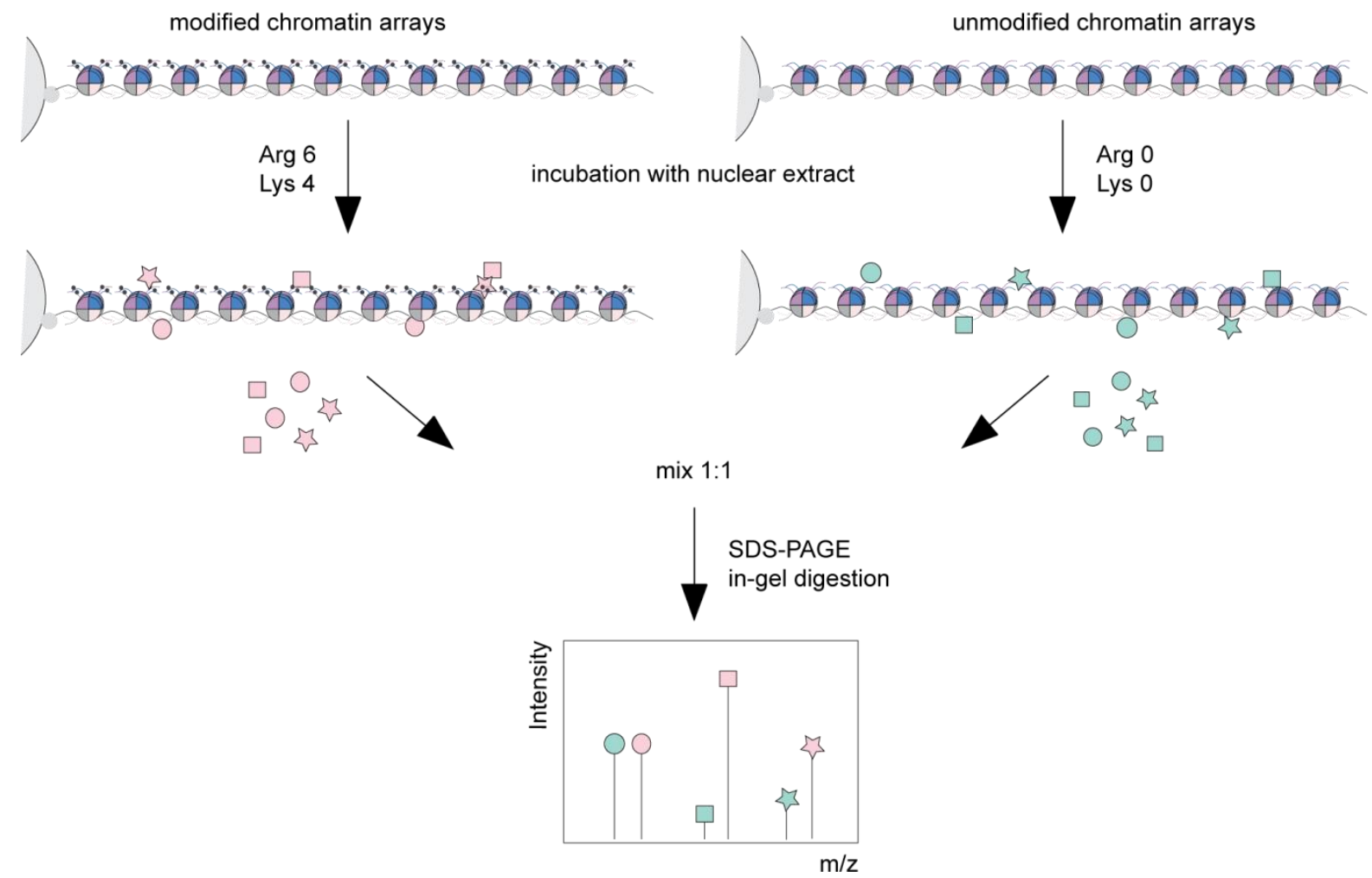

Figure 3.3 Workflow of SILAC-based chromatin affinity purification coupled with mass spectrometry.

Unmodified and modified chromatin arrays are incubated with light and heavy SILAC-labeled nuclear extracts from HeLa cells, respectively. Chromatin arrays and their associated proteins of both pull-downs are eluted, mixed in a 1:1 ratio, treated with benzonase and finally separated by SDS-PAGE. Proteins are in-gel digested using trypsin. The resulting peptides are analyzed by LC-MS/MS. Identification and quantification is achieved using the MaxQuant software. 
To determine the impact of histone PTMs on the chromatin-binding interactome, the proteins associated with modified and unmodified chromatin arrays were compared and their relative enrichment level was determined. Quantification using SILAC-labeled nuclear extracts assumes that unspecific binders, i.e. proteins binding to both templates to the same extent, feature a heavy/light ratio $(\mathrm{H} / \mathrm{L}$ ratio) value of 1 . However, due to small variations inherent to all biological preparations this value might vary from one experiment to another. Considering all performed experiments this theoretical heavy/light ratio of $1\left(\log _{2} 1=0\right)$ for unspecific binders in fact ranged from -3.2 $\log _{2}$ up to $2.3 \log _{2}$ (visualized in chapter 3.3 for each individual experiment) as calculated by applying a statistical significance test as described in the following.

The definition of a fixed fold enrichment cutoff is not sufficient to faithfully reflect which proteins are significantly enriched or depleted when two different data sets are compared. To overcome this problem, the statistical significance of individual quantification results was defined using the Perseus' "significance A" algorithm (Cox and Mann, 2008). This algorithm determines the values that are significant outliers relative to a certain population. The "significance A" algorithm was used by applying a two-sided False Discovery Rate estimation to a p-value of 0.1 and a Benjamini-Hochberg correction.

To determine significant enrichment values, SILAC ratios of the forward and the reverse experiment were averaged. This resulted in a narrowed distribution range of $\mathrm{H} / \mathrm{L}$ ratios of the protein population bound to chromatin. This can be visualized when comparing the distribution of $\mathrm{H} / \mathrm{L}$ ratios of proteins resulting from the individual experiments with the distribution of the averaged forward and reverse experiments (Figure $3.4 \mathrm{~A}-\mathrm{C}$ ). Consequently, an increased number of significant outliers was calculated compared to calculations using non-averaged SILAC ratios, as significant values are determined as outliers relative to the spread of the input population (Figure 3.4 A-C).

In order to visualize the results, SILAC ratios of forward and reverse experiments were scatter plotted on the $x$ - and $y$ - axis, respectively (Figure 3.4 D). The advantage of this presentation style is the complexity of information that can be visualized.

First, the protein-binding affinity to modified chromatin arrays is demonstrated. Therefore, $\mathrm{H} / \mathrm{L}$ ratios of the reverse experiment were inversed due to the label-swap approach. Data were $\log _{2}$ scaled, where positive values of the $\mathrm{H} / \mathrm{L}$ ratio represent an increased protein-binding affinity, whereas negative $\mathrm{H} / \mathrm{L}$ ratios represent a lower binding affinity to modified chromatin arrays compared to the unmodified chromatin species. Thus, the scatter plots display proteins whose binding is either increased or decreased as a consequence of a specific histone modification state. 
A)

forward experiment

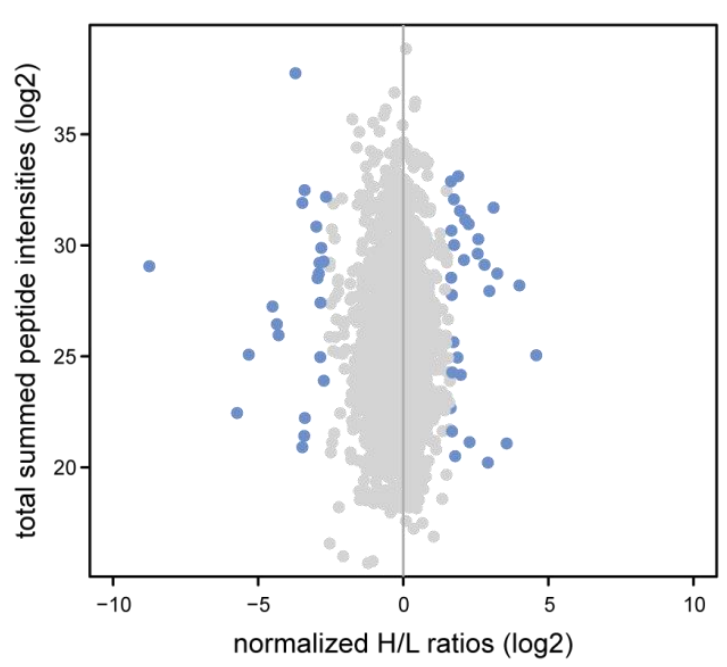

C)

forward and reverse experiments averaged

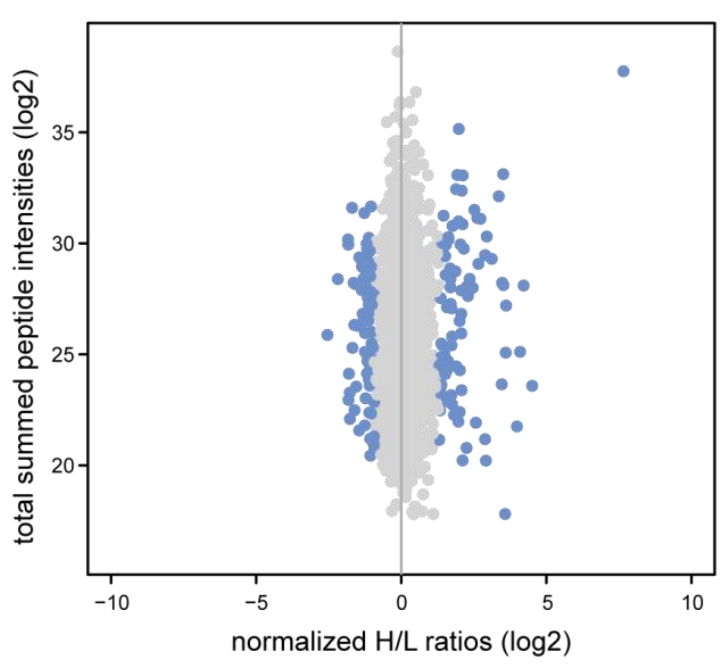

B)

reverse experiment

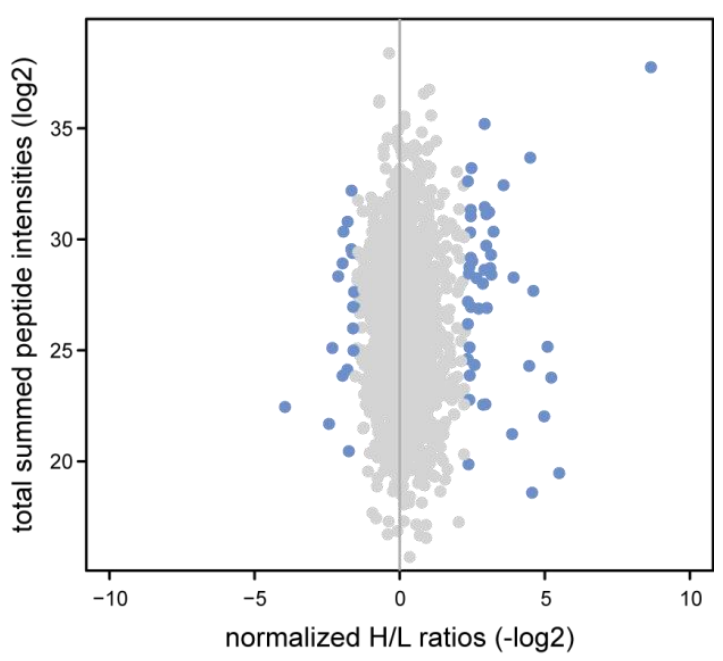

D)

forward and reverse experiments averaged

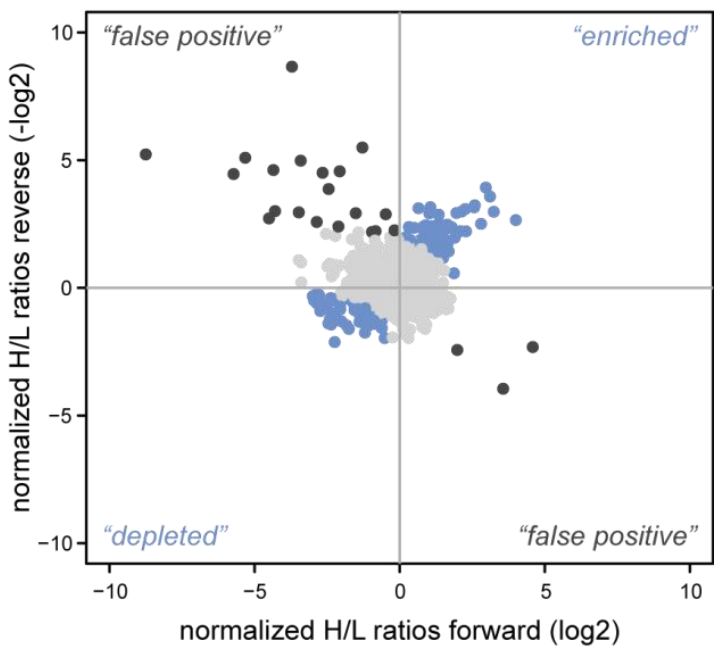

Figure 3.4 Determination and visualization of significantly regulated proteins using the example of the H3K9me3 interactome.

A) $\log _{2}$ SILAC ratios of proteins identified in the forward experiment are plotted on the $x$-axis. Total summed peptide intensities of the forward experiment are plotted on the $y$-axis in $\log _{2}$ scale. Proteins identified as significant outliers by application of the "significance A" algorithm are marked in blue. B) The same as in A) but with values of the reverse experiment. SILAC ratios are inverted. C) The same as in A) but with averaged values of the forward and the reverse experiments. D) $\log _{2}$ scaled $H / L$ ratios of proteins of the forward experiment are plotted on the $x$-axis and of the reverse experiment on the $y$-axis. Statistical analyses were performed with averaged $\mathrm{H} / \mathrm{L}$ ratios of the datasets. Proteins determined as significantly enriched are displayed in the upper right square, while proteins significantly depleted are displayed in the lower left square of the plot. Proteins determined as false positives are displayed in the upper left and the lower right square. Significantly recruited and depleted proteins are colored in blue. False positive proteins are marked in black. Proteins that were significantly regulated but only identified in either the forward or the reverse experiment are not plotted. 
Second, the scatter plots visualize the correlation of both biological replicates. In case of positive correlation, for both replicates proteins displaying the same trend in terms of binding affinity to modified chromatin arrays and are located in the upper right (increased binding) and in the lower left square of the scatter plot (decreased binding). Negative correlation is observed for proteins displaying an opposed $\mathrm{H} / \mathrm{L}$ ratio in both biological replicates. Those proteins are located in the upper left and the lower right square of the scatter plot (Figure $3.4 \mathrm{D}$ ). Accordingly, negative correlation is indicative of false positives. Therefore, significant outliers that displayed a diametrically opposed significance score in the forward and reverse experiments were eliminated from further analysis (Figure 3.4 D).

Third, statistically evaluated information is represented by a color code. Significant outliers that correspond to proteins significantly regulated by a certain modification state are marked in blue, whereas proteins constituting the background are labeled in grey. Proteins identified as false positive are colored in black.

With this statistical evaluation strategy all further introduced chromatin-binding interactomes were analyzed and the results were visualized as described.

\subsection{ChAP coupled to quantitative MS enables the investigation of chromatin- protein interactomics and provides insights into chromatin modification crosstalk}

The first steps towards investigating whether, and even more importantly, how posttranslational chromatin modifications affect the structure of chromatin and downstream biological processes, such as transcription, replication and cell division, is the investigation of the impact modifications have on the protein environment of the chromatin loci they mark. Heterochromatin is characterized by a tightly packed form of chromatin and thought to be mainly transcriptional silenced. However, it is specifically marked by a variety of posttranslational modifications and yet, it is not fully understood how and whether these modifications contribute solely to the molecular mechanisms of establishment and maintenance of the heterochromatic state. To investigate the extend of biological functions correlating with heterochromatin I focused on chemical modifications in literature so far associated with this chromatin stage. The following chapter introduces the protein-binding interactomes mapped in the context of different histone methylations, DNA methylation and combinations thereof. 


\subsubsection{Individual chromatin modification states recruit specific chromatin-binding interactomes}

Interactomes associated with 10 different chromatin modifications were characterized. For each modification state between 1,117 and 2,785 chromatin-bound proteins, including their isoforms, were identified. All analyses together identified more than 6,000 proteins. Of these, close to 500 were found to be regulated by the chromatin modifications. Each modification state impacted the binding properties of a specific set of factors. Moreover, each dataset included several proteins that were not known before to associate with specific histone modifications. A comprehensive list of factors whose binding to chromatin was impacted by the presence of a certain modification is provided in table 3.1. These data shed light on how extensively the chromatin interactomes are regulated by histone posttranslational modifications. The individual protein-binding interactomes for each histone modification state will be discussed in the following sections.

\section{H3K9me1 interactome}

Genome wide high resolution maps revealed that H3K9me1 is mainly associated with transcriptional active gene loci (Barski et al., 2007; Wang et al., 2008). The predominant absence of H3K9me1 at constitutive heterochromatin has been demonstrated before by using chromatin immunoprecipitation (ChIP) and antibody based detection methods in ES cells (Peters et al., 2003). In contrast, antibody based staining of MEF cells demonstrated that H3K9me1 is located at pericentric heterochromatic regions (Sims et al., 2006). To my knowledge, proteomic studies investigating protein interactions to H3K9me1 on a global scale have not been performed.

In this proteomic study, H3K9me1-modified chromatin arrays recruited six and repelled seven factors from binding (Figure $3.5 \mathrm{~A}$, Table 3.1). Although H3K9 mono-methylation was mainly shown to be linked to gene activation, several factors connected to heterochromatin were identified to be specifically recruited by H3K9me1. Among them were the heterochromatin protein HP1a (CBX5) and UHRF1. Both are known to be involved in heterochromatin establishment and maintenance and have been shown to bind preferentially to K9me3 of histone H3 (Lachner et al., 2001; Nady et al., 2011). The splicing co-activator SRRM1 (Blencowe et al., 1998) was also recruited to H3K9me1-modified chromatin, indicating that not only heterochromatin associated factors were recruited.

To my knowledge UHRF1 is the only factor of the interactome that has been described so far to directly interact with H3K9me1 (Nady et al., 2011). Protein interaction analysis using the database STRING (Jensen et al., 2009) predicted no protein-protein interactions besides the interaction between CBX5 and PRMT5 (Figure 3.5 B, upper panel). Thus, it remains unclear, whether the six proteins bind as single factors or form a multi subunit complex. 
In contrast, the transcription factors NFIB, NFIC, NFIX and E4F1 were significantly repelled from binding, as was INO80E, a component of the INO80 chromatin remodeling complex. The protein-protein interactions between NFIB, NFIC and NFIX and between INO80E and MCRS1 were predicted by STRING analysis (Figure $3.5 \mathrm{~B}$, lower panel), suggesting that H3K9me1 regulated factors are biologically linked, which verifies the specificity of the approach.

A)

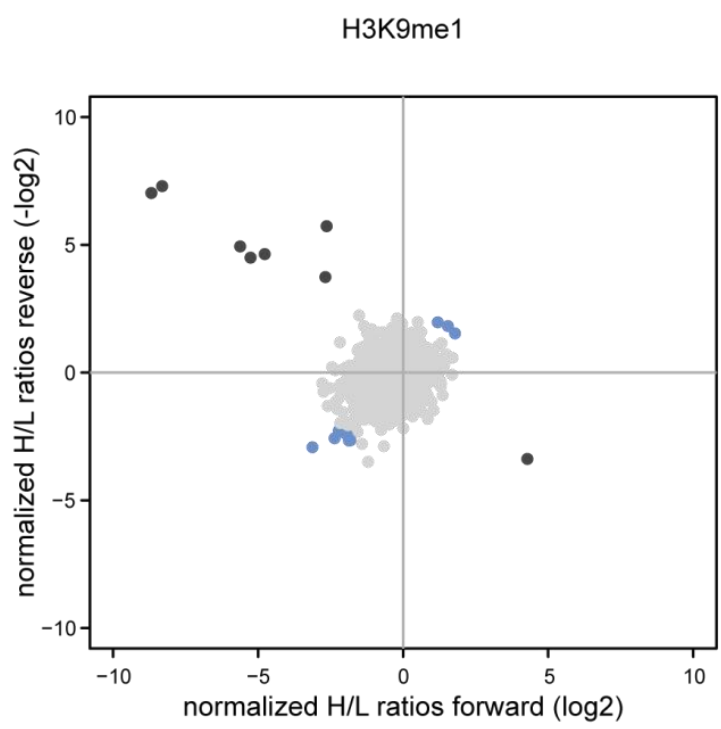

C)

H3K9me2

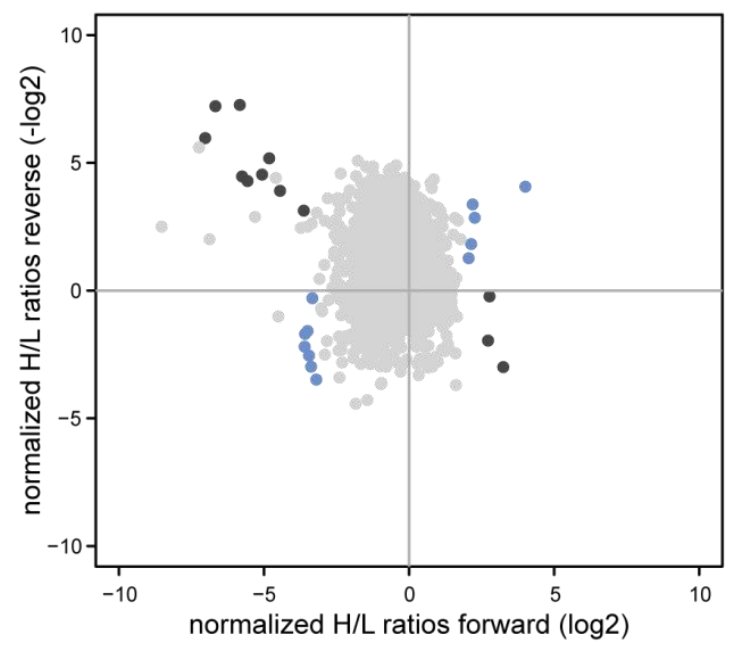

B)

H3K9me1 enriched proteins

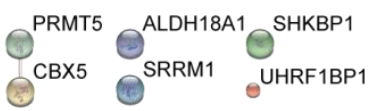

H3K9me1 excluded proteins

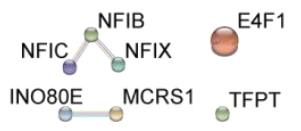

D)

H3K9me2 enriched proteins

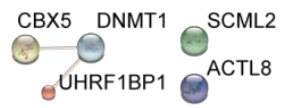

H3K9me2 excluded proteins

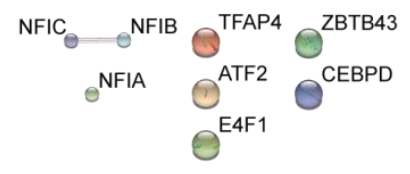

Figure 3.5 Protein-binding interactomes of H3K9me1 and H3K9me2 chromatin.

A) H3K9me1 interactome. Proteins are plotted by their $\log _{2}$ SILAC ratios of the forward experiment on the $\mathrm{x}$-axis and the reverse experiment on the $\mathrm{y}$-axis. Significantly recruited and depleted proteins are colored in blue. False positive proteins are marked in black. B) STRING protein-protein interaction networks of factors significantly recruited by H3K9me1 (upper part of the panel) and excluded proteins (lower part of the panel). C) H3K9me2 interactome. Data are represented as described in A. D) Protein-protein interaction networks from STRING analysis of proteins significantly affected by H3K9me2. Recruited 
proteins are shown in the upper part of the panel, whereas excluded proteins are represented in the lower part of the panel.

\section{H3K9me2}

The chromatin-binding interactome of $\mathrm{H} 3 \mathrm{~K} 9 \mathrm{me} 2$ introduced here is represented by the results of the forward experiment only, as data evaluation revealed a non-normal distribution of SILAC ratios of chromatin-bound proteins identified in the reverse experiment.

Chromatin di-methylated at lysine 9 of histone $\mathrm{H} 3$ recruited five and depleted eight factors from binding (Figure 3.5 C, Table 3.1). As the modification is known to be linked to gene repression (Wang et al., 2008), it is not surprising that nearly all factors recruited have been shown to be involved in maintenance of a repressive transcriptional state. These proteins comprise CBX5, UHFR1, DNMT1 and the putative polycomb group protein SCML2 (Montini et al., 1999). HP1 proteins, such as CBX5 have been shown to bind to H3K9me2 N-terminal peptides (Jacobs and Khorasanizadeh, 2002; Lachner et al., 2001). Also, UHRF1 has been demonstrated to bind to H3K9me2/-me3 (Liu et al., 2013). Moreover, UHRF1 was identified to interact with DNMT1, (Bostick et al., 2007; Sharif et al., 2007), suggesting a role for UHRF1 in DNMT1 recruitment to H3K9me2-marked chromatin regions. A link between CBX5, UHRF1 and DNMT1 was further suggested by STRING analysis (Figure 3.5 D, upper panel). There is one published study that screened for $\mathrm{H} 3 \mathrm{~K} 9 \mathrm{me}$ 2-binding proteins using $\mathrm{N}$-terminal modified peptides of $\mathrm{H} 3$ (Chan et al., 2009). Comparing the findings of this study to mine, CBX5 was found to be the only overlapping factor identified to bind in the context of H3K9me2.

The transcription factors CEBPD, TFAP4, ATF2 and the zinc finger protein ZBTB43 were depleted from binding to $\mathrm{H} 3 \mathrm{~K} 9 \mathrm{me} 2$ in addition to the transcription factors also excluded from binding to H3K9me1-modified chromatin arrays. STRING analysis did not reveal any association of these factors (Figure $3.5 \mathrm{D}$, lower panel).

\section{H3K9me3}

$\mathrm{H} 3 \mathrm{~K} 9 \mathrm{me} 3$ is the most intensively investigated repressive histone modification. It marks pericentric heterochromatin (Barski et al., 2007; Fischle et al., 2003a; Lachner et al., 2003; Wang et al., 2008). In my study, no other modification state affected as many proteins as trimethylated lysine 9 of histone H3. Altogether, 59 proteins were specifically recruited and 72 repelled from binding (Figure 3.6 A, Table 3.1). Previous studies using different methodologies, already identify a set of $\mathrm{H} 3 \mathrm{~K} 9 \mathrm{me} 3$-binding factors comprising among others the different isoforms of HP1, CBX1, CBX3 and CBX5 (Lachner et al., 2001), ADNP (Mosch et al., 2011) as well as UHRF1 (Karagianni et al., 2008) and MPHOSPH8 (Kokura et al., 2010). Besides single 
Table 3.1 Proteins recruited and excluded from binding to chemically modified chromatin.

Proteins are listed according to their enrichment ratios $(\mathrm{H} / \mathrm{L}$ ratios) starting with the highest value on top. Proteins significantly recruited by a certain modification state are written in black, proteins excluded are written in blue. Grey marked proteins indicate that the proteins were already found to interact with the respective modification in one of the following studies (Bartke et al., 2010; Bluhm et al., 2016; Bostick et al., 2007; Engelen et al., 2015; Kunowska et al., 2015; Nady et al., 2011; Nikolov et al., 2011; Oda et al., 2010; Vermeulen et al., 2010)

\begin{tabular}{|c|c|c|c|c|c|c|c|c|c|c|c|c|}
\hline H3K9me1 & H3K9me2 & H3K9me3 & HЗK27me1 & H3K27me2 & H3K27me3 & $\mathrm{H} 3 \Delta 1-20$ & H4K20me1 & H4K20me3 & H4R3me2 & $\mathrm{meCpG}$ & $\begin{array}{l}\text { H3K9me3- } \\
\text { H4K20me3 }\end{array}$ & $\begin{array}{c}\text { H3K9me3- } \\
\text { meCpG }\end{array}$ \\
\hline SHKBP1 & UHRF1 & CBX1 & GCC2 & JADE2 & EIF2B5 & FAM114A1 & SCML2 & DNAH8 & POLR3H & UHRF1 & DNAH8 & DSC3 \\
\hline SRRM1 & CBX5 & UHRF2 & CNOT2 & KIAA1524 & PHF1 & CAPN1 & LCN1 & ORC2 & POLR3D & DNMT1 & LRWD1 & CALML5 \\
\hline ALDH18A1 & ACTL8 & CBX3 & BAG6 & LGALS7 & FGF2 & TALDO1 & HDGFRP2 & LRWD1 & SNC73 & SCML2 & CBX1 & TGM3 \\
\hline PRMT5 & DNMT1 & CBX5 & RSF1 & PHF1 & FRMPD3 & MIF & ZRANB2 & ORC3 & POLR3G & RALGAPA2 & CBX5 & CSTA \\
\hline UHRF1 & SCML2 & USP3 & DYM & GBP1 & LRWD1 & ZGPAT & PC4 & HMGN5 & POLR1C & ZHX2 & ORC3 & CDK2AP1 \\
\hline CBX5 & NFIC & SPIN2B & DFNA5 & SLC25A3 & ORC2 & HDGF & RMND1 & ARG1 & E2F6 & ZBTB33 & CHAF1B & DNCL1 \\
\hline MCRS1 & CEBPD & POGZ & PRIC295 & MAGOH & ORC3 & CAST & SREK1 & ORC5 & NFIC & USP7 & ORC2 & TRAM1L1 \\
\hline NFIB & NFIB & CHAF1A & CNOT1 & SHPRH & ORC5 & PSAT1 & USP7 & SIRT6 & POLR3E & ACTL8 & POGZ & \\
\hline NFIX & ZBTB43 & CHAMP1 & IP011 & PFN1 & ORC3L & WARS & ENO1 & EIF5 & POLR2E & ZHX1 & CBX3 & \\
\hline E4F1 & NFIA & CHAF1B & KIF11 & PRPF40A & CBX8 & SYAP1 & RSRC2 & APEX1 & ACTL8 & ZHX3 & ORC5 & \\
\hline INO80E & TFAP4 & NIPBL & CNOT11 & SRSF6 & PRC1 & PARK7 & CIR & ORC3L & USP7 & UHRF2 & SPIN2B & \\
\hline TFPT & E4F1 & SEPT7 & XPO1 & PGK1 & ABCF1 & FMNL1 & NAP1L4 & SYAP1 & NOLC1 & SEMG1 & NIPBL & \\
\hline NFIC & ATF2 & SEPT9 & FBXL6 & SAP18 & EIF2S2 & ANXA5 & RPRC1 & PHF1 & NAA40 & CUEDC1 & CHAF1A & \\
\hline & & SIRT6 & UBA5 & EIF4A3 & TOPBP1 & POU2F1 & PSMD3 & RNF213 & LMNA & MBD2 & UHRF2 & \\
\hline & & SEPT2 & SIRT6 & $\mathrm{ACIN} 1$ & WDR5B & TPT1 & PHF8 & EIF5B & $\mathrm{NCL}$ & ZBTB12 & SPIN1 & \\
\hline & & SPIN1 & RAVER1 & PHF21A & TCOF1 & BCLAF1 & INO80C & USP3 & CHD1 & MTA2 & CHAMP1 & \\
\hline & & ACTL8 & GET4 & CLIC1 & EIF2S3 & NBN & MYC & EIF2S2 & SCML2 & UBE2D2 & ORC3L & \\
\hline & & DNMT1 & TTI1 & $\mathrm{ZC} 3 \mathrm{H} 11 \mathrm{~A}$ & PRC1 & PNP & MLXIPL & EMG1 & $\mathrm{ZCCHC} 10$ & GATAD2B & ADNP & \\
\hline & & ADNP2 & CNOT7 & RNPS1 & EIF2S1 & CKB & POLG & EIF2S1 & TADA1 & CDK2AP1 & MAU2 & \\
\hline & & LRWD1 & ZKSCAN3 & TKT & MAFK & ALDOC & TKT & ORC6 & RAD51AP1 & HYDIN & NOLC1 & \\
\hline & & USP7 & SPTY2D1 & PAXIP1 & PBX2 & GDI2 & PGK1 & TXLNA & & ZNF687 & JADE2 & \\
\hline & & HMGN5 & PSMA7 & CHAF1B & ELF2 & $\mathrm{AHCY}$ & PNISR & GOLT1B & & $\mathrm{FIZ1}$ & ING5 & \\
\hline & & ORC3L & POLR3K & ISL2 & KIF2A & EIF4H & PHF21A & EIF2S3 & & ZMYND8 & PHF5A & \\
\hline & & ORC2 & PRC1 & E4F1 & CDYL2 & CXorf38 & ZNF580 & KIF2A & & RBBP7 & SF3B1 & \\
\hline & & $\mathrm{TCHH}$ & VWA9 & AEBP2 & AAR2 & ZNF451 & RFXANK & RAD51AP1 & & CTSD & SHPRH & \\
\hline & & ORC3 & CHD1 & INO80E & PLK1 & MAGOH & HES1 & KIF2C & & GATAD2A & UBTF & \\
\hline & & UHRF1 & TAF11 & TFPT & RBM7 & FAM48A & INO80D & ABCF1 & & CHD3 & SF3A1 & \\
\hline & & RAD51AP1 & RECQL & SP3 & XPO5 & VRK3 & DHX36 & BCR/ABL f. & & RBBP7 & SF3B4 & \\
\hline & & ADNP & MED10 & NR2C1 & KIF5B-ALK & DLG3 & NCAPD2 & TXLNG & & PGBD3 & PMVK & \\
\hline & & ZHX1 & HSP90AB1 & HEATR2 & EXOC2 & FAM134C & NCOR2 & KIFC1 & & MTA3 & FRMPD3 & \\
\hline & & EIF5 & TPI1 & ZMYM1 & RPLP2 & NACA & NEIL2 & ZNF598 & & BLMH & SF3A3 & \\
\hline & & ORC5 & CENPF & SP1 & NOLC1 & TMPO & CHTOP & PDIA5 & & CHD4 & SEPT10 & \\
\hline & & MIER2 & SETX & ZNF629 & SPATA5L1 & CEBPD & $\mathrm{ZC} 3 \mathrm{H} 14$ & NFIX & & RBM4 & SF3B2 & \\
\hline & & EMG1 & CKAP2 & NFIB & OXSR1 & GOLGB1 & SNRPD3 & C19orf47 & & NCOA6 & SEPT9 & \\
\hline & & SEPT6 & PFN1 & ZNF770 & NPM1 & FMNL1 & SRSF6 & NFIA & & ZBTB4 & PGK1 & \\
\hline & & MPHOSPH8 & MED16 & CD3EAP & RPRC1 & SARS & SAP18 & POLR1B & & POGZ & SEPT6 & \\
\hline & & ATRX & GTF3C4 & POLR1E & PCMT1 & ATL3 & PNN & PKP2 & & RBBP4 & C9orf78 & \\
\hline & & MAU2 & HSPA9 & КMT2C & JADE2 & LMNA & DDX21 & ANKRD12 & & MECP2 & LSM2 & \\
\hline & & KIFC1 & GTF3C5 & PRDM10 & YKT6 & ARPC2 & MAGOH & BRCA1 & & MTA1 & USP3 & \\
\hline & & SMCHD1 & LDHB & POLR1A & EPPK1 & ALDH18A1 & RPL7L1 & E4F1 & & SEMG2 & EIF4G1 & \\
\hline & & SEPT8 & ZBTB1 & PARD3 & TINF2 & UIMC1 & SF3B4 & ANKRD32 & & $\mathrm{BACH} 1$ & EIF5 & \\
\hline & & CDYL & MARK2 & POLR1B & NAP1L1 & CBR1 & SNRPB2 & BARD1 & & CHAMP1 & ING4 & \\
\hline & & SCML2 & PRDX1 & ESRRA & UBL4A & ASNS & PURB & AMY1A & & WHSC1 & RAB6A & \\
\hline & & KIF2A & LDHA & & SYDE1 & CPNE1 & CGGBP1 & & & POLR3F & BRCA1 & \\
\hline & & ABCF1 & ACTN4 & & SCYL2 & CUL1 & MAP4 & & & E2F2 & SEPT7 & \\
\hline & & EIF2S2 & PKM & & HTRA1 & TADA3 & ZNF354A & & & SIN3A & CHD1L & \\
\hline & & MORC2 & TKT & & MAP2K2 & ARHGEF3 & SNRPA1 & & & CLOCK & JADE3 & \\
\hline & & EIF2S1 & EEF2 & & CHERP & BPTF & $\mathrm{ZC} 2 \mathrm{HC} 1 \mathrm{~A}$ & & & POLR1C & RAD51AP1 & \\
\hline & & EIF2S3 & PRDX6 & & CBFB & ARPC4 & SF3A3 & & & BHLHB2 & BARD1 & \\
\hline & & MIER1 & PNP & & UBTF & PES1 & ZBED6 & & & RPRD1B & BABAM1 & \\
\hline & & ELF1 & EEF1B2 & & PCSK9 & POM121C & PURA & & & ZNF131 & SEPT2 & \\
\hline & & CDYL2 & PGAM1 & & C11orf57 & LIG4 & SF3B2 & & & BRMS1L & BRCC3 & \\
\hline & & ZC3HAV1 & PSMA6 & & PHF8 & SHCBP1 & SF3B1 & & & MITF & ZBED1 & \\
\hline & & JMJD1C & ENO1 & & ACACA & MTF2 & ANKRD12 & & & ELF1 & SDHB & \\
\hline & & LRIF1 & G2E3 & & GTF2E2 & POLD3 & SF3A1 & & & SAP30 & ACACA & \\
\hline & & WHSC1L1 & PPIA & & TYMS & PUM1 & RSL1D1 & & & SUPT3H & BRE & \\
\hline & & ZNF280D & IGHG4 & & ZHX2 & RIOK3 & ATRIP & & & SAP130 & CSTA & \\
\hline & & CDKN1A & ALDOA & & MBTPS1 & RSL1D1 & ACACA & & & ARNTL & ASF1B & \\
\hline & & ZMYM2 & GNB2L1 & & AEBP2 & SMARCA5 & BRE & & & SUDS3 & & \\
\hline & & RNF213 & DSC2 & & JARID2 & CCDC59 & BRCA1 & & & CGGBP1 & & \\
\hline & & ETV6 & ANXA1 & & G2E3 & MRPL13 & BARD1 & & & ELF2 & & \\
\hline & & TINF2 & SERPINB3 & & & PUM1 & ANKRD11 & & & TAF1D & & \\
\hline & & INO80D & MMS22L & & & COBL & & & & ZSCAN20 & & \\
\hline & & PCNA & TGM3 & & & MOB4 & & & & MAX & & \\
\hline & & CACYBP & SFN & & & NFATC2IP & & & & ARID4A & & \\
\hline & & TAF1 & CDSN & & & FBXW11 & & & & BRMS1 & & \\
\hline & & TAF5 & EPPK1 & & & POP4 & & & & AP $2 A 1$ & & \\
\hline & & TAF6 & POF1B & & & PLEKHA4 & & & & MLXIPL & & \\
\hline & & WDR77 & TADA2B & & & CENPV & & & & ING2 & & \\
\hline & & CEBPG & DSC3 & & & HMGB3 & & & & E2F1 & & \\
\hline & & ZNF644 & TF & & & HMG20A & & & & ING1 & & \\
\hline
\end{tabular}




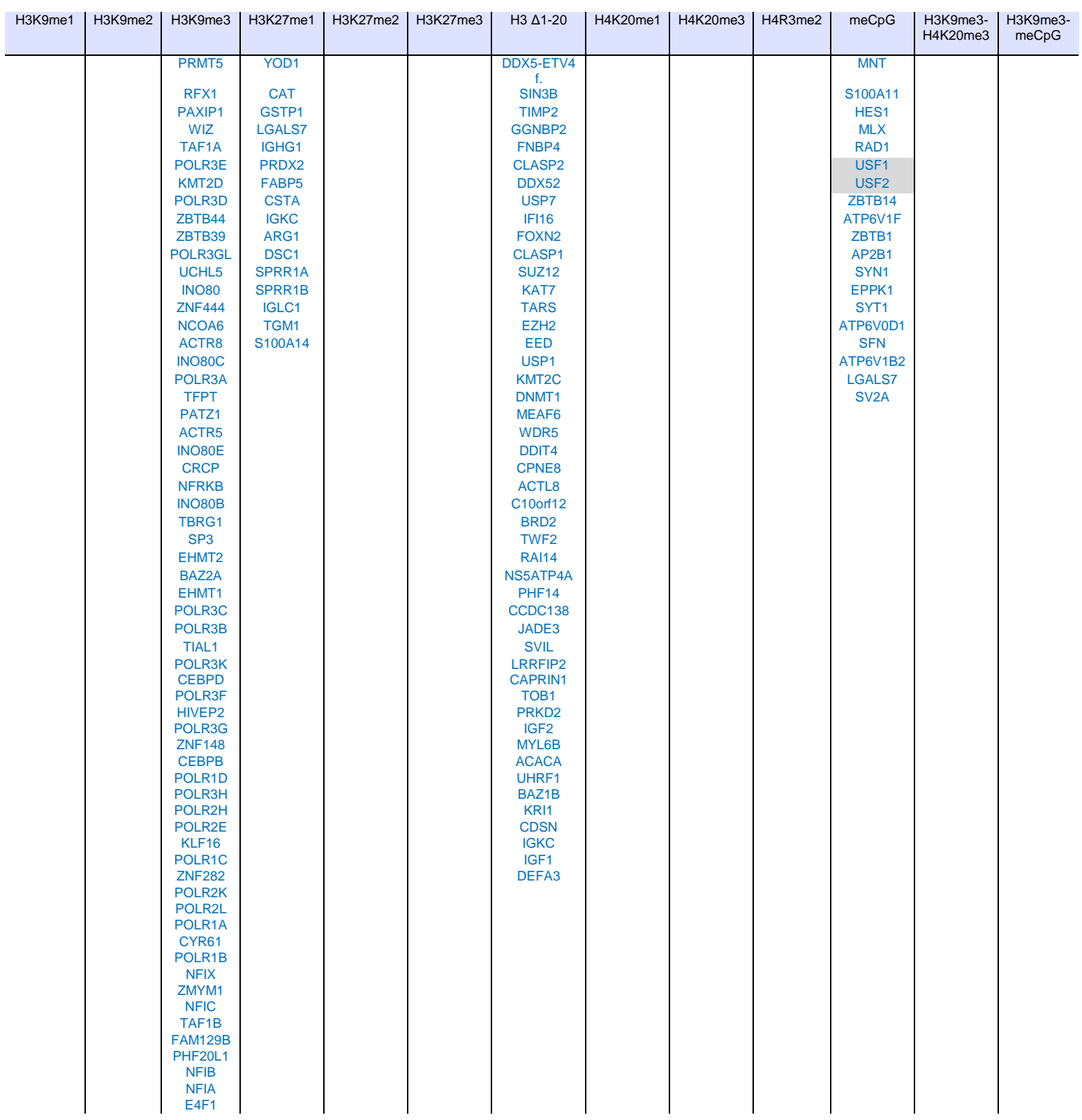

factors, protein complexes were also recruited by H3K9me3. In agreement with previous findings (Bartke et al., 2010; Vermeulen et al., 2010), several members of the human ORC complex were found in the dataset. Additionally, a set of factors not shown to bind to H3K9me3-modified chromatin before was identified. For example, four out of the five subunits of the septin complex were found. Protein-protein interaction analysis using the STRING database predicted an indirect connection of SEPT2 to CBX5 via ADNP (Figure $3.6 \mathrm{~B}$ ). Moreover, this analysis predicted a network including most of the proteins specifically recruited by $\mathrm{H} 3 \mathrm{~K} 9 \mathrm{me} 3$, thus validating the approach in terms of specificity and known biological context. Furthermore, the STRING analysis indicated recruitment of factors completely new in the context of H3K9me3 
binding and therefore argued for a gain in knowledge using chromatin arrays over e.g. histone peptides for identification of histone PTM-binding proteins.

Proteins depleted from binding seemed to be organized in complexes as well. For example, seven subunits of the INO80 complex and several members of the BRAC1-core RNA polymerase II complex were prevented from binding. These findings appeared to be directly connected, as 57 of the 72 factors excluded from binding to H3K9me3 are included in an extensive protein-protein binding network based on STRING analysis (Figure 3.6 C).

\section{$H 3 K_{C} 27 m e 1$}

The mono-methylation of lysine 27 of histone $\mathrm{H} 3$ has been shown to have a higher prevalence at active promoters compared to silent ones (Barski et al., 2007). Other studies mapped the modification to be significantly present in heterochromatin (Jacob et al., 2010). Chromatin affinity purification using $\mathrm{H}_{3} \mathrm{~K}_{\mathrm{C}} 27$ me1 modified chromatin revealed 19 factors significantly recruited and 68 excluded from binding (Figure 3.7 A, Table 3.1). Among them, four members of the CCR4NOT transcription complex, which is a key regulator of gene expression (Collart, 2016), were found (Figure $3.7 \mathrm{C}$ ). Other proteins identified are also involved in positive regulation of gene expression. These include the chromatin assembly factor RFS1 (LeRoy et al., 1998; Shamay et al., 2002) and GCN1L1 (Marton et al., 1997). Also recruited were the deacetylase SIRT6, the ribonucleoprotein RAVR1, which acts as a splicing co-repressor and thereby modulates alternative splicing events (Gromak et al., 2003; Plafker and Macara, 2000), and the proteins XPO1 and IPO11 that function in nuclear export and import, respectively (Kudo et al., 1997). Surprisingly, three of the identified factors, DYM, GET4, and GCC2, are involved in processes connected to the Golgi apparatus and are known to locate to the cytoplasm (Dimitrov et al., 2009; Mariappan et al., 2010; Reddy et al., 2006).

Factors excluded from binding to $\mathrm{H}_{3} \mathrm{~K}_{\mathrm{C}} 27$ mono-methylated chromatin feature a broad range of enzymatic activities and thus functional diversity. For instance, there were proteins depleted from binding that promote transcription such as GTF3C5, GTF3C4 or POLR3K and proteins connected to RNA polymerase II activity such as TAF11 and MED10. Many factors are connected to cytoskeleton associated functions i.e. PFN1, CKAP2, PRC1 and CENPF. Enzymatically active proteins included for example the pyruvate kinase PKM, the hydrolase YOD1 that can remove conjugated ubiquitin from proteins, and the G2/M-phase specific E3 ubiquitin protein ligase G2E3. Two members of the $20 \mathrm{~S}$ proteasome were identified as well. Even though there was a high diversity of expelled factors, protein-protein interactions predicted by STRING indicated that 34 of them are connected to one another (Figure 3.7 B). 
A)

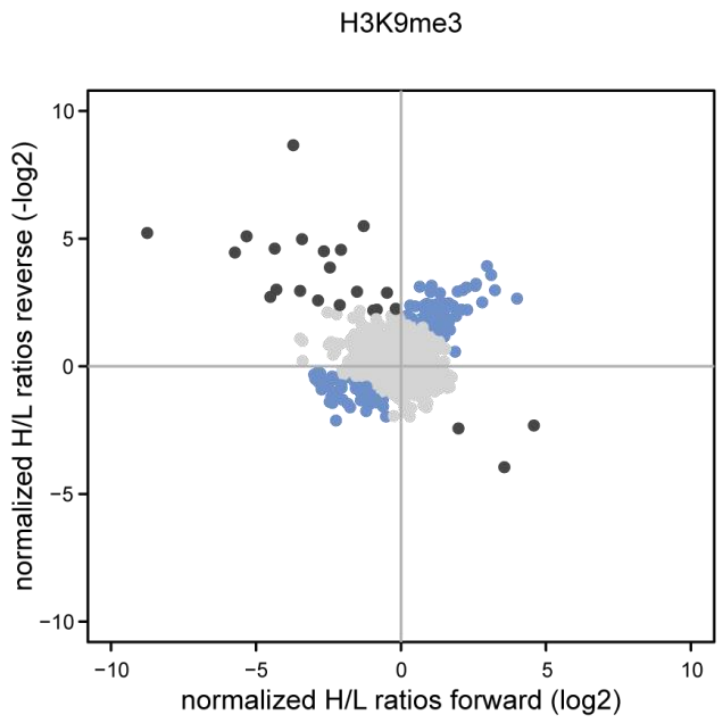

B)

H3K9me3 enriched proteins

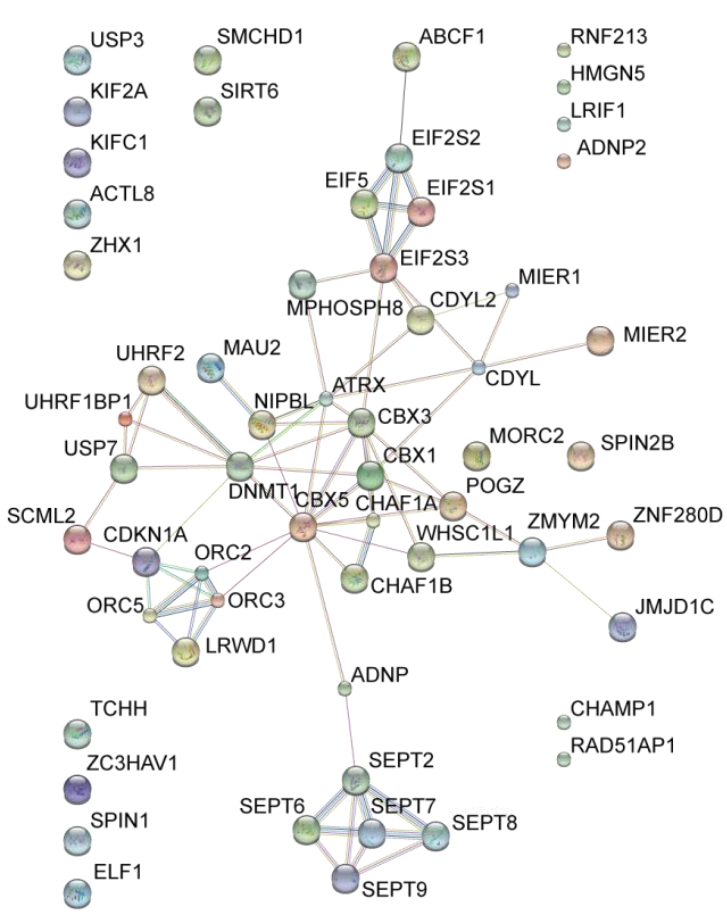

C)

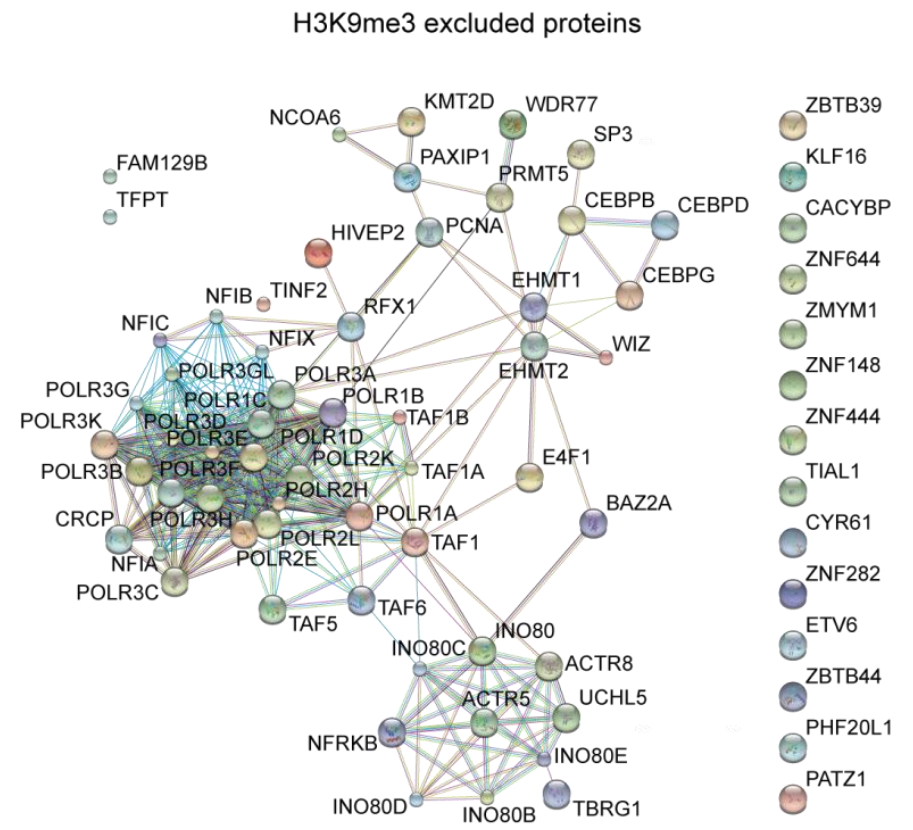

Figure 3.6 Protein-binding interactome of H3K9me3-modified chromatin.

A) H3K9me3 interactome. Proteins are plotted by their $\log _{2}$ SILAC ratios of the forward experiment on the $x$-axis and the reverse experiment on the $y$-axis. Proteins significantly recruited and excluded from binding to chromatin are colored in blue. False positive proteins are marked in black. B) STRING protein interactome of proteins significantly recruited by H3K9me3. C) STRING protein-protein interactions of factors significantly excluded by H3K9me3. 
$H 3 K_{\mathrm{c}} 27 m e 2$

The di-methylated state of $\mathrm{H}_{3} \mathrm{~K}_{\mathrm{C}} 27$ has been shown to be linked to transcriptional repression and localizes to heterochromatin and enhancers (Barski et al., 2007; Wang et al., 2008). 20 factors were recruited whereas 23 factors were repelled from binding to $\mathrm{H} 3 \mathrm{~K}_{\mathrm{C}} 27 \mathrm{me} 2$-modified chromatin (Figure 3.7 D, Table 3.1).

Several PHD finger proteins were recruited to $\mathrm{H}_{3} \mathrm{~K}_{\mathrm{C}} 27 \mathrm{me}$. Prominent examples are (i) the PHF21A protein, which is a component of the BHC co-repressor complex, (ii) the PHF15 (JADE2) protein, which is part of the HBO1 complex that displays histone $\mathrm{H} 4$ acetylation activity and (iii) PHF1, a protein, which is a component of the polycomb group. Remarkably, eight of the enriched factors are related to the spliceosome. SRRM1, MAGOH, EIF4A3, and SRRM2 are known to interact with the assembly intermediate $C$ of the spliceosome complex, while the proteins SRSF6, PRPF40A, RNPS1 and SAP18 interact with the spliceosome at different stages of assembly. Interestingly, SAP18 is also part of the (chromatin) repressor complex SIN3, suggesting a crosstalk between the two processes. Known associations among the recruited proteins are shown in figure $3.7 \mathrm{E}$, upper panel.

RNA splicing occurs either co-transcriptionally or immediately after transcription. Surprisingly, contrary to recruitment of spliceosomal factors, several subunits of the RNA polymerase I were excluded from binding to $\mathrm{H}_{3} \mathrm{~K}_{\mathrm{C}} 27 \mathrm{me} 2$. In addition, INO80, a component of the remodeling complex INO80, was also excluded from binding to $\mathrm{H}_{3} \mathrm{~K}_{\mathrm{C}} 27 \mathrm{me} 2$.

INO80 was additionally excluded from H3K9 mono- and tri- methylated chromatin. A similar binding profile was observed for the nuclear factors NFIB and E4F1 that were besides $\mathrm{H} 3 \mathrm{~K} 9 \mathrm{me} 1 /-\mathrm{me} 2 /-\mathrm{me} 3$ also excluded from $\mathrm{H} 3 \mathrm{~K}_{\mathrm{C}} 27 \mathrm{me} 2$. These findings support a linkage of biological functions facilitated by $\mathrm{H} 3 \mathrm{~K} 9 \mathrm{me} 1 /-\mathrm{me} 2 /-\mathrm{me} 3$ and $\mathrm{H} 3 \mathrm{~K}_{\mathrm{C}} 27 \mathrm{me} 2$ and indicate the exclusion of these factors from most heterochromatic regions. In general, the fact that several factors were regulated the same way by different modification states (Table 3.1) support the existence of a set of factors generally associated with the establishment and maintenance of certain chromatin stages.

An example of proteins underlying opposed regulation by different modifications is CHAF1B, a member of the chromatin assembly complex $1(\mathrm{CAF}-1)$. The protein was excluded from binding to $\mathrm{H}_{3} \mathrm{~K}_{\mathrm{C}} 27 \mathrm{me} 2$ but enriched by H3K9me3.

STRING analysis indicated that a protein complex comprised of the transcription factors SP1, SP3, ESRRA and NR2C1 was significantly depleted from $\mathrm{H}_{3} \mathrm{~K}_{\mathrm{C}} 27$ me2-modified chromatin arrays (Figure $3.7 \mathrm{E}$, lower panel). Another interaction among expelled proteins was identified between MLL3, a H3K4 methyltransferase, and the protein PAXIP1, which is involved in DNA damage response and transcriptional regulation (Wang et al., 2010). 
A)

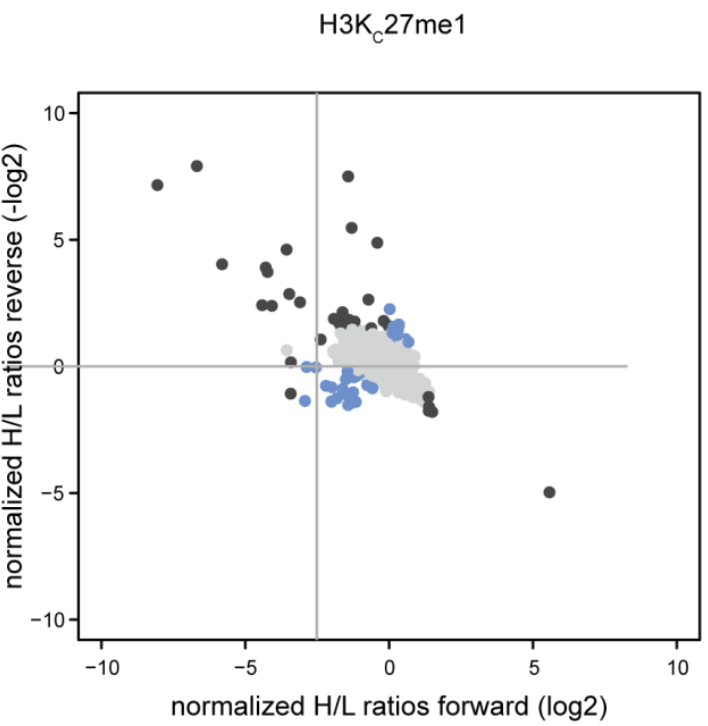

C)

$\mathrm{H} 3 \mathrm{~K}_{\mathrm{C}} 27 \mathrm{me} 1$ enriched proteins

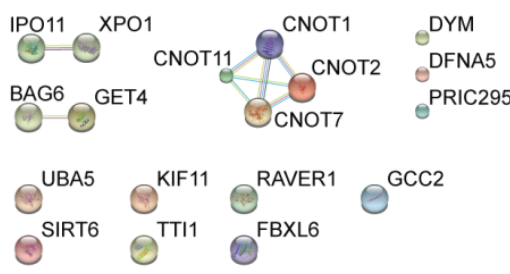

D)

H3K $27 m e 2$

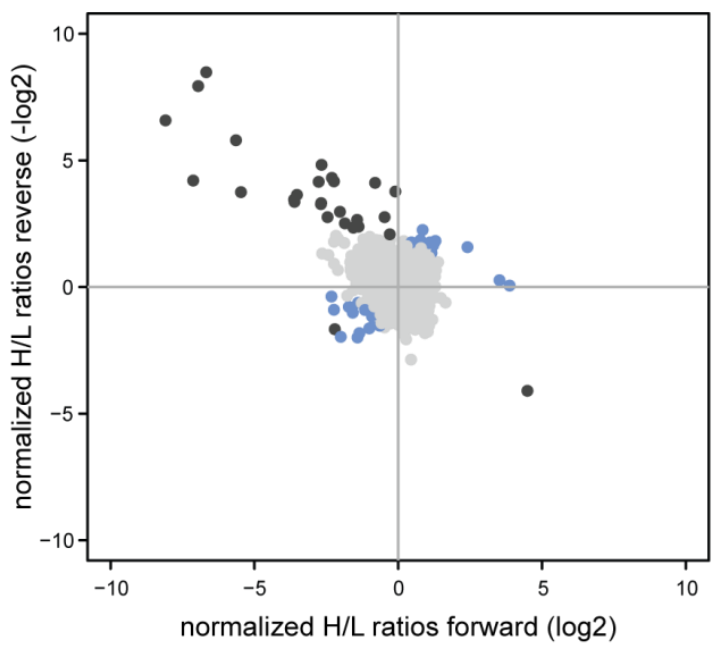

B)

$\mathrm{H} 3 \mathrm{~K}_{\mathrm{c}} 27 \mathrm{me} 1$ excluded proteins

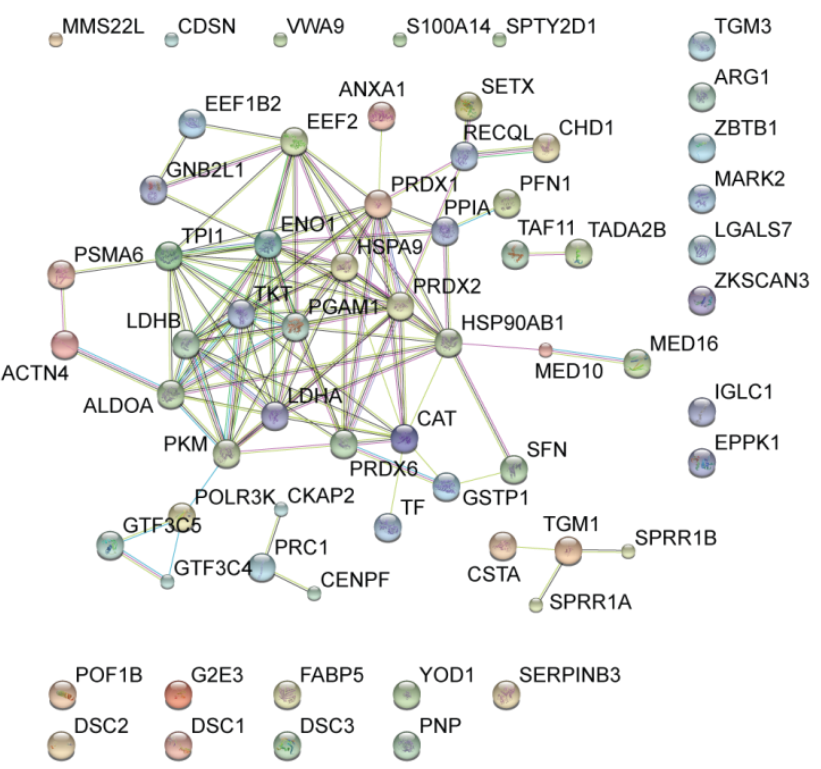

E)

$\mathrm{H} 3 \mathrm{~K}_{\mathrm{C}} 27 \mathrm{me} 2$ enriched proteins

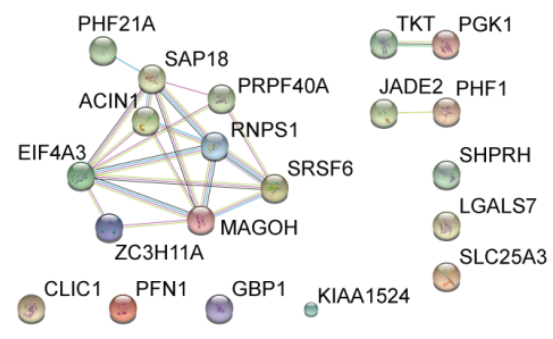

$\mathrm{H} 3 \mathrm{~K}_{\mathrm{c}} 27 \mathrm{me} 2$ excluded proteins

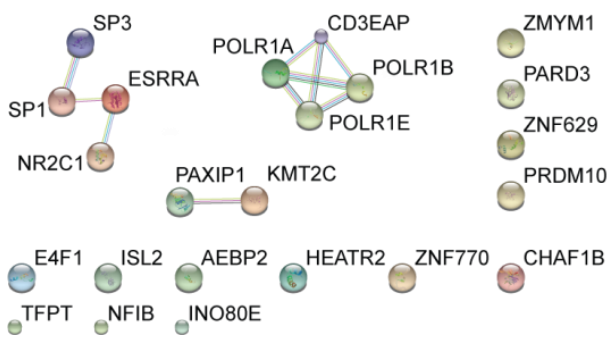


Figure 3.7 Protein-binding interactomes of $\mathrm{H}_{3} \mathrm{~K}_{\mathrm{c}} 27$ mono- and di-methylated chromatin.

A) $\mathrm{H}_{3} \mathrm{~K}_{\mathrm{C}} 27 \mathrm{me} 1$ interactome. Proteins are plotted by their $\log _{2}$ SILAC ratios of the forward experiment on the $x$-axis and the reverse experiment on the $y$-axis. Proteins that are significantly recruited or depleted from binding to chromatin by the modification are colored in blue. Proteins that were identified as false positive are marked in black. B) Protein-protein interaction network of proteins significantly excluded by

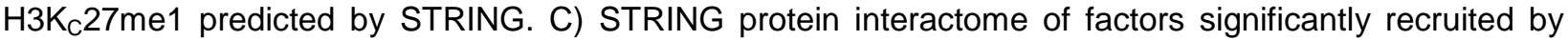
$\mathrm{H} 3 \mathrm{~K}_{\mathrm{C}} 27 m e 1$. D) $\mathrm{H} 3 \mathrm{~K}_{\mathrm{C}} 27 m e 2$ interactome. Data are represented as described in A. E) STRING predicted protein interactome of factors significantly regulated by $\mathrm{H}_{3} \mathrm{~K}_{\mathrm{c}} 27 \mathrm{me} 2$. Recruited factors are shown in the upper part of the panel, whereas excluded factors are shown in the lower part of the panel.

\section{$H 3 K_{C} 27 m e 3$}

The histone modification H3K27me3 is mainly present at silent promoters and is known to be related to gene silencing (Barski et al., 2007; Ringrose and Paro, 2004; Wang et al., 2008).

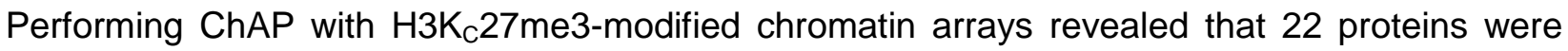
recruited and 37 proteins were excluded from binding to this modification in the context of chromatin (Figure 3.8 A, Table 3.1).

Protein-protein interaction analysis using STRING predicted three multi subunit complexes among the proteins recruited to $\mathrm{H}_{3} \mathrm{~K}_{\mathrm{C}} 27 \mathrm{me} 3$. The complex encompassing the highest number of proteins is formed by several translation initiation factors, connected to the chromo domain protein CDYL2 (Figure 3.8 B, upper panel). A second complex is formed by the members of the ORC complex that was also shown to be recruited to H3K9me3-modified chromatin (Figure 3.8 $\mathrm{B}$, upper panel). In a recent study, members of the ORC complex were shown to bind to both modification states, H3K9me3 and H3K27me3, in the context of mononucleosomes (Bartke et al., 2010). TOPBP1, which is required for DNA replication (Makiniemi et al., 2001), was also recruited but not shown to be in association with the ORC complex. A third complex was formed by the proteins PRC1, KIF2A and two members of the repressive polycomb group, CBX8 and PHF1 (Figure $3.8 \mathrm{~B}$, upper panel). The polycomb group proteins are well studied and known to interact with H3K27 methylation states (van Kruijsbergen et al., 2015), therefore they were expected to bind to H3K27me3-modified chromatin.

The protein-binding interactome of H3K27me3 was already investigated in the context of mononucleosomes (Bartke et al., 2010). CBX8 was the only protein found to be recruited by H3K27me3-modified mononucleosomes and in the here presented study. No further overlap was observed, as in the context of mononucleosomes factors depleted from binding to H3K27me3 were not identified.

Factors like G2E3 and NOLC1 were identified to be excluded from binding to $\mathrm{H}_{3} \mathrm{~K}_{\mathrm{C}} 27 \mathrm{me}$ modified chromatin. These factors have been identified in more than one of the chromatinbinding interactomes, always in the context of exclusion from chromatin binding. Additionally, in contrast to $\mathrm{H}_{3} \mathrm{~K}_{\mathrm{C}} 27 \mathrm{me} 2$, factors related to the spliceosome are depleted by $\mathrm{H}_{3} \mathrm{~K}_{\mathrm{C}} 27 \mathrm{me} 3$, demonstrating an opposed protein-binding regulation by different modification states as 
described for other factors before. Other expelled proteins are (i) transcriptional activators, (ii) factors functionally related to the cytoskeleton, (iii) proteins with kinase activity. The PHD finger proteins PHF8 and PH15 were found to be depleted as well.

The interactome of $\mathrm{H}_{3} \mathrm{~K}_{\mathrm{C}} 27 \mathrm{me} 3$ revealed the recruitment as well as repulsion of transcription factors from chromatin, which indicates the possibility of existing fine-tuning mechanisms of protein-binding properties mediated by a distinct PTM.

A)

$\mathrm{H} 3 \mathrm{~K}_{\mathrm{c}} 27 \mathrm{me} 3$

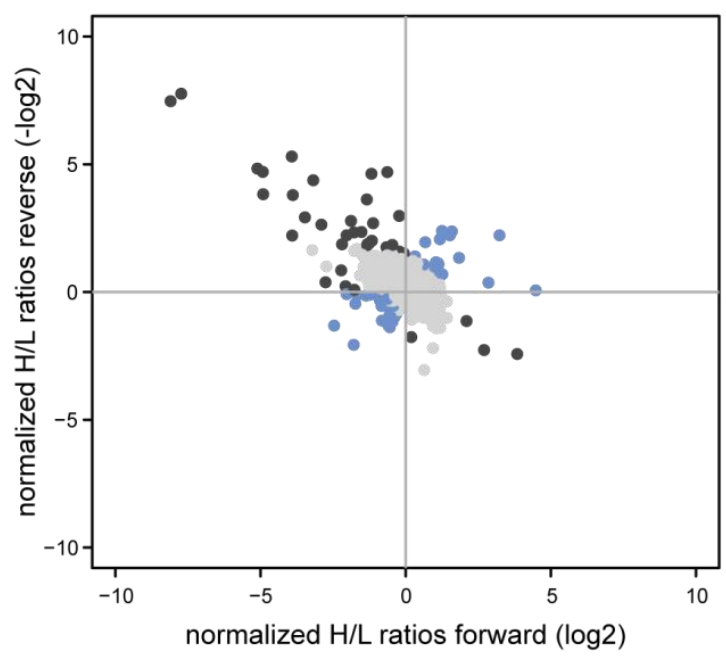

B) $\mathrm{H} 3 \mathrm{~K}_{\mathrm{C}} 27 \mathrm{me} 3$ enriched proteins

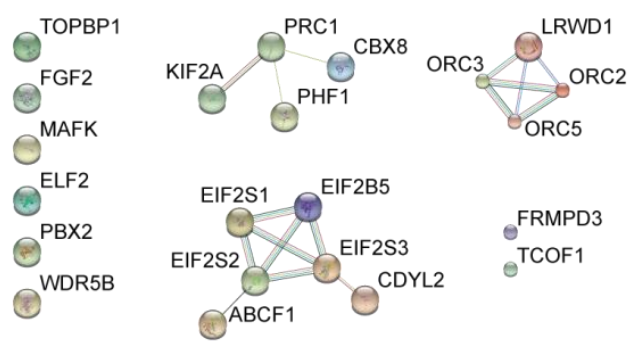

$\mathrm{H} 3 \mathrm{~K}_{\mathrm{c}} 27 \mathrm{me} 3$ excluded proteins

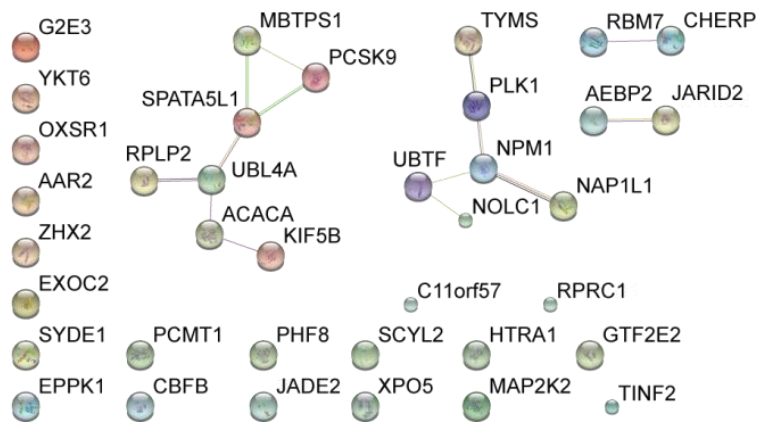

Figure $3.8 \mathrm{H} 3 \mathrm{~K}_{\mathrm{c}} 27 \mathrm{me} 3$ chromatin-binding interactome.

A) $\mathrm{H}_{3} \mathrm{~K}_{\mathrm{C}} 27 \mathrm{me} 3$ interactome. Data are represented as described in figure $3.7 \mathrm{~A}$. B) STRING predicted protein-protein interaction networks of factors significantly recruited (upper part of the panel) and depleted (lower part of the panel) by $\mathrm{H} 3 \mathrm{~K}_{\mathrm{c}} 27 \mathrm{me} 3$.

\section{H4K20me1}

Similar to all other investigated mono-methylation marks it was shown that H4K20me1 is linked to gene activation and co-localizes with H3K9me1 in vivo (Barski et al., 2007; Wang et al., 2008). 19 factors were enriched while 35 factors were excluded from binding to H4K20me1modified chromatin (Figure 3.9 A, Table 3.1). Two components of the INO80 complex were recruited as well as the nucleosome assembly protein NAP1L4 and the RNA polymerase subunit gamma-1. Several factors involved in transcriptional repression were found to be recruited as well. Among them I identified PHF21A, HES1 and CIR1 (Figure 3.9 B, upper panel). 
Protein-protein interaction analysis using STRING indicated that two complexes were depleted from binding to mono-methylated H4K20 (Figure 3.9 B, lower panel). The larger protein complex predicted by STRING consists of 18 proteins. 16 of these proteins are spliceosomal factors, mainly connected to the $C$ complex. The second protein network includes members of the BRCA1-A complex, BARD1, BRCA1 and BRE, which are responsible for the maintenance of genome stability. The proteins ACACA and ATRIP have been shown to associate with this complex as well (Figure $3.9 \mathrm{~B}$, lower panel). Altogether, the predicted STRING interactome connected 25 of the 35 excluded factors, suggesting most of the proteins to be linked to similar biological functionalities.

\section{H4K20me3}

Tri-methylated H4K20 has been shown to localize to heterochromatin and is connected to transcriptional repression (Barski et al., 2007; Schotta et al., 2004; Wang et al., 2008). Performing ChAP 32 factors were enriched and 11 factors excluded from binding to H4K20me3 chromatin (Figure 3.9 C, Table 3.1). The modification recruited the ORC complex together with its binding components LRWD1 and APEX1. With the exception of APEX1 the complex was also recruited to tri-methylated $\mathrm{H} 3 \mathrm{~K} 9$ and $\mathrm{H}_{3} \mathrm{~K}_{\mathrm{C}} 27$ (Table 3.1). The co-purification of the ORC complex with all three methylation sites has been shown before (Vermeulen et al., 2010). Additionally, STRING analysis predicted protein-protein associations between several recruited translation initiation factors (Figure $3.9 \mathrm{D}$, upper panel), which were also recruited to chromatin by $\mathrm{H}_{3} \mathrm{~K}_{\mathrm{C}} 27 \mathrm{me} 3$. This complex showed additional association with two taxilin proteins connected to the hydrolase USP3, which is known to deubiquitinate monoubiquitinated target proteins, such as histone $\mathrm{H} 2 \mathrm{~A}$ and $\mathrm{H} 2 \mathrm{~B}$. Several kinesin family members in association with DNAH8 and PHF1 formed a third complex predicted by STRING (Figure 3.9 D, upper panel).

H4K20me3 excluded several factors from binding to chromatin that have been shown to be excluded by other modification states investigated in this study as well. For instant, besides H4K20me3 all three methylation states of H3K9 excluded factors of the NFI gene family and the protein E4F1. The heterodimer BARD1-BRCA1 was excluded from binding to H4K20me1 as well as H4K20me3. Additionally, an identical regulation pattern was identified for a third protein, ANKRD12, which was regulated the same way by both introduced H4K20 methylation degrees. 
A)

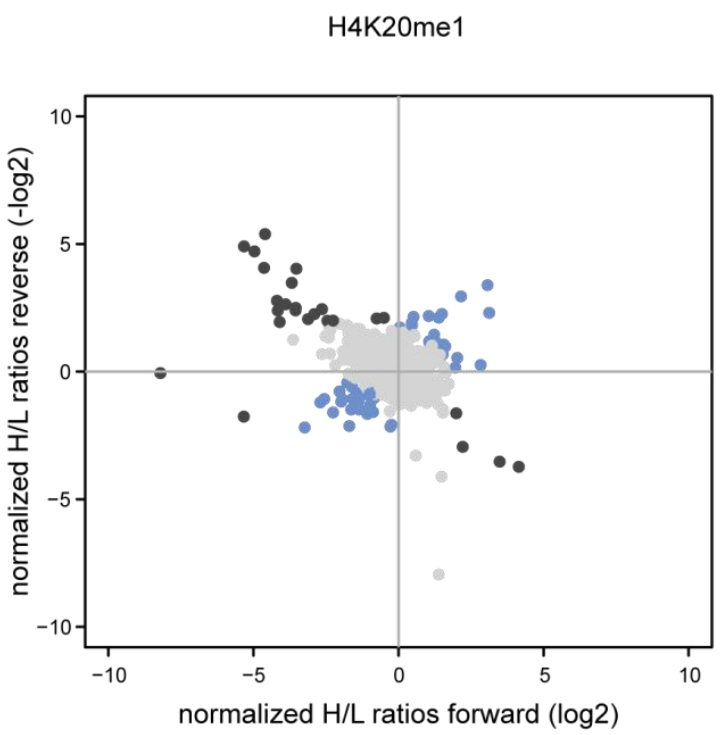

C)

H4K2Ome3

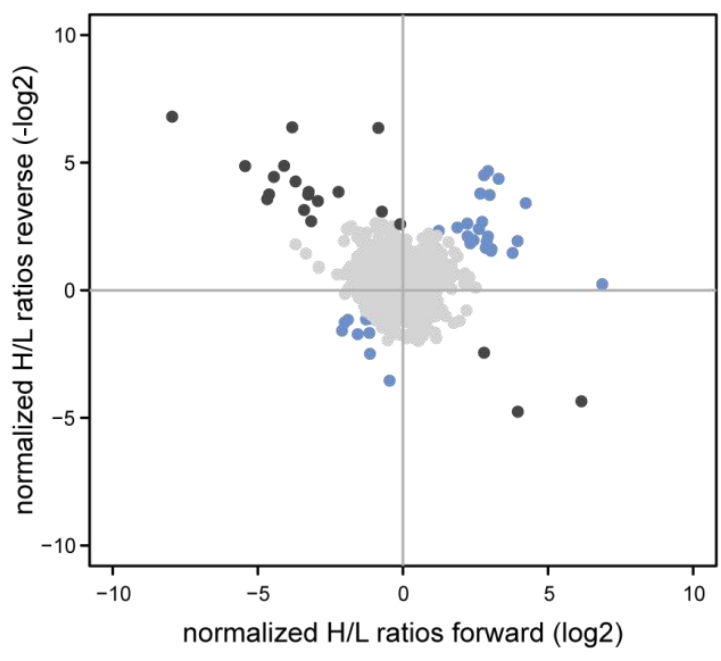

B)

H4K20me1 enriched proteins

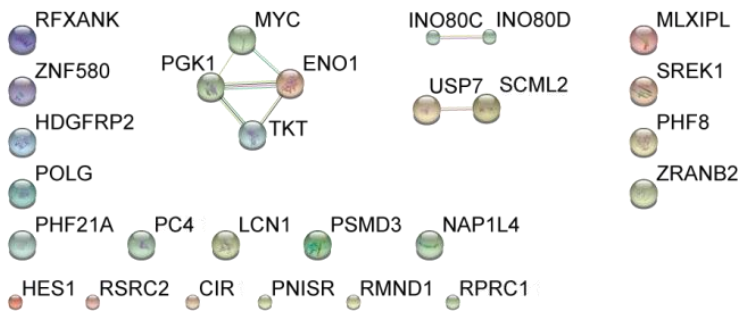

H4K20me1 excluded proteins

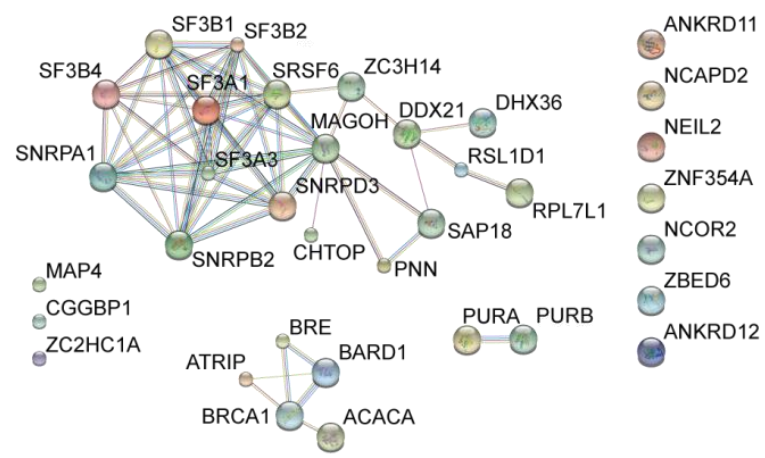

D)

H4K20me3 enriched proteins

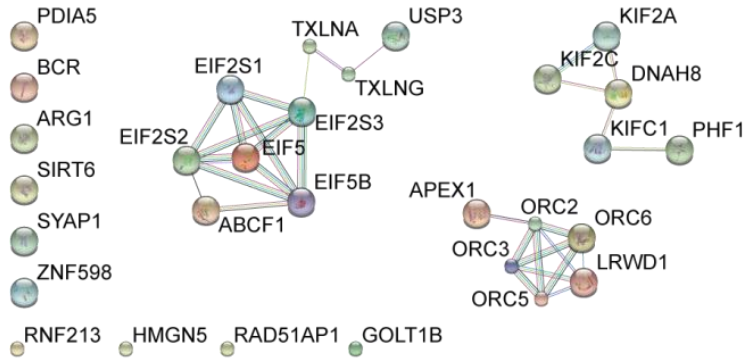

H4K20me3 excluded proteins

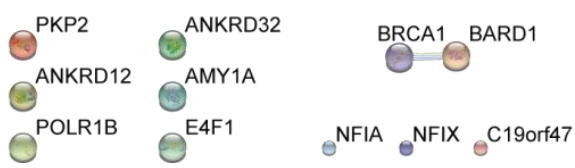

Figure 3.9 Chromatin-associated protein interactomes of histone H4K20 mono- and tri-methylation. A) $\mathrm{H} 4 \mathrm{~K} 20$ me1 interactome. $\log _{2} \mathrm{H} / \mathrm{L}$ ratios of proteins identified in the forward experiment are plotted on the $x$-axis and of the reverse experiment on the $y$-axis. Proteins that are significantly recruited or depleted from binding to chromatin arrays are colored in blue. False positive proteins are marked in black. B) STRING interactome of proteins significantly enriched (upper part of the panel) and excluded (lower part of the panel) by H4K20me1. C) H4K20me3 interactome. Data are represented as described in A. D) STRING protein-protein interaction network of proteins significantly recruited (upper part of the panel) and depleted (lower part of the panel) by H4K20me3. 


\section{H4R3me2}

Arginine 3 of histone $\mathrm{H} 4$ is symmetrically di-methylated by PRMT5, a methyltransferase that is part of the repressive MB2/NURD complex. I found this complex recruited by H3K9me1 and depleted with H3K9me3 (Table 3.1). According to the literature, H4R3me2 was expected to be a mark associated with heterochromatin (Le Guezennec et al., 2006; Zhao et al., 2009). Nine factors were recruited to H4R3me2-modified chromatin and 11 factors were found to be excluded from binding (Figure 3.10 A, Table 3.1). Surprisingly, six of the recruited factors are DNA-directed polymerases, suggesting an association to transcriptional activity for H4R3me2. Additionally, two transcription factors and the protein SNC73 were recruited, supporting the assumption that this modification is rather associated with transcriptional activity and therefore unlikely to be linked to pericentric heterochromatin. This assumption is further supported by the fact that H4R3me2 excluded the proteins ACTL8, USP7, SCML2 and RAD51AP1 from binding to chromatin, as all proteins have been shown to be recruited by H3K9me3, a well-studied marker for pericentric heterochromatin.

\section{$H 3 \Delta 1-20$}

ChAP was performed with chromatin arrays containing truncated histones $\mathrm{H} 3$ that were missing the first $20 \mathrm{~N}$-terminal amino acids (Figure $3.10 \mathrm{~B}$ ). This experiment gave indications of protein binding to $\mathrm{H} 3$ influenced by the unmodified histone tail.

The experiment revealed 71 factors with a higher affinity to the wt $\mathrm{H} 3$, and thus these proteins were depleted from binding to chromatin when the $\mathrm{N}$-terminus of $\mathrm{H} 3$ was missing (Table 3.1). USP7, DNMT1, ACTL8, and UHRF1 were found to be depleted by H3A1-20, which was not surprising as they have been demonstrated to be highly enriched in the context of several ChAP experiments with methylated $\mathrm{H} 3$ tails (Table 3.1). Other proteins excluded from binding to $\mathrm{H} 3 \Delta 1$ 20 were JADE3 (PHF16), several factors of the polycomb group, such as SUZ12, and the helicase SMARCA5. All these proteins are known to be associated with chromatin in the context of PTMs of the $\mathrm{H} 3 \mathrm{~N}$-terminus and therefore are not unlikely to be excluded from binding to $\mathrm{H} 3 \Delta \mathrm{\Delta}-20$ containing chromatin.

The proteins CDSN, IGKC and the histone methyltransferase KMT2C (MLL3) were found to be depleted by $\mathrm{H} 3 \Delta 1-20$ as well as by chromatin arrays containing methylated $\mathrm{H} 3$. This finding indicates that these proteins preferentially associate with chromatin in presence of the unmodified $\mathrm{H} 3$ tail.

Interestingly, the $\mathrm{N}$-terminal tail of $\mathrm{H} 3$ seems also to have a role in prevention of protein binding to chromatin. I identified 45 proteins that were significantly recruited to chromatin containing tailless histone H3 (Figure 3.10 B, Table 3.1). Most likely, these proteins showed enrichment in 
consequence of a better accessibility to the globular domain of $\mathrm{H} 3$ or other parts of the nucleosome that might be sterically blocked by the presence of the $\mathrm{H} 3 \mathrm{~N}$-terminal tail.

A)

H4R3me2

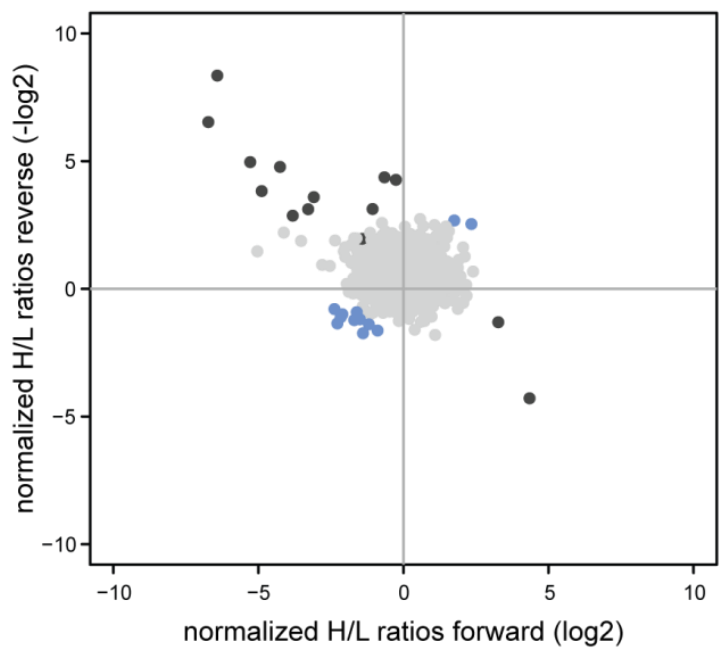

B)

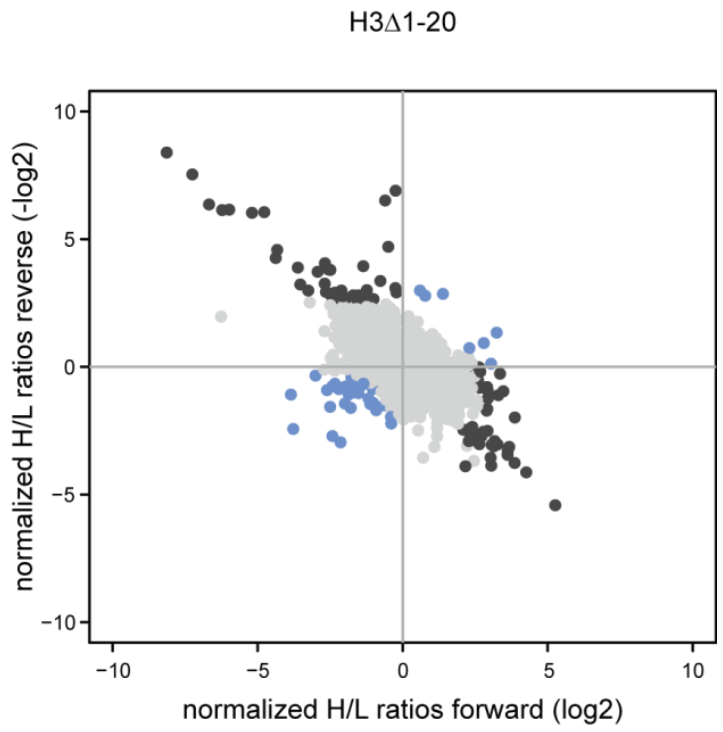

Figure 3.10 Chromatin-binding proteins regulated by H4R3me2 and H3A1-20.

Proteins are plotted by their $\log _{2}$ SILAC ratios of the forward experiment on the $x$-axis and the reverse experiment on the $y$-axis. Proteins that are significantly recruited or depleted from binding to chromatin by the modification are colored in blue. Proteins that were identified as false positive are marked in black. A) H4R3me2 protein-binding interactome. B) H3 $\Delta$ 1-20 interactome.

\section{Methylated DNA (meCpG)}

Chromatin arrays carrying methyl at position 5 of the cytosine pyrimidine ring within $\mathrm{CpG}$ stretches of the underlying DNA template recruited 42 factors and depleted 47 factors significantly from binding to nucleosomal arrays (Figure 3.11 A, Table 3.1). As DNA methylation has mainly been described as a repressive mark, I expected to find an overlap with factors also regulated by other repressive marks. Surprisingly, the only overlap of recruited factors was found between meCpG and H3K9me3. Among the overlapping nine factors were UHRF1, DNMT1, SCML2, USP7 and ACTL8. None of the factors regulated by $\mathrm{H}_{3} \mathrm{~K}_{\mathrm{C}} 27 \mathrm{me} 3$ or H4K20me3 were regulated by methylated DNA (Table 3.1). 19 of the factors recruited by meCpG were predicted by the STRING database to be associated with each other (Figure $3.11 \mathrm{~B}$ ). Among these proteins are chromo domain helicase binding proteins, metastasis associated 1 family members, the methyl $\mathrm{CpG}$ binding protein 2 and zinc finger proteins.

In the context of mononucleosomes affinity purification was performed with methylated DNA using a DNA template based on the 601 sequence (Bartke et al., 2010) similar to what was used in the here presented study. Five out of nine recruited factors that have been identified to be recruited in that study, namely, MECP2, MBD2, MTA2, CHD4, GATAD2A, were recruited by 
DNA methylated oligonucleosomal arrays as well. The proteins MAX, USF2, BHLHB2 and USF1 were depleted from binding to both, mono- and oligonucleosomal arrays.

Protein-protein interaction analysis of excluded proteins indicated that only ten proteins were not associated with the predicted network (Figure $3.11 \mathrm{C}$ ). Several factors of the ING2 complex were excluded from binding including ING1 and its associated factor SFN. An additional complex was predicted by three proteins connected to the V-ATPase. DNA-dependent RNA polymerase and transcriptional activators were depleted from binding, supporting an association with gene silencing. The overlap observed among proteins excluded from meCpG and H3K9me3 binding is unique to polymerase subunits, namely POLR3F and POLR1C.

A)

meCpG

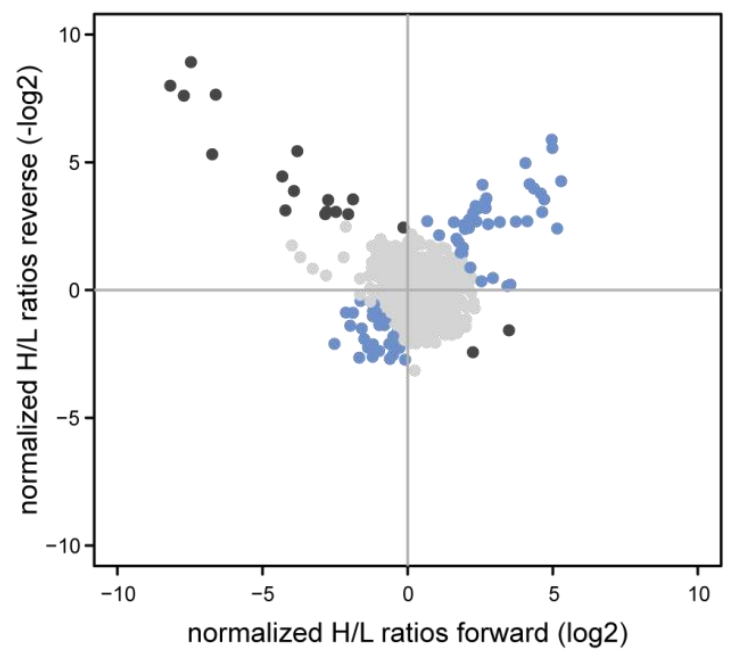

B)

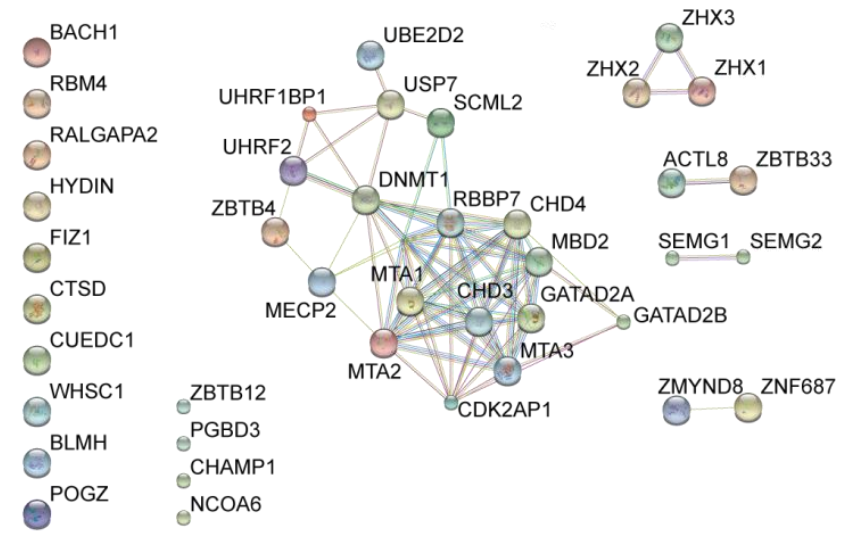

C)

meCpG excluded proteins

$e^{\text {EPPK100A11 }}$
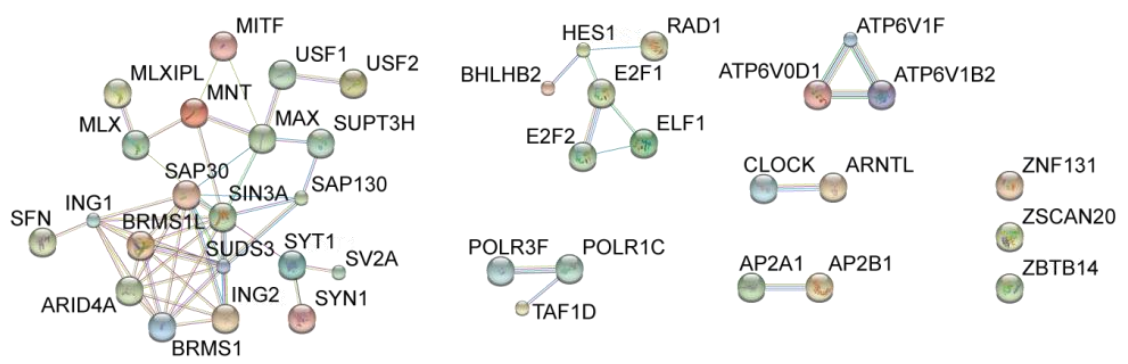

Figure 3.11 Protein-binding interactome of chromatin containing methylated DNA.

A) Protein-binding interactome of $\mathrm{meCpG}$ containing chromatin. $\log _{2}$ scaled $\mathrm{H} / \mathrm{L}$ ratios of proteins identified in the forward experiment were plotted on the $x$-axis and the reverse experiment on the $y$-axis. Factors determined as significant outliers are marked in blue. False positives are marked in black. B) STRING protein-protein interaction analysis of factors significantly recruited by meCpG. C) Protein-protein network of proteins significantly depleted from meCpG chromatin predicted by STRING. 


\subsubsection{The methylation degrees of lysine residues affect protein binding to different extends}

The functional significance of different methylation degrees of lysine residues within histones is still under discussion. The lower methylation degrees, mono- and di-methylation, might act as platforms that enable the establishment of the respective higher methylation state, leading to the tri-methylation mark that affects downstream processes. Alternatively, each of the three methylation degrees has their own biological significance and thus lead to distinct functional outcomes themselves.

In the following section I compared the protein-binding interactomes of different methylation degrees of H3K9, H3K27 and H4K20 to obtain more insights on how individual methylation degrees regulate chromatin-binding properties of individual factors and protein complexes.

A)

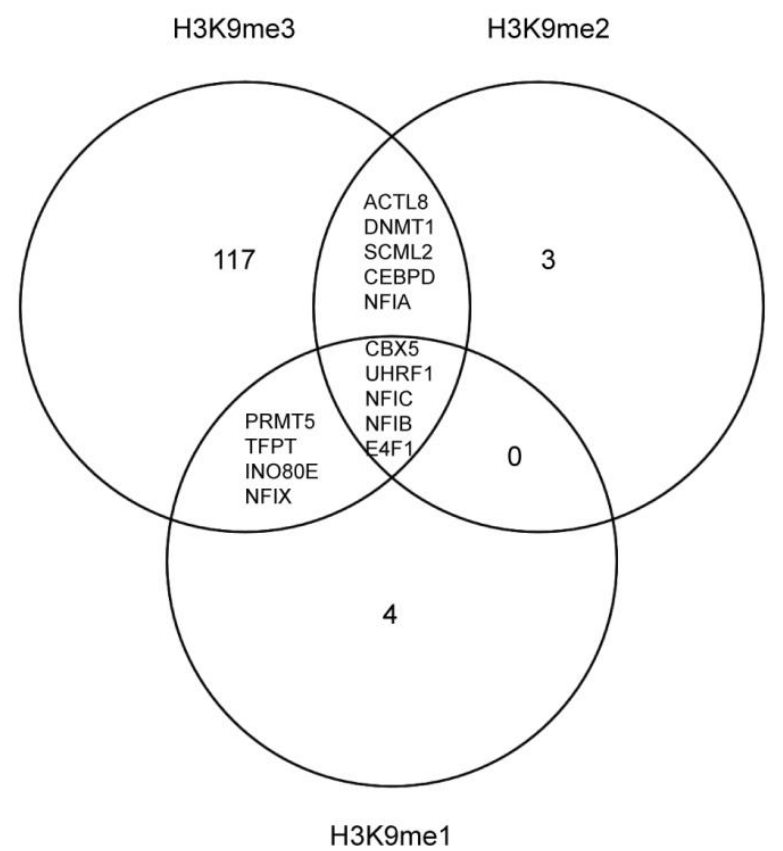

B)

overlap of significantly regulated proteins

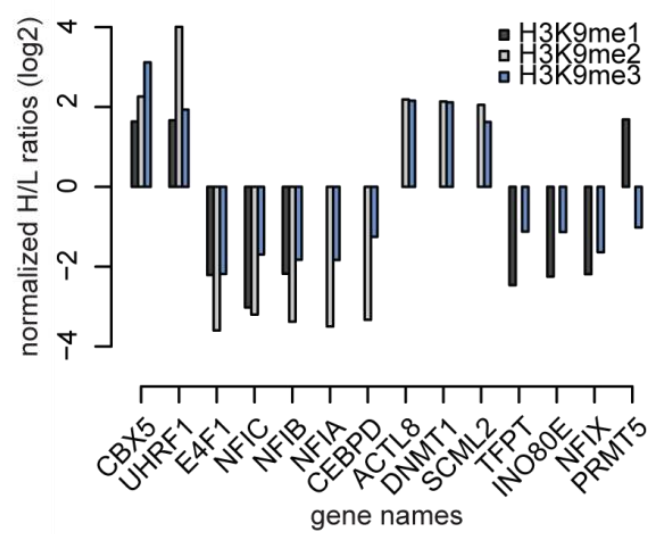

Figure 3.12 Overlap of proteins regulated by the methylation degrees of H3K9.

A) Venn diagram of all factors significantly regulated by mon-, di- and tri-methylation of H3K9. B) Bar plot of proteins regulated by at least two modification states of H3K9. $\log _{2}$ scaled SILAC ratios are plotted on the $y$-axis. Gene names are plotted on the x-axis.

Mono-, di- and tri-methylation of $\mathrm{H} 3 \mathrm{~K} 9$

Each of the methylation states of H3K9 recruited and repelled a specific set of factors from binding to the modified chromatin arrays (Figure 3.5, 3.6, Table 3.1). Remarkably, the number of factors regulated by $\mathrm{H} 3 \mathrm{~K} 9 \mathrm{me} 3$ was 10 times higher than those regulated by $\mathrm{H} 3 \mathrm{~K} 9 \mathrm{me} 1$ and 
H3K9me2. In fact, mono- and di-methylation of H3K9 affected the binding behavior of 13 proteins, whereas H3K9me3 had an impact on 131 proteins. The overlap of significantly regulated factors is shown in figure $3.12 \mathrm{~A}$. A set of five proteins, CBX5, UHRF1, NFIB, NFIC, and E4F1 was affected by all three modification states. These proteins were regulated the same way. They were either recruited to all modification states or excluded from binding to chromatin (Figure 3.12 B). Several proteins displayed an overlap between two of the modification states (Figure 3.12). With the exception of one factor, PRMT5, all proteins were regulated in the same direction by the overlapping methylation states. PRMT5 is the only factor found to display an opposed binding profile, as the protein was significantly recruited to $\mathrm{H} 3 \mathrm{~K} 9 \mathrm{me} 1$ but repelled from binding to $\mathrm{H} 3 \mathrm{~K} 9 \mathrm{me} 3$ (Figure $3.12 \mathrm{~B}$ ).

\section{Mono-, di- and tri-methylation of H3K27}

The three different methylation states of $\mathrm{H}_{3} \mathrm{~K}_{\mathrm{C}} 27$ affected different protein interactomes, indicating the specificity of each modification state on protein-binding properties to chromatin (Figure 3.7, 3.8, Table 3.1). Notably, none of the modified lysine residues within histones investigated in this study demonstrated such a high divergence of protein binding regulated by different methylation degrees. The different H3K27 methylation states have been shown to localize to different genomic regions, with a greater diversity than H3K9 and H4K2O methylation states (Rosenfeld et al., 2009). Contrary to H3K9 methylation, not a single factor was found to

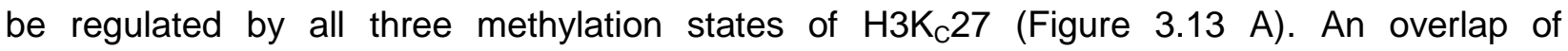
significantly regulated proteins was observed only between two of the H3K27 methylation states (Figure $3.13 \mathrm{~A}$ ). The three factors, LGALS7, PFN1 and TKT were significantly affected by

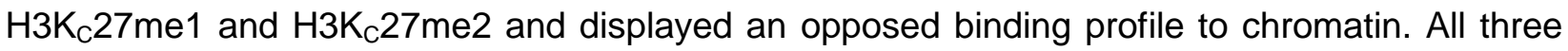
were significantly repelled from binding to $\mathrm{H}_{3} \mathrm{~K}_{\mathrm{C}} 27$ me1-modified chromatin while $\mathrm{H} 3 \mathrm{~K}_{\mathrm{C}} 27 \mathrm{me} 2$ significantly recruited these proteins (Table 3.1). JADE2 and the protein PRC1 also showed an opposed binding to chromatin in the context of different H3K27 methylation states. I also observed PHF1, AEBP2, EPPK1 and G2E3, to exhibit an opposed binding profile, thus, recruited and depleted from binding by two different H3K27 methylation states (Table 3.1).

\section{Mono- and tri-methylation of H4K20}

For H4K20 only mono- and tri-methylation states were investigated. Significantly regulated proteins of both modification degrees showed a high divergence (Figure 3.9, Table 3.1). An overlap was observed for only three factors, including ANKRD12, BRCA1 and BARD1 (Figure $3.13 \mathrm{~B})$. All three proteins were excluded from binding independently of the H4K20 methylation state investigated. 
Taken together, only a small set of common factors was observed to show the same binding profile to chromatin containing different modification degrees of a certain methylation site. The H3K9 methylation degrees showed an exceptionally high overlap of factors regulated the same way (Figure 3.12 A, Table 3.1). At least $70 \%$ of H3K9me1 and of $\mathrm{H} 3 \mathrm{~K} 9 \mathrm{me} 2$ regulated proteins were found to display the same binding profile as to H3K9me3. These findings suggest a functional link between the lower modification states and H3K9me3.

In contrast, diverse examples of proteins affected by the modification degrees of $\mathrm{H} 3 \mathrm{~K} 27$ and H4K20 highlighted the varying effect methylation degrees can have on the regulation of chromatin-binding interactomes that was not given for the H3K9 methylation states.

A)

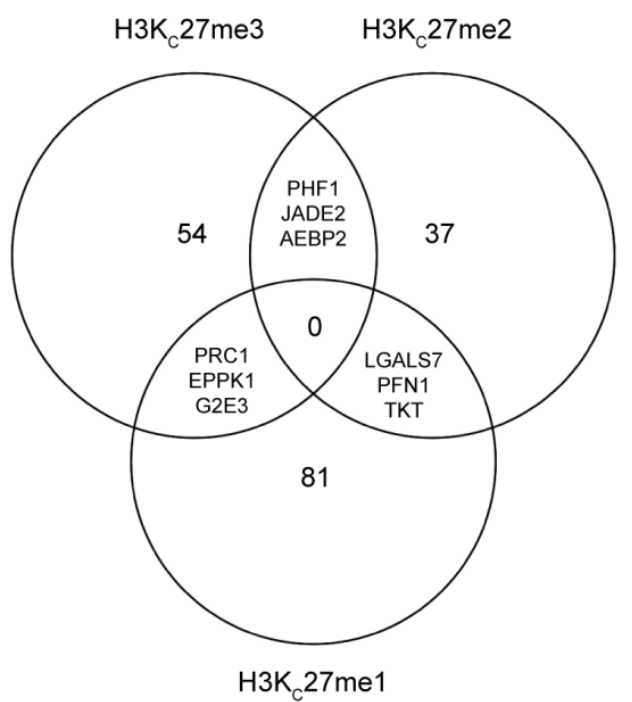

B)

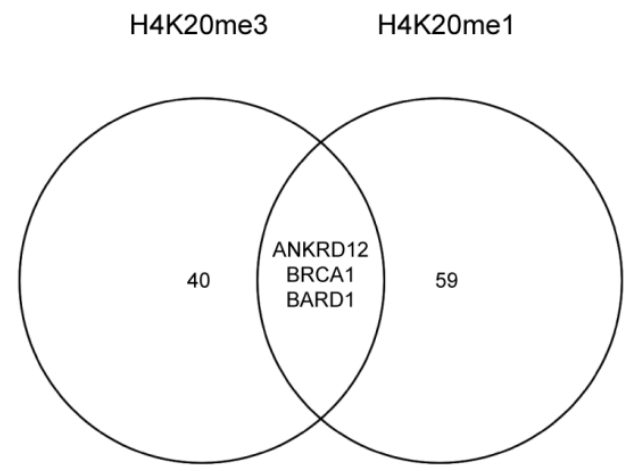

Figure 3.13 Overlap of significant proteins regulated by different methylation states.

A) Venn diagram of proteins significantly regulated by mono-, di- and tri-methylation of $\mathrm{H}_{3} \mathrm{~K}_{\mathrm{C}} 27$. B) Venn diagram of proteins significantly regulated by H4K20me1 and H4K20me3.

\subsubsection{Two chromatin modifications in trans do not only constitute the sum of their single counterparts but demonstrate an independent impact on chromatin-binding interactomes}

Crosstalk between histone modifications is referred to as the influence one modification has on the biological impact of a second one. It is mainly observed between two chemical modifications, resulting in changed chromatin-binding properties for a limited number of factors (Lee et al., 2010).

I hypothesized that crosstalk of two modifications does not only affect individual proteins but changes chromatin-binding properties of proteins on larger scale. Thus, I assumed that 
chromatin modification crosstalk is a process of its own functional significance that extends the variety of chromatin regulating mechanisms.

In order to investigate chromatin modification crosstalk I extended the approach and applied ChAP by combining two posttranslational chromatin modifications. On one end I incorporated two modifications in trans, i.e. on different histones within one nucleosome. To this end we investigated the communication between modifications of $\mathrm{H} 3$ and $\mathrm{H} 4$. On the other end, I analyzed crosstalk between histone PTMs and DNA methylation in the context of chromatin arrays.

\subsubsection{Chromatin arrays carrying a combination of $\mathrm{H} 3 \mathrm{~K} 9 \mathrm{me} 3$ and $\mathrm{H} 4 \mathrm{~K} 2 \mathrm{Ome} 3$ result in a specific protein interactome}

To take advantage of the possibilities provided by the oligonucleosomal arrays I decided to investigate histone modification crosstalk between $\mathrm{H} 3$ and $\mathrm{H} 4$. Crosstalk in trans cannot be investigated using modified $\mathrm{N}$-terminal peptides of histones, as it requires a nucleosomal context. Such analyses have not been done before on a proteomic scale. The combination of H3K9me3 and H4K20me3 was chosen as these modifications co-localize in pericentric heterochromatin in vivo and seem to be functionally linked (Mikkelsen et al., 2007; Schotta et al., 2004; Sims et al., 2006).

Performing ChAP using chromatin arrays carrying both modifications, H3K9me3 and H4K20me3 in combination (H3K9me3|H4K20me3), resulted in a protein interactome displaying 18 factors significantly enriched and 39 factors significantly repelled from binding to chromatin (Figure 3.14 A, Table 3.1). Interestingly, the interactome of the double modification was not just the sum or the average of the interactomes obtained with the individual modifications H3K9me3 and H4K20me3 (Figure 3.14 B). While all proteins specifically recruited by the double modification were also specifically recruited by at least one of the individual modifications, H3K9me3|H4K20me3 had a strong impact on exclusion of protein binding to chromatin. Here, overlapping proteins excluded from binding by H3K9me3|H4K20me3 and the individual modifications were rare and in fact only represented $5.1 \%$ of all excluded proteins. Thus, most factors excluded by the double modification were uniquely depleted from binding to chromatin only in presence of both, H3K9me3 and H4K20me3.

STRING analysis indicated that proteins repelled from binding to H3K9me3|H4K2Ome3 essentially clustered in four main protein complexes (Figure 3.14 C). Five members of the septin complex, four members of the HBO1 complex, responsible for the bulk of histone $\mathrm{H} 4$ acetylation, at least seven factors involved in splicing as well as several components of the BRCA1-A complex were identified. Interestingly, the components of the BRCA1-A complex, BARD1 and BRCA1, had been found to be excluded from binding to H4K20me3 as well (3.9 D, lower panel, 
Table 3.1). These proteins are located in the background of the H3K9me3 interactome. In contrast, the members of the septin complex were significantly enriched by H3K9me3-modified chromatin (3.6 B, Table 3.1), demonstrating an opposed binding profile in the context of the double modification. These findings motivated me to investigate the binding profiles of proteins affected by all three modification states in more detail.

A)

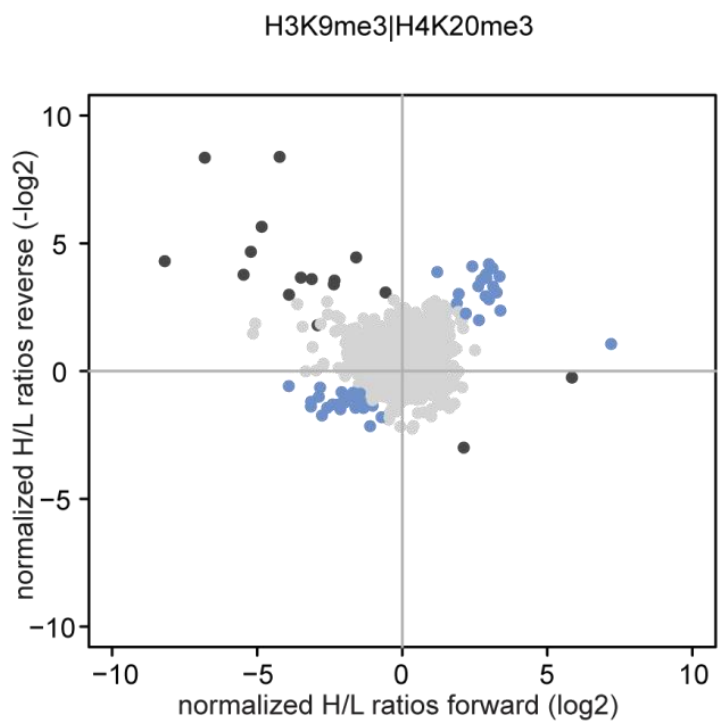

B)

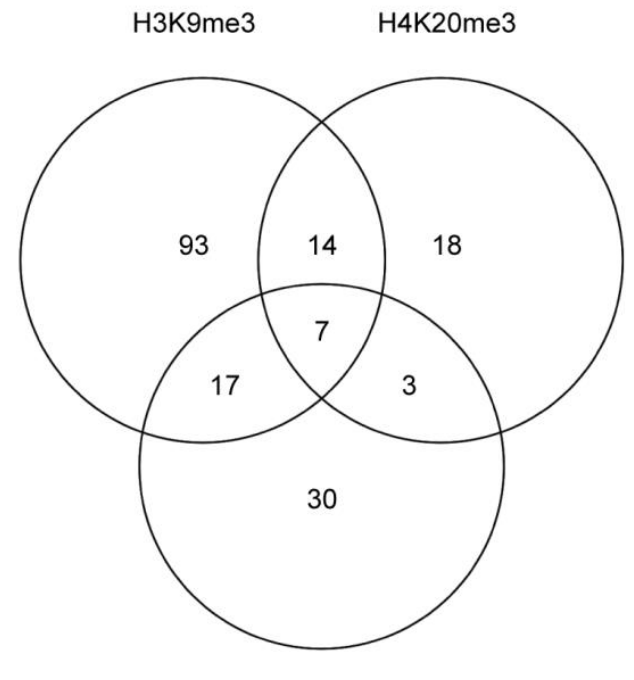

H3K9me3|H4K20me3

C)

H3K9me3|H4K2Ome3 excluded proteins
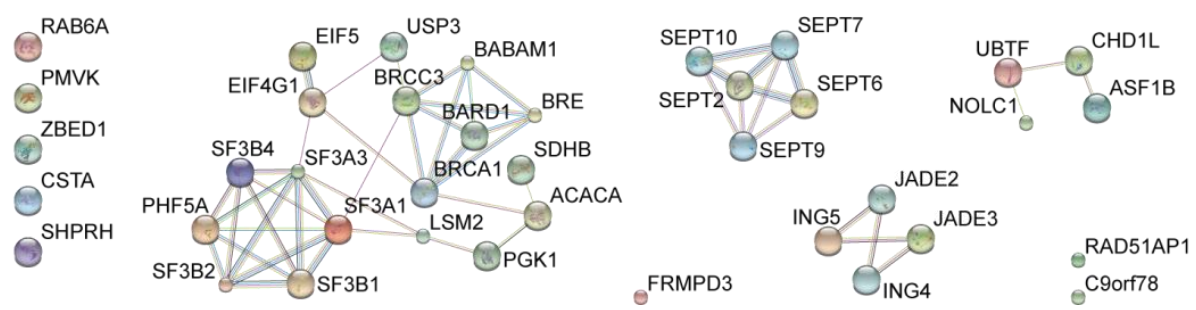

Figure 3.14 Protein-binding interactome of H3K9me3|H4K20me3-modified chromatin.

A) H3K9me3|H4K20me3 interactome. Proteins are plotted by their $\log _{2}$ SILAC ratios of the forward experiment on the $x$-axis and the reverse experiment on the $y$-axis. Significantly recruited and depleted proteins are colored in blue. False positive proteins are marked in black. B) Venn diagram of factors significantly regulated by $\mathrm{H} 3 \mathrm{~K} 9 \mathrm{me} 3$, H4K20me3 and $\mathrm{H} 3 \mathrm{~K} 9 \mathrm{me} 3 \mid \mathrm{H} 4 \mathrm{~K} 20 \mathrm{me}$. C) STRING protein-protein interaction network of proteins significantly depleted from binding to $\mathrm{H} 3 \mathrm{~K} 9 \mathrm{me} 3 \mid \mathrm{H} 4 \mathrm{~K} 20 \mathrm{me} 3$ chromatin.

\subsubsection{The double modification $\mathrm{H} 3 \mathrm{~K} 9 \mathrm{me} 3 / \mathrm{H} 4 \mathrm{~K} 2 \mathrm{Ome} 3$ indicates positive and negative crosstalk}

The communication between different chemical chromatin modifications can occur on several levels. A modification can either promote or prevent the addition or the removal of a second modification. Furthermore, a modification can promote or prevent functional properties of a 
second modification, e.g. the promotion or blockage of protein binding to chromatin and thereby functional consequences associated with these proteins.

The latter I investigated in the context of H3K9me3 and H4K20me3. Potential crosstalk was addressed by comparing the protein-binding profiles affected by the single modifications to the binding profile obtained with $\mathrm{H} 3 \mathrm{~K} 9 \mathrm{me} 3 \mid \mathrm{H} 4 \mathrm{~K} 20 \mathrm{me} 3$. I started with proteins found to be significantly recruited or excluded from binding to the three different chromatin species (Table 3.1).

Considering only proteins significantly recruited by $\mathrm{H} 3 \mathrm{~K} 9 \mathrm{me} 3 \mathrm{H} 4 \mathrm{~K} 20 \mathrm{me} 3 \mathrm{I}$ found all proteins recruited by at least one of the single modifications as well (Figure $3.15 \mathrm{~A}$ ). This finding suggested that protein recruitment in the context of H3K9me3 and H4K2Ome3 is defined by the presence of the individual modifications. The combination of both PTMs had no effect on protein recruitment independent $\mathrm{H} 3 \mathrm{~K} 9 \mathrm{me} 3$ and $\mathrm{H} 4 \mathrm{~K} 20 \mathrm{me} 3$.

Next, proteins significantly excluded from H3K9me3|H4K20me3 were compared to proteins excluded by the single modifications (Figure $3.15 \mathrm{~B}$ ). Surprisingly, only 2 of the factors excluded by H3K9me3|H4K20me3 were also found to be excluded by one of the single modifications, in fact by H4K20me3.

When comparing $\mathrm{H} 3 \mathrm{~K} 9 \mathrm{me} 3 \mid \mathrm{H} 4 \mathrm{~K} 20$ me3 excluded factors to significantly enriched factors of the two single modifications I found an overlap of 7 proteins (Figure $3.15 \mathrm{C}$ ). USP3, RAD51AP1 and EIF5 were recruited to both single modifications. Additionally, the members of the septin complex recruited by $\mathrm{H} 3 \mathrm{~K} 9 \mathrm{me} 3$ were excluded by the double modification. These findings show for the first time that the presence of two modifications in trans result in an inverse binding pattern of proteins to chromatin. The combination of both PTMs had an opposing effect on protein binding in comparison to the individual modifications, H3K9me3 and H4K2Ome3.

I was wondering whether this effect was limited to a small number of H3K9me3|H4K2Ome3 excluded proteins. Therefore, I compared the SILAC ratios of all H3K9me3|H4K20me3 significantly excluded proteins with proteins identified in the experiments of H3K9me3- and H4K20me3-modified chromatin (Figure 3.16 A). Indeed, 32 of H3K9me3|H4K2Ome3 significantly excluded factors displayed an opposed binding affinity to chromatin compared to the individual modifications, indicating negative crosstalk between H3K9me3 and H4K20me3.

So far I only considered proteins that were significantly affected by the modifications. As I found proteins opposingly regulated by the double modification I was questioning whether this effect is limited to proteins excluded from binding. Proteins significantly recruited to H3K9me3|H4K20me3 showed no crosstalk. Therefore, I decided to extend the analysis and included proteins displaying an $\mathrm{H} / \mathrm{L}$ ratio larger than $\log _{2} 1.5$ in the $\mathrm{H} 3 \mathrm{~K} 9 \mathrm{me} / \mathrm{H} 4 \mathrm{~K} 20 \mathrm{me} 3$ dataset. These proteins were not significantly recruited to the double modification but showed an 
increased affinity to the modified chromatin arrays. 18 of these proteins displayed opposed binding compared to the single modifications (Figure $3.16 \mathrm{~B}$ ), indicating also positive crosstalk between H3K9me3 and H4K20me3. Notably, almost all factors affected are in association with transcriptional activity, such as the DNA-directed RNA polymerase III subunits and the transcription factors NFIA and NFIC.

A)

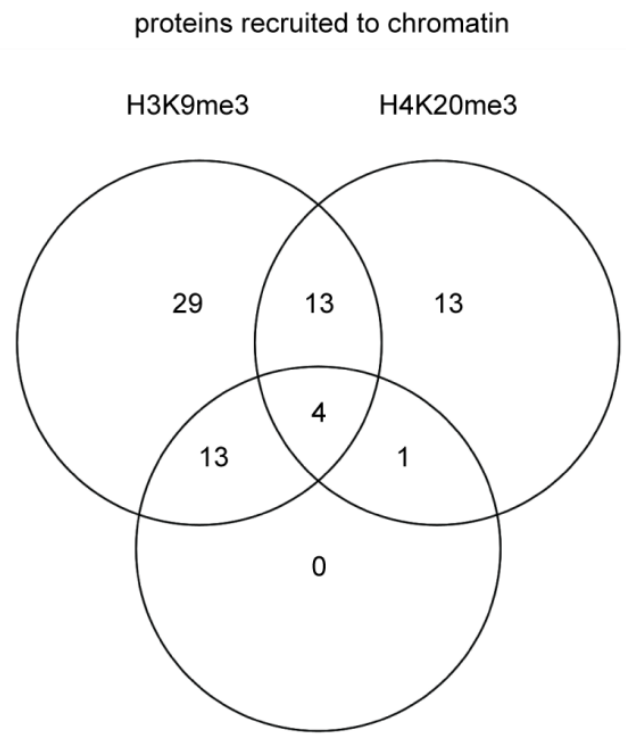

H3K9me3|H4K2Ome3
B)

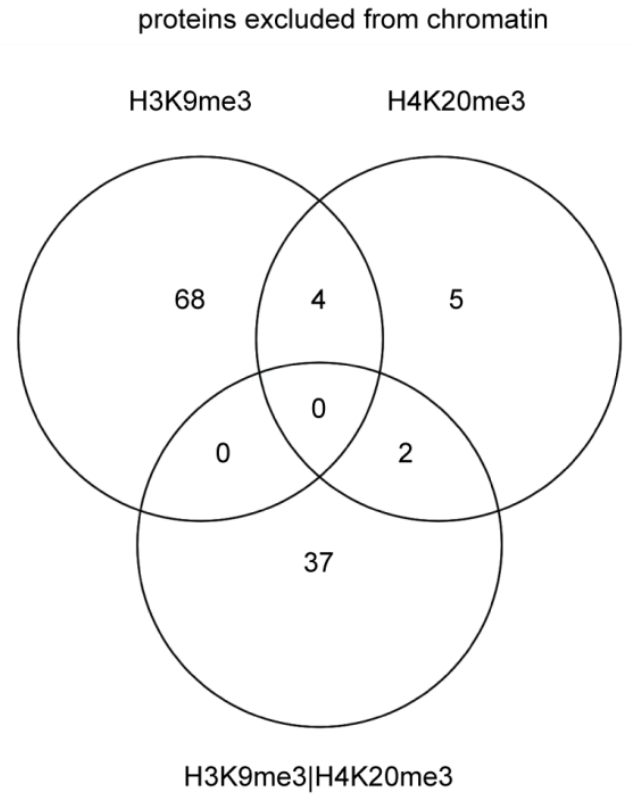

C)

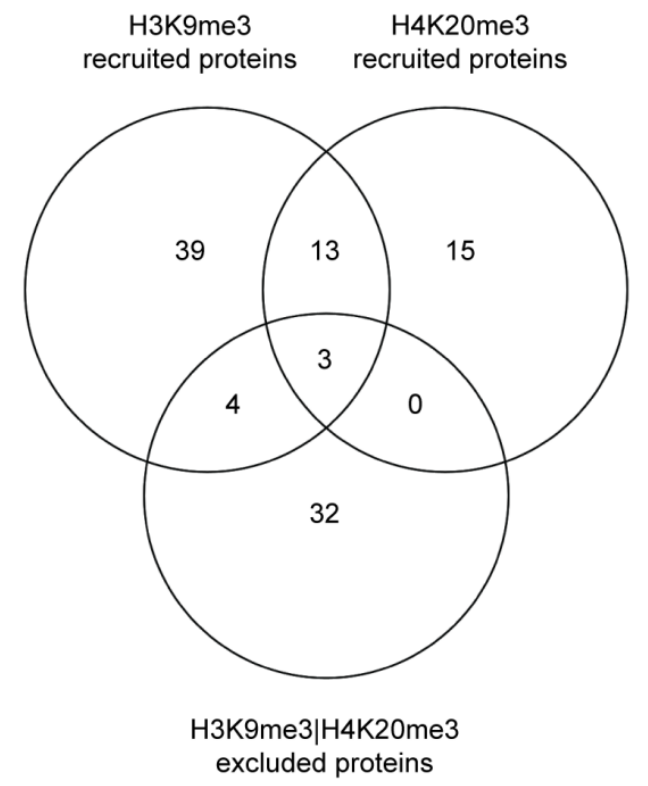

Figure 3.15 Comparison of significant protein binding to chromatin affected by H3K9me3, H4K2Ome3 and H3K9me3|H4K20me3. 
A) Venn diagram of proteins recruited to chromatin by all three modification states. B) Venn diagram of proteins excluded from binding to chromatin by all three modification states. C) Venn diagram of proteins significantly recruited to $\mathrm{H} 3 \mathrm{~K} 9 \mathrm{me} 3$ and $\mathrm{H} 4 \mathrm{~K} 2 \mathrm{Ome} 3$ and excluded from binding to $\mathrm{H} 3 \mathrm{~K} 9 \mathrm{me} 3 \mid \mathrm{H} 4 \mathrm{~K} 20 \mathrm{me} 3$ chromatin.

Taken together, the data showed three effects of H3K9me3 and H4K20me3 crosstalk. In the first category, the double modification had "no effect" on protein binding. "No effect" refers to the finding that the protein-binding properties were similar over all three modification patterns. In the second category, negative crosstalk between H3K9me3 and H4K20me3 was observed. Negative crosstalk was defined as decreased protein-binding affinities in the context of the double modification but enriched affinities with individual modifications. In the third category, H3K9me3 and H4K20me3 showed positive crosstalk. Positive crosstalk was given when the double modification displayed increased protein-binding affinities, while the individual modifications displayed decreased binding affinities.

\subsubsection{Communication of histone and DNA modifications is indicated by positive and negative crosstalk}

In many model organisms DNA methylation and histone PTMs have been found to be linked (Du et al., 2015) in the context of co-localization at certain chromatin regions, but also in terms of crosstalk. This made the combination of $\mathrm{H} 3 \mathrm{~K} 9 \mathrm{me} 3$ and $\mathrm{meCpG}$ attractive to prove, whether the crosstalk observed between $\mathrm{H} 3$ and $\mathrm{H} 4$ is also given in the context of further combinations, i.e. DNA methylation and posttranslational histone modifications.

The analysis of the meCpG protein-binding interactome revealed an overlap of factors also significantly affected by the H3K9me3 interactome (3.17 B, Table 3.1). It appeared that a set of nine proteins is similarly regulated by $\mathrm{H} 3 \mathrm{~K} 9 \mathrm{me} 3$ and $\mathrm{meCpG}$, suggesting that these two modifications may be involved in the same regulation pathways. This argument was in agreement with a strong association between these chromatin modifications described in the literature (Du et al., 2015; Fuks et al., 2003; Lehnertz et al., 2003; Liu et al., 2013).

In consequence, I wanted to characterize in more detail, how the combination of both, histone posttranslational modifications and DNA methylation, regulates the chromatin-binding interactome. I wanted to investigate potential crosstalk, as seen with the combinatorial readout of $\mathrm{H} 3$ and $\mathrm{H} 4$ methylation. Therefore, ChAP experiments were performed using oligonucleosomal arrays carrying both H3K9me3 and CpG-methylated DNA. 
A)

negative crosstalk

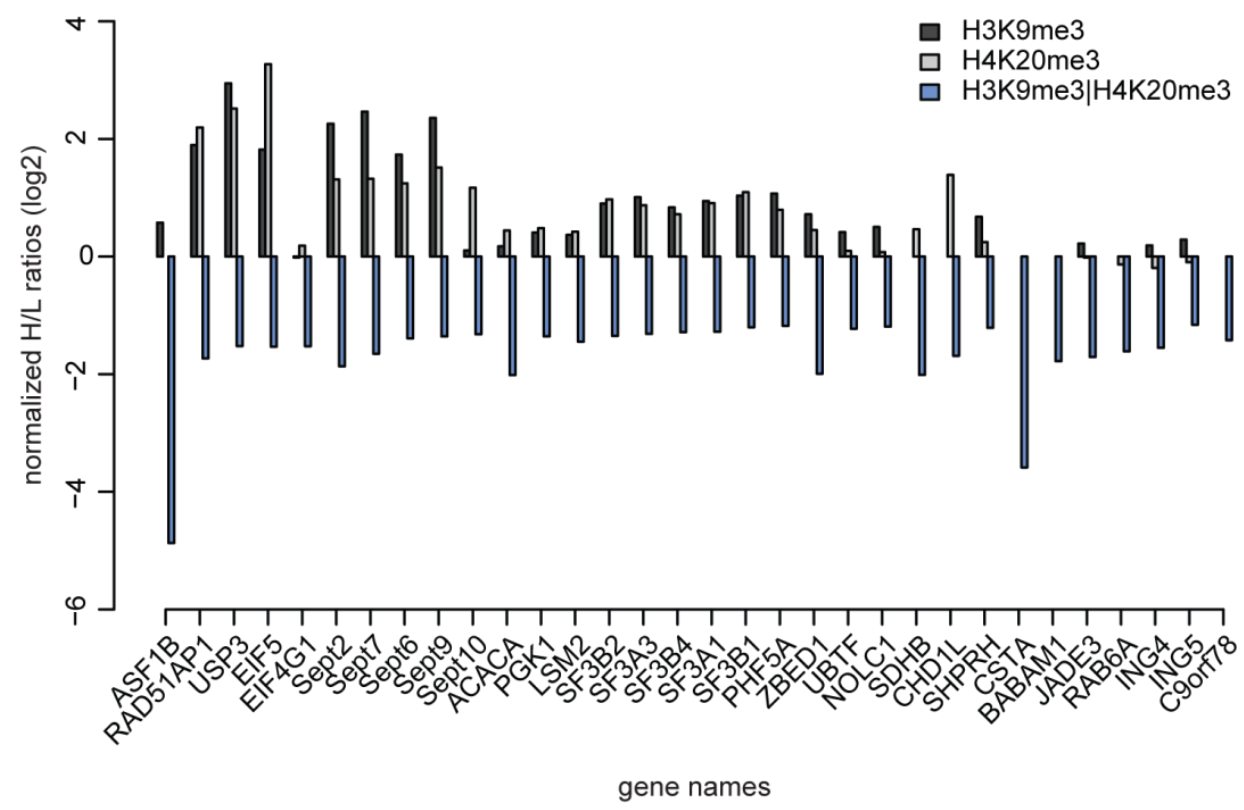

B)

positive crosstalk

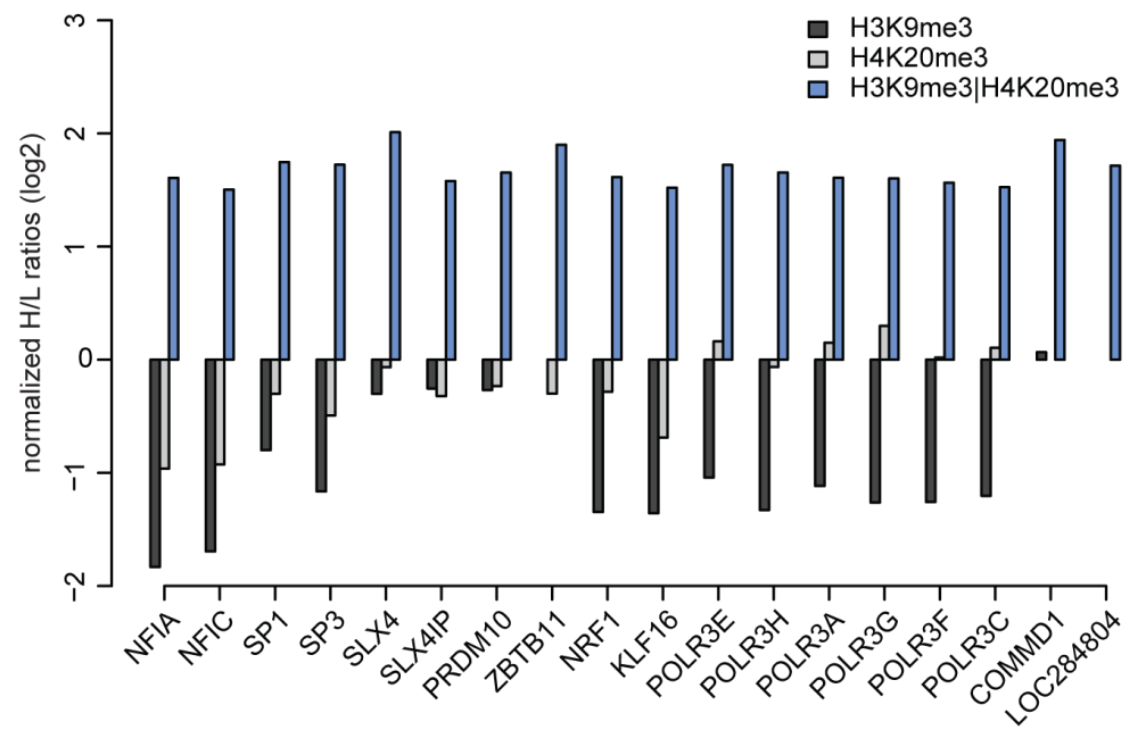

gene names

Figure 3.16 Crosstalk between H3K9me3 and H4K20me3.

A) Negative crosstalk between $\mathrm{H} 3 \mathrm{~K} 9 \mathrm{me} 3$ and $\mathrm{H} 4 \mathrm{~K} 20 \mathrm{me} 3$. $\log _{2}$ scaled SILAC ratios of proteins significantly repelled from binding and displaying negative crosstalk are indicated on the $y$-axis. There gene names are indicated on the x-axis. B) Positive crosstalk affected by H3K9me3 and H4K2Ome3. Proteins displaying an $\mathrm{H} / \mathrm{L}$ ratio larger than $\log _{2} 1.5$ in the dataset of the double modification and displaying positive crosstalk are plotted as described in A). 
The protein-binding interactome obtained from ChAP using chromatin arrays carrying both modifications, H3K9me3 and meCpG, in combination (H3K9me3/meCpG), displayed 7 factors significantly excluded from binding to chromatin but no factors significantly recruited (Figure 3.17 A, Table 3.1). The comparison of significantly affected proteins of all three datasets, H3K9me3, meCpG and the combination of both modifications, revealed an overlap of one factor, CDK2AP1, which was recruited to meCpG-modified chromatin and excluded from binding to H3K9me3|meCpG-modified chromatin (Figure $3.17 \mathrm{~B}$ ). The opposed binding behavior to chromatin indicated negative crosstalk between meCpG and H3K9me3.

Despite the results of the significance test, an unusual high number of proteins displayed a higher affinity to $\mathrm{H} 3 \mathrm{~K} 9 \mathrm{me} 3 \mathrm{meCpG}$ than to unmodified chromatin arrays, whereas the number of depleted proteins was similar to other ChAP-MS experiments (Figure 3.17 A). The possibility that this effect resulted from experimental variability is low, as only proteins displaying an increased $\mathrm{H} / \mathrm{L}$ ratio are affected in both replicates. The high number of recruited proteins shifted the overall distribution towards high $\mathrm{H} / \mathrm{L}$ ratios (Figure $3.17 \mathrm{~A}$ ). With this large number of enriched proteins significant outliers could not be determined by the described significance test (section 3.2). However, considering the increased $\mathrm{H} / \mathrm{L}$ ratios of both replicates, H3K9me3/meCpG-modified chromatin clearly displayed an impact on binding of a very large number of proteins to chromatin.

A)

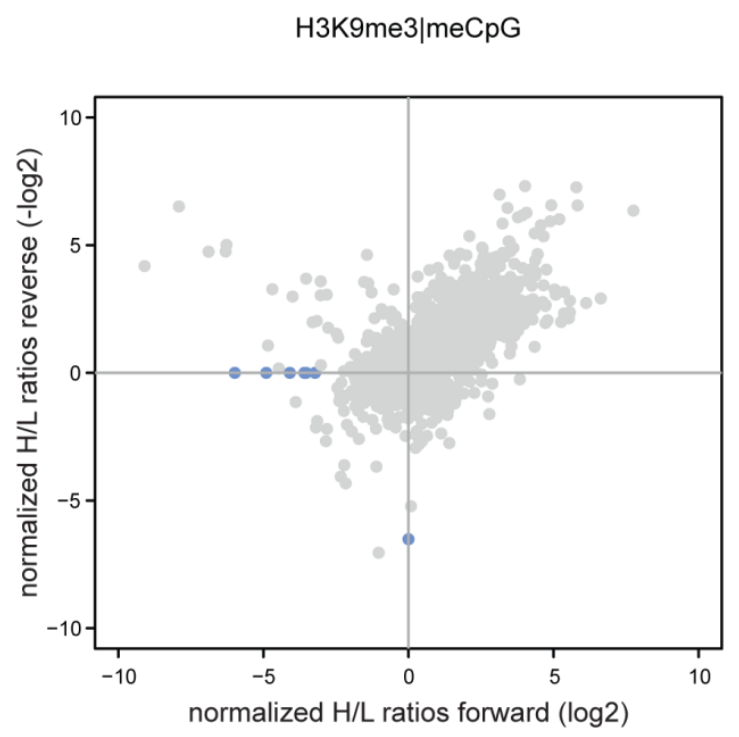

B)

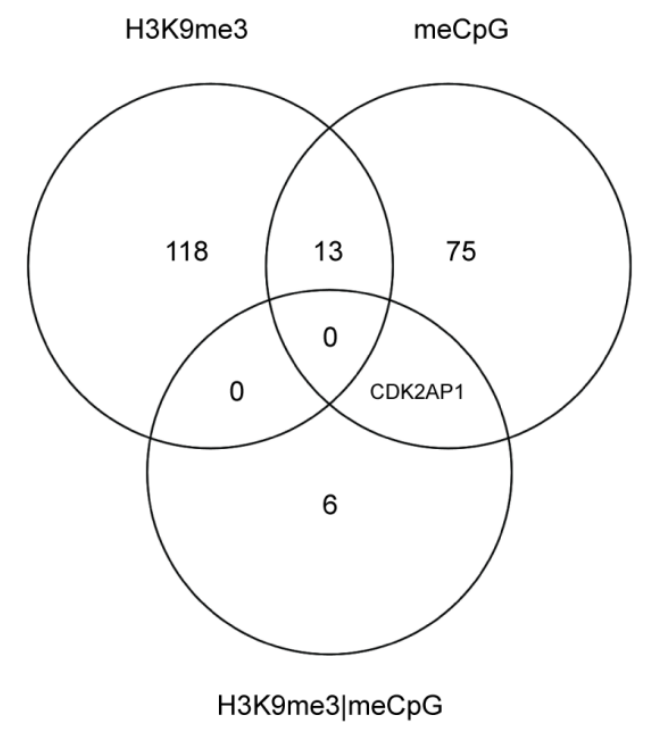

Figure 3.17 Chromatin-binding interactome of $\mathrm{H} 3 \mathrm{~K} 9 \mathrm{me} 3 / \mathrm{meCpG}$ in comparison to the individual modifications.

A) $\mathrm{H} 3 \mathrm{~K} 9 \mathrm{me} 3 / \mathrm{meCpG}$ interactome. Protein enrichment ratios are plotted as described in figure 3.14 A. B) Venn diagram of proteins significantly regulated by H3K9me3, CpG-methylated DNA and H3K9me3|meCpG-modified chromatin. 
The combinatorial readout of $\mathrm{H} 3 \mathrm{~K} 9 \mathrm{me} 3 \mid \mathrm{meCpG}$ has already been performed in the context of mononucleosomes (Bartke et al., 2010). In comparison to the interactome revealed with chromatin arrays no overlap of significantly excluded factors was observed. Considering the 40 factors displaying the strongest enrichment to $\mathrm{H} 3 \mathrm{~K} 9 \mathrm{me} / \mathrm{meCpG}$ chromatin and comparing these to the factors enriched with $\mathrm{H} 3 \mathrm{~K} 9 \mathrm{me} 3 \mid \mathrm{meCpG}$ mononucleosomes, an overlap of five factors was observed, namely UHRF1, CBX3, MBD2, MTA2 and CHD4. All five proteins have been shown to be enriched by at least one of the single modifications in the context of chromatin arrays as well.

In order to investigate positive and negative crosstalk I extended the analysis to proteins that showed no significance but increased and decreased binding affinities to H3K9me3|meCpG. Thus, all proteins displaying an $\mathrm{H} / \mathrm{L}$ ratio larger than $\log _{2} 3.5$ and smaller than $\log _{2}-2$ in the H3K9me3/meCpG dataset were included in the analysis.

Next to CDK2AP1, I found 14 more proteins showing a decreased binding affinity to $\mathrm{H} 3 \mathrm{~K} 9 \mathrm{me} 3 \mid \mathrm{meCpG}$ and an increased affinity to at least one of the individual modifications (Figure $3.18 \mathrm{~A})$. The binding profiles of these proteins indicated negative crosstalk between $\mathrm{H} 3 \mathrm{~K} 9 \mathrm{me} 3$ and meCpG. These proteins included USP3 and UBTF, which displayed negative crosstalk between both, H3K9me3 and meCpG and also H3K9me3 and H4K20me3. Other proteins affected were NUMA1, a component of the nuclear matrix, CENPF, which is involved in kinetochore function and chromosome segregation, the histone acetyltransferase KAT7 and the nucleolar protein NCL.

CpG-methylated DNA and H3K9me3 also showed positive crosstalk (Figure $3.18 \mathrm{~B}$ ). The majority of affected factors are known to promote transcriptional activity. Interestingly, there was an overlap of 5 factors that displayed positive crosstalk between H3K9me3 and H4K2Ome3 as well. NFIA, NFIC, PRDM10 and the polymerase subunits POLR3A and POLR3H showed positive crosstalk between both investigated combinations of chromatin modifications. Additionally, three components of the chromatin remodeling complex INO80 and several transcription factors showed positive crosstalk in presence of $\mathrm{H} 3 \mathrm{~K} 9 \mathrm{me} 3$ and methylated DNA. 
A)

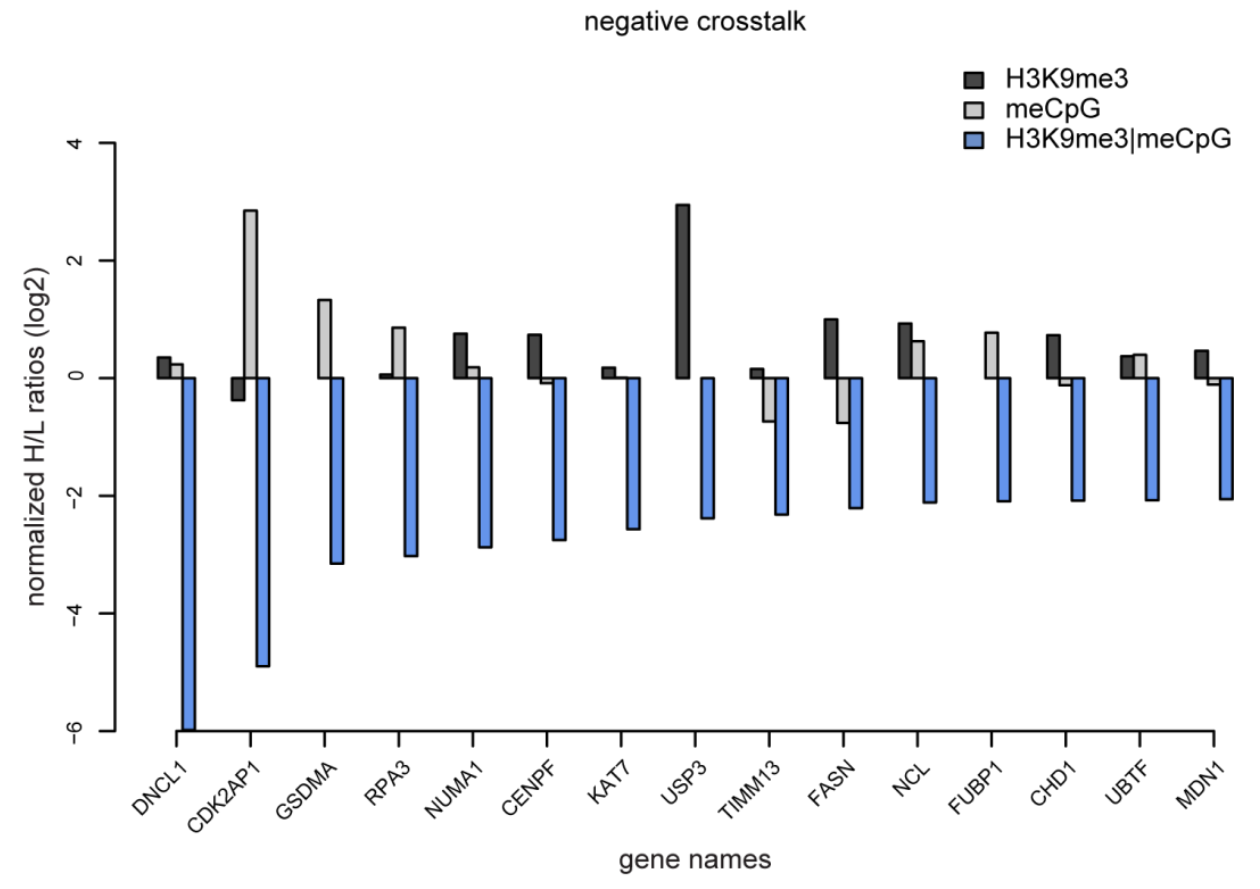

B)

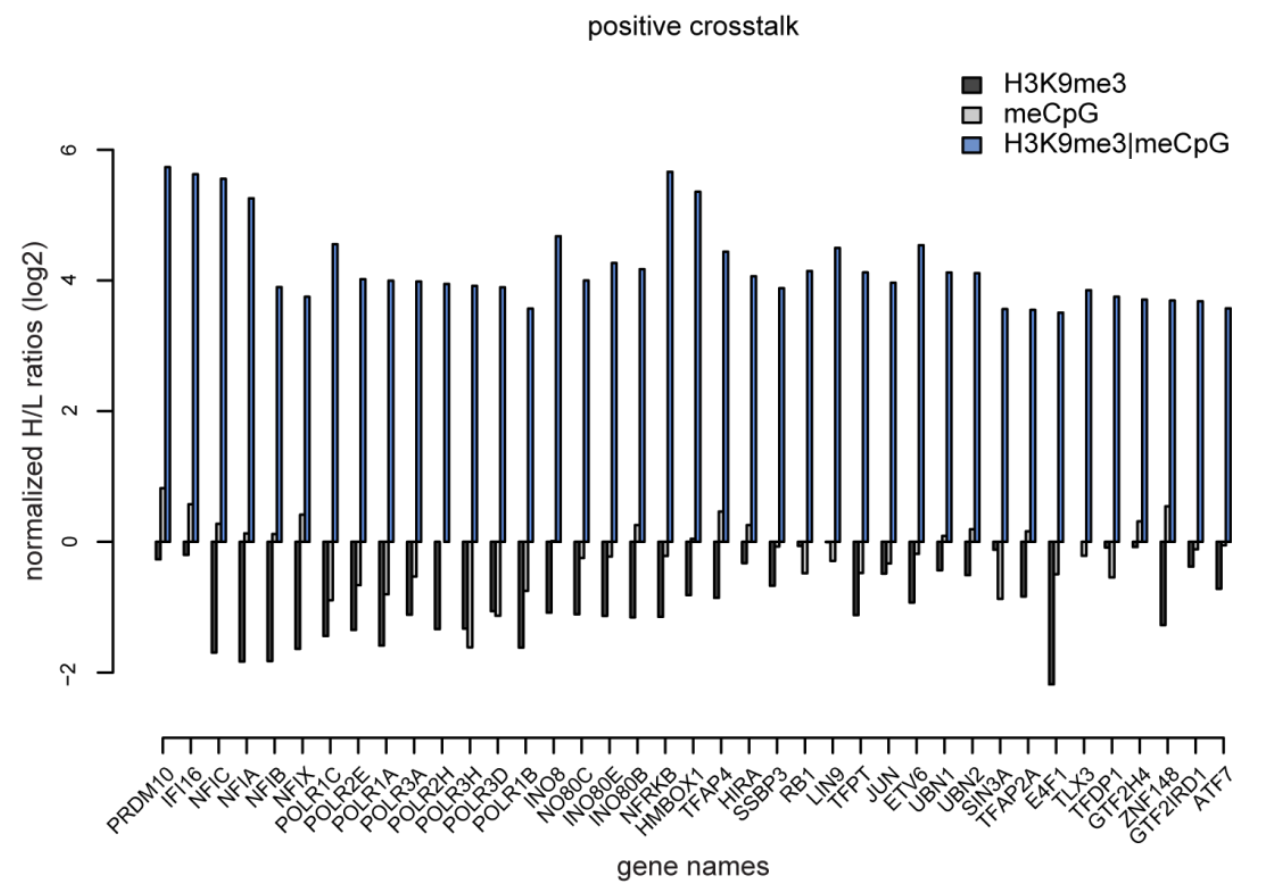

Figure 3.18 Crosstalk of H3K9me3 and CpG-methylated DNA.

A) Negative crosstalk of $\mathrm{H} 3 \mathrm{~K} 9 \mathrm{me} 3$ and meCpG. $\mathrm{Log}_{2}$ SILAC ratios are plotted on the $y$-axis. Gene names are plotted on the $x$-axis. Proteins with a SILAC ratio less than $\log _{2}-2$ were considered for the analysis. $B$ ) Positive crosstalk of H3K9me3 and meCpG. SILAC ratios and gene names were plotted as described for A). Proteins with a SILAC ratio greater than $\log _{2} 3.5$ were considered for the analysis. 


\subsection{Annotation analysis functionally correlates chromatin marks}

High resolution maps based on genome wide analyses of chemical chromatin modifications provided insights into the distribution of these marks (Barski et al., 2007; Mikkelsen et al., 2007; Wang et al., 2008). Distinct modification patterns have been shown to correlate with certain chromatin states, leading to the assumption that a functional correlation between modifications exists. To investigate a functional correlation of chromatin modifications based on the protein interactomes (3.3), I applied gene annotation enrichment analyses. These analyses resulted in identification of overrepresented biological processes and functions related to individual chromatin modification marks.

As general strategy, significantly enriched and repelled proteins of a certain modification mark, introduced in chapter 3.3, were considered individually for gene annotation enrichment analyses. Gene names were mapped to associated biological annotations using the functional annotation chart of the online software package DAVID (Huang da et al., 2009a, b). Results represented in the following chapter were obtained by using default statistic parameters of DAVID with the exception of the EASE score (enrichment) cutoff that was set to 0.05 to increase stringency. Annotation categories included in these analyses were GO terms for molecular functions (MF) and biological pathways. For the annotation of cellular pathways, the databases Panther_Pathways, KEGG_Pathway, BBID, Biocarta and Reactome_Pathway were screened. GO term annotation MF was performed including all terms. For each annotation term category two output lists per modification state were generated. One list of enriched annotation terms for recruited proteins $(\uparrow)$ and one for proteins excluded from binding $(\downarrow)$. Terms were only considered for enrichment analysis when at least two genes were assigned to a particular annotation term. Only significantly enriched annotation terms (matching the EASE score) were included in further analyses.

To enable global comparison of individual modification marks, the enriched annotation terms of recruited and depleted proteins of each chromatin modification mark were correlated by determining Pearson product-moment correlation coefficients (Pearson correlation coefficient). Modification marks showing a strong linear association of P-values of annotated terms displayed a high correlation coefficient. To compare annotated terms that were not enriched in each dataset missing P-values were set to 1.

Correlation coefficients obtained for enriched biological pathways are displayed in a heat map (Figure 3.19 A). Three clusters of strong correlation between modification states were observed. The largest cluster includes the datasets of recruited proteins of H3K9me3, H4K2Ome3, $\mathrm{H}_{3} \mathrm{~K}_{\mathrm{c}} 27 \mathrm{me} 3$ and $\mathrm{H} 3 \mathrm{~K} 9 \mathrm{me} 3 \mid \mathrm{H} 4 \mathrm{~K} 20 \mathrm{me}$, indicating a comparable impact on biological functions. 
All four chromatin modification marks have been shown to co-localize to pericentric heterochromatic regions and to be absent from transcriptionally active chromatin (Barski et al., 2007; Wang et al., 2008). Thus, functional similarities between these modifications might trigger related biological outcomes.

A second cluster of correlation coefficients was obtained for enriched biological pathways for the datasets of $\mathrm{H} 3 \mathrm{~K} 9 \mathrm{me} 3$ and $\mathrm{H}_{3} \mathrm{~K}_{\mathrm{C}} 27 \mathrm{me} 2$ depleted and $\mathrm{H} 4 \mathrm{R} 3 \mathrm{me} 2$ recruited proteins (Figure 3.19 A). These findings were additionally supported by a strong correlation of $\mathrm{H} 3 \mathrm{~K} 9 \mathrm{me} 3$ and $\mathrm{H}_{3} \mathrm{~K}_{\mathrm{C}} 27 \mathrm{me} 2$ depleted and H4R3me2 recruited proteins by annotation enrichment analyses of $\mathrm{GO}$ terms of MF (Figure $3.19 \mathrm{~B}$ ). The results clearly indicated an antagonistic regulation of biological functions impacted by these modification marks. These biological functions are depleted by $\mathrm{H} 3 \mathrm{~K} 9$ me3- and $\mathrm{H}_{3} \mathrm{~K}_{\mathrm{C}} 27$ me2-modified chromatin and given with H4R3me2-modified chromatin. On the individual protein level, I found several DNA-dependent RNA polymerase subunits excluded from binding to $\mathrm{H} 3 \mathrm{~K} 9 \mathrm{me} 3$ - and $\mathrm{H}_{3} \mathrm{~K}_{\mathrm{C}} 27$ me2-modified chromatin, while they were recruited by $\mathrm{H} 4 \mathrm{R} 3 \mathrm{me}$, suggesting transcriptional activity of chromatin regions marked with H4R3me2.

Another antagonistic regulation is highlighted by the third cluster of correlation coefficients obtained for enriched biological pathways of proteins recruited to chromatin modified at $\mathrm{H}_{3} \mathrm{~K}_{\mathrm{C}} 27$ me2 but depleted from binding to H3K9me3|H4K20me3 and H4K20me1 (Figure 3.19 A). All datasets were connected to the spliceosome, with the particularity that $\mathrm{H} 3 \mathrm{~K}_{\mathrm{C}} 27 \mathrm{me} 2$ attracted, while the presence of H3K9me3|H4K20me3 and H4K20me1 excluded spliceosomal activity. This was attributed to the binding properties of several spliceosome associated factors that were shown to be opposingly regulated by $\mathrm{H}_{3} \mathrm{~K}_{\mathrm{C}} 27 \mathrm{me} 2$ compared to $\mathrm{H} 3 \mathrm{~K} 9 \mathrm{me} 3 \mid \mathrm{H} 4 \mathrm{~K} 20 \mathrm{me} 3$ and H4K20me1 (section 3.3).

Correlation of P-values obtained from annotation term enrichment of GO terms MF pointed to a relation between the datasets of $\mathrm{H} 4 \mathrm{~K} 20 \mathrm{me} 1$ recruited factors and factors depleted from binding to chromatin by H3K9me1/-me2, H3K $27 m e 1 /-m e 2$ and H4K20me3 (Figure 3.19 B). These data suggested an antagonistic regulation of biological functions of H4K2Ome1 in comparison to the mono-methylation states of $\mathrm{H} 3 \mathrm{~K} 9$ and $\mathrm{H} 3 \mathrm{~K} 27$, which is surprising as they have been shown to be located at the same type of genomic regions (Barski et al., 2007; Mikkelsen et al., 2007; Rosenfeld et al., 2009; Wang et al., 2008). The correlation of opposed binding profiles of proteins to chromatin of $\mathrm{H} 4 \mathrm{~K} 20 \mathrm{me} 1$ and the modifications $\mathrm{H} 3 \mathrm{~K} 9 \mathrm{me} 2, \mathrm{H} 3 \mathrm{~K}_{\mathrm{C}} 27 \mathrm{me} 2$ and H4K20me3 in contrast, is in agreement with previous findings. In genome wide distribution profiles H4K20me1 had its significance at transcriptional active sites whereas $\mathrm{H} 3 \mathrm{~K} 9 \mathrm{me}$, $\mathrm{H}_{3} \mathrm{~K}_{\mathrm{C}} 27 \mathrm{me} 2$ and H4K20me3 accumulated at transcriptionally silenced regions (Barski et al., 2007; Mikkelsen et al., 2007; Rosenfeld et al., 2009; Wang et al., 2008). 
A)

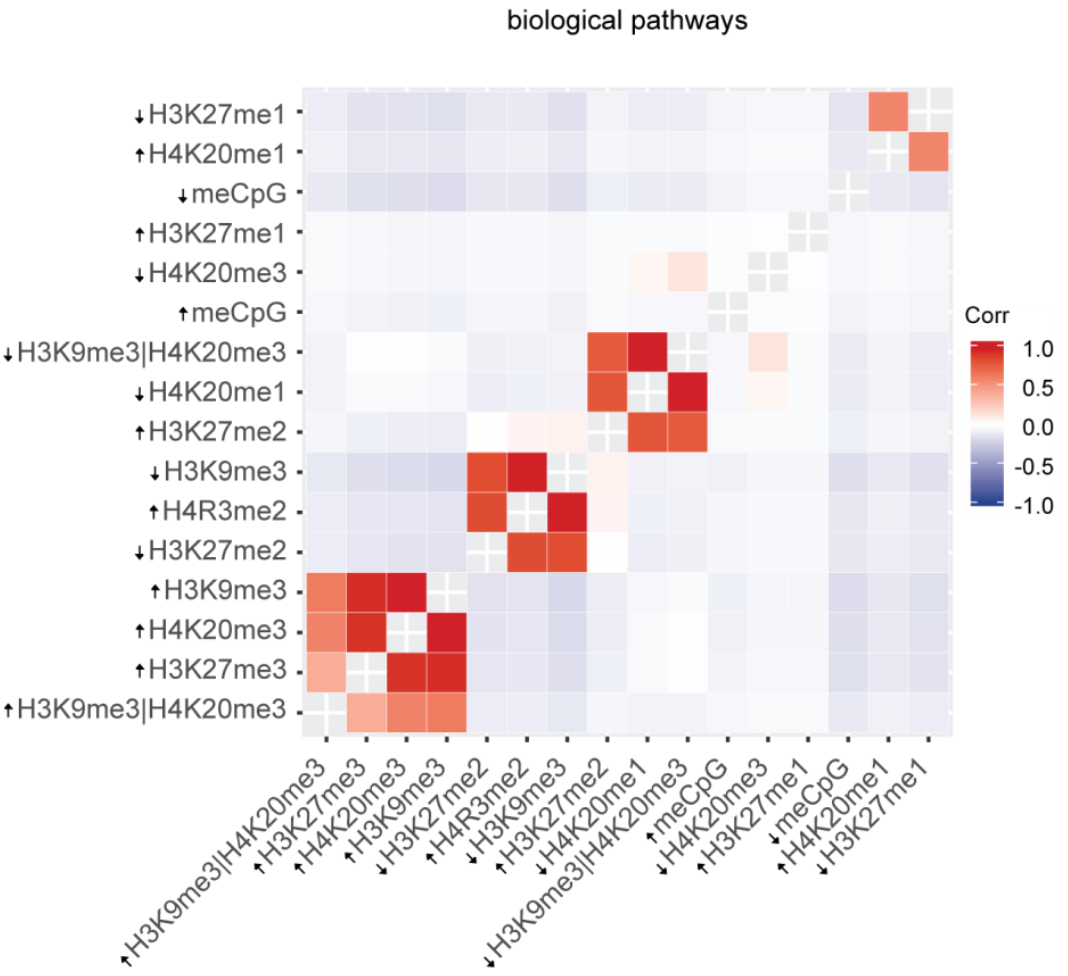

B)

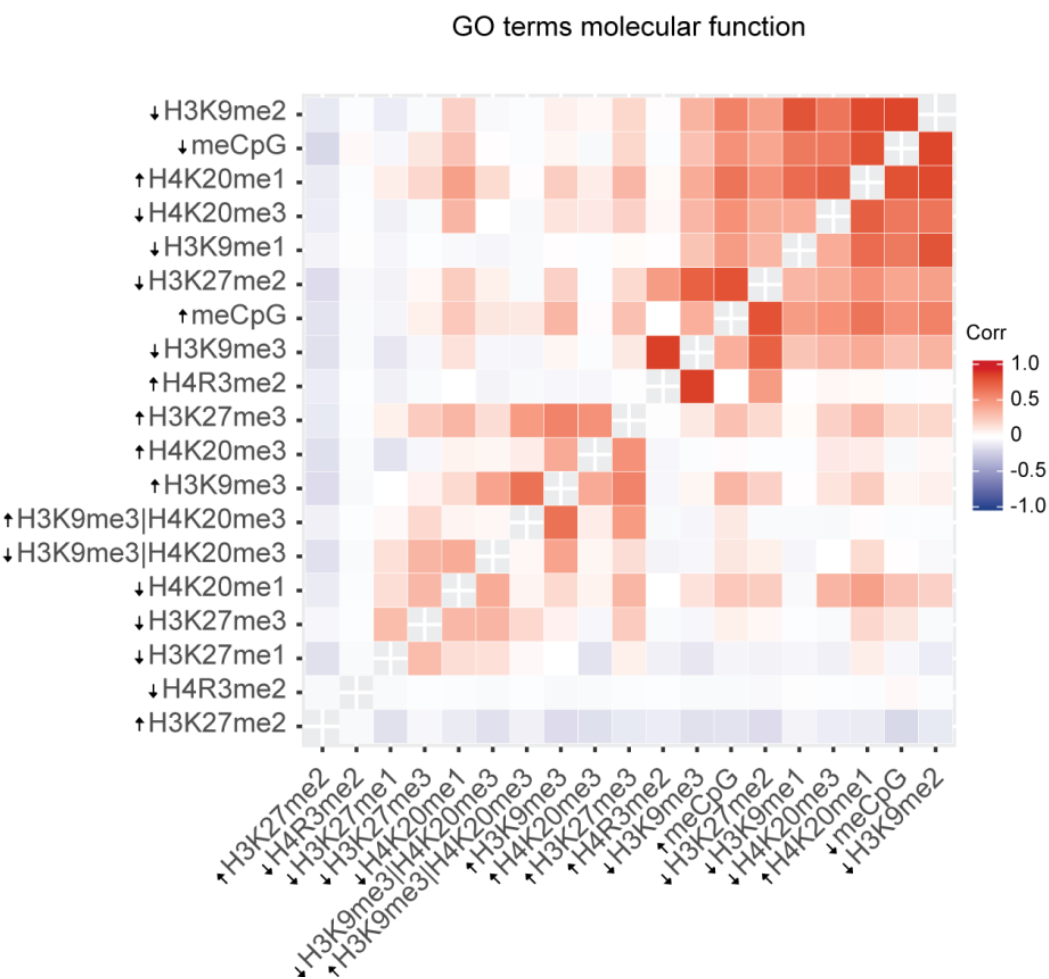

Figure 3.19 Annotation enrichment analyses of biological pathways and GO terms of molecular functions.

Significantly enriched $(\uparrow)$ and repelled proteins $(\downarrow)$ of all investigated chromatin modifications (section 3.3 ) were included in the analyses. Protein lists (enriched or excluded) resulted from the respective 
modification states were applied to annotation term enrichment analysis. P-values of enriched annotation terms resulted from the modified Fisher's exact test performed by DAVID (Huang da et al., 2009a, b) were taken as input for calculation and visualization of Pearson correlation coefficients. P-values of annotated terms that displayed no significance were set to 1. A) Pearson correlation coefficients (Corr) of enriched pathway terms annotated to proteins recruited and excluded from binding to modified chromatin are listed on the $y$ - and $x$-axis. Positive correlation is indicated in red shades while negative correlation is indicated in blue shades. B) Pearson correlation coefficients of enriched GO terms of molecular functions. Data are represented as described for $A$.

Notably, for both datasets of meCpG, proteins recruited and excluded from binding to chromatin, displayed a strong correlation (Figure $3.19 \mathrm{~B}$ ). The functional classification of meCpG is still controversial. In recent years, more and more evidence has accumulated that connects methylated DNA with transcriptional activity (Hu et al., 2013; Jin et al., 2012; Wu et al., 2010). However, meCpG has been mainly correlated with transcriptional inactivity (Breiling and Lyko, 2015). In my analyses, proteins depleted as well as recruited to meCpG-modified chromatin were associated with similar biological functions, supporting a role for methylated DNA in the context of transcriptional activation as well as repression.

In summary, the results of gene annotation enrichment analyses of different modification patterns, based on significantly enriched and depleted proteins, suggested four groups of functionally related modification patterns. H3K9me3, H4K20me3, $\mathrm{H}_{3} \mathrm{~K}_{\mathrm{c}} 27 \mathrm{me} 3$ and the double modification H3K9me3|H4K20me3 showed a strong correlation in the context of annotated pathway terms, suggesting a functional link between these modifications. Furthermore, the analyses indicated an opposing functional background for H4R3me2 compared to H3K9me3 and $\mathrm{H}_{3} \mathrm{~K}_{\mathrm{C}} 27 \mathrm{me} 2$. The fact that $\mathrm{H} 3 \mathrm{~K} 9 \mathrm{me} 3$ and $\mathrm{H} 3 \mathrm{~K}_{\mathrm{C}} 27 \mathrm{me} 2$ are associated with transcriptional inactivity and that H4R3me2 recruited several subunits of the polymerase (Table 3.1 ) strongly indicates that H4R3me2 is associated with transcriptional activity. The correlation analyses of enriched annotated terms also implied that H4K20me1 impacts protein binding to chromatin and associated functionalities opposed to $\mathrm{H}_{3} \mathrm{~K}_{\mathrm{C}} 27 \mathrm{me} 2$. This finding is based on the inverse regulation of binding properties of spliceosomal factors (Table 3.1, Figure 3.7 E, upper panel,

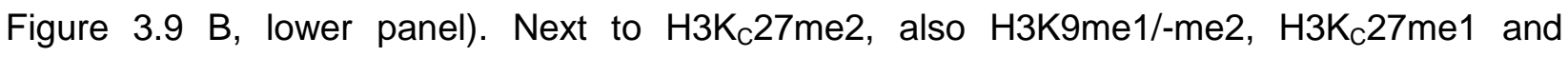
H4K20me3 seem to trigger opposed biological functions than H4K20me1. A last group of modifications that seemed to be functionally linked, constituted H3K9me1/-me2, H4K20me3 and to a lesser extend meCpG. These modification states showed a correlation of terms annotated to proteins that were significantly depleted from binding to chromatin. 


\subsection{Protein-protein cross-linking of the chromatin-binding interactome}

My results to that point demonstrated that chromatin affinity purification coupled to mass spectrometry is a powerful method for the identification of chromatin-associated factors. It allows determination of chromatin-binding interactomes in the context of different chromatin modifications and combinations thereof. The method allows studying the impact of chromatin modification patterns on recruitment and exclusion of protein binding to chromatin in addition to crosstalk effects of modifications (3.3). Yet, the approach does not provide information about factors that primarily bind to chromatin and factors that piggypack on other factors for association. To address the hierarchical binding pattern of chromatin-associated proteins we extended ChAP-MS by protein-protein cross-linking of the chromatin-binding interactome. A workflow combining ChAP, chemical cross-linking and mass spectrometry was established. This allowed the mapping of specific protein-protein interaction sites and thereby providing structural information of binding patterns of chromatin-associated factors and multi protein complexes.

\subsubsection{Protein-protein cross-linking on assembled chromatin arrays}

To identify physical interaction sites of specific chromatin-associated factors chemical crosslinking was applied. Chemical cross-linking results in permanent connections of protein interaction sites. Thus, proteins assembled in a non-covalent complex that are in close proximity, spanning the reach of the cross-linker, can be covalently linked.

In this study we used bis(sulfosuccinimidyl) suberate (BS3), a chemical cross-linker with a spacer arm length of $11.4 \AA$. It is a homobifunctional cross-linker that reacts with primary amines in the side chains of lysine $(\mathrm{K})$ residues and the $\mathrm{N}$-terminus of polypeptide chains resulting in stable amide bonds (Sinz, 2006). The appropriate BS3 concentration for efficient cross-linking was determined by titrating the cross-linker to affinity purified protein complexes bound to recombinant chromatin arrays (M. Nikolov, MPI-bpc). We used a concentration of $200 \mu \mathrm{M}$ BS3 to sufficiently cross-link specific chromatin-binding interactomes.

The complete workflow established for cross-linking of chromatin-binding interactomes is summarized in figure 3.20. Briefly, specific chromatin-binding interactomes were obtained by ChAP. Biotin-tagged 12mer chromatin arrays were assembled as described before and validated for sufficient saturation levels (Figure 3.21). The ChAP workflow was extended by the addition of BS3 during affinity purification. Cross-linking was found to be most efficient when a small concentration of BS3 $(20 \mu \mathrm{M})$ was already added to the pull down while chromatin arrays were still incubated with nuclear extract. The specific chromatin-binding interactomes were cross- 
linked after protein-bound chromatin arrays were separated from nuclear extract and unspecific bound proteins were washed away.

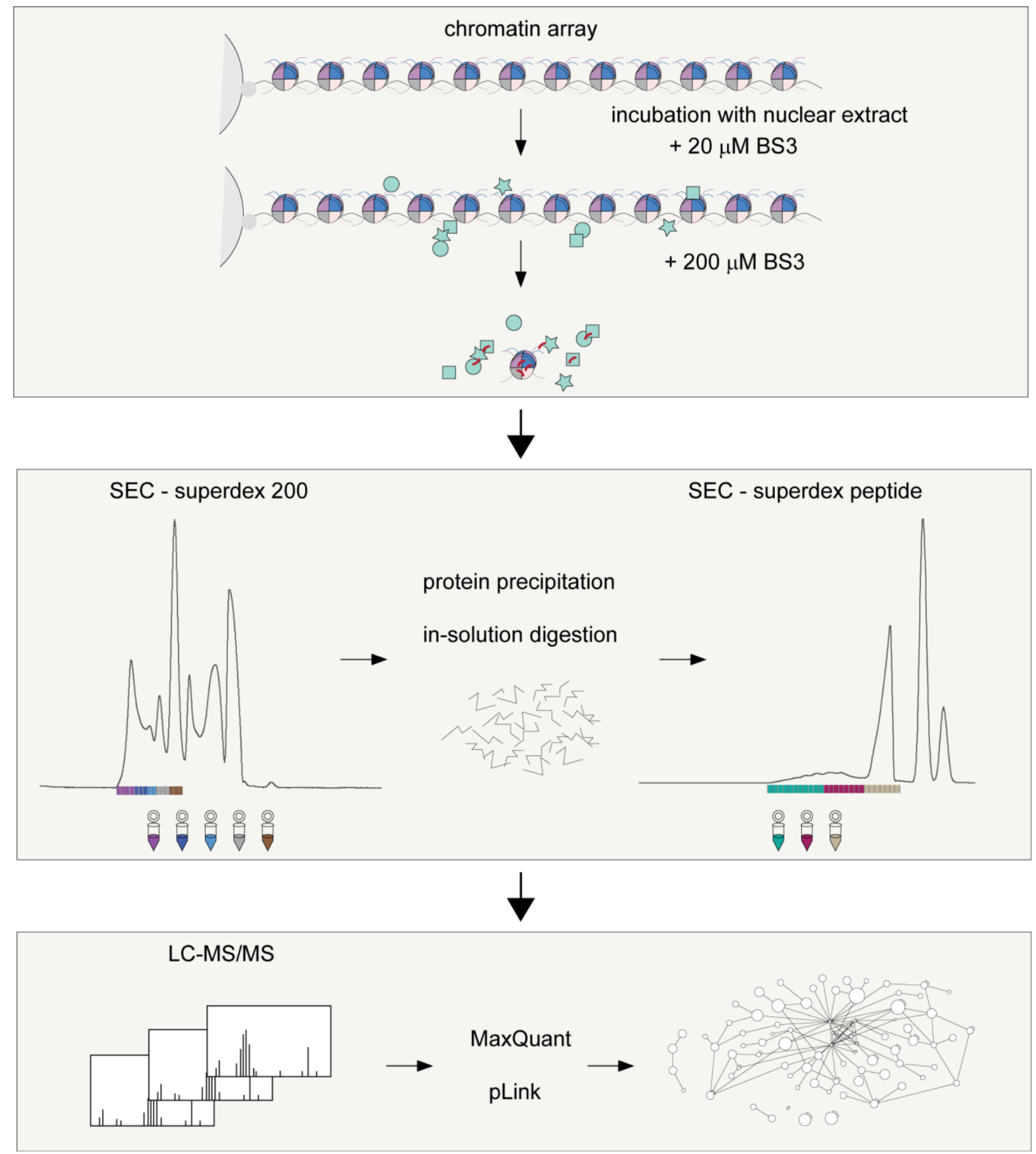

Figure 3.20 General workflow of protein-protein cross-link coupled with ChAP-MS. 
An important step of the purification procedure is the removal of proteins cross-linked to streptavidin of the magnetic beats used for the chromatin pull-down. To exclude such cross-links and cross-links occurring between the streptavidin beads themselves from the analysis, the cross-linked chromatin-binding interactome was separated from magnetic beats. Therefore, the biotin-tagged DNA of the chromatin arrays was degraded by the nuclease benzonase resulting in the release of histone octamers and associated proteins from magnetic beads.

I prepared a negative control that allowed direct comparison of cross-linked with non-crosslinked samples, to prove that ChAP coupled with cross-linking enabled efficient and specific cross-linking on-beads of chromatin-associated proteins (Figure 3.22).

A)

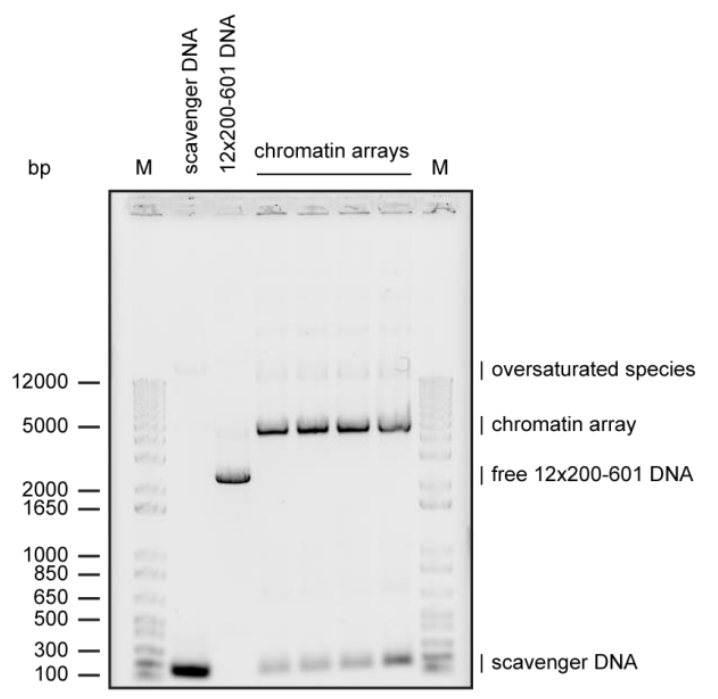

B)

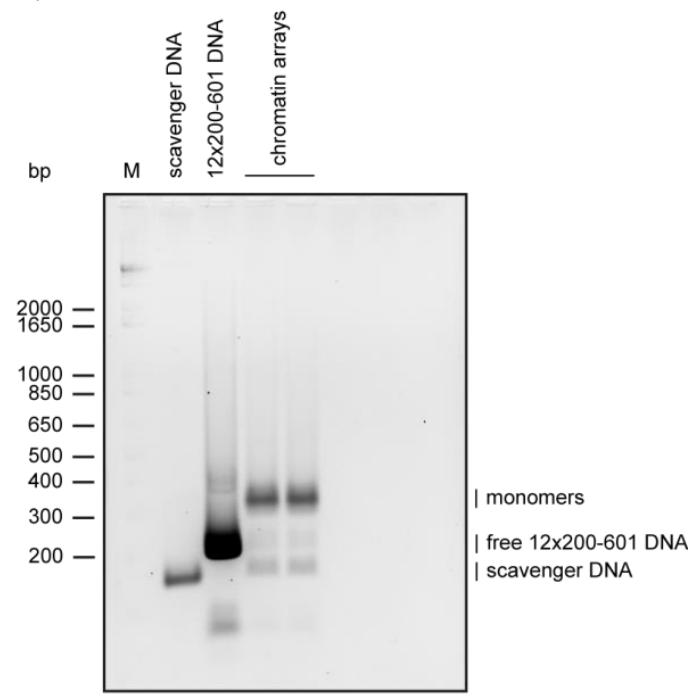

Figure 3.21 Quality control of reconstituted nucleosomal arrays.

(A) Agarose gel electrophoresis of free DNA and reconstituted nucleosomal 12mer arrays. (B) Aval digestion of nucleosomal 12mer arrays shown in (A). The saturation level of the nucleosomal arrays is above $90 \%$. M defines the DNA size marker.

The identification of cross-links from complex protein mixtures is challenging since cross-linked species are underrepresented. Therefore, it is necessary to reduce the complexity of the sample and enrich for cross-linked species before mass spectrometric measurements.

Separation of cross-linked and non-cross-linked species was performed by size exclusion chromatography (SEC) on the protein as well as on the peptide level. On the protein level, SEC elution profiles monitored by UV absorption at $215 \mathrm{~nm}$ featured 6 peaks (Figure $3.22 \mathrm{~A}$, left panel). The protein mixture of chromatin associated factors was separated and several fractions were pooled according to eluted peaks. Cross-linked proteins eluted within the first three peaks from the column, visible by SDS-PAGE and Western blot analysis (Figure $3.22 \mathrm{~A}$, middle and right panel). The analysis of elution fractions by SDS-PAGE validated the separation of proteins 
according to their size and indicated the presence of large protein aggregates by a diffuse separation pattern of proteins (Figure $3.22 \mathrm{~A}$, middle panel). The success of cross-linking was additionally demonstrated by upwards shifted signals of histone $\mathrm{H} 3$ as detected by Western blot analysis using an antibody against $\mathrm{H} 3$ (Figure 3.22 A, right panel). Fractions comprised of crosslinked material were used for further analyses. Proteins were digested in-solution using the protease trypsin.
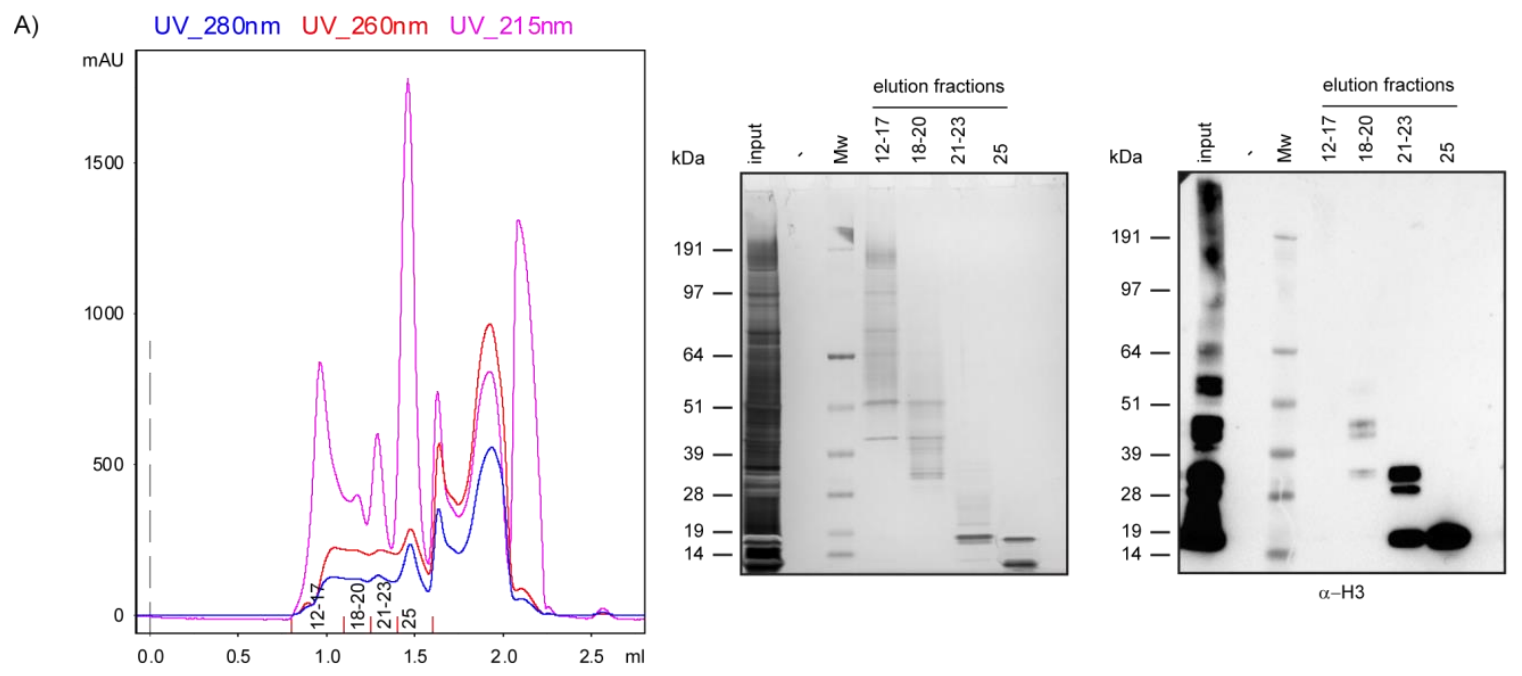

B)
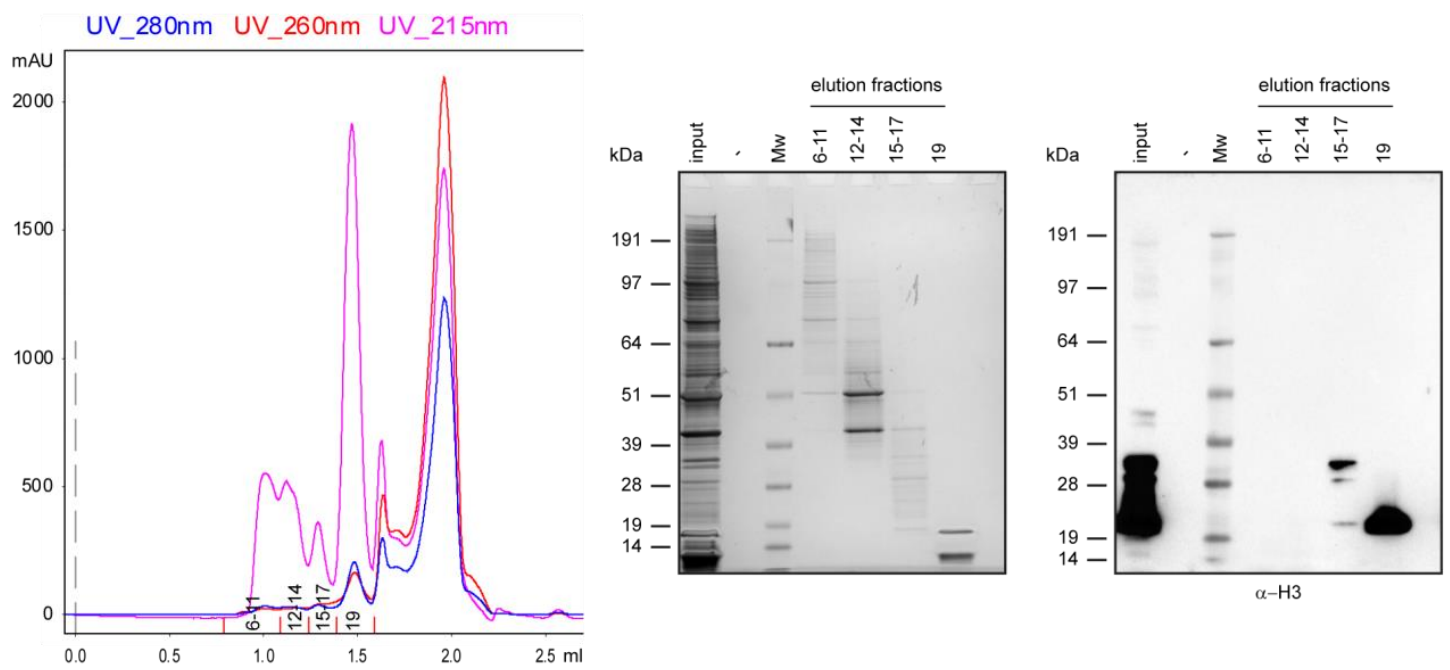

Figure 3.22 Enrichment for protein-protein cross-links by size exclusion chromatography (SEC).

Elution profiles (monitored by UV absorption) of proteins associated with unmodified chromatin that were separated according to their size using a Superdex 200 column (left panel). The protein contents of displayed peaks were analyzed by SDS-PAGE (middle panel) and Western blot analysis using apolyclonal $\alpha-\mathrm{H} 3$ antibody (right panel). SDS-PAGE gels were stained with silver nitrate. Mw defines the molecular weight marker. (A) BS3 treated chromatin-associated proteins. (B) Negative control. All experimental steps of the negative control were performed equally to the cross-link experiment but the sample was not treated with BS3. 
The negative control displayed a similar elution profile. Proteins started to elute with a shift of 0.1 $\mathrm{ml}$ (Figure $3.22 \mathrm{~B}$, left panel), indicating larger protein complexes in the cross-linked samples. Elution fractions were pooled according to the cross-linked experiment (Figure $3.22 \mathrm{~A}$, left panel).

The cross-linked protein samples treated with proteases were composed of three subpopulations of peptides, (i) cross-linked peptides, (ii) single peptide chains and (iii) noncross-linked peptides. Single peptide chains can either be linked to a cross-linker reacted with water or ammonia (mono-links) or with a cross-linker that reacted with both ends of the same peptide (loop cross-links). Cross-linked peptides can occur as intramolecular bonds within one protein molecule (intra-cross-links) or as intermolecular bonds between two different protein molecules (inter-cross-links) (Figure $3.23 \mathrm{~B}$ ) that are able to create high-molecular weight aggregates (Tran et al., 2016). Leitner and colleagues demonstrated that SEC at the peptide level of samples treated with cross-linkers facilitates the identification of cross-linked peptides by MS (Leitner et al., 2012). To ensure comprehensive detection of cross-linked peptides by MS, trypsinized protein mixtures were enriched for cross-linked peptides by another SEC step. The elution of peptides started with the void volume, indicating the presence of large peptides and the separation of cross-linked peptide aggregates from smaller peptide species. Elution fractions were pooled according to eluted peaks. Each of the resulting samples was analyzed by LCMS/MS on a QExactive mass spectrometer. The majority of cross-linked peptides identified in the samples resulted from the first two peaks of the elution profile, additionally indicating a successful separation of cross-linked and non-cross-linked peptide species.

A)

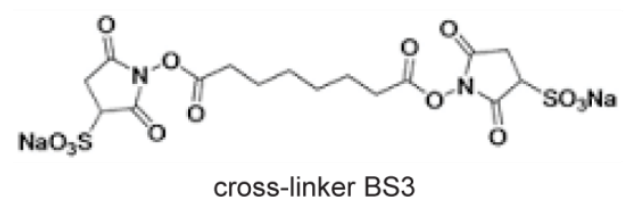

B)

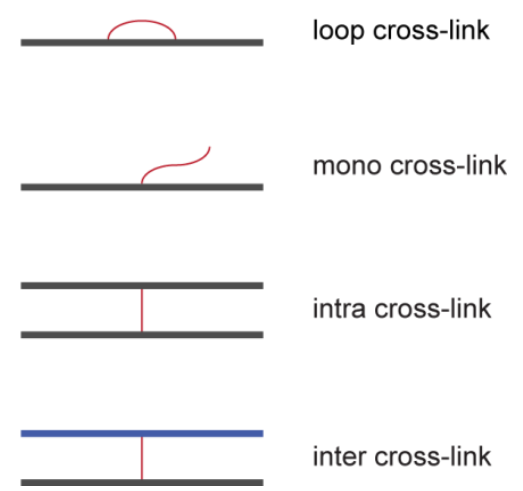

Figure 3.23 Chemical cross-links of peptides using the cross-linker BS3.

A) Chemical structure of bis(sulfosuccinimidyl) suberate (BS3). B) Cross-linked peptide species that can be obtained from proteolysis of cross-linked proteins (adapted from (Tran et al., 2016)). 


\subsubsection{Protein-protein cross-links of complex protein samples are specifically detected by the search algorithm pLink in combination with a reference database}

One of the major challenges in XL-MS is the identification of cross-linked peptides and proteins. The main reason is due to the fact that obtained MS spectra do not correspond to one peptide but to all possible combinations of a cross-linked peptide pair. The consequence is a dramatic increase of the database used for the search associated with increased risk of random assignments and higher false discovery rates. These increases are exponential with the number of proteins included for the search. Thus, it is necessary to determine those proteins having a high probability to be detected in the search. These proteins will constitute the reference database.

To define a specific database, protein identification from MS measurements, were obtained using the MaxQuant software (Cox and Mann, 2008; Cox et al., 2011). Only proteins detected by at least 3 "razor and unique peptides" were included. The most abundant proteins were determined by dividing the number of detected peptide counts by their molecular weight.

To increase specificity of cross-link identification a FDR of 0.01 was set for the search using the pLink software (Purcell et al., 2007).

To test the specificity of protein-protein cross-link identification using pLink, I applied the algorithm to the mass spectra obtained from the negative control that should not show any cross-links. In total three pLink analyses were done, each time using a reference database including either the most 100 abundant proteins, the most 150 or all proteins identified in the sample. Depending on the size of the reverence database that was used between 13 and 21 cross-links were identified. Three of those cross-links were also found to be positive identifications in the cross-linked sample and were excluded from further analysis. As the restriction settings for the detection of cross-linked peptides in combination with purification and enrichment strategies still included the identification of false positives the filtering stringency for the dataset was increased.

Therefore, the median of the pLink scores of all spectra that were identified for the negative control was determined. Spectra identified for the cross-linked samples displaying a score less than this median were excluded from further analyses. In total I found $0.6 \%$ false positive spectra that had to be excluded from further analysis. As the interest of investigation was focused on the identification of the binding hierarchy of proteins associated with chromatin, ambiguous spectra were generally excluded from analyses.

To further validate the specificity of cross-links I mapped the identified intra-cross-links of proteins against available crystal structures. The spacer arm of BS3 is 11.4- $\AA$ long. Lysines have a flexible side chain of $6-\AA . \AA$. Therefore, the two $C a$ atoms of specifically cross-linked lysines 
should be within a 24- $\AA$ distance. I selected the proteins Poly [ADP-ribose] polymerase 1 (PARP1) and DNA topoisomerase 2-alpha (TOP2A) from the dataset as they displayed several intra-cross-links and for both proteins crystal structures were available.

Using the Xlink Analyzer software (Kosinski et al., 2015; Pettersen et al., 2004) cross-links of PARP1 were mapped to the crystal structure of PARP1 with the PDB code 4DQY (Langelier et al., 2012). The crystal structure includes the amino acid sequences 6-91, 224-359, 531-575, 584-644, and 662-761. Hence, only 5 out of 20 cross-links could be mapped to the structure. All 5 cross links displayed a distance below 24- $\AA$ (Figure $3.24 \mathrm{~A}$ ) validating the specificity of the detected cross-links. The same analysis was performed with the identified intra-cross-links of the human DNA topoisomerase 2-alpha (TOP2A), using the 2.9-Å resolution structure with the PDB code 4FM9 (Wendorff et al., 2012). This structure includes the amino acid sequences 433-1092 and 1124-1190 of the protein. Here, 5 out of 13 cross-links could be mapped. Only one crosslink featured a distance longer than $24-\AA$ (33- $\AA$ ). This cross-link is spanning the DNA-binding domain (Figure $3.24 \mathrm{~B}$ ), which might be conformationally changed when TOP2A is not bound to DNA. Both, PARP1 and TOP2A are DNA-binding proteins (Figure 3.24) but have been identified to be cross-linked to proteins that themselves are directly cross-linked to one of the histones of the histone octamer (Figure 3.26 A).

A)

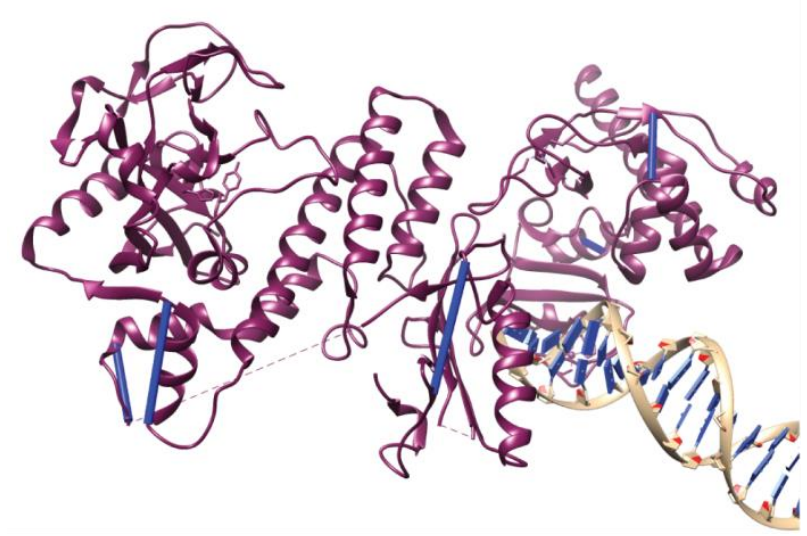

B)

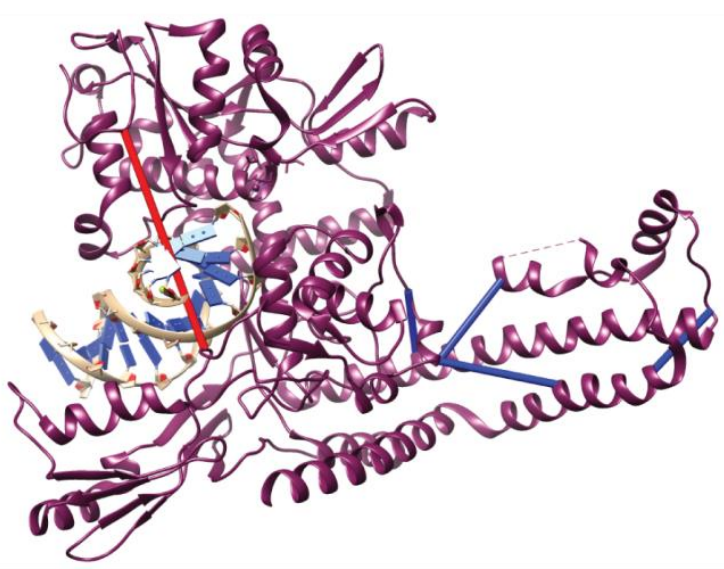

Figure 3.24 Protein-protein cross-links in the context of three-dimensional protein structures.

Identified intra-cross-links of Poly [ADP-ribose] polymerase 1 (PARP1) and DNA topoisomerase 2-alpha (TOP2A) were mapped to corresponding crystal structures using the Xlink Analyzer software (Kosinski et al., 2015; Pettersen et al., 2004). The tertiary protein structure is shown in purple. Cross-links are represented as blue bars when the distance is below 24- $\AA$ and in red when the distance is more than 24 $\AA$. DNA bound by the protein is indicated by a golden alpha helix surrounding the sugar backbone in blue. A) Crystal structure of human PARP1 (PDB code 4DQY (Langelier et al., 2012)) demonstrating the position of all identified cross-links corresponding to the amino acid sequence of the shown structure. $B$ ) 2.9-Å resolution structure of human TOP2A (PDB code 4FM9 (Wendorff et al., 2012)). The position of identified cross-links corresponding to the amino acid sequence is shown. 
In conclusion, with one exception I could demonstrate that all mapped cross-links lie within a 24A distance, suggesting that these cross-links are specific. The analysis of the negative control in parallel enabled the identification of false positive cross-links and therefore reduced the false positive rate. With the identification of only $0.6 \%$ false positive spectra, that were excluded from the dataset, and the mapping of identified cross-links to crystal structures as positive controls, it was shown that the stringency of data filtering is sufficient to provide reliable cross-link identifications.

\subsection{ChAP-MS coupled with XL provides information of the binding hierarchy beyond primary binding proteins recruited to chromatin}

ChAP-MS in combination with cross-linking was applied to unmodified nucleosomal arrays. For each experiment more than 200 protein species were identified. To ensure specificity and integrity of cross-link identification three different databases were used for pLink searches. The databases either comprised the top 100 abundant proteins, the top 150 or all positive identified proteins of one experiment. All together I obtained 1363 spectra with 521 different cross-link sites from two biological replicates. $45.7 \%$ of all identified cross-links localized to the nucleosome core particle.

\section{Protein-protein cross-links of the core nucleosome}

238 cross-links were identified within the core nucleosome, annotated in 792 spectra. These represented 464 inter- and 328 intra-cross-links. Most of these cross-links were mapped to the lysine-rich $\mathrm{N}$-terminal or $\mathrm{C}$-terminal tails of the histones (Figure $3.25 \mathrm{~A}$ ). These terminal tails of the histones are thought to be unstructured, flexible and protrude outwards from the nucleosome core particle (Figure 1.1). It is known that the tails interact with the DNA wrapped around the nucleosome (Angelov et al., 2001; Mutskov et al., 1998) as well as with acidic patches of nucleosomes in close proximity (Davey et al., 2002; Dorigo et al., 2004; Luger et al., 1997). Hence, it is very likely that each of the histone tails can be temporarily in close proximity to each other.

As consequence, lysines far apart from each other in the crystal structure of the core nucleosome can come in close proximity and therefore can be cross-linked by BS3. This might explain that only 57 of all protein-protein cross-links identified within the core nucleosome were shorter than $24-\AA$ whereas 174 of the cross-links were longer than $24-\AA$ (Figure $3.25 \mathrm{~B}$ ). In particular for histone $\mathrm{H} 3$ many cross-links were observed with a distance above 24- $\AA$. As shown in figure $3.25 \mathrm{~A}$ cross-links to histone $\mathrm{H} 3$ were mainly found within the $\mathrm{N}$-terminus of the protein. Similarly, for the histones $\mathrm{H} 2 \mathrm{~A}, \mathrm{H} 2 \mathrm{~B}$ and $\mathrm{H} 4$ cross-link sites were concentrated within the 
unstructured domains of the proteins (Figure $3.25 \mathrm{~A}$ ). Therefore, no conclusion regarding structural information can be drawn from the cross-links obtained for the histone octamer.

A)

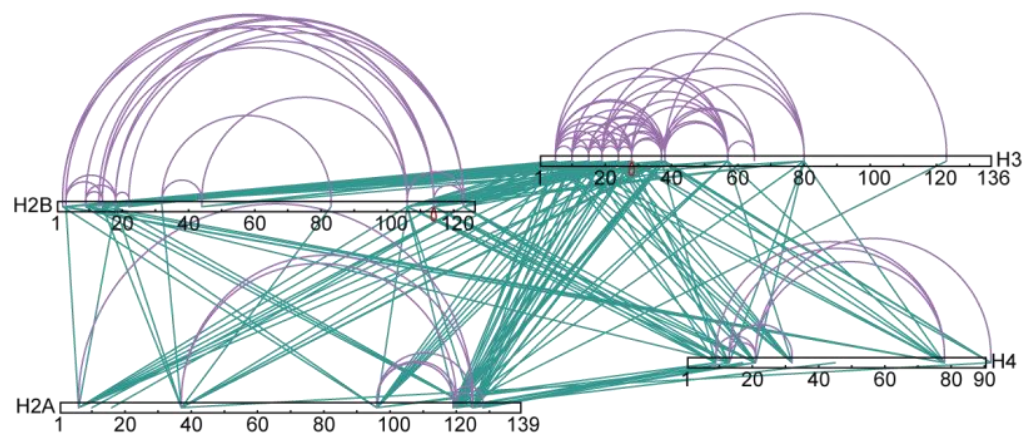

B)

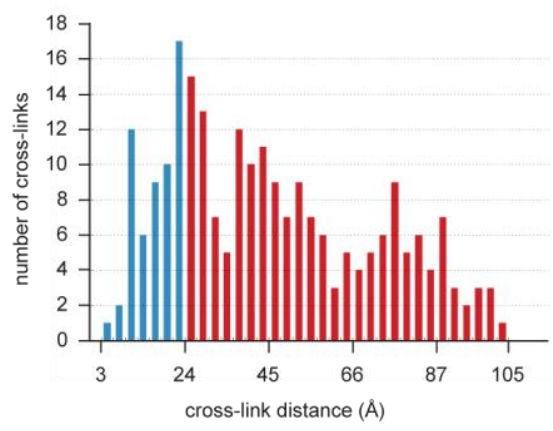

Figure 3.25 Protein-protein cross-link analysis of the core nucleosome.

A) Cross-links identified for the core nucleosome. The map was created using xiNet (Combe et al., 2015). Intra-cross-links are represented by purple lines. Inter-cross-links are colored in green. Red loops indicate that the same peptide was cross-linked to itself (multimerization). B) Histogram of cross-link distances of all identified cross-links within the four histone proteins mapped onto the crystal structure of the core nucleosome (PDB_1KX5 (Davey et al., 2002)) using the Xlink Analyzer software (Kosinski et al., 2015; Pettersen et al., 2004). Distances below 24- $\AA$ are labelled in blue. Distances longer then $24-\AA$ are colored in red.

\section{Protein-protein cross-links of chromatin-associated factors}

In order to address the hierarchical binding patterning of chromatin-associated proteins and their physical interaction sites all filtered spectra of identified cross-links were uploaded to xiNET (Combe et al., 2015). The information of these spectra is summarized in a node-link diagram (Figure 3.26 A). 75 proteins were found to be cross-linked. Eight of them displayed only intracross-links, whereas all others exhibit at least one inter-cross-link (Figure 3.26 A). As cross-links covalently link proteins in close proximity, it was not surprising that 238 cross-links out of 521 different cross-link sites were identified within the histone octamer. 18 proteins showed a direct physical interaction to at least one of the core histones (Figure $3.26 \mathrm{~A}$ ). Accordingly, these proteins were identified as primary binders to chromatin. A subset of these proteins was found to be cross-linked to a second protein. For instance, the protein HP1BP3 was cross-linked to histone $\mathrm{H} 3$ as well as to SMARCA5. Thus, this approach allowed the identification of secondary binders to the core nucleosome, in this case SMARCA5 (Figure $3.26 \mathrm{~A}$ ). This result clearly demonstrate that SMARCA5 can be recruited to chromatin via HP1BP3 when not binding directly to chromatin by itself. The same is true for RPL6 and RREB1 as they were also identified to be cross-linked to HP1BP3. The protein SMARCA5 itself was cross-linked to a second protein, HSPA5, that can be considered as a tertiary binder to the core nucleosome. This protein again is connected to a multi subunit complex of proteins identified to be cross-linked to each other (Figure $3.26 \mathrm{~A}$ ). 
Not all cross-linked proteins could be assigned to one of the core histones. A protein complex of SNRNP200, HLTF, DNMT1, KDM2A, BAZ1B and HNRNPC was cross-linked to each other. In this case, the connector to chromatin was either not identified or the complex was directly bound to DNA via one of the proteins, for instance, such as DNMT1.

To prove that I cross-linked proteins with a biological background to chromatin that show expected functional associations, I investigated the 75 proteins identified to be cross-linked using the STRING database (Figure 3.26 B). Only six proteins were not included in the proteinprotein map drawn by STRING, verifying the biological association of those proteins. The physical interaction sites of the four largest protein complexes of cross-linked proteins (Figure 3.26 A) were compared to the protein-protein interactions shown by STRING (Figure 3.26 B). Altogether, 17 protein-protein interactions overlapped between the two maps (Figure 3.26). For instance, the protein-protein interactions between the core histones as well as to histone $\mathrm{H} 1$ proteins have been also predicted by STRING. STRING also showed interactions between histone H2A and TOP2A. The complex of UBF1, TOP1, PARP1 and OGT1 was found in both maps as well.

Taken together, I could demonstrate that the established workflow is suitable for (i) identification of chromatin associated proteins, (ii) identification of physical interactions between proteins and proteins with the nucleosome, (iii) giving insights into the binding hierarchy of chromatinassociated proteins and their complexes, and (iv) identification of discrete interaction (cross-link) sites within proteins. 


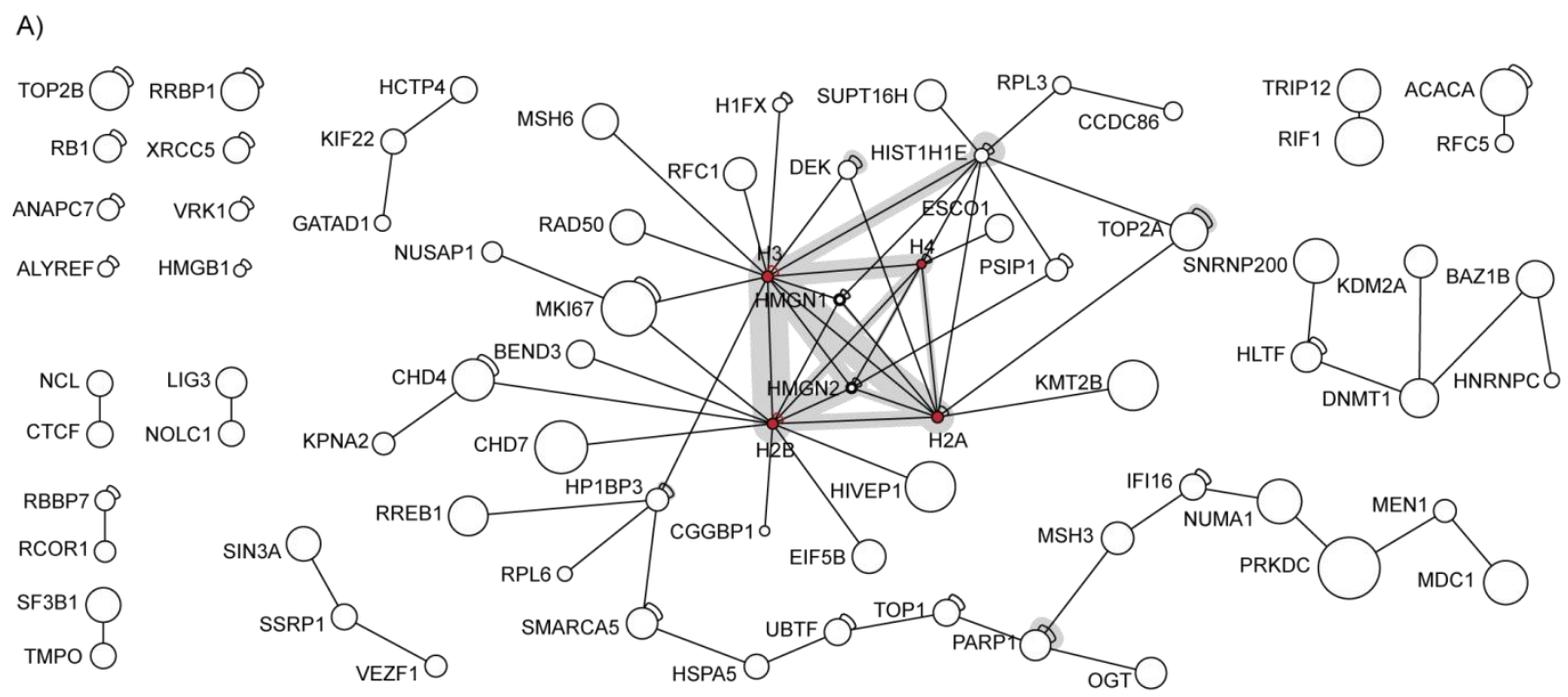

B)

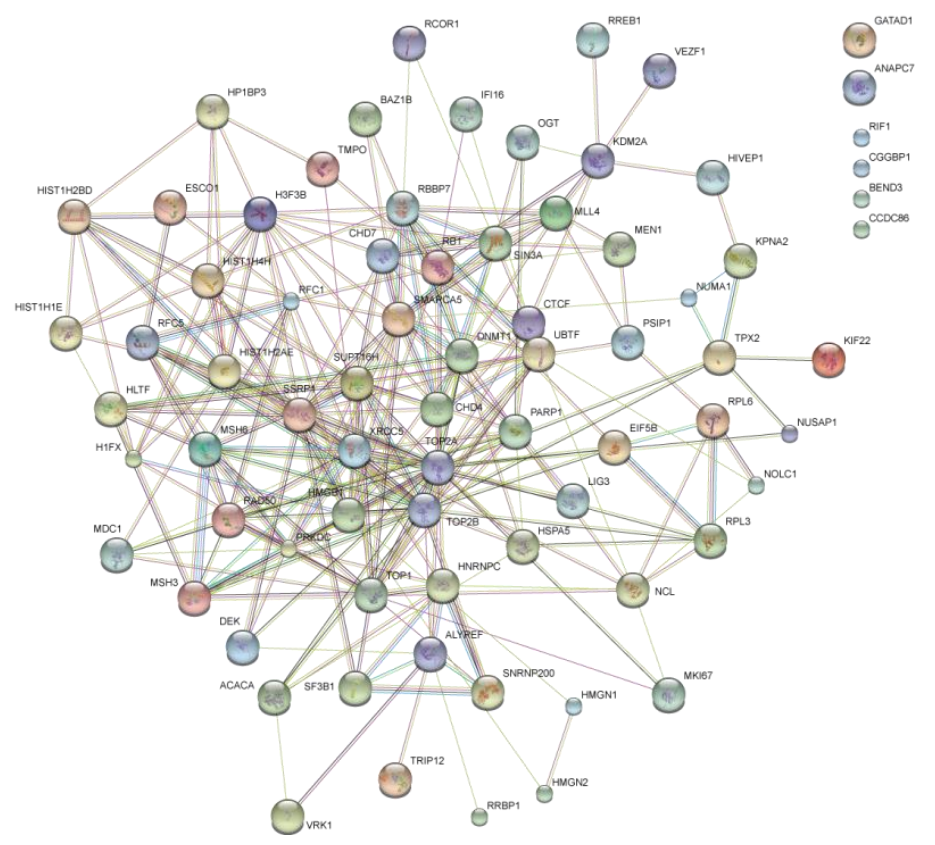

Figure 3.26 Interaction network of cross-linked factors associated with chromatin.

A) Node-link diagram of identified cross-links of chromatin-associated proteins. The network map was drawn in xiNET (Combe et al., 2015). Proteins are indicated by a node while cross-linked partner proteins are connected by a straight line between two nodes. Intra-cross-links are indicated by a loop connected to the respective node. Core histone proteins are marked in red. A large number of identified cross-links for a certain protein pair is roughly indicated by a grey shadow around the connection line between the two proteins. B) Protein-protein interaction network of known and predicted protein-protein interactions of the set of proteins found to be cross-linked in A. The network was generated by the database STRING (Jensen et al., 2009) using the default settings of the database. 


\section{Discussion}

This study is based on a strategy combining recombinant homogenously modified chromatin arrays and quantitative mass spectrometry in order to characterize the protein-binding profiles in the context of 12 different histone modification patterns. This thesis work has established comprehensive lists of proteins whose binding to nucleosomes is regulated by histone modifications specifically found at heterochromatin. The raw proteomic data were analyzed by using a statistical approach, which allowed the comparison of different interactomes side by side. Conclusions were drawn regarding the biological functions, which are actively regulated by histone posttranslational modifications. Furthermore, the approach was extended by the incorporation of two different histone modifications in combination. For the first time, the crosstalk between one modification on $\mathrm{H} 3$ and one on $\mathrm{H} 4$ was analyzed on a global scale. Also, positive as well as negative crosstalk between histone modifications and methylated DNA was investigated. Lastly, a new approach combing chromatin affinity purification, cross-linking and mass spectrometry was developed to characterize the hierarchy of physical protein-protein interactions within a chromatin-binding interactome.

\subsection{Individual chromatin-binding interactomes are comparable based on significant enrichment cutoffs}

Affinity purification using $12 \mathrm{mer}$ chromatin arrays resulted in a high number of recruited proteins. In fact, I found between 1,117 and 2,785 proteins associated with chromatin depending on the incorporated modification. Most of the proteins were binding to the chromatin arrays independently of the histone modification state. To define these modification specific regulated factors the binding of proteins to modified and unmodified chromatin arrays were quantitatively determined and compared by their relative enrichment levels (SILAC ratio). Proteins significantly affected by a modification were identified by applying a statistical scoring function ("significance A") (Cox and Mann, 2008) to the distribution of log SILAC ratios in the corresponding experiment. Assuming a symmetric Gaussian distribution of variables (i.e. $\log$ SILAC ratios) this function calculates and scores the probability that a random individual variable has a defined distance from the mean of the distribution, which identifies it as a significant outlier. In average, approximately $3.5 \%$ of chromatin-bound proteins appeared to be directly affected by a chromatin modification. 
The advantage of this statistical tool is a fold enrichment cutoff calculated based on the proteinbinding properties of each individual experiment. Thus, significantly affected proteins could be determined independently of the variations introduced by the many experimental steps during sample preparation, stringently required for direct comparison of proteins affected by different histone modifications.

The drawback of this statistical tool is the requirement for a Gaussian distribution with small variance. In fact, when the binding properties of a very large number of proteins are affected by the underlying chromatin modification the set of SILAC ratios will spread out around the mean of the Gaussian distribution. In consequence, even the data indicate a very large number of proteins displaying an increased binding affinity to the modified chromatin arrays, no significant outlier will be determined.

Such a case was observed for H3K9me3|meCpG-modified chromatin. More than $30 \%$ of chromatin-bound proteins appeared to be affected by the double modification. In consequence, significant outliers could not be determined. Thus, for the interactome of the double modification H3K9m3/meCpG significantly recruited proteins could not be determined, (Figure 3.17 A). Hence, proteins recruited to $\mathrm{H} 3 \mathrm{~K} 9 \mathrm{~m} 3 \mid \mathrm{meCpG}$ chromatin were not comparable to proteins significantly impacted by other modification states.

\subsection{Interactomics of chromatin-binding proteins provide new insights into the functional impact of individual chromatin modifications}

The complex language of posttranslational histone modifications is interpreted by proteins having reader domains. These histone-interacting domains, by recognizing their target sites, are thought to facilitate the recruitment of effector proteins and their connected biological functions to specific chromatin loci. The PTMs investigated here have a considerable impact on the composition of chromatin-bound proteins in the context of recombinant chromatin arrays. I investigated the protein-binding profiles in the context of 10 individual chromatin modifications. The first general conclusion from these datasets is the confirmation that PTMs as well as methylated DNA, promote and inhibit protein binding to chromatin. Second, independent of the methylation site and the degree of methylation, protein recruitment and exclusion from chromatin were regulated specifically, as shown by individual chromatin-binding interactomes for each modification. Although in the literature all investigated modification states were associated with heterochromatin, and therefore to be expected to have a certain overlap in regulated factors and functionality, a high diversity of factors was impacted by the different modification states. These factors display a limited set of common factors overlapping only partially between the different modification states. The observations support the notion of the existence of a histone PTM 
language, guiding different biological functions, dependent on the underlying chromatin modification pattern, to specific genome loci (Jenuwein and Allis, 2001; Strahl and Allis, 2000).

Mono-, di- and tri-methylation of H3K9

The three methylation degrees of H3K9 show a strong contrast in numbers of proteins they significantly regulated. Considering all modification patterns investigated, H3K9me3 modulated the binding properties of the largest set of proteins. In contrast, H3K9me1/-me2 impacted the lowest number of proteins (Table 3.1). The finding indicates that lower methylation degrees of H3K9 affect chromatin in a more subtle way, likely regulating a more restricted set of functions.

At least $70 \%$ of the proteins affected by H3K9me1/-me2 were also affected by $\mathrm{H} 3 \mathrm{~K} 9 \mathrm{me} 3$ (Figure $3.12 \mathrm{~A}$ ), suggesting a functional link between H3K9me1/-me2 and the tri-methylation state. The common functions regulated by these modifications might be heterochromatin establishment and maintenance. This assumption is supported by the facts that first, the methylation degrees of $\mathrm{H} 3 \mathrm{~K} 9$ are known to be involved in heterochromatin formation and maintenance (Loyola et al., 2009; Towbin et al., 2012). Second, in my study I found the well characterized human HP1 1 (CBX5) and the heterochromatic protein UHRF1 to be recruited to chromatin by all three modification degrees (Table 3.1). Both proteins have been previously shown to bind to the different H3K9 methylation degrees and are primarily involved in heterochromatin formation and maintenance (Lachner et al., 2001; Nady et al., 2011).

Another common feature of the three H3K9 methylation degrees was the significant decrease in chromatin binding of transcription factors of the NFI gene family as well as E4F1 (Table 3.1). These factors correlated with the regulation pattern of polymerase subunits in the context of several modifications (Table 3.1). More precisely, the heterochromatic PTMs H3K9me3, $\mathrm{H}_{3} \mathrm{~K}_{\mathrm{C}} 27 \mathrm{me} 2$ and $\mathrm{H} 4 \mathrm{~K} 20 \mathrm{me} 3$ repelled part of these transcription factors together with polymerase subunits from binding to chromatin (3.7 E, lower panel and 3.9 D, lower panel) whereas H4R3me2 specifically recruited polymerase subunits and NIFC (Table 3.1). Additionally, analysis using the database STRING predicted the factors of the NFI gene family to be in association with polymerase subunits (Figure $3.6 \mathrm{C}$ ). These findings suggest that the transcription factors of the NFI gene family work in the context of transcriptional activation and thereby support the heterochromatic context of the H3K9 methylation degrees.

The low number of $\mathrm{H} 3 \mathrm{~K} 9 \mathrm{me} 1 /-\mathrm{me} 2$ regulated proteins raises the question whether these modification degrees have their own biological significance or mainly function as substrates necessary for the establishment of H3K9me3 as suggested before (Loyola et al., 2009; Towbin et al., 2012). Indeed, my data implicate that H3K9me1/-me2 do not autonomously promote functional outcomes and are related to H3K9me3. This supports the suggestion of H3K9me1/- 
me2 being substrates for establishment of H3K9 tri-methylation. However, the independent genomic distribution of the modification degrees indicates the contribution to various chromatin related functions (Barski et al., 2007; Mikkelsen et al., 2007). Therefore, it cannot be ruled out that the lower methylation states of H3K9 act in concert with other modification sites to promote downstream functions at chromatin regions free of $\mathrm{H} 3 \mathrm{~K} 9 \mathrm{me} 3$.

Mono-, di- and tri-methylation of $\mathrm{H}_{3} \mathrm{~K}_{C} 27$

In comparison to $\mathrm{H} 3 \mathrm{~K} 9$, the characteristics of the three methylation degrees of $\mathrm{H}_{3} \mathrm{~K}_{\mathrm{c}} 27$ feature three main distinctions. First, there was not a single factor found to be commonly regulated by all three methylation degrees of $\mathrm{H}_{3} \mathrm{~K}_{\mathrm{C}} 27$ (Figure $3.13 \mathrm{~A}$ ). Second, the factors regulated by each of the modification degrees were highly divergent in functionality. Third, chromatin binding of several factors was regulated in an antagonistic manner by two of the $\mathrm{H}_{3} \mathrm{~K}_{\mathrm{c}} 27$ methylation degrees (Table 3.1). These observations indicate a specific biological significance for each of the methylation degrees. This point of view agrees with previous findings demonstrating that the different H3K27 methylation states are located in different regions of the genome, with a diversity much greater than for H3K9 and H4K20 methylation marks (Rosenfeld et al., 2009).

The mono-methylation of $\mathrm{H}_{3} \mathrm{~K}_{\mathrm{C}} 27$ recruited several components of the CCR4-NOT complex, which has been shown to be involved in transcription initiation and elongation (Collart, 2016). In this context the complex was shown to control $\mathrm{H} 3$ and $\mathrm{H} 4$ acetylation and $\mathrm{H} 3 \mathrm{~K} 4$ tri-methylation (Peng et al., 2008), indicating a role for H3K27me1 in transcriptional activation. This would be in agreement with the fact that H3K27me1 is enriched in gene bodies of actively transcribed genes (Barski et al., 2007; Wang et al., 2008). The CCR4-NOT complex has also been shown to act in the cytoplasm, regulating mRNA translation as well as degradation. Surprisingly, there are three more proteins recruited to $\mathrm{H}_{3} \mathrm{~K}_{\mathrm{C}} 27 \mathrm{me} 1$ that also show cytoplasmic localization. These proteins seem to be associated with a correct organization of the Golgi apparatus and transport processes between the Golgi apparatus and the endoplasmic reticulum or endosomes (Dimitrov et al., 2009; Mariappan et al., 2010; Reddy et al., 2006). It is unclear how these proteins are related to H3K27me1. These proteins might represent cytosolic contaminants found in the nuclear extracts due to the method used for the preparation of the extracts (Dignam et al., 1983). I think this is not very likely. First of all, light and heavy nuclear extracts were prepared individually. Secondly, for each of the two extracts three individual preparations were performed and pooled in order to average potential abnormalities introduced by experimental handling. Thirdly, some of the cytoplasmic proteins identified in this study were also identified by affinity purification in the context of $\mathrm{N}$-terminal histone peptides using HeLa nuclear extracts (Vermeulen et al., 2010). From my point of view these facts demonstrate the improbability of contaminants in 
the nuclear extracts used for this study and support the discovery of so far cytoplasmic classified proteins as positive identifications.

Remarkably, $40 \%$ of the proteins recruited to $\mathrm{H}_{3 \mathrm{~K}_{\mathrm{C}}} 27 \mathrm{me} 2$ chromatin are spliceosomal proteins. A link between regulation of alternative splicing and chromatin modifications has already been described (Luco et al., 2011; Luco et al., 2010; Zhou et al., 2014). The nucleosome density and consequently the presence of histone modifications was found to be higher over exons compared to introns (Schwartz et al., 2009; Tilgner et al., 2009). The presence of histone modifications is thought to locally impact alternative splicing by either, recruitment of splicing factors to the transcription site, or by marking alternative exons and/or the surrounding chromosomal regions and influencing the elongation rate of polymerase II and therefore splice site choices. The presence of H3K9me3 and HP1Y was shown to slow down polymerase II resulting in increased inclusion of transcribed alternative exons (Saint-Andre et al., 2011). In contrast, hyperacetylation of $\mathrm{H} 3$ and $\mathrm{H} 4$ increased the elongation rate of polymerase II and favored skipping of alternative exons (Hnilicova et al., 2011).

H3K27me2 was not extensively investigated in the context of alternative splicing so far but have been found to be specifically enriched on exons, with the strongest presence at low expressed exons, also shown for H3K27me3 (Andersson et al., 2009; Spies et al., 2009).

The finding that most of the factors recruited by $\mathrm{H} 3 \mathrm{~K}_{\mathrm{C}} 27 \mathrm{me} 2$ are associated with the spliceosome strongly correlates this modification site with splicing activities and implicates an active functional association. Contrary to the literature, in my study an association with the splicing process was exclusively found for $\mathrm{H}_{3} \mathrm{~K}_{\mathrm{C}} 27 \mathrm{me} 2$. As chromatin structure and recruitment of effector proteins by distinct histone modification patterns seem to correlate with alternative splicing it is very likely that the presence of H3K27me2 at certain chromatin regions might contribute to splice sites selection or even promote splicing events by slowing down transcriptional elongation as shown in the context of H3K9me3.

H3K27me3 is one of the best studied chromatin modification that is catalyzed by the repressive multi-subunit complex PRC2 (Schuettengruber et al., 2007). Several polycomb group proteins, the components of the PRC2 and PRC1 complexes, have been shown to co-localize with H3K27me3 and directly targeting this modification mark (Morey and Helin, 2010; Schuettengruber et al., 2007). Surprisingly, the $\mathrm{H}_{3} \mathrm{~K}_{\mathrm{c}} 27 \mathrm{me} 3$ interactome characterized here did not show an increased affinity for most of the polycomb group proteins expected to bind this mark. While I only found CBX8 and PHF1, which were significantly recruited by $\mathrm{H}_{3} \mathrm{~K}_{\mathrm{C}} 27 \mathrm{me} 3$, the majority of the polycomb proteins were identified below the P-value that was set for statistical significance or they were not quantified. The mechanisms that recruit the PRC complexes to their target genes are not fully understood yet, but they are thought to contain specific and 
complex elements, i.e. DNA, RNA and DNA-associated proteins (Morey and Helin, 2010). Those elements might have been absent from the chromatin template or the nuclear extracts. The preparation of nuclear extracts used for the ChAP experiments were based on high salt extraction of isolated nuclei followed by the removal of the insoluble chromatin fraction by centrifugation (Dignam et al., 1983). Thus, proteins displaying a very strong affinity to the chromatin, which resists to high salt treatment, might have been lost or reduced together with the chromatin during nuclear extract preparation.

\section{Histone H4 methylation sites}

The interactomes of H4K20me1 and H4K20me3 showed high divergence (Figure 3.9, Table 3.1) since only an overlap of three factors were found to be regulated by both modification degrees. These factors were excluded from binding to the modified chromatin templates (Figure 3.13 B).

In contrast to most of the other investigated PTMs, H4K20me1 recruited predominantly factors associated with positive regulation of transcription (Figure 3.9 B, upper panel, Table 3.1). Among them are members of the INO80 remodeling complex, which have been shown to be excluded from binding to chromatin by $\mathrm{H} 3 \mathrm{~K} 9 \mathrm{me} 1 /-\mathrm{me} 3$ and $\mathrm{H} 3 \mathrm{~K}_{\mathrm{C}} 27 \mathrm{me} 2$ histone modifications. In addition, an antagonistic regulation was observed of splicing associated factors: while $\mathrm{H}_{3} \mathrm{~K}_{\mathrm{C}} 27 \mathrm{me} 2$ recruited several spliceosomal factors, H4K20me1 excluded 16 factors mainly associated with the $\mathrm{C}$ spliceosomal complex (Figure $3.9 \mathrm{~B}$ lower panel, Table 3.1). The data are indicative of a connection of H4K20me1 to transcriptional activity and euchromatin, which would be in agreement with previous findings that found H4K20me1 located at gene bodies (Barski et al., 2007; Vakoc et al., 2006). Additionally, H4K20me1 has been shown to be involved in regulation of many cellular processes like genome stability, DNA replication, mitosis, and transcription (Beck et al., 2012; Lv et al., 2016). The broad spectrum of biological functions linked to H4K20me1 is in good agreement with the variety of functions connected to the proteins regulated by H4K20me1 as well as the stronger differences and smaller similarities to the other histone modifications investigated here.

The second modification in this study that showed a unique protein-binding regulation when compared to the other modifications was H4R3me2. Although this modification was expected to be in association with heterochromatin (Le Guezennec et al., 2006; Zhao et al., 2009), six of nine recruited proteins were polymerase subunits. Additionally, several proteins that were recruited to $\mathrm{H} 3 \mathrm{~K} 9 \mathrm{me} 3$, the hallmark of pericentric heterochromatin, were excluded from binding to H4R3me2 (Table 3.1). These findings strongly suggest an association of H4R3me2 with transcriptional activation. In agreement, it was shown that dependent on the methyltransferase 
that set the di-methylation of H4R3, either PRMT1 or PRMT5, H4R3me2 can be associated with transcriptional activation or repression, respectively (Barrero and Malik, 2006; Le Guezennec et al., 2006).

\subsection{Protein-binding interactomes of modified chromatin arrays display only moderate overlap compared to those revealed with different modified templates}

In recent years several laboratories have made attempts to identify proteins specifically associated with distinct histone modifications by using templates such as $\mathrm{N}$-terminal histone peptides, mononucleosomes and chromatin arrays (Bartke et al., 2010; Bluhm et al., 2016; Engelen et al., 2015; Kunowska et al., 2015; Nikolov et al., 2011; Oda et al., 2010; Vermeulen et al., 2010).

In the core of $\mathrm{H} 3 \mathrm{~K} 9 \mathrm{me} 3$, the proteins $\mathrm{CBX} 5, \mathrm{CBX} 3$ and POGZ were identified in six independent studies (including this one) using different templates, cell lines and organisms (Bartke et al., 2010; Bluhm et al., 2016; Engelen et al., 2015; Kunowska et al., 2015; Nikolov et al., 2011; Vermeulen et al., 2010). This high level of convergence suggests a strong affinity to chromatin for these proteins, which is in contrast to the finding that the interaction of POGZ and CBX5 weaken the interaction of CBX5 to chromatin (Nozawa et al., 2010). Overall, nearly half of the proteins I found to be recruited by $\mathrm{H} 3 \mathrm{~K} 9 \mathrm{me} 3$ were identified at least in one of the published studies mentioned above.

The overlap of $\mathrm{H}_{3} \mathrm{~K}_{\mathrm{C}} 27 \mathrm{me} 3$ recruited factors with published data was much lower. Common factors were CBX8 and several members of the ORC complex (Bartke et al., 2010; Vermeulen et al., 2010). The ORC complex seems to have a very strong affinity for heterochromatic modifications as the complex was also recruited to H3K9me3 and H4K2Ome3, which was observed using N-terminal histone peptides and mononucleosomes as well (Bartke et al., 2010; Vermeulen et al., 2010).

Next to the ORC complex I found another common feature of H3K9me3, H3K27me3 and H4K20me3 that had not been described before. The interactomes showed the recruitment of several eukaryotic translation initiation factors (Table 3.1). This was unexpected since these factors function in translation initiation within the cytoplasm and were previously shown to be absent from the nucleus (Bohnsack et al., 2002). As already discussed earlier, it is unlikely but I cannot rule out that such observation could be attributed to contaminations of the nuclear extracts by cytosolic proteins. Notably, several translation initiation factors were also found as background binders in the context of peptide pull-downs of H3K9me3, H3K27me3 and 
H4K20me3 (Vermeulen et al., 2010), indicating that these factors seem to be general constituents of (HeLa) nuclear extracts. The functional association of the translation initiation factors to the heterochromatic modifications H3K9me3, H3K27me3 and H4K2Ome3 in contrast remains to be in investigated.

Most of the differences observed are likely attributed to the different types of templates used in the previous studies. In contrast to $\mathrm{N}$-terminal histone peptides, chromatin arrays are formed by an underlying DNA sequence and histone octamers. Thus, chromatin arrays allow the identification of proteins that require a binding platform consisting of more than one interaction site that can be provided by e.g. the globular domain of a histone, the DNA or a second nucleosome in the case of inter-nucleosome binding of histone modification in trans. The latter binding mode can also not be addressed using mononucleosomes.

Some of the differences can also likely be attributed to the different experimental procedures used in the different studies. For example, nuclear extracts derived from different organism and cell types have been used, different mass spectrometer devices with varying sensitivity, the number of technical and biological replicates, and statistical evaluations contribute to divergent protein identifications.

However, in comparison to the interactomes of defined posttranslational chromatin modifications published so far, the number of proteins we found to be affected using modified chromatin arrays is much larger. Only a moderate overlap was observed between my thesis work and the studies available in the literature (Table 3.1). This is especially true regarding proteins showing decreased affinities to a certain modification state. Thus, the data presented here offer a close to comprehensive list of factors whose binding to chromatin is affected by posttranslational modifications comprising also the excluded proteins, which in previous studies have been essentially kept out of focus. These extended dataset provide not only new functional relations of histone modifications but also enables the investigation of chromatin modification crosstalk. All these facts validate the gain of additional information provided with the here presented study and strongly support the usage of a more complex template closer to the native form of chromatin.

\subsection{Individual heterochromatic marks display positive as well as negative functional correlation}

Since all investigated modification states have been shown to be associated with heterochromatin and transcriptional repression (Table 1.1) I expected a certain level of overlap of regulated factors. However, due to the vast number and diversity of proteins regulated by the 
individual chromatin modifications, it turned out to be very challenging to draw any general conclusions. Therefore, the functional relationship between individual modifications was investigated by using gene annotation enrichment analyses. These analyses showed positive as well as negative functional correlation of chromatin modifications and highlighted groups, which appeared to be functionally linked.

$\mathrm{H} 3 \mathrm{~K} 9 \mathrm{me} 3, \mathrm{H} 4 \mathrm{~K} 20 \mathrm{me} 3, \mathrm{H} 3 \mathrm{~K}_{\mathrm{C}} 27 \mathrm{me}$, and $\mathrm{H} 3 \mathrm{~K} 9 \mathrm{me} 3 \mid \mathrm{H} 4 \mathrm{~K} 20 \mathrm{me} 3$ showed a strong correlation in the context of annotated pathway terms (Figure $3.19 \mathrm{~A}$ ), suggesting a functional link between these modifications. All these modifications have been shown to localize to pericentric heterochromatin and to be involved in transcriptional repression (Barski et al., 2007; Fischle et al., 2003a; Lachner et al., 2003; Ringrose and Paro, 2004; Schotta et al., 2004; Sims et al., 2006; Wang et al., 2008). This observation is consistent with the strong correlation on one hand and validates the analytical approach on the other hand.

Negative functional correlation was observed between $\mathrm{H} 4 \mathrm{R} 3 \mathrm{me} 2$ and the two modification states $\mathrm{H} 3 \mathrm{~K} 9 \mathrm{me} 3$ and $\mathrm{H}_{3} \mathrm{~K}_{\mathrm{C}} 27 \mathrm{me} 2$. While $\mathrm{H} 4 \mathrm{R} 3 \mathrm{me} 2$ recruited several polymerase subunits these factors were excluded from binding to $\mathrm{H} 3 \mathrm{~K} 9 \mathrm{me} 3$ and $\mathrm{H}_{3} \mathrm{~K}_{\mathrm{c}} 27 \mathrm{me} 2$, strongly suggesting an association of H4R3me2 with transcriptional activity. H4R3me2 has been found in the context of both, transcriptional activation and repression as described earlier.

Additionally, $\mathrm{H} 3 \mathrm{~K}_{\mathrm{C}} 27 m e 2$ displayed a negative correlation to $\mathrm{H} 4 \mathrm{~K} 20 \mathrm{me}$. While $\mathrm{H} 3 \mathrm{~K}_{\mathrm{c}} 27 \mathrm{me} 2$ showed an increased binding affinity for several spliceosomal factors, in turn H4K20me1 significantly excluded factors associated with the spliceosome from binding to chromatin. This is the first time that such functional relationship between both modifications is described and suggested that H4K20me1 might mark sequences at the gene bodies with decreased splicing activity, while $\mathrm{H} 3 \mathrm{~K}_{\mathrm{c}} 27 \mathrm{me} 2$ might promote splicing events as suggested in chapter 4.2.

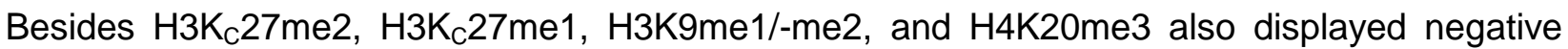
correlation to H4K20me1 (Figure $3.19 \mathrm{~B}$ ), which indicate a unique biological significance for H4K20me1 compared to the other investigated modification patterns.

\subsection{Combinatorial readout in trans provides new insights into crosstalk between chemical chromatin modifications}

The identification of proteins binding in the context of the combination of modifications is still challenging despite vast technical developments. Thus, the multivalent interactions of chromatinbinding proteins with combinatorial histone modification patterns were only touched upon in a limited number of studies. Here, I took the opportunity offered by our chromatin system to investigate the effect of combinatorial readout of $\mathrm{H} 3 \mathrm{~K} 9 \mathrm{me} 3$ and $\mathrm{H} 4 \mathrm{~K} 2 \mathrm{Ome} 3$ as well as $\mathrm{H} 3 \mathrm{~K} 9 \mathrm{me} 3$ and CpG-methylated DNA on a global scale. Both combinations of chromatin modifications were 
shown to be not only functionally linked but also displayed co-occurrence in several model organisms (Du et al., 2015; Mikkelsen et al., 2007; Schotta et al., 2004; Sims et al., 2006).

The data collected highlight three general effects of combinatorial readout that were observed for both combinations, H3K9me3|H4K20me3 and H3K9me3|meCpG.

In the first category, the double modification appeared to have "no effect" on protein-binding regulation. "No effect" was determined for the majority of proteins and referred to the finding that the protein-binding properties were similar over all three modification patterns, the two single modification sites and the combination thereof. These findings suggest that the regulation of protein recruitment to chromatin essentially relies on single modification sites.

In the second category, I observed antagonistic binding properties for several proteins when comparing the double modification to the single ones. In the context of the double modification, the decrease in binding of factors that were found otherwise to be enriched with the individual modifications was defined as negative crosstalk. In relation to H3K9me3 and H4K20me3 negative crosstalk was observed for several members of the septin family and spliceosomal factors, ASF1B, EIF5 and EIF4G1 (Figure 3.16 A). These findings give insights into the extent of consequences brought about by crosstalk. The effects of positive and negative crosstalk were not only limited to individual proteins but also were applied to entire protein complexes. Thus, it seems that not only direct binding proteins but also secondary interacting proteins were affected by crosstalk. Additionally, individual factors displayed negative crosstalk between H3K9me3 and meCpG as well as H3K9me3 and H4K2Ome3, namely USP3 and UBTF (Figure 3.16 A and 3.18 A).

Opposed binding was also determined in the reverse direction: crosstalk was considered as positive when the double modification displayed increased protein binding, while the individual modifications displayed decreased binding to chromatin. Several polymerase subunits and members of the NFI family displayed positive crosstalk between both H3K9me3 and meCpG as well as $\mathrm{H} 3 \mathrm{~K} 9 \mathrm{me} 3$ and H4K20me3. Additionally, each of the modification combinations showed individual transcription factors that were affected. Recently, positive crosstalk was shown in context of $\mathrm{H} 3$ methylation and $\mathrm{H} 4$ acetylation. It was shown that the presence of $\mathrm{H} 4 \mathrm{~K} 5 \mathrm{ac}$ next to H3K4me3 enhances the binding of BPTF and p300 to nucleosomes by at least seven-fold, probably mediated by simultaneous binding of the PHD and the bromodomain (Nguyen et al., 2014).

In the present study, conclusions about the binding mechanisms of single factors could not be drawn, as the experimental design does not allow determining whether a given protein binds directly or indirectly to the chromatin arrays. However, it appeared that positive crosstalk of chromatin modifications, mainly located at pericentric heterochromatin, tends to recruit factors 
and protein complexes in association with transcriptional activity and thus, might be involved in the reversion of transcriptional repression at defined chromatin regions.

Proteins showing negative crosstalk are inconclusively transcriptional repressors or in association with heterochromatin. In the context of negative crosstalk there seemed to be a trend of excluding factors or protein complexes involved in mitosis and proteins associated with the cytoskeleton in presence of one of the here investigated double modifications H3K9me3|meCpG and H3K9me3|H4K20me3.

\subsection{Protein-protein cross-linking coupled to MS maps the binding hierarchy of chromatin-bound proteins}

So far the identification of specific protein interactions using affinity purification based strategies coupled with cross-linking and mass spectrometry were challenging, mainly because of the lack in database engines capable to search for cross-links against large databases, which has restricted cross-linking approaches to the study of small protein complexes for a long time.

Now, with important technological developments of recent years, it has become possible to set up a workflow that combines proteomic and protein-specific XL-MS. This offers the possibility to analyze topological information of the protein interactome associated with chromatin. As I have demonstrated, both the protein-binding interactome as well as the hierarchy of protein binding to chromatin can be analyzed.

With the established workflow I found 75 cross-linked chromatin-associated proteins. As crosslinks covalently link proteins that are in close physical proximity, the proteins directly cross-linked to histones were considered as primary interactors. 18 of the cross-linked proteins showed a direct physical interaction to at least one of the core histones (Figure $3.26 \mathrm{~A}$ ) while 20 factors were identified as indirect binders, not cross-linked to histones, but recruited to the chromatin arrays via primary binders. The remaining identified proteins could not be assigned to one of the core histones. The connecting factors may have not been detected or, alternatively, these proteins are recruited to chromatin by interacting with the DNA.

STRING analysis of cross-linked proteins confirmed the biological association of these proteins and came as a support regarding the specificity of the cross-links and of the approach on a more general way (Figure $3.26 \mathrm{~B}$ ).

In contrast to the fact that more than 1000 proteins were identified to bind to the chromatin arrays using ChAP-MS, only a small number of proteins were found to be cross-linked. This low number might be attributed to limited availability and accessibility of interaction mediating sequences qualified for cross-linking. In brief, the BS3 cross-linker, which comprises two amine 
reactive groups separated by a spacer arm of $11.4-\AA$ in length, reacts with primary amines in the side chains of lysine $(K)$ residues and the N-terminus of polypeptides (Sinz, 2006; Tran et al., 2016). Thus, only interacting protein surfaces exhibiting two lysines within a distance of $24-\AA$ can be cross-linked. As a consequence, interaction interface that do not have lysines matching this criteria cannot be cross-linked, which result in the impossibility to identify actual interactions. This limitation may account, at least in part, for the rather small number of cross-linked proteins which were identified. Moreover, BS3 tend mostly to react with easily accessible lysine residues localized at the surfaces of protein complexes, since diffusion within the rather hydrophobic globular part of complexes is hindered by its hydrophilic nature (Huang et al., 2004). As a consequence, this may lower the number of cross-links and therefore reduce the number of final interacting partner identified. Additionally, the two enrichment steps on the protein as well as the peptide level by size exclusion chromatography are known to lead to loss of biological material.

Another limitation is related to on-bead cross-linking. Streptavidin coated beads that were used in this workflow have the potential to cross-link purified proteins to the beads itself, potentially leading to false positive identifications. To overcome this issue, the underlying biotinylated DNA of the cross-linked sample was degraded, which resulted in the release of histone octamers and their associated proteins from the beads.

In conclusion, we established a robust workflow that allows the highly specific identification of protein-protein cross-links in the context of chromatin affinity purification. The workflow enables the mapping of specific protein-protein interaction sites on one hand and provides information regarding the hierarchy of protein binding with each other and to chromatin on the other hand. The workflow identifies the abundant chromatin-interacting proteins and therefore has a strong potential for the investigation of physical protein-protein interaction sites of protein interactomes of modified chromatin arrays. 


\section{Conclusions and future perspectives}

I have shown here, that ChAP-MS is suitable for the elucidation of the interactomes of single and complex chromatin modification patterns. My results provide a step towards functional characterization of individual modifications deposited on an oligonucleosomal chromatin template. Each of the modification patterns studied here regulated a specific set of factors, underscoring their distinct biological significance. The protein interactomes of individual modifications have revealed novel functional associations of specific marks. This was shown for $\mathrm{H} 3 \mathrm{~K}_{\mathrm{C}} 27 \mathrm{me} 2$ that recruits several splicing factors, H4R3me2, which appears to have mainly impact on the recruitment of polymerase subunits and $\mathrm{H}_{3} \mathrm{~K}_{\mathrm{C}} 27 \mathrm{me} 1$, which was associated with transcriptional initiation and elongation by the CCR4-NOT complex. The assignment of biological functions to the different chromatin-binding interactomes on a global scale allowed the identification of novel relationships between individual modifications and indicated positive and negative functional correlation of PTMs. One example is the inverse regulation of the binding properties of several spliceosomal factors by $\mathrm{H}_{3} \mathrm{~K}_{\mathrm{c}} 27 \mathrm{me} 2$ and $\mathrm{H} 4 \mathrm{~K} 20 \mathrm{me} 1$.

My work shows that combinations of different modifications in the context of chromatin can reveal important aspects of the nature and the complexity of the language of histone PTMs. Indeed, the combinatorial experiments presented here identified a subset of factors regulated only in presence of two modification sites. Thus, my experimental design gives novel, unpredicted insights into mechanisms of crosstalk between two chromatin modifications. Both, $\mathrm{H} 3 \mathrm{~K} 9 \mathrm{me} 3$ and meCpG as well as H3K9me3 and H4K20me3 display positive as well as negative crosstalk, which underlines the importance of testing further combinations of modifications whose co-occurrence have been proved in vivo already.

The performed experiments have proven to provide new insights into specific functions and regulation mechanisms of individual histone modification patterns. Extending this approach to euchromatic marks would significantly increase the knowledge of pathways underlying epigenetic regulations. Furthermore, ChAP-MS can determine the protein-binding interactomes in the context of distinct biological circumstances by using e.g. a native DNA sequence instead of an artificial one or cell extracts from different cell types, organisms or different developmental stages.

The new cross-linking approach we have established provides a workflow for the identification of specific protein-protein interaction of chromatin-bound proteins. I expect this method will contribute to our understanding of the hierarchy of protein recruitment and deliver detailed information of physical interactions sites, allowing the identification of protein domains involved in protein-protein interactions. Expanding the workflow to modified chromatin arrays will in the near future provide insights into the formation of protein complexes in the context of different chromatin modification patterns. 


\section{References}

Akhtar, A., and Becker, P.B. (2000). Activation of transcription through histone $\mathrm{H} 4$ acetylation by MOF, an acetyltransferase essential for dosage compensation in Drosophila. Mol Cell 5, 367375.

Andersson, R., Enroth, S., Rada-Iglesias, A., Wadelius, C., and Komorowski, J. (2009). Nucleosomes are well positioned in exons and carry characteristic histone modifications. Genome research 19, 1732-1741.

Angelov, D., Vitolo, J.M., Mutskov, V., Dimitrov, S., and Hayes, J.J. (2001). Preferential interaction of the core histone tail domains with linker DNA. Proceedings of the National Academy of Sciences of the United States of America 98, 6599-6604.

Arita, K., Isogai, S., Oda, T., Unoki, M., Sugita, K., Sekiyama, N., Kuwata, K., Hamamoto, R., Tochio, H., Sato, M., et al. (2012). Recognition of modification status on a histone H3 tail by linked histone reader modules of the epigenetic regulator UHRF1. Proceedings of the National Academy of Sciences of the United States of America 109, 12950-12955.

Bannister, A.J., and Kouzarides, T. (2011). Regulation of chromatin by histone modifications. Cell Res 21, 381-395.

Bantscheff, M., Lemeer, S., Savitski, M.M., and Kuster, B. (2012). Quantitative mass spectrometry in proteomics: critical review update from 2007 to the present. Anal Bioanal Chem 404, 939-965.

Barrero, M.J., and Malik, S. (2006). Two functional modes of a nuclear receptor-recruited arginine methyltransferase in transcriptional activation. Mol Cell 24, 233-243.

Barski, A., Cuddapah, S., Cui, K., Roh, T.Y., Schones, D.E., Wang, Z., Wei, G., Chepelev, I., and Zhao, K. (2007). High-resolution profiling of histone methylations in the human genome. Cell 129, 823-837.

Bartke, T., Vermeulen, M., Xhemalce, B., Robson, S.C., Mann, M., and Kouzarides, T. (2010). Nucleosome-interacting proteins regulated by DNA and histone methylation. Cell 143, 470-484.

Beck, D.B., Oda, H., Shen, S.S., and Reinberg, D. (2012). PR-Set7 and H4K20me1: at the crossroads of genome integrity, cell cycle, chromosome condensation, and transcription. Genes \& development 26, 325-337.

Berger, S.L., Kouzarides, T., Shiekhattar, R., and Shilatifard, A. (2009). An operational definition of epigenetics. Genes \& development 23, 781-783.

Bernstein, B.E., Mikkelsen, T.S., Xie, X., Kamal, M., Huebert, D.J., Cuff, J., Fry, B., Meissner, A., Wernig, M., Plath, K., et al. (2006). A bivalent chromatin structure marks key developmental genes in embryonic stem cells. Cell 125, 315-326.

Blencowe, B.J., Issner, R., Nickerson, J.A., and Sharp, P.A. (1998). A coactivator of pre-mRNA splicing. Genes \& development 12, 996-1009. 
Bluhm, A., Casas-Vila, N., Scheibe, M., and Butter, F. (2016). Reader interactome of epigenetic histone marks in birds. Proteomics 16, 427-436.

Blum H., B.H., Gross h.J. (1987). Improved silver staining of plant proteins, RNA and DNA in polyacrylamide gels. Electrophoresis 8, 93-99.

Bohnsack, M.T., Regener, K., Schwappach, B., Saffrich, R., Paraskeva, E., Hartmann, E., and Gorlich, D. (2002). Exp5 exports eEF1A via tRNA from nuclei and synergizes with other transport pathways to confine translation to the cytoplasm. The EMBO journal 21, 6205-6215.

Bonasio, R., Lecona, E., and Reinberg, D. (2010). MBT domain proteins in development and disease. Semin Cell Dev Biol 21, 221-230.

Bostick, M., Kim, J.K., Esteve, P.O., Clark, A., Pradhan, S., and Jacobsen, S.E. (2007). UHRF1 plays a role in maintaining DNA methylation in mammalian cells. Science 317, 1760-1764.

Brasher, S.V., Smith, B.O., Fogh, R.H., Nietlispach, D., Thiru, A., Nielsen, P.R., Broadhurst, R.W., Ball, L.J., Murzina, N.V., and Laue, E.D. (2000). The structure of mouse HP1 suggests a unique mode of single peptide recognition by the shadow chromo domain dimer. The EMBO journal 19, 1587-1597.

Breiling, A., and Lyko, F. (2015). Epigenetic regulatory functions of DNA modifications: 5methylcytosine and beyond. Epigenetics Chromatin 8, 24.

Bronner, C., Achour, M., Arima, Y., Chataigneau, T., Saya, H., and Schini-Kerth, V.B. (2007). The UHRF family: oncogenes that are drugable targets for cancer therapy in the near future? Pharmacol Ther 115, 419-434.

Chan, D.W., Wang, Y., Wu, M., Wong, J., Qin, J., and Zhao, Y. (2009). Unbiased proteomic screen for binding proteins to modified lysines on histone H3. Proteomics 9, 2343-2354.

Chen, X., Wei, S., Ji, Y., Guo, X., and Yang, F. (2015). Quantitative proteomics using SILAC: Principles, applications, and developments. Proteomics 15, 3175-3192.

Collart, M.A. (2016). The Ccr4-Not complex is a key regulator of eukaryotic gene expression. Wiley interdisciplinary reviews RNA.

Combe, C.W., Fischer, L., and Rappsilber, J. (2015). xiNET: cross-link network maps with residue resolution. Molecular \& cellular proteomics : MCP 14, 1137-1147.

Cowieson, N.P., Partridge, J.F., Allshire, R.C., and McLaughlin, P.J. (2000). Dimerisation of a chromo shadow domain and distinctions from the chromodomain as revealed by structural analysis. Curr Biol 10, 517-525.

Cox, J., and Mann, M. (2008). MaxQuant enables high peptide identification rates, individualized p.p.b.-range mass accuracies and proteome-wide protein quantification. Nature biotechnology 26, 1367-1372.

Cox, J., Neuhauser, N., Michalski, A., Scheltema, R.A., Olsen, J.V., and Mann, M. (2011). Andromeda: a peptide search engine integrated into the MaxQuant environment. Journal of proteome research $10,1794-1805$. 
Cui, X.J., and Shi, C.X. (2016). Combinations of Histone Modifications for Pattern Genes. Acta Biotheor.

Davey, C.A., Sargent, D.F., Luger, K., Maeder, A.W., and Richmond, T.J. (2002). Solvent mediated interactions in the structure of the nucleosome core particle at 1.9 a resolution. Journal of molecular biology 319, 1097-1113.

Demeler, B. UltraScan (current version), A comprehensive software package for the analysis of sedimentation experiments. Dept of Biochemistry, The University of Texas Health Science Center.

Di Palma, S., Hennrich, M.L., Heck, A.J., and Mohammed, S. (2012). Recent advances in peptide separation by multidimensional liquid chromatography for proteome analysis. J Proteomics 75, 3791-3813.

Dignam, J.D., Martin, P.L., Shastry, B.S., and Roeder, R.G. (1983). Eukaryotic gene transcription with purified components. Methods in enzymology $101,582-598$.

Dillon, N., and Festenstein, R. (2002). Unravelling heterochromatin: competition between positive and negative factors regulates accessibility. Trends Genet 18, 252-258.

Dimitrov, A., Paupe, V., Gueudry, C., Sibarita, J.B., Raposo, G., Vielemeyer, O., Gilbert, T., Csaba, Z., Attie-Bitach, T., Cormier-Daire, V., et al. (2009). The gene responsible for DyggveMelchior-Clausen syndrome encodes a novel peripheral membrane protein dynamically associated with the Golgi apparatus. Hum Mol Genet 18, 440-453.

Dorigo, B., Schalch, T., Kulangara, A., Duda, S., Schroeder, R.R., and Richmond, T.J. (2004). Nucleosome arrays reveal the two-start organization of the chromatin fiber. Science 306, 15711573.

Du, J., Johnson, L.M., Jacobsen, S.E., and Patel, D.J. (2015). DNA methylation pathways and their crosstalk with histone methylation. Nat Rev Mol Cell Biol 16, 519-532.

Dyer, P.N., Edayathumangalam, R.S., White, C.L., Bao, Y., Chakravarthy, S., Muthurajan, U.M., and Luger, K. (2004). Reconstitution of nucleosome core particles from recombinant histones and DNA. Methods in enzymology 375, 23-44.

Elgin, S.C., and Grewal, S.I. (2003). Heterochromatin: silence is golden. Curr Biol 13, R895-898.

Engelen, E., Brandsma, J.H., Moen, M.J., Signorile, L., Dekkers, D.H., Demmers, J., Kockx, C.E., Ozgur, Z., van, I.W.F., van den Berg, D.L., et al. (2015). Proteins that bind regulatory regions identified by histone modification chromatin immunoprecipitations and mass spectrometry. Nat Commun 6, 7155.

Fairley, J.A., Scott, P.H., and White, R.J. (2003). TFIIIB is phosphorylated, disrupted and selectively released from tRNA promoters during mitosis in vivo. The EMBO journal 22, 58415850 .

Ferri, E., Petosa, C., and McKenna, C.E. (2016). Bromodomains: Structure, function and pharmacology of inhibition. Biochem Pharmacol 106, 1-18. 
Fischle, W., Wang, Y., and Allis, C.D. (2003a). Binary switches and modification cassettes in histone biology and beyond. Nature 425, 475-479.

Fischle, W., Wang, Y., and Allis, C.D. (2003b). Histone and chromatin cross-talk. Curr Opin Cell Biol 15, 172-183.

Fritzsche, R., Ihling, C.H., Gotze, M., and Sinz, A. (2012). Optimizing the enrichment of crosslinked products for mass spectrometric protein analysis. Rapid Commun Mass Spectrom 26, 653-658.

Fuks, F., Hurd, P.J., Deplus, R., and Kouzarides, T. (2003). The DNA methyltransferases associate with HP1 and the SUV39H1 histone methyltransferase. Nucleic acids research 31, 2305-2312.

Gallagher, S.R. (2006). One-dimensional SDS gel electrophoresis of proteins. Current protocols in molecular biology / edited by Frederick M Ausubel [et al] Chapter 10, Unit $1012 \mathrm{~A}$.

Gayatri, S., and Bedford, M.T. (2014). Readers of histone methylarginine marks. Biochimica et biophysica acta 1839, 702-710.

Geiger, T., Cox, J., Ostasiewicz, P., Wisniewski, J.R., and Mann, M. (2010). Super-SILAC mix for quantitative proteomics of human tumor tissue. Nat Methods 7, 383-385.

Geiger, T., Wisniewski, J.R., Cox, J., Zanivan, S., Kruger, M., Ishihama, Y., and Mann, M. (2011). Use of stable isotope labeling by amino acids in cell culture as a spike-in standard in quantitative proteomics. Nature protocols 6, 147-157.

Glover, J.N. (2006). Insights into the molecular basis of human hereditary breast cancer from studies of the BRCA1 BRCT domain. Fam Cancer 5, 89-93.

Goll, M.G., and Bestor, T.H. (2005). Eukaryotic cytosine methyltransferases. Annual review of biochemistry $74,481-514$.

Gromak, N., Rideau, A., Southby, J., Scadden, A.D., Gooding, C., Huttelmaier, S., Singer, R.H., and Smith, C.W. (2003). The PTB interacting protein raver1 regulates alpha-tropomyosin alternative splicing. The EMBO journal 22, 6356-6364.

Guenther, M.G., Levine, S.S., Boyer, L.A., Jaenisch, R., and Young, R.A. (2007). A chromatin landmark and transcription initiation at most promoters in human cells. Cell 130, 77-88.

Heemskerk, A.A., Deelder, A.M., and Mayboroda, O.A. (2016). CE-ESI-MS for bottom-up proteomics: Advances in separation, interfacing and applications. Mass spectrometry reviews 35, 259-271.

Heitz, E. (1928). Das Heterochromatin der Moose. I Jahrb Wiss Bot 69, 762-819.

Hnilicova, J., Hozeifi, S., Duskova, E., Icha, J., Tomankova, T., and Stanek, D. (2011). Histone deacetylase activity modulates alternative splicing. PloS one 6, e16727.

Hou, Z., Peng, H., Ayyanathan, K., Yan, K.P., Langer, E.M., Longmore, G.D., and Rauscher, F.J., 3rd (2008). The LIM protein AJUBA recruits protein arginine methyltransferase 5 to mediate SNAIL-dependent transcriptional repression. Molecular and cellular biology 28, 3198-3207. 
Hu, S., Wan, J., Su, Y., Song, Q., Zeng, Y., Nguyen, H.N., Shin, J., Cox, E., Rho, H.S., Woodard, C., et al. (2013). DNA methylation presents distinct binding sites for human transcription factors. Elife 2, e00726.

Huang, B.X., Kim, H.Y., and Dass, C. (2004). Probing three-dimensional structure of bovine serum albumin by chemical cross-linking and mass spectrometry. Journal of the American Society for Mass Spectrometry 15, 1237-1247.

Huang da, W., Sherman, B.T., and Lempicki, R.A. (2009a). Bioinformatics enrichment tools: paths toward the comprehensive functional analysis of large gene lists. Nucleic acids research $37,1-13$.

Huang da, W., Sherman, B.T., and Lempicki, R.A. (2009b). Systematic and integrative analysis of large gene lists using DAVID bioinformatics resources. Nature protocols 4, 44-57.

Huynh, V.A., Robinson, P.J., and Rhodes, D. (2005). A method for the in vitro reconstitution of a defined "30 nm" chromatin fibre containing stoichiometric amounts of the linker histone. Journal of molecular biology 345, 957-968.

Illingworth, R.S., and Bird, A.P. (2009). CpG islands--'a rough guide'. FEBS Lett 583, 1713-1720.

Izzo, A., and Schneider, R. (2010). Chatting histone modifications in mammals. Brief Funct Genomics 9, 429-443.

Jacob, Y., Stroud, H., Leblanc, C., Feng, S., Zhuo, L., Caro, E., Hassel, C., Gutierrez, C., Michaels, S.D., and Jacobsen, S.E. (2010). Regulation of heterochromatic DNA replication by histone H3 lysine 27 methyltransferases. Nature 466, 987-991.

Jacobs, S.A., and Khorasanizadeh, S. (2002). Structure of HP1 chromodomain bound to a lysine 9-methylated histone H3 tail. Science 295, 2080-2083.

Jensen, L.J., Kuhn, M., Stark, M., Chaffron, S., Creevey, C., Muller, J., Doerks, T., Julien, P., Roth, A., Simonovic, M., et al. (2009). STRING 8--a global view on proteins and their functional interactions in 630 organisms. Nucleic acids research 37, D412-416.

Jenuwein, T., and Allis, C.D. (2001). Translating the histone code. Science 293, 1074-1080.

Jin, B., Ernst, J., Tiedemann, R.L., Xu, H., Sureshchandra, S., Kellis, M., Dalton, S., Liu, C., Choi, J.H., and Robertson, K.D. (2012). Linking DNA methyltransferases to epigenetic marks and nucleosome structure genome-wide in human tumor cells. Cell Rep 2, 1411-1424.

Jones, P.A. (2012). Functions of DNA methylation: islands, start sites, gene bodies and beyond. Nat Rev Genet 13, 484-492.

Karagianni, P., Amazit, L., Qin, J., and Wong, J. (2008). ICBP90, a novel methyl K9 H3 binding protein linking protein ubiquitination with heterochromatin formation. Molecular and cellular biology 28, 705-717.

Kim, D., Blus, B.J., Chandra, V., Huang, P., Rastinejad, F., and Khorasanizadeh, S. (2010). Corecognition of DNA and a methylated histone tail by the MSL3 chromodomain. Nature structural \& molecular biology 17, 1027-1029. 
Kim, J., Daniel, J., Espejo, A., Lake, A., Krishna, M., Xia, L., Zhang, Y., and Bedford, M.T. (2006). Tudor, MBT and chromo domains gauge the degree of lysine methylation. EMBO Rep 7, 397-403.

Kimura, H. (2013). Histone modifications for human epigenome analysis. J Hum Genet 58, 439445.

Kokura, K., Sun, L., Bedford, M.T., and Fang, J. (2010). Methyl-H3K9-binding protein MPP8 mediates E-cadherin gene silencing and promotes tumour cell motility and invasion. The EMBO journal 29, 3673-3687.

Kornberg, R.D. (1974). Chromatin structure: a repeating unit of histones and DNA. Science 184, 868-871.

Kosinski, J., von Appen, A., Ori, A., Karius, K., Muller, C.W., and Beck, M. (2015). Xlink Analyzer: software for analysis and visualization of cross-linking data in the context of threedimensional structures. Journal of structural biology 189, 177-183.

Kouzarides, T. (2007). Chromatin modifications and their function. Cell 128, 693-705.

Kruithof, M., Chien, F.T., Routh, A., Logie, C., Rhodes, D., and van Noort, J. (2009). Singlemolecule force spectroscopy reveals a highly compliant helical folding for the 30 -nm chromatin fiber. Nature structural \& molecular biology 16, 534-540.

Kudo, N., Khochbin, S., Nishi, K., Kitano, K., Yanagida, M., Yoshida, M., and Horinouchi, S. (1997). Molecular cloning and cell cycle-dependent expression of mammalian CRM1, a protein involved in nuclear export of proteins. The Journal of biological chemistry 272, 29742-29751.

Kunowska, N., Rotival, M., Yu, L., Choudhary, J., and Dillon, N. (2015). Identification of protein complexes that bind to histone $\mathrm{H} 3$ combinatorial modifications using super-SILAC and weighted correlation network analysis. Nucleic acids research 43, 1418-1432.

Kuo, A.J., Song, J., Cheung, P., Ishibe-Murakami, S., Yamazoe, S., Chen, J.K., Patel, D.J., and Gozani, O. (2012). The BAH domain of ORC1 links H4K20me2 to DNA replication licensing and Meier-Gorlin syndrome. Nature 484, 115-119.

Lachner, M., O'Carroll, D., Rea, S., Mechtler, K., and Jenuwein, T. (2001). Methylation of histone H3 lysine 9 creates a binding site for HP1 proteins. Nature 410, 116-120.

Lachner, M., O'Sullivan, R.J., and Jenuwein, T. (2003). An epigenetic road map for histone lysine methylation. Journal of cell science 116, 2117-2124.

Laemmli, U.K. (1970). Cleavage of structural proteins during the assembly of the head of bacteriophage T4. Nature 227, 680-685.

Langelier, M.F., Planck, J.L., Roy, S., and Pascal, J.M. (2012). Structural basis for DNA damage-dependent poly(ADP-ribosyl)ation by human PARP-1. Science 336, 728-732.

Lawrence, M., Daujat, S., and Schneider, R. (2016). Lateral Thinking: How Histone Modifications Regulate Gene Expression. Trends Genet 32, 42-56. 
Le Guezennec, X., Vermeulen, M., Brinkman, A.B., Hoeijmakers, W.A., Cohen, A., Lasonder, E., and Stunnenberg, H.G. (2006). MBD2/NuRD and MBD3/NuRD, two distinct complexes with different biochemical and functional properties. Molecular and cellular biology 26, 843-851.

Lee, J.S., Smith, E., and Shilatifard, A. (2010). The language of histone crosstalk. Cell 142, 682685.

Lehnertz, B., Ueda, Y., Derijck, A.A., Braunschweig, U., Perez-Burgos, L., Kubicek, S., Chen, T., Li, E., Jenuwein, T., and Peters, A.H. (2003). Suv39h-mediated histone H3 lysine 9 methylation directs DNA methylation to major satellite repeats at pericentric heterochromatin. Curr Biol 13, $1192-1200$.

Leitner, A., Reischl, R., Walzthoeni, T., Herzog, F., Bohn, S., Forster, F., and Aebersold, R. (2012). Expanding the chemical cross-linking toolbox by the use of multiple proteases and enrichment by size exclusion chromatography. Molecular \& cellular proteomics : MCP 11, M111 014126.

LeRoy, G., Orphanides, G., Lane, W.S., and Reinberg, D. (1998). Requirement of RSF and FACT for transcription of chromatin templates in vitro. Science 282, 1900-1904.

Li, Z., Dai, H., Martos, S.N., Xu, B., Gao, Y., Li, T., Zhu, G., Schones, D.E., and Wang, Z. (2015). Distinct roles of DNMT1-dependent and DNMT1-independent methylation patterns in the genome of mouse embryonic stem cells. Genome biology 16, 115.

Liao, J., Karnik, R., Gu, H., Ziller, M.J., Clement, K., Tsankov, A.M., Akopian, V., Gifford, C.A., Donaghey, J., Galonska, C., et al. (2015). Targeted disruption of DNMT1, DNMT3A and DNMT3B in human embryonic stem cells. Nature genetics 47, 469-478.

Licklider, L.J., Thoreen, C.C., Peng, J., and Gygi, S.P. (2002). Automation of nanoscale microcapillary liquid chromatography-tandem mass spectrometry with a vented column. Analytical chemistry 74, 3076-3083.

Lis, J.T., and Schleif, R. (1975). Size fractionation of double-stranded DNA by precipitation with polyethylene glycol. Nucleic acids research 2, 383-389.

Litt, M., Qiu, Y., and Huang, S. (2009). Histone arginine methylations: their roles in chromatin dynamics and transcriptional regulation. Biosci Rep 29, 131-141.

Liu, X., Gao, Q., Li, P., Zhao, Q., Zhang, J., Li, J., Koseki, H., and Wong, J. (2013). UHRF1 targets DNMT1 for DNA methylation through cooperative binding of hemi-methylated DNA and methylated H3K9. Nat Commun 4, 1563.

Loyola, A., Tagami, H., Bonaldi, T., Roche, D., Quivy, J.P., Imhof, A., Nakatani, Y., Dent, S.Y., and Almouzni, G. (2009). The HP1alpha-CAF1-SetDB1-containing complex provides H3K9me1 for Suv39-mediated K9me3 in pericentric heterochromatin. EMBO Rep 10, 769-775.

Lu, R., and Wang, G.G. (2013). Tudor: a versatile family of histone methylation 'readers'. Trends Biochem Sci 38, 546-555.

Lu, X., Simon, M.D., Chodaparambil, J.V., Hansen, J.C., Shokat, K.M., and Luger, K. (2008). The effect of H3K79 dimethylation and H4K20 trimethylation on nucleosome and chromatin structure. Nature structural \& molecular biology 15, 1122-1124. 
Luco, R.F., Allo, M., Schor, I.E., Kornblihtt, A.R., and Misteli, T. (2011). Epigenetics in alternative pre-mRNA splicing. Cell 144, 16-26.

Luco, R.F., Pan, Q., Tominaga, K., Blencowe, B.J., Pereira-Smith, O.M., and Misteli, T. (2010). Regulation of alternative splicing by histone modifications. Science 327, 996-1000.

Luger, K., Mader, A.W., Richmond, R.K., Sargent, D.F., and Richmond, T.J. (1997). Crystal structure of the nucleosome core particle at 2.8 A resolution. Nature 389, 251-260.

Luger, K., Rechsteiner, T.J., and Richmond, T.J. (1999). Preparation of nucleosome core particle from recombinant histones. Methods in enzymology 304, 3-19.

Lv, X., Han, Z., Chen, H., Yang, B., Yang, X., Xia, Y., Pan, C., Fu, L., Zhang, S., Han, H., et al. (2016). A positive role for polycomb in transcriptional regulation via H4K20me1. Cell Res.

Makiniemi, M., Hillukkala, T., Tuusa, J., Reini, K., Vaara, M., Huang, D., Pospiech, H., Majuri, I., Westerling, T., Makela, T.P., et al. (2001). BRCT domain-containing protein TopBP1 functions in DNA replication and damage response. The Journal of biological chemistry 276, 30399-30406.

Margueron, R., Trojer, P., and Reinberg, D. (2005). The key to development: interpreting the histone code? Curr Opin Genet Dev 15, 163-176.

Mariappan, M., Li, X., Stefanovic, S., Sharma, A., Mateja, A., Keenan, R.J., and Hegde, R.S. (2010). A ribosome-associating factor chaperones tail-anchored membrane proteins. Nature $466,1120-1124$.

Marton, M.J., Vazquez de Aldana, C.R., Qiu, H., Chakraburtty, K., and Hinnebusch, A.G. (1997). Evidence that GCN1 and GCN20, translational regulators of GCN4, function on elongating ribosomes in activation of elF2alpha kinase GCN2. Molecular and cellular biology 17, 44744489.

Maurer-Stroh, S., Dickens, N.J., Hughes-Davies, L., Kouzarides, T., Eisenhaber, F., and Ponting, C.P. (2003). The Tudor domain 'Royal Family': Tudor, plant Agenet, Chromo, PWWP and MBT domains. Trends Biochem Sci 28, 69-74.

McBryant, S.J., and Hansen, J.C. (2012). Dynamic fuzziness during linker histone action. Adv Exp Med Biol 725, 15-26.

Mikkelsen, T.S., Ku, M., Jaffe, D.B., Issac, B., Lieberman, E., Giannoukos, G., Alvarez, P., Brockman, W., Kim, T.K., Koche, R.P., et al. (2007). Genome-wide maps of chromatin state in pluripotent and lineage-committed cells. Nature 448, 553-560.

Montini, E., Buchner, G., Spalluto, C., Andolfi, G., Caruso, A., den Dunnen, J.T., Trump, D., Rocchi, M., Ballabio, A., and Franco, B. (1999). Identification of SCML2, a second human gene homologous to the Drosophila sex comb on midleg (Scm): A new gene cluster on Xp22. Genomics 58, 65-72.

Morey, L., and Helin, K. (2010). Polycomb group protein-mediated repression of transcription. Trends Biochem Sci 35, 323-332.

Mosch, K., Franz, H., Soeroes, S., Singh, P.B., and Fischle, W. (2011). HP1 recruits activitydependent neuroprotective protein to $\mathrm{H} 3 \mathrm{~K} 9 \mathrm{me} 3$ marked pericentromeric heterochromatin for silencing of major satellite repeats. PloS one 6, e15894. 
Musselman, C.A., and Kutateladze, T.G. (2011). Handpicking epigenetic marks with PHD fingers. Nucleic acids research 39, 9061-9071.

Musselman, C.A., Lalonde, M.E., Cote, J., and Kutateladze, T.G. (2012). Perceiving the epigenetic landscape through histone readers. Nature structural \& molecular biology 19, 12181227.

Mutskov, V., Gerber, D., Angelov, D., Ausio, J., Workman, J., and Dimitrov, S. (1998). Persistent interactions of core histone tails with nucleosomal DNA following acetylation and transcription factor binding. Molecular and cellular biology 18, 6293-6304.

Nady, N., Lemak, A., Walker, J.R., Avvakumov, G.V., Kareta, M.S., Achour, M., Xue, S., Duan, S., Allali-Hassani, A., Zuo, X., et al. (2011). Recognition of multivalent histone states associated with heterochromatin by UHRF1 protein. The Journal of biological chemistry 286, 24300-24311.

Nan, X., Meehan, R.R., and Bird, A. (1993). Dissection of the methyl-CpG binding domain from the chromosomal protein MeCP2. Nucleic acids research 21, 4886-4892.

Nguyen, U.T., Bittova, L., Muller, M.M., Fierz, B., David, Y., Houck-Loomis, B., Feng, V., Dann, G.P., and Muir, T.W. (2014). Accelerated chromatin biochemistry using DNA-barcoded nucleosome libraries. Nat Methods 11, 834-840.

Nikolov, M., Stutzer, A., Mosch, K., Krasauskas, A., Soeroes, S., Stark, H., Urlaub, H., and Fischle, W. (2011). Chromatin affinity purification and quantitative mass spectrometry defining the interactome of histone modification patterns. Molecular \& cellular proteomics : MCP 10, M110 005371.

Nozawa, R.S., Nagao, K., Masuda, H.T., Iwasaki, O., Hirota, T., Nozaki, N., Kimura, H., and Obuse, C. (2010). Human POGZ modulates dissociation of HP1alpha from mitotic chromosome arms through Aurora B activation. Nat Cell Biol 12, 719-727.

Oda, H., Hubner, M.R., Beck, D.B., Vermeulen, M., Hurwitz, J., Spector, D.L., and Reinberg, D. (2010). Regulation of the histone H4 monomethylase PR-Set7 by CRL4(Cdt2)-mediated PCNAdependent degradation during DNA damage. Mol Cell 40, 364-376.

Ohki, I., Shimotake, N., Fujita, N., Jee, J., Ikegami, T., Nakao, M., and Shirakawa, M. (2001). Solution structure of the methyl-CpG binding domain of human MBD1 in complex with methylated DNA. Cell 105, 487-497.

Olins, A.L., and Olins, D.E. (1974). Spheroid chromatin units (v bodies). Science 183, 330-332.

Ong, S.E., Blagoev, B., Kratchmarova, I., Kristensen, D.B., Steen, H., Pandey, A., and Mann, M. (2002). Stable isotope labeling by amino acids in cell culture, SILAC, as a simple and accurate approach to expression proteomics. Molecular \& cellular proteomics : MCP 1, 376-386.

Ong, S.E., and Mann, M. (2005). Mass spectrometry-based proteomics turns quantitative. Nat Chem Biol 1, 252-262.

Owen, D.J., Ornaghi, P., Yang, J.C., Lowe, N., Evans, P.R., Ballario, P., Neuhaus, D., Filetici, P., and Travers, A.A. (2000). The structural basis for the recognition of acetylated histone $\mathrm{H} 4$ by the bromodomain of histone acetyltransferase gcn5p. The EMBO journal 19, 6141-6149. 
Paramelle, D., Miralles, G., Subra, G., and Martinez, J. (2013). Chemical cross-linkers for protein structure studies by mass spectrometry. Proteomics 13, 438-456.

Patel, D.J. (2016). A Structural Perspective on Readout of Epigenetic Histone and DNA Methylation Marks. Cold Spring Harb Perspect Biol 8.

Peng, W., Togawa, C., Zhang, K., and Kurdistani, S.K. (2008). Regulators of cellular levels of histone acetylation in Saccharomyces cerevisiae. Genetics 179, 277-289.

Peters, A.H., Kubicek, S., Mechtler, K., O'Sullivan, R.J., Derijck, A.A., Perez-Burgos, L., Kohlmaier, A., Opravil, S., Tachibana, M., Shinkai, Y., et al. (2003). Partitioning and plasticity of repressive histone methylation states in mammalian chromatin. Mol Cell 12, 1577-1589.

Pettersen, E.F., Goddard, T.D., Huang, C.C., Couch, G.S., Greenblatt, D.M., Meng, E.C., and Ferrin, T.E. (2004). UCSF Chimera--a visualization system for exploratory research and analysis. Journal of computational chemistry 25, 1605-1612.

Plafker, S.M., and Macara, I.G. (2000). Importin-11, a nuclear import receptor for the ubiquitinconjugating enzyme, UbcM2. The EMBO journal 19, 5502-5513.

Prokhortchouk, A., Hendrich, B., Jorgensen, H., Ruzov, A., Wilm, M., Georgiev, G., Bird, A., and Prokhortchouk, E. (2001). The p120 catenin partner Kaiso is a DNA methylation-dependent transcriptional repressor. Genes \& development 15, 1613-1618.

Purcell, S., Neale, B., Todd-Brown, K., Thomas, L., Ferreira, M.A., Bender, D., Maller, J., Sklar, P., de Bakker, P.I., Daly, M.J., et al. (2007). PLINK: a tool set for whole-genome association and population-based linkage analyses. American journal of human genetics 81, 559-575.

R Development-Core-Team. (2011). R: A Language and Enviroment for Statistical Computing. (Vienna, Austria: R Foundation for Statistical Computing).

Ramon-Maiques, S., Kuo, A.J., Carney, D., Matthews, A.G., Oettinger, M.A., Gozani, O., and Yang, W. (2007). The plant homeodomain finger of RAG2 recognizes histone H3 methylated at both lysine-4 and arginine-2. Proceedings of the National Academy of Sciences of the United States of America 104, 18993-18998.

Reddy, J.V., Burguete, A.S., Sridevi, K., Ganley, I.G., Nottingham, R.M., and Pfeffer, S.R. (2006). A functional role for the GCC185 golgin in mannose 6-phosphate receptor recycling. Molecular biology of the cell 17, 4353-4363.

Ringrose, L., and Paro, R. (2004). Epigenetic regulation of cellular memory by the Polycomb and Trithorax group proteins. Annual review of genetics 38, 413-443.

Rinner, O., Seebacher, J., Walzthoeni, T., Mueller, L.N., Beck, M., Schmidt, A., Mueller, M., and Aebersold, R. (2008). Identification of cross-linked peptides from large sequence databases. Nat Methods 5, 315-318.

Rosenfeld, J.A., Wang, Z., Schones, D.E., Zhao, K., DeSalle, R., and Zhang, M.Q. (2009). Determination of enriched histone modifications in non-genic portions of the human genome. BMC Genomics 10, 143.

Rothbart, S.B., and Strahl, B.D. (2014). Interpreting the language of histone and DNA modifications. Biochimica et biophysica acta 1839, 627-643. 
Ruthenburg, A.J., Li, H., Milne, T.A., Dewell, S., McGinty, R.K., Yuen, M., Ueberheide, B., Dou, Y., Muir, T.W., Patel, D.J., et al. (2011). Recognition of a mononucleosomal histone modification pattern by BPTF via multivalent interactions. Cell 145, 692-706.

Saint-Andre, V., Batsche, E., Rachez, C., and Muchardt, C. (2011). Histone H3 lysine 9 trimethylation and HP1gamma favor inclusion of alternative exons. Nature structural \& molecular biology 18, 337-344.

Sambrook, J., Russell, D. (2001). Molecular Cloning: A Laboratory Manual (Cold Spring Harbor Laboratory Press).

Sanchez, R., Meslamani, J., and Zhou, M.M. (2014). The bromodomain: from epigenome reader to druggable target. Biochimica et biophysica acta 1839, 676-685.

Schalch, T., Duda, S., Sargent, D.F., and Richmond, T.J. (2005). X-ray structure of a tetranucleosome and its implications for the chromatin fibre. Nature 436, 138-141.

Schmidt, C. (2010). Absolute and relative quantification of proteins in large protein-RNA assemblies by mass spectrometry. In Bioanalytical Mass Spectrometry Group (Göttingen: Georg August University).

Schotta, G., Lachner, M., Sarma, K., Ebert, A., Sengupta, R., Reuter, G., Reinberg, D., and Jenuwein, T. (2004). A silencing pathway to induce H3-K9 and H4-K20 trimethylation at constitutive heterochromatin. Genes \& development 18, 1251-1262.

Schuettengruber, B., Chourrout, D., Vervoort, M., Leblanc, B., and Cavalli, G. (2007). Genome regulation by polycomb and trithorax proteins. Cell 128, 735-745.

Schwartz, S., Meshorer, E., and Ast, G. (2009). Chromatin organization marks exon-intron structure. Nature structural \& molecular biology 16, 990-995.

Shamay, M., Barak, O., Doitsh, G., Ben-Dor, I., and Shaul, Y. (2002). Hepatitis B virus pX interacts with HBXAP, a PHD finger protein to coactivate transcription. The Journal of biological chemistry $277,9982-9988$.

Shannon, P., Markiel, A., Ozier, O., Baliga, N.S., Wang, J.T., Ramage, D., Amin, N., Schwikowski, B., and Ideker, T. (2003). Cytoscape: a software environment for integrated models of biomolecular interaction networks. Genome research 13, 2498-2504.

Sharif, J., Muto, M., Takebayashi, S., Suetake, I., Iwamatsu, A., Endo, T.A., Shinga, J., MizutaniKoseki, Y., Toyoda, T., Okamura, K., et al. (2007). The SRA protein Np95 mediates epigenetic inheritance by recruiting Dnmt1 to methylated DNA. Nature 450, 908-912.

Sharma, S., Wongpalee, S.P., Vashisht, A., Wohlschlegel, J.A., and Black, D.L. (2014). Stemloop 4 of U1 snRNA is essential for splicing and interacts with the U2 snRNP-specific SF3A1 protein during spliceosome assembly. Genes \& development 28, 2518-2531.

Shevchenko, A., Tomas, H., Havlis, J., Olsen, J.V., and Mann, M. (2006). In-gel digestion for mass spectrometric characterization of proteins and proteomes. Nature protocols 1, 2856-2860.

Shevchenko, A., Wilm, M., Vorm, O., and Mann, M. (1996). Mass spectrometric sequencing of proteins silver-stained polyacrylamide gels. Analytical chemistry 68, 850-858. 
Shogren-Knaak, M., Ishii, H., Sun, J.M., Pazin, M.J., Davie, J.R., and Peterson, C.L. (2006). Histone H4-K16 acetylation controls chromatin structure and protein interactions. Science 311, 844-847.

Shogren-Knaak, M.A., and Peterson, C.L. (2004). Creating designer histones by native chemical ligation. Methods in enzymology 375, 62-76.

Simon, M.D. (2010). Installation of site-specific methylation into histones using methyl lysine analogs. Current protocols in molecular biology / edited by Frederick M Ausubel [et al] Chapter 21, Unit $211821-10$.

Simon, M.D., Chu, F., Racki, L.R., de la Cruz, C.C., Burlingame, A.L., Panning, B., Narlikar, G.J., and Shokat, K.M. (2007). The site-specific installation of methyl-lysine analogs into recombinant histones. Cell 128, 1003-1012.

Sims, J.K., Houston, S.I., Magazinnik, T., and Rice, J.C. (2006). A trans-tail histone code defined by monomethylated H4 Lys-20 and H3 Lys-9 demarcates distinct regions of silent chromatin. The Journal of biological chemistry 281, 12760-12766.

Sinz, A. (2006). Chemical cross-linking and mass spectrometry to map three-dimensional protein structures and protein-protein interactions. Mass spectrometry reviews 25, 663-682.

Spies, N., Nielsen, C.B., Padgett, R.A., and Burge, C.B. (2009). Biased chromatin signatures around polyadenylation sites and exons. Mol Cell 36, 245-254.

Strahl, B.D., and Allis, C.D. (2000). The language of covalent histone modifications. Nature 403, 41-45.

Surka, M.C., Tsang, C.W., and Trimble, W.S. (2002). The mammalian septin MSF localizes with microtubules and is required for completion of cytokinesis. Molecular biology of the cell 13, 3532-3545.

Tilgner, H., Nikolaou, C., Althammer, S., Sammeth, M., Beato, M., Valcarcel, J., and Guigo, R. (2009). Nucleosome positioning as a determinant of exon recognition. Nature structural \& molecular biology 16, 996-1001.

Torres, I.O., and Fujimori, D.G. (2015). Functional coupling between writers, erasers and readers of histone and DNA methylation. Curr Opin Struct Biol 35, 68-75.

Towbin, B.D., Gonzalez-Aguilera, C., Sack, R., Gaidatzis, D., Kalck, V., Meister, P., Askjaer, P., and Gasser, S.M. (2012). Step-wise methylation of histone H3K9 positions heterochromatin at the nuclear periphery. Cell 150, 934-947.

Tran, B.Q., Goodlett, D.R., and Goo, Y.A. (2016). Advances in protein complex analysis by chemical cross-linking coupled with mass spectrometry (CXMS) and bioinformatics. Biochimica et biophysica acta 1864, 123-129.

Tremethick, D.J. (2007). Higher-order structures of chromatin: the elusive $30 \mathrm{~nm}$ fiber. Cell 128, 651-654.

Tsai, W.W., Wang, Z., Yiu, T.T., Akdemir, K.C., Xia, W., Winter, S., Tsai, C.Y., Shi, X., Schwarzer, D., Plunkett, W., et al. (2010). TRIM24 links a non-canonical histone signature to breast cancer. Nature 468, 927-932. 
Ucar, D., Hu, Q., and Tan, K. (2011). Combinatorial chromatin modification patterns in the human genome revealed by subspace clustering. Nucleic acids research 39, 4063-4075.

Unoki, M., Nishidate, T., and Nakamura, Y. (2004). ICBP90, an E2F-1 target, recruits HDAC1 and binds to methyl-CpG through its SRA domain. Oncogene 23, 7601-7610.

Vakoc, C.R., Sachdeva, M.M., Wang, H., and Blobel, G.A. (2006). Profile of histone lysine methylation across transcribed mammalian chromatin. Molecular and cellular biology 26, 91859195.

Van Bortle, K., Ramos, E., Takenaka, N., Yang, J., Wahi, J.E., and Corces, V.G. (2012). Drosophila CTCF tandemly aligns with other insulator proteins at the borders of H3K27me3 domains. Genome research 22, 2176-2187.

van Kruijsbergen, I., Hontelez, S., and Veenstra, G.J. (2015). Recruiting polycomb to chromatin. Int J Biochem Cell Biol 67, 177-187.

Vastenhouw, N.L., and Schier, A.F. (2012). Bivalent histone modifications in early embryogenesis. Curr Opin Cell Biol 24, 374-386.

Vermeulen, M., Eberl, H.C., Matarese, F., Marks, H., Denissov, S., Butter, F., Lee, K.K., Olsen, J.V., Hyman, A.A., Stunnenberg, H.G., et al. (2010). Quantitative interaction proteomics and genome-wide profiling of epigenetic histone marks and their readers. Cell 142, 967-980.

Vezzoli, A., Bonadies, N., Allen, M.D., Freund, S.M., Santiveri, C.M., Kvinlaug, B.T., Huntly, B.J., Gottgens, B., and Bycroft, M. (2010). Molecular basis of histone H3K36me3 recognition by the PWWP domain of Brpf1. Nature structural \& molecular biology 17, 617-619.

Waddington, C.H. (1953). Epigenetics and evolution. Symposia of the Society for Experimental Biology 7, 186-199.

Walzthoeni, T., Claassen, M., Leitner, A., Herzog, F., Bohn, S., Forster, F., Beck, M., and Aebersold, R. (2012). False discovery rate estimation for cross-linked peptides identified by mass spectrometry. Nat Methods 9, 901-903.

Wang, X., Takenaka, K., and Takeda, S. (2010). PTIP promotes DNA double-strand break repair through homologous recombination. Genes Cells 15, 243-254.

Wang, Z., Schones, D.E., and Zhao, K. (2009). Characterization of human epigenomes. Curr Opin Genet Dev 19, 127-134.

Wang, Z., Zang, C., Rosenfeld, J.A., Schones, D.E., Barski, A., Cuddapah, S., Cui, K., Roh, T.Y., Peng, W., Zhang, M.Q., et al. (2008). Combinatorial patterns of histone acetylations and methylations in the human genome. Nature genetics 40, 897-903.

Wendorff, T.J., Schmidt, B.H., Heslop, P., Austin, C.A., and Berger, J.M. (2012). The structure of DNA-bound human topoisomerase II alpha: conformational mechanisms for coordinating intersubunit interactions with DNA cleavage. Journal of molecular biology 424, 109-124.

Woodcock, C.L., Skoultchi, A.I., and Fan, Y. (2006). Role of linker histone in chromatin structure and function: $\mathrm{H} 1$ stoichiometry and nucleosome repeat length. Chromosome Res 14, 17-25. 
Wu, H., Coskun, V., Tao, J., Xie, W., Ge, W., Yoshikawa, K., Li, E., Zhang, Y., and Sun, Y.E. (2010). Dnmt3a-dependent nonpromoter DNA methylation facilitates transcription of neurogenic genes. Science 329, 444-448.

Wysocka, J. (2006). Identifying novel proteins recognizing histone modifications using peptide pull-down assay. Methods 40, 339-343.

Wysocka, J., Swigut, T., Xiao, H., Milne, T.A., Kwon, S.Y., Landry, J., Kauer, M., Tackett, A.J., Chait, B.T., Badenhorst, P., et al. (2006). A PHD finger of NURF couples histone H3 lysine 4 trimethylation with chromatin remodelling. Nature 442, 86-90.

Xi, Q., Wang, Z., Zaromytidou, A.I., Zhang, X.H., Chow-Tsang, L.F., Liu, J.X., Kim, H., Barlas, A., Manova-Todorova, K., Kaartinen, V., et al. (2011). A poised chromatin platform for TGF-beta access to master regulators. Cell 147, 1511-1524.

Xie, H., Surka, M., Howard, J., and Trimble, W.S. (1999). Characterization of the mammalian septin H5: distinct patterns of cytoskeletal and membrane association from other septin proteins. Cell motility and the cytoskeleton $43,52-62$.

Xu, X., Hoang, S., Mayo, M.W., and Bekiranov, S. (2010). Application of machine learning methods to histone methylation ChIP-Seq data reveals H4R3me2 globally represses gene expression. BMC Bioinformatics 11, 396.

Yang, N., and Xu, R.M. (2013). Structure and function of the BAH domain in chromatin biology. Crit Rev Biochem Mol Biol 48, 211-221.

Yap, K.L., and Zhou, M.M. (2010). Keeping it in the family: diverse histone recognition by conserved structural folds. Crit Rev Biochem Mol Biol 45, 488-505.

Yates, J.R., 3rd (2004). Mass spectral analysis in proteomics. Annu Rev Biophys Biomol Struct 33, 297-316.

Yu, Y.Q., Gilar, M., Lee, P.J., Bouvier, E.S., and Gebler, J.C. (2003). Enzyme-friendly, mass spectrometry-compatible surfactant for in-solution enzymatic digestion of proteins. Analytical chemistry 75, 6023-6028.

Zeng, L., Zhang, Q., Li, S., Plotnikov, A.N., Walsh, M.J., and Zhou, M.M. (2010). Mechanism and regulation of acetylated histone binding by the tandem PHD finger of DPF3b. Nature 466, 258262.

Zhao, Q., Rank, G., Tan, Y.T., Li, H., Moritz, R.L., Simpson, R.J., Cerruti, L., Curtis, D.J., Patel, D.J., Allis, C.D., et al. (2009). PRMT5-mediated methylation of histone H4R3 recruits DNMT3A, coupling histone and DNA methylation in gene silencing. Nature structural \& molecular biology 16, 304-311.

Zhou, H.L., Luo, G., Wise, J.A., and Lou, H. (2014). Regulation of alternative splicing by local histone modifications: potential roles for RNA-guided mechanisms. Nucleic acids research 42, 701-713. 


\section{Appendix}

Appendix 1 Proteins significantly recruited or excluded by one of the chromatin modification patterns investigated by chromatin affinity purification. The gene names, the uniprot identifier (Protein IDs) and the normalized heavy to light ratios of the forward (F) and the reverse $(R)$ experiments are shown as reported by MaxQuant for each chromatin modification state.

\begin{tabular}{|c|c|c|c|c|c|c|c|c|c|c|c|c|c|c|c|c|c|c|c|c|c|c|c|c|c|c|c|}
\hline Gene names & Protein IDs & $\begin{array}{l}\text { H3 } \Delta 1- \\
20 \_F\end{array}$ & $\begin{array}{l}H 3 \Delta 1- \\
20 \_R\end{array}$ & $\begin{array}{l}\text { H4K20 } \\
\text { me1_F }\end{array}$ & $\mid \begin{array}{l}\mathrm{H} 4 \mathrm{~K} 20 \\
\mathrm{me1} \text {. } \mathrm{R}\end{array}$ & $\begin{array}{l}\mathrm{H} 4 \mathrm{~K} 20 \\
\mathrm{me3} F \mathrm{~F}\end{array}$ & $\begin{array}{l}\text { H4K } 20 \\
\text { me3_R }\end{array}$ & $\begin{array}{c}\mathrm{H}_{3} \mathrm{~K}_{\mathrm{C}} 27 \\
\mathrm{me} \_\mathrm{F}\end{array}$ & $\begin{array}{l}\mathrm{H} 3 \mathrm{~K}_{\mathrm{C}} 27 \\
\mathrm{me1} \_\mathrm{R}\end{array}$ & $\begin{array}{l}\mathrm{H} 3 \mathrm{~K}_{\mathrm{c}}^{\mathrm{C} 27} \\
\text { me_F }\end{array}$ & $\begin{array}{c}H 3 \mathrm{H}_{\mathrm{C}} 27 \\
\mathrm{me2} 2 \mathrm{R}\end{array}$ & $\begin{array}{l}\mathrm{H} 3 \mathrm{~K}_{\mathrm{C}} 27 \\
\mathrm{me3} \_\mathrm{F}\end{array}$ & $\begin{array}{c}\mathrm{H}_{\mathrm{H}}^{\mathrm{K}} \mathrm{C} 27 \\
\mathrm{me3} \_\mathrm{R}\end{array}$ & $\begin{array}{c}\mathrm{H} 3 \mathrm{~K} 9 \mathrm{~m} \\
\text { e1_F }\end{array}$ & $\mid \begin{array}{c}\text { H3K9m } \\
\text { e1_R }\end{array}$ & $\begin{array}{c}\text { H3К9m } \\
\text { e2_F }\end{array}$ & $\begin{array}{c}\mathrm{H} 3 \mathrm{~K} 9 \mathrm{~m} \\
\mathrm{e} 2 \_\mathrm{R}\end{array}$ & $\begin{array}{l}\text { H3K9m } \\
\text { e3_F }\end{array}$ & $\begin{array}{c}\mathrm{H} 3 \mathrm{~K} 9 \mathrm{~m} \\
\text { e3_R }\end{array}$ & \begin{tabular}{|c|} 
Hзк $3 \mathrm{~m}$ \\
e3|meC \\
pG_F
\end{tabular} & $\begin{array}{c}\text { H3K9m } \\
\text { e3|meC } \\
\text { pG_R }\end{array}$ & $\begin{array}{c}\mathrm{H} 3 \mathrm{~K} 9 \mathrm{~m} \\
\mathrm{e} 3 \mid \mathrm{H} 4 \mathrm{~K} \\
20 \mathrm{me} \\
\mathrm{F}\end{array}$ & $\mid \begin{array}{c}\mathrm{H} 3 \mathrm{~K} 9 \mathrm{~m} \\
\mathrm{e} \mid \mathrm{|H} 4 \mathrm{~K} \\
20 \mathrm{me}{ }_{-} \\
\mathrm{R}\end{array}$ & $\begin{array}{c}\text { meCpg } \\
-\mathrm{F} \\
\end{array}$ & $\begin{array}{c}\mathrm{meCpG} \\
-\mathrm{R} \\
\end{array}$ & \begin{tabular}{|c|} 
H4R3m \\
e2_F \\
\end{tabular} & $\begin{array}{c}\text { H4R3m } \\
\text { e2_R }\end{array}$ \\
\hline AAK1 & D6W5G0 & 0.75 & $\mathrm{NaN}$ & $\mathrm{NaN}$ & $\mathrm{NaN}$ & $\mathrm{NaN}$ & $\mathrm{NaN}$ & $\mathrm{NaN}$ & $\mathrm{NaN}$ & $\mathrm{NaN}$ & $\mathrm{NaN}$ & $\mathrm{NaN}$ & $\mathrm{NaN}$ & $\mathrm{NaN}$ & $\mathrm{NaN}$ & $\begin{array}{ll}1.24 \\
\end{array}$ & 0.27 & $\mathrm{NaN}$ & $\mathrm{NaN}$ & $\mathrm{NaN}$ & \begin{tabular}{|l|}
$\mathrm{NaN}$ \\
\end{tabular} & $\mathrm{NaN}$ & $\mathrm{NaN}$ & $\mathrm{NaN}$ & $\mathrm{NaN}$ & $\mathrm{NaN}$ & $\mathrm{NaN}$ \\
\hline AR2 & 31 & & & & & & 0.4 & 1.27 & & 0.5 & 0.86 & & & 0.89 & & 1.03 & 0.23 & & 1.87 & $\mathrm{NaN}$ & $\mathrm{NaN}$ & 0.77 & 1.31 & $\mathrm{NaN}$ & $\mathrm{NaN}$ & 1.26 & 0.57 \\
\hline RSS & PK7 & & & $\mathrm{NaN}$ & $\mathrm{NaN}$ & & $\mathrm{NaN}$ & $\mathrm{NaN}$ & $\mathrm{NaN}$ & $\mathrm{NaN}$ & $\mathrm{NaN}$ & $\mathrm{NaN}$ & $\mathrm{NaN}$ & $\mathrm{NaN}$ & $\mathrm{NaN}$ & $\mathrm{NaN}$ & 0.24 & $\mathrm{NaN}$ & $\mathrm{NaN}$ & $\mathrm{NaN}$ & $\mathrm{NaN}$ & $\mathrm{NaN}$ & $\mathrm{NaN}$ & $\mathrm{NaN}$ & $\mathrm{NaN}$ & $\mathrm{NaN}$ & $\mathrm{NaN}$ \\
\hline BCF1 & PPK7 & & 2.13 & 0.50 & 0.85 & & 0.28 & 0.89 & $\mathrm{NaN}$ & 0.91 & 0.49 & 1.60 & 0.26 & 0.42 & 0.57 & 1.18 & 1.40 & 1.78 & 0.25 & 5.02 & 0.19 & 0.74 & 0.63 & 0.92 & 1.63 & 0.67 & 0.41 \\
\hline CACA & 2790 & & & & 2.31 & & & 0.45 & 73 & 1.05 & 0.41 & 0.30 & 1.37 & 1.22 & .43 & 1.02 & 2.03 & .57 & 0.59 & 0.44 & 1.27 & 0.11 & 2.62 & 0.74 & 0.56 & 0.44 & 0.72 \\
\hline ACACB & 0763 & $\mathrm{Na}$ & & & $\mathrm{Na}$ & & & $\mathrm{Na}$ & & 0.1 & 0.8 & $\mathrm{Na}$ & $\mathrm{Nal}$ & $\mathrm{NaI}$ & $\mathrm{NaN}$ & $\mathrm{Na}$ & $\mathrm{Na}$ & 0.5 & & 0.6 & 1.24 & $\mathrm{aN}$ & & 0.68 & 0.82 & 0.31 & 0.46 \\
\hline ACIN1 & JKVZ-5 & NaN & $\mathrm{NaN}$ & $\mathrm{NaN}$ & $\mathrm{NaN}$ & $\mathrm{NaN}$ & $\mathrm{NaN}$ & $\mathrm{NaN}$ & $\mathrm{NaN}$ & $\mathrm{NaN}$ & $\mathrm{NaN}$ & $\mathrm{NaN}$ & $\mathrm{NaN}$ & $\mathrm{NaN}$ & $\mathrm{NaN}$ & $\mathrm{NaN}$ & $\mathrm{NaN}$ & $\mathrm{NaN}$ & $\mathrm{NaN}$ & $\mathrm{NaN}$ & $\mathrm{NaN}$ & $\mathrm{NaN}$ & $\mathrm{NaN}$ & $\mathrm{NaN}$ & $\mathrm{NaN}$ & $\mathrm{NaN}$ & $\mathrm{NaN}$ \\
\hline ACIN1 & EQT4 & 7.62 & 1.91 & 0.50 & 1.46 & 1.04 & 0.67 & 0.73 & 0.94 & 2.27 & 0.33 & 1.12 & 0.93 & 1.92 & 0.57 & 2.49 & 1.55 & 1.08 & 0.53 & 1.74 & 3.87 & 0.54 & 1.45 & 1.96 & 1.84 & 0.36 & 0.44 \\
\hline 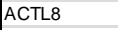 & & & & & & & & 104 & $\mathrm{aN}$ & & & 1.60 & & 2.30 & 0.49 & & 0.1 & & & 29.52 & 0.02 & & & 18.41 & & 0.54 & 3.09 \\
\hline & & & & & & & & & & & & & & & & & & & & & & & & & & & $\mathrm{NaN}$ \\
\hline CTR5 & & & & 2.21 & 0.49 & & 1.51 & 1.14 & & 0.6 & 2.29 & 1.35 & 1.04 & 0.25 & 3.79 & 0.20 & 1.28 & 0.50 & 2.40 & & 0.21 & 2.89 & 0.52 & 1.10 & 1.62 & $\mathrm{NaN}$ & $\mathrm{NaN}$ \\
\hline ACTR8 & & & & 2.0 & 0.58 & & 1.42 & 1.37 & $\mathrm{~N}$ & 0.70 & 2.28 & 3 & 1.21 & 0.27 & 4.17 & 21 & 1.49 & 0.51 & 2.37 & 12.93 & 0.20 & 2.77 & 0.53 & 1.05 & 1.42 & 3.04 & 0.47 \\
\hline & BM8 & & & & 0.79 & & 1.0 & 73 & 73 & 0.7 & 0.92 & 0.94 & 0.76 & 1.03 & 1.18 & 0.88 & 0.23 & 2.82 & 0.22 & 55.25 & 0.01 & 3.69 & 0.16 & 0.99 & 1.55 & 1.61 & 0.18 \\
\hline & & & & & & & & & & & & & & & & & & & & & & & & & & & $\mathrm{aN}$ \\
\hline BP2 & & & & 0.5 & $\mathrm{Na}$ & & 1.1 & $\mathrm{NaN}$ & $N$ & 0.5 & $\mathrm{aN}$ & 0.30 & $\mathrm{NaN}$ & $\mathrm{NaN}$ & $\mathrm{aN}$ & & 0.2 & 1.73 & 0.49 & & 0.84 & 3.03 & 0.34 & $\mathrm{NaN}$ & $\mathrm{NaN}$ & $\mathrm{aN}$ & 1.21 \\
\hline & & & & $\mathrm{Nal}$ & $\mathrm{NaN}$ & & $\mathrm{Na}$ & $\mathrm{NaN}$ & & $\mathrm{NaN}$ & $\mathrm{NaN}$ & $\mathrm{N}$ & $\mathrm{N}$ & $\mathrm{NaN}$ & $\mathrm{NaN}$ & 39 & $\mathrm{NaN}$ & 0.03 & 1.01 & 3 & 1.76 & 06 & 1.16 & 0.99 & 1.01 & $\mathrm{NaN}$ & $\mathrm{NaN}$ \\
\hline $18 \mathrm{~A}$ & & & & & & & & & & & & & & & 0.27 & & & $\mathrm{NaN}$ & $\mathrm{Na}$ & & $\mathrm{NaN}$ & & & & $\mathrm{NaN}$ & $\mathrm{NaN}$ & $\mathrm{NaN}$ \\
\hline & & & & & & & & & & & & & & & & & & & & & & & & & & & $\mathrm{aN}$ \\
\hline & & & & & & & & & & & & & & & & & & & & & & & & & & & $\mathrm{aN}$ \\
\hline AMN1 & & & & $\mathrm{Nal}$ & $\mathrm{NaN}$ & $\mathrm{NaN}$ & $\mathrm{NaN}$ & $\mathrm{NaN}$ & $\mathrm{NaN}$ & $\mathrm{NaN}$ & $\mathrm{NaN}$ & $\mathrm{NaN}$ & & $\mathrm{NaN}$ & 0.37 & 0.48 & 0.15 & $\mathrm{NaI}$ & $\mathrm{NaN}$ & $\mathrm{NaN}$ & $\mathrm{NaN}$ & $\mathrm{NaN}$ & $\mathrm{NaN}$ & $\mathrm{NaN}$ & $\mathrm{NaN}$ & $\mathrm{NaN}$ & $\mathrm{NaN}$ \\
\hline & & & & & & & & & & & & & & & $\mathrm{NaN}$ & & & & & & & & & & & & $\mathrm{NaN}$ \\
\hline & & & & & & & & & & & & & & & & & & & & & & & & & & & $\mathrm{aN}$ \\
\hline & & & & & & & & & & & & & & & & & & & & & & & & & & & $\mathrm{N}$ \\
\hline & & & & & & & & & & & & & & 1.0 & & & & & & & & & & & & 55 & 39 \\
\hline & & & & & & & & & & & & & & & & & & & & & & & & & & & $\mathrm{N}$ \\
\hline & & & & & & & & & & & & & & & & & & $\mathrm{NaN}$ & $\mathrm{NaN}$ & & $\mathrm{NaN}$ & & & 1.10 & $\mathrm{NaN}$ & $\mathrm{NaN}$ & $\mathrm{aN}$ \\
\hline & & & & & & & & & & & & & & & & & & & & & & & & & & & 70 \\
\hline & & & & & & & & & & & & & & & & & & & & & & & & & & & 73 \\
\hline & & & & & & & & & & & & & & & & & & & & & & & & & & & 22 \\
\hline & & & & & & & & & & & & & & & & & & $\mathrm{Na}$ & & & $\mathrm{NaN}$ & & & $\mathrm{NaN}$ & $\mathrm{NaN}$ & $\mathrm{NaN}$ & 95 \\
\hline HGE & & 0.5 & $\mathrm{Na}$ & $\mathrm{Na}$ & & & $\mathrm{Na}$ & $\mathrm{Na}$ & $\mathrm{NaN}$ & $\mathrm{Na}$ & $\mathrm{Na}$ & $\mathrm{NaN}$ & & $\mathrm{Na}$ & & 0.44 & 0.10 & $\mathrm{NaN}$ & 1.26 & & $\mathrm{NaN}$ & & 61 & $\mathrm{NaN}$ & $\mathrm{NaN}$ & $\mathrm{NaN}$ & $\mathrm{aN}$ \\
\hline & & & & & & & & & & & & & & & & & & & & 20 & & & & & & & \\
\hline & & & 0.8 & & & & 1. & & & 1.0 & & & 1.0 & 0.8 & & & 1.5 & & 1. & 1.5 & 0.4 & 1. & & 0.44 & 1.77 & 1.23 & 54 \\
\hline$P($ & & & $\mathrm{Na}$ & $\mathrm{NaN}$ & $\mathrm{NaN}$ & $\mathrm{Na}$ & $\mathrm{NaN}$ & $\mathrm{NaN}$ & $\mathrm{NaN}$ & $\mathrm{Nal}$ & $\mathrm{Nal}$ & $\mathrm{NaN}$ & $\mathrm{NaN}$ & 0.9 & $\mathrm{NaN}$ & $\mathrm{NaN}$ & $\mathrm{NaN}$ & $\mathrm{Nal}$ & $\mathrm{NaN}$ & $\mathrm{Na}$ & $\mathrm{NaN}$ & $\mathrm{Nal}$ & $\mathrm{Na}$ & $\mathrm{NaN}$ & $\mathrm{NaN}$ & $\mathrm{NaN}$ & $\mathrm{NaN}$ \\
\hline $\mathrm{PPC} 4$ & 9998 & 0.49 & 1.82 & $\mathrm{Nal}$ & $\mathrm{NaN}$ & & $\mathrm{Na}$ & $\mathrm{NaN}$ & $\mathrm{NaN}$ & $\mathrm{NaN}$ & $\mathrm{NaN}$ & $\mathrm{NaN}$ & $\mathrm{NaN}$ & 1.71 & 0.56 & 0.49 & 0.36 & $\mathrm{NaN}$ & $\mathrm{NaN}$ & 0.69 & 3.52 & $\mathrm{NaN}$ & $\mathrm{NaN}$ & 2.30 & 2.61 & $\mathrm{NaN}$ & $\mathrm{IaN}$ \\
\hline & & & & & & & & & & & & & & & & & & & & & & & & & & & \\
\hline & & $\mathrm{Na}$ & 0.2 & $\mathrm{NaN}$ & $\mathrm{NaN}$ & & 1.3 & $\mathrm{NaN}$ & & $\mathrm{Na}$ & $\mathrm{Na}$ & & $\mathrm{Na}$ & $\mathrm{Na}$ & & 0.7 & 0.3 & 1.0 & $\mathrm{NaN}$ & & $\mathrm{Na}$ & $\mathrm{Nal}$ & $\mathrm{Na}$ & 0.93 & 0.55 & $\mathrm{NaN}$ & \\
\hline ATF2 & 77U9 & $\mathrm{NaN}$ & $\mathrm{NaN}$ & $\mathrm{NaN}$ & $\mathrm{NaN}$ & $\mathrm{NaN}$ & $\mathrm{NaN}$ & $\mathrm{NaN}$ & $\mathrm{NaN}$ & $\mathrm{NaN}$ & $\mathrm{NaN}$ & $\mathrm{NaN}$ & $\mathrm{NaN}$ & $\mathrm{NaN}$ & $\mathrm{NaN}$ & $\mathrm{NaN}$ & $\mathrm{NaN}$ & $\mathrm{NaN}$ & $\mathrm{NaN}$ & $\mathrm{NaN}$ & $\mathrm{NaN}$ & $\mathrm{NaN}$ & $\mathrm{NaN}$ & $\mathrm{NaN}$ & 0.99 & $\mathrm{NaN}$ & $\mathrm{VaN}$ \\
\hline ATF2 & F5H629 & $\mathrm{NaN}$ & $\mathrm{NaN}$ & $\mathrm{NaN}$ & $\mathrm{NaN}$ & $\mathrm{NaN}$ & $\mathrm{NaN}$ & $\mathrm{NaN}$ & 0.87 & $\mathrm{NaN}$ & $\mathrm{NaN}$ & $\mathrm{NaN}$ & $\mathrm{NaN}$ & $\mathrm{NaN}$ & $\mathrm{NaN}$ & $\mathrm{NaN}$ & $\mathrm{NaN}$ & $\mathrm{NaN}$ & $\mathrm{NaN}$ & $\mathrm{NaN}$ & $\mathrm{NaN}$ & $\mathrm{NaN}$ & $\mathrm{NaN}$ & $\mathrm{NaN}$ & $\mathrm{NaN}$ & $\mathrm{NaN}$ & $\mathrm{NaN}$ \\
\hline
\end{tabular}




\begin{tabular}{|c|c|c|c|c|c|c|c|c|c|c|c|c|c|c|c|c|c|c|c|c|c|c|c|c|c|c|c|}
\hline Gene names & Protein IDs & $\begin{array}{l}H 3 \Delta 1- \\
20 \_F\end{array}$ & $\begin{array}{l}H 3 \Delta 1- \\
20 \_R\end{array}$ & $\begin{array}{l}\text { H4K20 } \\
\text { me1_F }\end{array}$ & $\begin{array}{l}\text { H4K20 } \\
\text { me1_R }\end{array}$ & $\begin{array}{l}\text { H4K20 } \\
\text { me3_F }\end{array}$ & $\begin{array}{l}\text { H4K20 } \\
\text { me3_R }\end{array}$ & $\begin{array}{r}\mathrm{H} 3 \mathrm{~K}_{\mathrm{C}} 27 \\
\text { e1_F }\end{array}$ & $\begin{array}{l}\mathrm{H} 3 \mathrm{~K}_{\mathrm{C}} 27 \\
\mathrm{me1} \mathrm{R}\end{array}$ & $\begin{array}{l}\mathrm{H} 3 \mathrm{~K}_{\mathrm{C}} 27 \\
\mathrm{me} 2 \mathrm{~F}\end{array}$ & $\begin{array}{l}\mathrm{H} 3 K_{\mathrm{c}} 27 \\
\mathrm{me2} \_\mathrm{R}\end{array}$ & $\begin{array}{l}\mathrm{H} 3 \mathrm{~K}_{\mathrm{c}} 27 \\
\mathrm{me3} 3 \mathrm{~F}\end{array}$ & $\begin{array}{l}\mathrm{H} 3 \mathrm{~K}_{\mathrm{C}} 27 \\
\mathrm{me3} \text {.R }\end{array}$ & $\begin{array}{c}\text { НзК9m } \\
\text { e1_F }\end{array}$ & $\begin{array}{c}\text { HзК9m } \\
\text { e1_R }\end{array}$ & $\begin{array}{c}\text { Нзкаm } \\
\text { e2_F }\end{array}$ & $\begin{array}{c}\text { H3K9m } \\
\text { e2_R }\end{array}$ & $\begin{array}{c}\text { HзK9m } \\
\text { e3_F }\end{array}$ & $\begin{array}{c}\text { НзК9m } \\
\text { e3_R }\end{array}$ & $\begin{array}{c}\text { HзK9m } \\
\text { e3|meC } \\
\text { pG_F }\end{array}$ & $\begin{array}{c}\text { H3к9m } \\
\text { e3|meC } \\
\text { pG_R }\end{array}$ & $\mid \begin{array}{c}\mathrm{H} 3 \mathrm{~K} 9 \mathrm{~m} \\
\mathrm{e} 3 \mathrm{H} 4 \mathrm{~K} \\
20 \mathrm{me} 3 \\
\mathrm{~F}\end{array}$ & $\begin{array}{c}\mathrm{H} 3 \mathrm{~K} 9 \mathrm{~m} \\
\mathrm{e} 3 \mid \mathrm{H} 4 \mathrm{~K} \\
20 \mathrm{me3} \\
\mathrm{R}\end{array}$ & $\underset{\mathrm{F}}{\mathrm{meCpG}}$ & $\underset{-R}{\operatorname{meCpG}}$ & $\begin{array}{c}\text { H4R3m } \\
\text { e2_F }\end{array}$ & $\begin{array}{c}\text { H4R3n } \\
\text { e2_R }\end{array}$ \\
\hline \begin{tabular}{|l|} 
ATF2 \\
\end{tabular} & P15336-5 & 0.28 & 0.37 & $\mathrm{NaN}$ & $\mathrm{NaN}$ & 0.49 & 0.94 & 0.63 & 0.59 & 0.56 & 1.10 & 0.71 & 0.64 & $\mathrm{NaN}$ & $\mathrm{NaN}$ & 0.06 & $\mathrm{NaN}$ & 0.39 & 1.13 & 3.35 & 0.21 & 1.08 & 0.37 & 0.83 & 1.02 & $\mathrm{NaN}$ & $\mathrm{NaN}$ \\
\hline ATF2 & P 15336-8 & $\mathrm{NaN}$ & $\mathrm{NaN}$ & $\mathrm{NaN}$ & $\mathrm{NaN}$ & 0.46 & $\mathrm{NaN}$ & $\mathrm{NaN}$ & $\mathrm{NaN}$ & $\mathrm{NaN}$ & $\mathrm{NaN}$ & $\mathrm{NaN}$ & $\mathrm{NaN}$ & $\mathrm{NaN}$ & $\mathrm{NaN}$ & $\mathrm{NaN}$ & $\mathrm{NaN}$ & $\mathrm{NaN}$ & $\mathrm{NaN}$ & $\mathrm{NaN}$ & $\mathrm{NaN}$ & $\mathrm{NaN}$ & $\mathrm{NaN}$ & $\mathrm{NaN}$ & $\mathrm{NaN}$ & $\mathrm{NaN}$ & $\mathrm{NaN}$ \\
\hline ATL3 & Q6DD88 & $\mathrm{NaN}$ & 0.24 & 0.60 & $\mathrm{NaN}$ & $\mathrm{NaN}$ & 0.29 & $\mathrm{NaN}$ & $\mathrm{NaN}$ & 0.81 & $\mathrm{NaN}$ & $\mathrm{NaN}$ & $\mathrm{NaN}$ & $\mathrm{NaN}$ & $\mathrm{NaN}$ & $\mathrm{NaN}$ & 2.15 & 2.21 & $\mathrm{NaN}$ & 0.94 & $\mathrm{NaN}$ & $\mathrm{NaN}$ & 1.05 & $\mathrm{NaN}$ & $\mathrm{NaN}$ & $\mathrm{NaN}$ & $\mathrm{NaN}$ \\
\hline ATP6VOD1 & B2R7M1 & 1.15 & 1.51 & $\mathrm{NaN}$ & $\mathrm{NaN}$ & $\mathrm{NaN}$ & 1.02 & $\mathrm{NaN}$ & $\mathrm{NaN}$ & $\mathrm{NaN}$ & $\mathrm{NaN}$ & $\mathrm{NaN}$ & $\mathrm{NaN}$ & 1.04 & 0.99 & 0.78 & 0.18 & $\mathrm{NaN}$ & $\mathrm{NaN}$ & $\mathrm{NaN}$ & $\mathrm{NaN}$ & $\mathrm{NaN}$ & $\mathrm{NaN}$ & 0.13 & $\mathrm{NaN}$ & $\mathrm{NaN}$ & $\mathrm{NaN}$ \\
\hline ATP6VIB2 & P21281 & 0.97 & 0.80 & $\mathrm{NaN}$ & $\mathrm{NaN}$ & $\mathrm{NaN}$ & $\mathrm{NaN}$ & $\mathrm{NaN}$ & $\mathrm{NaN}$ & $\mathrm{NaN}$ & $\mathrm{NaN}$ & $\mathrm{NaN}$ & $\mathrm{NaN}$ & 0.82 & 0.62 & 0.78 & 0.38 & $\mathrm{NaN}$ & $\mathrm{NaN}$ & NaN & $\mathrm{NaN}$ & $\mathrm{NaN}$ & $\mathrm{NaN}$ & 0.12 & $\mathrm{NaN}$ & $\mathrm{NaN}$ & $\mathrm{NaN}$ \\
\hline ATP6VIF & A4D1K0 & $\mathrm{NaN}$ & $\mathrm{NaN}$ & $\mathrm{NaN}$ & $\mathrm{NaN}$ & $\mathrm{NaN}$ & $\mathrm{NaN}$ & $\mathrm{NaN}$ & $\mathrm{NaN}$ & $\mathrm{NaN}$ & $\mathrm{NaN}$ & $\mathrm{NaN}$ & $\mathrm{NaN}$ & $\mathrm{NaN}$ & $\mathrm{NaN}$ & $\mathrm{NaN}$ & $\mathrm{NaN}$ & $\mathrm{NaN}$ & $\mathrm{NaN}$ & $\mathrm{NaN}$ & $\mathrm{NaN}$ & $\mathrm{NaN}$ & $\mathrm{NaN}$ & 0.21 & $\mathrm{NaN}$ & $\mathrm{NaN}$ & $\mathrm{NaN}$ \\
\hline ATRIP & Q8WXE1-2 & 0.85 & 0.81 & 0.32 & $\mathrm{NaN}$ & 0.70 & 0.66 & $\mathrm{NaN}$ & $\mathrm{NaN}$ & 0.84 & $\mathrm{NaN}$ & 0.96 & $\mathrm{NaN}$ & 0.84 & 0.45 & 0.62 & 0.36 & 0.81 & 0.79 & NaN & 0.38 & 0.46 & 0.61 & $\mathrm{NaN}$ & $\mathrm{NaN}$ & 1.67 & $\mathrm{NaN}$ \\
\hline ATRX & A4LAA3 & 0.65 & 0.48 & 0.33 & 0.55 & 0.39 & 0.40 & 0.34 & 0.49 & 0.48 & 0.50 & 0.44 & 0.55 & 0.51 & 1.07 & 0.67 & 0.35 & 1.41 & 0.20 & 1.25 & 0.13 & 0.85 & 0.25 & 0.50 & 0.32 & 0.45 & 0.39 \\
\hline BABAM1 & MORO10 & 0.84 & 0.40 & $\mathrm{NaN}$ & 3.17 & $\mathrm{NaN}$ & $\mathrm{NaN}$ & $\mathrm{NaN}$ & $\mathrm{NaN}$ & 1.34 & 0.55 & $\mathrm{NaN}$ & $\mathrm{NaN}$ & $\mathrm{NaN}$ & 0.89 & $\mathrm{NaN}$ & 0.25 & $\mathrm{NaN}$ & $\mathrm{NaN}$ & 0.63 & 4.44 & 0.23 & 2.81 & $\mathrm{NaN}$ & $\mathrm{NaN}$ & $\mathrm{NaN}$ & $\mathrm{NaN}$ \\
\hline BACH1 & Q6ICU0 & $\mathrm{NaN}$ & $\mathrm{NaN}$ & $\mathrm{NaN}$ & $\mathrm{NaN}$ & $\mathrm{NaN}$ & $\mathrm{NaN}$ & $\mathrm{NaN}$ & $\mathrm{NaN}$ & $\mathrm{NaN}$ & $\mathrm{NaN}$ & $\mathrm{NaN}$ & $\mathrm{NaN}$ & $\mathrm{NaN}$ & $\mathrm{NaN}$ & $\mathrm{NaN}$ & $\mathrm{NaN}$ & $\mathrm{NaN}$ & $\mathrm{NaN}$ & 3.74 & 0.17 & 0.94 & $\mathrm{NaN}$ & 3.68 & 0.37 & $\mathrm{NaN}$ & $\mathrm{NaN}$ \\
\hline BAG6 & BOUX83 & 1.79 & 0.50 & 0.61 & 1.03 & 0.99 & 0.31 & 1.27 & 0.32 & 0.84 & 0.72 & 0.72 & 0.40 & 2.43 & 0.95 & 1.53 & 0.21 & 1.34 & 0.52 & $\mathrm{NaN}$ & $\mathrm{NaN}$ & 0.93 & 1.07 & $\mathrm{NaN}$ & $\mathrm{NaN}$ & 1.52 & 0.40 \\
\hline BARD1 & F6MDH7 & 0.64 & 0.37 & 0.11 & 4.55 & 0.23 & 2.99 & 0.75 & 0.75 & 1.02 & 0.67 & 0.97 & 0.77 & 0.60 & 1.09 & 0.59 & 1.17 & 0.74 & 0.84 & 0.63 & 1.22 & 0.19 & 2.47 & 1.04 & 0.64 & 0.64 & 0.92 \\
\hline BAZ1B & Q9UIG0-2 & 0.07 & 5.41 & 1.21 & 0.93 & 1.31 & 1.00 & 0.98 & 0.86 & 0.93 & 1.19 & 1.04 & 1.10 & 1.08 & 1.38 & 0.67 & 1.02 & 1.10 & 0.96 & 1.60 & 2.27 & 1.00 & 1.07 & 1.39 & 1.81 & 0.56 & 1.38 \\
\hline BAZ2A & J30K86 & $\mathrm{NaN}$ & $\mathrm{NaN}$ & 0.54 & 0.77 & 0.34 & 1.11 & $\mathrm{NaN}$ & $\mathrm{NaN}$ & 0.72 & 0.48 & 0.85 & 0.58 & 0.50 & $\mathrm{NaN}$ & $\mathrm{NaN}$ & NaN & 0.34 & 1.83 & $\mathrm{NaN}$ & $\mathrm{NaN}$ & 0.60 & 0.94 & $\mathrm{NaN}$ & $\mathrm{NaN}$ & 0.83 & $\mathrm{NaN}$ \\
\hline BCLAF1 & E9PK91 & 0.23 & 0.24 & $\mathrm{NaN}$ & $\mathrm{NaN}$ & $\mathrm{NaN}$ & $\mathrm{NaN}$ & 0.81 & $\mathrm{NaN}$ & $\mathrm{NaN}$ & $\mathrm{NaN}$ & $\mathrm{NaN}$ & $\mathrm{NaN}$ & $\mathrm{NaN}$ & $\mathrm{NaN}$ & 2.21 & $\mathrm{NaN}$ & $\mathrm{NaN}$ & $\mathrm{NaN}$ & $\mathrm{NaN}$ & $\mathrm{NaN}$ & $\mathrm{NaN}$ & $\mathrm{NaN}$ & $\mathrm{NaN}$ & 1.41 & $\mathrm{NaN}$ & $\mathrm{NaN}$ \\
\hline BCLAF1 & Q9NYF8-2 & 9.47 & 0.39 & 3.98 & 3.10 & 0.69 & 0.83 & 0.80 & 1.37 & 0.85 & 0.66 & 4.28 & 2.20 & $\mathrm{NaN}$ & $\mathrm{NaN}$ & 0.98 & 0.78 & 0.89 & 0.97 & 1.13 & 1.31 & 0.58 & 0.72 & 1.38 & 1.51 & 0.68 & 0.79 \\
\hline BCR/ABLf. & A9UEZ6 & 0.91 & $\mathrm{NaN}$ & NaN & $\mathrm{NaN}$ & 4.19 & $\mathrm{NaN}$ & $\mathrm{NaN}$ & $\mathrm{NaN}$ & NaN & $\mathrm{NaN}$ & $\mathrm{NaN}$ & $\mathrm{NaN}$ & $\mathrm{NaN}$ & $\mathrm{NaN}$ & $\mathrm{NaN}$ & $\mathrm{NaN}$ & $\mathrm{NaN}$ & $\mathrm{NaN}$ & $\mathrm{NaN}$ & $\mathrm{NaN}$ & $\mathrm{NaN}$ & $\mathrm{NaN}$ & $\begin{array}{l}1.00 \\
1.11\end{array}$ & $\mathrm{NaN}$ & $\mathrm{NaN}$ & $\mathrm{NaN}$ \\
\hline BCR/ABLf. & P11274-2 & 1.12 & 0.65 & $\mathrm{NaN}$ & $\mathrm{NaN}$ & $\mathrm{NaN}$ & $\mathrm{NaN}$ & $\mathrm{NaN}$ & $\mathrm{NaN}$ & $\mathrm{NaN}$ & $\mathrm{NaN}$ & $\mathrm{NaN}$ & $\mathrm{NaN}$ & $\mathrm{NaN}$ & $\mathrm{NaN}$ & 0.92 & 0.13 & $\mathrm{NaN}$ & $\mathrm{NaN}$ & $\mathrm{NaN}$ & $\mathrm{NaN}$ & $\mathrm{NaN}$ & $\mathrm{NaN}$ & $\mathrm{NaN}$ & $\mathrm{NaN}$ & $\mathrm{NaN}$ & $\mathrm{NaN}$ \\
\hline BHLHB2 & Q6IB83 & 0.62 & 0.59 & 0.56 & 0.36 & 0.46 & 0.55 & 0.46 & 0.36 & 0.45 & 0.50 & 0.43 & 0.47 & $\mathrm{NaN}$ & $\mathrm{NaN}$ & 0.68 & 1.19 & 0.39 & 0.45 & 1.78 & 0.26 & 0.40 & 0.51 & 0.32 & 1.34 & 0.68 & 0.30 \\
\hline BLMH & Q13867 & $\mathrm{NaN}$ & $\mathrm{NaN}$ & & $\mathrm{NaN}$ & 0.47 & & 0.17 & $\mathrm{NaN}$ & 40.10 & $\mathrm{NaN}$ & 0.73 & $\mathrm{NaN}$ & $\mathrm{NaN}$ & $\mathrm{NaN}$ & $\mathrm{NaN}$ & 11.03 & 5.54 & $\mathrm{NaN}$ & 0.45 & & $\mathrm{NaN}$ & $\mathrm{NaN}$ & 4.60 & $\mathrm{NaN}$ & $\mathrm{NaN}$ & $\mathrm{NaN}$ \\
\hline BPTF & E9PE19 & $\mathrm{NaN}$ & $\mathrm{NaN}$ & $\mathrm{NaN}$ & $\mathrm{NaN}$ & $\mathrm{NaN}$ & 0.74 & $\mathrm{NaN}$ & $\mathrm{NaN}$ & $\mathrm{NaN}$ & $\mathrm{NaN}$ & $\mathrm{NaN}$ & $\mathrm{NaN}$ & $\mathrm{NaN}$ & $\mathrm{NaN}$ & $\mathrm{NaN}$ & $\mathrm{NaN}$ & $\mathrm{NaN}$ & $\mathrm{NaN}$ & $\mathrm{NaN}$ & $\mathrm{NaN}$ & 0.71 & 0.66 & $\mathrm{NaN}$ & $\mathrm{NaN}$ & $\mathrm{NaN}$ & $\mathrm{NaN}$ \\
\hline BPTF & F5GXF5 & $\mathrm{NaN}$ & $\mathrm{NaN}$ & $\mathrm{NaN}$ & 0.06 & 0.56 & 0.32 & $\mathrm{NaN}$ & $\mathrm{NaN}$ & 0.34 & 0.20 & 0.31 & 0.32 & $\mathrm{NaN}$ & $\mathrm{NaN}$ & $\mathrm{NaN}$ & $\mathrm{NaN}$ & 0.32 & 0.27 & $\mathrm{NaN}$ & $\mathrm{NaN}$ & 0.25 & 0.33 & $\mathrm{NaN}$ & $\mathrm{NaN}$ & $\mathrm{NaN}$ & 0.58 \\
\hline BPTF & $\mathrm{F} 5 \mathrm{H} 176$ & $\mathrm{NaN}$ & $\mathrm{NaN}$ & $\mathrm{NaN}$ & $\mathrm{NaN}$ & $\mathrm{NaN}$ & $\mathrm{NaN}$ & $\mathrm{NaN}$ & $\mathrm{NaN}$ & $\mathrm{NaN}$ & $\mathrm{NaN}$ & $\mathrm{NaN}$ & $\mathrm{NaN}$ & $\mathrm{NaN}$ & $\mathrm{NaN}$ & $\mathrm{NaN}$ & 0.40 & $\mathrm{NaN}$ & $\mathrm{NaN}$ & $\mathrm{NaN}$ & $\mathrm{NaN}$ & 0.86 & 0.79 & $\mathrm{NaN}$ & $\mathrm{NaN}$ & $\mathrm{NaN}$ & $\mathrm{NaN}$ \\
\hline BPTF & Q12830 & 0.29 & 1.33 & 0.54 & 0.79 & 0.64 & 0.67 & 0.57 & 0.58 & 0.93 & 0.45 & 0.72 & 0.63 & 0.37 & 1.10 & 0.61 & 0.75 & 0.70 & 0.64 & 2.01 & 0.39 & 0.63 & 0.61 & 1.04 & 0.71 & 0.74 & 0.44 \\
\hline BPTF & Q12830-2 & 0.22 & $\mathrm{NaN}$ & $\mathrm{NaN}$ & $\mathrm{NaN}$ & $\mathrm{NaN}$ & 1.32 & $\mathrm{NaN}$ & $\mathrm{NaN}$ & $\mathrm{NaN}$ & $\mathrm{NaN}$ & $\mathrm{NaN}$ & $\mathrm{NaN}$ & $\mathrm{NaN}$ & $\mathrm{NaN}$ & $\mathrm{NaN}$ & & $\mathrm{NaN}$ & 1.03 & $\mathrm{NaN}$ & $\mathrm{NaN}$ & 0.88 & 0.91 & $\mathrm{NaN}$ & $\mathrm{NaN}$ & $\mathrm{NaN}$ & $\mathrm{NaN}$ \\
\hline BRCA1 & $\begin{array}{l}\text { Q12000- } \\
\text { Q3LRH8 }\end{array}$ & $\begin{array}{l}0.22 \\
0.86\end{array}$ & 0.39 & 0.31 & 4.37 & 0.25 & $\begin{array}{l}2.05 \\
2.39\end{array}$ & 0.78 & 0.65 & 1.09 & 0.62 & 0.95 & 0.85 & $\mathrm{NaN}$ & 1.03 & 0.43 & 0.12 & 0.75 & 0.91 & 1.18 & 1.10 & 0.23 & 2.40 & 1.11 & 0.47 & 0.62 & 0.72 \\
\hline BRCC3 & P46736-2 & 0.55 & 0.21 & $\mathrm{NaN}$ & $\mathrm{NaN}$ & 0.42 & 1.68 & 0.43 & $\mathrm{NaN}$ & 1.37 & 0.48 & 0.75 & 0.88 & 0.61 & 0.88 & 0.33 & 0.27 & 0.98 & 0.65 & $\mathrm{NaN}$ & 0.29 & 0.17 & 2.71 & $\mathrm{NaN}$ & 0.38 & 0.64 & 0.79 \\
\hline $\begin{array}{l}\text { BRD2 } \\
\text { BRD }\end{array}$ & HOYGK2 & 0.29 & 3.05 & 1.22 & 1.45 & 1.29 & $\begin{array}{l}1.00 \\
2.47\end{array}$ & $\begin{array}{l}0.40 \\
1.13\end{array}$ & 1.42 & $\begin{array}{l}1.01 \\
1.26\end{array}$ & $\begin{array}{l}0.40 \\
1.38\end{array}$ & 1.15 & $\begin{array}{l}0.00 \\
2.12\end{array}$ & 0.83 & $\begin{array}{l}2.00 \\
2.19\end{array}$ & 0.87 & 1.67 & 1.44 & 1.55 & 1.39 & 1.80 & 1.30 & 2.09 & 1.86 & $\begin{array}{l}.00 \\
1.94\end{array}$ & $\begin{array}{l}0.04 \\
1.09\end{array}$ & 1.76 \\
\hline BRE & NXXR7-4 & 0.48 & 0.26 & 0.2 & 3.04 & 0.42 & 1.6 & 0.61 & $\mathrm{NaN}$ & 1.46 & 0.45 & 0.75 & 0.70 & 0.61 & & & 0.12 & 0.97 & 0.63 & & & 0.15 & 3.33 & & & 0.59 & 0.62 \\
\hline BRMS1 & Q9Y3T1 & 0.59 & 0.81 & 1.51 & 1.22 & 0.71 & 2.90 & 1.53 & 1.53 & 1.35 & 1.64 & 1.29 & 1.06 & 0.46 & $\mathrm{NaN}$ & $\mathrm{NaN}$ & 1.89 & 1.07 & 1.44 & 10.22 & 0.23 & 1.04 & 1.55 & 0.64 & 4.30 & $\mathrm{NaN}$ & $\mathrm{NaN}$ \\
\hline BRMS1L & KKU43 & 0.80 & 1.03 & 1.51 & 1.56 & 0.81 & 3.14 & 1.74 & 1.25 & 1.68 & 1.92 & 1.66 & 1.33 & 0.63 & 3.27 & 0.51 & 0.87 & 1.30 & 1.77 & 6.12 & 0.36 & 1.22 & 2.08 & 0.82 & 4.80 & 3.32 & 0.97 \\
\hline C10orf 12 & $3 N 655$ & 0.25 & 2.70 & $\mathrm{NaN}$ & $\mathrm{NaN}$ & 0.79 & 0.6 & $\mathrm{NaN}$ & $\mathrm{NaN}$ & $\mathrm{NaN}$ & $\mathrm{NaN}$ & 0.61 & $\mathrm{~N}$ & $\mathrm{NaN}$ & $\mathrm{NaN}$ & 0.96 & 0.59 & $\mathrm{NaN}$ & 0.66 & $\begin{array}{l}1.74 \\
1.74\end{array}$ & 2. & 0.94 & 0.39 & $\begin{array}{l}.02 \\
1.09\end{array}$ & 1.90 & 0.54 & $\mathrm{NaN}$ \\
\hline & & $\mathrm{N}$ & & 2. & 0.8 & & 1. & 1.50 & 2.01 & 1.41 & 1.62 & 0. & & & & & & & & & & & 2.08 & & & 1.14 & 2.26 \\
\hline C19orf 47 & Q8N9M1-2 & $\mathrm{NaN}$ & $\mathrm{NaN}$ & $\mathrm{Na}$ & $\mathrm{NaN}$ & 0.46 & $\mathrm{NaN}$ & $\mathrm{Nal}$ & $\mathrm{NaN}$ & $\mathrm{NaN}$ & $\mathrm{NaN}$ & $\mathrm{NaN}$ & $\mathrm{NaN}$ & $\mathrm{NaN}$ & $\mathrm{NaN}$ & $\mathrm{NaN}$ & $\mathrm{NaN}$ & $\mathrm{NaN}$ & 0.86 & 6.53 & 0.33 & 0.51 & 0.24 & $\mathrm{NaN}$ & $\mathrm{NaN}$ & $\mathrm{NaN}$ & $\mathrm{NaN}$ \\
\hline C9orf78 & Q9NZ63 & $\mathrm{NaN}$ & 1.16 & $\mathrm{NaN}$ & $\mathrm{NaN}$ & $\mathrm{NaN}$ & $\mathrm{NaN}$ & $\mathrm{NaN}$ & $\mathrm{NaN}$ & $\mathrm{NaN}$ & $\mathrm{NaN}$ & $\mathrm{NaN}$ & $\mathrm{NaN}$ & $\mathrm{NaN}$ & $\mathrm{NaN}$ & $\mathrm{NaN}$ & 1.18 & $\mathrm{NaN}$ & $\mathrm{NaN}$ & $\mathrm{NaN}$ & $\mathrm{NaN}$ & 0.37 & $\mathrm{NaN}$ & $\mathrm{NaN}$ & $\mathrm{NaN}$ & $\mathrm{NaN}$ & $\mathrm{NaN}$ \\
\hline CACYBP & Q9HB71-3 & 1.44 & $\mathrm{NaN}$ & $\mathrm{Na}$ & NaN & 1.13 & 0. & 0.65 & $\mathrm{NaN}$ & 1.12 & 0.71 & 0.61 & 0.66 & 0.68 & 0.86 & 1.42 & 0.65 & 0.52 & $\mathrm{NaN}$ & $\mathrm{NaN}$ & $\mathrm{NaN}$ & 0.67 & 0.68 & $\mathrm{NaN}$ & 0.86 & $\mathrm{NaN}$ & $\mathrm{NaN}$ \\
\hline & P07384 & $\mathrm{NaN}$ & 0.05 & 0.38 & 0.49 & 0.27 & 0.1 & 0.3 & 0.33 & 0.34 & 0.67 & $\mathrm{NaN}$ & $\mathrm{NaN}$ & $\mathrm{NaN}$ & $\mathrm{NaN}$ & 0.37 & 6. & 1.45 & 0.76 & $\mathrm{NaN}$ & $\mathrm{NaN}$ & 0.55 & 0.66 & $\mathrm{NaN}$ & $\mathrm{NaN}$ & $\mathrm{NaN}$ & 0.45 \\
\hline CAPRIN & Q14444-2 & $\mathrm{NaN}$ & 4.34 & $\mathrm{Na}$ & $\mathrm{Na}$ & & & 0. & $\mathrm{NaN}$ & & $\mathrm{Na}$ & $\mathrm{N}$ & & $\mathrm{N}$ & $\mathrm{Na}$ & & 1. & $\mathrm{Na}$ & & & & & 0.57 & & $\mathrm{NaN}$ & $\mathrm{NaN}$ & $\mathrm{NaN}$ \\
\hline CAST & B7Z468 & $\mathrm{NaN}$ & 0.11 & $\mathrm{Nal}$ & $\mathrm{Nal}$ & $\mathrm{NaN}$ & $\mathrm{NaI}$ & $\mathrm{Na}$ & $\mathrm{NaN}$ & $\mathrm{N}$ & $\mathrm{Nal}$ & $\mathrm{NaN}$ & $\mathrm{NaN}$ & $\mathrm{NaN}$ & $\mathrm{NaN}$ & $\mathrm{NaN}$ & $\mathrm{NaN}$ & $\mathrm{NaN}$ & $\mathrm{NaN}$ & $\mathrm{NaN}$ & $\mathrm{NaN}$ & $\mathrm{NaN}$ & $\mathrm{NaN}$ & $\mathrm{NaN}$ & $\mathrm{NaN}$ & $\mathrm{NaN}$ & $\mathrm{NaN}$ \\
\hline CAST & & & $\mathrm{NaN}$ & $\mathrm{Na}$ & $\mathrm{Na}$ & & $\mathrm{Na}$ & $\mathrm{Na}$ & $\mathrm{NaN}$ & $\mathrm{NaN}$ & $\mathrm{Na}$ & $\mathrm{NaN}$ & & $\mathrm{NaN}$ & $\mathrm{Nal}$ & & $\mathrm{NaN}$ & $\mathrm{NaN}$ & $\mathrm{NaN}$ & $\mathrm{NaN}$ & $\mathrm{NaN}$ & $\mathrm{NaN}$ & $\mathrm{NaN}$ & $\mathrm{NaN}$ & $\mathrm{NaN}$ & $\mathrm{NaN}$ & $\mathrm{NaN}$ \\
\hline CAT & P04040 & $\mathrm{NaN}$ & $\mathrm{NaN}$ & $\mathrm{NaN}$ & $\mathrm{NaN}$ & $\mathrm{NaN}$ & NaN & 0.10 & $\mathrm{NaN}$ & NaN & $\mathrm{NaN}$ & $\mathrm{NaN}$ & $\mathrm{NaN}$ & $\mathrm{NaN}$ & NaN & $\mathrm{NaN}$ & $\mathrm{NaN}$ & NaN & $\mathrm{NaN}$ & 0.08 & $\mathrm{NaN}$ & $\mathrm{NaN}$ & $\mathrm{NaN}$ & $\mathrm{NaN}$ & $\mathrm{NaN}$ & $\mathrm{NaN}$ & 1.01 \\
\hline CBFB & Q13951 & 0.85 & 2.02 & 1.1 & 0.7 & 0.71 & 1.2 & & $\mathrm{NaN}$ & & & 0. & & & & & & 0.54 & & & & & 1.10 & & & & \\
\hline CBFB & 3951-2 & $\mathrm{NaN}$ & $\mathrm{Na}$ & $\mathrm{Na}$ & $\mathrm{Na}$ & & $\mathrm{N}$ & No & $\mathrm{Na}$ & & $\mathrm{N}$ & $\mathrm{Na}$ & & $\mathrm{N}$ & $\mathrm{NaN}$ & $\mathrm{NaN}$ & & $\mathrm{N}$ & & & & & $\mathrm{NaN}$ & & 94 & $\mathrm{NaN}$ & $\mathrm{NaN}$ \\
\hline CBR & & & 0. & $\mathrm{~N}=$ & & & $\mathrm{Na}$ & $\mathrm{N}=$ & $\mathrm{Na}$ & & 1.3 & $\mathrm{NaN}$ & & $\mathrm{NaN}$ & $\mathrm{NaN}$ & $\mathrm{NaN}$ & $\mathrm{Na}$ & $\mathrm{NaN}$ & $\mathrm{N}=$ & 0. & $\mathrm{Na}$ & $\mathrm{NaN}$ & $\mathrm{NaN}$ & $\mathrm{NaN}$ & $\mathrm{NaN}$ & $\mathrm{NaN}$ & $\mathrm{NaN}$ \\
\hline CBX1 & Q61B & $\mathrm{NaN}$ & $\mathrm{N}$ & 1.1 & 0.74 & & 1.2 & 0.78 & 0.79 & 0. & 1.0 & 1.04 & 0.89 & 1.18 & 0.62 & 1.84 & 0.42 & 7.84 & 0.07 & 26.41 & 0.16 & 8.65 & 0.06 & $\mathrm{NaN}$ & 0.80 & 2.66 & 0.32 \\
\hline CBX3 & AD177 & 0.68 & 0.50 & 1.3 & 0.89 & 0.87 & 1.5 & 1.06 & 0.99 & 0. & 1.4 & 1.32 & 1.08 & 1.69 & 0.78 & 1.76 & 0.23 & 8.64 & 0.08 & 16.22 & 0.01 & 8.76 & 0.10 & 1.55 & 0.92 & 2.83 & 0.25 \\
\hline $\mathrm{CBX}$ & & $\mathrm{NaN}$ & $\mathrm{NaN}$ & 0. & & 0. & 0. & & 0. & & & & & 2.2 & 0.26 & & & & & 20.30 & & 10.24 & & & & & \\
\hline CBX8 & & 0.7 & $\mathrm{~N}$ & 1.2 & 0. & 1. & 1.2 & 0. & 1.0 & & 0.5 & 3. & & 0. & 1.9 & & 0 & 1.89 & 0. & 6. & 0 & 2.31 & 0.56 & 2. & 1.16 & 1.86 & 0.91 \\
\hline $\mathrm{CCDC1}$ & & 0.26 & & $\mathrm{Na}$ & $\mathrm{NaN}$ & $\mathrm{NaN}$ & $\mathrm{Ne}$ & $\mathrm{Na}$ & $\mathrm{NaN}$ & $\mathrm{NaN}$ & $\mathrm{NaN}$ & $\mathrm{NaN}$ & $\mathrm{NaN}$ & $\mathrm{NaN}$ & $\mathrm{Na}$ & 0.21 & 0.2 & $\mathrm{NaN}$ & $\mathrm{NaN}$ & $\mathrm{NaN}$ & $\mathrm{NaN}$ & $\mathrm{NaN}$ & $\mathrm{NaN}$ & $\mathrm{NaN}$ & $\mathrm{NaN}$ & $\mathrm{NaN}$ & $\mathrm{NaN}$ \\
\hline CCDC59 & Q9P 031 & 0.49 & $\mathrm{NaN}$ & 1.16 & 1.23 & 1.18 & 1.23 & $\mathrm{NaN}$ & $\mathrm{NaN}$ & 1.22 & 1.03 & 1.09 & 1.23 & 0.87 & $\mathrm{NaN}$ & 0.36 & 0.33 & 1.12 & 0.53 & $\mathrm{NaN}$ & 1.01 & 1.02 & 1.43 & 0.96 & 1.17 & 1.43 & 1.38 \\
\hline CD3EAP & A & $\mathrm{NaN}$ & $\mathrm{NaN}$ & $\mathrm{NaN}$ & 0.79 & 0.61 & 0.58 & 0.75 & 0.59 & & $\mathrm{NaN}$ & 1.42 & & $\mathrm{NaN}$ & $\mathrm{NaN}$ & & 6.25 & 0.32 & 0.95 & 5.46 & & & & & & & \\
\hline & & 1.47 & 1. & 0.7 & 0.9 & 0 & 0. & 1. & & & 2. & 1.4 & & $\mathrm{~N}$ & $\mathrm{~N}$ & & 7. & 0. & 0 & & & & 0.30 & $\mathrm{~N}$ & $\mathrm{NaN}$ & $\mathrm{NaN}$ & $\mathrm{NaN}$ \\
\hline CDK2AP1 & |014519-2 & $\mathrm{NaN}$ & $\mathrm{NaN}$ & $\mathrm{NaN}$ & $\mathrm{NaN}$ & 1.31 & 1.28 & $\mathrm{NaN}$ & $\mathrm{NaN}$ & $\mathrm{NaN}$ & 1.40 & $\mathrm{NaN}$ & $\mathrm{NaN}$ & $\mathrm{NaN}$ & $\mathrm{NaN}$ & $\mathrm{NaN}$ & $\mathrm{NaN}$ & 0.88 & 1.51 & 0.03 & $\mathrm{NaN}$ & 1.04 & 1.83 & 5.32 & 0.11 & $\mathrm{NaN}$ & $\mathrm{NaN}$ \\
\hline CDKN1A & Q96LE1 & $\mathrm{NaN}$ & $\mathrm{NaN}$ & $\mathrm{NaN}$ & 0.53 & $\mathrm{NaN}$ & $\mathrm{NaN}$ & $\mathrm{NaN}$ & $\mathrm{NaN}$ & $\mathrm{NaN}$ & $\mathrm{NaN}$ & $\mathrm{NaN}$ & $\mathrm{NaN}$ & $\mathrm{NaN}$ & $\mathrm{NaN}$ & $\mathrm{NaN}$ & $\mathrm{NaN}$ & $\mathrm{NaN}$ & 0.40 & $\mathrm{NaN}$ & $\mathrm{NaN}$ & $\mathrm{NaN}$ & 0.59 & $\mathrm{NaN}$ & $\mathrm{NaN}$ & $\mathrm{NaN}$ & $\mathrm{NaN}$ \\
\hline CDSN & Q0EFA5 & 0.08 & $\mathrm{NaN}$ & $\mathrm{NaN}$ & $\mathrm{NaN}$ & $\mathrm{NaN}$ & $\mathrm{NaN}$ & 0.14 & $\mathrm{NaN}$ & 0.21 & 0.06 & 0.25 & $\mathrm{NaN}$ & $\mathrm{NaN}$ & $\mathrm{NaN}$ & & $\mathrm{NaN}$ & $\mathrm{NaN}$ & & . & $\mathrm{NaN}$ & 0.07 & & $\mathrm{NaN}$ & $\mathrm{NaN}$ & & $\mathrm{NaN}$ \\
\hline CD & & 0.86 & & 0.4 & 0.6 & & 1. & 0.5 & & 0. & 0. & 0. & 0.50 & 0. & 0.58 & & 0.1 & 1.80 & 0.23 & 3.90 & & 1.89 & 0.20 & 1.23 & & 03 & \\
\hline CDY2 & Q8N8U2 & $\mathrm{NaN}$ & $\mathrm{NaN}$ & 0.86 & 0.86 & 0.40 & 1.53 & $\mathrm{NaN}$ & $\mathrm{NaN}$ & $\mathrm{NaN}$ & $\mathrm{NaN}$ & 1.54 & 0.47 & 1.37 & 1.27 & $\mathrm{NaN}$ & $\mathrm{NaN}$ & 2.50 & 0.37 & 6.51 & 0.09 & 2.74 & 0.25 & 1.32 & 0.97 & $\mathrm{NaN}$ & $\mathrm{NaN}$ \\
\hline CEBPB & P17676 & 0.61 & 0.40 & 1.52 & 1.03 & 0.50 & 2.04 & 1.44 & 1.19 & 0.99 & 1.84 & 1.10 & 1.16 & $\mathrm{NaN}$ & $\mathrm{NaN}$ & 0.20 & 1.95 & 0.45 & 2.74 & 4.22 & 0.21 & 1.47 & 0.86 & 1.57 & 1.05 & $\mathrm{NaN}$ & 0.56 \\
\hline
\end{tabular}




\begin{tabular}{|c|c|c|c|c|c|c|c|c|c|c|c|c|c|c|c|c|c|c|c|c|c|c|c|c|c|c|c|}
\hline Genenames & Protein IDs & $\begin{array}{l}\mathrm{H} 3 \Delta 1- \\
20 \_\mathrm{F}\end{array}$ & $\begin{array}{l}\mathrm{H} 3 \Delta \mathrm{1}- \\
20 \_\mathrm{R}\end{array}$ & $\begin{array}{l}\text { H4K20 } \\
\text { me1_F }\end{array}$ & $\begin{array}{l}\text { H4K20 } \\
\text { me1_R }\end{array}$ & $\begin{array}{l}\text { H4K20 } \\
\text { me3_F }\end{array}$ & $\begin{array}{l}\text { H4K20 } \\
\text { me3_R }\end{array}$ & $\begin{array}{r}\mathrm{H} 3 \mathrm{~K}_{\mathrm{C}} 27 \\
\mathrm{me1} F\end{array}$ & $\begin{array}{l}\mathrm{H} 3 \mathrm{~K}_{\mathrm{C}} 27 \\
\mathrm{me} 1 \mathrm{R}\end{array}$ & $\begin{array}{l}\mathrm{H} 3 \mathrm{~K}_{\mathrm{c}} 27 \\
\text { me2_F }\end{array}$ & $\begin{array}{c}\mathrm{H} 3 \mathrm{~K}_{\mathrm{c}} 27 \\
\mathrm{me2} \_\mathrm{R}\end{array}$ & $\begin{array}{l}\mathrm{H} 3 \mathrm{~K}_{\mathrm{c}} 27 \\
\mathrm{me3} \_\mathrm{F}\end{array}$ & $\begin{array}{l}\mathrm{H} 3 \mathrm{~K}_{\mathrm{C}} 27 \\
\mathrm{me3} 3 \mathrm{R}\end{array}$ & $\begin{array}{c}\text { Н3К9m } \\
\text { e1_F }\end{array}$ & $\begin{array}{c}\text { HзК9m } \\
\text { e1_R }\end{array}$ & \begin{tabular}{|c} 
H3к9m \\
e2_F
\end{tabular} & $\begin{array}{c}\text { H3k9m } \\
\text { e2_R }\end{array}$ & $\begin{array}{c}\text { H3К9m } \\
\text { e3_F }\end{array}$ & $\begin{array}{c}\text { H3K9m } \\
\text { e3_R }\end{array}$ & $\begin{array}{c}\text { H3k9m } \\
\text { e3|mec } \\
\text { pG_F }\end{array}$ & $\begin{array}{c}\text { HзK9m } \\
\text { eз3/meC } \\
\text { pG_R }\end{array}$ & $\mid \begin{array}{c}\mathrm{H} 3 \mathrm{~K} 9 \mathrm{~m} \\
\mathrm{e} \mid \mathrm{|H} 4 \mathrm{~K} \\
20 \mathrm{me}{ }_{-} \\
\mathrm{F}\end{array}$ & $\begin{array}{c}\mathrm{H} 3 \mathrm{~K} / \mathrm{m} \\
\mathrm{e} \mid \mathrm{H} 4 \mathrm{~K} \\
20 \mathrm{me}{ }_{-} \\
\mathrm{R}\end{array}$ & $\underset{-F}{\mathrm{meCp}}$ & $\begin{array}{c}\mathrm{meCpG} \\
-\mathrm{R}\end{array}$ & $\begin{array}{c}\text { H4R3m } \\
\text { e2_F }\end{array}$ & $\begin{array}{c}\mathrm{H} 4 \mathrm{R} 3 \mathrm{~m} \\
\text { e2_R }\end{array}$ \\
\hline CEBPD & P49716 & $\mathrm{NaN}$ & 0.23 & 1.60 & 0.91 & 0.60 & 2.75 & $\mathrm{NaN}$ & $\mathrm{NaN}$ & $\mathrm{NaN}$ & 1.92 & 1.26 & 1.32 & \begin{tabular}{ll|} 
\\
$\mathrm{NaN}$
\end{tabular} & $\mathrm{NaN}$ & \begin{tabular}{|l|}
0.10 \\
\end{tabular} & 1.23 & \begin{tabular}{|l|} 
\\
\end{tabular} & \begin{tabular}{|l|}
2.77 \\
\end{tabular} & \begin{tabular}{|l|}
5.85 \\
\end{tabular} & \begin{tabular}{|l|} 
\\
\end{tabular} & \begin{tabular}{|l|}
1.69 \\
\end{tabular} & 0.92 & $\begin{array}{l}1.26 \\
\end{array}$ & \begin{tabular}{|l|l|}
1.37 \\
\end{tabular} & $\mathrm{NaN}$ & $\mathrm{NaN}$ \\
\hline CEBPG & P53567 & $\mathrm{NaN}$ & $\mathrm{NaN}$ & 1.47 & 0.79 & 0.60 & $\mathrm{NaN}$ & $\mathrm{NaN}$ & $\mathrm{NaN}$ & $\mathrm{NaN}$ & $\mathrm{NaN}$ & 1.11 & $\mathrm{NaN}$ & $\mathrm{NaN}$ & $\mathrm{NaN}$ & $\mathrm{NaN}$ & 2.99 & 0.60 & 2.45 & $\mathrm{NaN}$ & $\mathrm{NaN}$ & 1.85 & $\mathrm{NaN}$ & $\mathrm{NaN}$ & $\mathrm{NaN}$ & 2.14 & $\mathrm{NaN}$ \\
\hline CENPF & P49454 & 1.08 & 0.70 & 1.29 & 0.70 & 1.76 & 0.58 & 0.17 & 1.03 & 1.12 & 0.69 & 0.73 & 1.15 & $\mathrm{NaN}$ & $\mathrm{NaN}$ & 1.31 & 0.60 & 1.74 & 0.63 & 0.22 & 12.26 & 0.78 & 1.20 & 1.18 & 1.42 & 0.52 & 1.52 \\
\hline CENPV & Q7Z7K6 & 1.87 & 1.97 & 0.87 & 0.65 & 0.88 & 0.92 & 0.84 & 0.80 & 0.77 & 0.86 & 0.78 & 0.77 & 0.72 & 0.97 & 2.21 & 4.94 & 0.73 & 0.75 & 1.40 & 1.22 & 0.89 & 0.77 & 0.92 & 1.02 & 0.77 & 1.14 \\
\hline CENPV & Q7Z7K6-3 & $\mathrm{NaN}$ & 2.18 & $\mathrm{NaN}$ & NaN & $\mathrm{NaN}$ & $\mathrm{NaN}$ & 0.93 & 0.92 & NaN & $\mathrm{NaN}$ & $\mathrm{NaN}$ & $\mathrm{NaN}$ & $\mathrm{NaN}$ & $\mathrm{NaN}$ & 2.26 & $\mathrm{NaN}$ & $\mathrm{NaN}$ & $\mathrm{NaN}$ & $\mathrm{NaN}$ & 0.83 & 1.22 & 1.13 & 0.75 & 1.28 & $\mathrm{NaN}$ & $\mathrm{NaN}$ \\
\hline CGGBP1 & Q9UFW8 & 0.71 & 1.11 & 0.44 & $\mathrm{NaN}$ & 2.30 & 0.45 & $\mathrm{NaN}$ & $\mathrm{NaN}$ & 1.03 & 0.90 & 0.96 & 0.44 & $\mathrm{NaN}$ & $\mathrm{NaN}$ & 0.45 & 0.53 & 1.67 & 0.35 & 6.52 & 0.18 & 1.32 & 0.61 & 0.50 & 2.13 & $\mathrm{NaN}$ & $\mathrm{NaN}$ \\
\hline CHAF1A & D6W625 & 0.49 & 1.07 & 0.96 & 0.48 & 1.34 & 0.83 & 0.55 & 0.82 & 0.41 & 1.55 & 0.76 & 0.92 & $\mathrm{NaN}$ & 0.64 & 0.86 & 1.02 & 4.77 & 0.12 & 5.08 & 0.37 & 6.15 & 0.10 & 1.50 & 1.54 & $\mathrm{NaN}$ & $\mathrm{NaN}$ \\
\hline CHAF1B & B2R7X3 & 0.58 & 0.82 & 0.89 & 0.41 & 1.10 & 0.83 & 0.54 & 0.78 & 0.38 & 1.54 & 0.81 & 0.75 & 0.71 & 0.59 & 0.85 & 1.61 & 4.40 & 0.13 & 2.30 & 0.59 & 7.41 & 0.07 & 1.30 & 1.25 & 2.10 & 0.18 \\
\hline CHAMP1 & Q96JM3 & 1.07 & 0.71 & 1.09 & 0.85 & 1.06 & 1.04 & 0.61 & $\mathrm{NaN}$ & 0.77 & 1.66 & 1.22 & 1.35 & 1.21 & $\mathrm{NaN}$ & 0.63 & 0.14 & 6.98 & 0.18 & 11.02 & 0.12 & 7.92 & 0.14 & 4.49 & 0.54 & 1.06 & 0.34 \\
\hline CHD1 & |014646-2 & 1.40 & 0.47 & 0.81 & 0.54 & 1.32 & 0.48 & 0.67 & 1.82 & 1.08 & 0.57 & $\mathrm{NaN}$ & 0.99 & $\mathrm{NaN}$ & $\mathrm{NaN}$ & 1.33 & 0.46 & 1.09 & 0.45 & 0.31 & 6.01 & 0.54 & 1.03 & 0.78 & 0.95 & 0.23 & 1.99 \\
\hline CHD1L & Q86WJ1 & $\mathrm{NaN}$ & 0.51 & $\mathrm{NaN}$ & $\mathrm{NaN}$ & $\mathrm{NaN}$ & 0.38 & $\mathrm{NaN}$ & $\mathrm{NaN}$ & $\mathrm{NaN}$ & $\mathrm{NaN}$ & $\mathrm{NaN}$ & $\mathrm{NaN}$ & $\mathrm{NaN}$ & 1.35 & 0.69 & 0.56 & $\mathrm{NaN}$ & $\mathrm{NaN}$ & 0.34 & 0.68 & 0.31 & $\mathrm{NaN}$ & 0.63 & 0.40 & $\mathrm{NaN}$ & $\mathrm{NaN}$ \\
\hline CHD3 & H7COJ3 & $\mathrm{NaN}$ & $\mathrm{NaN}$ & $\mathrm{NaN}$ & $\mathrm{NaN}$ & $\mathrm{NaN}$ & 1.15 & $\mathrm{NaN}$ & $\mathrm{NaN}$ & $\mathrm{NaN}$ & $\mathrm{NaN}$ & $\mathrm{NaN}$ & $\mathrm{NaN}$ & $\mathrm{NaN}$ & $\mathrm{NaN}$ & 0.68 & $\mathrm{NaN}$ & $\mathrm{NaN}$ & $\mathrm{NaN}$ & $\mathrm{NaN}$ & $\mathrm{NaN}$ & $\mathrm{NaN}$ & $\mathrm{NaN}$ & $\mathrm{NaN}$ & $\mathrm{NaN}$ & $\mathrm{NaN}$ & $\mathrm{NaN}$ \\
\hline CHD3 & Q12873 & 0.53 & $\mathrm{NaN}$ & 0.60 & 0.49 & 0.59 & 0.41 & 0.48 & 0.46 & 0.69 & 0.49 & 0.68 & 0.41 & 0.44 & 1.34 & 0.42 & 0.16 & 0.38 & 0.46 & 4.21 & 0.28 & 0.61 & 0.41 & 4.32 & 0.15 & 0.76 & 0.22 \\
\hline CHD4 & F5GWX5 & 0.58 & 1.00 & & 0.7 & 0.89 & & 0.7 & 0.73 & & 0.6 & 0.87 & .71 & 0.56 & 1.06 & 0.79 & 0.4 & 1.12 & 0.52 & 11.85 & 0.6 & 1.31 & 0.44 & 34 & 0.19 & 1.17 & 0.43 \\
\hline CHD4 & Q14839 & $\mathrm{NaN}$ & $\mathrm{NaN}$ & $\mathrm{NaN}$ & $\mathrm{NaN}$ & $\mathrm{NaN}$ & $\mathrm{NaN}$ & $\mathrm{NaN}$ & $\mathrm{NaN}$ & $\mathrm{NaN}$ & $\mathrm{NaN}$ & $\mathrm{NaN}$ & $\mathrm{NaN}$ & $\mathrm{NaN}$ & $\mathrm{NaN}$ & $\mathrm{NaN}$ & $\mathrm{NaN}$ & 1.60 & 0.53 & 8.52 & 0.26 & 2.15 & 0.32 & $\mathrm{NaN}$ & $\mathrm{NaN}$ & $\mathrm{NaN}$ & $\mathrm{NaN}$ \\
\hline CHERP & Q8IWX8 & 1.76 & 2.12 & 0.86 & 1.82 & 1.60 & 0.83 & 0.84 & 1.16 & 1.52 & 1.37 & $\mathrm{NaN}$ & 1.66 & $\mathrm{NaN}$ & $\mathrm{NaN}$ & 1.26 & 0.62 & 1.61 & 0.94 & 1.38 & 1.03 & 0.88 & 2.02 & 1.16 & 1.13 & 0.91 & 1.36 \\
\hline CHTOP & Q9Y3Y2-4 & 2.23 & 0.46 & 0.50 & 1.83 & 0.81 & 0.77 & 0.70 & 1.02 & 2.25 & 0.76 & 1.31 & 1.03 & 1.19 & $\mathrm{NaN}$ & 0.77 & 0.23 & 0.92 & 0.73 & 3.44 & 1.26 & 0.99 & 1.06 & 2.32 & $\mathrm{NaN}$ & 0.80 & 0.49 \\
\hline CIR & AOPJI7 & $\mathrm{Nal}$ & $\mathrm{Nal}$ & 1.4 & 0.2 & $\mathrm{~N}$ & & $\mathrm{Ne}$ & $\mathrm{Na}$ & & 0.6 & $\mathrm{NaN}$ & & $\mathrm{Na}$ & $\mathrm{Na}$ & & & $\mathrm{N}$ & & 1.0 & & & $\mathrm{NaN}$ & & 145 & & $\mathrm{NaN}$ \\
\hline CKAP2 & B2RMQ4 & 3.76 & 2.14 & 0.41 & 0.64 & 0.53 & 0.50 & 0.55 & $\mathrm{NaN}$ & 0.64 & 0.71 & 0.72 & 0.92 & 0.53 & $\mathrm{NaN}$ & 0.01 & 0.25 & 0.53 & 0.75 & 0.35 & 1.36 & 0.50 & 0.71 & 0.87 & 0.67 & 0.38 & 0.52 \\
\hline CKAP2 & E9PD90 & $\mathrm{NaN}$ & $\mathrm{NaN}$ & $\mathrm{NaN}$ & $\mathrm{NaN}$ & $\mathrm{NaN}$ & $\mathrm{NaN}$ & $\mathrm{NaN}$ & $\mathrm{NaN}$ & $\mathrm{NaN}$ & $\mathrm{NaN}$ & $\mathrm{NaN}$ & $\mathrm{NaN}$ & $\mathrm{NaN}$ & $\mathrm{NaN}$ & $\mathrm{NaN}$ & 0.63 & $\mathrm{NaN}$ & $\mathrm{NaN}$ & $\mathrm{NaN}$ & $\mathrm{NaN}$ & $\mathrm{NaN}$ & $\mathrm{NaN}$ & $\mathrm{NaN}$ & $\mathrm{NaN}$ & $\mathrm{NaN}$ & $\mathrm{NaN}$ \\
\hline CKB & P 12277 & $\mathrm{NaN}$ & 0.18 & $\mathrm{NaN}$ & $\mathrm{NaN}$ & $\mathrm{NaN}$ & $\mathrm{NaN}$ & $\mathrm{NaN}$ & $\mathrm{NaN}$ & $\mathrm{NaN}$ & $\mathrm{NaN}$ & $\mathrm{NaN}$ & $\mathrm{NaN}$ & $\mathrm{NaN}$ & $\mathrm{NaN}$ & 0.30 & $\mathrm{NaN}$ & $\mathrm{NaN}$ & $\mathrm{NaN}$ & 0.29 & $\mathrm{NaN}$ & $\mathrm{NaN}$ & $\mathrm{NaN}$ & 1.18 & 0.39 & $\mathrm{NaN}$ & $\mathrm{NaN}$ \\
\hline CLASP1 & B7ZLLX3 & $\mathrm{NaN}$ & $\mathrm{NaN}$ & $\mathrm{Na}$ & $\mathrm{NaN}$ & 1.59 & 0.70 & $\mathrm{NaN}$ & $\mathrm{NaN}$ & $\mathrm{NaN}$ & $\mathrm{NaN}$ & $\mathrm{NaN}$ & $\mathrm{NaN}$ & $\mathrm{NaN}$ & $\mathrm{NaN}$ & 0.42 & 0.03 & 0.75 & 0.55 & $\mathrm{NaN}$ & $\mathrm{NaN}$ & 1.40 & 0.50 & $\mathrm{NaN}$ & $\mathrm{NaN}$ & $\mathrm{NaN}$ & $\mathrm{NaN}$ \\
\hline CLASP1 & Q7Z460 & 0.53 & 3.25 & 0.69 & 0.42 & 1.29 & 0.52 & 0.74 & 0.52 & 0.87 & 0.47 & 0.82 & 0.35 & 1.54 & 0.55 & 0.54 & 0.08 & 0.79 & 0.51 & 2.96 & 0.31 & 1.04 & 0.40 & 0.53 & 0.67 & 1.16 & 0.25 \\
\hline CLASP2 & $\mathrm{F} 5 \mathrm{H} 604$ & 0.49 & 2.66 & 0.70 & 0.54 & 1.54 & 0.54 & 0.91 & 0.68 & 0.66 & 0.73 & 1.01 & 0.45 & 1.73 & 0.60 & 0.51 & 0.08 & 1.04 & 0.58 & 1.92 & 0.25 & 0.70 & 0.77 & 0.69 & $\mathrm{NaN}$ & 1.24 & 0.45 \\
\hline CLIC1 & Q5SRT3 & 0.18 & 0.15 & $\mathrm{NaN}$ & $\mathrm{NaN}$ & $\mathrm{NaN}$ & $\mathrm{NaN}$ & $\mathrm{NaN}$ & $\mathrm{NaN}$ & 1.79 & 0.32 & $\mathrm{NaN}$ & $\mathrm{NaN}$ & $\mathrm{NaN}$ & $\mathrm{NaN}$ & 0.25 & $\mathrm{NaN}$ & $\mathrm{NaN}$ & $\mathrm{NaN}$ & 0.38 & 1.79 & $\mathrm{NaN}$ & $\mathrm{NaN}$ & 1.15 & 0.31 & $\mathrm{NaN}$ & $\mathrm{NaN}$ \\
\hline CLOCK & 3EUO & 1.24 & $\mathrm{NaN}$ & 0.91 & $\mathrm{NaN}$ & 1.62 & 2.15 & 1.18 & 1.14 & 0.81 & 1.26 & 1.25 & $\mathrm{NaN}$ & $\mathrm{NaN}$ & 2.57 & $\mathrm{NaN}$ & $\mathrm{NaN}$ & 1.40 & 1.38 & 3.10 & 0.40 & 1.52 & 1.38 & 0.63 & 2.19 & 1.67 & 1.54 \\
\hline CLTC & & & & & 1.5 & & & & & & & & & & & & & & & & & & & & & & $\mathrm{NaN}$ \\
\hline CLUH & I3L2BO & $\mathrm{NaN}$ & $\mathrm{NaN}$ & $\mathrm{NaN}$ & $\mathrm{NaN}$ & $\mathrm{NaN}$ & $\mathrm{NaN}$ & $\mathrm{NaN}$ & $\mathrm{NaN}$ & $\mathrm{NaN}$ & $\mathrm{NaN}$ & $\mathrm{NaN}$ & $\mathrm{NaN}$ & $\mathrm{NaN}$ & $\mathrm{NaN}$ & $\mathrm{NaN}$ & $\mathrm{NaN}$ & $\mathrm{NaN}$ & $\mathrm{NaN}$ & $\mathrm{NaN}$ & $\mathrm{NaN}$ & $\mathrm{NaN}$ & $\mathrm{NaN}$ & $\mathrm{NaN}$ & $\mathrm{NaN}$ & $\mathrm{NaN}$ & $\mathrm{NaN}$ \\
\hline CLUH & K7EIG1 & $\mathrm{NaN}$ & $\mathrm{NaN}$ & $\mathrm{NaN}$ & $\mathrm{NaN}$ & $\mathrm{NaN}$ & $\mathrm{NaN}$ & $\mathrm{NaN}$ & $\mathrm{NaN}$ & $\mathrm{NaN}$ & $\mathrm{NaN}$ & $\mathrm{NaN}$ & $\mathrm{NaN}$ & $\mathrm{NaN}$ & $\mathrm{NaN}$ & $\mathrm{NaN}$ & $\mathrm{NaN}$ & $\mathrm{NaN}$ & $\mathrm{NaN}$ & $\mathrm{NaN}$ & $\mathrm{NaN}$ & $\mathrm{NaN}$ & $\mathrm{NaN}$ & $\mathrm{NaN}$ & $\mathrm{NaN}$ & $\mathrm{NaN}$ & $\mathrm{NaN}$ \\
\hline CNBP & P62633-2 & 1.04 & 0.79 & 1.07 & 0.57 & $\mathrm{NaN}$ & $\mathrm{NaN}$ & $\mathrm{NaN}$ & $\mathrm{NaN}$ & $\mathrm{NaN}$ & $\mathrm{NaN}$ & $\mathrm{NaN}$ & $\mathrm{NaN}$ & $\mathrm{NaN}$ & $\mathrm{NaN}$ & 0.78 & 2.12 & $\mathrm{NaN}$ & $\mathrm{NaN}$ & 0.88 & 0.73 & 0.50 & $\mathrm{NaN}$ & 1.24 & $\mathrm{NaN}$ & $\mathrm{NaN}$ & $\mathrm{NaN}$ \\
\hline $\mathrm{CNO}$ & YKK6 & 0.91 & 1.60 & 0.5 & 0.7 & 1.01 & 0.30 & 1.05 & 0.34 & 0.52 & 0.6 & 0.46 & & 1.42 & 0.33 & 0.7 & 0. & 1.17 & 0.67 & & & 48 & 0.96 & & $\mathrm{NaN}$ & 1.10 & 0.47 \\
\hline CNOT1 & H3BMZ2 & 1.05 & $\mathrm{NaN}$ & $\mathrm{N} a$ & $\mathrm{Nal}$ & $\mathrm{NaN}$ & $\mathrm{N}$ & $\mathrm{Na}$ & $\mathrm{NaN}$ & & $\mathrm{N}$ & $\mathrm{NaN}$ & $\mathrm{aN}$ & $\mathrm{NaN}$ & $\mathrm{NaN}$ & $\mathrm{NaN}$ & $\mathrm{NaN}$ & $\mathrm{NaN}$ & $\mathrm{NaN}$ & $\mathrm{NaN}$ & $\mathrm{NaN}$ & $\mathrm{NaN}$ & $\mathrm{NaN}$ & $\mathrm{aN}$ & $\mathrm{NaN}$ & $\mathrm{NaN}$ & $\mathrm{NaN}$ \\
\hline CNOT10 & BH9A5 & 0.87 & 1.17 & 0.4 & 0.66 & 1.07 & 0. & 0.86 & $\mathrm{NaN}$ & 0. & 0.64 & 0.42 & 55 & 1.16 & 0.48 & 0.72 & 0.18 & 1.05 & 0.69 & 0.82 & $\mathrm{NaN}$ & 0.50 & 0.84 & $\mathrm{NaN}$ & $\mathrm{NaN}$ & 0.86 & 0.51 \\
\hline CNOT11 & QS & 0.67 & 0.77 & 0.7 & 0.61 & 0.88 & $0.4 C$ & 1.23 & 0.42 & 0.52 & 0.7 & 0.51 & 0.46 & 1.02 & 0.42 & 0. & 0.15 & 1.20 & 0.72 & 1.30 & $\mathrm{NaN}$ & 0.56 & 0.95 & $\mathrm{NaN}$ & $\mathrm{NaN}$ & 1.33 & 0.55 \\
\hline $\mathrm{CNO}$ & & $\mathrm{N}$ & $\mathrm{Nal}$ & 0.8 & 0.9 & 0.8 & 0. & $\mathrm{NaN}$ & 0. & 0. & 0. & 0.8 & 0.85 & 0.8 & 1.3 & $\mathrm{NaN}$ & $\mathrm{N}$ & 0. & 0. & $\mathrm{Na}$ & & 0.89 & 0.78 & 0.85 & 0.78 & 0.72 & 0.72 \\
\hline CINC & & 0.4 & 0.9 & & 0. & & & 1.2 & 0. & & & & & & 0. & 0.53 & & & 0.61 & & & 0.37 & 0.8 & & & 0.84 & 0.52 \\
\hline CNOT3 & $\mathrm{H}$ & $\mathrm{NaN}$ & $\mathrm{NaN}$ & $\mathrm{Nc}$ & $\mathrm{Nal}$ & $\mathrm{NaN}$ & $\mathrm{Nc}$ & 0.6 & $\mathrm{NaN}$ & $\mathrm{N}$ & $\mathrm{N}$ & $\mathrm{NaN}$ & $N$ & $\mathrm{~N}$ & $\mathrm{Nal}$ & $\mathrm{NaN}$ & $N$ & $\mathrm{NaN}$ & $\mathrm{NaN}$ & $\mathrm{NaN}$ & $\mathrm{NaN}$ & $\mathrm{NaN}$ & $\mathrm{NaN}$ & $\mathrm{NaN}$ & $\mathrm{NaN}$ & $\mathrm{NaN}$ & $\mathrm{NaN}$ \\
\hline CNOT7 & & 0.86 & 1.18 & 0. & 0.7 & 1. & & 1.19 & 0. & & 1.02 & 0.5 & & 0.94 & 0.49 & & 0. & 1.08 & 0.72 & 1.87 & $\mathrm{NaN}$ & 0.62 & 1.08 & $\mathrm{NaN}$ & $\mathrm{NaN}$ & 1.72 & 0.57 \\
\hline $\mathrm{COB}$ & & 0. & 1.68 & $\mathrm{~N} c$ & $\mathrm{Na}$ & $\mathrm{NaN}$ & $\mathrm{NaN}$ & $\mathrm{NaN}$ & $\mathrm{NaN}$ & $\mathrm{NaN}$ & $\mathrm{NaN}$ & $\mathrm{Na}$ & 0.70 & $\mathrm{NaN}$ & $\mathrm{NaN}$ & 0.32 & 0.05 & $\mathrm{NaN}$ & $\mathrm{NaN}$ & $\mathrm{NaN}$ & $\mathrm{NaN}$ & $\mathrm{NaN}$ & $\mathrm{NaN}$ & $\mathrm{NaN}$ & $\mathrm{NaN}$ & $\mathrm{NaN}$ & 0.39 \\
\hline CPN & & $\mathrm{Na}$ & 0.3 & $\mathrm{~N} a$ & $\mathrm{Na}$ & $\mathrm{Nal}$ & $\mathrm{Na}$ & $\mathrm{Na}$ & $\mathrm{Na}$ & $\mathrm{N}$ & $\mathrm{Na}$ & $\mathrm{Na}$ & $\mathrm{Nal}$ & $\mathrm{Na}$ & $\mathrm{Na}$ & 0.6 & $\mathrm{~N}$ & $\mathrm{Na}$ & $\mathrm{NaN}$ & & $\mathrm{Na}$ & $\mathrm{NaN}$ & $\mathrm{Na}$ & $\mathrm{NaN}$ & $\mathrm{NaN}$ & $\mathrm{NaN}$ & $\mathrm{NaN}$ \\
\hline CPNE8 & Q886Q8 & $\mathrm{Nal}$ & 2.8 & & $\mathrm{Na}$ & $\mathrm{N}$ & & $\mathrm{N}$ & $\mathrm{Na}$ & & $\mathrm{N}$ & $\mathrm{N}$ & & $\mathrm{N}$ & $\mathrm{Na}$ & & 1. & $\mathrm{Na}$ & $\mathrm{NaN}$ & 0.62 & & $\mathrm{NaN}$ & & & 0.94 & $\mathrm{NaN}$ & $\mathrm{NaN}$ \\
\hline CRCP & & $\mathrm{Na}$ & $\mathrm{N}$ & $\mathrm{Nc}$ & $\mathrm{Na}$ & & & 0. & 0. & & 0. & 0.6 & & $\mathrm{~N}$ & $\mathrm{Na}$ & & & 0.2 & 1.4 & 22 & $\mathrm{~N}$ & 1.44 & 0.25 & $\mathrm{NaN}$ & $\mathrm{NaN}$ & $\mathrm{NaN}$ & $\mathrm{NaN}$ \\
\hline CSTA & & & $\mathrm{Ne}$ & 0. & $\mathrm{Na}$ & & & 0. & $\mathrm{Na}$ & & $\mathrm{Ne}$ & $\mathrm{Nc}$ & & $\mathrm{N}$ & $\mathrm{Na}$ & & $\mathrm{N}$ & $\mathrm{Na}$ & $\mathrm{NaN}$ & & $\mathrm{Na}$ & 0.08 & $\mathrm{NaN}$ & $\mathrm{NaN}$ & 0.21 & $\mathrm{NaN}$ & $\mathrm{NaN}$ \\
\hline CTSL & P07339 & $\mathrm{NaN}$ & $\mathrm{NaN}$ & $\mathrm{NaN}$ & $\mathrm{Nal}$ & $\mathrm{NaN}$ & $\mathrm{Nal}$ & $\mathrm{NaN}$ & $\mathrm{NaN}$ & $\mathrm{NaN}$ & $\mathrm{Nal}$ & $\mathrm{NaN}$ & $\mathrm{NaN}$ & $\mathrm{NaN}$ & $\mathrm{NaN}$ & $\mathrm{NaN}$ & $\mathrm{NaN}$ & $\mathrm{NaN}$ & $\mathrm{NaN}$ & 0.18 & $\mathrm{NaN}$ & $\mathrm{NaN}$ & $\mathrm{NaN}$ & $\mathrm{NaN}$ & 0.18 & $\mathrm{NaN}$ & $\mathrm{NaN}$ \\
\hline CUEDC & & $\mathrm{Na}$ & $\mathrm{Na}$ & & & 1.8 & 0. & & & & & & & & & & & & & & & & & & & & $\mathrm{VaN}$ \\
\hline CUL1 & & 4.9 & 0. & 1. & $\mathrm{~N}$ & 1. & 1. & $\mathrm{~N}$ & $\mathrm{~N}$ & & $\mathrm{~N}$ & $\mathrm{~N}$ & & & 0. & & & $\mathrm{~N}$ & & & & 1. & & & & $\mathrm{NaN}$ & $\mathrm{NaN}$ \\
\hline CXor & & 4. & & $\mathrm{Ne}$ & & & & $\mathrm{NaN}$ & & & $\mathrm{Na}$ & $\mathrm{NaN}$ & & $\mathrm{NaN}$ & $\mathrm{Na}$ & & $\mathrm{NaN}$ & $\mathrm{Na}$ & $\mathrm{NaN}$ & $\mathrm{NaN}$ & $\mathrm{NaN}$ & $\mathrm{NaN}$ & $\mathrm{NaN}$ & $\mathrm{NaN}$ & $\mathrm{NaN}$ & $\mathrm{NaN}$ & $\mathrm{NaN}$ \\
\hline CYR61 & B4DI6 & 12.33 & 9.81 & 0.73 & 0.75 & 0.61 & 0.47 & 0.54 & $\mathrm{NaN}$ & $\mathrm{NaN}$ & $\mathrm{Na}$ & 0.67 & 0.94 & 0.36 & $\mathrm{NaN}$ & 9.50 & 7.97 & 0.33 & $\mathrm{NaN}$ & 1.11 & 1.14 & $\mathrm{NaN}$ & 0.55 & 1.04 & 0.96 & $\mathrm{NaN}$ & $\mathrm{NaN}$ \\
\hline DDIT4 & Q9NXC & 0.35 & $\mathrm{Nan}$ & $\mathrm{Na}$ & $\mathrm{Na}$ & $\mathrm{NaN}$ & $\mathrm{Na}$ & $\mathrm{Nal}$ & $\mathrm{NaN}$ & $\mathrm{Na}$ & $\mathrm{Na}$ & $\mathrm{NaN}$ & $\mathrm{NaN}$ & $\mathrm{Na}$ & $\mathrm{NaN}$ & 0. & 0.12 & $\mathrm{NaN}$ & $\mathrm{NaN}$ & $\mathrm{NaN}$ & $\mathrm{NaN}$ & $\mathrm{NaN}$ & $\mathrm{NaN}$ & $\mathrm{NaN}$ & $\mathrm{NaN}$ & $\mathrm{NaN}$ & $\mathrm{NaN}$ \\
\hline DDX & & 1.5 & 3. & 0. & 1. & 1.8 & $1 . C$ & 1.0 & & & & 1. & & 0 & 1. & & & 0. & & & & & & & & & \\
\hline DDX52 & & 0. & & 1.3 & 1.0 & 1. & 0. & & & 1. & 0.5 & & & 1. & 0. & & 0. & 1.00 & 0.92 & 1.4 & 0. & 1.18 & 1.00 & 1.65 & 0.93 & 0.90 & 2.38 \\
\hline DDX5-ET & C11K54 & 0.45 & $\mathrm{NaN}$ & $\mathrm{NaN}$ & $\mathrm{NaN}$ & 0.11 & 0.31 & $\mathrm{NaN}$ & $\mathrm{NaN}$ & $\mathrm{NaN}$ & $\mathrm{Nal}$ & $\mathrm{NaN}$ & $\mathrm{NaN}$ & $\mathrm{NaN}$ & $\mathrm{NaN}$ & $\mathrm{NaN}$ & 0.17 & $\mathrm{NaN}$ & $\mathrm{NaN}$ & 0.04 & 0.28 & $\mathrm{NaN}$ & $\mathrm{NaN}$ & $\mathrm{NaN}$ & $\mathrm{NaN}$ & $\mathrm{NaN}$ & $\mathrm{NaN}$ \\
\hline DEFA3 & Q6EZE9 & 0.02 & $\mathrm{NaN}$ & $\mathrm{NaN}$ & $\mathrm{NaN}$ & $\mathrm{NaN}$ & $\mathrm{NaN}$ & $\mathrm{NaN}$ & $\mathrm{NaN}$ & $\mathrm{NaN}$ & $\mathrm{NaN}$ & $\mathrm{NaN}$ & $\mathrm{NaN}$ & $\mathrm{NaN}$ & $\mathrm{NaN}$ & $\mathrm{NaN}$ & $\mathrm{NaN}$ & $\mathrm{NaN}$ & $\mathrm{NaN}$ & $\mathrm{NaN}$ & $\mathrm{NaN}$ & $\mathrm{NaN}$ & $\mathrm{NaN}$ & $\mathrm{NaN}$ & $\mathrm{NaN}$ & $\mathrm{NaN}$ & $\mathrm{NaN}$ \\
\hline DFNA5 & H7C147 & 0.17 & $\mathrm{NaN}$ & & & & & & & & & & & & & & & & & & & & & & & & \\
\hline DFNA5 & & 0.41 & 0.15 & 0.66 & 0.81 & 0.73 & 0.25 & 1.17 & 0.3 & 0.5 & 0.71 & 0.38 & 0.52 & 0.68 & 0.88 & 0.34 & 0.13 & 1.45 & 0.53 & $\mathrm{NaN}$ & $\mathrm{NaN}$ & 0.57 & 1.03 & $\mathrm{NaN}$ & $\mathrm{NaN}$ & 1.25 & 0.49 \\
\hline DHX36 & Q9H $2 \mathrm{U} 1$ & 1.14 & 0.77 & 0.54 & 1.78 & 1.00 & 1.22 & 0.97 & 0.58 & 1.11 & 0.97 & 1.68 & 0.69 & 0.35 & 2.24 & 1.19 & 0.98 & 1.10 & 0.82 & 1.39 & 0.61 & 1.34 & 0.85 & 1.09 & 0.89 & 1.28 & 0.51 \\
\hline DLG & Q92796-3 & $\mathrm{NaN}$ & 0.22 & 0.62 & 0.66 & $\mathrm{NaN}$ & $\mathrm{NaN}$ & $\mathrm{NaN}$ & $\mathrm{NaN}$ & $\mathrm{NaN}$ & $\mathrm{NaN}$ & $\mathrm{NaN}$ & $\mathrm{NaN}$ & $\mathrm{NaN}$ & $\mathrm{NaN}$ & 0.35 & 0.36 & $\mathrm{NaN}$ & $\mathrm{NaN}$ & $\mathrm{NaN}$ & 0.76 & 51 & $\mathrm{NaN}$ & $\mathrm{NaN}$ & $\mathrm{NaN}$ & $\mathrm{NaN}$ & $\mathrm{NaN}$ \\
\hline DNAH8 & HoY7V4 & $\mathrm{NaN}$ & $\mathrm{NaN}$ & $\mathrm{NaN}$ & $\mathrm{NaN}$ & 116.99 & 0.85 & $\mathrm{NaN}$ & $\mathrm{NaN}$ & 1.02 & 1.04 & $\mathrm{NaN}$ & $\mathrm{NaN}$ & $\mathrm{NaN}$ & $\mathrm{NaN}$ & $\mathrm{NaN}$ & $\mathrm{NaN}$ & $\mathrm{NaN}$ & $\mathrm{NaN}$ & $\mathrm{NaN}$ & $\mathrm{NaN}$ & 146.80 & 0.48 & $\mathrm{NaN}$ & $\mathrm{NaN}$ & $\mathrm{NaN}$ & $\mathrm{NaN}$ \\
\hline
\end{tabular}




\begin{tabular}{|c|c|c|c|c|c|c|c|c|c|c|c|c|c|c|c|c|c|c|c|c|c|c|c|c|c|c|c|}
\hline Gene names & Protein IDs & $\begin{array}{l}\text { H3 } \Delta 1- \\
20 \_F\end{array}$ & $\begin{array}{c}\mathrm{H} 3 \Delta 1-1- \\
20 \_\mathrm{R}\end{array}$ & $\begin{array}{l}\text { H4K20 } \\
\text { me1_F }\end{array}$ & $\begin{array}{l}\text { H4K20 } \\
\text { me1_R }\end{array}$ & $\begin{array}{l}\text { H4K20 } \\
\text { me3_F }\end{array}$ & $\begin{array}{l}\text { H4K } 20 \\
\text { me3_R }\end{array}$ & $\begin{array}{c}\mathrm{H} 3 \mathrm{~K}_{\mathrm{C}} 27 \\
\text { me1_F }\end{array}$ & $\begin{array}{c}\mathrm{H}_{3} \mathrm{~K}_{\mathrm{C}} 27 \\
\text { me1_R }\end{array}$ & $\begin{array}{l}\mathrm{H} 3 \mathrm{~K}_{\mathrm{c}} 27 \\
\text { me2_F }\end{array}$ & $\mid \begin{array}{c}\mathrm{H}_{3} \mathrm{~K}_{\mathrm{C}} 27 \\
\mathrm{me2} \_\mathrm{R}\end{array}$ & $\begin{array}{l}\mathrm{H} 3 \mathrm{~K}_{\mathrm{C}} 27 \\
\mathrm{me3} \_\mathrm{F}\end{array}$ & $\begin{array}{l}\mathrm{H} 3 \mathrm{~K}_{\mathrm{C}} 27 \\
\text { me3_R }\end{array}$ & $\begin{array}{c}\mathrm{H} 3 \mathrm{~K} 9 \mathrm{~m} \\
\text { e1_F }\end{array}$ & \begin{tabular}{|c} 
H3K9m \\
e1_R
\end{tabular} & $\begin{array}{c}\text { H3k9m } \\
\text { e2_F }\end{array}$ & $\begin{array}{c}\text { H3K9m } \\
\text { e2_R }\end{array}$ & $\begin{array}{c}\text { HзК9m } \\
\text { e3_F }\end{array}$ & $\begin{array}{c}\text { H3K9m } \\
\text { e3_R }\end{array}$ & $\begin{array}{c}\text { H3K9m } \\
\text { e3|meC } \\
\text { pG_F }\end{array}$ & \begin{tabular}{|c|} 
HзK9m \\
e3/meC \\
pG_R
\end{tabular} & \begin{tabular}{|c}
$\mathrm{H} 3 \mathrm{~K} 9 \mathrm{~m}$ \\
$\mathrm{e}$ e3/H4K \\
$20 \mathrm{me} 3$ \\
$\mathrm{~F}$
\end{tabular} & $\mid \begin{array}{c}\mathrm{H} 3 \mathrm{~K} / \mathrm{m} \\
\mathrm{e}|\mathrm{H}| \mathrm{HK} \\
20 \mathrm{me}{ }_{-} \\
\mathrm{R}\end{array}$ & $\begin{array}{c}\mathrm{meCpG} \\
-\mathrm{F}\end{array} \mid$ & $\begin{array}{c}\mathrm{meCpG} \\
\_\mathrm{R}\end{array}$ & $\begin{array}{c}\text { H4R3m } \\
\text { e2_F }\end{array}$ & $\begin{array}{c}\text { H4R3m } \\
\text { e2_R }\end{array}$ \\
\hline DNMT1 & F5GX68 & 0.19 & 1.69 & 1.18 & 0.42 & 2.58 & 0.46 & 0.60 & 0.96 & 0.83 & 0.78 & 1.04 & 0.69 & 1.45 & 0.46 & 4.40 & 0.28 & 3.14 & 0.18 & \begin{tabular}{|l|}
56.86 \\
\end{tabular} & 0.01 & 1.82 & 0.38 & \begin{tabular}{|l|l|}
31.78 \\
\end{tabular} & 0.02 & 0.38 & 1.61 \\
\hline DNMT1 & P26358 & $\mathrm{NaN}$ & $\mathrm{NaN}$ & 1.48 & 0.38 & 2.75 & 0.50 & $\mathrm{NaN}$ & $\mathrm{NaN}$ & $\mathrm{NaN}$ & $\mathrm{NaN}$ & 0.95 & 0.70 & 1.41 & $\mathrm{NaN}$ & $\mathrm{NaN}$ & $\mathrm{NaN}$ & 3.32 & 0.20 & $\mathrm{NaN}$ & $\mathrm{NaN}$ & 1.96 & 0.44 & $\mathrm{NaN}$ & $\mathrm{NaN}$ & $\mathrm{NaN}$ & $\mathrm{NaN}$ \\
\hline DNMT1 & P26358-2 & $\mathrm{NaN}$ & $\mathrm{NaN}$ & 1.29 & 0.36 & 2.44 & 0.44 & $\mathrm{NaN}$ & $\mathrm{NaN}$ & $\mathrm{NaN}$ & $\mathrm{NaN}$ & 1.05 & 0.66 & $\mathrm{NaN}$ & 0.23 & $\mathrm{NaN}$ & $\mathrm{NaN}$ & 2.92 & 0.18 & 215.33 & 0.01 & 1.88 & 0.36 & 39.19 & 0.05 & $\mathrm{NaN}$ & $\mathrm{NaN}$ \\
\hline DSC1 & Q9HB00 & 0.08 & 0.07 & $\mathrm{NaN}$ & $\mathrm{NaN}$ & 0.13 & $\mathrm{NaN}$ & 0.06 & $\mathrm{NaN}$ & 0.37 & 0.09 & $\mathrm{NaN}$ & $\mathrm{NaN}$ & 0.68 & 1.08 & 0.08 & $\mathrm{NaN}$ & $\mathrm{NaN}$ & $\mathrm{NaN}$ & 0.06 & 0.13 & 0.13 & $\mathrm{NaN}$ & 0.17 & $\mathrm{NaN}$ & $\mathrm{NaN}$ & $\mathrm{NaN}$ \\
\hline DSC2 & Q68DY8 & $\mathrm{NaN}$ & $\mathrm{NaN}$ & $\mathrm{NaN}$ & $\mathrm{NaN}$ & $\mathrm{NaN}$ & $\mathrm{NaN}$ & 0.21 & $\mathrm{NaN}$ & $\mathrm{NaN}$ & $\mathrm{NaN}$ & $\mathrm{NaN}$ & $\mathrm{NaN}$ & $\mathrm{NaN}$ & $\mathrm{NaN}$ & $\mathrm{NaN}$ & $\mathrm{NaN}$ & $\mathrm{NaN}$ & $\mathrm{NaN}$ & $\mathrm{NaN}$ & $\mathrm{NaN}$ & $\mathrm{NaN}$ & $\mathrm{NaN}$ & $\mathrm{NaN}$ & $\mathrm{NaN}$ & $\mathrm{NaN}$ & $\mathrm{NaN}$ \\
\hline DSC3 & АВК6ТЗ & $\mathrm{NaN}$ & $\mathrm{NaN}$ & $\mathrm{NaN}$ & $\mathrm{NaN}$ & $\mathrm{NaN}$ & 1.00 & 0.12 & $\mathrm{NaN}$ & $\mathrm{NaN}$ & $\mathrm{NaN}$ & $\mathrm{NaN}$ & $\mathrm{NaN}$ & $\mathrm{NaN}$ & $\mathrm{NaN}$ & $\mathrm{NaN}$ & $\mathrm{NaN}$ & $\mathrm{NaN}$ & $\mathrm{NaN}$ & 0.11 & $\mathrm{NaN}$ & $\mathrm{NaN}$ & $\mathrm{NaN}$ & $\mathrm{NaN}$ & $\mathrm{NaN}$ & $\mathrm{NaN}$ & $\mathrm{NaN}$ \\
\hline DYM & J3QSE7 & $\mathrm{NaN}$ & $\mathrm{NaN}$ & $\mathrm{NaN}$ & $\mathrm{NaN}$ & $\mathrm{NaN}$ & $\mathrm{NaN}$ & $\mathrm{NaN}$ & $\mathrm{NaN}$ & $\mathrm{NaN}$ & $\mathrm{NaN}$ & $\mathrm{NaN}$ & $\mathrm{NaN}$ & $\mathrm{NaN}$ & $\mathrm{NaN}$ & $\mathrm{NaN}$ & 0.16 & $\mathrm{NaN}$ & $\mathrm{NaN}$ & $\mathrm{NaN}$ & $\mathrm{NaN}$ & $\mathrm{NaN}$ & $\mathrm{NaN}$ & $\mathrm{NaN}$ & $\mathrm{NaN}$ & $\mathrm{NaN}$ & $\mathrm{NaN}$ \\
\hline DYM & Q7RTS9 & 0.79 & 0.91 & 0.44 & 0.72 & 0.91 & 0.32 & 1.11 & 0.34 & 0.44 & 0.65 & 0.45 & 0.38 & 0.76 & 0.51 & 0.73 & 0.14 & 1.28 & 0.71 & 0.71 & $\mathrm{NaN}$ & 0.49 & 0.99 & $\mathrm{NaN}$ & $\mathrm{NaN}$ & 1.27 & 0.51 \\
\hline E2F1 & Q01094 & $\mathrm{NaN}$ & $\mathrm{NaN}$ & $\mathrm{NaN}$ & $\mathrm{NaN}$ & 0.65 & 0.50 & 0.62 & $\mathrm{NaN}$ & 0.46 & 0.46 & 0.54 & 0.74 & $\mathrm{NaN}$ & $\mathrm{NaN}$ & $\mathrm{NaN}$ & $\mathrm{NaN}$ & 0.52 & 0.36 & $\mathrm{NaN}$ & 0.38 & 0.51 & 0.45 & 0.23 & 1.85 & $\mathrm{NaN}$ & $\mathrm{NaN}$ \\
\hline E2F2 & Q5UOJO & $\mathrm{NaN}$ & $\mathrm{NaN}$ & $\mathrm{NaN}$ & $\mathrm{NaN}$ & 1.04 & 0.74 & $\mathrm{NaN}$ & $\mathrm{NaN}$ & 0.58 & $\mathrm{NaN}$ & $\mathrm{NaN}$ & $\mathrm{NaN}$ & $\mathrm{NaN}$ & $\mathrm{NaN}$ & $\mathrm{NaN}$ & $\mathrm{NaN}$ & 0.65 & 0.51 & $\mathrm{NaN}$ & $\mathrm{NaN}$ & 0.57 & 0.65 & 0.45 & 1.49 & $\mathrm{NaN}$ & $\mathrm{NaN}$ \\
\hline E2F6 & 075461 & 75 & $\mathrm{NaN}$ & 3.04 & 1.67 & 1.61 & 3.48 & 2.27 & 2.24 & 1.77 & 3.06 & 2.02 & 2.42 & 1.10 & 2.77 & 0.78 & 0.49 & 2.47 & 1.83 & 2.05 & 0.13 & 3.44 & 1.48 & 1.71 & 3.74 & 5.28 & $\mathrm{NaN}$ \\
\hline E4F1 & H3BUJ7 & $\mathrm{NaN}$ & 0.51 & 1.28 & 0.77 & 0.34 & 3.30 & 0.65 & $\mathrm{NaN}$ & 0.58 & 2.27 & 1.28 & 1.30 & 0.27 & 6.34 & 0.08 & 4.58 & 0.21 & 4.35 & 12.56 & 0.10 & 1.64 & 0.73 & 0.93 & 2.05 & $\mathrm{NaN}$ & $\mathrm{NaN}$ \\
\hline EED & 075530 & 0.29 & 1.91 & 0.89 & 0.64 & 0.90 & 0.66 & 0.65 & 0.90 & 0.81 & 0.74 & 0.76 & 0.77 & 0.50 & 1.51 & 1.31 & 0.76 & 1.46 & 0.33 & 2.34 & 0.29 & 1.41 & 0.35 & 1.01 & 1.60 & 0.71 & 0.60 \\
\hline EEF1B2 & P24534 & 0.65 & 0.34 & 1.08 & $\mathrm{NaN}$ & 0.52 & 1.02 & 0.40 & 2.66 & 0.76 & 0.49 & 0.79 & 0.61 & $\mathrm{NaN}$ & $\mathrm{NaN}$ & $\mathrm{NaN}$ & 0.80 & 0.33 & 1.20 & 0.66 & 0.87 & 0.90 & 0.57 & 1.35 & $\mathrm{NaN}$ & 1.22 & $\mathrm{NaN}$ \\
\hline EEF2 & P13639 & 0.88 & 0.41 & 1.39 & $\mathrm{NaN}$ & 1.10 & 0.97 & 0.40 & $\mathrm{NaN}$ & 1.35 & 0.60 & 1.12 & $\mathrm{NaN}$ & 1.50 & 0.93 & 0.74 & 0.39 & 1.02 & 0.85 & 0.18 & 1.50 & 0.61 & 0.86 & 0.97 & 0.38 & $\mathrm{NaN}$ & 0.88 \\
\hline EHMT1 & Q9H9B1 & 0.45 & 0.51 & 1.05 & 0.92 & 0.68 & 1.60 & 0.85 & 1.06 & 0.94 & 1.27 & 1.14 & 0.98 & 2.16 & 0.74 & 1.03 & 0.25 & 0.38 & 2.02 & 8.61 & 0.20 & 1.05 & 0.87 & 2.20 & 2.01 & 2.29 & 0.34 \\
\hline EHMT2 & A2ABF8 & 0.54 & 0.56 & 0.92 & 0.93 & 0.72 & 1.64 & 0.82 & 0.95 & 0.92 & 1.15 & 1.11 & 0.92 & 2.24 & 0.73 & 1.24 & 0.48 & 0.37 & 1.94 & 7.75 & 0.23 & 1.08 & 0.85 & 2.24 & 1.91 & 1.68 & 0.28 \\
\hline EIF2B5 & Q13144 & $\mathrm{NaN}$ & 0.73 & $\mathrm{NaN}$ & $\mathrm{NaN}$ & $\mathrm{NaN}$ & $\mathrm{NaN}$ & $\mathrm{NaN}$ & $\mathrm{NaN}$ & $\mathrm{NaN}$ & $\mathrm{NaN}$ & 22.40 & 0.95 & 1.34 & 1.12 & $\mathrm{NaN}$ & $\mathrm{NaN}$ & $\mathrm{NaN}$ & $\mathrm{NaN}$ & $\mathrm{NaN}$ & $\mathrm{NaN}$ & $\mathrm{NaN}$ & $\mathrm{NaN}$ & $\mathrm{NaN}$ & $\mathrm{NaN}$ & $\mathrm{NaN}$ & $\mathrm{NaN}$ \\
\hline EIF2S1 & Q53 XC० & 1.52 & 2.07 & 1.09 & 1.30 & 8.22 & 0.34 & 0.89 & 1.13 & 1.29 & 0.76 & 1.96 & 0.50 & 0.55 & 0.46 & 1.32 & 1.23 & 2.84 & 0.35 & $\mathrm{NaN}$ & 0.26 & 0.97 & 1.00 & 1.09 & 2.28 & 1.57 & 0.64 \\
\hline EIF2S2 & Q61BR8 & 0.93 & 1.39 & 0.71 & 0.83 & 8.30 & 0.33 & 1.11 & $\mathrm{NaN}$ & 1.28 & 0.74 & 2.18 & 0.46 & 0.45 & 0.43 & 1.18 & 2.24 & 2.71 & 0.33 & 2.23 & 0.28 & 0.95 & 1.08 & 0.90 & 1.06 & 1.50 & 0.53 \\
\hline EIF2S3 & P41091 & 0.82 & 1.35 & 1.01 & 1.02 & 7.23 & 0.32 & 0.88 & 1.04 & 1.16 & 0.67 & 2.08 & 0.52 & 0.79 & 0.72 & 1.15 & 0.98 & 2.37 & 0.34 & 2.61 & 0.62 & 0.88 & 1.03 & 1.23 & 1.24 & 0.95 & 0.52 \\
\hline EIF4A3 & P38919 & 4.31 & 0.92 & 0.48 & 1.40 & 0.83 & 0.61 & 0.79 & 0.90 & 2.11 & 0.30 & 1.08 & 0.97 & 1.37 & 0.99 & 2.02 & 1.56 & 1.05 & 0.56 & 1.37 & 2.38 & 0.58 & 1.24 & 1.96 & 1.34 & 0.56 & 0.55 \\
\hline EIF4G1 & E7EX73 & 1.30 & 1.24 & $\mathrm{NaN}$ & $\mathrm{NaN}$ & 0.66 & 0.62 & $\mathrm{NaN}$ & 0.71 & 1.14 & 0.38 & $\mathrm{NaN}$ & $\mathrm{NaN}$ & $\mathrm{NaN}$ & 0.61 & 1.50 & 0.14 & 0.63 & 0.74 & 1.17 & 1.34 & 0.35 & $\mathrm{NaN}$ & $\mathrm{NaN}$ & 0.88 & 0.62 & $\mathrm{NaN}$ \\
\hline EIF4H & Q15056 & $\mathrm{NaN}$ & 0.20 & $\mathrm{NaN}$ & $\mathrm{NaN}$ & $\mathrm{NaN}$ & $\mathrm{NaN}$ & $\mathrm{NaN}$ & $\mathrm{NaN}$ & $\mathrm{NaN}$ & $\mathrm{NaN}$ & $\mathrm{NaN}$ & $\mathrm{NaN}$ & $\mathrm{NaN}$ & $\mathrm{NaN}$ & 0.36 & 0.56 & $\mathrm{NaN}$ & $\mathrm{NaN}$ & 0.43 & 1.00 & $\mathrm{NaN}$ & $\mathrm{NaN}$ & 0.89 & 0.87 & $\mathrm{NaN}$ & $\mathrm{NaN}$ \\
\hline EIF5 & P55010 & $\mathrm{NaN}$ & 0.40 & $\mathrm{NaN}$ & $\mathrm{NaN}$ & 15.49 & 0.26 & $\mathrm{NaN}$ & $\mathrm{NaN}$ & $\mathrm{NaN}$ & $\mathrm{NaN}$ & $\mathrm{NaN}$ & $\mathrm{NaN}$ & $\mathrm{NaN}$ & $\mathrm{NaN}$ & $\mathrm{NaN}$ & $\mathrm{NaN}$ & 1.76 & 0.19 & $\mathrm{NaN}$ & 0.28 & $\mathrm{NaN}$ & 2.89 & 0.73 & 0.68 & $\mathrm{NaN}$ & $\mathrm{NaN}$ \\
\hline EFFB & Q8N5A0 & $\mathrm{NaN}$ & $\mathrm{NaN}$ & $\mathrm{NaN}$ & $\mathrm{NaN}$ & 6.17 & 0.19 & $\mathrm{NaN}$ & 0.86 & $\mathrm{NaN}$ & $\mathrm{NaN}$ & 0.86 & 0.62 & $\mathrm{NaN}$ & $\mathrm{NaN}$ & $\mathrm{NaN}$ & $\mathrm{NaN}$ & 0.88 & 0.21 & $\mathrm{NaN}$ & 0.24 & 0.39 & 1.02 & $\mathrm{NaN}$ & 1.53 & $\mathrm{NaN}$ & $\mathrm{NaN}$ \\
\hline ELF1 & P32519-2 & 0.80 & $\mathrm{NaN}$ & $\mathrm{NaN}$ & 1.02 & 3.68 & 0.40 & $\mathrm{NaN}$ & $\mathrm{NaN}$ & $\mathrm{NaN}$ & $\mathrm{NaN}$ & $\mathrm{NaN}$ & $\mathrm{NaN}$ & $\mathrm{NaN}$ & $\mathrm{NaN}$ & 0.19 & 3.47 & 2.27 & 0.34 & 3.10 & 0.24 & 2.38 & 0.57 & 0.47 & 1.85 & $\mathrm{NaN}$ & $\mathrm{NaN}$ \\
\hline ELF2 & B7Z720 & & 0.40 & $\mathrm{NaN}$ & $\mathrm{NaN}$ & 3.04 & 0.66 & $\mathrm{NaN}$ & & $\mathrm{NaN}$ & $\mathrm{NaN}$ & 1.99 & 0.55 & $\mathrm{NaN}$ & & 0.12 & 0.73 & & & 8.91 & & 2.59 & 0.55 & 0.58 & 2.58 & $\mathrm{NaN}$ & $\mathrm{NaN}$ \\
\hline ELF2 & Q15723-1 & NaN & $\mathrm{NaN}$ & $\mathrm{NaN}$ & $\mathrm{NaN}$ & $\mathrm{NaN}$ & $\mathrm{NaN}$ & $\mathrm{NaN}$ & $\mathrm{NaN}$ & $\mathrm{NaN}$ & $\mathrm{NaN}$ & $\mathrm{NaN}$ & $\mathrm{NaN}$ & $\mathrm{NaN}$ & $\mathrm{NaN}$ & $\mathrm{NaN}$ & $\mathrm{NaN}$ & $\mathrm{NaN}$ & $\mathrm{NaN}$ & $\mathrm{NaN}$ & $\mathrm{NaN}$ & $\mathrm{NaN}$ & $\mathrm{NaN}$ & $\mathrm{NaN}$ & $\mathrm{NaN}$ & $\mathrm{NaN}$ & $\mathrm{NaN}$ \\
\hline EMG1 & Q92979 & 0.51 & 0.79 & 0.84 & 0.87 & 7.42 & 0.27 & 1.02 & 0.93 & 1.61 & 2.89 & 1.04 & 0.62 & 0.75 & 0.62 & 0.53 & 0.15 & 2.79 & 0.25 & 2.15 & 0.49 & 0.72 & 0.96 & 1.43 & 1.85 & 0.85 & $\mathrm{NaN}$ \\
\hline ENO1 & P06733 & 0.18 & 0.07 & 3.44 & $\mathrm{NaN}$ & 1.91 & 0.98 & 0.25 & 2.61 & 1.90 & 0.36 & $\mathrm{NaN}$ & $\mathrm{NaN}$ & $\mathrm{NaN}$ & 1.03 & 0.16 & 0.16 & 0.79 & $\mathrm{NaN}$ & 0.32 & 1.67 & 0.46 & 0.45 & 1.01 & 0.27 & $\mathrm{NaN}$ & $\mathrm{NaN}$ \\
\hline EPPK1 & P58107 & $\mathrm{NaN}$ & 0.69 & 1.42 & 1.10 & 1.01 & 0.48 & 0.13 & $\mathrm{NaN}$ & $\mathrm{NaN}$ & 0.38 & 0.70 & 1.68 & 0.75 & 0.41 & 0.75 & 0.57 & 0.63 & & $\mathrm{NaN}$ & & 0.45 & 0.58 & 0.16 & $\mathrm{NaN}$ & $\mathrm{NaN}$ & $\mathrm{NaN}$ \\
\hline ESRRA & Q569H8 & $\mathrm{NaN}$ & $\mathrm{Nal}$ & $\mathrm{NaN}$ & 0.56 & 2.2 & 0.83 & 0.83 & 0.95 & 0.25 & 3.92 & 0.78 & 0.87 & $\mathrm{NaN}$ & $\mathrm{NaN}$ & $\mathrm{NaN}$ & $\mathrm{NaN}$ & 0.80 & 1.42 & 4.48 & 0.22 & 1.82 & 0.54 & $\mathrm{NaN}$ & $\mathrm{NaN}$ & 1.46 & 0.50 \\
\hline ETV6 & P41212 & 0.57 & 0.56 & 1.66 & 1.44 & 0.83 & 2.60 & 1.27 & 1.96 & 0.97 & 2.56 & 1.31 & 1.17 & 0.78 & 2.38 & 0.27 & 0.71 & 0.65 & 2.49 & 10.92 & 0.03 & 1.86 & 0.84 & 0.80 & 1.04 & 2.01 & 1.12 \\
\hline EXOC2 & Q96KP1 & 0.8 & 1.19 & 0.84 & 0.95 & 0.9 & 0.53 & 1.13 & 0.7 & 0.94 & 0.99 & 0.74 & 1.46 & 0.77 & 0.64 & 0.77 & 0.21 & 1.33 & 1.05 & $\mathrm{NaN}$ & $\mathrm{NaN}$ & 0.65 & 1.22 & $\mathrm{NaN}$ & $\mathrm{NaN}$ & 2.44 & 0.60 \\
\hline $\mathrm{EZH} 2$ & Q15910 & 0.32 & 1.99 & 0.77 & 0.71 & 1.06 & 0.94 & 0.73 & 0.94 & 0.87 & 0.98 & 0.90 & 1.03 & 0.59 & 1.42 & 1.34 & 1.02 & 1.49 & 0.45 & 1.85 & 0.94 & 1.38 & 0.42 & 1.13 & 1.69 & 0.99 & 0.74 \\
\hline FABP5 & E7DWW5 & $\mathrm{NaN}$ & $\mathrm{NaN}$ & $\mathrm{NaN}$ & $\mathrm{NaN}$ & $\mathrm{NaN}$ & $\mathrm{NaN}$ & 0.07 & & $\mathrm{NaN}$ & $\mathrm{NaN}$ & & $\mathrm{NaN}$ & $\mathrm{NaN}$ & $\mathrm{NaN}$ & & 0.18 & & $\mathrm{NaN}$ & & & & & & & $\mathrm{NaN}$ & $\mathrm{NaN}$ \\
\hline FAM114A1 & Q8IWE2 & $\mathrm{NaN}$ & 0.05 & $\mathrm{NaN}$ & $\mathrm{NaN}$ & $\mathrm{Nal}$ & $\mathrm{NaN}$ & $\mathrm{NaN}$ & $\mathrm{NaN}$ & $\mathrm{NaN}$ & $\mathrm{NaN}$ & $\mathrm{NaN}$ & $\mathrm{NaN}$ & $\mathrm{NaN}$ & $\mathrm{NaN}$ & $\mathrm{NaN}$ & $\mathrm{NaN}$ & $\mathrm{NaN}$ & $\mathrm{NaN}$ & $\mathrm{NaN}$ & $\mathrm{NaN}$ & $\mathrm{NaN}$ & $\mathrm{NaN}$ & $\mathrm{NaN}$ & $\mathrm{NaN}$ & $\mathrm{NaN}$ & $\mathrm{NaN}$ \\
\hline FAM129B & Q96TA1-2 & 1.31 & 1.04 & $\mathrm{NaN}$ & $\mathrm{Na}$ & 0.8 & 0.50 & 0.80 & $\mathrm{Na}$ & $\mathrm{Na}$ & $\mathrm{NaN}$ & 1.2 & $\mathrm{Nal}$ & $\mathrm{NaN}$ & $\mathrm{NaN}$ & 1.46 & 0.93 & 0.29 & $\mathrm{NaN}$ & 0.26 & $\mathrm{NaN}$ & 0.90 & 1.12 & 0.92 & 1.09 & 0.01 & 0.56 \\
\hline FAM 134C & Q86VR2 & $\mathrm{NaN}$ & 0.22 & $\mathrm{NaN}$ & $\mathrm{N}$ & $\mathrm{Na}$ & $\mathrm{NaN}$ & $\mathrm{NaN}$ & $\mathrm{NaN}$ & $\mathrm{NaN}$ & $\mathrm{NaN}$ & $\mathrm{NaN}$ & $\mathrm{NaN}$ & $\mathrm{NaN}$ & $\mathrm{NaN}$ & 0.62 & $\mathrm{NaN}$ & $\mathrm{NaN}$ & $\mathrm{NaN}$ & $\mathrm{NaN}$ & $\mathrm{NaN}$ & $\mathrm{NaN}$ & $\mathrm{NaN}$ & $\mathrm{NaN}$ & $\mathrm{NaN}$ & $\mathrm{NaN}$ & $\mathrm{NaN}$ \\
\hline FAM48A & B3KN11 & $\mathrm{NaN}$ & 0.2 & 0.54 & 0.41 & 0.4 & 0.9 & $\mathrm{NaN}$ & $\mathrm{NaN}$ & $\mathrm{NaN}$ & $\mathrm{NaN}$ & 0.94 & 0.65 & $\mathrm{NaN}$ & $\mathrm{NaN}$ & 0.33 & $\mathrm{NaN}$ & 0.50 & 0.93 & $\mathrm{NaN}$ & $\mathrm{N}$ & 1.16 & 0.32 & $\mathrm{NaN}$ & $\mathrm{NaN}$ & $\mathrm{NaN}$ & $\mathrm{NaN}$ \\
\hline FBXL6 & Q8N531 & 0.68 & 0.3 & 0.95 & 0.54 & 0.6 & 0.8 & 1.09 & 0.40 & 0.69 & 0.64 & 0.90 & 0.41 & 0.42 & 1.61 & 0.62 & $\mathrm{NaN}$ & 0.6 & 0.65 & 2.90 & 0. & 0.75 & 0.67 & 0.60 & 1.03 & 0.49 & 0.72 \\
\hline FBXW11 & B4DH70 & $\mathrm{Na}$ & 2.1 & 1.02 & & 0.6 & 1.1 & 0.82 & 0.8 & 0.7 & 1.0 & & 0.8 & 0.45 & 2.58 & $\mathrm{Na}$ & $\mathrm{NaN}$ & 0.5 & 1.11 & 8.04 & 0.2 & 0.91 & 0.77 & 1.16 & 1.11 & $\mathrm{NaN}$ & 0.53 \\
\hline FGF2 & D9ZGF5 & & 1.98 & 0.50 & & 0. & 0.84 & 0.81 & $\mathrm{Na}$ & 1.2 & 4.1 & & $\mathrm{Nal}$ & $\mathrm{Nan}$ & $\mathrm{NaN}$ & 1.5 & 4.47 & 0.82 & 0.72 & 0.7 & 0.4 & 0.70 & & 0.86 & 0.46 & 0.44 & 0.76 \\
\hline $\mathrm{FIZ1}$ & Q96SL8 & 0.81 & 0.39 & 0.64 & 0.82 & 0.46 & 1.52 & 0.80 & 0.77 & 0.52 & 0.64 & 0.71 & 0.62 & 0.29 & 1.40 & 0.62 & 6.13 & 0.28 & 1.13 & 9.62 & 0.04 & 0.58 & 0.50 & 6.84 & 0.17 & 1.19 & 0.30 \\
\hline FMNL1 & $16 \mathrm{~L} 919$ & $\mathrm{NaN}$ & 0.15 & $\mathrm{NaN}$ & $\mathrm{NaN}$ & $\mathrm{NaN}$ & $\mathrm{NaN}$ & $\mathrm{NaN}$ & & $\mathrm{NaN}$ & $\mathrm{NaN}$ & & & $\mathrm{NaN}$ & & & $\mathrm{NaN}$ & & $\mathrm{NaN}$ & & & & & & & 1.30 & 0.63 \\
\hline FMN & O95466-2 & 0.8 & 0.13 & 0.70 & 1.0 & 0.8 & 0.34 & $\mathrm{NaN}$ & $\mathrm{Na}$ & $\mathrm{Nal}$ & $\mathrm{NaN}$ & 0.84 & $\mathrm{Na}$ & $\mathrm{NaN}$ & $\mathrm{NaN}$ & 0.6 & 2.51 & 1.12 & 1.00 & 1.21 & 0.99 & 1.05 & 1.18 & $\mathrm{NaN}$ & $\mathrm{NaN}$ & $\mathrm{NaN}$ & 0.60 \\
\hline FNBP4 & D3DQS4 & $\mathrm{Na}$ & 2.29 & $\mathrm{NaN}$ & $\mathrm{NaN}$ & $\mathrm{NaN}$ & $\mathrm{NaN}$ & $\mathrm{NaN}$ & $\mathrm{NaN}$ & $\mathrm{NaN}$ & $\mathrm{NaN}$ & $\mathrm{NaN}$ & $\mathrm{NaN}$ & $\mathrm{NaN}$ & $\mathrm{NaN}$ & 1.9 & 0.96 & $\mathrm{NaN}$ & $\mathrm{NaN}$ & $\mathrm{NaN}$ & $\mathrm{NaN}$ & $\mathrm{NaN}$ & $\mathrm{NaN}$ & $\mathrm{NaN}$ & $\mathrm{NaN}$ & $\mathrm{NaN}$ & $\mathrm{NaN}$ \\
\hline FOXN2 & Q6IS90 & 0.42 & $\mathrm{NaN}$ & $\mathrm{NaN}$ & $\mathrm{NaN}$ & $\mathrm{NaN}$ & $\mathrm{NaN}$ & $\mathrm{NaN}$ & $\mathrm{NaN}$ & $\mathrm{NaN}$ & $\mathrm{NaN}$ & $\mathrm{NaN}$ & 0.84 & 1.17 & 0.78 & $\mathrm{NaN}$ & 0.34 & $\mathrm{NaN}$ & $\mathrm{NaN}$ & $\mathrm{NaN}$ & $\mathrm{NaN}$ & 0.70 & $\mathrm{NaN}$ & $\mathrm{NaN}$ & $\mathrm{NaN}$ & $\mathrm{NaN}$ & $\mathrm{NaN}$ \\
\hline FRMPD3 & Q5JV73 & 0.07 & $\mathrm{NaN}$ & 0.95 & 0.85 & 0.29 & 0.88 & $\mathrm{NaN}$ & $\mathrm{NaN}$ & $\mathrm{NaN}$ & 0.04 & 7.21 & 0.78 & 7.64 & 1.26 & 0.94 & $\mathrm{NaN}$ & 0.56 & 0.21 & 0.38 & 0.09 & 0.40 & 2.45 & 0.10 & $\mathrm{NaN}$ & 0.33 & 0.25 \\
\hline G2E3 & F5GX24 & & 0.3 & 1.30 & 1.13 & 1.2 & & 0.2 & 2.6 & 0.5 & 1.3 & 0.2 & 4.19 & 1.36 & & 0.7 & 0.6 & & & 0.46 & 12.76 & & 1.18 & 2.66 & 1.57 & 0.66 & 1.18 \\
\hline GATAD2A & Q86YP4 & 0.39 & 0.90 & 1.01 & 0.72 & 0.95 & 0.84 & 0.98 & 0.96 & 1.01 & 0.82 & 0.92 & 0.85 & 0.41 & 1.45 & 0.67 & 0.87 & 0.68 & 1.10 & 5.61 & 0.05 & 0.69 & 1.03 & 4.53 & 0.15 & 1.45 & 0.52 \\
\hline GATAD2B & Q8WX19 & 0.43 & 0.85 & 0.81 & 0.61 & 0.85 & 0.61 & 0.66 & 0.72 & 0.82 & 0.69 & 0.76 & 0.68 & 0.34 & 1.32 & 0.69 & 0.50 & 0.58 & 0.92 & 5.81 & 0.03 & 0.57 & 0.90 & 5.07 & 0.10 & 0.99 & 0.42 \\
\hline GBP1 & Q5D1D5 & $\mathrm{NaN}$ & $\mathrm{NaN}$ & $\mathrm{NaN}$ & $\mathrm{NaN}$ & $\mathrm{NaN}$ & $\mathrm{NaN}$ & 0.04 & 0.50 & $\mathrm{NaN}$ & 0.25 & 0.91 & $\mathrm{NaN}$ & $\mathrm{NaN}$ & $\mathrm{NaN}$ & $\mathrm{NaN}$ & $\mathrm{NaN}$ & $\mathrm{NaN}$ & $\mathrm{NaN}$ & 0.10 & $\mathrm{NaN}$ & $\mathrm{NaN}$ & 0.04 & $\mathrm{NaN}$ & $\mathrm{NaN}$ & $\mathrm{NaN}$ & $\mathrm{NaN}$ \\
\hline GCC2 & Q8IWJ2 & $\mathrm{NaN}$ & 0.33 & 1.10 & $\mathrm{NaN}$ & $\mathrm{NaN}$ & 0.66 & 1.02 & 0.21 & $\mathrm{Nal}$ & 0.89 & 0.78 & $\mathrm{Nal}$ & $\mathrm{NaN}$ & $\mathrm{NaN}$ & $\mathrm{Na}$ & 0.14 & 1.77 & 1.16 & $\mathrm{NaN}$ & 0.13 & 0.99 & 1.10 & $\mathrm{NaN}$ & & 0.85 & 1.58 \\
\hline GDI2 & Q5S X86 & $\mathrm{NaN}$ & $\mathrm{NaN}$ & $\mathrm{NaN}$ & $\mathrm{NaN}$ & $\mathrm{NaN}$ & $\mathrm{NaN}$ & $\mathrm{NaN}$ & $\mathrm{NaN}$ & $\mathrm{NaN}$ & $\mathrm{NaN}$ & 0.71 & $\mathrm{NaN}$ & $\mathrm{NaN}$ & $\mathrm{NaN}$ & $\mathrm{NaN}$ & $\mathrm{NaN}$ & $\mathrm{NaN}$ & $\mathrm{NaN}$ & $\mathrm{NaN}$ & $\mathrm{NaN}$ & $\mathrm{NaN}$ & $\mathrm{NaN}$ & $\mathrm{NaN}$ & $\mathrm{NaN}$ & $\mathrm{NaN}$ & 1.91 \\
\hline GDI2 & Q6IAT1 & 2.61 & 0.14 & 1.74 & $\mathrm{NaN}$ & $\mathrm{NaN}$ & $\mathrm{NaN}$ & 0.23 & $\mathrm{NaN}$ & $\mathrm{NaN}$ & $\mathrm{NaN}$ & $\mathrm{NaN}$ & $\mathrm{NaN}$ & $\mathrm{NaN}$ & $\mathrm{NaN}$ & 0.71 & $\mathrm{NaN}$ & $\mathrm{NaN}$ & $\mathrm{NaN}$ & 0.20 & 1.37 & $\mathrm{NaN}$ & $\mathrm{NaN}$ & 0.87 & 0.26 & $\mathrm{NaN}$ & $\mathrm{NaN}$ \\
\hline GET4 & Q7L5D6 & 2.59 & 0.73 & 0.63 & 0.82 & 1.11 & 0.53 & 1.26 & 0.46 & 0.57 & 0.76 & 0.51 & 0.83 & 1.65 & 0.84 & 1.88 & 0.17 & 1.30 & 0.55 & $\mathrm{NaN}$ & $\mathrm{NaN}$ & 0.98 & 0.99 & $\mathrm{NaN}$ & $\mathrm{NaN}$ & 2.23 & 0.70 \\
\hline
\end{tabular}




\begin{tabular}{|c|c|c|c|c|c|c|c|c|c|c|c|c|c|c|c|c|c|c|c|c|c|c|c|c|c|c|c|}
\hline Gene names & Protein IDs & $\begin{array}{l}\mathrm{H} 3 \Delta \mathrm{H}^{-} \\
20 \_\mathrm{F}\end{array}$ & $\begin{array}{l}\mathrm{H} 3 \Delta \Delta-1- \\
20 \_R\end{array}$ & $\begin{array}{l}\text { H4K20 } \\
\text { me1_F }\end{array}$ & $\begin{array}{l}\text { H4K20 } \\
\text { me1_R }\end{array}$ & $\begin{array}{l}\text { H4K20 } \\
\text { me3_F }\end{array}$ & $\begin{array}{l}\mathrm{H} 4 \mathrm{~K} 20 \\
\text { me3_R }\end{array}$ & $\begin{array}{c}\mathrm{H}^{\mathrm{K}} \mathrm{K}_{\mathrm{C}} 27 \\
\text { me1_F }\end{array}$ & $\begin{array}{l}\mathrm{H} 3 \mathrm{~K}_{\mathrm{C}} 27 \\
\mathrm{me1} \mathrm{R}\end{array}$ & $\begin{array}{l}\mathrm{H}_{3} \mathrm{~K}_{\mathrm{c}} 27 \\
\text { me2_F }\end{array}$ & $\begin{array}{l}\mathrm{H} 3 \mathrm{~K}_{\mathrm{c}} 27 \\
\mathrm{me2} \_\mathrm{R}\end{array}$ & 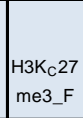 & $\begin{array}{c}\mathrm{H}_{3} \mathrm{~K}_{\mathrm{C}} 27 \\
\mathrm{me} \_\mathrm{R}\end{array}$ & $\begin{array}{c}\text { H3к9m } \\
\text { e1_F }\end{array}$ & \begin{tabular}{|c} 
НзК9m \\
e1_R
\end{tabular} & \begin{tabular}{|c|}
$\mathrm{H} 3 \mathrm{~K} 9 \mathrm{~m}$ \\
e2_F
\end{tabular} & $\begin{array}{c}\text { H3K9m } \\
\text { e2_R }\end{array}$ & \begin{tabular}{|c|} 
H3K9m \\
e3_F
\end{tabular} & \begin{tabular}{|c} 
HзкK9m \\
e3_R
\end{tabular} & \begin{tabular}{|c|}
$\mathrm{H} 3 \mathrm{~K} 9 \mathrm{~m}$ \\
$\mathrm{e} 3 \mathrm{meC}$ \\
$\mathrm{pG} \_\mathrm{F}$
\end{tabular} & $\begin{array}{c}\text { HзKलm } \\
\text { e3/meC } \\
\text { pG_R }\end{array}$ & $\mid \begin{array}{c}\mathrm{H} 3 \mathrm{~K} 9 \mathrm{~m} \\
\mathrm{e}|\mathrm{H}| \mathrm{KK} \\
20 \mathrm{me} 3 \\
\mathrm{~F}\end{array}$ & $\begin{array}{c}\mathrm{H} 3 \mathrm{~K} 9 \mathrm{~m} \\
\mathrm{e} 3 \mid \mathrm{H} / \mathrm{K} \\
20 \mathrm{me3} \\
\mathrm{R}\end{array}$ & $\begin{array}{c}\mathrm{meCpG} \\
-\mathrm{F}\end{array}$ & $\begin{array}{c}\mathrm{meCpg} \\
{ }^{\mathrm{R}}\end{array}$ & $\begin{array}{c}\text { H4R3m } \\
\text { e2_F }\end{array}$ & $\begin{array}{c}\text { H4R3m } \\
\text { e2_R }\end{array}$ \\
\hline GGNBP2 & A8K3S2 & 0.44 & $\mathrm{NaN}$ & $\mathrm{NaN}$ & $\mathrm{NaN}$ & $\mathrm{NaN}$ & $\mathrm{NaN}$ & $\mathrm{NaN}$ & $\mathrm{NaN}$ & $\mathrm{NaN}$ & $\mathrm{NaN}$ & $\mathrm{NaN}$ & $\mathrm{NaN}$ & $\mathrm{NaN}$ & $\mathrm{NaN}$ & 0.63 & 0.46 & $\mathrm{NaN}$ & $\mathrm{NaN}$ & $\mathrm{NaN}$ & $\mathrm{NaN}$ & $\mathrm{NaN}$ & $\mathrm{NaN}$ & $\mathrm{NaN}$ & $\mathrm{NaN}$ & $\mathrm{NaN}$ & $\mathrm{NaN}$ \\
\hline GNB2L1 & E9KL35 & 1.08 & 1.05 & $\mathrm{NaN}$ & $\mathrm{NaN}$ & 1.24 & 0.99 & 0.25 & $\mathrm{NaN}$ & $\mathrm{NaN}$ & 0.43 & $\mathrm{NaN}$ & $\mathrm{NaN}$ & 0.64 & 1.33 & 1.01 & 0.23 & 1.26 & $\mathrm{NaN}$ & $\mathrm{NaN}$ & 1.02 & 1.28 & 1.31 & 0.68 & 0.68 & 1.40 & $\mathrm{NaN}$ \\
\hline GOLGB1 & Q14789 & $\mathrm{NaN}$ & 0.24 & 0.08 & 0.42 & 0.60 & 0.12 & $\mathrm{NaN}$ & $\mathrm{NaN}$ & $\mathrm{NaN}$ & $\mathrm{NaN}$ & 0.05 & $\mathrm{NaN}$ & $\mathrm{NaN}$ & 1.33 & $\mathrm{NaN}$ & $\mathrm{NaN}$ & 1.12 & 0.67 & $\mathrm{NaN}$ & $\mathrm{NaN}$ & 0.67 & 0.81 & $\mathrm{NaN}$ & $\mathrm{NaN}$ & 0.97 & 1.29 \\
\hline GOLT1B & G3VIUL & $\mathrm{NaN}$ & $\mathrm{NaN}$ & $\mathrm{NaN}$ & $\mathrm{NaN}$ & $\mathrm{NaN}$ & 0.19 & $\mathrm{NaN}$ & $\mathrm{NaN}$ & $\mathrm{NaN}$ & $\mathrm{NaN}$ & $\mathrm{NaN}$ & $\mathrm{NaN}$ & $\mathrm{NaN}$ & $\mathrm{NaN}$ & $\mathrm{NaN}$ & 0.04 & $\mathrm{NaN}$ & 1.39 & $\mathrm{NaN}$ & $\mathrm{NaN}$ & $\mathrm{NaN}$ & $\mathrm{NaN}$ & $\mathrm{NaN}$ & $\mathrm{NaN}$ & $\mathrm{NaN}$ & $\mathrm{NaN}$ \\
\hline GSTP1 & P09211 & $\mathrm{NaN}$ & $\mathrm{NaN}$ & $\mathrm{NaN}$ & $\mathrm{NaN}$ & $\mathrm{NaN}$ & $\mathrm{NaN}$ & 0.10 & $\mathrm{NaN}$ & $\mathrm{NaN}$ & $\mathrm{NaN}$ & $\mathrm{NaN}$ & $\mathrm{NaN}$ & $\mathrm{NaN}$ & $\mathrm{NaN}$ & $\mathrm{NaN}$ & 0.70 & $\mathrm{NaN}$ & $\mathrm{NaN}$ & $\mathrm{NaN}$ & $\mathrm{NaN}$ & $\mathrm{NaN}$ & $\mathrm{NaN}$ & $\mathrm{NaN}$ & $\mathrm{NaN}$ & $\mathrm{NaN}$ & $\mathrm{NaN}$ \\
\hline GTF2E2 & P29084 & $\mathrm{NaN}$ & $\mathrm{NaN}$ & $\mathrm{NaN}$ & 0.59 & 1.47 & 0.50 & 0.79 & 2.24 & 0.94 & 1.66 & 0.57 & 2.17 & 0.31 & 3.64 & 0.68 & 0.79 & $\mathrm{NaN}$ & $\mathrm{NaN}$ & $\mathrm{NaN}$ & $\mathrm{NaN}$ & 1.66 & 0.95 & $\mathrm{NaN}$ & 1.73 & $\mathrm{NaN}$ & $\mathrm{NaN}$ \\
\hline GTF3C4 & Q9UKN8 & 0.81 & 0.55 & 0.51 & 0.55 & 0.71 & 0.66 & 0.51 & $\mathrm{NaN}$ & 0.51 & 0.89 & 0.81 & 0.56 & 0.53 & 0.95 & 0.80 & 0.33 & 0.83 & 0.46 & 5.58 & 0.34 & 1.82 & 0.17 & 0.80 & $\mathrm{NaN}$ & $\mathrm{NaN}$ & $\mathrm{NaN}$ \\
\hline GTF3C5 & Q5T7U4 & $\mathrm{NaN}$ & $\mathrm{NaN}$ & $\mathrm{NaN}$ & $\mathrm{NaN}$ & $\mathrm{NaN}$ & $\mathrm{NaN}$ & $\mathrm{NaN}$ & $\mathrm{NaN}$ & $\mathrm{NaN}$ & $\mathrm{NaN}$ & $\mathrm{NaN}$ & $\mathrm{NaN}$ & $\mathrm{NaN}$ & $\mathrm{NaN}$ & $\mathrm{NaN}$ & $\mathrm{NaN}$ & $\mathrm{NaN}$ & $\mathrm{NaN}$ & $\mathrm{NaN}$ & $\mathrm{NaN}$ & $\mathrm{NaN}$ & $\mathrm{NaN}$ & $\mathrm{NaN}$ & $\mathrm{NaN}$ & $\mathrm{NaN}$ & $\mathrm{NaN}$ \\
\hline GTF3C5 & Q9Y5Q8 & 0.62 & 0.47 & 0.45 & 0.7 & 0.66 & 0.53 & 0.47 & $\mathrm{Nal}$ & 0.37 & 0.84 & 0.70 & 0.51 & 0.40 & 1.24 & 0.68 & 0.18 & 0.96 & 0.37 & 9.10 & 0.11 & 2.15 & 17 & 0.81 & 1.55 & $\mathrm{NaN}$ & 0.69 \\
\hline HDGF & P51858 & $\mathrm{NaN}$ & 0.10 & $\mathrm{NaN}$ & $\mathrm{NaN}$ & $\mathrm{NaN}$ & $\mathrm{NaN}$ & $\mathrm{NaN}$ & $\mathrm{NaN}$ & $\mathrm{NaN}$ & $\mathrm{NaN}$ & $\mathrm{NaN}$ & $\mathrm{NaN}$ & $\mathrm{NaN}$ & $\mathrm{NaN}$ & $\mathrm{NaN}$ & $\mathrm{NaN}$ & $\mathrm{NaN}$ & $\mathrm{NaN}$ & $\mathrm{NaN}$ & $\mathrm{NaN}$ & $\mathrm{NaN}$ & $\mathrm{NaN}$ & $\mathrm{NaN}$ & $\mathrm{NaN}$ & $\mathrm{NaN}$ & $\mathrm{NaN}$ \\
\hline HDGFRP2 & Q7Z4V5-2 & $\mathrm{NaN}$ & 0.57 & 8.74 & 0.20 & 0.70 & 0.77 & $\mathrm{NaN}$ & 0.65 & 0.78 & $\mathrm{NaN}$ & 0.52 & $\mathrm{NaN}$ & $\mathrm{NaN}$ & $\mathrm{NaN}$ & $\mathrm{NaN}$ & $\mathrm{NaN}$ & 0.92 & 0.47 & $\mathrm{NaN}$ & $\mathrm{NaN}$ & $\mathrm{NaN}$ & $\mathrm{NaN}$ & $\mathrm{NaN}$ & $\mathrm{NaN}$ & $\mathrm{NaN}$ & $\mathrm{NaN}$ \\
\hline HEATR2 & Q86Y56 & 1.08 & 0.72 & 0.88 & 0.99 & 1.21 & 0.53 & 1.72 & $\mathrm{NaN}$ & 0.20 & 1.30 & 0.42 & 0.85 & 1.14 & 0.98 & 0.93 & 0.56 & 1.86 & 0.99 & 0.33 & $\mathrm{NaN}$ & 0.67 & 1.21 & $\mathrm{NaN}$ & $\mathrm{NaN}$ & 2.21 & 0.77 \\
\hline HES1 & |Q14469 & 0.79 & 1.39 & 2.08 & 0.44 & 1.02 & 1.41 & 1.23 & 1.26 & 0.82 & 1.50 & 1.13 & 1.04 & $\mathrm{NaN}$ & $\mathrm{NaN}$ & 0.52 & 0.95 & 0.79 & 1.38 & $\mathrm{NaN}$ & 0.22 & 1.53 & 0.81 & 0.44 & 4.35 & 3.09 & $\mathrm{NaN}$ \\
\hline HIVEP2 & P31629 & 1.30 & 3.32 & 0.69 & $\mathrm{NaN}$ & 0.44 & 1.34 & $\mathrm{NaN}$ & $\mathrm{NaN}$ & $\mathrm{NaN}$ & $\mathrm{NaN}$ & 0.63 & 0.52 & 0.55 & $\mathrm{NaN}$ & $\mathrm{NaN}$ & $\mathrm{NaN}$ & 0.42 & $\mathrm{NaN}$ & 4.31 & 0.58 & 0.73 & 0.42 & $\mathrm{NaN}$ & $\mathrm{NaN}$ & $\mathrm{NaN}$ & $\mathrm{NaN}$ \\
\hline HMG20A & Q9NP66 & 0.12 & 1.27 & 1.51 & 1.00 & 1.16 & 1.34 & 1.07 & 1.23 & 1.07 & 1.59 & 1.13 & 1.29 & $\mathrm{NaN}$ & $\mathrm{NaN}$ & 0.49 & 0.64 & 1.09 & 1.16 & 0.84 & 1.42 & 1.11 & 1.02 & 1.01 & 1.12 & 1.17 & 1.90 \\
\hline HMGA1 & P17096 & 2.15 & 2.37 & 0.55 & 0.93 & 0.93 & 0.78 & 0.54 & 0.54 & 0.67 & 0.59 & 0.91 & 0.65 & 0.69 & 0.76 & 2.09 & 2.24 & 0.92 & 0.64 & 1.28 & 1.31 & 0.75 & 0.64 & 1.37 & 1.27 & 0.49 & 0.90 \\
\hline HMGB3 & 015347 & 0.30 & 1.62 & 1.14 & 1.80 & 5.09 & 0.89 & 1.06 & 1.57 & 1.47 & 0.95 & 0.46 & $\mathrm{NaN}$ & $\mathrm{NaN}$ & $\mathrm{NaN}$ & 0.28 & $\mathrm{NaN}$ & 1.23 & 0.50 & 4.72 & 0.91 & 1.25 & 0.81 & 2.74 & 1.92 & $\mathrm{NaN}$ & 0.93 \\
\hline HMGN5 & Q5JSLO & $\mathrm{NaN}$ & $\mathrm{NaN}$ & $\mathrm{NaN}$ & $\mathrm{NaN}$ & 18.80 & 0.09 & $\mathrm{NaN}$ & $\mathrm{NaN}$ & $\mathrm{NaN}$ & $\mathrm{N}$ & $\mathrm{Na}$ & $\mathrm{NaN}$ & $\mathrm{NaN}$ & $\mathrm{Na}$ & $\mathrm{NaN}$ & $\mathrm{NaN}$ & & & & $\mathrm{NaN}$ & & & & & $\mathrm{NaN}$ & $\mathrm{NaN}$ \\
\hline HSP9OAB & P08238 & 1.29 & 0.95 & 1.17 & 1.09 & 1.25 & 0.75 & 0.59 & 1.67 & 1.12 & 0.52 & 0.70 & 0.80 & 0.94 & 1.35 & 1.60 & 1.27 & 0.96 & 0.84 & 0.28 & 2.48 & 0.74 & 0.75 & 1.11 & 0.62 & 1.08 & 0.72 \\
\hline HSPA9 & B7Z4V2 & 1.19 & 1.28 & 0.48 & 0.59 & 0.50 & 0.51 & 0.48 & $\mathrm{NaN}$ & 0.40 & 0.55 & 0.39 & 0.43 & $\mathrm{NaN}$ & 0.81 & 1.11 & 0.97 & 0.39 & 0.50 & $\mathrm{NaN}$ & 0.48 & 0.57 & 0.31 & $\mathrm{NaN}$ & $\mathrm{NaN}$ & $\mathrm{NaN}$ & 2.30 \\
\hline HTRA1 & Q05DJ8 & 0.15 & 0.24 & 0.33 & 0.44 & 0.34 & 0.40 & 0.32 & 0.61 & 0.73 & 0.60 & 0.32 & 1.08 & 0.42 & 0.34 & $\mathrm{NaN}$ & $\mathrm{NaN}$ & 0.45 & 0.83 & 0.24 & $\mathrm{NaN}$ & 0.39 & 0.37 & $\mathrm{NaN}$ & $\mathrm{NaN}$ & 0.29 & 0.30 \\
\hline HYDIN & $\mathrm{Q}$ & $\mathrm{Na}$ & $\mathrm{NaN}$ & & 1.73 & 0.7 & & 1.3 & 1.54 & & & 1.72 & 1.47 & & $\mathrm{Na}$ & $\mathrm{NaN}$ & $\mathrm{NaN}$ & 1.1 & & & & & & & 0.12 & & $\mathrm{NaN}$ \\
\hline IFI16 & D3DUZ3 & 0.26 & 1.71 & 0.45 & 1.04 & 0.58 & 0.89 & 0.44 & 0.63 & 0.69 & 0.69 & 0.85 & 0.72 & 0.49 & 1.09 & 0.32 & 7.31 & 0.59 & 0.78 & 3.99 & 0.04 & 0.66 & 0.68 & 0.96 & 0.44 & 0.87 & 0.30 \\
\hline IFI16 & Q16666 & 0.29 & 1.81 & 0.54 & 1.12 & 0.65 & 0.97 & 0.55 & 0.75 & 0.79 & 0.79 & 0.94 & 0.81 & 0.57 & 1.29 & 0.33 & 4.45 & 0.63 & 0.90 & 10.65 & 0.01 & 0.76 & 0.78 & 0.99 & 0.50 & 1.13 & 0.34 \\
\hline IGF1 & Q9NP 10 & 0.06 & $\mathrm{NaN}$ & $\mathrm{NaN}$ & $\mathrm{NaN}$ & $\mathrm{NaN}$ & $\mathrm{NaN}$ & $\mathrm{NaN}$ & $\mathrm{NaN}$ & $\mathrm{NaN}$ & $\mathrm{NaN}$ & $\mathrm{NaN}$ & $\mathrm{NaN}$ & $\mathrm{NaN}$ & $\mathrm{NaN}$ & 0.04 & $\mathrm{NaN}$ & $\mathrm{NaN}$ & $\mathrm{NaN}$ & $\mathrm{NaN}$ & $\mathrm{NaN}$ & $\mathrm{NaN}$ & $\mathrm{NaN}$ & $\mathrm{NaN}$ & $\mathrm{NaN}$ & $\mathrm{NaN}$ & $\mathrm{NaN}$ \\
\hline IGF2 & E3UN46 & 0.19 & $\mathrm{NaN}$ & & & $\mathrm{NaN}$ & & $\mathrm{NaN}$ & $\mathrm{Nan}$ & $\mathrm{NaN}$ & & $\mathrm{NaN}$ & $\mathrm{NaN}$ & & $\mathrm{NaN}$ & 0.34 & $\mathrm{NaN}$ & & & $\mathrm{NaN}$ & & & & & $\mathrm{NaN}$ & $\mathrm{NaN}$ & $\mathrm{NaN}$ \\
\hline & & & $\mathrm{NaN}$ & & $\mathrm{N}+\mathrm{c}$ & 0.9 & & 0. & $\mathrm{Na}$ & $\mathrm{N}$ & & $\mathrm{Na}$ & 0.90 & & & $\mathrm{NaN}$ & & & & $\mathrm{aN}$ & & & & & $\mathrm{NaN}$ & $\mathrm{NaN}$ & $\mathrm{NaN}$ \\
\hline IGHG4 & P01861 & $\mathrm{NaN}$ & $\mathrm{NaN}$ & $\mathrm{NaN}$ & $\mathrm{NaN}$ & $\mathrm{NaN}$ & $\mathrm{NaN}$ & 0.29 & $\mathrm{NaN}$ & $\mathrm{NaN}$ & $\mathrm{NaN}$ & $\mathrm{NaN}$ & $\mathrm{NaN}$ & $\mathrm{NaN}$ & $\mathrm{NaN}$ & $\mathrm{NaN}$ & 0.13 & $\mathrm{NaN}$ & $\mathrm{NaN}$ & $\mathrm{NaN}$ & $\mathrm{NaN}$ & $\mathrm{NaN}$ & $\mathrm{NaN}$ & $\mathrm{NaN}$ & $\mathrm{NaN}$ & $\mathrm{NaN}$ & $\mathrm{NaN}$ \\
\hline IGKC & Q0KKI6 & 0.06 & $\mathrm{NaN}$ & $\mathrm{Na}$ & $\mathrm{NaN}$ & $\mathrm{NaN}$ & $\mathrm{NaN}$ & 0.07 & $\mathrm{NaN}$ & $\mathrm{NaN}$ & 0.42 & $\mathrm{NaN}$ & $\mathrm{NaN}$ & $\mathrm{NaN}$ & $\mathrm{NaN}$ & $\mathrm{NaN}$ & 0.16 & $\mathrm{NaN}$ & $\mathrm{NaN}$ & $\mathrm{NaN}$ & $\mathrm{NaN}$ & $\mathrm{NaN}$ & $\mathrm{NaN}$ & 0.28 & $\mathrm{NaN}$ & $\mathrm{NaN}$ & 0.57 \\
\hline IGLC1 & NUT2 & $\mathrm{NaN}$ & $\mathrm{NaN}$ & $\mathrm{NaN}$ & $\mathrm{NaN}$ & $\mathrm{NaN}$ & $\mathrm{NaN}$ & 0.04 & $\mathrm{NaN}$ & $\mathrm{NaN}$ & $\mathrm{NaN}$ & $\mathrm{NaN}$ & $\mathrm{NaN}$ & $\mathrm{NaN}$ & $\mathrm{NaN}$ & $\mathrm{NaN}$ & 0.22 & $\mathrm{NaN}$ & $\mathrm{NaN}$ & $\mathrm{NaN}$ & $\mathrm{NaN}$ & $\mathrm{NaN}$ & $\mathrm{NaN}$ & $\mathrm{NaN}$ & $\mathrm{NaN}$ & $\mathrm{NaN}$ & $\mathrm{NaN}$ \\
\hline ING1 & & & 1.18 & & 1.3 & 0.7 & & & 1.62 & 1.5 & & 1.3 & 1.12 & & & & & & & & & & & & & 1.67 & $\mathrm{NaN}$ \\
\hline ING2 & B2RA 15 & $\mathrm{NaN}$ & 0.71 & 1.5 & 1.5 & 0.94 & 3. & 1.92 & 2.15 & 1.69 & 2. & 1.62 & 1.44 & 0.69 & 3.35 & $\mathrm{NaN}$ & $\mathrm{NaN}$ & 1.59 & 1.92 & $\mathrm{NaN}$ & 0.25 & 1.60 & 2.09 & 0.66 & 6.47 & 2.70 & 1.47 \\
\hline ING4 & KKYM7 & $\mathrm{NaN}$ & $\mathrm{NaN}$ & & 0.81 & 0.68 & 0.93 & 0.73 & 0.90 & 1.16 & & 0.69 & 1.07 & 52 & 1.94 & 0.65 & $\mathrm{NaN}$ & 0.98 & 0.77 & 0.36 & $\mathrm{NaN}$ & 0.25 & 33 & 1.43 & 2.34 & 1.02 & 1.22 \\
\hline ING5 & E9PENO & $\mathrm{NaN}$ & $\mathrm{NaN}$ & 1.14 & 1.1 & 1.32 & & 1. & 1.06 & 1.48 & 1.03 & $\mathrm{NaN}$ & $\mathrm{NaN}$ & 0.97 & $\mathrm{NaN}$ & 0.70 & $\mathrm{NaN}$ & 1.81 & 1.58 & 0.52 & 6.29 & 0.61 & 50 & 1.95 & 2.74 & 1.55 & 1.48 \\
\hline & & 0.6 & 0.47 & & 0. & 0. & & 1.0 & & & 2. & 1.2 & 1.07 & & & 0. & 2.8 & & 2. & 45.99 & & & & 1.5 & & 2.54 & 1.03 \\
\hline INO8 & Q9 & 0.3 & 0.2 & & & 0.7 & & & & & & 1.3 & 1. & & & 0. & & & 2.5 & 25.2 & & & & 1.13 & .80 & .10 & 0.38 \\
\hline INOBC & Q6P198 & 0.71 & 0.4 & 2. & 0.4 & 0.8 & 2.02 & 1.68 & 0.9 & 0.7 & 3. & 1.50 & 1.27 & 0 & 4.4 & 0.25 & & 0. & 2.6 & 18.02 & 0.07 & 3.32 & 0.59 & 1.08 & 1.64 & 2.83 & 0.47 \\
\hline & TQ3-2 & $\mathrm{Na}$ & $\mathrm{N}$ & & 0. & $0 . \varepsilon$ & & $\mathrm{N}_{c}$ & $\mathrm{Na}$ & & & 1.48 & 1.42 & & $\mathrm{Na}$ & $\mathrm{NaN}$ & & & 2.0 & $\mathrm{NaN}$ & 0.25 & & 0.53 & $\mathrm{NaN}$ & $\mathrm{NaN}$ & $\mathrm{NaN}$ & $\mathrm{NaN}$ \\
\hline & Q8NBZ0 & 0.41 & 0.29 & & 0.6 & 0.77 & & 1.45 & 0.9 & 0.64 & & 1.37 & 1.26 & 0.21 & 4.8 & 0.25 & 0.93 & 0.52 & 2.5 & 11.44 & 0.04 & 3.09 & 0.54 & 1.08 & 1.59 & $\mathrm{NaN}$ & 0.52 \\
\hline IPO1 & Q9UI2 & 1.2 & 1.1 & & 0. & 0.8 & & 1.1 & & & & 0. & 1.2 & & & 1. & & & & & & & & & $\mathrm{NaN}$ & 1.95 & 0.57 \\
\hline ISL2 & & $\mathrm{NaN}$ & $\mathrm{Nal}$ & & 0.7 & 1.0 & & 1. & $\mathrm{Na}$ & & & 0.8 & 0.96 & $\mathrm{~N}$ & & $\mathrm{NaN}$ & & & $\mathrm{N}$ & 15.38 & & & & 0.96 & 1.32 & $\mathrm{aN}$ & $\mathrm{NaN}$ \\
\hline & & 0.19 & 0. & & & & & & 1. & & & 0. & 1.8 & & 1.3 & $\mathrm{NaN}$ & & & 1. & & & 0.46 & & $\mathrm{NaN}$ & $\mathrm{NaN}$ & $\mathrm{aN}$ & 1.37 \\
\hline JAD & Q926 & 0.18 & 2.96 & & 0.9 & 0.87 & & & 1.11 & & & 1.03 & 1.50 & & 1. & 1.44 & 0. & & 0.69 & 0.27 & & 0.21 & 48 & 1.26 & 1.80 & 0.60 & 0.77 \\
\hline JARID2 & Q92833 & $\mathrm{NaN}$ & $\mathrm{NaN}$ & 0.42 & 0.9 & 0.68 & 0. & $\mathrm{NaN}$ & $\mathrm{Nal}$ & 0.5 & 0.8 & 0.18 & 2.49 & $\mathrm{NaN}$ & 1.05 & $\mathrm{NaN}$ & $\mathrm{NaN}$ & 0.56 & 0.8 & $\mathrm{NaN}$ & 0.5 & 0.64 & 0.6 & 1.42 & 1.15 & $\mathrm{NaN}$ & $\mathrm{NaN}$ \\
\hline JMJD1C & & 1.2 & $\mathrm{Na}$ & & & 1.2 & & & & & & & & & & 0.53 & & & & & & & & & & & \\
\hline & & 0.2 & 1.5 & & 0. & 0. & & 0. & 0. & & & 0. & 0.7 & & 0. & 1. & & & 0. & 0 & & & & 1.12 & 1.12 & 0.50 & 0.76 \\
\hline AA1524 & |а8тC & 0. & 0.7 & & 0.7 & 0 & & $\mathrm{~N}_{c}$ & $\mathrm{Na}$ & 11 & 0.8 & $\mathrm{NaN}$ & $\mathrm{NaN}$ & & 1.0 & 0.76 & & 1.46 & 0.75 & 0.18 & $\mathrm{NaN}$ & 0.5 & 1.51 & $\mathrm{NaN}$ & $\mathrm{NaN}$ & 2.77 & 0.64 \\
\hline & P52732 & 1.28 & 0.94 & 0.77 & 1.45 & 0.67 & 0.19 & 1.07 & 0.38 & 0.55 & 0.73 & 0.40 & $\mathrm{NaN}$ & 0.93 & 0.75 & 1.13 & 0.15 & 1.31 & 0.73 & $\mathrm{NaN}$ & $\mathrm{NaN}$ & 0.65 & 1.23 & $\mathrm{NaN}$ & $\mathrm{NaN}$ & 1.11 & 0.43 \\
\hline & 00 & $\mathrm{NaN}$ & $\mathrm{NaN}$ & & & $\mathrm{Na}$ & & & & & & & & & & & & $\mathrm{NaN}$ & & & & & & $\mathrm{NaN}$ & $\mathrm{NaN}$ & & \\
\hline & & $\mathrm{NaN}$ & $\mathrm{N}$ & & $\mathrm{N}$ & & & $\mathrm{N}$ & & & & 1.2 & & & & 0. & & & & & & & & & & $\mathrm{aN}$ & \\
\hline & A8K3 & 1.01 & 0.78 & & 0.6 & 4.6 & & $\mathrm{NaN}$ & $\mathrm{Na}$ & & 0.45 & 0.7 & $\mathrm{NaN}$ & $\mathrm{NaN}$ & 0.89 & $\mathrm{NaN}$ & 0.33 & 1.0 & 0.48 & $\mathrm{NaN}$ & 0.90 & 0.61 & 0.65 & $\mathrm{NaN}$ & $\mathrm{NaN}$ & $\mathrm{NaN}$ & 0.45 \\
\hline KIF5B-A & C1PHA2 & $\mathrm{NaN}$ & $\mathrm{NaN}$ & 1.16 & $\mathrm{NaN}$ & $\mathrm{NaN}$ & $\mathrm{NaN}$ & $\mathrm{NaN}$ & 0.59 & 0.28 & $\mathrm{NaN}$ & 0.73 & $\mathrm{NaN}$ & $\mathrm{NaN}$ & $\mathrm{NaN}$ & $\mathrm{NaN}$ & $\mathrm{NaN}$ & 1.56 & $\mathrm{NaN}$ & $\mathrm{NaN}$ & $\mathrm{NaN}$ & $\mathrm{NaN}$ & $\mathrm{NaN}$ & $\mathrm{NaN}$ & $\mathrm{NaN}$ & $\mathrm{NaN}$ & $\mathrm{NaN}$ \\
\hline KIFC1 & Q9BW19 & 0.62 & 0.57 & 0.37 & $\mathrm{NaN}$ & 2.36 & 0.20 & $\mathrm{NaN}$ & $\mathrm{NaN}$ & 0.61 & 0.25 & 0.73 & 0.28 & $\mathrm{NaN}$ & 0.43 & 0.42 & 0.12 & 1.25 & 0.19 & 1.86 & $\mathrm{NaN}$ & 0.67 & 0.37 & $\mathrm{NaN}$ & $\mathrm{NaN}$ & $\mathrm{NaN}$ & $\mathrm{NaN}$ \\
\hline & & 0.82 & 0.58 & 1.60 & 0.8 & & & 1.18 & & & & & & & & & & & & & & & & & & & \\
\hline AT2C & Q8NE & 0.39 & $\mathrm{NaN}$ & $\mathrm{NaN}$ & $\mathrm{NaN}$ & 0.54 & 0.79 & $\mathrm{NaN}$ & $\mathrm{NaN}$ & 0.38 & $\mathrm{NaN}$ & 0.95 & 0.88 & $\mathrm{NaN}$ & $\mathrm{NaN}$ & 0.18 & 0.22 & $\mathrm{NaN}$ & $\mathrm{NaN}$ & $\mathrm{NaN}$ & 0.32 & 2.04 & 0.31 & 0.71 & 0.76 & $\mathrm{NaN}$ & $\mathrm{NaN}$ \\
\hline KMT2D & 014686 & 0.45 & 0.65 & 1.59 & 0.57 & 0.95 & 1.15 & 0.31 & $\mathrm{NaN}$ & 0.73 & 1.18 & 1.19 & 1.06 & 0.78 & 1.25 & 0.24 & 0.22 & 0.46 & 2.00 & $\mathrm{NaN}$ & 0.44 & 1.35 & 0.76 & 2.10 & 1.44 & $\mathrm{NaN}$ & 0.10 \\
\hline KRI1 & HOYFD2 & 0.11 & $\mathrm{NaN}$ & $\mathrm{NaN}$ & $\mathrm{NaN}$ & $\mathrm{NaN}$ & $\mathrm{NaN}$ & $\mathrm{NaN}$ & $\mathrm{NaN}$ & $\mathrm{NaN}$ & $\mathrm{NaN}$ & $\mathrm{NaN}$ & $\mathrm{NaN}$ & $\mathrm{NaN}$ & $\mathrm{NaN}$ & $\mathrm{NaN}$ & $\mathrm{NaN}$ & $\mathrm{NaN}$ & $\mathrm{NaN}$ & 1.42 & $\mathrm{NaN}$ & $\mathrm{NaN}$ & $\mathrm{NaN}$ & $\mathrm{NaN}$ & $\mathrm{NaN}$ & $\mathrm{NaN}$ & $\mathrm{NaN}$ \\
\hline$l_{L C}$ & P31025 & $\mathrm{NaN}$ & $\mathrm{NaN}$ & $\mathrm{NaN}$ & 0.14 & $\mathrm{NaN}$ & $\mathrm{NaN}$ & $\mathrm{NaN}$ & $\mathrm{NaN}$ & $\mathrm{NaN}$ & $\mathrm{NaN}$ & $\mathrm{NaN}$ & $\mathrm{NaN}$ & 0.04 & $\mathrm{NaN}$ & $\mathrm{NaN}$ & $\mathrm{NaN}$ & $\mathrm{NaN}$ & $\mathrm{NaN}$ & $\mathrm{NaN}$ & $\mathrm{NaN}$ & $\mathrm{NaN}$ & $\mathrm{NaN}$ & $\mathrm{NaN}$ & 0.12 & $\mathrm{NaN}$ & $\mathrm{NaN}$ \\
\hline
\end{tabular}




\begin{tabular}{|c|c|c|c|c|c|c|c|c|c|c|c|c|c|c|c|c|c|c|c|c|c|c|c|c|c|c|c|}
\hline Genenames & Protein IDs & $\begin{array}{l}\text { H3 } \Delta 1- \\
20 \_F\end{array}$ & $\begin{array}{l}H 3 \Delta 1- \\
20 \_R\end{array}$ & $\begin{array}{l}\text { H4K20 } \\
\text { me1_F }\end{array}$ & $\begin{array}{l}\text { H4K20 } \\
\text { me1_R }\end{array}$ & $\begin{array}{l}\text { H4K20 } \\
\text { me3_F }\end{array}$ & $\begin{array}{l}\text { H4K20 } \\
\text { me3_R }\end{array}$ & $\mid \begin{array}{c}\mathrm{H}_{3} \mathrm{~K}_{\mathrm{C}} 27 \\
\mathrm{me1}_{1} \mathrm{~F}\end{array}$ & $\left|\begin{array}{c}\mathrm{H} 3 \mathrm{~K}_{\mathrm{c}} 27 \\
\mathrm{me1} \_\mathrm{R}\end{array}\right|$ & $\mid \begin{array}{l}\mathrm{H}_{3} \mathrm{~K}_{\mathrm{C}} 27 \\
\mathrm{me} 2 \mathrm{~F}\end{array}$ & $\mid \begin{array}{r}\mathrm{H}_{3} \mathrm{~K}_{\mathrm{c}} 27 \\
\mathrm{me2} 2 \mathrm{R}\end{array}$ & $\mid \begin{array}{c}\mathrm{H} 3 \mathrm{~K}_{\mathrm{c}} 27 \\
\text { me3_F }\end{array}$ & $\begin{array}{l}\mathrm{H} 3 \mathrm{~K}_{\mathrm{C}} 27 \\
\mathrm{me} 3 \mathrm{R}\end{array}$ & $\begin{array}{c}\text { H3K9m } \\
\text { e1_F }\end{array}$ & $\mid \begin{array}{c}\text { HзK9m } \\
\text { e1_R }\end{array}$ & $\mid \begin{array}{c}\mathrm{H} 3 \mathrm{~K} 9 \mathrm{~m} \\
\text { e2_F }\end{array}$ & $\begin{array}{c}\text { HзK9m } \\
\text { e2_R }\end{array}$ & $\begin{array}{c}\text { H3K9m } \\
\text { e3_F }\end{array}$ & $\begin{array}{c}\text { Hзкаm } \\
\text { e3_R }\end{array}$ & \begin{tabular}{|c} 
HзK9m \\
eз|meC \\
pG_F
\end{tabular} & $\begin{array}{c}\text { Hзкаm } \\
\text { e3/meC } \\
\text { pG_R R }\end{array}$ & $\begin{array}{c}\mathrm{H3K9m} \\
\text { e3|H4K } \\
20 \mathrm{me3} \\
\mathrm{F}\end{array}$ & $\begin{array}{c}\mathrm{H3K9m} \\
\mathrm{e} 3 \mid \mathrm{H} 4 \mathrm{~K} \\
20 \mathrm{me} 3 \\
\mathrm{R}\end{array}$ & $\begin{array}{c}\mathrm{meCpG} \\
-\mathrm{F}\end{array}$ & $\begin{array}{c}\mathrm{meCpG} \\
-\mathrm{R}\end{array}$ & \begin{tabular}{|c|} 
H4R3m \\
e2_F
\end{tabular} & $\begin{array}{c}\text { H4R3m } \\
\text { e2_R }\end{array}$ \\
\hline LDHA & P00338 & 0.34 & 0.17 & 1.63 & 1.45 & 1.42 & 1.11 & 0.45 & 2.65 & 1.51 & 0.58 & 1.13 & 1.24 & 1.24 & 1.02 & 0.27 & 0.22 & 1.24 & 1.08 & 0.78 & 1.94 & \begin{tabular}{|l|} 
\\
\end{tabular} & 1.00 & 1.41 & 0.72 & 2.07 & 1.10 \\
\hline LDHB & Q5U077 & 0.99 & 0.52 & 1.28 & 1.04 & 0.86 & 0.69 & 0.42 & 2.03 & 1.20 & 0.52 & 0.96 & 1.07 & 1.15 & 0.95 & 0.82 & 0.22 & 1.00 & 0.94 & 0.51 & 1.48 & 0.66 & 0.84 & 1.18 & 0.69 & 1.77 & 0.55 \\
\hline LGALS7 & P47929 & $\mathrm{NaN}$ & $\mathrm{NaN}$ & $\mathrm{NaN}$ & $\mathrm{NaN}$ & $\mathrm{NaN}$ & $\mathrm{NaN}$ & 0.08 & $\mathrm{NaN}$ & $\mathrm{NaN}$ & 0.20 & $\mathrm{NaN}$ & $\mathrm{NaN}$ & $\mathrm{NaN}$ & $\mathrm{NaN}$ & $\mathrm{NaN}$ & $\mathrm{NaN}$ & $\mathrm{NaN}$ & $\mathrm{NaN}$ & $\mathrm{NaN}$ & $\mathrm{NaN}$ & $\mathrm{NaN}$ & $\mathrm{NaN}$ & 0.06 & $\mathrm{NaN}$ & $\mathrm{NaN}$ & $\mathrm{NaN}$ \\
\hline LIG4 & P49917 & $\mathrm{NaN}$ & 1.95 & 1.64 & 1.27 & 0.87 & 1.65 & 1.30 & 1.22 & 0.82 & 2.19 & 1.37 & 1.52 & 0.65 & 2.57 & $\mathrm{NaN}$ & $\mathrm{NaN}$ & 0.82 & 1.62 & 4.67 & 0.16 & 1.65 & 1.02 & 1.06 & 0.83 & 2.15 & 0.78 \\
\hline LMNA & P02545 & 0.69 & 0.42 & 1.15 & 0.57 & 1.99 & 0.35 & 0.72 & 0.49 & 1.10 & 0.86 & 0.78 & 1.06 & 0.96 & 1.12 & 0.56 & 0.62 & 1.24 & 0.60 & 0.07 & 2.21 & 0.79 & 0.97 & 0.10 & 0.56 & 0.19 & 1.73 \\
\hline LMNA & P02545-2 & $\mathrm{NaN}$ & 0.25 & $\mathrm{NaN}$ & $\mathrm{NaN}$ & $\mathrm{NaN}$ & $\mathrm{NaN}$ & $\mathrm{NaN}$ & $\mathrm{NaN}$ & $\mathrm{NaN}$ & $\mathrm{NaN}$ & $\mathrm{NaN}$ & $\mathrm{NaN}$ & $\mathrm{NaN}$ & $\mathrm{NaN}$ & 0.31 & $\mathrm{NaN}$ & $\mathrm{NaN}$ & $\mathrm{NaN}$ & 0.09 & 1.46 & $\mathrm{NaN}$ & $\mathrm{NaN}$ & $\mathrm{NaN}$ & $\mathrm{NaN}$ & $\mathrm{NaN}$ & $\mathrm{NaN}$ \\
\hline LMNA & P02545-5 & $\mathrm{NaN}$ & $\mathrm{NaN}$ & $\mathrm{NaN}$ & $\mathrm{NaN}$ & $\mathrm{NaN}$ & $\mathrm{NaN}$ & $\mathrm{NaN}$ & 35.49 & $\mathrm{NaN}$ & $\mathrm{NaN}$ & $\mathrm{NaN}$ & $\mathrm{NaN}$ & $\mathrm{NaN}$ & $\mathrm{NaN}$ & $\mathrm{NaN}$ & $\mathrm{NaN}$ & $\mathrm{NaN}$ & $\mathrm{NaN}$ & $\mathrm{NaN}$ & $\mathrm{NaN}$ & $\mathrm{NaN}$ & $\mathrm{NaN}$ & $\mathrm{NaN}$ & $\mathrm{NaN}$ & $\mathrm{NaN}$ & $\mathrm{NaN}$ \\
\hline LRIF1 & Q5T3J3 & $\mathrm{NaN}$ & $\mathrm{NaN}$ & 1.54 & $\mathrm{NaN}$ & 1.22 & 1.22 & $\mathrm{NaN}$ & $\mathrm{NaN}$ & 0.91 & $\mathrm{NaN}$ & 2.35 & 1.15 & $\mathrm{NaN}$ & $\mathrm{NaN}$ & $\mathrm{NaN}$ & $\mathrm{NaN}$ & 3.65 & 0.67 & 5.72 & 0.13 & 2.75 & 0.38 & 2.52 & $\mathrm{NaN}$ & $\mathrm{NaN}$ & $\mathrm{NaN}$ \\
\hline LRRFIP2 & A8MXR0 & $\mathrm{NaN}$ & 4.13 & $\mathrm{NaN}$ & $\mathrm{NaN}$ & $\mathrm{NaN}$ & $\mathrm{NaN}$ & $\mathrm{NaN}$ & $\mathrm{NaN}$ & $\mathrm{NaN}$ & $\mathrm{NaN}$ & $\mathrm{NaN}$ & $\mathrm{NaN}$ & $\mathrm{NaN}$ & $\mathrm{NaN}$ & 0.36 & 1.04 & $\mathrm{NaN}$ & $\mathrm{NaN}$ & $\mathrm{NaN}$ & $\mathrm{NaN}$ & $\mathrm{NaN}$ & $\mathrm{NaN}$ & $\mathrm{NaN}$ & $\mathrm{NaN}$ & $\mathrm{NaN}$ & $\mathrm{NaN}$ \\
\hline LRWD1 & Q9UFC0 & 0.76 & 0.71 & 1.12 & 1.09 & 9.82 & 0.05 & 1.47 & 1.05 & 0.91 & 1.15 & 3.02 & 0.19 & 0.99 & 0.93 & 0.78 & 0.25 & 3.16 & 0.18 & 3.42 & 0.61 & 7.94 & 0.05 & 2.90 & 1.09 & 1.35 & 1.11 \\
\hline LSM2 & Q9Y333 & 1.43 & 0.87 & $\mathrm{NaN}$ & $\mathrm{NaN}$ & 1.31 & 0.73 & $\mathrm{NaN}$ & 1.09 & $\mathrm{NaN}$ & $\mathrm{NaN}$ & $\mathrm{NaN}$ & $\mathrm{NaN}$ & $\mathrm{NaN}$ & $\mathrm{NaN}$ & 0.95 & 0.40 & $\mathrm{NaN}$ & 0.77 & 0.61 & 1.13 & 0.07 & 1.50 & $\mathrm{NaN}$ & $\mathrm{NaN}$ & 0.63 & 0.70 \\
\hline MAFK & A2VCQ5 & $\mathrm{NaN}$ & $\mathrm{NaN}$ & $\mathrm{NaN}$ & $\mathrm{NaN}$ & $\mathrm{NaN}$ & 0.28 & 0.09 & $\mathrm{NaN}$ & $\mathrm{NaN}$ & $\mathrm{NaN}$ & $\mathrm{NaN}$ & $\mathrm{NaN}$ & $\mathrm{NaN}$ & $\mathrm{NaN}$ & $\mathrm{NaN}$ & $\mathrm{NaN}$ & $\mathrm{NaN}$ & $\mathrm{NaN}$ & $\mathrm{NaN}$ & $\mathrm{NaN}$ & $\mathrm{NaN}$ & $\mathrm{NaN}$ & $\mathrm{NaN}$ & $\mathrm{NaN}$ & $\mathrm{NaN}$ & $\mathrm{NaN}$ \\
\hline MAFK & A8WFP5 & 0.87 & $\mathrm{NaN}$ & 0.70 & 0.67 & $\mathrm{NaN}$ & $\mathrm{NaN}$ & $\mathrm{NaN}$ & $\mathrm{NaN}$ & $\mathrm{NaN}$ & $\mathrm{NaN}$ & $\mathrm{NaN}$ & 0.51 & $\mathrm{NaN}$ & $\mathrm{NaN}$ & 0.34 & 1.88 & $\mathrm{NaN}$ & $\mathrm{NaN}$ & 5.77 & 0.12 & $\mathrm{NaN}$ & 0.57 & 2.28 & 0.49 & $\mathrm{NaN}$ & $\mathrm{NaN}$ \\
\hline MAGOH & P61326 & 8.28 & 0.92 & 0.39 & 1.79 & 0.76 & 0.47 & 0.71 & 1.01 & 1.80 & 0.21 & 1.13 & 1.15 & $\mathrm{NaN}$ & 0.41 & 2.21 & 0.57 & 1.20 & 0.46 & $\mathrm{NaN}$ & $\mathrm{NaN}$ & 0.48 & 1.28 & $\mathrm{NaN}$ & $\mathrm{NaN}$ & 0.42 & 0.64 \\
\hline MAP2K2 & P36507 & 0.81 & 0.61 & 0.84 & & 0.66 & 0.48 & 1.03 & 0.65 & 0.77 & 1.17 & 0.68 & 1.85 & 0.58 & 0.66 & 0.75 & 0.18 & 1.19 & 0.97 & $\mathrm{NaN}$ & $\mathrm{NaN}$ & 0.61 & 1.02 & $\mathrm{NaN}$ & $\mathrm{NaN}$ & 2.17 & 0.68 \\
\hline MAP4 & E7EVAO & $\mathrm{NaN}$ & 0.58 & 0.54 & 3.00 & 1.97 & 0.82 & $\mathrm{NaN}$ & $\mathrm{NaN}$ & 1.40 & $\mathrm{NaN}$ & 1.64 & 0.76 & $\mathrm{NaN}$ & $\mathrm{NaN}$ & 0.47 & 0.12 & 1.23 & 0.76 & 3.75 & $\mathrm{NaN}$ & 1.39 & 0.99 & 2.56 & 2.22 & $\mathrm{NaN}$ & $\mathrm{NaN}$ \\
\hline MAP4 & P27816-5 & $\mathrm{NaN}$ & $\mathrm{NaN}$ & $\mathrm{NaN}$ & $\mathrm{NaN}$ & $\mathrm{NaN}$ & $\mathrm{NaN}$ & $\mathrm{NaN}$ & $\mathrm{NaN}$ & $\mathrm{NaN}$ & $\mathrm{NaN}$ & $\mathrm{NaN}$ & $\mathrm{NaN}$ & $\mathrm{NaN}$ & $\mathrm{NaN}$ & $\mathrm{NaN}$ & $\mathrm{NaN}$ & $\mathrm{NaN}$ & $\mathrm{NaN}$ & $\mathrm{NaN}$ & $\mathrm{NaN}$ & $\mathrm{NaN}$ & $\mathrm{NaN}$ & $\mathrm{NaN}$ & $\mathrm{NaN}$ & $\mathrm{NaN}$ & $\mathrm{NaN}$ \\
\hline MARK2 & Q7KZI7-8 & 0.66 & 0.01 & $\mathrm{NaN}$ & $\mathrm{NaN}$ & $\mathrm{NaN}$ & $\mathrm{NaN}$ & $\mathrm{NaN}$ & $\mathrm{NaN}$ & $\mathrm{NaN}$ & $\mathrm{NaN}$ & $\mathrm{NaN}$ & $\mathrm{NaN}$ & $\mathrm{NaN}$ & $\mathrm{NaN}$ & $\mathrm{NaN}$ & 0.72 & $\mathrm{NaN}$ & $\mathrm{NaN}$ & $\mathrm{NaN}$ & $\mathrm{NaN}$ & $\mathrm{NaN}$ & $\mathrm{NaN}$ & $\mathrm{NaN}$ & $\mathrm{NaN}$ & $\mathrm{NaN}$ & $\mathrm{NaN}$ \\
\hline MAU2 & Q9Y6X3 & 0.86 & 0.62 & $\mathrm{NaN}$ & $\mathrm{NaN}$ & 0.93 & 1.30 & 0.92 & $\mathrm{NaN}$ & 1.07 & $\mathrm{NaN}$ & 1.25 & 0.92 & $\mathrm{NaN}$ & 1.67 & 1.08 & 0.28 & 3.14 & 0.30 & 12.24 & 0.08 & 4.56 & 0.21 & $\mathrm{NaN}$ & 0.80 & $\mathrm{NaN}$ & $\mathrm{NaN}$ \\
\hline MAU2 & Q9Y6X3-2 & $\mathrm{NaN}$ & $\mathrm{NaN}$ & $\mathrm{aN}$ & $\mathrm{Na}$ & 1.38 & 1.35 & & $\mathrm{NaN}$ & $\mathrm{NaN}$ & $\mathrm{NaN}$ & $\mathrm{NaN}$ & $\mathrm{NaN}$ & $\mathrm{NaN}$ & $\mathrm{NaN}$ & $\mathrm{NaN}$ & $\mathrm{NaN}$ & 3.22 & 0.3 & 8.03 & 0.14 & 1.93 & 0.26 & $\mathrm{NaN}$ & $\mathrm{NaN}$ & $\mathrm{NaN}$ & $\mathrm{NaN}$ \\
\hline MAX & Q8TAX8 & 1.02 & 1.71 & 3.10 & 1.03 & $\begin{array}{l}1.30 \\
1.38\end{array}$ & $\begin{array}{l}1.05 \\
1.89\end{array}$ & 2.24 & 1.63 & 1.66 & 2.27 & 1.99 & 1.67 & $\mathrm{NaN}$ & $\mathrm{NaN}$ & 0.75 & 4.76 & 1.56 & 1.69 & 6.02 & 0.17 & 2.33 & 1.37 & 0.51 & 2.58 & 2.48 & NaN \\
\hline MBD2 & Q9UBB5 & 0.38 & 0.79 & 1.48 & 0.71 & 0.97 & 1.04 & 1.00 & 1.02 & 1.11 & 0.89 & 1.06 & 0.87 & 0.33 & 2.55 & 0.68 & 0.32 & 0.65 & 1.46 & 15.14 & 0.01 & 0.86 & 1.17 & 6.56 & 0.08 & 2.02 & 0.54 \\
\hline MBTPS1 & Q14703 & $\mathrm{NaN}$ & $\mathrm{NaN}$ & $\mathrm{NaN}$ & $\mathrm{NaN}$ & 0.35 & 0.49 & $\mathrm{NaN}$ & $\mathrm{NaN}$ & $\mathrm{NaN}$ & $\mathrm{NaN}$ & 0.32 & $\mathrm{NaN}$ & $\mathrm{NaN}$ & $\mathrm{NaN}$ & $\mathrm{NaN}$ & $\mathrm{NaN}$ & 0.61 & $\mathrm{NaN}$ & $\mathrm{NaN}$ & $\mathrm{NaN}$ & 0.27 & 0.62 & $\mathrm{NaN}$ & $\mathrm{NaN}$ & 0.69 & 0.69 \\
\hline MCR & Q96EZ8-3 & 0.58 & 0.33 & & 0.7 & 1.08 & 1.26 & 1.35 & 1.15 & 0.89 & 1.62 & 1.4 & 1.22 & 0.26 & 5.42 & 0.37 & 0.53 & 0.80 & 157 & 7.29 & 0.18 & & 0.62 & 1.71 & 1.12 & $\mathrm{NaN}$ & 0.49 \\
\hline MEAF6 & Q9HAF1-2 & 0.22 & 1.83 & 1.12 & 1.14 & 0.99 & 1.27 & 0.78 & 1.04 & 1.07 & 0.87 & 1.17 & 1.51 & $\mathrm{NaN}$ & $\mathrm{NaN}$ & 0.75 & 0.65 & 1.16 & 1.15 & 0.70 & 2.39 & 0.86 & 1.77 & 1.86 & 2.03 & 0.88 & 0.93 \\
\hline MECP2 & P51608-2 & 1.04 & 1.09 & 0.50 & 0.93 & 0.55 & 0.91 & 0.58 & 0.70 & 0.88 & 0.65 & 0.75 & 0.71 & 0.62 & 0.78 & 0.60 & 5.77 & 0.62 & 0.98 & 5.15 & 0.10 & 0.77 & 0.74 & 3.74 & 0.32 & 0.58 & 0.58 \\
\hline MED10 & Q9BTT4 & 0.67 & $\mathrm{NaN}$ & $\mathrm{NaN}$ & $\mathrm{NaN}$ & 0.89 & 0.54 & 0.60 & $\mathrm{NaN}$ & 0.60 & 1.10 & 0.83 & 0.76 & $\mathrm{NaN}$ & $\mathrm{NaN}$ & 0.22 & $\mathrm{NaN}$ & 0.52 & 0.90 & 0.22 & $\mathrm{NaN}$ & 1.25 & 0.53 & $\mathrm{NaN}$ & $\mathrm{NaN}$ & $\mathrm{NaN}$ & $\mathrm{NaN}$ \\
\hline MED16 & Q9Y2X0-2 & 0.53 & 0.64 & 0.71 & 0.56 & 0.84 & 0.41 & 0.51 & $\mathrm{NaN}$ & 0.49 & 0.98 & 0.8 & 0.64 & 0.52 & 1.13 & 0.27 & 0.99 & 0.52 & 0.6 & 1.59 & 0.47 & 0.88 & 0.34 & 0.74 & 1.15 & $\mathrm{NaN}$ & $\mathrm{NaN}$ \\
\hline MIER1 & Q8N108-19 & $\mathrm{NaN}$ & $\mathrm{NaN}$ & & 0.6 & 0.72 & 1.49 & & $\mathrm{Na}$ & 0.87 & 1.0 & & $\mathrm{Na}$ & & $\mathrm{NaN}$ & $\mathrm{NaN}$ & 0.84 & 2.15 & & & 0.09 & 2.39 & 0.32 & $\mathrm{NaN}$ & & $\mathrm{NaN}$ & $\mathrm{NaN}$ \\
\hline MIER2 & Q8N344 & $\mathrm{NaN}$ & $\mathrm{NaN}$ & $\mathrm{NaN}$ & $\mathrm{NaN}$ & 0.53 & $\mathrm{NaN}$ & $\mathrm{NaN}$ & $\mathrm{NaN}$ & $\mathrm{NaN}$ & $\mathrm{NaN}$ & $\mathrm{NaN}$ & NaN & $\mathrm{NaN}$ & $\mathrm{NaN}$ & NaN & $\mathrm{NaN}$ & 2.08 & 0.21 & 3.85 & $\mathrm{NaN}$ & 2.66 & 0.20 & $\mathrm{NaN}$ & NaN & $\mathrm{NaN}$ & NaN \\
\hline MIF & 14AY87 & $\mathrm{NaN}$ & 0.09 & $\mathrm{NaN}$ & $\mathrm{NaN}$ & $\mathrm{NaN}$ & $\mathrm{NaN}$ & $\mathrm{NaN}$ & $\mathrm{NaN}$ & $\mathrm{NaN}$ & $\mathrm{NaN}$ & $\mathrm{NaN}$ & $\mathrm{NaN}$ & $\mathrm{NaN}$ & $\mathrm{NaN}$ & $\mathrm{NaN}$ & $\mathrm{NaN}$ & $\mathrm{NaN}$ & $\mathrm{NaN}$ & 1.44 & $\mathrm{NaN}$ & $\mathrm{NaN}$ & $\mathrm{NaN}$ & $\mathrm{NaN}$ & $\mathrm{NaN}$ & $\mathrm{NaN}$ & $\mathrm{NaN}$ \\
\hline MITF & A8K5K3 & $\mathrm{NaN}$ & $\mathrm{NaN}$ & $\mathrm{NaN}$ & $\mathrm{NaN}$ & $\mathrm{NaN}$ & $\mathrm{NaN}$ & 1.02 & $\mathrm{NaN}$ & 2.45 & 1.33 & $\mathrm{NaN}$ & $\mathrm{NaN}$ & $\mathrm{NaN}$ & $\mathrm{NaN}$ & $\mathrm{NaN}$ & $\mathrm{NaN}$ & 0.78 & $\mathrm{NaN}$ & 1.75 & 0.27 & $\mathrm{NaN}$ & 0.63 & 0.55 & 2.12 & $\mathrm{NaN}$ & $\mathrm{NaN}$ \\
\hline$M L$ & & 0.44 & 0.72 & & 0. & & 1.11 & 1.2 & 0.8 & 0.7 & 1.2 & & 0.8 & & 2.2 & & 0.31 & & 0.93 & 10.58 & 0.21 & & & 0.25 & 2.64 & 2.25 & 0.34 \\
\hline MLXIPL & H7C IV3 & $\mathrm{NaN}$ & 0.58 & 1.29 & 0.42 & 0.75 & 0.85 & $\mathrm{NaN}$ & $\mathrm{NaN}$ & $\mathrm{NaN}$ & $\mathrm{NaN}$ & 0.72 & 0.53 & $\mathrm{NaN}$ & $\mathrm{NaN}$ & $\mathrm{NaN}$ & $\mathrm{NaN}$ & 0.68 & 0.75 & $\mathrm{NaN}$ & 0.12 & 0.90 & 0.80 & $\mathrm{NaN}$ & $\mathrm{NaN}$ & $\mathrm{NaN}$ & $\mathrm{NaN}$ \\
\hline MLXIPL & Q9NP71-3 & 0.31 & 0.55 & 1.38 & 0.28 & 0.94 & 1.04 & 0.94 & 0.65 & 0.60 & 1.17 & 0.8 & 0.80 & 0.46 & 2.00 & 0.22 & 0.21 & 0.71 & 0.83 & 10.76 & 0.17 & 1.11 & 0.74 & 0.27 & 1.85 & $\mathrm{NaN}$ & 0.44 \\
\hline MMS22L & E2QRD4 & $\mathrm{NaN}$ & $\mathrm{NaN}$ & $\mathrm{NaN}$ & 0.7 & 0.38 & 1.24 & 0.19 & $\mathrm{NaN}$ & $\mathrm{NaN}$ & $\mathrm{NaN}$ & 0.98 & 0.72 & & 0.90 & $\mathrm{NaN}$ & $\mathrm{NaN}$ & & 0.6 & 1.27 & 0.5 & 0.26 & 0.90 & 0.78 & 0.70 & 0.91 & $\mathrm{NaN}$ \\
\hline & Q99583 & 0.43 & 1.20 & 1.60 & 0.82 & 1.14 & 1.74 & 1.11 & 1.11 & 1.18 & 1.49 & 1.19 & 1.27 & 0.74 & 2.24 & 0.37 & 6.72 & 1.26 & 1.41 & 3.72 & 0.23 & 1.23 & 1.13 & 0.33 & 2.83 & 2.65 & 0.51 \\
\hline MOB4 & Q9Y3АЗ-3 & 0.48 & $\mathrm{NaN}$ & $\mathrm{NaN}$ & $\mathrm{NaN}$ & $\mathrm{NaN}$ & $\mathrm{NaN}$ & $\mathrm{NaN}$ & $\mathrm{NaN}$ & $\mathrm{NaN}$ & & $\mathrm{NaN}$ & $\mathrm{NaN}$ & $\mathrm{NaN}$ & $\mathrm{NaN}$ & $\mathrm{NaN}$ & 32.32 & $\mathrm{NaN}$ & $\mathrm{NaN}$ & $\mathrm{NaN}$ & $\mathrm{NaN}$ & $\mathrm{NaN}$ & $\mathrm{NaN}$ & $\mathrm{NaN}$ & $\mathrm{NaN}$ & $\mathrm{NaN}$ & $\mathrm{NaN}$ \\
\hline MORC2 & Q9Y6X9-2 & 1.04 & 1.36 & $\mathrm{NaN}$ & $\mathrm{NaN}$ & 1.77 & 0.30 & $\mathrm{NaN}$ & $\mathrm{NaN}$ & $\mathrm{NaN}$ & $\mathrm{NaN}$ & $\mathrm{NaN}$ & $\mathrm{NaN}$ & $\mathrm{NaN}$ & $\mathrm{NaN}$ & 1.08 & 0.67 & $\mathrm{NaN}$ & 0.35 & 1.07 & 1.16 & $\mathrm{NaN}$ & 0.63 & 1.27 & 1.22 & $\mathrm{NaN}$ & $\mathrm{NaN}$ \\
\hline MPHOSPH8 & Q99549 & $\mathrm{NaN}$ & $\mathrm{NaN}$ & $\mathrm{Na}$ & $\mathrm{Na}$ & $\mathrm{NaN}$ & & & $\mathrm{NaN}$ & $\mathrm{NaN}$ & & $\mathrm{Na}$ & 2.07 & & $\mathrm{NaN}$ & 12.07 & $\mathrm{NaN}$ & 3.00 & 0.2 & $\mathrm{NaN}$ & 0.08 & 2.63 & 0.22 & $\mathrm{NaN}$ & $\mathrm{NaN}$ & $\mathrm{NaN}$ & $\mathrm{NaN}$ \\
\hline MRPL13 & Q9BYD1 & 0.49 & $\mathrm{NaN}$ & $\mathrm{NaN}$ & $\mathrm{NaN}$ & $\mathrm{NaN}$ & $\mathrm{NaN}$ & NaN & NaN & $\mathrm{NaN}$ & $\mathrm{NaN}$ & $\mathrm{NaN}$ & $\mathrm{NaN}$ & $\mathrm{NaN}$ & $\mathrm{NaN}$ & $\mathrm{NaN}$ & 0.28 & $\mathrm{NaN}$ & $\mathrm{NaN}$ & NaN & $\mathrm{NaN}$ & $\mathrm{NaN}$ & $\mathrm{NaN}$ & NaN & NaN & $\mathrm{NaN}$ & NaN \\
\hline MTA1 & Q13330 & $\mathrm{NaN}$ & $\mathrm{NaN}$ & $\mathrm{NaN}$ & $\mathrm{NaN}$ & $\mathrm{Nal}$ & 0.39 & $\mathrm{NaN}$ & $\mathrm{NaN}$ & $\mathrm{NaN}$ & & 0.6 & 0.57 & 0.42 & $\mathrm{NaN}$ & $\mathrm{NaN}$ & & 0.34 & 1.09 & & 0.48 & 0.42 & 0.95 & Navir & 0.25 & $\mathrm{NaN}$ & $\mathrm{NaN}$ \\
\hline MTA & Q13330-3 & 0.19 & 0.84 & 0.9 & 0.5 & 0.8 & 0.69 & 0.7 & 0.8 & 0.8 & 0. & 0. & 0.7 & & 1. & 0.5 & 0.6 & 0. & 1.17 & 3.36 & 0.11 & 0.50 & 1.15 & 2.12 & 0.23 & 1.05 & 0.47 \\
\hline MTA2 & o94776 & 0.45 & 1.04 & 1.6 & 0.7 & 0.99 & & $0.7-2>$ & 0.83 & 0.9 & $0.7>-2>$ & 0.9 & 0.82 & 0.42 & 1.5 & 0.82 & 0.65 & 0.6 & 1.11 & 5.90 & 0.03 & 0.70 & 1.05 & 6.43 & 0.11 & 1.34 & 0.45 \\
\hline & E7EQY4 & 0.34 & 0.91 & 0.71 & 0.68 & 0.77 & 0.83 & 0.86 & 0.80 & 0.81 & 0.75 & 0.79 & 0.66 & 0.31 & 1.77 & 0.57 & 0.55 & 0.67 & 0.85 & 8.18 & 0.04 & 0.66 & 0.87 & 3.02 & 0.16 & 1.02 & 0.51 \\
\hline MTF2 & Q9Y483 & 0.3 & 1.58 & 0.8 & 0.6 & 0.85 & 0.97 & 0.71 & 0.73 & 0.8 & 1.01 & 0.7 & 1.11 & 0.51 & $\mathrm{Na}$ & 1.3 & 1.6 & 1.06 & 0.8 & 1.2 & 0.50 & 108 & 0.8 & & 1.94 & & \\
\hline & & $\mathrm{Na}$ & $\mathrm{NaN}$ & 3.8 & 0. & 1.9 & 2.08 & 2.3 & & 1.6 & 1.7 & 2. & 2. & & & $\mathrm{~N}$ & $\mathrm{~N}_{2}$ & & 1. & & & & & & 1 & $\mathrm{NaN}$ & \\
\hline MYL6B & 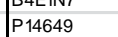 & $\mathrm{NaN}$ & 5.52 & N & $\mathrm{NaN}$ & $\mathrm{Na}$ & $\mathrm{N}$ & $\mathrm{NaN}$ & NaN & $\begin{array}{l}1.0 \\
\mathrm{Na}\end{array}$ & $\mathrm{Na}$ & $\mathrm{Na}$ & $\mathrm{NaN}$ & $\mathrm{NaN}$ & $\mathrm{NaN}$ & 0.64 & 1.31 & $\begin{array}{l}1.00 \\
\mathrm{NaN}\end{array}$ & $\mathrm{Na}$ & NaN & NaN & $\mathrm{NaN}$ & $\begin{array}{l}\mathrm{NaN} \\
\mathrm{NaN}\end{array}$ & $\mathrm{NaN}$ & $\begin{array}{l}\text { NaN } \\
\mathrm{NaN}\end{array}$ & $\mathrm{NaN}$ & $\mathrm{NaN}$ \\
\hline NAA40 & Q86UY6 & 0.80 & 0.62 & 0.80 & 0.84 & 0.91 & 0.95 & 0.91 & 0.72 & 0.80 & 0.98 & 0.87 & 0.84 & 0.57 & 1.21 & 0.89 & 1.07 & 0.90 & 0.77 & 0.97 & 0.90 & 0.89 & 0.77 & 0.89 & 0.97 & 0.35 & 2.29 \\
\hline NACA & E9PAV3 & 6.94 & 0.53 & 1.73 & 1.66 & 1.39 & 1.01 & 0.79 & $\mathrm{NaN}$ & 0.42 & 0.8 & 1.03 & 0.93 & $\mathrm{NaN}$ & $\mathrm{NaN}$ & 0.72 & 0.37 & 1.13 & $\mathrm{NaN}$ & $\mathrm{NaN}$ & 1.59 & 1.10 & 1.05 & 1.37 & 0.78 & $\mathrm{NaN}$ & $\mathrm{NaN}$ \\
\hline & F8VX35 & 1.04 & 2.68 & 2.0 & 0.9 & 2.30 & 0.84 & 1.09 & 1.74 & 1.85 & 1.1 & 0.76 & 1.98 & & 1.90 & 1.95 & 1.35 & 2.12 & 0.71 & 1.19 & 1.2 & 1.85 & & & & & \\
\hline NAPIL4 & B7ZAF & $\mathrm{NaN}$ & 0.21 & 4.07 & 0.69 & 2.71 & 0.49 & $\mathrm{NaN}$ & $\mathrm{NaN}$ & $\begin{array}{l}1.03 \\
1.03\end{array}$ & 1.2 & $\mathrm{Na}$ & $\mathrm{NaN}$ & $\mathrm{NaN}$ & $\mathrm{Nal}$ & 1.25 & 0.50 & $\mathrm{NaN}$ & $\mathrm{NaN}$ & 1.13 & 0.57 & 1.01 & $\mathrm{NaN}$ & 1.52 & $\mathrm{NaN}$ & $\mathrm{NaN}$ & $\mathrm{NaN}$ \\
\hline NBN & 060934 & $\mathrm{NaN}$ & 0.17 & 1.10 & 1.69 & 3.36 & 2.43 & 2.31 & 2.33 & 2.03 & 2.83 & 2.09 & 1.80 & 0.95 & 2.48 & 0.32 & 0.17 & 3.19 & 1.28 & 0.19 & 0.38 & 1.75 & 1.56 & 0.49 & 0.29 & 2.03 & 1.61 \\
\hline NCAPD2 & B3KMSO & 1.41 & 1.16 & 0.84 & 4.23 & 1.04 & 0.54 & 1.07 & 0.53 & 0.87 & 1.04 & 0.81 & 0.86 & 1.05 & 1.16 & 1.41 & 0.70 & 1.08 & 1.08 & 2.40 & 0.37 & 0.85 & 0.77 & 1.02 & 0.93 & 1.30 & 0.57 \\
\hline CL & P19338 & 0.60 & 1.27 & 0.99 & 0.86 & 2.39 & 0.50 & 0.62 & 1.07 & 1.32 & 0.77 & 0.86 & 1.11 & 1.21 & 0.88 & 1.26 & 0.34 & 2.10 & 0.58 & 0.26 & 4.90 & 0.86 & & 1.30 & 0.56 & 0.31 & 2.33 \\
\hline & Q14686 & $\mathrm{NaN}$ & $\mathrm{NaN}$ & 1.4 & 1.30 & 1.18 & 1.29 & $\mathrm{NaN}$ & $\mathrm{NaN}$ & 0.89 & 2.25 & 1.0 & 1.04 & $\mathrm{NaN}$ & $\mathrm{NaN}$ & 0.35 & 0.4 & $0.4-4>$ & 2.12 & 5.02 & 0.31 & 1.8 & & 1.60 & 0.15 & $\mathrm{aN}$ & \\
\hline NCOR2 & C9JE98 & 0.94 & 0.58 & 0.52 & $\mathrm{NaN}$ & 0.51 & 0.77 & 0.60 & $\mathrm{NaN}$ & 1.22 & 0.52 & 0.95 & 0.80 & $\mathrm{NaN}$ & 0.89 & 0.56 & 0.07 & 0.63 & 0.92 & $\mathrm{NaN}$ & $\mathrm{NaN}$ & 1.02 & 0.69 & $\mathrm{NaN}$ & $\mathrm{NaN}$ & $\mathrm{NaN}$ & $\mathrm{NaN}$ \\
\hline NEIL2 & Q969S2-2 & $\mathrm{NaN}$ & $\mathrm{NaN}$ & 0.82 & 4.46 & 1.60 & 0.96 & 1.32 & 2.03 & 1.39 & 0.88 & 1.02 & 1.01 & $\mathrm{NaN}$ & 1.58 & 1.05 & $\mathrm{NaN}$ & 1.46 & 0.81 & 1.71 & 0.78 & 0.97 & 1.22 & 1.55 & 1.13 & 1.29 & 1.25 \\
\hline
\end{tabular}




\begin{tabular}{|c|c|c|c|c|c|c|c|c|c|c|c|c|c|c|c|c|c|c|c|c|c|c|c|c|c|c|c|}
\hline Gene names & Protein IDs & $\begin{array}{l}H 3 \Delta 1- \\
20 \_F\end{array}$ & $\begin{array}{l}H 3 \Delta 1- \\
20 \_R\end{array}$ & $\begin{array}{l}\text { H4K2O } \\
\text { me1_F }\end{array}$ & $\begin{array}{l}\text { H4K20 } \\
\text { me1_R }\end{array}$ & $\begin{array}{l}\text { H4K20 } \\
\text { me3_F }\end{array}$ & $\begin{array}{l}\text { H4K20 } \\
\text { me3_R }\end{array}$ & $\begin{array}{r}\mathrm{H}_{3} \mathrm{~K}_{\mathrm{c}} 27 \\
\text { e1_F }\end{array}$ & $\begin{array}{l}\mathrm{H} 3 \mathrm{~K}_{\mathrm{C}} 27 \\
\mathrm{me1} \mathrm{R}\end{array}$ & $\begin{array}{l}\mathrm{H} 3 \mathrm{~K}_{\mathrm{C}} 27 \\
\mathrm{me} 2 \mathrm{~F}\end{array}$ & $\begin{array}{l}\mathrm{H} 3 K_{\mathrm{c}} 27 \\
\mathrm{me2} \_\mathrm{R}\end{array}$ & $\begin{array}{l}\mathrm{H} 3 \mathrm{~K}_{\mathrm{c}} 27 \\
\mathrm{me3} 3 \mathrm{~F}\end{array}$ & $\begin{array}{l}\mathrm{H} 3 \mathrm{~K}_{\mathrm{C}} 27 \\
\mathrm{me3} \text {.R }\end{array}$ & $\begin{array}{c}\text { НзК9m } \\
\text { e1_F }\end{array}$ & $\begin{array}{c}\text { HзК9m } \\
\text { e1_R }\end{array}$ & $\begin{array}{c}\text { Нзкаm } \\
\text { e2_F }\end{array}$ & $\begin{array}{c}\text { H3K9m } \\
\text { e2_R }\end{array}$ & $\begin{array}{c}\text { HзK9m } \\
\text { e3_F }\end{array}$ & $\begin{array}{c}\text { НзК9m } \\
\text { e3_R }\end{array}$ & $\begin{array}{c}\text { HзK9m } \\
\text { e3|meC } \\
\text { pG_F }\end{array}$ & $\begin{array}{c}\text { H3к9m } \\
\text { e3|meC } \\
\text { pG_R }\end{array}$ & $\mid \begin{array}{c}\mathrm{H} 3 \mathrm{~K} 9 \mathrm{~m} \\
\mathrm{e} 3 \mathrm{H} 4 \mathrm{~K} \\
20 \mathrm{me} 3 \\
\mathrm{~F}\end{array}$ & $\begin{array}{c}\mathrm{H} 3 \mathrm{~K} 9 \mathrm{~m} \\
\mathrm{e} 3 \mid \mathrm{H} 4 \mathrm{~K} \\
20 \mathrm{me3} \\
\mathrm{R}\end{array}$ & $\underset{\mathrm{F}}{\mathrm{meCpG}}$ & $\underset{-R}{\operatorname{meCpG}}$ & $\begin{array}{c}\text { H4R3m } \\
\text { e2_F }\end{array}$ & $\begin{array}{c}\text { H4R3n } \\
\text { e2_R }\end{array}$ \\
\hline NFATC2IP & Q8NCF5 & 0.47 & $\mathrm{NaN}$ & $\mathrm{NaN}$ & $\mathrm{NaN}$ & $\mathrm{NaN}$ & $\mathrm{NaN}$ & $\mathrm{NaN}$ & $\mathrm{NaN}$ & $\mathrm{NaN}$ & $\mathrm{NaN}$ & $\mathrm{NaN}$ & $\mathrm{NaN}$ & $\mathrm{NaN}$ & $\mathrm{NaN}$ & 0.45 & 0.26 & $\mathrm{NaN}$ & $\mathrm{NaN}$ & $\mathrm{NaN}$ & $\mathrm{NaN}$ & $\mathrm{NaN}$ & $\mathrm{NaN}$ & $\mathrm{NaN}$ & $\mathrm{NaN}$ & $\mathrm{NaN}$ & $\mathrm{NaN}$ \\
\hline NFIA & B1AKN8 & 0.60 & 0.38 & 0.78 & 0.49 & 0.41 & 1.62 & $\mathrm{NaN}$ & $\mathrm{NaN}$ & 0.21 & 0.53 & 0.84 & 0.68 & 0.24 & 2.93 & 0.09 & 2.99 & 0.18 & 2.64 & 69.81 & 0.15 & 2.04 & 0.25 & 1.04 & 0.87 & $\mathrm{NaN}$ & 0.26 \\
\hline NFIA & Q12857-2 & $\mathrm{NaN}$ & $\mathrm{NaN}$ & 0.23 & 0.60 & 0.44 & $\mathrm{NaN}$ & $\mathrm{NaN}$ & $\mathrm{NaN}$ & $\mathrm{NaN}$ & $\mathrm{NaN}$ & 0.70 & 0.48 & $\mathrm{NaN}$ & $\mathrm{NaN}$ & 0.05 & $\mathrm{NaN}$ & 0.17 & $\mathrm{NaN}$ & 3.42 & 0.04 & 1.63 & 0.27 & 1.13 & 0.91 & $\mathrm{NaN}$ & $\mathrm{NaN}$ \\
\hline NFIB & 000712-5 & $\mathrm{NaN}$ & $\mathrm{NaN}$ & $\mathrm{NaN}$ & $\mathrm{NaN}$ & $\mathrm{NaN}$ & $\mathrm{NaN}$ & $\mathrm{NaN}$ & $\mathrm{NaN}$ & $\mathrm{NaN}$ & $\mathrm{NaN}$ & $\mathrm{NaN}$ & $\mathrm{NaN}$ & $\mathrm{NaN}$ & $\mathrm{NaN}$ & $\mathrm{NaN}$ & $\mathrm{NaN}$ & $\mathrm{NaN}$ & $\mathrm{NaN}$ & $\mathrm{NaN}$ & $\mathrm{NaN}$ & $\mathrm{NaN}$ & $\mathrm{NaN}$ & $\mathrm{NaN}$ & $\mathrm{NaN}$ & $\mathrm{NaN}$ & $\mathrm{NaN}$ \\
\hline NFIB & Q5WW27 & $\mathrm{NaN}$ & $\mathrm{NaN}$ & $\mathrm{NaN}$ & $\mathrm{NaN}$ & $\mathrm{NaN}$ & $\mathrm{NaN}$ & $\mathrm{NaN}$ & $\mathrm{NaN}$ & $\mathrm{NaN}$ & $\mathrm{NaN}$ & $\mathrm{NaN}$ & $\mathrm{NaN}$ & $\mathrm{NaN}$ & $\mathrm{NaN}$ & $\mathrm{NaN}$ & $\mathrm{NaN}$ & $\mathrm{NaN}$ & $\mathrm{NaN}$ & NaN & $\mathrm{NaN}$ & $\mathrm{NaN}$ & 0.43 & $\mathrm{NaN}$ & $\mathrm{NaN}$ & $\mathrm{NaN}$ & $\mathrm{NaN}$ \\
\hline NFIB & Q5WW28 & $\mathrm{NaN}$ & $\mathrm{NaN}$ & $\mathrm{NaN}$ & $\mathrm{NaN}$ & $\mathrm{NaN}$ & $\mathrm{NaN}$ & $\mathrm{NaN}$ & $\mathrm{NaN}$ & $\mathrm{NaN}$ & $\mathrm{NaN}$ & $\mathrm{NaN}$ & $\mathrm{NaN}$ & $\mathrm{NaN}$ & $\mathrm{NaN}$ & $\mathrm{NaN}$ & $\mathrm{NaN}$ & $\mathrm{NaN}$ & $\mathrm{NaN}$ & $\mathrm{NaN}$ & $\mathrm{NaN}$ & $\mathrm{NaN}$ & $\mathrm{NaN}$ & $\mathrm{NaN}$ & $\mathrm{NaN}$ & $\mathrm{NaN}$ & $\mathrm{NaN}$ \\
\hline NFIB & Q5WW30 & $\mathrm{NaN}$ & 0.37 & 0.98 & 0.59 & 0.43 & 1.74 & $\mathrm{NaN}$ & 0.64 & 0.43 & $\mathrm{NaN}$ & 0.83 & 0.73 & 0.28 & 6.29 & 0.10 & 7.91 & 0.19 & 2.70 & 17.59 & 0.08 & 1.90 & 0.31 & 1.07 & 0.91 & $\mathrm{NaN}$ & $\mathrm{NaN}$ \\
\hline NFIC & P08651 & 0.73 & 0.85 & 0.97 & 0.56 & 0.40 & 1.54 & 0.26 & 0.27 & 0.19 & 0.42 & 0.84 & 0.75 & 0.11 & 7.60 & 0.11 & 11.19 & 0.19 & 2.33 & 16.73 & 0.01 & 2.02 & 0.27 & 1.41 & 0.99 & $\mathrm{NaN}$ & 0.20 \\
\hline NFIC & P08651-5 & $\mathrm{NaN}$ & $\mathrm{NaN}$ & $\mathrm{NaN}$ & $\mathrm{NaN}$ & $\mathrm{NaN}$ & $\mathrm{NaN}$ & $\mathrm{NaN}$ & $\mathrm{NaN}$ & $\mathrm{NaN}$ & $\mathrm{NaN}$ & $\mathrm{NaN}$ & $\mathrm{NaN}$ & $\mathrm{NaN}$ & $\mathrm{NaN}$ & $\mathrm{NaN}$ & $\mathrm{NaN}$ & $\mathrm{NaN}$ & $\mathrm{NaN}$ & $\mathrm{NaN}$ & $\mathrm{NaN}$ & $\mathrm{NaN}$ & 0.75 & $\mathrm{NaN}$ & $\mathrm{NaN}$ & $\mathrm{NaN}$ & $\mathrm{NaN}$ \\
\hline NFIC & P08651-6 & $\mathrm{NaN}$ & $\mathrm{NaN}$ & $\mathrm{NaN}$ & $\mathrm{NaN}$ & 0.54 & 1.49 & $\mathrm{NaN}$ & $\mathrm{NaN}$ & $\mathrm{NaN}$ & $\mathrm{NaN}$ & 1.03 & 0.84 & $\mathrm{NaN}$ & $\mathrm{NaN}$ & $\mathrm{NaN}$ & $\mathrm{NaN}$ & 0.28 & $\mathrm{NaN}$ & $\mathrm{NaN}$ & $\mathrm{NaN}$ & 2.75 & 0.39 & $\mathrm{NaN}$ & $\mathrm{NaN}$ & $\mathrm{NaN}$ & $\mathrm{NaN}$ \\
\hline NFIX & B4DM25 & $\mathrm{NaN}$ & 0.76 & 1.17 & 0.80 & 0.47 & 2.17 & $\mathrm{NaN}$ & $\mathrm{NaN}$ & 0.66 & $\mathrm{NaN}$ & 1.02 & 0.98 & 0.24 & 5.04 & 0.16 & $\mathrm{NaN}$ & 0.28 & 2.77 & 19.53 & 0.14 & 1.68 & 0.48 & 1.52 & 0.87 & $\mathrm{NaN}$ & $\mathrm{NaN}$ \\
\hline NFIX & C9JWJ8 & $\mathrm{NaN}$ & $\mathrm{NaN}$ & $\mathrm{Na}$ & $\mathrm{NaN}$ & $\mathrm{NaN}$ & $\mathrm{NaN}$ & $\mathrm{NaN}$ & $\mathrm{NaN}$ & $\mathrm{NaN}$ & $\mathrm{NaN}$ & $\mathrm{NaN}$ & $\mathrm{NaN}$ & $\mathrm{NaN}$ & $\mathrm{NaN}$ & $\mathrm{NaN}$ & $\mathrm{NaN}$ & $\mathrm{NaN}$ & $\mathrm{NaN}$ & 6.98 & 0.63 & 1.73 & 0.44 & $\mathrm{NaN}$ & $\mathrm{NaN}$ & $\mathrm{NaN}$ & $\mathrm{NaN}$ \\
\hline NFIX & D2DXM9 & $\mathrm{NaN}$ & $\mathrm{NaN}$ & $\mathrm{NaN}$ & $\mathrm{NaN}$ & $\mathrm{NaN}$ & $\mathrm{NaN}$ & $\mathrm{NaN}$ & $\mathrm{NaN}$ & $\mathrm{NaN}$ & $\mathrm{NaN}$ & $\mathrm{NaN}$ & $\mathrm{NaN}$ & $\mathrm{NaN}$ & $\mathrm{NaN}$ & $\mathrm{NaN}$ & $\mathrm{NaN}$ & $\mathrm{NaN}$ & $\mathrm{NaN}$ & $\mathrm{NaN}$ & 0.24 & $\mathrm{NaN}$ & $\mathrm{NaN}$ & $\mathrm{NaN}$ & $\mathrm{NaN}$ & $\mathrm{NaN}$ & $\mathrm{NaN}$ \\
\hline NFRKB & Q6P4R8-3 & 0.70 & 0.44 & 1.87 & 0.60 & 0.88 & 1.62 & 1.18 & 0.87 & 0.65 & 2.45 & 1.27 & 1.08 & 0.38 & 6.92 & 0.27 & 0.56 & 0.48 & 2.35 & 36.59 & 0.02 & 2.53 & 0.50 & 1.35 & 2.70 & 1.91 & 0.32 \\
\hline NIPBL & Q6KC79 & 0.86 & 0.66 & 0.58 & 0.89 & 0.87 & 0.78 & $\mathrm{NaN}$ & 0.69 & 1.01 & 0.42 & 1.15 & 0.73 & 1.53 & 0.68 & 1.95 & 0.12 & 3.88 & 0.13 & 14.75 & 0.19 & 3.86 & 0.12 & $\mathrm{NaN}$ & 1.06 & 0.91 & 0.61 \\
\hline NIPBL & Q6KC79-2 & $\mathrm{NaN}$ & $\mathrm{NaN}$ & $\mathrm{NaN}$ & $\mathrm{NaN}$ & $\mathrm{NaN}$ & $\mathrm{NaN}$ & $\mathrm{NaN}$ & $\mathrm{NaN}$ & $\mathrm{NaN}$ & $\mathrm{NaN}$ & $\mathrm{NaN}$ & $\mathrm{NaN}$ & $\mathrm{NaN}$ & $\mathrm{NaN}$ & $\mathrm{NaN}$ & 0.16 & $\mathrm{NaN}$ & $\mathrm{NaN}$ & $\mathrm{NaN}$ & $\mathrm{NaN}$ & 2.31 & 0.07 & $\mathrm{NaN}$ & $\mathrm{NaN}$ & $\mathrm{NaN}$ & $\mathrm{NaN}$ \\
\hline NOLC1 & Q14978-2 & 0.60 & 0.61 & 0.68 & 1.21 & 0.91 & 0.76 & 0.98 & 1.23 & 1.02 & 0.81 & 0.65 & 1.34 & 0.89 & 0.73 & 0.83 & 0.66 & 1.21 & 0.56 & 1.12 & 2.64 & 0.37 & 1.85 & 2.18 & 1.32 & 0.44 & 2.62 \\
\hline NOLC1 & Q14978-3 & $\mathrm{NaN}$ & $\mathrm{NaN}$ & 0.90 & 1.22 & 1.21 & 1.11 & 1.17 & 1.66 & 1.38 & 1.17 & 0.81 & 1.48 & 1.15 & 1.11 & $\mathrm{NaN}$ & $\mathrm{NaN}$ & 1.38 & 0.69 & 1.21 & 3.02 & 0.46 & 2.39 & 2.42 & 1.51 & 0.38 & 3.33 \\
\hline NPM1 & E5RI98 & $\mathrm{NaN}$ & $\mathrm{NaN}$ & $\mathrm{NaN}$ & $\mathrm{NaN}$ & $\mathrm{NaN}$ & $\mathrm{NaN}$ & $\mathrm{NaN}$ & $\mathrm{NaN}$ & $\mathrm{NaN}$ & $\mathrm{NaN}$ & $\mathrm{NaN}$ & $\mathrm{NaN}$ & $\mathrm{NaN}$ & $\mathrm{NaN}$ & $\mathrm{NaN}$ & $\mathrm{NaN}$ & $\mathrm{NaN}$ & $\mathrm{NaN}$ & $\mathrm{NaN}$ & $\mathrm{NaN}$ & $\mathrm{NaN}$ & $\mathrm{NaN}$ & $\mathrm{NaN}$ & $\mathrm{NaN}$ & $\mathrm{NaN}$ & $\mathrm{NaN}$ \\
\hline PM1 & P06748 & 1.11 & 1.51 & & 0.97 & 2.06 & & 0.84 & 1.34 & 1.20 & 1.02 & 0.80 & 1.81 & 1.14 & 1.42 & 1.06 & 0.47 & 1.60 & 1.03 & & & 1.07 & 1.66 & 0.95 & 0.83 & 0.98 & 1.43 \\
\hline NR2C1 & H9NIM2 & 0.43 & 1.18 & 0.85 & 0.60 & 1.32 & 1.40 & $\begin{array}{l}1.34 \\
1.34\end{array}$ & $\mathrm{NaN}$ & 0.53 & 2.23 & 1.19 & 0.86 & 1.121 & 0.91 & 0.32 & 0.41 & 0.96 & 1.18 & 3.06 & 0.36 & 2.20 & 0.58 & 0.56 & 0.95 & $\mathrm{NaN}$ & $\mathrm{NaN}$ \\
\hline NS5ATP4A & Q09GNo & 0.27 & $\mathrm{NaN}$ & $\mathrm{NaN}$ & $\mathrm{NaN}$ & $\mathrm{NaN}$ & $\mathrm{NaN}$ & $\mathrm{NaN}$ & $\mathrm{NaN}$ & $\mathrm{NaN}$ & $\mathrm{NaN}$ & $\mathrm{NaN}$ & $\mathrm{NaN}$ & $\mathrm{NaN}$ & $\mathrm{NaN}$ & 0.49 & 0.18 & $\mathrm{NaN}$ & $\mathrm{NaN}$ & $\mathrm{NaN}$ & $\mathrm{NaN}$ & $\mathrm{NaN}$ & $\mathrm{NaN}$ & $\mathrm{NaN}$ & $\mathrm{NaN}$ & $\mathrm{NaN}$ & $\mathrm{NaN}$ \\
\hline ORC2 & Q13416 & 0.66 & 0.73 & 1.03 & 1.03 & 7.63 & 0.04 & 1. 18 & 0.88 & 0.84 & 0.94 & 2.93 & 0.20 & 1.07 & 0.89 & 0.76 & 0.19 & 2.86 & 0.19 & 3.24 & 0.78 & 7.26 & 0.08 & 2.47 & 1.05 & 0.82 & 0.95 \\
\hline ORC3 & Q9UBD5-2 & 0.82 & 0.83 & 0.89 & 0.96 & 6.95 & 0.04 & 1.00 & 0.87 & 0.83 & 0.8 & 2.39 & 0.19 & 1.06 & 0.79 & 0.88 & 0.44 & & & 2.98 & & 5.33 & 0.06 & 2.79 & 1.10 & 0.75 & 0.97 \\
\hline ORC3L & Q9UBD5 & $\mathrm{NaN}$ & $\mathrm{NaN}$ & 1.85 & $\mathrm{NaN}$ & 6.65 & 0.16 & $\mathrm{NaN}$ & 0.68 & 1.47 & 1.45 & 2.26 & 0.24 & $\mathrm{NaN}$ & $\mathrm{NaN}$ & 0.68 & 0.10 & $\mathrm{NaN}$ & 0.24 & $\mathrm{NaN}$ & 1.29 & 6.24 & 0.25 & $\mathrm{NaN}$ & $\mathrm{NaN}$ & $\mathrm{NaN}$ & 0.75 \\
\hline ORC5 & A4DOP7 & 0.59 & 0.74 & $\begin{array}{l}1.05 \\
1.25\end{array}$ & 1.14 & $\begin{array}{l}.05 \\
7.95\end{array}$ & 0.08 & 1.48 & $\begin{array}{l}0.00 \\
1.05\end{array}$ & 0.99 & $\begin{array}{l}1.45 \\
1.07\end{array}$ & 2.88 & $\begin{array}{l}0.24 \\
0.21\end{array}$ & 1.00 & 0.96 & 0.64 & 0.41 & 2.66 & $\begin{array}{l}0.24 \\
0.24\end{array}$ & 6.95 & 0.40 & $\begin{array}{l}0.44 \\
6.65\end{array}$ & 0.08 & 2.84 & 1.07 & 1.34 & 1.27 \\
\hline ORC6 & B $3 K M P 9$ & 0.67 & 0.60 & $\mathrm{NaN}$ & $\mathrm{NaN}$ & 4.67 & 0.16 & 1.20 & $\mathrm{NaN}$ & $\mathrm{NaN}$ & 0.82 & 0.97 & 0.58 & $\mathrm{NaN}$ & 0.59 & 0.50 & 0.07 & 1.70 & 0.34 & 1.09 & 1.06 & $\mathrm{NaN}$ & 0.49 & $\mathrm{NaN}$ & $\mathrm{NaN}$ & $\mathrm{NaN}$ & $\mathrm{NaN}$ \\
\hline OXSR1 & 095747 & 1.88 & 1.50 & $\mathrm{NaN}$ & $\mathrm{NaN}$ & 0.66 & 0.50 & 0.82 & 0.86 & 0.82 & 1.30 & 0.69 & 1.45 & 0.66 & 1.07 & 1.51 & 0.25 & 1.21 & 1.16 & $\mathrm{NaN}$ & $\mathrm{NaN}$ & 0.64 & 0.85 & $\mathrm{NaN}$ & $\mathrm{NaN}$ & 1.51 & 0.70 \\
\hline PARD3 & TEWO-1 & 1.90 & $\mathrm{~N}$ & $\mathrm{~N}$ & $\mathrm{Na}$ & $\mathrm{aN}$ & & 0.4 & 0.49 & & $\mathrm{Nal}$ & & & $\mathrm{NaN}$ & $\mathrm{NaN}$ & 0. & & & $\mathrm{JaN}$ & 1.24 & & 1.08 & $\mathrm{NaN}$ & 0.48 & 1.02 & $\mathrm{NaN}$ & 0.79 \\
\hline PARK7 & Q99497 & $\mathrm{NaN}$ & 0.12 & $\mathrm{NaN}$ & $\mathrm{NaN}$ & $\mathrm{NaN}$ & $\mathrm{NaN}$ & $\mathrm{NaN}$ & $\mathrm{NaN}$ & $\mathrm{NaN}$ & $\mathrm{NaN}$ & $\mathrm{NaN}$ & $\mathrm{NaN}$ & $\mathrm{NaN}$ & $\mathrm{NaN}$ & 0.16 & $\mathrm{NaN}$ & $\mathrm{NaN}$ & $\mathrm{NaN}$ & 0.21 & $\mathrm{NaN}$ & $\mathrm{NaN}$ & $\mathrm{NaN}$ & 0.84 & 0.39 & $\mathrm{NaN}$ & $\mathrm{NaN}$ \\
\hline PATZ1 & $99 \mathrm{HH} 1$ & 1.01 & 0.50 & 0.92 & 1.34 & 0.54 & 1.78 & $\mathrm{NaN}$ & $\mathrm{NaN}$ & 1.55 & 1.18 & 0.73 & 0.71 & $\mathrm{NaN}$ & $\mathrm{NaN}$ & 0.57 & 3.04 & 0.43 & 2.06 & 2.87 & 0.62 & 1.10 & 0.73 & 1.03 & 0.90 & 3.95 & $\mathrm{NaN}$ \\
\hline PAXIP1 & B4DEQ6 & 0.63 & 1.15 & 2.01 & 0.54 & 1.06 & 1.16 & $\mathrm{NaN}$ & $\mathrm{NaN}$ & 0.50 & 1.89 & 1.20 & 1. & 0.90 & 1.72 & 0.43 & 0.07 & 0.51 & 2.13 & 6.18 & 0. & 1.71 & 0.88 & 2.02 & 1.34 & 0.67 & $\mathrm{NaN}$ \\
\hline $\mathrm{PB} \times 2$ & P404: & $\mathrm{NaN}$ & $\mathrm{NaN}$ & $\mathrm{NaN}$ & $\mathrm{Na}$ & 2.33 & 0.51 & $\mathrm{Nal}$ & $\mathrm{NaN}$ & & $\mathrm{NaN}$ & 1.2 & & & & 0.6 & & & & & & & 0.45 & & 50 & $\mathrm{NaN}$ & $\mathrm{NaN}$ \\
\hline PC4 & Q61BA2 & 1.04 & 1.15 & 7.1 & 0.83 & 0.84 & 0.95 & 0.98 & 0.96 & 0. & 0.92 & 0.88 & 0 & 0.87 & 0.88 & 0.96 & 1.43 & 0.77 & 0.94 & 0.98 & 1.27 & 0.72 & 0.95 & $\begin{array}{l}1.07 \\
1.07\end{array}$ & 1.00 & 0.87 & 1.08 \\
\hline PCMT1 & BY58 & 1.18 & 1.40 & 0.3 & 0.5 & 0. & 0.44 & 0. & 0.23 & & 0. & 0.54 & 1 & $\mathrm{NaN}$ & $\mathrm{NaN}$ & 1.22 & 4.14 & 0.21 & 0.67 & .38 & 0.36 & 0.26 & 0.46 & 0.49 & 0.37 & 0.76 & 0.32 \\
\hline PCNA & $\mathrm{P}$ & 0.38 & 0. & 1.0 & 0.63 & & 1.3 & 0.8 & 1.0 & & 1.68 & 0. & & 0.34 & 1.78 & 0. & & 0. & 1. & & & 2.09 & & 0.93 & 1.06 & 1.28 & 0.41 \\
\hline PCSK9 & NBP7 & $\mathrm{NaN}$ & $\mathrm{NaN}$ & $\mathrm{NaN}$ & 0.45 & 0.46 & 0.51 & $\mathrm{NaN}$ & $\mathrm{NaN}$ & 0.3 & 0.44 & 0.55 & $\mathrm{NaN}$ & 0.62 & 0.33 & $\mathrm{NaN}$ & 0.77 & 0.40 & 0.48 & $\mathrm{NaN}$ & $\mathrm{NaN}$ & 0.32 & 0.40 & $\mathrm{NaN}$ & $\mathrm{NaN}$ & $\mathrm{NaN}$ & $\mathrm{NaN}$ \\
\hline PDIA5 & $Q$ & $\mathrm{NaI}$ & $\mathrm{NaN}$ & $\mathrm{Na}$ & $\mathrm{Nal}$ & 3.5 & & $\mathrm{Na}$ & $\mathrm{NaN}$ & & $\mathrm{Nal}$ & $\mathrm{NaN}$ & $\mathrm{NaN}$ & $\mathrm{NaN}$ & $\mathrm{Nal}$ & $\mathrm{NaN}$ & NaN & $\mathrm{Na}$ & & $\mathrm{NaN}$ & $\mathrm{Na}$ & $\mathrm{NaN}$ & $\mathrm{NaN}$ & $\mathrm{NaN}$ & $\mathrm{NaN}$ & $\mathrm{NaN}$ & $\mathrm{NaN}$ \\
\hline PES1 & B5MCF9 & $\mathrm{NaN}$ & 1.93 & $\mathrm{NaN}$ & $\mathrm{NaN}$ & $\mathrm{NaN}$ & $\mathrm{NaN}$ & $\mathrm{Nal}$ & $\mathrm{NaN}$ & $\mathrm{NaN}$ & $\mathrm{Na}$ & 1.25 & 0.71 & $\mathrm{NaN}$ & $\mathrm{NaN}$ & 1.92 & $\mathrm{NaN}$ & $\mathrm{NaN}$ & $\mathrm{NaN}$ & 1.16 & 1.42 & $\mathrm{NaN}$ & $\mathrm{NaN}$ & 1.21 & $\mathrm{NaN}$ & $\mathrm{NaN}$ & $\mathrm{NaN}$ \\
\hline PFN1 & & 0 & 0. & $\mathrm{~N}$ & $\mathrm{Na}$ & & & 0.3 & 1.4 & $N_{c}$ & 0.3 & $\mathrm{NaN}$ & & $\mathrm{Na}$ & $\mathrm{Nal}$ & $\mathrm{NaN}$ & $\mathrm{NaN}$ & $0 . \varepsilon$ & $\mathrm{NaN}$ & $\mathrm{NaN}$ & $\mathrm{Na}$ & $\mathrm{NaN}$ & $\mathrm{NaN}$ & $\mathrm{NaN}$ & $\mathrm{NaN}$ & 0.15 & $\mathrm{NaN}$ \\
\hline PGAM1 & QE & 0.30 & 0.16 & $\mathrm{NaN}$ & $\mathrm{NaN}$ & $\mathrm{NaN}$ & 0.88 & 0.37 & 2.87 & 0.31 & 0.51 & $\mathrm{NaN}$ & $\mathrm{NaN}$ & $\mathrm{NaN}$ & $\mathrm{NaN}$ & 0.24 & 0.25 & $\mathrm{NaN}$ & 2.91 & 0.58 & 1.33 & $\mathrm{NaN}$ & $\mathrm{NaN}$ & 0.26 & $\mathrm{NaN}$ & $\mathrm{NaN}$ & $\mathrm{NaN}$ \\
\hline PGBD3 & & $\mathrm{NaN}$ & $\mathrm{NaN}$ & 0.9 & 0.5 & 0.79 & 0.6 & 0.6 & 0.5 & 0. & 0. & 0.7 & & 0. & 1.99 & & $\mathrm{NaN}$ & 0. & 0.86 & 9.50 & & & 0.65 & 4.32 & 019 & 0.85 & 0.24 \\
\hline PGK1 & & 0.4 & 0.1 & 2. & $\mathrm{Na}$ & & 0. & No & 4.6 & 2. & 0. & $\mathrm{~N} a$ & & $\mathrm{~N}$ & $\mathrm{Na}$ & & 0 & 1.8 & 0. & 0. & & & $\mathrm{~N}$ & 1.10 & & $\mathrm{aN}$ & $\mathrm{NaN}$ \\
\hline PHF1 & & $\mathrm{N}$ & & $\mathrm{N}=$ & & & 0. & $\mathrm{NaN}$ & $\mathrm{NaN}$ & 5. & 0. & 9.4 & & $\mathrm{Na}$ & $\mathrm{Nal}$ & & $\mathrm{Na}$ & $\mathrm{NaN}$ & $\mathrm{NaN}$ & $\mathrm{Na}$ & $\mathrm{Na}$ & $\mathrm{NaN}$ & 2.81 & $\mathrm{NaN}$ & $\mathrm{NaN}$ & $\mathrm{NaN}$ & $\mathrm{NaN}$ \\
\hline PHF14 & o94880 & 0.07 & 2.12 & 1.45 & 1.13 & 1.4 & 1.3 & 1.00 & 1.26 & 1.0 & 1.51 & $\begin{array}{l}. .40 \\
1.08\end{array}$ & 1.29 & 0.67 & 3.93 & 0.50 & 0.95 & 1.25 & 1.24 & 1.23 & 2.00 & 1.11 & 1.43 & 1.27 & 1.95 & 0.61 & 1.92 \\
\hline PHF14 & O94880-2 & 0.12 & $\mathrm{NaN}$ & $\mathrm{NaN}$ & $\mathrm{NaN}$ & 2.02 & 1.47 & 1.25 & 2.05 & 1.16 & 2.45 & 1.52 & 2.00 & $\mathrm{NaN}$ & $\mathrm{NaN}$ & $\mathrm{NaN}$ & $\mathrm{NaN}$ & $\mathrm{NaN}$ & 2.32 & $\mathrm{NaN}$ & 1.97 & $\mathrm{NaN}$ & 1.40 & 1.36 & $\mathrm{NaN}$ & $\mathrm{NaN}$ & 1.72 \\
\hline PHF20L & & $\mathrm{NaN}$ & $\mathrm{NaN}$ & $\mathrm{NaN}$ & 1.2 & & 1.92 & & & & & & & & $\mathrm{NaN}$ & & & & & & & & 2.72 & & & & \\
\hline PHF2 & & 0.27 & 0. & $\mathrm{Na}$ & 0.4 & 0 & 0. & $\mathrm{Na}$ & $\mathrm{Na}$ & $\mathrm{N}$ & 0. & 0.5 & & $\mathrm{Na}$ & $\mathrm{NaN}$ & & 0.5 & 0.6 & 0.4 & 0. & 0. & 0.67 & 0.38 & $\mathrm{NaN}$ & 0.80 & $\mathrm{NaN}$ & $\mathrm{NaN}$ \\
\hline PHF5A & Q7RTVO & 1.51 & 1.0 & $\mathrm{NaN}$ & $\mathrm{NaN}$ & 1.62 & 0.54 & 0.86 & 1.37 & 2.1 & 0.51 & $\mathrm{NaN}$ & $\mathrm{NaN}$ & 1.97 & 0.63 & 0.84 & 1.0 & 2.2 & 0.52 & $\mathrm{NaN}$ & $\mathrm{NaN}$ & 0.49 & 2.55 & $\mathrm{NaN}$ & $\mathrm{NaN}$ & 0.43 & $\mathrm{NaN}$ \\
\hline PHF8 & Q9UPP1-2 & 1.28 & 2.06 & 3.05 & 0.50 & 1.79 & 0.54 & 1.13 & 1.76 & 1.63 & 1.23 & 0.66 & 2.50 & $\mathrm{NaN}$ & 3.17 & $\mathrm{NaN}$ & $\mathrm{NaN}$ & 1.41 & 0.42 & $\mathrm{NaN}$ & $\mathrm{NaN}$ & 1.17 & 0.53 & 1.28 & 1.05 & 0.48 & 1.90 \\
\hline PKM & P14618 & 0.30 & 0.26 & 0.62 & 1.34 & 1.57 & 0.83 & 0.25 & 1.77 & 1.05 & 0.88 & 0.81 & 0.50 & 0.82 & 1. & 0.43 & 0.47 & 0.67 & & 0.46 & & & & & & & 0.92 \\
\hline PKM & & $\mathrm{NaN}$ & $\mathrm{Na}$ & $\mathrm{Na}$ & $\mathrm{Na}$ & & $\mathrm{N}$ & $\mathrm{Na}$ & $\mathrm{Na}$ & $\mathrm{N}$ & $\mathrm{N}$ & $\mathrm{Na}$ & & $\mathrm{N}$ & $\mathrm{N}$ & & $\mathrm{N}$ & $\mathrm{NaN}$ & & $\mathrm{N}$ & & & $\mathrm{NaN}$ & $\mathrm{aN}$ & $\mathrm{aN}$ & $\mathrm{NaN}$ & $\mathrm{NaN}$ \\
\hline PKP2 & Q99959-2 & 0.49 & 0.35 & 0.77 & $\mathrm{NaN}$ & 0.73 & 11.63 & 0.71 & 0.56 & 0.86 & 0.86 & 0.83 & 0.80 & 0.61 & 0.91 & 0.34 & 0.22 & 0.74 & 1.06 & 0.47 & 0.89 & 0.88 & 0.60 & 0.61 & 0.49 & 0.87 & 0.63 \\
\hline PLEKHA4 & Q59H51 & 0.46 & $\mathrm{NaN}$ & $\mathrm{NaN}$ & $\mathrm{NaN}$ & $\mathrm{NaN}$ & $\mathrm{NaN}$ & $\mathrm{NaN}$ & $\mathrm{NaN}$ & $\mathrm{NaN}$ & $\mathrm{NaN}$ & $\mathrm{NaN}$ & $\mathrm{NaN}$ & $\mathrm{NaN}$ & $\mathrm{NaN}$ & $\mathrm{NaN}$ & $\mathrm{NaN}$ & $\mathrm{NaN}$ & $\mathrm{NaN}$ & $\mathrm{NaN}$ & $\mathrm{NaN}$ & $\mathrm{NaN}$ & $\mathrm{NaN}$ & $\mathrm{NaN}$ & $\mathrm{NaN}$ & $\mathrm{NaN}$ & $\mathrm{NaN}$ \\
\hline PLK1 & B2R841 & 1.18 & $\mathrm{NaN}$ & $\mathrm{NaN}$ & 0.92 & 0.98 & 0.63 & $\mathrm{NaN}$ & $\mathrm{NaN}$ & $\mathrm{NaN}$ & $\mathrm{NaN}$ & 0.73 & $\mathrm{NaN}$ & 0.95 & 0.51 & 1.12 & 0.21 & 0.90 & 0.65 & NN & NAN & 0.98 & 00 & $\mathrm{NaN}$ & & & \\
\hline & & 1.01 & 0. & 0.73 & 0.70 & & 0. & $\mathrm{NaN}$ & & & $\mathrm{Na}$ & $\mathrm{N}$ & & & 0.59 & & 0.15 & & & & & & & & & & \\
\hline $\begin{array}{l}\text { PNISR } \\
\text { PNIR }\end{array}$ & $\begin{array}{l}\text { O8TF01 } \\
\text { Q8TFo }\end{array}$ & $\begin{array}{l}1.00 \\
1.00\end{array}$ & $\begin{array}{l}0.65 \\
1.25\end{array}$ & 2.45 & 0.45 & $\begin{array}{l}\mathrm{NaN} \\
\end{array}$ & $\mathrm{NaN}$ & $\mathrm{NaN}$ & $\mathrm{NaN}$ & $\mathrm{NaN}$ & $\mathrm{NaN}$ & $\mathrm{NaN}$ & $\mathrm{NaN}$ & $\mathrm{NaN}$ & $\mathrm{NaN}$ & $\begin{array}{l}1.52 \\
1.55\end{array}$ & $\begin{array}{l}0.10 \\
2.12\end{array}$ & $\mathrm{NaN}$ & $\mathrm{NaN}$ & $\mathrm{NaN}$ & $\mathrm{NaN}$ & $\begin{array}{l}\mathrm{NaN} \\
\mathrm{Na}\end{array}$ & $\mathrm{NaN}$ & $\mathrm{NaN}$ & $\mathrm{NaN}$ & $\mathrm{NaN}$ & $\mathrm{NaN}$ \\
\hline PNN & A8K964 & 9.86 & 2.15 & 0.46 & 1.83 & 0.91 & 0.63 & 1.53 & $\mathrm{NaN}$ & 2.15 & 0.41 & 1.26 & 1.18 & 1.69 & 0.63 & 2.83 & 1.72 & 1.29 & 0.50 & 1.51 & 2.20 & 0.59 & 1.61 & 1.78 & 1.67 & 0.45 & 0.51 \\
\hline
\end{tabular}




\begin{tabular}{|c|c|c|c|c|c|c|c|c|c|c|c|c|c|c|c|c|c|c|c|c|c|c|c|c|c|c|c|}
\hline Genenames & Protein IDs & $\begin{array}{l}\mathrm{H} 3 \Delta-1- \\
20 \_\mathrm{F}\end{array}$ & $\begin{array}{l}\mathrm{H} 3 \Delta{ }^{-1-} \\
20 \_\mathrm{R}\end{array}$ & $\begin{array}{l}\text { H4K20 } \\
\text { me1_F }\end{array}$ & $\begin{array}{l}\text { H4K20 } \\
\text { me1_R }\end{array}$ & $\begin{array}{l}\text { H4K20 } \\
\text { me3_F }\end{array}$ & $\begin{array}{l}\text { H4K20 } \\
\text { me3_R }\end{array}$ & $\begin{array}{l}\mathrm{H} 3 \mathrm{~K}_{\mathrm{C}} 27 \\
\text { me1_F }\end{array}$ & $\begin{array}{c}\mathrm{H} 3 \mathrm{~K}_{\mathrm{C}} 27 \\
\mathrm{me1} \_\mathrm{R}\end{array}$ & $\begin{array}{l}\mathrm{H} 3 \mathrm{~K}_{\mathrm{c}}^{\mathrm{m} 27} \\
\text { me2_F }\end{array}$ & $\begin{array}{l}\mathrm{H} 3 \mathrm{~K}_{\mathrm{C}} 27 \\
\mathrm{me2} \_\mathrm{R}\end{array}$ & $\begin{array}{l}\mathrm{H} 3 \mathrm{~K}_{\mathrm{c}}^{\mathrm{c} 27} \\
\mathrm{me} \_\mathrm{F}\end{array}$ & $\begin{array}{l}\mathrm{H} 3 \mathrm{~K}_{\mathrm{C}} 27 \\
\mathrm{me3} \_\mathrm{R}\end{array}$ & $\begin{array}{c}\text { НзК9m } \\
\text { e1_F }\end{array}$ & \begin{tabular}{|c} 
H3K9m \\
e1_R
\end{tabular} & \begin{tabular}{|} 
H3K9m \\
e2_F
\end{tabular} & $\begin{array}{c}\mathrm{H} 3 \mathrm{k} 9 \mathrm{~m} \\
\mathrm{e} 2 \_\mathrm{R}\end{array}$ & \begin{tabular}{|c} 
H3K9m \\
e3_F
\end{tabular} & $\begin{array}{c}\text { H3K9m } \\
\text { e3_R }\end{array}$ & $\begin{array}{c}\text { H3K9m } \\
\text { e3|mec } \\
\text { pG_F }\end{array}$ & $\begin{array}{c}\text { HзK9m } \\
\text { e3|meC } \\
\text { pG_R }\end{array}$ & \begin{tabular}{|c|c}
$\mathrm{H} 3 \mathrm{~K} 9 \mathrm{~m}$ \\
$\mathrm{e} \mid \mathrm{|H} 4 \mathrm{~K}$ \\
$20 \mathrm{me}{ }_{-}$ \\
$\mathrm{F}$
\end{tabular} & $\mid \begin{array}{c}\mathrm{H} 3 \mathrm{~K} / \mathrm{m} \\
\mathrm{e}|\mathrm{H}| \mathrm{HK} \\
20 \mathrm{me}{ }_{-} \\
\mathrm{R}\end{array}$ & $\underset{-F}{\mathrm{meCpG}}$ & $\begin{array}{c}\mathrm{meCpG} \\
-\mathrm{R}\end{array}$ & $\begin{array}{c}\text { H4R3m } \\
\text { e2_F }\end{array}$ & $\begin{array}{c}\text { H4R3m } \\
\text { e2_R }\end{array}$ \\
\hline PNP & Q8N7G1 & $\mathrm{NaN}$ & 0.18 & $\mathrm{NaN}$ & $\mathrm{NaN}$ & $\mathrm{NaN}$ & $\mathrm{NaN}$ & 0.39 & $\mathrm{NaN}$ & $\mathrm{NaN}$ & $\mathrm{NaN}$ & $\mathrm{NaN}$ & $\mathrm{NaN}$ & $\mathrm{NaN}$ & $\mathrm{NaN}$ & 0.47 & 0.59 & $\mathrm{NaN}$ & $\mathrm{NaN}$ & \begin{tabular}{|l|l|}
0.27 \\
\end{tabular} & $\mathrm{NaN}$ & $\mathrm{NaN}$ & $\mathrm{NaN}$ & 1.01 & $\mathrm{NaN}$ & $\mathrm{NaN}$ & $\mathrm{NaN}$ \\
\hline POF1B & Q8WV4 & $\mathrm{NaN}$ & $\mathrm{NaN}$ & $\mathrm{NaN}$ & $\mathrm{NaN}$ & $\mathrm{NaN}$ & $\mathrm{NaN}$ & 0.12 & $\mathrm{NaN}$ & $\mathrm{NaN}$ & $\mathrm{NaN}$ & $\mathrm{NaN}$ & $\mathrm{NaN}$ & $\mathrm{NaN}$ & $\mathrm{NaN}$ & 0.15 & $\mathrm{NaN}$ & $\mathrm{NaN}$ & $\mathrm{NaN}$ & 0.21 & $\mathrm{NaN}$ & $\mathrm{NaN}$ & $\mathrm{NaN}$ & $\mathrm{NaN}$ & $\mathrm{NaN}$ & $\mathrm{NaN}$ & $\mathrm{NaN}$ \\
\hline POGZ & Q7ZЗКз-5 & $\mathrm{NaN}$ & 0.69 & 1.51 & 0.93 & 1.07 & 1.35 & 0.86 & $\mathrm{NaN}$ & 0.59 & 1.84 & 1.13 & 1.16 & 2.13 & 1.51 & 0.77 & $\mathrm{NaN}$ & 5.91 & 0.11 & 47.32 & 0.14 & 7.48 & 0.08 & 5.82 & 0.79 & $\mathrm{NaN}$ & $\mathrm{NaN}$ \\
\hline POGZ & Q7Z3Кз-7 & $\mathrm{NaN}$ & $\mathrm{NaN}$ & $\mathrm{NaN}$ & $\mathrm{NaN}$ & $\mathrm{NaN}$ & $\mathrm{NaN}$ & $\mathrm{NaN}$ & $\mathrm{NaN}$ & $\mathrm{NaN}$ & $\mathrm{NaN}$ & $\mathrm{NaN}$ & $\mathrm{NaN}$ & $\mathrm{NaN}$ & $\mathrm{NaN}$ & $\mathrm{NaN}$ & $\mathrm{NaN}$ & 2.77 & $\mathrm{NaN}$ & 15.19 & $\mathrm{NaN}$ & $\mathrm{NaN}$ & $\mathrm{NaN}$ & 6.14 & $\mathrm{NaN}$ & $\mathrm{NaN}$ & $\mathrm{NaN}$ \\
\hline POLD3 & B7ZAQ5 & 0.51 & $\mathrm{NaN}$ & $\mathrm{NaN}$ & 0.59 & 0.42 & 0.48 & $\mathrm{NaN}$ & $\mathrm{NaN}$ & $\mathrm{NaN}$ & 0.84 & 0.77 & 0.66 & $\mathrm{NaN}$ & 0.40 & 0.70 & 0.45 & $\mathrm{NaN}$ & $\mathrm{NaN}$ & $\mathrm{NaN}$ & 1.00 & 0.58 & 0.53 & 1.72 & $\mathrm{NaN}$ & $\mathrm{NaN}$ & 0.54 \\
\hline POLG & E5KNX5 & $\mathrm{NaN}$ & $\mathrm{NaN}$ & $\mathrm{NaN}$ & 0.41 & 0.38 & 0.38 & 0.29 & $\mathrm{NaN}$ & 0.73 & 1.00 & 0.37 & 0.51 & $\mathrm{NaN}$ & $\mathrm{NaN}$ & 0.45 & $\mathrm{NaN}$ & 0.19 & 0.52 & $\mathrm{NaN}$ & $\mathrm{NaN}$ & 0.49 & 0.47 & $\mathrm{NaN}$ & $\mathrm{NaN}$ & $\mathrm{NaN}$ & 0.42 \\
\hline POLR1A & B7ZKR9 & $\mathrm{NaN}$ & $\mathrm{NaN}$ & $\mathrm{NaN}$ & $\mathrm{NaN}$ & $\mathrm{NaN}$ & $\mathrm{NaN}$ & 1.13 & $\mathrm{NaN}$ & $\mathrm{NaN}$ & $\mathrm{NaN}$ & $\mathrm{NaN}$ & $\mathrm{NaN}$ & $\mathrm{NaN}$ & $\mathrm{NaN}$ & $\mathrm{NaN}$ & 2.76 & 0.34 & $\mathrm{NaN}$ & $\mathrm{NaN}$ & 0.37 & $\mathrm{NaN}$ & 0.51 & $\mathrm{NaN}$ & $\mathrm{NaN}$ & $\mathrm{NaN}$ & $\mathrm{NaN}$ \\
\hline POLRTA & | 095602 & 1.85 & 2.21 & 1.02 & 1.08 & 0.47 & 1.98 & 1.03 & 0.76 & 0.39 & 3.52 & 1.80 & 0.75 & 0.76 & 1.40 & 1.15 & 2.91 & 0.21 & 2.19 & 27.77 & 0.24 & 1.94 & 0.41 & 0.73 & 2.40 & 0.75 & 0.95 \\
\hline POLR1A & B7ZKR9 & $\mathrm{NaN}$ & $\mathrm{NaN}$ & $\mathrm{NaN}$ & $\mathrm{NaN}$ & $\mathrm{NaN}$ & $\mathrm{NaN}$ & 1.13 & $\mathrm{NaN}$ & $\mathrm{NaN}$ & $\mathrm{NaN}$ & $\mathrm{NaN}$ & $\mathrm{NaN}$ & $\mathrm{NaN}$ & $\mathrm{NaN}$ & $\mathrm{NaN}$ & 2.76 & 0.34 & $\mathrm{NaN}$ & $\mathrm{NaN}$ & 0.37 & $\mathrm{NaN}$ & 0.51 & $\mathrm{NaN}$ & $\mathrm{NaN}$ & $\mathrm{NaN}$ & $\mathrm{NaN}$ \\
\hline POLR1A & 095602 & 1.85 & 2.21 & 1.02 & 1.08 & 0.47 & 1.98 & 1.03 & 0.76 & 0.39 & 3.52 & 1.80 & 0.75 & 0.76 & 1.40 & 1.15 & 2.91 & 0.21 & 2.19 & 27.77 & 0.24 & 1.94 & 0.41 & 0.73 & 2.40 & 0.75 & 0.95 \\
\hline POLR1B & Q9H9Ү६ & 1.94 & 2.83 & 1.08 & 1.06 & 0.42 & 2.20 & 1.21 & 0.82 & 0.38 & 3.98 & 2.30 & 1.06 & 0.40 & 3.60 & 1.22 & 3.63 & 0.25 & 2.48 & 16.97 & 0.15 & 2.54 & 0.54 & 0.75 & 2.26 & $\mathrm{NaN}$ & $\mathrm{NaN}$ \\
\hline POLRIC & Q96HT3 & 1.60 & 1.26 & 0.70 & 0.69 & 0.34 & 0.94 & 0.74 & 0.81 & 0.47 & 0.89 & 0.86 & 0.68 & 0.16 & 2.47 & 0.70 & 3.79 & 0.15 & 1.70 & 21.83 & 0.04 & 1.54 & 0.28 & 0.79 & 3.44 & 5.07 & 0.17 \\
\hline POLR1D & Q9Y2S0 & $\mathrm{Nal}$ & 0.8 & $\mathrm{Na}$ & $\mathrm{Nal}$ & 0.56 & 1.1 & 0.8 & 0.89 & 0.5 & 0.8 & 0.92 & 0.76 & $\mathrm{NaN}$ & $\mathrm{NaN}$ & 0.48 & 2. & 0.2 & 1.80 & 0.05 & & 1.97 & & & $\mathrm{NaN}$ & $\mathrm{NaN}$ & $\mathrm{NaN}$ \\
\hline POLRIE & Q9GZS1-2 & $\mathrm{NaN}$ & 1.67 & 1.01 & 1.06 & 0.51 & 0.97 & 1.28 & 0.89 & 0.50 & 3.10 & 1.75 & 0.69 & 0.26 & $\mathrm{NaN}$ & 1.14 & $\mathrm{NaN}$ & 0.31 & 1.10 & 5.58 & 0.12 & 1.78 & 0.36 & 0.74 & $\mathrm{NaN}$ & $\mathrm{NaN}$ & $\mathrm{NaN}$ \\
\hline POLR2E & E5KT65 & 1.06 & 0.96 & 0.74 & 0.69 & 0.38 & 1.00 & 0.74 & 0.85 & 0.47 & 0.94 & 0.93 & 0.70 & 0.21 & 2.04 & 0.39 & 3.56 & 0.17 & 1.63 & 15.20 & 0.06 & 1.67 & 0.30 & 0.73 & 1.89 & 3.37 & 0.16 \\
\hline POLR2H & C9JLU1 & 1.41 & 1.40 & 0.67 & 1.01 & 0.46 & 0.98 & 0.83 & 0.96 & 0.52 & 0.93 & 1.00 & 0.80 & 0.20 & 2.22 & 0.36 & 1.39 & 0.17 & 1.61 & 15.42 & $\mathrm{NaN}$ & 1.70 & 0.30 & $\mathrm{NaN}$ & $\mathrm{NaN}$ & $\mathrm{NaN}$ & $\mathrm{NaN}$ \\
\hline POLR2K & P5380 & $\mathrm{NaN}$ & & & $\mathrm{Nal}$ & & & 0.7 & 0.95 & & $1 . C$ & 1.03 & & & $\mathrm{Na}$ & & $\mathrm{N}$ & 0.2 & 1.99 & & & 1.73 & & & & & $\mathrm{NaN}$ \\
\hline POLR2L & P62875 & 1.01 & 0.92 & $\mathrm{NaN}$ & $\mathrm{NaN}$ & 0.37 & 1.01 & 0.77 & 0.87 & 0.55 & 1.11 & 0.82 & $\mathrm{NaN}$ & $\mathrm{NaN}$ & $\mathrm{NaN}$ & 0.45 & 1.29 & 0.15 & 1.88 & $\mathrm{NaN}$ & $\mathrm{NaN}$ & 1.85 & 0.30 & 0.77 & $\mathrm{NaN}$ & $\mathrm{NaN}$ & $\mathrm{NaN}$ \\
\hline POLR3A & 014802 & 1.03 & 0.93 & 0.36 & 0.44 & 0.37 & 0.54 & 0.26 & 0.66 & 0.45 & 0.67 & 0.52 & 0.52 & 0.26 & 1.80 & 0.44 & 2.46 & 0.13 & 1.25 & 24.78 & 0.15 & 1.15 & 0.20 & 1.05 & 3.00 & 2.76 & 0.18 \\
\hline POLR3B & Q7Z3R8 & 1.16 & 1.02 & 0.42 & 0.54 & 0.36 & 0.56 & 0.27 & 0.67 & 0.44 & 0.71 & 0.51 & 0.67 & 0.28 & $\mathrm{NaN}$ & 0.41 & 1.78 & 0.14 & 1.37 & 4.80 & 0.21 & 1.29 & 0.26 & 1.19 & 2.45 & $\mathrm{NaN}$ & 0.25 \\
\hline POLR3C & Q9BUI4 & 0.98 & 0.91 & 0.32 & 0.52 & 0.36 & 0.5 & 0.31 & 0.71 & 0.47 & 0.60 & 0.50 & 0.51 & 0.35 & 0.78 & 0.53 & 1.53 & 0.14 & 1.37 & 9.50 & 0.3 & 1.17 & & 1.04 & 2.03 & 2.96 & 0.21 \\
\hline POLR3D & P05423 & 0.34 & 0.50 & 0.45 & 0.46 & 0.34 & 0.58 & 0.28 & 0.71 & 0.46 & 0.67 & 0.56 & 0.54 & 0.22 & 1.67 & 0.34 & 2.6 & 0.13 & 1.21 & 14.87 & $\mathrm{NaN}$ & 1.16 & 0.23 & $\mathrm{NaN}$ & 2.19 & $\mathrm{NaN}$ & 0.11 \\
\hline POLR3E & Q9NVUO & 0.94 & 0.83 & 0.30 & 0.37 & 0.38 & 0.54 & 0.26 & 0.66 & 0.45 & 0.45 & 0.51 & 0.53 & 0.35 & 1.59 & 0.41 & 3.68 & 0.15 & 1.21 & 1.97 & 0.20 & 1.06 & 0.18 & 1.24 & $\mathrm{NaN}$ & $\mathrm{NaN}$ & 0.20 \\
\hline POLR3F & Q53FI8 & 1.43 & 0.82 & 0.36 & 0.47 & 0.31 & 0.58 & 0.30 & 0.79 & 0.45 & 0.73 & 0.45 & 0.53 & $\mathrm{NaN}$ & $\mathrm{NaN}$ & 0.37 & 5.03 & 0.13 & 1.41 & $\mathrm{NaN}$ & 0.18 & 1.25 & 0.21 & 0.79 & 3.01 & 2.57 & 0.21 \\
\hline POLR3G & R9U7 & 1.56 & 0.86 & 0.35 & 0.40 & 0.34 & 0.47 & 0.34 & 0.77 & 0.46 & 0.7 & 0.56 & 0.53 & $\mathrm{NaN}$ & $\mathrm{NaN}$ & 0.39 & 1.90 & 0.18 & 1.52 & 11.83 & 0.32 & 1.39 & 0.21 & $\mathrm{NaN}$ & 2.53 & $\mathrm{NaN}$ & 0.17 \\
\hline POLR3GL & & $\mathrm{NaN}$ & & & & 0.2 & & & 0. & & & 0.54 & & & & & & & & & & & & & $\mathrm{NaN}$ & $\mathrm{NaN}$ & $\mathrm{NaN}$ \\
\hline POLR3H & E7ERZ2 & $\mathrm{NaN}$ & $\mathrm{NaN}$ & $\mathrm{NaN}$ & $\mathrm{NaN}$ & $\mathrm{NaN}$ & $\mathrm{NaN}$ & $\mathrm{NaN}$ & $\mathrm{NaN}$ & 0.85 & $\mathrm{NaN}$ & $\mathrm{NaN}$ & $\mathrm{NaN}$ & $\mathrm{NaN}$ & $\mathrm{NaN}$ & $\mathrm{NaN}$ & $\mathrm{NaN}$ & $\mathrm{NaN}$ & $\mathrm{NaN}$ & $\mathrm{NaN}$ & $\mathrm{NaN}$ & $\mathrm{NaN}$ & $\mathrm{NaN}$ & $\mathrm{NaN}$ & $\mathrm{NaN}$ & $\mathrm{NaN}$ & $\mathrm{NaN}$ \\
\hline POLR3H & Q9Y535 & 1.52 & 0.67 & 0.33 & 0.45 & 0.35 & 0.64 & 0.33 & 0.76 & 0.50 & 0.75 & 0.60 & 0.56 & 0.15 & 1.68 & 0.36 & 1.62 & 0.14 & 1.52 & 26.33 & 0.26 & 1.39 & 0.20 & $\mathrm{NaN}$ & 3.06 & $\mathrm{NaN}$ & 0.10 \\
\hline POLR3H & E7ERZ2 & $\mathrm{NaN}$ & $\mathrm{NaN}$ & $\mathrm{NaN}$ & $\mathrm{NaN}$ & $\mathrm{NaN}$ & $\mathrm{NaN}$ & $\mathrm{NaN}$ & $\mathrm{NaN}$ & 0.85 & $\mathrm{NaN}$ & $\mathrm{NaN}$ & $\mathrm{NaN}$ & $\mathrm{NaN}$ & $\mathrm{NaN}$ & $\mathrm{NaN}$ & $\mathrm{NaN}$ & $\mathrm{NaN}$ & $\mathrm{NaN}$ & $\mathrm{NaN}$ & $\mathrm{NaN}$ & $\mathrm{NaN}$ & $\mathrm{NaN}$ & $\mathrm{NaN}$ & $\mathrm{NaN}$ & $\mathrm{NaN}$ & $\mathrm{NaN}$ \\
\hline POLR: & Q9Y535 & 1.52 & 0.67 & 0. & 0.4 & 0.35 & 0.6 & 0.3 & 0.76 & & & 0.60 & & 0.15 & 1.68 & 0.36 & & 0.14 & 1.52 & 26 & & & & & 3.06 & $\mathrm{NaN}$ & 0.10 \\
\hline POLR3K & Q9Y2Y1 & $\mathrm{NaN}$ & $\mathrm{NaN}$ & $\mathrm{N}$ & $\mathrm{NaN}$ & 0.44 & 0. & 0.3 & 1.14 & & 0.9 & 0.64 & 0 & $\mathrm{NaN}$ & $\mathrm{NaN}$ & $\mathrm{NaN}$ & 1.62 & 0.24 & 1.66 & $\mathrm{NaN}$ & $\mathrm{NaN}$ & 1.61 & 0.27 & $\mathrm{NaN}$ & $\mathrm{NaN}$ & $\mathrm{NaN}$ & $\mathrm{NaN}$ \\
\hline POM121C & A8CG34 & 0.51 & $\mathrm{NaN}$ & $\mathrm{NaN}$ & $\mathrm{NaN}$ & $\mathrm{NaN}$ & $\mathrm{NaN}$ & 0.04 & $\mathrm{NaN}$ & $\mathrm{N}$ & $\mathrm{NaN}$ & $\mathrm{NaN}$ & $\mathrm{NaN}$ & $\mathrm{NaN}$ & $\mathrm{NaN}$ & $\mathrm{NaN}$ & $\mathrm{NaN}$ & $\mathrm{NaN}$ & $\mathrm{NaN}$ & $\mathrm{NaN}$ & $\mathrm{NaN}$ & $\mathrm{NaN}$ & $\mathrm{NaN}$ & $\mathrm{NaN}$ & $\mathrm{NaN}$ & $\mathrm{NaN}$ & $\mathrm{NaN}$ \\
\hline POP4 & 55707 & 0.47 & $\mathrm{NaN}$ & $\mathrm{NaN}$ & $\mathrm{NaN}$ & $\mathrm{NaN}$ & 1.6 & $\mathrm{NaN}$ & $\mathrm{NaN}$ & & $\mathrm{NaN}$ & 0.92 & & 0.79 & 0.80 & 0.52 & 0.24 & 0.93 & $\mathrm{NaN}$ & 1.53 & 1.37 & 1.28 & 1.03 & 0.78 & $\mathrm{NaN}$ & $\mathrm{NaN}$ & $\mathrm{NaN}$ \\
\hline POU2F1 & & $\mathrm{NaN}$ & 0.16 & $\mathrm{Na}$ & $\mathrm{NaN}$ & $\mathrm{NaN}$ & $\mathrm{NaN}$ & $\mathrm{Na}$ & $\mathrm{NaN}$ & $\mathrm{NaN}$ & $\mathrm{Nal}$ & $\mathrm{NaN}$ & $\mathrm{NaN}$ & $\mathrm{Na}$ & $\mathrm{NaN}$ & 0.19 & $\mathrm{NaN}$ & $\mathrm{NaN}$ & $\mathrm{NaN}$ & $\mathrm{Na}$ & $\mathrm{NaN}$ & $\mathrm{NaN}$ & $\mathrm{NaN}$ & $\mathrm{NaN}$ & $\mathrm{NaN}$ & $\mathrm{NaN}$ & $\mathrm{NaN}$ \\
\hline PPIA & & 0.16 & 0.1 & 1.7 & 1.6 & 1.5 & 1. & 0. & $\mathrm{Na}$ & & 0. & $1 . C$ & 0. & & 0. & 0. & 0 & 0. & 0.6 & & $\mathrm{Na}$ & 0.73 & 0.5 & .79 & 47 & $\mathrm{NaN}$ & $\mathrm{NaN}$ \\
\hline PRC1 & H9KV59 & 0.70 & $\mathrm{NaN}$ & $\mathrm{Na}$ & $\mathrm{Nal}$ & 0.54 & 0. & $\mathrm{Nal}$ & $\mathrm{NaN}$ & $\mathrm{NaN}$ & $\mathrm{Na}$ & $\mathrm{NaN}$ & 0 & $\mathrm{NaN}$ & $\mathrm{NaN}$ & $\mathrm{NaN}$ & 0.43 & 0.5 & 0.22 & $\mathrm{NaN}$ & 0.58 & $\mathrm{NaN}$ & $\mathrm{NaN}$ & $\mathrm{NaN}$ & $\mathrm{NaN}$ & $\mathrm{NaN}$ & $\mathrm{NaN}$ \\
\hline PRC1 & & 0.5 & & & 0. & & & 0. & 1.3 & & 0.4 & 0.63 & & 0.3 & 1.35 & & 0. & 0.8 & 0. & 1.90 & 0.3 & 0.75 & 26 & 0.42 & $\mathrm{NaN}$ & 0.46 & 0.26 \\
\hline PRDM10 & & $\mathrm{NaN}$ & $\mathrm{NaN}$ & $\mathrm{Ne}$ & 0.4 & 0.65 & & $\mathrm{Ne}$ & $\mathrm{NaN}$ & & 1.87 & 0.6 & & 0.57 & $\mathrm{NaN}$ & 0.23 & $\mathrm{NaN}$ & 0.41 & 0. & 98.83 & 0.13 & & & 2.67 & 1.16 & $\mathrm{NaN}$ & $\mathrm{NaN}$ \\
\hline PRD & & 0.6 & 0.3 & 1.5 & 1.7 & & & 0.3 & 1.8 & 0. & 0.4 & 0.7 & 0.8 & 0.8 & 1.13 & 0.6 & 1.0 & 1.3 & 1.04 & 0.4 & 1. & 0.93 & & 1.00 & 0.52 & 0.87 & 0.57 \\
\hline PRDX & $\mathrm{P}_{3}$ & 0.4 & & 1.5 & & & & & $\mathrm{~N} a$ & & & & & & & & & $\mathrm{Na}$ & & & & & & & & & $\mathrm{NaN}$ \\
\hline PRDX6 & P300 & 0.47 & 0. & 2.8 & 2. & & & 0. & 2. & & 0. & 0.7 & & 0. & 0.8 & 0.4 & & 1.16 & 1. & & 1. & 0. & 0. & 1.13 & 0.30 & 1.65 & 0.64 \\
\hline PRIC295 & & 1.50 & & & 0.8 & & & 1.2 & 0.36 & & 0.6 & 0.48 & & & 0.6 & 1.11 & 0. & 1.02 & 0.64 & 0. & 0.3 & 0.41 & 0.77 & $\mathrm{NaN}$ & $\mathrm{NaN}$ & 1.05 & 0.44 \\
\hline PRKD2 & Q8NCK8 & 0.19 & $\mathrm{NaN}$ & $\mathrm{Nal}$ & $\mathrm{Nal}$ & $\mathrm{NaN}$ & $\mathrm{Na}$ & $\mathrm{NaN}$ & $\mathrm{NaN}$ & $\mathrm{N}$ & $\mathrm{NaN}$ & $\mathrm{NaN}$ & $\mathrm{NaN}$ & $\mathrm{NaN}$ & $\mathrm{NaN}$ & $\mathrm{NaN}$ & $\mathrm{NaN}$ & $\mathrm{NaN}$ & $\mathrm{NaN}$ & $\mathrm{NaN}$ & $\mathrm{NaN}$ & $\mathrm{NaN}$ & $\mathrm{NaN}$ & $\mathrm{NaN}$ & $\mathrm{NaN}$ & $\mathrm{NaN}$ & $\mathrm{NaN}$ \\
\hline PRMT5 & O147 & 1.0 & 0.8 & 0.8 & 0.6 & 0.7 & & $\mathrm{Na}$ & $\mathrm{N}$ & & 0. & 0.9 & & & 0.2 & 1.18 & 0.4 & 0.3 & & & & & & 1.15 & 0.89 & & 0.28 \\
\hline & & 2.16 & 1.5 & 0. & 0. & 0 & 0. & 1.0 & 0. & & 0. & 1.5 & & $\mathrm{~N}$ & 0. & & 1. & 1.0 & & & & & 0 & 15 & $\mathrm{aN}$ & 0.30 & 0.75 \\
\hline PRPF $40 A$ & |07: & $\mathrm{NaN}$ & $\mathrm{Na}$ & $\mathrm{N}_{\mathrm{c}}^{\mathrm{z}}$ & $\mathrm{Na}$ & & & $\mathrm{N}_{\mathrm{c}}^{\mathrm{a}}$ & $N_{c}$ & & $\mathrm{Na}$ & $\mathrm{Na}$ & & $\mathrm{Na}$ & $\mathrm{Na}$ & & $\mathrm{Na}$ & $\mathrm{Na}$ & $\mathrm{N}$ & $\mathrm{NaN}$ & $\mathrm{NaN}$ & $\mathrm{NaN}$ & $\mathrm{NaN}$ & $\mathrm{NaN}$ & $\mathrm{NaN}$ & $\mathrm{NaN}$ & $\mathrm{NaN}$ \\
\hline PSAT1 & B4DHQ3 & $\mathrm{NaN}$ & 0.11 & $\mathrm{NaN}$ & $\mathrm{NaN}$ & $\mathrm{NaN}$ & $\mathrm{NaN}$ & $\mathrm{Nal}$ & $\mathrm{NaN}$ & $\mathrm{NaN}$ & $\mathrm{NaN}$ & $\mathrm{NaN}$ & $\mathrm{NaN}$ & $\mathrm{NaN}$ & $\mathrm{NaN}$ & $\mathrm{NaN}$ & $\mathrm{NaN}$ & $\mathrm{NaN}$ & $\mathrm{NaN}$ & 0.28 & 1.81 & $\mathrm{NaN}$ & $\mathrm{NaN}$ & 0.94 & $\mathrm{NaN}$ & $\mathrm{NaN}$ & $\mathrm{NaN}$ \\
\hline PSM & & 0.2 & 0. & $\mathrm{Na}$ & & $\mathrm{Na}$ & $\mathrm{N}$ & 0.2 & 2.3 & & 0.6 & 0.5 & & $\mathrm{Na}$ & $\mathrm{Na}$ & & 0. & 1.1 & 0. & 0 & & & 10 & 1.12 & 0.74 & $\mathrm{IaN}$ & $\mathrm{NaN}$ \\
\hline PSN & & 0 & & 1. & & & 1. & & & & & & & & 0 & & & & & & & & & & & & \\
\hline PSN & & 0 & & 2. & 0. & & & & & & 0. & $\mathrm{~N}$ & & & 2. & & 0. & 1.23 & 1.12 & 0.8 & 0. & $\mathrm{NaN}$ & 1.02 & $\mathrm{NaN}$ & 1.02 & $\mathrm{NaN}$ & $\mathrm{NaN}$ \\
\hline PUM1 & Q14671 & 0.76 & 4.67 & $\mathrm{NaN}$ & $\mathrm{NaN}$ & $\mathrm{NaN}$ & $\mathrm{NaN}$ & $\mathrm{NaN}$ & $\mathrm{NaN}$ & $\mathrm{NaN}$ & $\mathrm{NaN}$ & $\mathrm{NaN}$ & $\mathrm{NaN}$ & $\mathrm{NaN}$ & $\mathrm{NaN}$ & 0.90 & 0.29 & $\mathrm{NaN}$ & $\mathrm{NaN}$ & $\mathrm{NaN}$ & $\mathrm{NaN}$ & $\mathrm{NaN}$ & $\mathrm{NaN}$ & $\mathrm{NaN}$ & $\mathrm{NaN}$ & $\mathrm{NaN}$ & $\mathrm{NaN}$ \\
\hline PUM1 & Q5T1Z4 & 0.76 & 3.89 & 1.60 & $\mathrm{NaN}$ & 1.30 & 1.26 & 1.40 & $\mathrm{NaN}$ & 0.72 & 0.95 & $\mathrm{NaN}$ & $\mathrm{NaN}$ & 1.65 & 0.67 & 0.92 & 0.09 & 1.28 & 1.34 & $\mathrm{NaN}$ & $\mathrm{NaN}$ & 0.49 & 1.35 & $\mathrm{NaN}$ & $\mathrm{NaN}$ & 1.95 & 0.97 \\
\hline PURA & Q2NLD4 & 1.73 & 0.83 & 0.47 & 3.16 & 0.60 & 2.00 & & $\mathrm{NaN}$ & $\mathrm{NaN}$ & $\mathrm{NaN}$ & $\mathrm{NaN}$ & 1.29 & $\mathrm{NaN}$ & $\mathrm{NaN}$ & 0.90 & 2.93 & 0.66 & 1.64 & 3.50 & & & & 427 & & & \\
\hline PURB & Q966 & 1.32 & 0.75 & 0.5 & 2.5 & 0.57 & 2. & 1.09 & 1.31 & 1.2 & 1.49 & 1.47 & 1.0 & 0.4 & 1.83 & 0.74 & 4.17 & 0.65 & 1.74 & 3.11 & 0.6 & 1.42 & 1.08 & 3.72 & 0.96 & 1.12 & 1.24 \\
\hline RABGA & P20340 & 0.53 & 1.72 & 2.79 & 17.37 & 0.81 & 2.83 & $\mathrm{NaN}$ & $\mathrm{NaN}$ & 0.70 & $\mathrm{NaN}$ & $\mathrm{NaN}$ & 0.74 & 0.86 & 0.23 & 0.53 & 0.15 & 0.67 & $\mathrm{NaN}$ & $\mathrm{NaN}$ & $\mathrm{NaN}$ & 0.15 & 0.82 & $\mathrm{NaN}$ & $\mathrm{NaN}$ & $\mathrm{NaN}$ & $\mathrm{NaN}$ \\
\hline RAB6A & Q66FGX3 & 0.75 & 1.60 & $\mathrm{NaN}$ & $\mathrm{NaN}$ & 1.45 & 2.67 & $\mathrm{NaN}$ & $\mathrm{NaN}$ & $\mathrm{NaN}$ & $\mathrm{NaN}$ & $\mathrm{NaN}$ & $\mathrm{NaN}$ & 1.54 & 0.33 & 0.24 & 0.32 & $\mathrm{NaN}$ & $\mathrm{NaN}$ & $\mathrm{NaN}$ & $\mathrm{NaN}$ & 0.33 & $\mathrm{NaN}$ & $\mathrm{NaN}$ & $\mathrm{NaN}$ & $\mathrm{NaN}$ & $\mathrm{NaN}$ \\
\hline Ran & 060671 & $\mathrm{NaN}$ & & $\mathrm{NaN}$ & $\mathrm{NaN}$ & 1.23 & 0.86 & $\mathrm{NaN}$ & $\mathrm{NaN}$ & & $\mathrm{NaN}$ & 1.20 & 079 & & $\mathrm{NaN}$ & $\mathrm{NaN}$ & $\mathrm{NaN}$ & 1.00 & $\mathrm{NaN}$ & $\mathrm{NaN}$ & 0.26 & & 065 & (20) & & & $\mathrm{NaN}$ \\
\hline RAD51 & Q96B01-2 & $\mathrm{NaN}$ & $\mathrm{NaN}$ & $\mathrm{NaN}$ & $\mathrm{NaN}$ & 3.67 & 0.18 & $\mathrm{NaN}$ & $\mathrm{NaN}$ & $\mathrm{NaN}$ & $\mathrm{NaN}$ & $\mathrm{NaN}$ & $\mathrm{aN}$ & $\mathrm{NaN}$ & $\mathrm{NaN}$ & $\mathrm{NaN}$ & $\mathrm{NaN}$ & 2.14 & 0.19 & 0.39 & 3.09 & 0.30 & $\mathrm{NaN}$ & $\mathrm{NaN}$ & $\mathrm{NaN}$ & 0.21 & 2.55 \\
\hline
\end{tabular}




\begin{tabular}{|c|c|c|c|c|c|c|c|c|c|c|c|c|c|c|c|c|c|c|c|c|c|c|c|c|c|c|c|}
\hline Gene names & Protein IDs & $\begin{array}{l}\mathrm{H} 3 \Delta \mathrm{t}- \\
20 \_\mathrm{F}\end{array}$ & $\begin{array}{l}H 3 \Delta 1- \\
20 \_R\end{array}$ & $\begin{array}{l}\text { H4K20 } \\
\text { me1_F }\end{array}$ & $\begin{array}{l}\text { H4K20 } \\
\text { me1_R }\end{array}$ & $\begin{array}{l}\text { H4K20 } \\
\text { me3_F }\end{array}$ & $\begin{array}{l}\text { H4k20 } \\
\text { me3_R }\end{array}$ & $\begin{array}{c}\mathrm{H}^{\mathrm{H}} \mathrm{K}_{\mathrm{c}} 27 \\
\text { me1_F }\end{array}$ & $\begin{array}{c}\mathrm{H} 3 \mathrm{~K}_{\mathrm{c}} 27 \\
\mathrm{me1} \mathrm{R}\end{array}$ & $\begin{array}{l}\mathrm{H}^{\mathrm{K}} \mathrm{K}_{\mathrm{c}} 27 \\
\mathrm{me2}{ }^{2} \mathrm{~F}\end{array}$ & $\begin{array}{c}\mathrm{H} 3 \mathrm{~K}_{\mathrm{c}} 27 \\
\mathrm{me2} \_\mathrm{R}\end{array}$ & $\begin{array}{l}\mathrm{H} 3 \mathrm{~K}_{\mathrm{c}} 27 \\
\mathrm{me} 3 \mathrm{~F}\end{array}$ & $\begin{array}{l}\mathrm{H} 3 \mathrm{~K}_{\mathrm{c}} 27 \\
\mathrm{me3} \_\mathrm{R}\end{array}$ & $\begin{array}{c}\text { HзкК9m } \\
\text { e1_F }\end{array}$ & \begin{tabular}{|c|} 
H3к \\
e1_R
\end{tabular} & \begin{tabular}{|c|} 
H3k 3 Kom \\
e2_F
\end{tabular} & $\begin{array}{c}\text { H3K9m } \\
\text { e2_R }\end{array}$ & \begin{tabular}{|c|} 
H3K9m \\
e3_F
\end{tabular} & $\begin{array}{c}\text { Hзкаm } \\
\text { e3_R }\end{array}$ & $\begin{array}{c}\mathrm{H} 3 \mathrm{k} 9 \mathrm{~m} \\
\mathrm{e} 3 \mathrm{meC} \\
\mathrm{pG} \_\mathrm{F}\end{array}$ & \begin{tabular}{|c|} 
HзK9m \\
e3/meC \\
pG_R
\end{tabular} & $\begin{array}{c}\mathrm{H} 3 \mathrm{~K} / \mathrm{m} \\
\mathrm{e} \mid \mathrm{H} / \mathrm{KK} \\
20 \mathrm{me} 3 \\
\mathrm{~F}\end{array}$ & $\begin{array}{c}\mathrm{H} 3 \mathrm{~K} 9 \mathrm{~m} \\
\mathrm{e} \mid \mathrm{H} / \mathrm{k} \\
20 \mathrm{me} 3 \\
\mathrm{R}\end{array}$ & $\begin{array}{c}\mathrm{meCpG} \\
-\mathrm{F}\end{array}$ & $\begin{array}{c}\mathrm{meCpG} \\
\mathrm{R}\end{array}$ & $\begin{array}{c}\text { H4R3m } \\
\text { e2_F }\end{array}$ & $\begin{array}{c}\text { H4R3m } \\
\text { e2_R }\end{array}$ \\
\hline RAl14 & Q9P0K7-2 & $\mathrm{NaN}$ & 3.57 & 1.03 & $\mathrm{NaN}$ & $\mathrm{NaN}$ & $\mathrm{NaN}$ & $\mathrm{NaN}$ & $\mathrm{NaN}$ & $\mathrm{NaN}$ & $\mathrm{NaN}$ & $\mathrm{NaN}$ & $\mathrm{NaN}$ & $\mathrm{NaN}$ & $\mathrm{NaN}$ & $\mathrm{NaN}$ & 0.19 & $\mathrm{NaN}$ & 0.49 & $\mathrm{NaN}$ & $\mathrm{NaN}$ & $\mathrm{NaN}$ & 0.19 & $\mathrm{NaN}$ & $\mathrm{NaN}$ & $\mathrm{NaN}$ & $\mathrm{NaN}$ \\
\hline RALGAPA2 & Q2PPJ7-3 & 0.67 & $\mathrm{NaN}$ & $\mathrm{NaN}$ & 1.31 & $\mathrm{NaN}$ & $\mathrm{NaN}$ & $\mathrm{NaN}$ & $\mathrm{NaN}$ & $\mathrm{NaN}$ & $\mathrm{NaN}$ & 0.88 & $\mathrm{NaN}$ & $\mathrm{NaN}$ & $\mathrm{NaN}$ & 1.34 & 0.14 & $\mathrm{NaN}$ & $\mathrm{NaN}$ & $\mathrm{NaN}$ & $\mathrm{NaN}$ & $\mathrm{NaN}$ & $\mathrm{NaN}$ & 35.51 & 0.19 & $\mathrm{NaN}$ & $\mathrm{NaN}$ \\
\hline RAVER1 & E9PAU2 & 0.88 & 1.48 & 0.53 & 0.73 & 1.03 & 0.48 & 1.11 & 0.42 & 0.60 & 0.73 & 0.49 & 0.57 & 1.19 & 0.56 & 0.82 & 0.43 & 1.09 & 0.75 & 0.75 & $\mathrm{NaN}$ & 0.67 & 0.96 & $\mathrm{NaN}$ & $\mathrm{NaN}$ & 1.29 & 0.50 \\
\hline RBBP4 & Q09028-3 & 0.38 & 0.67 & 1.15 & 0.76 & 0.99 & 1.10 & 0.89 & 0.95 & 0.88 & 1.02 & 0.99 & 0.87 & 0.42 & 2.03 & 0.71 & 2.17 & 1.11 & 0.82 & 4.30 & 0.08 & 1.48 & 0.60 & 3.37 & 0.27 & 1.64 & 0.55 \\
\hline RBBP7 & Q16576-2 & 0.34 & 0.69 & 1.16 & 0.78 & 0.92 & 1.01 & 0.95 & 0.95 & 0.98 & 0.89 & 1.03 & 0.87 & 0.40 & 2.26 & 0.64 & 1.61 & 0.77 & 1.00 & 12.27 & 0.03 & 0.87 & 0.95 & 3.85 & 0.17 & 1.66 & 0.56 \\
\hline RBBP7 & Q6FHQ0 & 0.33 & 0.65 & 1.28 & 0.76 & 0.93 & 1.01 & 1.00 & 1.01 & 1.10 & 0.89 & 1.03 & 0.85 & 0.37 & 2.62 & 0.66 & 3.20 & 0.73 & 1.14 & 20.37 & 0.02 & 0.80 & 1.16 & 5.14 & 0.16 & 1.87 & 0.55 \\
\hline RBM4 & A8K9U0 & 1.64 & 0.98 & 0.68 & 1.27 & 1.02 & 1.15 & 0.87 & 0.92 & $\mathrm{NaN}$ & 0.89 & 0.95 & 0.89 & $\mathrm{NaN}$ & 0.81 & 1.17 & 0.55 & $\mathrm{NaN}$ & 0.73 & 5.52 & 0.58 & 1.10 & 0.96 & 7.67 & 0.72 & 0.84 & 0.91 \\
\hline RBM7 & J3KPD3 & 0.83 & 0.63 & 1.19 & 1.61 & 0.95 & 0.91 & 1.11 & 1.08 & 1.03 & 1.06 & $\mathrm{NaN}$ & 1.37 & 0.72 & 1.22 & 0.79 & 0.74 & 1.08 & $\mathrm{NaN}$ & $\mathrm{NaN}$ & $\mathrm{NaN}$ & 1.16 & 1.11 & 1.51 & $\mathrm{NaN}$ & $\mathrm{NaN}$ & $\mathrm{NaN}$ \\
\hline RBM7 & Q6IRX3 & 0.57 & 0.55 & $\mathrm{NaN}$ & $\mathrm{NaN}$ & $\mathrm{NaN}$ & $\mathrm{IaN}$ & $\mathrm{NaN}$ & $\mathrm{Nal}$ & $\mathrm{NaN}$ & $\mathrm{NaN}$ & $\mathrm{NaN}$ & $\mathrm{NaN}$ & $\mathrm{NaN}$ & $\mathrm{NaN}$ & $\mathrm{NaN}$ & $\mathrm{NaN}$ & $\mathrm{NaN}$ & $\mathrm{NaN}$ & $\mathrm{NaN}$ & $\mathrm{NaN}$ & $\mathrm{NaN}$ & $\mathrm{NaN}$ & $\mathrm{NaN}$ & $\mathrm{NaN}$ & $\mathrm{NaN}$ & $\mathrm{NaN}$ \\
\hline RECQL & P46063 & 0.48 & 0.81 & 0.33 & 0.54 & 0.79 & 0.68 & 0.60 & $\mathrm{NaN}$ & 0.51 & 0.57 & 0.56 & 0.43 & 0.25 & 1.24 & 0.62 & 2.59 & 0.38 & 0.81 & 1.45 & 0.59 & 1.11 & 0.26 & 1.50 & 1.15 & 0.55 & 0.34 \\
\hline RFX1 & P22670 & 0.71 & 0.87 & 0.80 & 0.89 & 0.43 & 1.18 & $\mathrm{NaN}$ & $\mathrm{NaN}$ & 0.62 & $\mathrm{NaN}$ & 0.90 & 0.84 & 0.84 & 0.59 & 0.70 & 0.08 & 0.45 & 1.89 & 11.12 & 0.14 & 1.54 & 0.50 & 2.16 & 0.55 & $\mathrm{NaN}$ & 0.53 \\
\hline RFXANK & 014593 & 0.54 & 1.26 & 1.03 & 0.30 & 0.83 & 0.58 & $\mathrm{NaN}$ & $\mathrm{NaN}$ & 0.34 & 1.10 & 0.58 & 0.55 & 0.25 & 1.55 & 0.27 & 0.43 & 0.57 & 0.56 & 20.68 & 0.05 & 1.34 & 0.26 & 1.58 & 0.59 & $\mathrm{NaN}$ & $\mathrm{NaN}$ \\
\hline RIOK3 & B4E1Q4 & 0.50 & $\mathrm{NaN}$ & $\mathrm{NaN}$ & $\mathrm{NaN}$ & $\mathrm{NaN}$ & $\mathrm{NaN}$ & $\mathrm{NaN}$ & $\mathrm{NaN}$ & $\mathrm{NaN}$ & $\mathrm{NaN}$ & $\mathrm{NaN}$ & $\mathrm{NaN}$ & $\mathrm{NaN}$ & $\mathrm{NaN}$ & 0.43 & 0.15 & $\mathrm{NaN}$ & $\mathrm{NaN}$ & $\mathrm{NaN}$ & $\mathrm{NaN}$ & $\mathrm{NaN}$ & $\mathrm{NaN}$ & $\mathrm{NaN}$ & $\mathrm{NaN}$ & $\mathrm{NaN}$ & $\mathrm{NaN}$ \\
\hline RMND1 & Q5SZ82 & 1.11 & $\mathrm{NaN}$ & 4.02 & $\mathrm{NaN}$ & $\mathrm{NaN}$ & $\mathrm{NaN}$ & 0.90 & $\mathrm{NaN}$ & $\mathrm{NaN}$ & 0.80 & 0.7 & 0.92 & $\mathrm{NaN}$ & $\mathrm{NaN}$ & $\mathrm{NaN}$ & $\mathrm{NaN}$ & 0.70 & 0.90 & 0.92 & $\mathrm{NaN}$ & 0.71 & $\mathrm{NaN}$ & $\mathrm{NaN}$ & $\mathrm{NaN}$ & $\mathrm{NaN}$ & $\mathrm{NaN}$ \\
\hline RNF213 & Q63HN8 & 0.78 & 0.42 & 0.72 & 0.98 & $\mathrm{NaN}$ & 0.17 & $\mathrm{NaN}$ & $\mathrm{NaN}$ & $\mathrm{NaN}$ & 0.45 & 0.74 & 0.57 & 1.13 & 0.42 & 0.54 & 0.23 & 1.28 & 0.27 & $\mathrm{NaN}$ & 0.32 & $\mathrm{NaN}$ & 0.79 & $\mathrm{NaN}$ & $\mathrm{NaN}$ & 1.54 & 0.41 \\
\hline RNPS1 & H3BV80 & 5.57 & 1.30 & 0.48 & 1.49 & 0.79 & 0.55 & 0.91 & 1.12 & 2.23 & 0.39 & 1.34 & 1.17 & $\mathrm{NaN}$ & 0.59 & 1.79 & 2.26 & 1.04 & 0.57 & 1.08 & 2.62 & 0.37 & 1.71 & $\mathrm{NaN}$ & $\mathrm{NaN}$ & 0.48 & 0.54 \\
\hline RPL7L1 & R4GMU7 & $\mathrm{NaN}$ & $\mathrm{NaN}$ & 0.37 & 1.78 & 0.95 & 0.70 & $\mathrm{NaN}$ & $\mathrm{NaN}$ & 1.72 & $\mathrm{NaN}$ & 0.98 & 0.62 & $\mathrm{NaN}$ & 0.88 & 0.86 & 0.35 & 1.00 & 0.50 & 0.85 & 0.72 & 0.60 & 1.07 & 1.09 & 0.66 & 0.67 & 1.03 \\
\hline RPLP2 & $\mathrm{P}$ & 1.30 & 1.51 & $\mathrm{NaN}$ & $\mathrm{Na}$ & 1.30 & 0.93 & & 1. & & 1. & 0. & $\mathrm{NaN}$ & $\mathrm{aN}$ & & 1.12 & & & & & 37.48 & & & & & & 0.72 \\
\hline RPRC1 & D3DPS3 & 1.19 & 1.71 & 1.37 & 0.27 & $\mathrm{NaN}$ & 0.60 & $\mathrm{NaN}$ & $\mathrm{NaN}$ & $\mathrm{NaN}$ & 0.58 & 0.43 & 1.08 & $\mathrm{NaN}$ & $\mathrm{NaN}$ & 1.43 & 1.00 & $\mathrm{NaN}$ & $\mathrm{NaN}$ & 0.86 & 0.67 & 1.47 & 0.36 & 0.85 & 0.78 & $\mathrm{NaN}$ & $\mathrm{NaN}$ \\
\hline RPRD1B & Q9NQG5 & 0.69 & 0.54 & $\mathrm{NaN}$ & $\mathrm{NaN}$ & $\mathrm{NaN}$ & $\mathrm{NaN}$ & $\mathrm{NaN}$ & $\mathrm{NaN}$ & $\mathrm{NaN}$ & $\mathrm{NaN}$ & $\mathrm{NaN}$ & $\mathrm{NaN}$ & $\mathrm{NaN}$ & 0.80 & 0.81 & 0.66 & $\mathrm{NaN}$ & 1.03 & $\mathrm{NaN}$ & 1.58 & 1.04 & 1.12 & $\mathrm{NaN}$ & 1.90 & 1.04 & $\mathrm{NaN}$ \\
\hline RSF1 & Q96Т23-2 & $\mathrm{NaN}$ & 0.60 & 0.16 & 0.62 & 0.41 & 0.45 & $\mathrm{NaN}$ & 0.48 & 0.54 & 0.39 & 0.35 & 0.36 & 0.55 & $\mathrm{NaN}$ & 0.56 & $\mathrm{NaN}$ & 0.42 & 0.53 & 0.43 & 1.43 & 0.38 & 0.71 & 0.07 & 0.02 & 0.03 & 0.36 \\
\hline RSL1D1 & & 0.6 & 2.8 & & 2.1 & 0.58 & & & $\mathrm{Nal}$ & 1.12 & & 0.7 & 0.45 & 0.70 & 0.6 & 2.07 & 0.60 & & & & & & & 1.68 & & 26 & 0.88 \\
\hline RSRC2 & Q7L4I2-2 & 0.60 & 0.80 & 2.06 & 0.22 & 0.66 & 0.56 & 1.47 & 0.58 & 0.50 & 0.35 & $\mathrm{NaN}$ & $\mathrm{NaN}$ & $\mathrm{NaN}$ & $\mathrm{NaN}$ & 0.56 & 0.93 & 0.55 & $\mathrm{NaN}$ & 0.68 & 1.02 & $\mathrm{NaN}$ & 0.70 & 1.05 & 0.78 & 0.37 & $\mathrm{NaN}$ \\
\hline S100A11 & P31949 & $\mathrm{NaN}$ & $\mathrm{NaN}$ & $\mathrm{NaN}$ & $\mathrm{NaN}$ & $\mathrm{NaN}$ & $\mathrm{NaN}$ & 0.17 & $\mathrm{NaN}$ & $\mathrm{NaN}$ & $\mathrm{NaN}$ & $\mathrm{NaN}$ & 0.70 & $\mathrm{NaN}$ & $\mathrm{NaN}$ & $\mathrm{NaN}$ & 0.39 & $\mathrm{NaN}$ & $\mathrm{NaN}$ & $\mathrm{NaN}$ & $\mathrm{NaN}$ & $\mathrm{NaN}$ & $\mathrm{NaN}$ & 0.34 & $\mathrm{NaN}$ & $\mathrm{NaN}$ & $\mathrm{NaN}$ \\
\hline S100A14 & Q9HCY8 & $\mathrm{NaN}$ & $\mathrm{NaN}$ & $\mathrm{NaN}$ & $\mathrm{NaN}$ & $\mathrm{NaN}$ & $\mathrm{NaN}$ & 0.01 & $\mathrm{NaN}$ & $\mathrm{NaN}$ & $\mathrm{NaN}$ & $\mathrm{NaN}$ & $\mathrm{NaN}$ & $\mathrm{NaN}$ & $\mathrm{NaN}$ & $\mathrm{NaN}$ & $\mathrm{NaN}$ & $\mathrm{NaN}$ & $\mathrm{NaN}$ & $\mathrm{NaN}$ & $\mathrm{NaN}$ & $\mathrm{NaN}$ & $\mathrm{NaN}$ & $\mathrm{NaN}$ & $\mathrm{NaN}$ & $\mathrm{NaN}$ & $\mathrm{NaN}$ \\
\hline SAP130 & H7BXF5 & 0.55 & 1.15 & & 1.26 & 0.77 & 2.28 & 1.47 & 1.3 & 1.72 & 1.35 & 1.24 & 1.10 & 0.60 & 2.95 & 0.40 & $\mathrm{NaN}$ & 1.01 & 1.19 & 4.46 & 0.29 & 0.88 & & 0.76 & 4.12 & 1.49 & 0.63 \\
\hline SAP18 & & 11. & 1.94 & & 1.4 & 0.7 & & & 0.8 & & & & & & & 3.14 & & & & & & & & & & & $\mathrm{NaN}$ \\
\hline SAP30 & 075446 & 0.71 & 0.79 & 1.47 & 1.38 & 0.83 & 3.28 & 1.73 & 1.41 & 1.63 & 1.92 & 1.62 & 1.22 & 0.64 & 2.90 & 0.54 & 0.82 & 1.29 & 1.60 & 10.80 & 0.19 & 1.23 & 1.77 & 0.81 & 4.83 & 3.45 & 0.87 \\
\hline SARS & Q53HA4 & $\mathrm{NaN}$ & 0.24 & $\mathrm{NaN}$ & $\mathrm{NaN}$ & $\mathrm{NaN}$ & $\mathrm{NaN}$ & $\mathrm{NaN}$ & $\mathrm{NaN}$ & $\mathrm{NaN}$ & $\mathrm{NaN}$ & $\mathrm{NaN}$ & $\mathrm{NaN}$ & $\mathrm{NaN}$ & $\mathrm{NaN}$ & $\mathrm{NaN}$ & $\mathrm{NaN}$ & $\mathrm{NaN}$ & $\mathrm{NaN}$ & 0.50 & $\mathrm{NaN}$ & $\mathrm{NaN}$ & $\mathrm{NaN}$ & $\mathrm{NaN}$ & $\mathrm{NaN}$ & $\mathrm{NaN}$ & $\mathrm{NaN}$ \\
\hline SCML2 & IDZR9 & $\mathrm{NaN}$ & $\mathrm{NaN}$ & $\mathrm{NaN}$ & $\mathrm{Na}$ & $\mathrm{NaN}$ & $\mathrm{NaN}$ & $\mathrm{NaN}$ & $\mathrm{NaI}$ & $\mathrm{NaN}$ & $\mathrm{NaN}$ & $\mathrm{NaN}$ & $\mathrm{NaN}$ & $\mathrm{NaN}$ & $\mathrm{NaN}$ & 1.14 & $\mathrm{NaN}$ & $\mathrm{NaN}$ & $\mathrm{NaN}$ & $\mathrm{NaN}$ & $\mathrm{NaN}$ & $\mathrm{NaN}$ & $\mathrm{NaN}$ & $\mathrm{NaN}$ & $\mathrm{NaN}$ & $\mathrm{NaN}$ & $\mathrm{NaN}$ \\
\hline SCML2 & & 0. & 1.53 & & 0. & 1.02 & & & & & & & 112 & & 0. & 4.14 & & & & & & & & & & 22 & 2.16 \\
\hline SCY22 & Q6P3W7 & 1.60 & 1.60 & 0.63 & 0.7 & 1.04 & 0.60 & 0.89 & 0.26 & 0.88 & 1.32 & 0.62 & $\mathrm{NaN}$ & 1.10 & 0.65 & 1.01 & 0.14 & 1.16 & 0.88 & $\mathrm{NaN}$ & $\mathrm{NaN}$ & 0.58 & 1.14 & $\mathrm{NaN}$ & $\mathrm{NaN}$ & 1.91 & 0.63 \\
\hline SDHB & QOQEY7 & $\mathrm{NaN}$ & $\mathrm{NaN}$ & $\mathrm{NaN}$ & 0.5 & 0.43 & 0.43 & $N_{c}$ & $\mathrm{NaN}$ & $\mathrm{aN}$ & $\mathrm{NaN}$ & $\mathrm{NaN}$ & $\mathrm{NaN}$ & $\mathrm{NaN}$ & $\mathrm{NaN}$ & $\mathrm{NaN}$ & $\mathrm{NaN}$ & $\mathrm{NaN}$ & $\mathrm{NaN}$ & $\mathrm{NaN}$ & $\mathrm{NaN}$ & 0.25 & $\mathrm{NaN}$ & $\mathrm{NaN}$ & $\mathrm{NaN}$ & $\mathrm{NaN}$ & $\mathrm{NaN}$ \\
\hline SEMG1 & P04279-2 & $\mathrm{NaN}$ & $\mathrm{NaN}$ & $\mathrm{NaN}$ & $\mathrm{N}$ & $\mathrm{NaN}$ & $\mathrm{NaN}$ & $\mathrm{Na}$ & $\mathrm{Na}$ & $\mathrm{NaN}$ & $\mathrm{NaN}$ & $\mathrm{Na}$ & $\mathrm{NaN}$ & $\mathrm{NaN}$ & $\mathrm{NaN}$ & $\mathrm{NaN}$ & $\mathrm{NaN}$ & $\mathrm{NaN}$ & $\mathrm{NaN}$ & $\mathrm{NaN}$ & $\mathrm{NaN}$ & $\mathrm{NaN}$ & $\mathrm{NaN}$ & $\mathrm{NaN}$ & 0.09 & $\mathrm{NaN}$ & $\mathrm{NaN}$ \\
\hline & & $\mathrm{NaN}$ & $\mathrm{NaN}$ & $\mathrm{Na}$ & & $\mathrm{Na}$ & $\mathrm{N}$ & $\mathrm{Na}$ & $\mathrm{Na}$ & & $\mathrm{N}$ & $\mathrm{Na}$ & $\mathrm{Nat}$ & & $\mathrm{Na}$ & $\mathrm{Na}$ & $\mathrm{NaN}$ & $\mathrm{Na}$ & & & & & & $\mathrm{NaN}$ & 31 & $\mathrm{NaN}$ & $\mathrm{NaN}$ \\
\hline SEPT10 & ETEWG & $\mathrm{Na}$ & $\mathrm{Nal}$ & & $\mathrm{Na}$ & 1.5 & & & $\mathrm{Na}$ & & & & $\mathrm{Na}$ & & 0.9 & $\mathrm{Na}$ & & 1.6 & & & & $0.2 \quad \mathrm{H}$ & & & aN & $\mathrm{NaN}$ & $\mathrm{NaN}$ \\
\hline SEPT2 & Q15019 & 1.17 & 1.78 & 0. & 0.8 & 1.69 & & 0. & 0.6 & 0 & $\mathrm{~N}$ & 0. & 0.62 & 0 & 0.51 & 0.76 & 0.23 & 2.03 & 0.13 & 0.58 & 0.54 & 0.11 & 2.30 & 0.81 & 0.89 & 0.49 & 0.44 \\
\hline SEPT6 & & $\mathrm{NaN}$ & $\mathrm{NaN}$ & & 1. & 1.89 & & & $\mathrm{~N}$ & & & $\mathrm{Na}$ & $\mathrm{NaN}$ & & & $\mathrm{NaN}$ & & & 0.23 & $\mathrm{aN}$ & $\mathrm{N}_{\mathrm{c}}$ & 0.40 & & $\mathrm{NaN}$ & $\mathrm{NaN}$ & $\mathrm{NaN}$ & $\mathrm{NaN}$ \\
\hline SEP & & 1.54 & 1.59 & $\mathrm{NaN}$ & $\mathrm{Na}$ & 1.67 & 0.30 & $\mathrm{NaN}$ & $\mathrm{Na}$ & $\mathrm{NaN}$ & $\mathrm{NaN}$ & 0.55 & 0.40 & $\mathrm{NaN}$ & 0.62 & $\mathrm{NaN}$ & $\mathrm{NaN}$ & 2.08 & 0.11 & 0.61 & $\mathrm{NaN}$ & 0.14 & 2.00 & $\mathrm{NaN}$ & $\mathrm{NaN}$ & $\mathrm{NaN}$ & $\mathrm{NaN}$ \\
\hline SEPT & $A$ & $\mathrm{NaN}$ & $\mathrm{NaN}$ & & $\mathrm{N}$ & $\mathrm{Nal}$ & & & $\mathrm{N}$ & & & & & & & & & & & & & & & & & & \\
\hline SEPT8 & B BZV & $\mathrm{NaN}$ & $\mathrm{NaN}$ & $\mathrm{Na}$ & $\mathrm{Na}$ & 1.7 & & $\mathrm{Na}$ & $\mathrm{Na}$ & & & $\mathrm{N}$ & $\mathrm{Na}$ & $\mathrm{N}$ & $\mathrm{N}$ & $\mathrm{Nal}$ & & & 0. & NaN & & 0.36 & $\mathrm{NaN}$ & $\mathrm{NaN}$ & $\mathrm{NaN}$ & $\mathrm{NaN}$ & $\mathrm{NaN}$ \\
\hline SEPT9 & & 1.29 & 2.84 & & & 1.59 & & & & & & 0. & 0.63 & & 0.26 & 0.91 & & 1.56 & 0.12 & & $\mathrm{NaN}$ & 0.14 & & $\mathrm{NaN}$ & $\mathrm{NaN}$ & 0.57 & 1.00 \\
\hline SERPINB3 & P295 & $\mathrm{NaN}$ & $\mathrm{NaN}$ & $\mathrm{Na}$ & $\mathrm{N}$ & $\mathrm{NaN}$ & & 0. & $\mathrm{NaN}$ & $\mathrm{NaN}$ & $\mathrm{NaN}$ & $\mathrm{Na}$ & $\mathrm{NaN}$ & $\mathrm{NaN}$ & $\mathrm{NaN}$ & $\mathrm{NaN}$ & $\mathrm{NaN}$ & $\mathrm{NaN}$ & $\mathrm{NaN}$ & & $\mathrm{NaN}$ & $\mathrm{NaN}$ & $\mathrm{aN}$ & $\mathrm{NaN}$ & 0.54 & $\mathrm{NaN}$ & $\mathrm{NaN}$ \\
\hline SETX & Q7Z333 & 0.72 & 0.83 & 1. & 0. & 1.27 & 0.47 & 0.1 & 1.02 & 1.01 & 1.13 & 1.1 & 1.14 & 1.0 & 0.74 & 0.71 & 0.06 & 0.89 & 0.94 & 0. & $\mathrm{NaN}$ & 0.58 & 0.6 & $\mathrm{NaN}$ & $\mathrm{NaN}$ & 1.21 & 0.31 \\
\hline $\mathrm{SF}_{3}$ & & 2.0 & 1.00 & 0.3 & 2. & 1.5 & & 0. & 1.0 & & & & 1.2 & & & 1.2 & & & & & & & & & & & 1.22 \\
\hline $\mathrm{SF} 3$ & & 1.4 & 1.0 & & 2. & 1. & & 0. & 1.8 & & 0. & 0. & 0. & 1. & & 1. & & 1. & 0. & & & & & 0.97 & 0.87 & 30 & 1.13 \\
\hline $\mathrm{SF}_{3}$ & & 1.53 & 1.09 & & 2. & 1.5 & & & 1.0 & & & 0.8 & 1.05 & & 0. & 1.2 & 1.2 & 1.5 & 0.3 & 1.29 & 1.4 & 0.3 & 1.8 & 1.06 & 1.12 & 0.27 & 1.08 \\
\hline SF3B2 & E91 & $\mathrm{NaN}$ & $\mathrm{NaN}$ & $\mathrm{NaN}$ & $\mathrm{NaN}$ & $\mathrm{NaN}$ & $\mathrm{NaN}$ & $\mathrm{NaN}$ & $\mathrm{Nal}$ & $\mathrm{NaN}$ & $\mathrm{NaN}$ & $\mathrm{NaN}$ & $\mathrm{NaN}$ & $\mathrm{NaN}$ & $\mathrm{NaN}$ & 0.83 & $\mathrm{NaN}$ & $\mathrm{NaN}$ & $\mathrm{NaN}$ & $\mathrm{NaN}$ & $\mathrm{NaN}$ & $\mathrm{NaN}$ & $\mathrm{NaN}$ & $\mathrm{NaN}$ & $\mathrm{NaN}$ & $\mathrm{NaN}$ & $\mathrm{NaN}$ \\
\hline SF3B4 & Q5: & 1.3 & 0.70 & 0.47 & $\mathrm{Na}$ & 1.1 & & 0. & & & 0.55 & 1.0 & 1.31 & & & 1.24 & & & & & & & & 108 & 0.87 & & \\
\hline & & 0.3 & 0.1 & & $\mathrm{~N}$ & $\mathrm{~N}$ & & 0 & & & & $\mathrm{~N}$ & & & & 0. & & & & & & & & & & $\mathrm{aN}$ & \\
\hline & & $\mathrm{NaN}$ & 1.95 & $\mathrm{Na}$ & $\mathrm{Na}$ & 1.20 & & $\mathrm{NaN}$ & $\mathrm{Na}$ & & $\mathrm{NaN}$ & $\mathrm{Nal}$ & $\mathrm{NaN}$ & $\mathrm{NaN}$ & $\mathrm{NaN}$ & 1.15 & $\mathrm{NaN}$ & $\mathrm{NaN}$ & 0.37 & $\mathrm{NaN}$ & $\mathrm{NaN}$ & $\mathrm{NaN}$ & $\mathrm{NaN}$ & $\mathrm{NaN}$ & $\mathrm{NaN}$ & $\mathrm{NaN}$ & $\mathrm{NaN}$ \\
\hline SHKBP1 & B2R6W9 & $\mathrm{NaN}$ & 2.05 & 2.61 & 247.82 & $\mathrm{NaN}$ & $\mathrm{NaN}$ & $\mathrm{NaN}$ & $\mathrm{NaN}$ & 18.49 & $\mathrm{NaN}$ & 8.02 & $\mathrm{NaN}$ & 7.50 & $\mathrm{NaN}$ & $\mathrm{NaN}$ & $\mathrm{NaN}$ & $\mathrm{NaN}$ & 14.85 & 10.88 & $\mathrm{NaN}$ & $\mathrm{NaN}$ & 7.93 & $\mathrm{NaN}$ & 12.58 & 9.65 & 2.47 \\
\hline SHPRH & Q149N8 & 0.54 & 0.39 & 0.55 & 1.15 & 1.13 & 0.80 & $\mathrm{NaN}$ & $\mathrm{NaI}$ & 2.46 & 0.28 & $\mathrm{NaN}$ & $\mathrm{NaN}$ & 0.99 & 0.71 & 0.04 & 2.02 & 1.25 & 0.51 & 0.78 & 1.63 & 0.31 & 1.80 & $\mathrm{NaN}$ & $\mathrm{NaN}$ & 1.07 & 0.59 \\
\hline & & 0.60 & 1.10 & 1.14 & 1.43 & 0.78 & & 1.2 & 1.2 & & 1.3 & 1. & & & & & 1. & & & & & & & 0. & & & \\
\hline SIN3B & |075182-2 & 0.45 & $\mathrm{NaN}$ & 1.51 & 1.25 & 1.10 & 1.37 & 0.91 & 1.02 & 1. & 0.8 & 1.62 & 1.18 & 0.76 & 3.14 & 0.56 & 0.70 & 1.02 & 1.40 & 6.63 & $\mathrm{NaN}$ & 2.23 & 0.78 & 1.75 & $\mathrm{NaN}$ & 2.19 & 0.36 \\
\hline SIRT6 & Q8N6T7 & $\mathrm{NaN}$ & 0.36 & 0.85 & 0.33 & 6.34 & 0.07 & $\mathrm{NaN}$ & 0.57 & $\mathrm{NaN}$ & 0.38 & 0.52 & 0.45 & 1.42 & 0.25 & 2.87 & 0.22 & 2.54 & 0.14 & 2.56 & 0.16 & 1.44 & 0.29 & 0.87 & 0.71 & 0.67 & 0.38 \\
\hline $\mathrm{C} 25 \mathrm{~A} 3$ & F8WM2 & $\mathrm{NaN}$ & $\mathrm{NaN}$ & $\mathrm{NaN}$ & 7.09 & $\mathrm{NaN}$ & $\mathrm{NaN}$ & $\mathrm{NaN}$ & $\mathrm{NaN}$ & $\mathrm{NaN}$ & 0.27 & 0.37 & $\mathrm{NaN}$ & $\mathrm{NaN}$ & $\mathrm{NaN}$ & $\mathrm{NaN}$ & $\mathrm{NaN}$ & $\mathrm{NaN}$ & $\mathrm{NaN}$ & 70.06 & $\mathrm{NaN}$ & $\mathrm{NaN}$ & 0.38 & $\mathrm{NaN}$ & $\mathrm{NaN}$ & 0.55 & $\mathrm{NaN}$ \\
\hline $\mathrm{AABCA}:$ & 060264 & 0.37 & 1.62 & 1.03 & 1.36 & 0.88 & 1.53 & 0.97 & 1.09 & 1.25 & 1.09 & 1.26 & 1.28 & 0.85 & 2.11 & 0.70 & 1.13 & 1.00 & 1.34 & 2.63 & 0.54 & 1.18 & 1.33 & 1.94 & 1.13 & 0.89 & 1.07 \\
\hline
\end{tabular}




\begin{tabular}{|c|c|c|c|c|c|c|c|c|c|c|c|c|c|c|c|c|c|c|c|c|c|c|c|c|c|c|c|}
\hline Gene names & Protein IDs & $\begin{array}{l}\mathrm{H} 3 \Delta \Delta- \\
20 \_\mathrm{F}\end{array}$ & $\begin{array}{l}H 3 \Delta 1- \\
20 \_R\end{array}$ & $\begin{array}{l}\text { H4K20 } \\
\text { me1_F }\end{array}$ & $\begin{array}{l}\text { H4K20 } \\
\text { me1_R }\end{array}$ & $\begin{array}{l}\text { H4K20 } \\
\text { me3_F }\end{array}$ & $\begin{array}{l}\text { H4K20 } \\
\text { me3_R }\end{array}$ & $\begin{array}{r}\mathrm{H}_{3} \mathrm{~K}_{\mathrm{c}} 27 \\
\text { e1_F }\end{array}$ & $\begin{array}{l}\mathrm{H} 3 \mathrm{~K}_{\mathrm{C}} 27 \\
\mathrm{me1} \mathrm{R}\end{array}$ & $\begin{array}{l}\mathrm{H} 3 \mathrm{~K}_{\mathrm{C}} 27 \\
\mathrm{me} 2 \mathrm{~F}\end{array}$ & $\begin{array}{l}\mathrm{H} 3 K_{\mathrm{c}} 27 \\
\mathrm{me2} \_\mathrm{R}\end{array}$ & $\begin{array}{l}\mathrm{H} 3 \mathrm{~K}_{\mathrm{c}} 27 \\
\mathrm{me3} 3 \mathrm{~F}\end{array}$ & $\begin{array}{l}\mathrm{H} 3 \mathrm{~K}_{\mathrm{C}} 27 \\
\mathrm{me3} \text {.R }\end{array}$ & $\begin{array}{c}\text { НзК9m } \\
\text { e1_F }\end{array}$ & $\begin{array}{c}\text { HзК9m } \\
\text { e1_R }\end{array}$ & $\begin{array}{c}\text { Нзкаm } \\
\text { e2_F }\end{array}$ & $\begin{array}{c}\text { H3K9m } \\
\text { e2_R }\end{array}$ & $\begin{array}{c}\text { HзK9m } \\
\text { e3_F }\end{array}$ & $\begin{array}{c}\text { НзК9m } \\
\text { e3_R }\end{array}$ & $\begin{array}{c}\text { HзK9m } \\
\text { e3|meC } \\
\text { pG_F }\end{array}$ & $\begin{array}{c}\text { H3к9m } \\
\text { e3|meC } \\
\text { pG_R }\end{array}$ & $\mid \begin{array}{c}\mathrm{H} 3 \mathrm{~K} 9 \mathrm{~m} \\
\mathrm{e} 3 \mathrm{H} 4 \mathrm{~K} \\
20 \mathrm{me} 3 \\
\mathrm{~F}\end{array}$ & $\begin{array}{c}\mathrm{H} 3 \mathrm{~K} 9 \mathrm{~m} \\
\mathrm{e} 3 \mid \mathrm{H} 4 \mathrm{~K} \\
20 \mathrm{me3} \\
\mathrm{R}\end{array}$ & $\underset{\mathrm{F}}{\mathrm{meCpG}}$ & $\underset{-R}{\operatorname{meCpG}}$ & $\begin{array}{c}\text { H4R3m } \\
\text { e2_F }\end{array}$ & $\begin{array}{c}\text { H4R3n } \\
\text { e2_R }\end{array}$ \\
\hline \begin{tabular}{|l|} 
SMCHD1 \\
\end{tabular} & A6NHR9 & 0.54 & 0.91 & 0.61 & 0.97 & 0.99 & 0.73 & 0.58 & $\mathrm{NaN}$ & 0.79 & 0.65 & 1.02 & 0.73 & 3.26 & 0.67 & 1.44 & 0.17 & 2.21 & 0.24 & 47.25 & 0.19 & 3.01 & 0.19 & $\mathrm{NaN}$ & $\begin{array}{l}1.06 \\
\end{array}$ & $\mathrm{NaN}$ & $\mathrm{NaN}$ \\
\hline SMCHD1 & LOR6P7 & $\mathrm{NaN}$ & $\mathrm{NaN}$ & $\mathrm{NaN}$ & $\mathrm{NaN}$ & $\mathrm{NaN}$ & $\mathrm{NaN}$ & $\mathrm{NaN}$ & $\mathrm{NaN}$ & $\mathrm{NaN}$ & $\mathrm{NaN}$ & $\mathrm{NaN}$ & $\mathrm{NaN}$ & 0.78 & $\mathrm{NaN}$ & $\mathrm{NaN}$ & $\mathrm{NaN}$ & $\mathrm{NaN}$ & $\mathrm{NaN}$ & $\mathrm{NaN}$ & $\mathrm{NaN}$ & $\mathrm{NaN}$ & $\mathrm{NaN}$ & $\mathrm{NaN}$ & $\mathrm{NaN}$ & $\mathrm{NaN}$ & $\mathrm{NaN}$ \\
\hline SNC73 & Q9UP60 & $\mathrm{NaN}$ & $\mathrm{NaN}$ & $\mathrm{NaN}$ & $\mathrm{NaN}$ & 47.70 & $\mathrm{NaN}$ & 0.06 & $\mathrm{NaN}$ & $\mathrm{NaN}$ & $\mathrm{NaN}$ & 0.05 & $\mathrm{NaN}$ & $\mathrm{NaN}$ & $\mathrm{NaN}$ & $\mathrm{NaN}$ & $\mathrm{NaN}$ & $\mathrm{NaN}$ & $\mathrm{NaN}$ & $\mathrm{NaN}$ & $\mathrm{NaN}$ & $\mathrm{NaN}$ & $\mathrm{NaN}$ & $\mathrm{NaN}$ & $\mathrm{NaN}$ & $\mathrm{NaN}$ & 0.12 \\
\hline SNRPA1 & P09661 & 1.80 & 0.90 & 0.39 & 2.21 & 1.43 & 0.47 & 0.65 & 1.06 & 1.57 & 0.58 & 0.90 & 0.99 & 1.68 & 0.56 & 1.10 & 0.99 & 1.57 & 0.43 & 0.94 & 1.41 & 0.38 & 1.79 & 1.17 & 1.01 & 0.44 & 1.17 \\
\hline SNRPB2 & P08579 & 1.62 & 0.96 & 0.37 & 1.84 & 1.40 & 0.47 & 0.69 & 1.03 & 1.53 & 0.48 & 0.77 & 0.89 & 1.68 & 0.57 & 1.06 & 1.03 & 1.53 & 0.43 & 0.98 & 1.26 & 0.41 & 1.65 & 1.08 & 1.07 & 0.49 & 1.14 \\
\hline SNRPD3 & B4DJP7 & 1.72 & 0.87 & 0.55 & 2.08 & 1.24 & 0.62 & 0.80 & 1.11 & 1.71 & 0.71 & 1.10 & 1.14 & $\mathrm{NaN}$ & 0.67 & 1.13 & 1.02 & 1.43 & 0.64 & 0.80 & $\mathrm{NaN}$ & 0.72 & 1.38 & 0.73 & 0.79 & 0.59 & 1.22 \\
\hline SP1 & G5E9M8 & 0.84 & $\mathrm{NaN}$ & $\mathrm{NaN}$ & 0.54 & $\mathrm{NaN}$ & 1.23 & $\mathrm{NaN}$ & $\mathrm{NaN}$ & 0.30 & 1.72 & 0.83 & 0.61 & $\mathrm{NaN}$ & $\mathrm{NaN}$ & 0.47 & 0.49 & $\mathrm{NaN}$ & 1.74 & 1.34 & $\mathrm{NaN}$ & 2.52 & 0.24 & $\mathrm{NaN}$ & $\mathrm{NaN}$ & $\mathrm{NaN}$ & $\mathrm{NaN}$ \\
\hline SP3 & Q8WWU3 & 0.70 & $\mathrm{NaN}$ & 0.79 & 0.55 & 0.79 & $\begin{array}{l}1.50 \\
1.58\end{array}$ & 0.96 & 1.01 & 0.45 & 1.88 & 0.94 & 0.81 & $\mathrm{NaN}$ & $\mathrm{NaN}$ & 0.39 & 3.36 & 0.43 & 2.14 & 1.40 & 0.82 & 3.09 & 0.28 & 1.10 & 0.70 & 2.61 & 0.28 \\
\hline SPATA5L1 & Q9BVQ7 & 1.54 & 1.33 & 1.50 & $\mathrm{NaN}$ & 0.89 & 0.60 & $\mathrm{NaN}$ & $\mathrm{NaN}$ & 0.97 & $\mathrm{NaN}$ & 0.71 & 1.46 & 1.15 & 0.86 & 1.11 & 0.45 & 0.88 & 1.20 & 0.91 & $\mathrm{NaN}$ & 0.82 & 0.78 & $\mathrm{NaN}$ & $\mathrm{NaN}$ & $\mathrm{NaN}$ & $\mathrm{NaN}$ \\
\hline SPIN1 & Q9Y657 & $\mathrm{NaN}$ & $\mathrm{NaN}$ & $\mathrm{NaN}$ & $\mathrm{NaN}$ & $\mathrm{NaN}$ & $\mathrm{NaN}$ & $\mathrm{NaN}$ & $\mathrm{NaN}$ & $\mathrm{NaN}$ & $\mathrm{NaN}$ & $\mathrm{NaN}$ & $\mathrm{NaN}$ & $\mathrm{NaN}$ & $\mathrm{NaN}$ & $\mathrm{NaN}$ & $\mathrm{NaN}$ & 4.88 & 0.22 & 3.36 & 0.38 & 7.35 & 0.13 & $\mathrm{NaN}$ & $\mathrm{NaN}$ & $\mathrm{NaN}$ & $\mathrm{NaN}$ \\
\hline SPIN2B & Q5JZB8 & $\mathrm{NaN}$ & $\mathrm{NaN}$ & $\mathrm{NaN}$ & $\mathrm{NaN}$ & $\mathrm{NaN}$ & $\mathrm{NaN}$ & $\mathrm{NaN}$ & $\mathrm{NaN}$ & $\mathrm{NaN}$ & $\mathrm{NaN}$ & $\mathrm{NaN}$ & $\mathrm{NaN}$ & $\mathrm{NaN}$ & $\mathrm{NaN}$ & $\mathrm{NaN}$ & $\mathrm{NaN}$ & 7.57 & $\mathrm{NaN}$ & 12.49 & 0.13 & 9.55 & 0.12 & $\mathrm{NaN}$ & $\mathrm{NaN}$ & $\mathrm{NaN}$ & $\mathrm{NaN}$ \\
\hline SPRRIA & B7ZLF8 & $\mathrm{NaN}$ & $\mathrm{NaN}$ & 0.04 & $\mathrm{NaN}$ & $\mathrm{NaN}$ & $\mathrm{NaN}$ & 0.06 & $\mathrm{NaN}$ & $\mathrm{NaN}$ & 0.03 & 0.14 & 0.18 & $\mathrm{NaN}$ & $\mathrm{NaN}$ & $\mathrm{NaN}$ & $\mathrm{NaN}$ & $\mathrm{NaN}$ & $\mathrm{NaN}$ & $\mathrm{NaN}$ & $\mathrm{NaN}$ & $\mathrm{NaN}$ & $\mathrm{NaN}$ & $\mathrm{NaN}$ & $\mathrm{NaN}$ & $\mathrm{NaN}$ & $\mathrm{NaN}$ \\
\hline $\begin{array}{l}\text { SPRRAB } \\
\text { SPRA }\end{array}$ & \begin{tabular}{|l} 
D1L250 \\
P22528
\end{tabular} & $\mathrm{NaN}$ & $\mathrm{NaN}$ & $\mathrm{NaN}$ & $\mathrm{NaN}$ & $\mathrm{NaN}$ & $\mathrm{NaN}$ & 0.05 & $\mathrm{NaN}$ & $\mathrm{NaN}$ & $\mathrm{NaN}$ & $\begin{array}{l}\mathrm{NaN} \\
\mathrm{NaN}\end{array}$ & $\begin{array}{l}.10 \\
\mathrm{NaN}\end{array}$ & $\mathrm{NaN}$ & $\mathrm{NaN}$ & $\mathrm{NaN}$ & $\mathrm{NaN}$ & $\mathrm{NaN}$ & $\mathrm{NaN}$ & $\mathrm{NaN}$ & $\mathrm{NaN}$ & $\mathrm{NaN}$ & $\mathrm{NaN}$ & $\mathrm{NaN}$ & $\mathrm{NaN}$ & $\mathrm{NaN}$ & $\mathrm{NaN}$ \\
\hline SPTY2D1 & Q68D10-2 & 1.07 & 1.61 & 0.58 & 0.62 & 0.53 & 1.01 & 0.49 & 1.21 & 1.18 & 0.77 & 0.71 & 0.91 & 2.42 & $\mathrm{NaN}$ & 1.21 & 0.62 & 0.55 & 0.89 & 0.65 & 0.69 & 0.76 & 0.52 & 0.86 & 0.47 & 0.67 & 1.30 \\
\hline SREK1 & B3KRJ9 & 1.25 & 0.69 & 2.82 & 0.21 & 1.15 & 0.53 & $\mathrm{NaN}$ & $\mathrm{NaN}$ & $\mathrm{NaN}$ & $\mathrm{NaN}$ & $\mathrm{NaN}$ & $\mathrm{NaN}$ & 2.27 & 0.79 & 0.96 & 0.83 & 1.22 & 0.54 & $\mathrm{NaN}$ & $\mathrm{NaN}$ & 0.57 & 1.04 & $\mathrm{NaN}$ & $\mathrm{NaN}$ & 0.45 & 0.70 \\
\hline SRRM1 & A9ZZ1X7 & 7.44 & 3.28 & 0.27 & 0.72 & 0.35 & 0.21 & 0.34 & 0.48 & 0.72 & 0.15 & 0.45 & 0.39 & $\mathrm{NaN}$ & 0.27 & 1.64 & 1.39 & 0.37 & 0.22 & 0.78 & 1.27 & 0.34 & 0.42 & $\mathrm{NaN}$ & $\mathrm{NaN}$ & 0.20 & 0.27 \\
\hline $\begin{array}{l}\text { SRSF6 } \\
\text { SR }\end{array}$ & A8K588 & $\begin{array}{l}7.44 \\
7.33\end{array}$ & $\begin{array}{l}0.60 \\
1.79\end{array}$ & 0.52 & $\begin{array}{l}0.16 \\
2.00\end{array}$ & 0.03 & 0.75 & 0.97 & $\begin{array}{l}.40 \\
1.25\end{array}$ & $\begin{array}{l}.112 \\
2.36\end{array}$ & 0.31 & $\begin{array}{l}0.45 \\
1.51\end{array}$ & 1.23 & 2.06 & 1.16 & $\begin{array}{l}1.04 \\
1.53\end{array}$ & $\begin{array}{l}1.05 \\
1.63\end{array}$ & 1.32 & 0.59 & $\begin{array}{l}0.63 \\
1.63\end{array}$ & 1.57 & 0.77 & $\begin{array}{l}0.48 \\
1.38\end{array}$ & $\mathrm{NaN}$ & 0.96 & 0.33 & 0.63 \\
\hline SUDS3 & Q9H7L9 & 0.60 & 0.77 & 1.45 & 1.34 & 0.72 & 3.05 & 1.47 & 1.43 & 1.51 & 1.69 & 1.52 & 1.10 & 0.58 & 3.30 & 0.41 & 3.57 & 1.19 & 1.47 & 9.63 & 0.24 & 1.11 & 1.76 & 0.71 & 3.53 & 2.46 & 1.07 \\
\hline SUPT3H & 075486 & 0.64 & 0.52 & 1.02 & 0.55 & 0.45 & 0.97 & 0.84 & $\mathrm{NaN}$ & 0.58 & 0.99 & 0.82 & 0.77 & 1.55 & 0.41 & 0.44 & 0.45 & 0.47 & 1.02 & $\mathrm{NaN}$ & 0.09 & 1.53 & 0.38 & 0.58 & 2.32 & 1.71 & 0.40 \\
\hline SUZ12 & Q15022 & 0.35 & 2.04 & & 0.65 & 0.89 & & 0.76 & 0.98 & & 0.89 & 0.92 & 0.84 & 0.61 & 1.37 & 1.44 & 1.45 & 1.33 & 0.50 & 1.80 & & & 0.44 & 1.07 & & 1.05 & 0.67 \\
\hline SV2A & A8K6Q3 & $\mathrm{NaN}$ & $\mathrm{NaN}$ & $\mathrm{NaN}$ & $\mathrm{NaN}$ & $\mathrm{NaN}$ & $\mathrm{NaN}$ & $\mathrm{NaN}$ & $\mathrm{NaN}$ & $\mathrm{NaN}$ & $\mathrm{NaN}$ & $\mathrm{NaN}$ & $\mathrm{NaN}$ & $\mathrm{NaN}$ & $\mathrm{NaN}$ & $\mathrm{NaN}$ & $\mathrm{NaN}$ & $\mathrm{NaN}$ & $\mathrm{NaN}$ & $\mathrm{NaN}$ & $\mathrm{NaN}$ & $\mathrm{NaN}$ & $\mathrm{NaN}$ & 0.03 & $\mathrm{NaN}$ & $\mathrm{NaN}$ & $\mathrm{NaN}$ \\
\hline SV2A & L8E840 & $\mathrm{NaN}$ & $\mathrm{NaN}$ & $\mathrm{NaN}$ & 0.96 & $\mathrm{NaN}$ & $\mathrm{NaN}$ & $\mathrm{NaN}$ & 0.83 & $\mathrm{NaN}$ & $\mathrm{NaN}$ & $\mathrm{NaN}$ & 1.01 & $\mathrm{NaN}$ & $\mathrm{NaN}$ & $\mathrm{NaN}$ & $\mathrm{NaN}$ & 1.06 & 1.22 & $\mathrm{NaN}$ & $\mathrm{NaN}$ & 0.85 & $\mathrm{NaN}$ & $\mathrm{NaN}$ & $\mathrm{NaN}$ & 0.83 & 0.84 \\
\hline SVIL & O95425-2 & $\mathrm{NaN}$ & 4.06 & $\mathrm{NaN}$ & $\mathrm{NaN}$ & $\mathrm{NaN}$ & $\mathrm{NaN}$ & $\mathrm{NaN}$ & $\mathrm{NaN}$ & $\mathrm{NaN}$ & $\mathrm{NaN}$ & $\mathrm{NaN}$ & $\mathrm{NaN}$ & $\mathrm{NaN}$ & $\mathrm{NaN}$ & 0.08 & 0.62 & $\mathrm{NaN}$ & $\mathrm{NaN}$ & $\mathrm{NaN}$ & $\mathrm{NaN}$ & $\mathrm{NaN}$ & $\mathrm{NaN}$ & $\mathrm{NaN}$ & $\mathrm{NaN}$ & $\mathrm{NaN}$ & $\mathrm{NaN}$ \\
\hline SYAP1 & B2RB12 & $\mathrm{NaN}$ & 0.12 & 0.88 & 1.23 & $\mathrm{NaN}$ & 0.17 & 0.82 & $\mathrm{NaN}$ & $\mathrm{NaN}$ & 0.78 & $\mathrm{NaN}$ & $\mathrm{NaN}$ & $\mathrm{NaN}$ & $\mathrm{NaN}$ & & & 1.36 & 0.90 & $\mathrm{NaN}$ & & $\mathrm{NaN}$ & 1.09 & $\mathrm{NaN}$ & $\mathrm{NaN}$ & 1.30 & 0.55 \\
\hline SYDE1 & B2RD93 & 1.00 & $\mathrm{NaN}$ & 2.41 & $\mathrm{NaN}$ & 3.42 & 0.36 & $\mathrm{NaN}$ & $\mathrm{NaN}$ & $\mathrm{NaN}$ & $\mathrm{NaN}$ & 0.56 & 1.46 & $\mathrm{NaN}$ & $\mathrm{NaN}$ & 0.68 & 0.13 & 1.17 & 0.62 & 1.37 & 0.75 & 0.80 & 1.05 & $\mathrm{NaN}$ & $\mathrm{NaN}$ & $\mathrm{NaN}$ & $\mathrm{NaN}$ \\
\hline $\begin{array}{l}\text { SYN1 } \\
\text { SYN }\end{array}$ & $\begin{array}{l}\mid l 17600 \\
P 1600\end{array}$ & $\mathrm{NaN}$ & $\mathrm{NaN}$ & $\begin{array}{l}2.41 \\
\mathrm{NaN}\end{array}$ & $\mathrm{NaN}$ & $\begin{array}{l}. .46 \\
\mathrm{NaN}\end{array}$ & $\mathrm{NaN}$ & $\mathrm{NaN}$ & NaN & $\mathrm{NaN}$ & 1.02 & $\mathrm{NaN}$ & $\mathrm{NaN}$ & $\mathrm{NaN}$ & $\mathrm{NaN}$ & NaN & $\mathrm{NaN}$ & $\mathrm{NaN}$ & $\mathrm{NaN}$ & $\mathrm{NaN}$ & $\mathrm{NaN}$ & $\mathrm{NaN}$ & $\mathrm{NaN}$ & 0.18 & $\mathrm{NaN}$ & $\mathrm{NaN}$ & $\mathrm{NaN}$ \\
\hline SYT1 & J3KQA0 & $\mathrm{NaN}$ & $\mathrm{NaN}$ & $\mathrm{NaN}$ & $\mathrm{NaN}$ & $\mathrm{NaN}$ & $\mathrm{NaN}$ & $\mathrm{NaN}$ & $\mathrm{NaN}$ & $\mathrm{NaN}$ & $\mathrm{NaN}$ & $\mathrm{NaN}$ & $\mathrm{NaN}$ & $\mathrm{NaN}$ & $\mathrm{NaN}$ & $\mathrm{NaN}$ & $\mathrm{NaN}$ & $\mathrm{NaN}$ & $\mathrm{NaN}$ & $\mathrm{NaN}$ & $\mathrm{NaN}$ & $\mathrm{NaN}$ & $\mathrm{NaN}$ & 0.14 & $\mathrm{NaN}$ & $\mathrm{NaN}$ & $\mathrm{NaN}$ \\
\hline TADA1 & Q96BN2 & 0.57 & 0.57 & 0.75 & 0.54 & 0.49 & 1.11 & 0.75 & $\mathrm{NaN}$ & 0.54 & 0.95 & 0.81 & 0.72 & $\mathrm{NaN}$ & $\mathrm{NaN}$ & 0.55 & 0.30 & 0.51 & 0.97 & 5.32 & 0.17 & 1.31 & 0.40 & 0.77 & 1.95 & 0.33 & $\mathrm{NaN}$ \\
\hline TADA2B & 作 & $\mathrm{NaN}$ & $\mathrm{NaN}$ & 0.9 & 0.5 & 0.49 & 1.0 & 0.12 & $\mathrm{NaN}$ & & 0.8 & 0.94 & 0.72 & 0.29 & 2.07 & $\mathrm{NaN}$ & & & 0.96 & 0. & & 1.39 & 0.38 & & & 0.16 & $\mathrm{NaN}$ \\
\hline TADA3 & 075528 & $\mathrm{NaN}$ & 0.30 & 1.76 & 0.83 & 1.15 & 1.15 & 1.20 & 0.99 & 0.89 & 1.45 & 1.10 & 0.94 & $\mathrm{NaN}$ & $\mathrm{NaN}$ & 0.29 & 1.42 & 0.94 & 1.13 & 1.85 & 0.76 & 1.40 & 0.79 & 1.22 & 1.29 & 1.57 & 0.92 \\
\hline TAF1 & P21675-6 & $\mathrm{NaN}$ & $\mathrm{NaN}$ & 0.53 & 0.62 & 0.51 & $\mathrm{NaN}$ & 0.71 & $\mathrm{NaN}$ & 0.48 & 0.83 & 0.67 & 0.79 & $\mathrm{NaN}$ & $\mathrm{NaN}$ & 0.18 & $\mathrm{NaN}$ & 0.29 & 1.34 & 4.80 & 0.28 & 1.47 & 0.32 & $\mathrm{NaN}$ & $\mathrm{NaN}$ & $\mathrm{NaN}$ & $\mathrm{NaN}$ \\
\hline TAF11 & Q15544 & $\mathrm{NaN}$ & $\mathrm{NaN}$ & $\mathrm{NaN}$ & $\mathrm{NaN}$ & 0.71 & $\mathrm{NaN}$ & 0.4 & 1.26 & 0.6 & 0.9 & 0.74 & 1.04 & $\mathrm{NaN}$ & $\mathrm{NaN}$ & $\mathrm{NaN}$ & $\mathrm{NaN}$ & 0.36 & 0.52 & & $\mathrm{NaN}$ & 1.76 & 0.42 & $\mathrm{NaN}$ & 0.80 & $\mathrm{NaN}$ & $\mathrm{NaN}$ \\
\hline TAF & Q15573 & & $\mathrm{N}$ & 0.4 & $\mathrm{NaN}$ & 0. & & 0. & 0.70 & & & & & & & & & & 33 & & & 1.02 & 0.48 & & & $\mathrm{NaN}$ & $\mathrm{NaN}$ \\
\hline TAF1B & Q53T94 & $\mathrm{NaN}$ & $\mathrm{NaN}$ & $\mathrm{NaN}$ & 1.00 & 0.84 & $\mathrm{NaN}$ & 0.5 & 0.78 & $\mathrm{~N}$ & $\mathrm{Na}$ & 0.99 & 75 & $\mathrm{NaN}$ & $\mathrm{NaN}$ & $\mathrm{NaN}$ & $\mathrm{NaN}$ & 0.29 & $\mathrm{NaN}$ & $\mathrm{NaN}$ & 0.22 & $\mathrm{NaN}$ & 0.40 & 0.48 & $\mathrm{NaN}$ & $\mathrm{NaN}$ & 0.98 \\
\hline TAF1D & H5J8 & $\mathrm{NaN}$ & $\mathrm{NaN}$ & 1.91 & 0.73 & $\mathrm{NaN}$ & $\mathrm{NaN}$ & 0.7 & 0.67 & 0. & 1.03 & 0.83 & 0 & $\mathrm{NaN}$ & $\mathrm{NaN}$ & 0.52 & $\mathrm{NaN}$ & 3.21 & 1.75 & 9.84 & 0.22 & 0.95 & 0.51 & 0.51 & 2.33 & $\mathrm{NaN}$ & $\mathrm{NaN}$ \\
\hline TAF5 & Q15542 & 0.24 & 1.04 & 0.4 & 0.75 & 0. & 1.10 & 0. & 0.91 & & 0.98 & 0.77 & 0.87 & $\mathrm{NaN}$ & 2.06 & 0.15 & 1.30 & 0.31 & 1. & 2.51 & 0.1 & 1.50 & 0.37 & 1.34 & 2.18 & $\mathrm{NaN}$ & $\mathrm{NaN}$ \\
\hline TAF6 & J3KR72 & 0.33 & 0.90 & 0.41 & 0.67 & 0.63 & 0.83 & 0.3 & 0.88 & 0.4 & 0.7 & 0.58 & 0.88 & 0.35 & 1.13 & 0.14 & 3.94 & 0.32 & 1.43 & 2.56 & 0.4 & 1.33 & 0.31 & $\mathrm{NaN}$ & 2.36 & $\mathrm{NaN}$ & 0.50 \\
\hline TALL & $P 3$ & $\mathrm{NaN}$ & 0.0 & & & 1.18 & & & & & 0. & & & $\mathrm{Na}$ & $\mathrm{NaN}$ & & $\mathrm{Na}$ & 1.8 & & 0. & & & & & $\mathrm{NaN}$ & $\mathrm{NaN}$ & $\mathrm{NaN}$ \\
\hline TARS & Q & 0.46 & 2.76 & $\mathrm{Nal}$ & $\mathrm{Nal}$ & $\mathrm{NaN}$ & $\mathrm{NaI}$ & $\mathrm{Na}$ & $\mathrm{NaN}$ & $\mathrm{Nan}$ & $\mathrm{N}$ & $\mathrm{NaN}$ & $\mathrm{NaN}$ & $\mathrm{NaN}$ & $\mathrm{NaN}$ & 0.54 & 0.42 & 1.0 & $\mathrm{NaN}$ & $\mathrm{NaN}$ & $\mathrm{NaN}$ & $\mathrm{NaN}$ & $\mathrm{NaN}$ & $\mathrm{NaN}$ & $\mathrm{NaN}$ & $\mathrm{NaN}$ & $\mathrm{NaN}$ \\
\hline TBR & & $\mathrm{NaN}$ & $\mathrm{NaN}$ & 2. & 0.7 & & & 3.2 & $\mathrm{NaN}$ & 1.06 & 2.9 & 1.70 & & 0.3 & 4.99 & & 1.3 & 0.49 & 2.50 & 6.11 & 0.1 & 2.92 & 0.64 & $\mathrm{NaN}$ & 1.46 & $\mathrm{NaN}$ & $\mathrm{NaN}$ \\
\hline TCHH & Q07283 & $\mathrm{NaN}$ & $\mathrm{NaN}$ & $\mathrm{NaN}$ & $\mathrm{NaN}$ & 2.83 & 0.54 & $\mathrm{Nal}$ & $\mathrm{NaN}$ & $\mathrm{NaN}$ & $\mathrm{NaN}$ & $\mathrm{NaN}$ & 0.78 & $\mathrm{NaN}$ & $\mathrm{NaN}$ & $\mathrm{NaN}$ & 0.86 & 3.06 & 0.20 & $\mathrm{NaN}$ & $\mathrm{NaN}$ & 1.80 & 0.34 & $\mathrm{NaN}$ & $\mathrm{NaN}$ & 0.15 & $\mathrm{NaN}$ \\
\hline TCOF1 & E7ETY2 & $\mathrm{NaN}$ & $\mathrm{NaN}$ & & $\mathrm{Nal}$ & 0.7 & 1.4 & 2.0 & 1.40 & 0.67 & 1.54 & 2.41 & 0.62 & $\mathrm{~N}$ & $\mathrm{NaN}$ & & $\mathrm{NaN}$ & & & & & & 0.52 & & & & $\mathrm{NaN}$ \\
\hline TCOF1 & & 0.94 & 1.1 & 0. & 0.9 & 0 & 0. & 1.3 & 1. & & 1.3 & 1.7 & & 0 & 0.94 & & 3 & 0. & & & & & & & 1.00 & 0.80 & 0.42 \\
\hline TCOF1 & & & $\mathrm{NaN}$ & 0. & & & & 1. & 0.9 & & 1.0 & 1.51 & & $\mathrm{~N}$ & $\mathrm{NaN}$ & & 5. & 0.3 & 0.79 & $\mathrm{~N}$ & $\mathrm{Na}$ & 2.02 & 0.47 & $\mathrm{NaN}$ & $\mathrm{NaN}$ & 1.08 & 0.25 \\
\hline TF & Q53 H & $\mathrm{NaN}$ & $\mathrm{NaN}$ & $\mathrm{NaN}$ & $\mathrm{NaN}$ & $\mathrm{NaN}$ & $\mathrm{NaN}$ & 0.11 & $\mathrm{NaN}$ & $\mathrm{NaN}$ & $\mathrm{Nal}$ & $\mathrm{NaN}$ & $\mathrm{NaN}$ & $\mathrm{NaN}$ & $\mathrm{NaN}$ & NaN & $\mathrm{NaN}$ & $\mathrm{NaN}$ & $\mathrm{NaN}$ & $\mathrm{NaN}$ & $\mathrm{NaN}$ & $\mathrm{NaN}$ & $\mathrm{NaN}$ & $\mathrm{NaN}$ & $\mathrm{NaN}$ & $\mathrm{NaN}$ & $\mathrm{NaN}$ \\
\hline TFAP4 & Q6FHM5 & 0.99 & 0.42 & 2.57 & 1.24 & 1.09 & 3.69 & 1.73 & 1.59 & 1.26 & 2.30 & 1.93 & 1.79 & $\mathrm{NaN}$ & $\mathrm{NaN}$ & 0.08 & 3.25 & 0.84 & 3.84 & 26.96 & 0.06 & 3.54 & 1.12 & 2.42 & 3.00 & 1.64 & $\mathrm{NaN}$ \\
\hline TFPT & $G$ & 0.65 & 0.36 & 2.24 & & & 1.6 & & & & 2.7 & & & 0.19 & 5.94 & & & 0.50 & 2. & & & & & & & & 0.42 \\
\hline TGM1 & & $\mathrm{Na}$ & $\mathrm{N}$ & $\mathrm{Na}$ & $\mathrm{Na}$ & $\mathrm{N}$ & $\mathrm{N}$ & 0. & $\mathrm{~N}$ & $\mathrm{~N}$ & $\mathrm{Na}$ & No & & $\mathrm{NaN}$ & $\mathrm{NaN}$ & $\mathrm{NaN}$ & $\mathrm{Na}$ & $\mathrm{Na}$ & $\mathrm{NaN}$ & $\mathrm{Nc}$ & $\mathrm{Na}$ & NaN & $\mathrm{NaN}$ & $\mathrm{NaN}$ & $\mathrm{NaN}$ & $\begin{array}{l}4.52 \\
\mathrm{NaN}\end{array}$ & $\mathrm{NaN}$ \\
\hline TGM & Q0818 & $\mathrm{NaN}$ & $\mathrm{NaN}$ & 0.23 & $\mathrm{NaN}$ & $\mathrm{NaN}$ & $\mathrm{NaN}$ & 0.18 & $\mathrm{NaN}$ & $\mathrm{N}$ & $\mathrm{Na}$ & $\mathrm{NaN}$ & $\mathrm{NaN}$ & $\mathrm{NaN}$ & $\mathrm{NaN}$ & $\mathrm{NaN}$ & $\mathrm{NaN}$ & $\mathrm{NaN}$ & $\mathrm{NaN}$ & 0.08 & $\mathrm{NaN}$ & $\mathrm{NaN}$ & $\mathrm{NaN}$ & $\mathrm{NaN}$ & $\mathrm{NaN}$ & $\mathrm{NaN}$ & $\mathrm{NaN}$ \\
\hline TIAL1 & $\mathrm{Q} 01085-2$ & 1.75 & 1.20 & 1.07 & 1.49 & 1.11 & 0.98 & $\mathrm{NaN}$ & $\mathrm{NaN}$ & NaN & $\mathrm{NaN}$ & 1.41 & 1.34 & 1.37 & 1.06 & 1.37 & 0.49 & $\mathrm{NaN}$ & 2.35 & $\mathrm{NaN}$ & 1.72 & 1.15 & 1.11 & $\mathrm{NaN}$ & $\mathrm{NaN}$ & 1.03 & 1.29 \\
\hline TIAL & Q2TSD2 & $\mathrm{NaN}$ & 1.01 & $\mathrm{NaN}$ & & & $\mathrm{NaN}$ & & $\mathrm{NaN}$ & & $\mathrm{NaN}$ & $\mathrm{NaN}$ & & $\mathrm{NaN}$ & $\mathrm{NaN}$ & & 1.14 & $\mathrm{NaN}$ & & & & & & & & & $\mathrm{NaN}$ \\
\hline & & $\mathrm{Na}$ & 2.2 & 1.2 & 1.1 & & 1.6 & 1.4 & 1. & & 1.8 & 1.32 & & 0. & $\mathrm{~N}$ & & & 1.19 & & $\mathrm{No}$ & $\mathrm{N}$ & & 1.58 & $\mathrm{NaN}$ & $\mathrm{NaN}$ & $\mathrm{NaN}$ & $\mathrm{NaN}$ \\
\hline TINF2 & B4DFJ1 & $\mathrm{NaN}$ & $\mathrm{NaN}$ & 1.16 & 1.30 & 1.16 & 2.40 & $\mathrm{NaN}$ & $\mathrm{NaN}$ & $\mathrm{NaN}$ & 2.61 & $\mathrm{NaN}$ & 1.55 & $\mathrm{NaN}$ & $\mathrm{NaN}$ & $\mathrm{NaN}$ & $\mathrm{NaN}$ & 0.62 & 2.35 & 2.44 & 1.02 & 1.66 & 1.50 & 1.60 & 2.38 & $\mathrm{NaN}$ & $\mathrm{NaN}$ \\
\hline TKT & P29401 & 0.16 & 0.10 & 2.42 & $\mathrm{NaN}$ & $\mathrm{NaN}$ & 0.44 & 0.22 & 1.70 & 1.37 & 0.30 & $\mathrm{NaN}$ & $\mathrm{NaN}$ & $\mathrm{NaN}$ & $\mathrm{NaN}$ & 0.23 & 0.54 & 0.89 & 0.63 & 0.19 & 2.15 & 0.62 & $\mathrm{NaN}$ & 0.85 & $\mathrm{NaN}$ & $\mathrm{NaN}$ & $\mathrm{NaN}$ \\
\hline TMPO & P42166 & 1.71 & 0.14 & 0.59 & 0.24 & 0.92 & 0.46 & 0.61 & 0.16 & 0.37 & 0.73 & 0.42 & 0.27 & $\mathrm{NaN}$ & (v) & 0.33 & 2.10 & 0.42 & 0.45 & 0.05 & 0.89 & & 024 & 0.08 & & & 0.52 \\
\hline TOB1 & & 0.20 & $\mathrm{NaN}$ & $\mathrm{NaN}$ & $\mathrm{NaN}$ & $\mathrm{NaN}$ & 0.75 & $\mathrm{NaN}$ & $\mathrm{NaN}$ & $\mathrm{NaN}$ & $\mathrm{NaN}$ & $\mathrm{N}$ & & $\mathrm{NaN}$ & $\mathrm{NaN}$ & & 0.08 & 0.33 & $\mathrm{NaN}$ & $\mathrm{NaN}$ & $\mathrm{NaN}$ & $\mathrm{NaN}$ & $\mathrm{NaN}$ & & & $\mathrm{JaN}$ & $\mathrm{NaN}$ \\
\hline TOPBP1 & Q92547 & 1.66 & $\mathrm{NaN}$ & $\mathrm{NaN}$ & $\mathrm{NaN}$ & 2.82 & 0.59 & $\mathrm{NaN}$ & $\mathrm{NaN}$ & $\mathrm{NaN}$ & $\mathrm{NaN}$ & 2.19 & 0.47 & $\mathrm{NaN}$ & $\mathrm{NaN}$ & 0.99 & 0.15 & 1.62 & $\mathrm{NaN}$ & 4.50 & 0.47 & 2.33 & 0.35 & $\mathrm{NaN}$ & $\mathrm{NaN}$ & $\mathrm{NaN}$ & $\mathrm{NaN}$ \\
\hline TPI1 & P60174-1 & 0.13 & 0.08 & 0.99 & $\mathrm{NaN}$ & $\mathrm{NaN}$ & $\mathrm{NaN}$ & 0.43 & 1.35 & 1.15 & 0.36 & $\mathrm{NaN}$ & $\mathrm{NaN}$ & $\mathrm{NaN}$ & $\mathrm{NaN}$ & 0.13 & 0.50 & $\mathrm{NaN}$ & $\mathrm{NaN}$ & 0.33 & 1.97 & $\mathrm{NaN}$ & $\mathrm{NaN}$ & 1.21 & 0.52 & $\mathrm{NaN}$ & $\mathrm{NaN}$ \\
\hline
\end{tabular}




\begin{tabular}{|c|c|c|c|c|c|c|c|c|c|c|c|c|c|c|c|c|c|c|c|c|c|c|c|c|c|c|c|}
\hline Gene names & Protein IDs & $\begin{array}{l}\text { H3 } \Delta \Delta 1- \\
20 \_F\end{array}$ & $\begin{array}{l}H 3 \Delta 1- \\
20 \_R\end{array}$ & $\begin{array}{l}\text { H4K20 } \\
\text { me1_F }\end{array}$ & $\begin{array}{l}\text { H4K20 } \\
\text { me1_R }\end{array}$ & $\begin{array}{l}\text { H4K20 } \\
\text { me3_F }\end{array}$ & $\begin{array}{l}\text { H4K20 } \\
\text { me3_R }\end{array}$ & $\begin{array}{l}\mathrm{H} 3 \mathrm{~K}_{\mathrm{C}} 27 \\
\mathrm{me1} \mathrm{F}\end{array}$ & $\begin{array}{l}\mathrm{H} 3 \mathrm{~K}_{\mathrm{C}} 27 \\
\mathrm{me1} \mathrm{R}\end{array}$ & $\begin{array}{l}\mathrm{H} 3 \mathrm{~K}_{\mathrm{C}} 27 \\
\mathrm{me} 2 \mathrm{~F}\end{array}$ & $\begin{array}{l}\mathrm{H} 3 K_{\mathrm{c}} 27 \\
\mathrm{me2} \_\mathrm{R}\end{array}$ & $\begin{array}{l}\mathrm{H} 3 \mathrm{~K}_{\mathrm{c}} 27 \\
\text { e3_F }\end{array}$ & $\begin{array}{l}\mathrm{H} 3 \mathrm{~K}_{\mathrm{C}} 27 \\
\mathrm{me3} \text {.R }\end{array}$ & $\begin{array}{c}\text { HзК9m } \\
\text { e1_F }\end{array}$ & $\begin{array}{c}\text { HзК9m } \\
\text { e1_R }\end{array}$ & $\begin{array}{c}\text { H3K9m } \\
\text { e2_F }\end{array}$ & $\begin{array}{c}\text { H3K9m } \\
\text { e2_R }\end{array}$ & $\begin{array}{c}\text { HзK9m } \\
\text { e3_F }\end{array}$ & $\begin{array}{c}\text { НзК9m } \\
\text { e3_R }\end{array}$ & $\begin{array}{c}\text { HзK9m } \\
\text { e3|meC } \\
\text { pG_F }\end{array}$ & $\begin{array}{c}\text { HзK9m } \\
\text { e3|meC } \\
\text { pG_R }\end{array}$ & {$\left[\begin{array}{c}\mathrm{H} 3 \mathrm{~K} 9 \mathrm{~m} \\
\mathrm{e} \mid \mathrm{H} 4 \mathrm{~K} \\
20 \mathrm{me} \\
\mathrm{F}\end{array}\right.$} & $\begin{array}{c}\mathrm{H} 3 \mathrm{~K} 9 \mathrm{~m} \\
\mathrm{e} \mid \mathrm{H} 4 \mathrm{~K} \\
20 \mathrm{me} 3 \\
\mathrm{R}\end{array}$ & $\underset{\mathrm{F}}{\mathrm{meCp}}$ & $\underset{R}{\operatorname{meCpG}}$ & $\begin{array}{c}\text { H4R3m } \\
\text { e2_F }\end{array}$ & $\begin{array}{r}\text { H4R3m } \\
\text { e2_R }\end{array}$ \\
\hline TPT1 & Q5WOH4 & $\mathrm{NaN}$ & 0.16 & $\mathrm{NaN}$ & $\mathrm{NaN}$ & $\mathrm{NaN}$ & $\mathrm{NaN}$ & $\mathrm{NaN}$ & $\mathrm{NaN}$ & $\mathrm{NaN}$ & $\mathrm{NaN}$ & $\mathrm{NaN}$ & $\mathrm{NaN}$ & $\mathrm{NaN}$ & $\mathrm{NaN}$ & $\mathrm{NaN}$ & $\mathrm{NaN}$ & $\mathrm{NaN}$ & $\mathrm{NaN}$ & 0.33 & $\mathrm{NaN}$ & $\mathrm{NaN}$ & $\mathrm{NaN}$ & 0.83 & $\mathrm{NaN}$ & $\mathrm{NaN}$ & $\mathrm{NaN}$ \\
\hline$\pi / 1$ & 043156 & 1.08 & 0.75 & 0.48 & 0.62 & 0.87 & 0.50 & 1.27 & 0.46 & 0.60 & 0.79 & 0.67 & $\mathrm{NaN}$ & 0.89 & 0.82 & 0.96 & 0.22 & 1.26 & 0.74 & 1.89 & 0.32 & 0.68 & 1.06 & $\mathrm{NaN}$ & $\mathrm{NaN}$ & 1.38 & 0.52 \\
\hline TWF2 & \begin{tabular}{|l|l|} 
D6RG15 \\
\end{tabular} & $\mathrm{NaN}$ & 3.42 & $\mathrm{NaN}$ & $\mathrm{NaN}$ & $\mathrm{NaN}$ & $\mathrm{NaN}$ & $\mathrm{NaN}$ & $\mathrm{NaN}$ & $\mathrm{NaN}$ & $\mathrm{NaN}$ & $\mathrm{NaN}$ & $\mathrm{NaN}$ & $\mathrm{NaN}$ & $\mathrm{NaN}$ & $\mathrm{NaN}$ & $\mathrm{NaN}$ & $\mathrm{NaN}$ & $\mathrm{NaN}$ & $\mathrm{NaN}$ & $\mathrm{NaN}$ & $\mathrm{NaN}$ & $\mathrm{NaN}$ & $\mathrm{NaN}$ & $\mathrm{NaN}$ & $\mathrm{NaN}$ & $\mathrm{NaN}$ \\
\hline TXLNA & P40222 & $\mathrm{NaN}$ & $\mathrm{NaN}$ & $\mathrm{NaN}$ & $\mathrm{NaN}$ & 5.37 & $\mathrm{NaN}$ & $\mathrm{NaN}$ & $\mathrm{NaN}$ & $\mathrm{NaN}$ & $\mathrm{NaN}$ & $\mathrm{NaN}$ & $\mathrm{NaN}$ & $\mathrm{NaN}$ & $\mathrm{NaN}$ & $\mathrm{NaN}$ & $\mathrm{NaN}$ & $\mathrm{NaN}$ & $\mathrm{NaN}$ & $\mathrm{NaN}$ & $\mathrm{NaN}$ & $\mathrm{NaN}$ & $\mathrm{NaN}$ & $\mathrm{NaN}$ & $\mathrm{NaN}$ & $\mathrm{NaN}$ & $\mathrm{NaN}$ \\
\hline TXLNG & Q9NUQ3 & $\mathrm{NaN}$ & 0.20 & $\mathrm{NaN}$ & $\mathrm{NaN}$ & 4.14 & $\mathrm{NaN}$ & $\mathrm{NaN}$ & $\mathrm{NaN}$ & $\mathrm{NaN}$ & $\mathrm{NaN}$ & $\mathrm{NaN}$ & $\mathrm{NaN}$ & $\mathrm{NaN}$ & $\mathrm{NaN}$ & $\mathrm{NaN}$ & $\mathrm{NaN}$ & $\mathrm{NaN}$ & $\mathrm{NaN}$ & NaN & $\mathrm{NaN}$ & $\mathrm{NaN}$ & $\mathrm{NaN}$ & $\mathrm{NaN}$ & $\mathrm{NaN}$ & $\mathrm{NaN}$ & $\mathrm{NaN}$ \\
\hline TYMS & Q53Y97 & 0.98 & 1.14 & 1.27 & 1.33 & 1.11 & 0.91 & $\mathrm{NaN}$ & $\mathrm{NaN}$ & $\mathrm{NaN}$ & $\mathrm{NaN}$ & $\mathrm{NaN}$ & 2.08 & 0.97 & 0.62 & 0.77 & 0.15 & $\mathrm{NaN}$ & 1.20 & $\mathrm{NaN}$ & $\mathrm{NaN}$ & 0.99 & 1.29 & $\mathrm{NaN}$ & $\mathrm{NaN}$ & $\mathrm{NaN}$ & $\mathrm{NaN}$ \\
\hline UBA5 & Q9G GZ79 & 0.72 & 0.88 & 0.60 & 0.83 & 1.15 & 0.75 & 1.60 & 0.51 & 0.62 & 0.83 & 0.50 & 0.51 & 1.07 & 0.74 & 0.69 & 0.37 & 1.45 & 1.02 & NaN & $\mathrm{NaN}$ & 0.56 & 1.31 & $\mathrm{NaN}$ & $\mathrm{NaN}$ & 1.97 & 0.60 \\
\hline UBE2D2 & P62837 & $\mathrm{NaN}$ & $\mathrm{NaN}$ & $\mathrm{NaN}$ & $\mathrm{NaN}$ & 1.04 & 0.60 & 0.23 & $\mathrm{NaN}$ & $\mathrm{NaN}$ & $\mathrm{NaN}$ & $\mathrm{NaN}$ & $\mathrm{NaN}$ & $\mathrm{NaN}$ & $\mathrm{NaN}$ & $\mathrm{NaN}$ & $\mathrm{NaN}$ & 1.14 & 0.45 & 11.11 & 0.03 & $\mathrm{NaN}$ & $\mathrm{NaN}$ & 9.02 & 0.16 & $\mathrm{NaN}$ & $\mathrm{NaN}$ \\
\hline UBL4A & P11441 & 2.03 & 0.41 & $\mathrm{NaN}$ & $\mathrm{NaN}$ & 1.01 & 0.45 & 1.24 & $\mathrm{NaN}$ & 1.44 & 0.67 & 0.63 & $\mathrm{NaN}$ & 1.91 & 0.67 & 1.08 & 0.14 & 1.03 & 0.54 & $\mathrm{NaN}$ & $\mathrm{NaN}$ & 0.64 & 1.01 & $\mathrm{NaN}$ & $\mathrm{NaN}$ & 1.73 & 0.50 \\
\hline UBTF & E9PKP7 & 0.75 & 0.75 & 0.87 & 1.20 & 1.22 & 1.09 & 0.92 & 1.43 & 1.36 & 1.24 & 0.73 & 2.26 & $\mathrm{NaN}$ & $\mathrm{NaN}$ & 1.44 & 0.76 & 1.41 & 0.80 & 0.14 & 4.57 & 0.45 & 2.45 & $\mathrm{NaN}$ & $\mathrm{NaN}$ & $\mathrm{NaN}$ & $\mathrm{NaN}$ \\
\hline UBTF & P17480 & 0.91 & 0.87 & 0.72 & 1.13 & 1.14 & 1.09 & 0.95 & 1.23 & 1.21 & 1.03 & 0.86 & 1.62 & 1.04 & 1.17 & 1.57 & 1.36 & 1.22 & 0.73 & 0.23 & 4.10 & 0.50 & 2.12 & 1.36 & 0.79 & 0.46 & 1.97 \\
\hline UCHL5 & Q9Y5K5 & $\mathrm{NaN}$ & $\mathrm{NaN}$ & $\mathrm{NaN}$ & $\mathrm{NaN}$ & $\mathrm{NaN}$ & $\mathrm{NaN}$ & $\mathrm{NaN}$ & $\mathrm{NaN}$ & 0.78 & 2.69 & 1.50 & 1.34 & $\mathrm{NaN}$ & $\mathrm{NaN}$ & $\mathrm{NaN}$ & $\mathrm{NaN}$ & $\mathrm{NaN}$ & $\mathrm{NaN}$ & 4.50 & $\mathrm{NaN}$ & 1.96 & 0.83 & $\mathrm{NaN}$ & $\mathrm{NaN}$ & $\mathrm{NaN}$ & $\mathrm{NaN}$ \\
\hline UCHL5 & Q9Y5K5-2 & 0.81 & 0.39 & 2.42 & 0.62 & 0.75 & 1.69 & 1.49 & 0.86 & 0.67 & 2.69 & 1.43 & 1.29 & 0.47 & 3.50 & 0.34 & 1.84 & 0.54 & 2.42 & 9.85 & 0.11 & 2.90 & 0.57 & 1.31 & 2.61 & 4.16 & 0.32 \\
\hline UHRF1 & Q & 0.19 & 6.56 & 0.85 & 0.97 & 1.41 & 1.03 & 0.68 & 1.07 & 1.51 & 0.69 & 1.75 & 0.69 & 3.45 & 0.34 & 16.08 & 0.06 & 3.72 & 0.26 & 8.81 & 0.01 & 4.06 & 0.25 & 31.17 & 0.02 & 1.21 & 1.00 \\
\hline UHRF2 & Q96PU4 & $\mathrm{NaN}$ & $\mathrm{NaN}$ & $\mathrm{NaN}$ & $\mathrm{NaN}$ & 3.81 & & $\mathrm{NaN}$ & $\mathrm{NaN}$ & $\mathrm{NaN}$ & $\mathrm{NaN}$ & $\mathrm{NaN}$ & $\mathrm{NaN}$ & $\mathrm{NaN}$ & $\mathrm{NaN}$ & 10.59 & $\mathrm{NaN}$ & 16.02 & 0.16 & 8.83 & 0.14 & 10.44 & 0.19 & 5.97 & 0.06 & $\mathrm{NaN}$ & $\mathrm{NaN}$ \\
\hline UIMC1 & F8VQY2 & $\mathrm{NaN}$ & 0.27 & $\mathrm{NaN}$ & 1.96 & 0.59 & 1.10 & $\mathrm{NaN}$ & $\mathrm{NaN}$ & 1.08 & 0.40 & 0.83 & 0.57 & $\mathrm{NaN}$ & $\mathrm{NaN}$ & $\mathrm{NaN}$ & $\mathrm{NaN}$ & 0.92 & 0.68 & 0.38 & 0.40 & 0.29 & $\mathrm{NaN}$ & 0.52 & 0.45 & $\mathrm{NaN}$ & $\mathrm{NaN}$ \\
\hline USF1 & B1AQP1 & 1.03 & 1.35 & 1.98 & 0.83 & 1.22 & 1.48 & 1.68 & 1.11 & 1.13 & 1.73 & 1.17 & 1.34 & 0.59 & 2.54 & 0.95 & 6.52 & 1.20 & 1.30 & 5.16 & 0.33 & 1.23 & 1.29 & 0.43 & 6.11 & 3.28 & 0.62 \\
\hline USF2 & Q15853 & 0.82 & 1.56 & 2.21 & 0.83 & 1.32 & 1.61 & 1.68 & 1.21 & 1.25 & 1.72 & 1.31 & 1.39 & 0.57 & 2.84 & 0.81 & 4.45 & 1.19 & 1.35 & 5.42 & 0.20 & 1.34 & 1.34 & 0.39 & 4.76 & 3.67 & 0.57 \\
\hline USF2 & Q15853-3 & $\mathrm{NaN}$ & $\mathrm{NaN}$ & & $\mathrm{NaN}$ & $\mathrm{NaN}$ & $\mathrm{NaN}$ & $\mathrm{NaN}$ & $\mathrm{NaN}$ & & $\mathrm{NaN}$ & NaN & & $\mathrm{NaN}$ & $\mathrm{NaN}$ & $\mathrm{NaN}$ & $\mathrm{NaN}$ & NaP & & $\mathrm{NaN}$ & & & $\mathrm{NaN}$ & & & $\mathrm{NaN}$ & $\mathrm{NaN}$ \\
\hline USP1 & B2R636 & 0.40 & $\mathrm{NaN}$ & $\mathrm{NaN}$ & $\mathrm{NaN}$ & $\mathrm{NaN}$ & $\mathrm{NaN}$ & $\mathrm{NaN}$ & $\mathrm{NaN}$ & $\mathrm{NaN}$ & $\mathrm{NaN}$ & $\mathrm{NaN}$ & $\mathrm{NaN}$ & 1.07 & 0.59 & $\mathrm{NaN}$ & 0.28 & $\mathrm{NaN}$ & $\mathrm{NaN}$ & $\mathrm{NaN}$ & $\mathrm{NaN}$ & $\mathrm{NaN}$ & $\mathrm{NaN}$ & $\mathrm{NaN}$ & $\mathrm{NaN}$ & $\mathrm{NaN}$ & $\mathrm{NaN}$ \\
\hline USP3 & Q9Y614 & 0.64 & 0.89 & 2.69 & 0.64 & 6.14 & 0.19 & $\mathrm{NaN}$ & $\mathrm{NaN}$ & $\mathrm{NaN}$ & $\mathrm{NaN}$ & $\mathrm{NaN}$ & $\mathrm{NaN}$ & $\mathrm{NaN}$ & 0.59 & 0.86 & 0.86 & 6.00 & 0.11 & 0.11 & 3.69 & 0.33 & 2.71 & $\mathrm{NaN}$ & $\mathrm{NaN}$ & 1.21 & 0.54 \\
\hline USP7 & Q93009 & 0.29 & 1.82 & 2.62 & 0.23 & 1.97 & 0.65 & 0.61 & 1.12 & 0.89 & 0.85 & 1.02 & 1.13 & 1.70 & 0.72 & 3.42 & 0.25 & 3.36 & 0.20 & 30.30 & 0.01 & 2.30 & 0.45 & 20.45 & 0.06 & 0.33 & 1.88 \\
\hline VRK3 & Q8IV63 & $\mathrm{NaN}$ & 0.22 & $\mathrm{NaN}$ & 1.47 & $\mathrm{NaN}$ & $\mathrm{NaN}$ & 1.08 & 0.64 & 0.46 & 1.33 & $\mathrm{NaN}$ & 1.09 & $\mathrm{NaN}$ & $\mathrm{NaN}$ & 0.49 & $\mathrm{NaN}$ & 0.63 & 1.07 & 0.52 & 1.08 & 0.95 & 0.70 & 0.59 & 0.97 & 0.49 & $\mathrm{NaN}$ \\
\hline WA9 & B & 0.91 & 0.48 & 0.66 & 0.86 & 0.74 & 0.77 & 0.61 & $\mathrm{NaN}$ & & 0.64 & 0.90 & 0.79 & 1.03 & 1.47 & & & & 0.88 & 4.74 & & & 0.54 & 1.06 & 0.89 & $\mathrm{NaN}$ & 0.35 \\
\hline WARS & P23381 & $\mathrm{NaN}$ & 0.11 & $\mathrm{NaN}$ & $\mathrm{NaN}$ & $\mathrm{NaN}$ & $\mathrm{NaN}$ & $\mathrm{NaN}$ & $\mathrm{NaN}$ & $\mathrm{NaN}$ & $\mathrm{NaN}$ & $\mathrm{NaN}$ & 2.37 & $\mathrm{NaN}$ & $\mathrm{NaN}$ & 0.38 & $\mathrm{NaN}$ & $\mathrm{NaN}$ & $\mathrm{NaN}$ & 0.35 & $\mathrm{NaN}$ & $\mathrm{NaN}$ & $\mathrm{NaN}$ & $\mathrm{NaN}$ & $\mathrm{NaN}$ & $\mathrm{NaN}$ & $\mathrm{NaN}$ \\
\hline WDR5 & P61964 & 0.28 & 2.13 & 1.47 & 1.11 & 1.25 & 1.37 & 1.83 & 0.98 & 1.60 & 1.23 & 1.50 & 1.19 & 0.62 & 2.71 & 0.58 & 1.12 & 1.43 & 1.11 & 1.14 & 1.14 & 1.48 & 1.37 & 1.69 & 1.79 & 1.59 & 1.00 \\
\hline WDR5B & Q86VZ2 & $\mathrm{NaN}$ & $\mathrm{NaN}$ & $\mathrm{NaN}$ & $\mathrm{NaN}$ & $\mathrm{NaN}$ & $\mathrm{NaN}$ & $\mathrm{NaN}$ & $\mathrm{NaN}$ & $\mathrm{NaN}$ & $\mathrm{NaN}$ & 2.06 & 0.45 & $\mathrm{NaN}$ & $\mathrm{NaN}$ & $\mathrm{NaN}$ & $\mathrm{NaN}$ & $\mathrm{NaN}$ & $\mathrm{NaN}$ & $\mathrm{NaN}$ & 0.32 & 1.65 & $\mathrm{NaN}$ & $\mathrm{NaN}$ & $\mathrm{NaN}$ & $\mathrm{NaN}$ & $\mathrm{NaN}$ \\
\hline WDR77 & & 1.47 & 0.98 & 1.1 & 0.77 & 0.77 & & & 0.72 & & 1.25 & 1.08 & & & 0.45 & & & & & & & & & & & $\mathrm{NaN}$ & $\mathrm{NaN}$ \\
\hline WHSC1 & 096028 & $\begin{array}{l}1.41 \\
\mathrm{NaN}\end{array}$ & $\mathrm{NaN}$ & $\mathrm{NaN}$ & $\mathrm{NaN}$ & $\mathrm{NaN}$ & $\mathrm{NaN}$ & $\mathrm{NaN}$ & $\mathrm{NaN}$ & $\mathrm{NaN}$ & $\mathrm{NaN}$ & $\mathrm{NaN}$ & 0.49 & $\mathrm{NaN}$ & $\mathrm{NaN}$ & NaN & $\begin{array}{l}.04 \\
\mathrm{NaN}\end{array}$ & $\mathrm{NaN}$ & $\mathrm{NaN}$ & $\mathrm{NaN}$ & $\mathrm{NaN}$ & $\begin{array}{l}0.04 \\
0.75\end{array}$ & $\mathrm{NaN}$ & $\mathrm{NaN}$ & $\begin{array}{l}\mathrm{NaO} \\
\mathrm{NaN}\end{array}$ & $\mathrm{NaN}$ & $\mathrm{NaN}$ \\
\hline WHSC1 & |096028-3 & 0.41 & 0.86 & 0.38 & 0.53 & 0.44 & 0.58 & 0.66 & 0.55 & 0.82 & 0.32 & 0.54 & 0.40 & 0.38 & 0.50 & $\mathrm{NaN}$ & $\mathrm{NaN}$ & 0.48 & 0.48 & 3.80 & 0.23 & 0.54 & 0.40 & 3.54 & 0.36 & 0.43 & 0.35 \\
\hline WHSC1 & O96028-5 & $\mathrm{NaN}$ & $\mathrm{NaN}$ & $\mathrm{NaN}$ & $\mathrm{NaN}$ & 0.55 & 0.9 & $\mathrm{NaN}$ & $\mathrm{NaN}$ & $\mathrm{NaN}$ & $\mathrm{NaN}$ & $\mathrm{NaN}$ & $\mathrm{NaN}$ & $\mathrm{NaN}$ & $\mathrm{NaN}$ & $\mathrm{NaN}$ & $\mathrm{NaN}$ & 0.40 & 0.42 & $\mathrm{NaN}$ & $\mathrm{NaN}$ & 0.47 & 0.37 & $\mathrm{NaN}$ & 0.53 & $\mathrm{NaN}$ & $\mathrm{NaN}$ \\
\hline WHSC1 & Q05CW4 & $\mathrm{NaN}$ & & $\mathrm{NaN}$ & $\mathrm{NaN}$ & $\mathrm{N}$ & 0 & $\mathrm{NaN}$ & $\mathrm{NaN}$ & NaN & $\mathrm{NaN}$ & $\mathrm{NaN}$ & $\mathrm{NaN}$ & $\mathrm{NaN}$ & $\mathrm{NaN}$ & $\mathrm{NaN}$ & $\mathrm{NaN}$ & $\mathrm{NaN}$ & & $\mathrm{NaN}$ & $\mathrm{NaN}$ & $\mathrm{NaN}$ & $\mathrm{NaN}$ & $\mathrm{NaN}$ & $\mathrm{NaN}$ & $\mathrm{NaN}$ & $\mathrm{NaN}$ \\
\hline WHSCIL1 & Q9BZ95-4 & 0.62 & 0.68 & 0.71 & 1.05 & 1.02 & 1. & 0.89 & 1.23 & 1.17 & 1.04 & 1.09 & 0.89 & $\mathrm{NaN}$ & $\mathrm{NaN}$ & $\mathrm{NaN}$ & 0.18 & 2.09 & 0.33 & 17.75 & 0.19 & 2.46 & 0.32 & 3.75 & 0.70 & 1.64 & 0.68 \\
\hline WIZ & B7ZM82 & 0.71 & 0.76 & 0.8 & 0.84 & 0.57 & 1.30 & 0.71 & 0.90 & 0.76 & 1.02 & 0.96 & 0.82 & 1.52 & 0.70 & 0.83 & 0.32 & 0.34 & 1.57 & 6.27 & 0.17 & 0.97 & 0.68 & 1.35 & 1.21 & 1.74 & 0.26 \\
\hline WIZ & B9EGQ5 & $\mathrm{NaN}$ & $\mathrm{NaN}$ & $\mathrm{Nal}$ & $\mathrm{NaN}$ & $\mathrm{NaN}$ & 1.17 & $\mathrm{NaN}$ & $\mathrm{NaN}$ & $\mathrm{Na}$ & $\mathrm{NaN}$ & 1.16 & 0.82 & $\mathrm{NaN}$ & $\mathrm{NaN}$ & $\mathrm{NaN}$ & $\mathrm{NaN}$ & $\mathrm{NaN}$ & $\mathrm{NaN}$ & $\mathrm{NaN}$ & $\mathrm{NaN}$ & 0.92 & 0.74 & $\mathrm{NaN}$ & $\mathrm{NaN}$ & $\mathrm{NaN}$ & $\mathrm{NaN}$ \\
\hline WIZ & 095785 & $\mathrm{NaN}$ & $\mathrm{NaN}$ & $\mathrm{Na}$ & $\mathrm{NaN}$ & $\mathrm{NaN}$ & $\mathrm{N}$ & $\mathrm{NaN}$ & $\mathrm{NaN}$ & $\mathrm{NaN}$ & $\mathrm{NaN}$ & $\mathrm{NaN}$ & $\mathrm{NaN}$ & $\mathrm{NaN}$ & $\mathrm{NaN}$ & $\mathrm{NaN}$ & $\mathrm{NaN}$ & $\mathrm{NaN}$ & $\mathrm{NaN}$ & $\mathrm{NaN}$ & $\mathrm{NaN}$ & $\mathrm{NaN}$ & 1.30 & $\mathrm{NaN}$ & $\mathrm{NaN}$ & $\mathrm{NaN}$ & $\mathrm{NaN}$ \\
\hline XPO1 & & 1.14 & 1.5 & 0. & 1.0 & & & 1.49 & 0.4 & & 0.9 & 0.5 & 0.78 & 1.27 & 0. & 0.92 & & 1.7 & 0.88 & 1.22 & 0.42 & 0.62 & 1.50 & $\mathrm{NaN}$ & 1.09 & 2.17 & 0.58 \\
\hline XPO5 & 3K5YY7 & 1.09 & 0. & 0.9 & 1.08 & & 0. & 1.17 & 0.63 & 0. & 1.0 & 0.48 & 1. & 0.93 & 1.08 & 0.9 & 0. & 1.3 & 1.02 & 0.93 & 0.40 & 0.68 & 1.33 & 0.77 & $\mathrm{NaN}$ & 1.72 & 0.54 \\
\hline YKT6 & Q9UESO & 0.77 & & 0. & 1.0 & & 0. & 1.06 & 0. & & 0.7 & 0.3 & & 0.5 & 0.50 & & 0. & 1.1 & 1.63 & $\mathrm{NaN}$ & $\mathrm{Na}$ & 0.35 & 1.08 & $\mathrm{NaN}$ & $\mathrm{NaN}$ & 1.60 & 0.55 \\
\hline YOL & Q5WQ6-2 & $\mathrm{NaN}$ & $\mathrm{N}$ & $\mathrm{N}$ & $\mathrm{NaN}$ & & & 0.11 & $\mathrm{NaN}$ & & $\mathrm{Na}$ & $\mathrm{NaN}$ & & $\mathrm{NaN}$ & $\mathrm{Nal}$ & $\mathrm{NaN}$ & $\mathrm{NaN}$ & $\mathrm{NaN}$ & $\mathrm{NaN}$ & $\mathrm{NaN}$ & $\mathrm{NaN}$ & $\mathrm{NaN}$ & $\mathrm{NaN}$ & $\mathrm{NaN}$ & $\mathrm{NaN}$ & $\mathrm{NaN}$ & $\mathrm{NaN}$ \\
\hline ZBED1 & 096006 & 1.27 & & & $\mathrm{NaN}$ & & 0. & & & $\mathrm{NaI}$ & & 0.54 & & & $\mathrm{Nal}$ & 1.12 & & 0.44 & & & NaN & 0.25 & $\mathrm{NaN}$ & $\mathrm{NaN}$ & $\mathrm{NaN}$ & $\mathrm{NaN}$ & $\mathrm{NaN}$ \\
\hline ZBED6 & \begin{tabular}{|l} 
P86452 \\
\end{tabular} & $\mathrm{NaN}$ & 0.2 & 0.4 & $\mathrm{Na}$ & 0. & 1.07 & 0.46 & $\mathrm{NaN}$ & 0. & 0.5 & 0.88 & & 0.46 & $\mathrm{NaN}$ & $\mathrm{Na}$ & & 0.31 & & $\mathrm{NaN}$ & $\mathrm{NaN}$ & & 0. & & $\mathrm{NaN}$ & 1.27 & 0.38 \\
\hline ZBTB1 & & 0.44 & 1.2 & 0 & 0.5 & & 1.29 & 0.44 & $\mathrm{NaN}$ & & 0.8 & 0.6 & & 0.35 & 1.92 & & 1.8 & 0.2 & 1.0 & 1.90 & 0.31 & 0.76 & 0.52 & 0.17 & 4.31 & 2.38 & 0.29 \\
\hline ZBTE & & & & & $\mathrm{Na}$ & & & $\mathrm{NaN}$ & $\mathrm{NaN}$ & & $\mathrm{Na}$ & $\mathrm{NaN}$ & $\mathrm{NaN}$ & $\mathrm{NaN}$ & $\mathrm{NaN}$ & & 0. & $\mathrm{Na}$ & 0.24 & 11. & 0. & $\mathrm{NaN}$ & $\mathrm{NaN}$ & 6.37 & 0.09 & $\mathrm{NaN}$ & $\mathrm{NaN}$ \\
\hline ZBTB14 & 043829 & 0.51 & 0.91 & 1.64 & 0.65 & 1.12 & 1.03 & 0.96 & 0.66 & 0.83 & 1.24 & 1.00 & 0.80 & 0.32 & 3.20 & 0.33 & 2.99 & 0.88 & 0.78 & 5.38 & 0.18 & 1.05 & 0.66 & 0.31 & 6.27 & 0.86 & 0.50 \\
\hline ZBTB33 & & 0.79 & 0.78 & $\mathrm{NaN}$ & 1.01 & & $\mathrm{NaN}$ & $\mathrm{NaN}$ & $\mathrm{NaN}$ & & $\mathrm{NaN}$ & 0.63 & $\mathrm{NaN}$ & 0.94 & 0.82 & & & 1.69 & 1.06 & & & & & 24.10 & & & $\mathrm{NaN}$ \\
\hline ZBTB? & & $\mathrm{Na}$ & $\mathrm{N}$ & 1.4 & 1.00 & & 2. & 1.15 & 1. & 1. & 1.87 & 1.0 & & $\mathrm{NaN}$ & $\mathrm{N}$ & $\mathrm{N}$ & $\mathrm{NaN}$ & 0.7 & 3.91 & NaN & $\mathrm{Na}$ & 1.29 & 1.36 & $\mathrm{NaN}$ & $\mathrm{NaN}$ & 1.53 & 0.89 \\
\hline ZBT & & $\mathrm{NaN}$ & $\mathrm{NaN}$ & $\mathrm{Na}$ & $\mathrm{NaN}$ & $\mathrm{NaN}$ & $\mathrm{Nal}$ & $\mathrm{NaN}$ & $\mathrm{NaN}$ & $\mathrm{NaN}$ & $\mathrm{NaN}$ & $\mathrm{NaN}$ & $\mathrm{NaN}$ & $\mathrm{NaN}$ & $\mathrm{Nal}$ & $\mathrm{NaN}$ & $\mathrm{NaN}$ & $\mathrm{NaN}$ & $\mathrm{NaN}$ & 3.19 & 0.20 & $\mathrm{NaN}$ & $\mathrm{NaN}$ & 3.21 & 0.25 & $\mathrm{NaN}$ & $\mathrm{NaN}$ \\
\hline ZBTB43 & 043298 & $\mathrm{NaN}$ & 1.50 & 1.34 & 0.62 & 2.07 & 1.33 & $\mathrm{NaN}$ & $\mathrm{NaN}$ & $\mathrm{NaN}$ & $\mathrm{NaN}$ & 2.29 & 0.90 & $\mathrm{NaN}$ & $\mathrm{NaN}$ & 0.09 & 5.84 & 0.95 & 1.63 & 11.00 & 0.16 & 2.30 & 1.00 & 1.07 & 1.06 & $\mathrm{NaN}$ & $\mathrm{NaN}$ \\
\hline ZBTB44 & H7BY22 & $\mathrm{NaN}$ & $\mathrm{NaN}$ & $\mathrm{NaN}$ & $\mathrm{NaN}$ & 0.68 & 1.68 & & $\mathrm{NaN}$ & NaN & $\mathrm{NaN}$ & 0.65 & & & $\mathrm{NaN}$ & & & 0.47 & & 2.55 & 0.51 & & & & 0.71 & $\mathrm{NaN}$ & $\mathrm{NaN}$ \\
\hline & & 0.5 & 0. & 0. & 1. & & 0. & & & 1. & 0. & 0. & & 0.25 & 0. & & & 0 & & & & & & $\mathrm{NaN}$ & & & 0.37 \\
\hline $\mathrm{ZC} 3 \mathrm{H}$ & & 4.78 & 0. & 0.43 & $\mathrm{NaN}$ & 1.25 & 0.54 & 0.64 & $\mathrm{NaN}$ & 1.99 & 0.35 & 1.32 & 0.98 & 1.34 & 0.80 & 0.51 & 0.23 & 1.27 & 0.50 & $\mathrm{NaN}$ & $\mathrm{NaN}$ & 0.62 & 1.07 & $\mathrm{NaN}$ & $\mathrm{NaN}$ & 0.55 & 0.51 \\
\hline ZC3H14 & B4DXU8 & 11.51 & $\mathrm{NaN}$ & $\mathrm{NaN}$ & $\mathrm{NaN}$ & $\mathrm{NaN}$ & $\mathrm{NaN}$ & $\mathrm{NaN}$ & $\mathrm{NaN}$ & $\mathrm{NaN}$ & $\mathrm{NaN}$ & $\mathrm{NaN}$ & $\mathrm{NaN}$ & $\mathrm{NaN}$ & $\mathrm{NaN}$ & $\mathrm{NaN}$ & $\mathrm{NaN}$ & $\mathrm{NaN}$ & $\mathrm{NaN}$ & $\mathrm{NaN}$ & $\mathrm{NaN}$ & $\mathrm{NaN}$ & $\mathrm{NaN}$ & $\mathrm{NaN}$ & $\mathrm{NaN}$ & $\mathrm{NaN}$ & $\mathrm{NaN}$ \\
\hline ZC3H14 & G3V4R5 & $\mathrm{NaN}$ & & $\mathrm{NaN}$ & $\mathrm{NaN}$ & $\mathrm{NaN}$ & $\mathrm{NaN}$ & & $\mathrm{NaN}$ & $\mathrm{NaN}$ & $\mathrm{NaN}$ & $\mathrm{NaN}$ & $\mathrm{NaN}$ & $\mathrm{NaN}$ & $\mathrm{NaN}$ & $\mathrm{NaN}$ & $\mathrm{NaN}$ & $\mathrm{NaN}$ & $\mathrm{NaN}$ & $\mathrm{NaN}$ & $\mathrm{NaN}$ & $\mathrm{NaN}$ & $\mathrm{NaN}$ & $\mathrm{NaN}$ & $\mathrm{NaN}$ & $\mathrm{NaN}$ & $\mathrm{NaN}$ \\
\hline tevint & Q6PJT7-5 & 6.20 & 1.01 & 0.29 & 1.35 & 0.74 & 0.49 & $\mathrm{NaN}$ & & 1.5 & 0.2 & 0. & & & 0.98 & 1.61 & 0. & 0.94 & 0.42 & 0.16 & & & 0.97 & $\mathrm{NaN}$ & $\mathrm{NaN}$ & 0.43 & 0.44 \\
\hline ZC3HAV1 & A8K9U6 & 1.23 & 1.45 & 0.96 & $\mathrm{NaN}$ & 0.78 & 0.61 & $\mathrm{NaN}$ & $\mathrm{NaN}$ & $\mathrm{NaN}$ & 0.50 & 0.82 & 0.96 & $\mathrm{NaN}$ & $\mathrm{NaN}$ & 1.16 & 1.09 & 1.59 & 0.28 & $\mathrm{NaN}$ & $\mathrm{NaN}$ & 1.03 & 0.49 & $\mathrm{NaN}$ & $\mathrm{NaN}$ & $\mathrm{NaN}$ & $\mathrm{NaN}$ \\
\hline $\mathrm{ZCCHC} 10$ & B3KVL5 & $\mathrm{NaN}$ & $\mathrm{NaN}$ & $\mathrm{NaN}$ & $\mathrm{NaN}$ & $\mathrm{NaN}$ & $\mathrm{NaN}$ & $\mathrm{NaN}$ & $\mathrm{NaN}$ & 2.96 & $\mathrm{NaN}$ & $\mathrm{NaN}$ & $\mathrm{NaN}$ & $\mathrm{NaN}$ & $\mathrm{NaN}$ & 1.37 & $\mathrm{NaN}$ & $\mathrm{NaN}$ & $\mathrm{NaN}$ & 0.42 & 1.42 & $\mathrm{NaN}$ & $\mathrm{NaN}$ & 0.75 & 0.78 & $\mathrm{NaN}$ & 2.93 \\
\hline ZGPAT & HoUl99 & 11.06 & $\mathrm{NaN}$ & $\mathrm{NaN}$ & $\mathrm{NaN}$ & 0.66 & 0.69 & $\mathrm{NaN}$ & $\mathrm{NaN}$ & $\mathrm{NaN}$ & $\mathrm{NaN}$ & $\mathrm{NaN}$ & $\mathrm{NaN}$ & $\mathrm{NaN}$ & $\mathrm{NaN}$ & 6.30 & $\mathrm{NaN}$ & 0.66 & 0.54 & $\mathrm{NaN}$ & $\mathrm{NaN}$ & 0.15 & 0.86 & $\mathrm{NaN}$ & $\mathrm{NaN}$ & $\mathrm{NaN}$ & $\mathrm{NaN}$ \\
\hline
\end{tabular}




\begin{tabular}{|c|c|c|c|c|c|c|c|c|c|c|c|c|c|c|c|c|c|c|c|c|c|c|c|c|c|c|c|}
\hline Gene names & Protein IDs & $\begin{array}{l}\mathrm{H} 3 \Delta 1- \\
20 \_\mathrm{F}\end{array}$ & $\begin{array}{l}\mathrm{H} 3 \Delta 1- \\
20 \_\mathrm{R}\end{array}$ & $\begin{array}{l}\text { H4K20 } \\
\text { me1_F }\end{array}$ & $\begin{array}{l}\text { H4K20 } \\
\text { me1_R }\end{array}$ & $\begin{array}{l}\text { H4K20 } \\
\text { me3_F }\end{array}$ & $\begin{array}{l}\text { H4K20 } \\
\text { me3_R }\end{array}$ & $\begin{array}{c}\mathrm{H}_{3} \mathrm{~K}_{\mathrm{C}} 27 \\
\mathrm{me1} \mathrm{F}\end{array}$ & $\begin{array}{l}\mathrm{H}_{3} \mathrm{~K}_{\mathrm{C}} 27 \\
\text { me1_R }\end{array}$ & $\begin{array}{l}\mathrm{H} 3 \mathrm{~K}_{\mathrm{c}} 27 \\
\text { me2_F }\end{array}$ & $\begin{array}{c}\mathrm{H}_{3} \mathrm{~K}_{\mathrm{C}} 27 \\
\mathrm{me2} \_\mathrm{R}\end{array}$ & $\begin{array}{l}\mathrm{H} 3 \mathrm{~K}_{\mathrm{c}} 27 \\
\mathrm{me3} 3 \mathrm{~F}\end{array}$ & $\mid \begin{array}{l}\mathrm{H}_{3 \mathrm{~K}}^{\mathrm{C}} 27 \\
\mathrm{me} \_\mathrm{R}\end{array}$ & $\begin{array}{c}\mathrm{H} 3 \mathrm{~K} 9 \mathrm{~m} \\
\text { e1_F }\end{array}$ & $\mid \begin{array}{c}\mathrm{H} 3 \mathrm{~K} 9 \mathrm{~m} \\
\text { e1_R }\end{array}$ & $\begin{array}{c}\text { H3K9m } \\
\text { e2_F }\end{array}$ & $\begin{array}{c}\text { H3K9m } \\
\text { e2_R }\end{array}$ & $\begin{array}{c}\text { H3K9m } \\
\text { e3_F }\end{array}$ & $\mid \begin{array}{c}\mathrm{H} 3 \mathrm{~K} 9 \mathrm{~m} \\
\text { e3_R }\end{array}$ & $\begin{array}{c}\text { HзK9m } \\
\text { e3|meC } \\
\text { pG_F }\end{array}$ & $\begin{array}{c}\text { Hзкаm } \\
\text { e3|meC } \\
\text { pG_R }\end{array}$ & 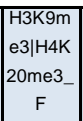 & $\begin{array}{c}\mathrm{H} 3 \mathrm{~K} 9 \mathrm{~m} \\
\mathrm{e} \mid \mathrm{H} / \mathrm{K} \\
20 \mathrm{me} 3 \\
\mathrm{R}\end{array}$ & $\underset{\mathrm{F}}{\mathrm{meCpG}}$ & $\begin{array}{c}\mathrm{meCpG} \\
\_\mathrm{R}\end{array}$ & $\begin{array}{l}\text { H4R3m } \\
\text { e2_F }\end{array}$ & $\begin{array}{c}\text { H4R3m } \\
\text { e2_R }\end{array}$ \\
\hline $\mathrm{ZHX1}$ & Q9UKY1 & 0.35 & 0.71 & 0.74 & \begin{tabular}{|l}
0.48 \\
\end{tabular} & 2.25 & 0.23 & $\mathrm{NaN}$ & $\mathrm{NaN}$ & 0.46 & 0.62 & 0.49 & 0.52 & \begin{tabular}{|l|}
$\mathrm{NaN}$ \\
\end{tabular} & \begin{tabular}{|l|}
$\mathrm{NaN}$ \\
\end{tabular} & \begin{tabular}{|l|}
0.29 \\
\end{tabular} & \begin{tabular}{|l|}
$\mathrm{NaN}$ \\
\end{tabular} & \begin{tabular}{|l|}
1.85 \\
\end{tabular} & \begin{tabular}{|l|}
0.18 \\
\end{tabular} & \begin{tabular}{|l|}
41.17 \\
\end{tabular} & 0.11 & \begin{tabular}{|l|}
0.47 \\
\end{tabular} & 0.64 & \begin{tabular}{|l|}
24.81 \\
\end{tabular} & \begin{tabular}{|l|}
0.12 \\
\end{tabular} & \begin{tabular}{|l|l}
$\mathrm{NaN}$ \\
\end{tabular} & $\mathrm{NaN}$ \\
\hline $\mathrm{ZHX2}$ & Q9Y6X8 & 0.42 & 0.57 & $\mathrm{NaN}$ & $\mathrm{NaN}$ & $\mathrm{NaN}$ & $\mathrm{NaN}$ & $\mathrm{NaN}$ & $\mathrm{NaN}$ & $\mathrm{NaN}$ & $\mathrm{NaN}$ & 0.48 & $\mathrm{NaN}$ & $\mathrm{NaN}$ & $\mathrm{NaN}$ & 0.25 & 0.56 & 0.42 & 0.71 & 33.67 & 0.12 & 0.96 & 0.34 & 26.09 & 0.09 & $\mathrm{NaN}$ & $\mathrm{NaN}$ \\
\hline $\mathrm{ZHX3}$ & A8K8Q0 & $\mathrm{NaN}$ & $\mathrm{NaN}$ & $\mathrm{NaN}$ & $\mathrm{NaN}$ & $\mathrm{NaN}$ & $\mathrm{NaN}$ & $\mathrm{NaN}$ & $\mathrm{NaN}$ & $\mathrm{NaN}$ & $\mathrm{NaN}$ & $\mathrm{NaN}$ & $\mathrm{NaN}$ & $\mathrm{NaN}$ & $\mathrm{NaN}$ & $\mathrm{NaN}$ & $\mathrm{NaN}$ & $\mathrm{NaN}$ & $\mathrm{NaN}$ & 19.41 & 0.14 & $\mathrm{NaN}$ & 0.39 & 17.43 & 0.15 & $\mathrm{NaN}$ & $\mathrm{NaN}$ \\
\hline ZKSCAN3 & Q9BRR0 & 0.52 & 0.46 & 0.53 & 0.92 & 0.62 & 0.85 & 0.67 & $\mathrm{NaN}$ & 0.60 & 0.69 & 0.68 & 0.53 & 0.53 & 0.88 & 0.53 & $\mathrm{NaN}$ & 0.55 & 0.73 & 4.22 & 0.50 & 0.59 & 0.59 & 0.84 & 0.83 & $\mathrm{NaN}$ & 0.50 \\
\hline ZMYM1 & Q5SVZ6 & 0.88 & 1.22 & 1.07 & $\mathrm{NaN}$ & 0.62 & 2.22 & 1.05 & 1.55 & 0.57 & 2.61 & 1.30 & 1.96 & NaN & 1.79 & 0.50 & 0.31 & 0.30 & 3.05 & 6.90 & 3.06 & 2.12 & 0.88 & 1.18 & 8.86 & $\mathrm{NaN}$ & $\mathrm{NaN}$ \\
\hline ZMYM2 & Q9UBW7 & 0.76 & 0.63 & $\mathrm{NaN}$ & $\mathrm{NaN}$ & 0.99 & $\mathrm{NaN}$ & $\mathrm{NaN}$ & $\mathrm{NaN}$ & 0.63 & 0.69 & 0.70 & 0.57 & $\mathrm{NaN}$ & $\mathrm{NaN}$ & 0.53 & 0.19 & 1.64 & 0.30 & 26.45 & 0.16 & 2.82 & 0.24 & 0.90 & $\mathrm{NaN}$ & $\mathrm{NaN}$ & $\mathrm{NaN}$ \\
\hline ZMYND8 & Q9ULU4-11 & $\mathrm{NaN}$ & 0.88 & 0.33 & 0.51 & 0.74 & 0.58 & $\mathrm{NaN}$ & $\mathrm{NaN}$ & 0.61 & 0.43 & 0.72 & 0.58 & 0.47 & 2.29 & 0.46 & $\mathrm{NaN}$ & 0.49 & 0.90 & 15.95 & 0.26 & 1.15 & 0.38 & 10.81 & 0.90 & $\mathrm{NaN}$ & $\mathrm{NaN}$ \\
\hline ZMYND8 & Q9ULU4-9 & $\mathrm{NaN}$ & $\mathrm{NaN}$ & $\mathrm{NaN}$ & $\mathrm{NaN}$ & $\mathrm{NaN}$ & $\mathrm{NaN}$ & $\mathrm{NaN}$ & $\mathrm{NaN}$ & $\mathrm{NaN}$ & $\mathrm{NaN}$ & NaN & $\mathrm{NaN}$ & $\mathrm{NaN}$ & $\mathrm{NaN}$ & $\mathrm{NaN}$ & $\mathrm{NaN}$ & $\mathrm{NaN}$ & $\mathrm{NaN}$ & $\mathrm{NaN}$ & $\mathrm{NaN}$ & $\mathrm{NaN}$ & $\mathrm{NaN}$ & 7.83 & NaN & $\mathrm{NaN}$ & $\mathrm{NaN}$ \\
\hline ZNF131 & P52739 & 0.44 & 1.21 & 1.17 & 0.70 & 1.12 & 1.07 & 0.96 & 0.92 & 0.84 & 1.02 & 0.97 & 1.02 & 0.58 & 1.36 & 0.48 & 3.26 & 1.03 & 0.81 & 1.63 & 0.85 & 0.92 & 0.85 & 0.59 & 2.17 & 0.91 & 0.74 \\
\hline ZNF148 & Q9UQR1 & 1.40 & 0.65 & 1.36 & 0.81 & 0.57 & 2.07 & 0.98 & 1.11 & 1.04 & 1.41 & 1.17 & 1.17 & 0.32 & 4.18 & 0.25 & 3.30 & 0.42 & 2.43 & 20.20 & 0.18 & 1.77 & 0.73 & 1.68 & 0.81 & 2.40 & 0.35 \\
\hline ZNF280D & A8K 124 & $\mathrm{NaN}$ & $\mathrm{NaN}$ & $\mathrm{NaN}$ & $\mathrm{NaN}$ & $\mathrm{NaN}$ & $\mathrm{NaN}$ & $\mathrm{NaN}$ & $\mathrm{NaN}$ & $\mathrm{NaN}$ & $\mathrm{NaN}$ & $\mathrm{NaN}$ & $\mathrm{NaN}$ & $\mathrm{NaN}$ & $\mathrm{NaN}$ & $\mathrm{NaN}$ & 0.72 & 2.82 & 0.45 & 8.04 & 0.19 & 2.19 & 0.15 & $\mathrm{NaN}$ & $\mathrm{NaN}$ & NaN & $\mathrm{NaN}$ \\
\hline ZNF282 & Q86YG2 & $\mathrm{NaN}$ & NaN & 1.77 & 0.77 & $\mathrm{NaN}$ & $\mathrm{NaN}$ & $\mathrm{NaN}$ & $\mathrm{NaN}$ & 0.69 & 2.37 & 1.12 & 1.14 & $\mathrm{NaN}$ & $\mathrm{NaN}$ & $\mathrm{NaN}$ & $\mathrm{NaN}$ & 0.44 & 3.37 & $\mathrm{NaN}$ & $\mathrm{NaN}$ & 2.22 & 0.66 & $\mathrm{NaN}$ & NaN & $\mathrm{NaN}$ & $\mathrm{NaN}$ \\
\hline ZNF354A & Q86Y64 & $\mathrm{NaN}$ & $\mathrm{NaN}$ & 0.43 & $\mathrm{NaN}$ & 0.54 & 0.92 & $\mathrm{NaN}$ & $\mathrm{NaN}$ & $\mathrm{NaN}$ & $\mathrm{NaN}$ & 0.71 & 0.62 & 0.41 & 0.88 & $\mathrm{NaN}$ & $\mathrm{NaN}$ & 0.48 & 0.64 & $\mathrm{NaN}$ & $\mathrm{NaN}$ & 0.74 & 0.56 & $\mathrm{NaN}$ & $\mathrm{NaN}$ & $\mathrm{NaN}$ & $\mathrm{NaN}$ \\
\hline ZNF444 & Q53F81 & 1.06 & 0.29 & 0.81 & 1.16 & 0.60 & 2.13 & 1.09 & 0.95 & 0.82 & 0.92 & 0.98 & 0.83 & 0.59 & 1.41 & 0.41 & 0.69 & 0.38 & 1.79 & 2.50 & 0.39 & 1.10 & 0.67 & 1.24 & 0.93 & $\mathrm{NaN}$ & $\mathrm{NaN}$ \\
\hline ZNF451 & Q4KMR5 & 1.51 & 0.13 & $\mathrm{NaN}$ & $\mathrm{NaN}$ & $\mathrm{NaN}$ & $\mathrm{NaN}$ & $\mathrm{NaN}$ & $\mathrm{NaN}$ & $\mathrm{NaN}$ & $\mathrm{NaN}$ & $\mathrm{NaN}$ & $\mathrm{NaN}$ & $\mathrm{NaN}$ & $\mathrm{NaN}$ & $\mathrm{NaN}$ & $\mathrm{NaN}$ & 0.45 & $\mathrm{NaN}$ & $\mathrm{NaN}$ & NaN & 0.10 & 0.41 & $\begin{array}{l}\text { NaN } \\
\mathrm{NaN}\end{array}$ & NaN & $\mathrm{NaN}$ & NaN \\
\hline ZNF580 & Q9UK33 & $\mathrm{NaN}$ & $\mathrm{NaN}$ & 2.87 & 0.63 & 1.17 & 1.34 & 1.37 & $\mathrm{NaN}$ & 0.96 & 1.29 & 1.18 & 1.13 & $\mathrm{NaN}$ & $\mathrm{NaN}$ & $\mathrm{NaN}$ & 0.18 & 2.47 & 0.68 & 5.07 & 0.15 & 3.22 & 0.55 & 1.86 & 2.04 & $\mathrm{NaN}$ & 0.52 \\
\hline ZNF598 & Q86UK7-2 & 1.10 & 1.44 & $\mathrm{NaN}$ & $\mathrm{NaN}$ & 3.62 & $\mathrm{NaN}$ & $\mathrm{NaN}$ & $\mathrm{NaN}$ & $\mathrm{NaN}$ & $\mathrm{NaN}$ & $\mathrm{NaN}$ & $\mathrm{NaN}$ & $\mathrm{NaN}$ & 1.24 & 0.55 & 0.12 & $\mathrm{NaN}$ & 1.19 & 1.03 & 0.65 & $\mathrm{NaN}$ & $\mathrm{NaN}$ & 1.00 & $\mathrm{NaN}$ & $\mathrm{NaN}$ & $\mathrm{NaN}$ \\
\hline ZNF629 & Q9UEG4 & 0.50 & 0.46 & $\mathrm{NaN}$ & $\mathrm{NaN}$ & $\mathrm{NaN}$ & $\mathrm{NaN}$ & 0.37 & $\mathrm{NaN}$ & 0.44 & $\mathrm{NaN}$ & 0.88 & 0.41 & $\mathrm{NaN}$ & 0.99 & 0.31 & $\mathrm{NaN}$ & 1.16 & 0.33 & 2.98 & 0.17 & 4.22 & 0.24 & $\mathrm{NaN}$ & NaN & $\mathrm{NaN}$ & $\mathrm{NaN}$ \\
\hline ZNF644 & Q9H582 & 0.41 & 0.47 & 1.92 & 1.27 & 1.12 & 2.01 & 1.28 & 1.38 & 1.35 & 1.86 & 1.68 & 1.43 & 2.46 & 1.46 & 0.55 & 0.20 & 0.65 & 2.98 & 13.72 & 0.16 & 1.65 & 1.29 & 2.56 & 1.72 & 1.87 & 0.58 \\
\hline ZNF687 & Q8N1G0 & $\mathrm{NaN}$ & $\mathrm{NaN}$ & 0.51 & 0.48 & 0.59 & 0.68 & $\mathrm{NaN}$ & $\mathrm{NaN}$ & 0.43 & 0.64 & 0.66 & 0.49 & 0.60 & $\mathrm{NaN}$ & 0.55 & 0.72 & 0.39 & 0.75 & 46.11 & 0.23 & 0.86 & 0.36 & 11.67 & 0.86 & $\mathrm{NaN}$ & $\mathrm{NaN}$ \\
\hline ZNF770 & A8K5X3 & $\mathrm{NaN}$ & $\mathrm{NaN}$ & $\mathrm{NaN}$ & $\mathrm{NaN}$ & $\mathrm{NaN}$ & $\mathrm{NaN}$ & $\mathrm{NaN}$ & $\mathrm{NaN}$ & 0.42 & $\mathrm{NaN}$ & 0.40 & 0.43 & $\mathrm{NaN}$ & $\mathrm{NaN}$ & $\mathrm{NaN}$ & $\mathrm{NaN}$ & $\mathrm{NaN}$ & $\mathrm{NaN}$ & 0.51 & 0.57 & $\mathrm{NaN}$ & $\mathrm{NaN}$ & $\mathrm{NaN}$ & NaN & $\mathrm{NaN}$ & $\mathrm{NaN}$ \\
\hline ZRANB2 & |095218-2 & 1.16 & 1.14 & 4.46 & 0.13 & $\mathrm{NaN}$ & $\mathrm{NaN}$ & 0.71 & 0.69 & 0.64 & 0.51 & $\mathrm{NaN}$ & $\mathrm{NaN}$ & $\mathrm{NaN}$ & $\mathrm{NaN}$ & 1.12 & 2.62 & 1.18 & 0.64 & 0.69 & 1.20 & 0.50 & 0.71 & $\mathrm{NaN}$ & 0.88 & 0.47 & 0.65 \\
\hline ZSCAN20 & P17040-3 & $\mathrm{NaN}$ & 1.13 & 1.05 & 0.71 & 0.80 & 1.08 & $\mathrm{NaN}$ & $\mathrm{NaN}$ & $\mathrm{NaN}$ & 1.26 & 1.06 & 1.06 & $\mathrm{NaN}$ & $\mathrm{NaN}$ & 0.29 & $\mathrm{NaN}$ & 1.00 & 1.08 & 6.98 & 0.17 & 1.26 & 0.84 & 0.44 & 2.04 & 15.72 & $\mathrm{NaN}$ \\
\hline
\end{tabular}




\section{Curriculum Vitae}

\section{Personal data}

Name:

Date of birth:

Place of birth:

Nationality:

\section{Education}

$04.2012-06.2016$

10.2011

$10.2005-10.2011$

06.2003

$08.1996-06.2003$

\section{Stipends}

$02.2012-06.2016$
Zimmermann, Nadin

20th January 1984

Berlin, Germany

German
GGNB Biomolecules: Structure - Function - Dynamics, Göttingen

PhD thesis in Chromatin Biochemistry Group,

Max Planck Institute for Biophysical Chemistry, Göttingen

"Systematic analysis of heterochromatin modification readout"

Diploma in Biology

Martin-Luther-University, Halle-Wittenberg

Diploma thesis in Department of Developmental Genetics, Martin-Luther-University, Halle-Wittenberg

"Reinigung differentieller dLSD1 Proteinkomplexe und Charakterisierung ihrer Funktion beim Silencing transponibler Elemente in der Keimbahn und im Soma von Drosophila melanogaster"

Abitur

High School “Jules-Verne-Oberschule”, Berlin, Germany 